\title{
Weak Hydrogen Bonds to Molecular Nitrogen and Oxygen as Experimental Benchmarks for Quantum Chemistry
}

\author{
Dissertation \\ to acquire the doctoral degree in mathematics and natural science \\ „Doctor rerum naturalium“ \\ at the Georg-August-Universität Göttingen \\ in the doctoral degree programme chemistry \\ at the Georg-August University School of Science (GAUSS)
}

Submitted by

Sönke Oswald

from Henstedt-Ulzburg

Göttingen, 2019 
Thesis Committee:

Prof. Dr. Martin A. Suhm, Institut für Physikalische Chemie

Prof. Dr. Alec M. Wodtke, Institut für Physikalische Chemie

Members of the Examination Board:

Referee: Prof. Dr. Martin A. Suhm

Co-referee: Prof. Dr. Alec M. Wodtke

Other members of the Examination Board:

Prof. Dr. Ricardo A. Mata, Institut für Physikalische Chemie

Prof. Dr. Jörg Behler, Institut für Physikalische Chemie

Dr. Sebastian Kruss, Institut für Physikalische Chemie

Prof. Dr. Dietmar Stalke, Institut für Anorganische Chemie

Date of the oral examination: 28.02.2019 


\section{Acknowledgements}

At the end of my PhD project, I wish to express my gratitude to many people. First and foremost, I would like to thank my family and friends, especially my parents, for their continued support over all these years. Martin Suhm provided excellent supervision already during my undergraduate studies and continued to do so for my entire time as a member of his research group. I am deeply grateful for his support and guidance throughout this entire project. I would also like to thank Alec Wodtke for the cosupervision.

In addition, I thank all members of the Suhm research group, past and current, for the pleasant working atmosphere, lots of cake, thrilling table football matches, evenings dedicated to board games and helpful discussions. Especially Katharina Meyer, Anja Poblotzki, Hannes Gottschalk and Thomas Forsting have accompanied and supported me through my entire time in the group and Petra Lawecki helped me through countless troubles with administrative tasks such as writing correct travel requests or ordering chemicals. Highly motivated and talented student co-workers included Mareike Wallrabe, Enno Meyer and Elsa Lang during their respective bachelor's theses as well as Niels Karschin and Fabian Hecker during research internships. I thank all of them for their hard work and dedication which resulted in valuable contributions to this work.

A lot of the work performed in the group would not be possible without the institute staff and the workshops. Especially the valuable contributions from the mechanical and electronics departments supervised by Volker Meyer, Reinhard Hildebrandt, Mike Zippert and Andreas Knorr are gratefully acknowledged as well as support from Markus Hold, Clemens Heymann, Michael Schlote and Josef Schikowski.

Successful collaborations included the groups of Yunjie Xu and Wolfgang Jäger at the University of Alberta in Edmonton, Canada as well as Stéphane Coussan at AixMarseille University in Marseille, France. I thank all of them for hosting me during my guest stays as well as their respective groups for welcoming me. Especially Matthias Heger and Nathan Seifert turned my lengthy trip to Canada into a very enjoyable experience. I thank the FCI (Fonds der Chemischen Industrie) for funding during most of my time as a doctoral candidate and especially for financial support during those visits to collaborating research groups.

Finally, I would like to thank Anja Poblotzki, Katharina Meyer, Hannes Gottschalk, Nils Lüttschwager and Matthias Heger for proofreading parts of this work. 



\section{Contents}

1. Experimental and Theoretical Methods 3

1.1. Vibrational Spectroscopy . . . . . . . . . . . . . . . . . . . . 3

1.1.1. FTIR Spectroscopy: filet-Jet . . . . . . . . . . . . . 3

1.1.2. Raman Spectroscopy: curry-Jet . . . . . . . . . . . . . 5

1.1.3. FTIR Spectroscopy in Cryomatrices . . . . . . . . . . . . . 6

1.2. Rotational spectroscopy . . . . . . . . . . . . . . . . 8

1.2.1. Chirped Pulse Fourier Transform Microwave Spectroscopy . . . . 10

1.2.2. Cavity-enhanced Fourier Transform Microwave Spectroscopy . . . 10

1.3. Theoretical Methods . . . . . . . . . . . . . . . . . . . 11

1.3.1. Benchmarking Theoretical Predictions . . . . . . . . . . . . 12

2. Carboxylic Acids 15

2.1. Introduction . . . . . . . . . . . . . . . . . . . . 15

2.2. Quantum Chemical Calculations and Nomenclature . . . . . . . . . 17

2.3. FTIR Spectra . . . . . . . . . . . . . . . . . . . 18

2.3.1. Formic Acid . . . . . . . . . . . . . . . . . . . 19

2.3.2. Formic Acid Nitrogen . . . . . . . . . . . . . . . . . . . 20

2.3.3. Formic Acid Oxygen . . . . . . . . . . . . . . . . . 25

2.3.4. Acetic Acid . . . . . . . . . . . . . . . . 26

2.3.5. Acetic Acid Nitrogen . . . . . . . . . . . . . . . . . . . 28

2.3.6. Acetic Acid Oxygen . . . . . . . . . . . . . . . . . . . . . 30

2.4. Benchmarking Quantum Chemical Calculations . . . . . . . . . 31

2.4.1. Binding Energy of $(\mathrm{FF})$. . . . . . . . . . . . . . . . 34

2.4.2. Harmonic Vibrational Wavenumbers of $\mathrm{F}$ and $\mathrm{FN}_{\mathrm{H}}$. . . . . . . . 34

2.4.3. Harmonic Vibrational Wavenumbers of $\mathrm{A}$ and $\mathrm{AN}_{\mathrm{H}} \ldots . . . . .38$

2.4.4. Anharmonic Vibrational Wavenumbers of $\mathrm{F}$ and $\mathrm{FN}_{\mathrm{H}}$. . . . . . . 40

2.4.5. Anharmonic Vibrational Wavenumbers of $\mathrm{A}$ and $\mathrm{AN}_{\mathrm{H}}$. . . . . . . 40

2.4.6. Theory Performance Evaluation . . . . . . . . . . . . . . . . 41

2.5. Summary . . . . . . . . . . . . . . . . . . . . . . 42

3. 1,1,1,3,3,3-Hexafluoro-2-propanol 45

3.1. Introduction . . . . . . . . . . . . . . . . . . . . . . . 45

3.2. Nomenclature and Experimental Details . . . . . . . . . . . . . . 46

3.3. Monomer . . . . . . . . . . . . . . . . . . . 46

3.4. Binary Complex with Nitrogen . . . . . . . . . . . . . . 48

3.5. Homodimer . . . . . . . . . . . . . . . . . . . 51

3.6. Homodimer with Nitrogen . . . . . . . . . . . . . . . 55 
3.7. Homotrimer . . . . . . . . . . . . . . . . . . . . 55

3.8. Oxygen Aggregates . . . . . . . . . . . . . . . . . . . . 61

3.9. Benchmarking Quantum Chemical Calculations . . . . . . . . . . 62

3.9.1. Rotational Constants . . . . . . . . . . . . . . . 62

3.9.2. Vibrational Band Centre Positions . . . . . . . . . . . . 65

3.9.3. Relative Conformational Energies . . . . . . . . . . . . . . 69

3.9.4. Theory Performance Evaluation . . . . . . . . . . . . . . . 72

3.10. Summary . . . . . . . . . . . . . . . . . . . . 73

4. Aliphatic Alcohols $\quad \mathbf{7 5}$

4.1. Introduction . . . . . . . . . . . . . . . . . . . . 75

4.2. Quantum Chemical Calculations and Nomenclature . . . . . . . . . 76

4.3. FTIR Spectra with Nitrogen . . . . . . . . . . . . . . . . . 78

4.3.1. Methanol . . . . . . . . . . . . . . . 81

4.3.2. tert-Butyl Alcohol . . . . . . . . . . . . . . . . . . . 81

4.3.3. Ethanol . . . . . . . . . . . . . . . . . 82

4.3.4. Alkylation Trends . . . . . . . . . . . . . . . . . . . . . . 87

4.4. FTIR Spectra with Oxygen . . . . . . . . . . . . . . . 90

4.4.1. Methanol .................... . . . 90

4.4.2. Ethanol . . . . . . . . . . . . . . . . . . . . . . . . . . . . . . . . . . .

4.5. Raman Spectra . . . . . . . . . . . . . . . . . . . . . 92

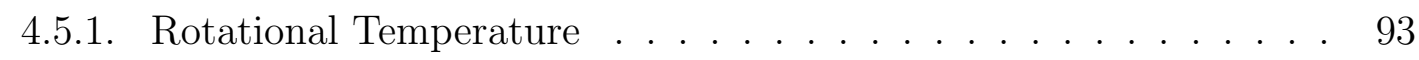

4.5.2. Conformational Temperature . . . . . . . . . . . . . . . . 95

4.6. Summary . . . . . . . . . . . . . . . . . . . . . . 97

5. Pyrrole 99

5.1. Introduction . . . . . . . . . . . . . . . . . . . . . . 99

5.2. Quantum Chemical Calculations and Nomenclature . . . . . . . . . . . 100

5.3. FTIR Spectra . . . . . . . . . . . . . . . . . . . . . . . . . . . . . . . . . . . . . . . .

5.3.1. Neon, Argon and Nitrogen Matrices . . . . . . . . . . . . . . 103

5.3.2. Nitrogen Added to Neon and Argon Matrices . . . . . . . . . . . 106

5.3.3. Supersonic Expansions . . . . . . . . . . . . . . . . . . . 109

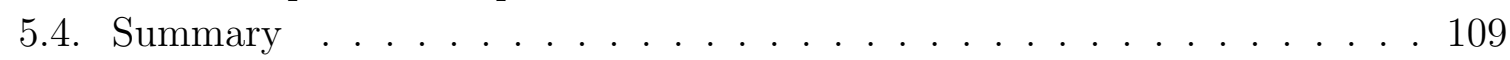

$\begin{array}{ll}\text { 6. Chloroform } & 113\end{array}$

6.1. Introduction . . . . . . . . . . . . . . . . . 113

6.2. Quantum Chemical Calculations and Nomenclature . . . . . . . . . . 114

6.3. FTIR Spectra . . . . . . . . . . . . . . . . . . 116

6.3.1. Neon, Argon and Nitrogen Matrices . . . . . . . . . . . . . . . 116

6.3.2. Nitrogen Added to Neon and Argon Matrices . . . . . . . . . . . 121

6.3.3. Supersonic Expansion . . . . . . . . . . . . . . . . 126

6.4. Summary . . . . . . . . . . . . . . . . . 127 
7.1. Introduction . . . . . . . . . . . . . . . . . . . . . . . . 129

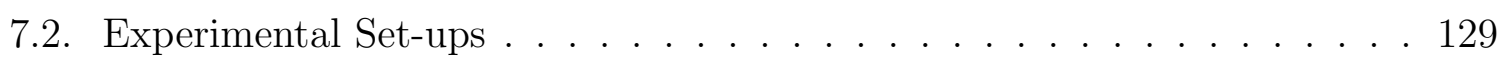

7.2.1. Power Enhancement Evaluation Set-ups . . . . . . . . . . . . . . 129

7.2.2. Raman Spectroscopy Set-up . . . . . . . . . . . . . . . . 131

7.3. Results and Discussion . . . . . . . . . . . . . . . . . . . 133

7.3.1. Laser Diode Characterisation . . . . . . . . . . . . . . . . 133

7.3.2. Power Enhancement Evaluations . . . . . . . . . . . . . . . 133

7.3.3. Raman Spectroscopy . . . . . . . . . . . . . . . . . . 136

7.4. Summary . . . . . . . . . . . . . . . . . . 138

$\begin{array}{lr}\text { 8. Conclusions and Outlook } & 139\end{array}$

$\begin{array}{ll}\text { A. Appendix } & \mathbf{1 7 5}\end{array}$

A.1. Experimental and Theoretical Methods . . . . . . . . . . . . . . . 175

A.2. Carboxylic Acids . . . . . . . . . . . . . . . . . . . . . . . . . . 179

A.3. 1,1,1,3,3,3-Hexafluoro-2-propanol . . . . . . . . . . . . . . 185

A.4. Aliphatic Alcohols . . . . . . . . . . . . . . . . . . . . 220

A.5. Pyrrole . . . . . . . . . . . . . . . . . . . 233

A.6. Chloroform . . . . . . . . . . . . . . . . . . 240

A.7. Cavity-enhanced Raman Spectroscopy . . . . . . . . . . . . . . 254

A.8. Conclusions and Outlook . . . . . . . . . . . . . . . . . 255 



\section{Contents}

\section{Introduction}

Progressing computational capabilities in both hardware ${ }^{1}$ and software ${ }^{2}$ enable the execution of electronic structure calculations with increasing accuracy ${ }^{3}$ for molecular systems of growing size. ${ }^{4}$ The Schrödinger equation with relativistic corrections can be considered as exact for most currently relevant applications ${ }^{5}$ with few exceptions such as parity violation. ${ }^{6}$ Hence, for certain observables like the dissociation energy of the hydrogen molecule theoretical predictions ${ }^{7}$ can in principle reach accuracy superior to experiment. ${ }^{8-10}$ Since analytical solutions of the Schrödinger equation are only available in the case of one electron and exact numerical solutions for multi-electron systems are only feasible for a very limited range of system sizes, different approximations and thus compromises between accuracy and computational effort are necessary. Hence, method hierarchies can be useful for choosing a suitable electronic structure method for a particular application or performance evaluations of newly developed methods. ${ }^{11}$ While establishing a ranking can be relatively simple for some wave-function based methods such as coupled cluster $(\mathrm{CC})^{12}$ which gain accuracy by including an increasing number of terms, other approaches like Møller-Plesset perturbation theory (MP) do not necessarily show the same convergence. ${ }^{13}$ For the vast variety of different density functional theory (DFT) methods, the Jacob's ladder ${ }^{14}$ provides a coarse ordering, although the performance of different functionals does not necessarily match the provided sequence. ${ }^{15}$

To examine the applicability of methods to a broad range of molecular systems and minimise the possibility of good method assessments due to fortuitous error cancellation, large benchmarking test data sets containing a variety of different molecules and their selected properties such as S22 ${ }^{16-18}$ or GMTKN30 $0^{19-21}$ are needed and typically used as reference values for performance evaluation. A drawback of these data sets is the small fraction of experimental reference data. ${ }^{11,22}$ The generally accepted $\operatorname{CCSD}(\mathrm{T})$ 'gold standard' 23,24 invites a variety of benchmarking studies using exclusively theoretical reference data, ${ }^{25,26}$ which can be helpful for comparison of different method implementations in varying quantum chemistry program packages. ${ }^{27}$ Ultimately however, testing theoretical predictions against experiment is indispensable, ${ }^{28}$ although this introduces a variety of effects aggravating accurate theoretical modelling such as large system sizes, thermal motion or interactions of the sample molecules with environments like solvents or cryogenic matrices. In some cases relatively easily predictable quantities like harmonic vibrational spectra ${ }^{29}$ are difficult to access experimentally, ${ }^{30}$ while in other cases readily available experimental data ${ }^{31,32}$ are simply ignored. ${ }^{33}$

Bridging the gap between experiment and theory requires efforts from both sides. On the experimental side, reducing large systems down to the essential features, ${ }^{34}$ or minimising environmental and thermal effects by exploiting cold, isolated gas phase conditions provided by supersonic expansions ${ }^{35,36}$ or molecular beams ${ }^{37}$ facilitates comparison 


\section{Contents}

between experiment and theory. On the theory side, one of the many remaining tasks in meeting experiment is accurate modelling of non-removable experimental phenomena such as zero-point vibrational motion ${ }^{38,39}$ and resulting structural relaxation. ${ }^{40,41}$

An aim of this work is the addition of experimental observables suitable for benchmarking purposes involving small multi-molecular complexes weakly bound by noncovalent interactions. An accurate description of the subtle interplay between dispersion forces, ${ }^{42}$ hydrogen bonding ${ }^{43}$ and various electric multipole interactions ${ }^{44}$ remains a difficult task for quantum chemistry. Here, the electric quadrupole moments of molecular nitrogen ${ }^{45}$ and oxygen ${ }^{46}$ serve as acceptors for weak hydrogen bonds from dipolar $\mathrm{OH}, \mathrm{NH}$ and $\mathrm{CH}$ donors. Experimental quantities used for benchmarking purposes include vibrational band centre positions, hydrogen bond induced spectral shifts, ${ }^{47}$ structural information obtained with microwave spectroscopy and relative conformational energies. ${ }^{48-54}$

Additionally, nitrogen is a widely used host molecule for inert cryogenic matrices, ${ }^{55-58}$ which induce characteristic frequency shifts to vibrational band positions of deposited guest molecules and their aggregates compared to corresponding counterparts in isolated gas phase conditions. ${ }^{59-62}$ Stepwise nitrogen decoration of hydrogen bond donor systems in the gas phase is employed for incremental reproduction of the gas-to-matrix shift. While the bulk matrix environment may be currently too large and complex for accurate theoretical modelling, neural network potentials ${ }^{63-65}$ enable future predictions for such large-scale systems, which can also be tested and benchmarked against the experimental data provided in this work.

Due to their high abundance, ${ }^{66}$ nitrogen and oxygen have an impact on important atmospherical processes such as particle formation ${ }^{67}$ and growth. ${ }^{68}$ Collisions with inorganic or organic components in cold stratospheric regions possibly results in formation of transient complexes and their spectral features have a possible impact on the planetary radiation balance, at least in case of water. ${ }^{69-83}$ Spectral shifts and infrared activation of the NN stretching vibration ${ }^{84-86}$ can be regarded as a small first step towards chemical nitrogen activation. ${ }^{87,88}$ 


\section{Experimental and Theoretical Methods}

The weakly bound molecular complexes investigated in this work are only stable in isolated, low temperature environments. These were provided by supersonic expansions ${ }^{35,36}$ in an inert carrier gas or by isolation in noble gas or nitrogen cryomatrices. ${ }^{55,57,89}$ The resulting vibrational and rotational cooling also simplifies spectra and facilitates their analysis. ${ }^{90}$ Additionally, all quantum chemical calculations carried out throughout this work assume vacuum-isolated molecules or molecular aggregates at a temperature of $0 \mathrm{~K}$, therefore the employed experimental methods, especially supersonic expansions, support comparability of experimental findings and theoretical predictions.

FTIR and Raman spectra in supersonic expansions were recorded in Göttingen at the Institute of Physical Chemistry. Complementary microwave spectra were obtained with set-ups at the University of Alberta, Canada, and for FTIR spectra of molecules trapped in cryomatrices the facilities at Aix-Marseille University, France, were utilised.

\subsection{Vibrational Spectroscopy}

Vibrational spectroscopy is a powerful and sensitive tool to elucidate the structure and dynamics of hydrogen bonded aggregates. ${ }^{91}$ Formation of XH $\cdots$ Y hydrogen bonds weakens the covalent XH bond and causes the most notable spectral signature of hydrogen bonding, a characteristic shift of the corresponding XH stretching vibration. ${ }^{47}$ In most cases a downshift to lower wavenumbers occurs, ${ }^{31,92}$ while upshifts have been observed in few cases as well. ${ }^{93}$ The spectral shifts are accompanied by an increased infrared band strength of the fundamental vibrational transitions due to a steeper dipole moment function along the XH stretching coordinate. ${ }^{47}$ Formation of cooperative hydrogen bond networks ${ }^{94}$ leads to a more pronounced manifestation of both effects in larger aggregates. ${ }^{95}$ Especially in investigations of symmetric molecules or clusters, infrared and Raman spectroscopy complement each other well because of their contrasting selection rules. ${ }^{96-98}$

\subsubsection{FTIR Spectroscopy: filet-Jet}

For FTIR spectra of molecules in supersonic expansions the filet-jet set-up ${ }^{99-101}$ (Figure 1.1) was used. Gas mixtures of analyte molecules in a large excess of a carrier gas, typically helium, were prepared by guiding a stream of helium through thermostatted glass saturators containing the sample substance in liquid or solid state. The vapour 


\section{Experimental and Theoretical Methods}

pressure and therefore concentration of this substance in the mixture could be controlled by the temperature of the glass vessel. A second sample preparation method, preferable for gaseous samples and thus predominantly utilised throughout this work, was the use of previously prepared gas mixtures with fixed composition in compressed gas bottles.

Through three separate inlets controllable via solenoid valves the gas mixtures were introduced into a $67 \mathrm{~L}$ Teflon-coated reservoir and stored at stagnation pressures of 0.40 to 0.75 bar. Another set of six high-throughput solenoid valves connected the reservoir to a $330 \mathrm{~cm}^{3}$ pre-expansion chamber. Afterwards, the gas entered the expansion chamber through a $(600 \times 0.2) \mathrm{mm}^{2}$ slit nozzle, which is referenced in the acronym of the setup (fine but lengthy). A sufficiently low background pressure of 0.1 mbar during the expansion was ensured by pumping time intervals of 25-120 s between pulses and an attached $23 \mathrm{~m}^{3}$ buffer volume, which was continuously evacuated by two series of roots and rotary vane pumps to minimise interferences by recompression shock waves due to background gas. ${ }^{35,102}$ The smaller series of pumps (Pfeiffer Vacuum, WKP 500 A, WKP 250 A, UniDry 050-3) provided a pumping speed of $500 \mathrm{~m}^{3} \mathrm{~h}^{-1}$, while the larger series (Pfeiffer Vacuum, WKP 2000 AD, WKP 250 A, UniDry 050) added $2000 \mathrm{~m}^{3} \mathrm{~h}^{-1}$.

The resulting, pulsed supersonic expansion was probed perpendicular to its propagation direction by an infrared beam from a Bruker IFS 66v/S FTIR spectrometer operated by the Bruker OPUS 7.0 software package at typically $2 \mathrm{~cm}^{-1}$ resolution. The beam was mildly focused to a diameter of $1-2 \mathrm{~cm}$ by a lens $(f=500 \mathrm{~mm})$ before passing the expansion and after an optical path of $776 \mathrm{~mm}$ collimated by a second lens $(f=250 \mathrm{~mm})$ before entering the detector chamber. Both the spectrometer and the detector chamber were continuously evacuated by a scroll pump (Edwards, XDS35i, $35 \mathrm{~m}^{3} \mathrm{~h}^{-1}$ ). The gaps between lenses and windows of the spectrometer and the detector chamber, respectively, were flushed with dried air at all times.

Depending on the desired spectral window, the spectrometer could be equipped with various light sources, optics, optical filters and detectors, allowing for a total spectral range from 8000 to $200 \mathrm{~cm}^{-1}$. For near-infrared measurements $7 \mathrm{~mm}^{2} \mathrm{InGaAs}$ and $3 \mathrm{~mm}^{2}$ InSb detector elements were available. Mid-infrared measurements were performed with a $4 \mathrm{~mm}^{2} \mathrm{HgCdTe}$ element, while a Si-bolometer could be employed for detections in the far-infrared spectral region. The windows, lenses and beam splitters could be varied from $\mathrm{CaF}_{2}$ for the near-infrared region to $\mathrm{KBr}$ in the mid-infrared. The far-infrared setup includes CsI windows and lenses as well as a Mylar beam splitter. The light source could be switched from a $\mathrm{SiC}$ globar to a $150 \mathrm{~W}$ tungsten lamp, which provided a higher intensity in the near-infrared region.

FTIR scans were synchronized to the gas pulses. Before the expansion, 20 background scans were recorded, followed by two pre-scans and the sample scan. Afterwards, two post-scans were added for diagnostic purposes. Gas pulse duration could be varied, but was fixed at $147 \mathrm{~ms}$ for all measurements included in this work. Typically, spectra were averaged from 300 up to 1000 gas pulses. Specific parameters for the measurements are listed in Table A.1 in appendix A.1.

Unlike some kinds of action spectroscopy, ${ }^{103,104}$ filet-jet FTIR spectroscopy does not provide size selectivity. Therefore, compositions and sizes of the investigated aggregates were determined by analysing changes in band intensities induced by variation of ex- 


\section{Experimental and Theoretical Methods}

perimental conditions such as analyte concentrations in the gas mixtures or stagnation pressures.

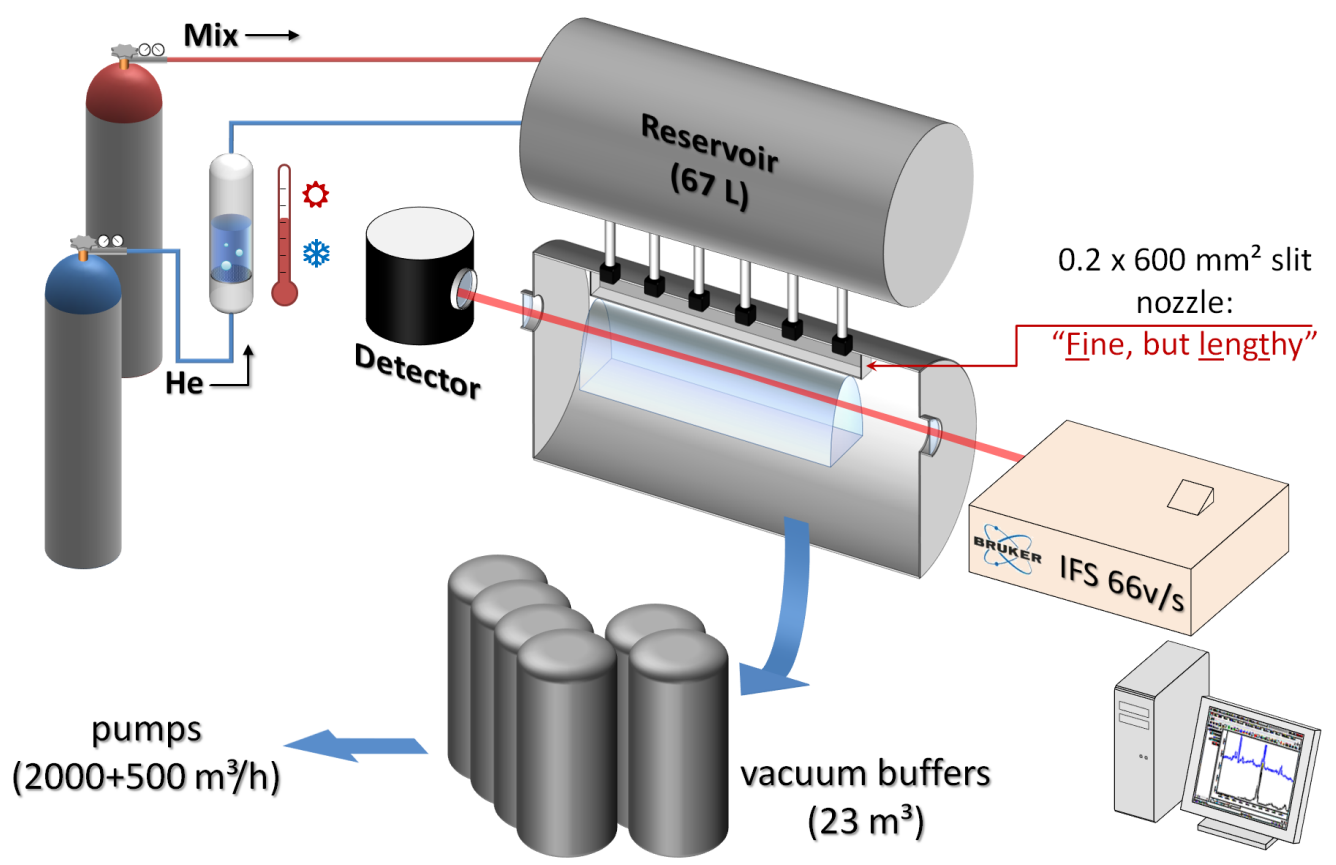

Figure 1.1.: Scheme of the filet-jet set-up (reprinted from reference 99 licensed under CC BY-NC-ND 4.0 with permission from Dr. M. Heger). The infrared beam is depicted in red for better visibility.

\subsubsection{Raman Spectroscopy: curry-Jet}

Complementary Raman spectra were obtained at the curry-jet set-up ${ }^{49,105,106}$ depicted in Figure 1.2. The available methods of sample preparation were very similar to the previously described filet-jet set-up with the addition of a heatable saturator and nozzle. The reservoir volume was smaller $(4.7 \mathrm{~L})$ and a continuous expansion through a $(0.15 \times 4) \mathrm{mm}^{2}$ slit nozzle occurred into the jet chamber which was evacuated by two roots pumps (Pfeiffer Vacuum, Okta 500 AM, WKP 250 AM) and a rotary vane pump (Dr. Ing. K. Busch GmbH, UNO $101 \mathrm{~S}$ ) at a maximum speed of $560 \mathrm{~m}^{3} \mathrm{~h}^{-1}$ (typically $500 \mathrm{~m}^{3} \mathrm{~h}^{-1}$ ), $270 \mathrm{~m}^{3} \mathrm{~h}^{-1}$ (typically $250 \mathrm{~m}^{3} \mathrm{~h}^{-1}$ ) and $100 \mathrm{~m}^{3} \mathrm{~h}^{-1}$, respectively, resulting in background pressures of approximately 1 mbar at reservoir pressures of 750 mbar. The expansion was crossed perpendicularly by the beam of a continuous-wave $532 \mathrm{~nm}$ laser (Spectra Physics Millenia eV, $25 \mathrm{~W}$ ). The resulting scattered light was collected at a $90^{\circ}$ angle by a collimating camera lens $(d=50 \mathrm{~mm}, f / 1.2)$, focused on the entrance slit of a monochromator (McPherson Inc. Model 2501, $1 \mathrm{~m}$ focal length, $f / 8.7$, ruled grating with 1200 grooves $\left.\mathrm{mm}^{-1}\right)$ by a convex lens $(d=50 \mathrm{~mm}, f / 7)$ while passing through a Raman edge filter (OD $6.0 @ 532.0 \mathrm{~nm}, T>90 \%$ @ $\lambda>533.7 \mathrm{~nm}$ ). Detection by a nitrogen 


\section{Experimental and Theoretical Methods}

cooled, back-illuminated CCD-camera (Princeton Instruments, PyLoN400B, $1340 \times 400$ pixel of size $20 \mu \mathrm{m} \times 20 \mu \mathrm{m})$ results in a spectral resolution of approximately $1 \mathrm{~cm}^{-1}$, depending on the investigated spectral range. Disruptive, randomly distributed spikes in a given scan due to cosmic background radiation were eliminated by comparing to two independent scans and substituting for the average value of all other scans. ${ }^{107,108}$ Wavelength calibration was achieved with vacuum atomic emission lines of neon (L.O.T.-Oriel, LSP032) and krypton (L.O.T.-Oriel, LSP031) light sources.

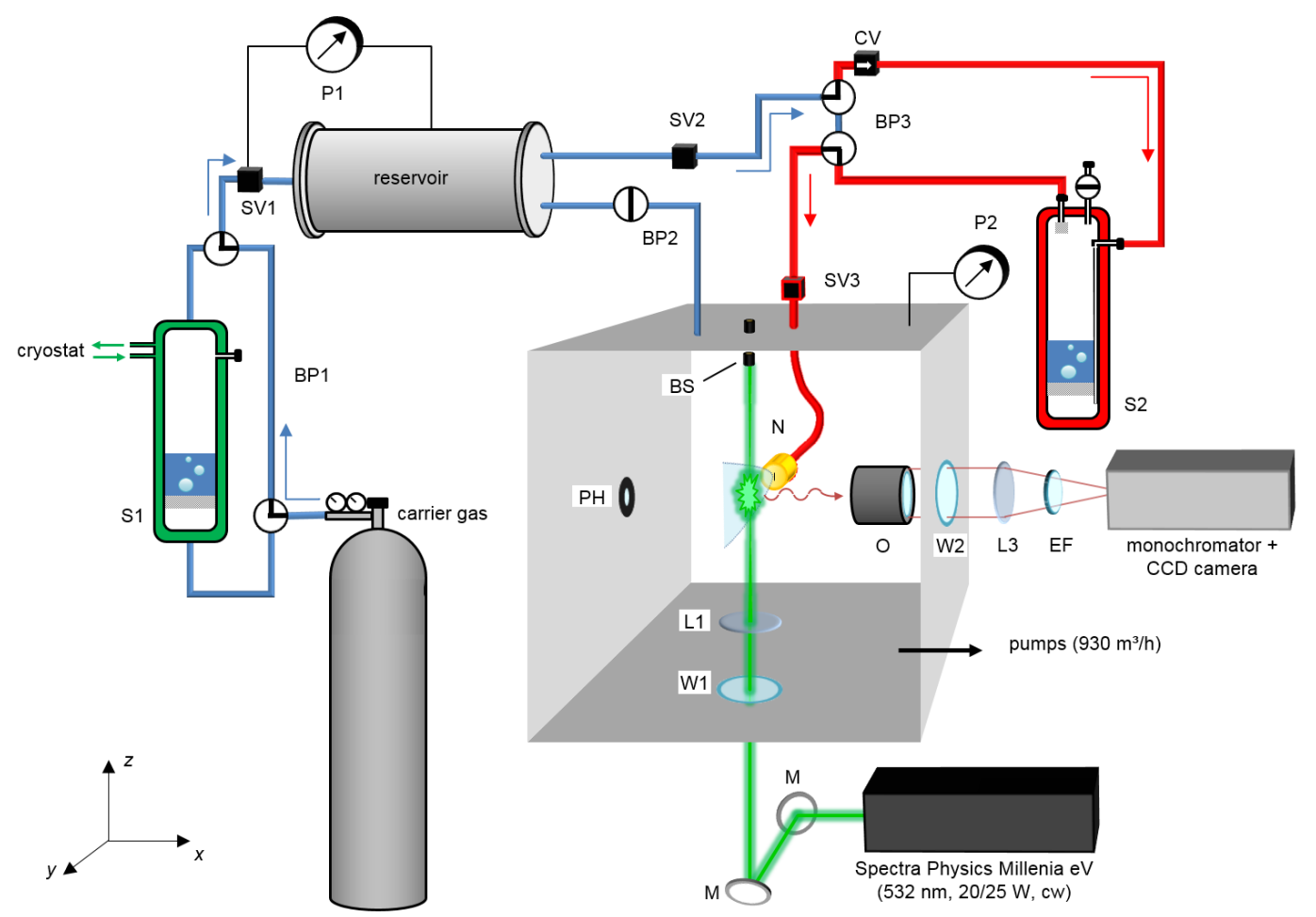

Figure 1.2.: Scheme of the curry-jet set-up (reprinted from reference 109 licensed under CC BY-NC-ND 4.0 with permission from T. Forsting). Abbreviations: S: saturator (S1:cooled, S2: heated), P: pressure gauge, SV: solenoid valves, CV: control valve, BP: bypass vacuum lines, M: mirror, W: windows, L: lens, PH: pinhole, BS: beamstop, N: nozzle, O: camera lens, EF: edge filter.

\subsubsection{FTIR Spectroscopy in Cryomatrices}

Cryomatrices are mostly amorphous solids of chemically inert molecules such as noble gases or nitrogen, which are prepared at very low temperatures, typically a few Kelvin and can be doped with analyte molecules of interest. ${ }^{55,57,89}$ Very efficient vibrational cooling is achieved for embedded molecules and molecular rotations are mostly suppressed ${ }^{110}$ with the exception of some very small molecules. ${ }^{111}$ Depending on the matrix host, optical transparency over a wide frequency range from far-infrared to vacuumultraviolet radiation ${ }^{112}$ allows for the combination of matrix isolation techniques with 


\section{Experimental and Theoretical Methods}

various spectroscopic methods. ${ }^{113-116}$ The solid state properties of the different matrix hosts used in this work, neon, argon and nitrogen, are listed in Table A.2 in appendix A.1.

Originally, the matrix isolation technique was employed for the stabilisation and subsequent investigation of unstable or reactive species like radicals. ${ }^{55}$ Since isolation in cryomatrices also stabilises weakly bound intermolecular complexes, it has been widely used in their investigation by means of vibrational spectroscopy. ${ }^{56,58-60,62,117-123}$ Matrix hosts may be chemically inert, but weak intermolecular interactions between host and analyte molecules are still present and can lead to significant shifts of vibrational bands compared to measurements in gas phase or supersonic expansions. ${ }^{112}$

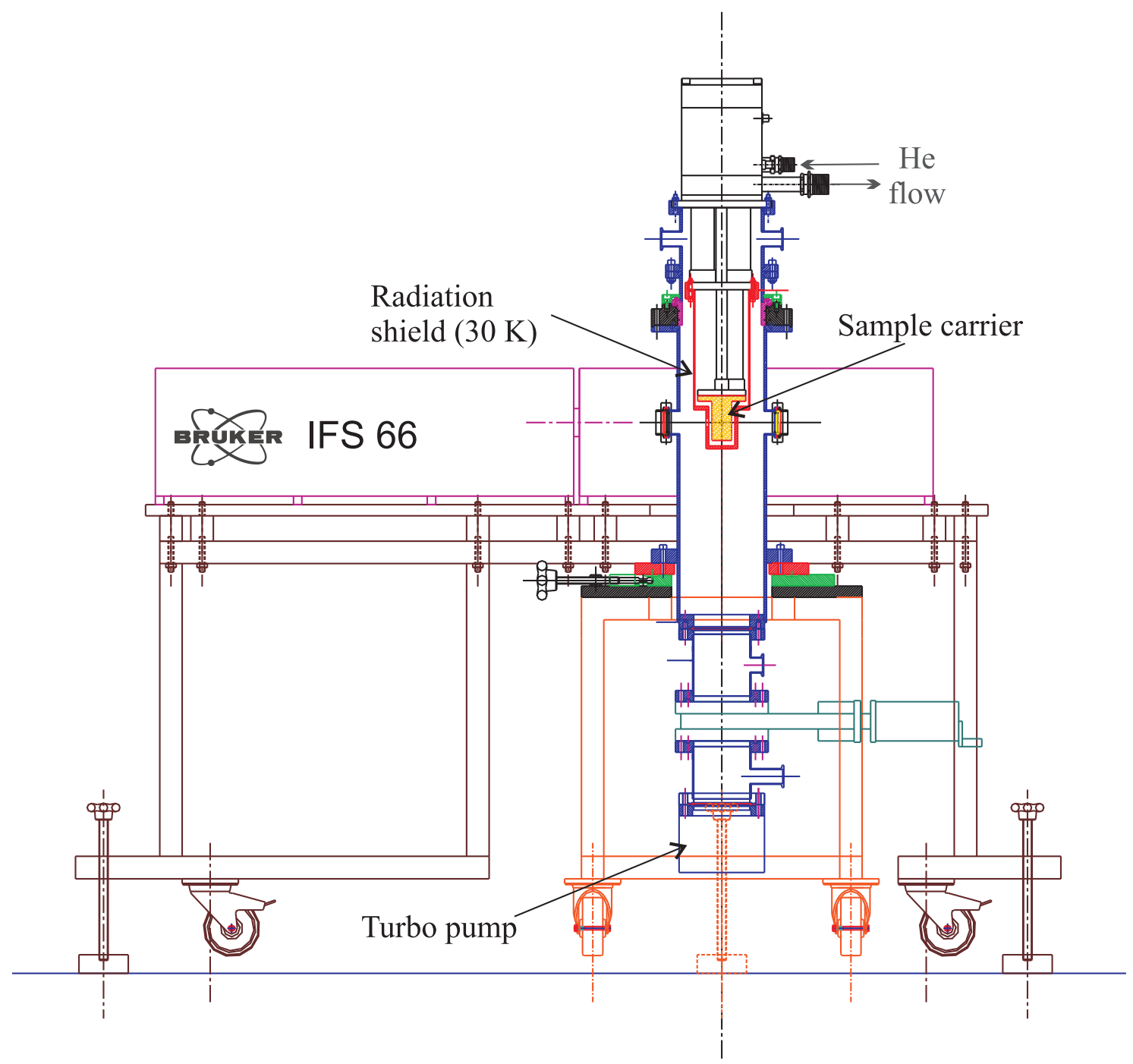

Figure 1.3.: Technical drawing of the set-up for matrix isolation FTIR spectroscopy at Aix-Marseille university (reprinted from reference 124 with permission from Dr. S. Coussan). ${ }^{125}$ 


\section{Experimental and Theoretical Methods}

The set-up used for FTIR spectroscopy in cryomatrices throughout this work designed by Dr. Stéphane Coussan at Aix-Marseille university is depicted in Figure 1.3 and a detailed description can be found in reference 125. Matrices were deposited on a goldcoated copper sample carrier $\left((34.6 \times 80 \times 34.6) \mathrm{mm}^{3}\right)$ cooled to temperatures as low as $4.3 \mathrm{~K}$ by an attached helium flow cryostat (Cryomech PT 405). Thermal conductivity was increased by an indium foil between cryostat and sample carrier. Controlled heating (Lakeshore Model 336) with a $50 \Omega$ resistor could be performed as well, while temperatures of the heat exchanger and sample carrier were monitored with silicon diodes (Lake Shore Cryotronics, DT-470-SD-13). For protection from thermal background radiation, cryostat and sample carrier were enclosed by a chrome-plated brass radiation shield, which was kept at a temperature of $30 \mathrm{~K}$. A small hole $(d=10 \mathrm{~mm})$ in the radiation shield served as inlet for the gas mixtures during matrix depositions. This assembly was placed in a vacuum chamber which was continuously evacuated by a turbomolecular pump (Alcatel, APT 100, $150 \mathrm{~L} \mathrm{~s}^{-1}$ ) and a rotary vane pump (Alcatel, Pascal 2005 $\mathrm{SD}, 6.5 \mathrm{~m}^{3} \mathrm{~h}^{-1}$ ) to ensure a pressure of $10^{-7} \mathrm{mbar}$. Gas mixtures for deposition were prepared in a $1 \mathrm{~L}$ glass flask attached to a stainless-steel mixing line. FTIR spectra from $5000-600 \mathrm{~cm}^{-1}$ at a resolution of $0.12 \mathrm{~cm}^{-1}$ were recorded with a Bruker IFS 66/S spectrometer equipped with a $\mathrm{SiC}$ globar radiation source, a $\mathrm{KBr}$ beam splitter, $\mathrm{KBr}$ windows and a $1 \mathrm{~mm}^{2} \mathrm{HgCdTe}$ detector.

\subsection{Rotational spectroscopy}

Rotational spectroscopy in combination with predictions from quantum chemical calculations is a very effective method for the determination of gas phase molecular structures, ${ }^{126}$ although it is mostly limited to aggregates with a permanent dipole moment, unless one is induced by centrifugal distortion in highly excited rotational states. ${ }^{127}$ The determined ground state rotational constants are directly linked to the moments of inertia for rotations around the principal axes of the molecule and therefore the molecular geometry. ${ }^{128}$ Even discrimination between the two enantiomers of a chiral compound is possible with nonlinear resonant phase-sensitive microwave spectroscopy. ${ }^{129}$

In cavity-enhanced Fourier transform microwave spectroscopy, ${ }^{130,131}$ a single frequency microwave excitation pulse resonant within a linear Fabry-Pérot cavity and with a molecular transition frequency causes coherent rotation of the ensemble of sample molecules introduced into the cavity by a supersonic expansion. This rotation results in molecular emission of the resonance frequency, which decays as the coherence of rotation is lost over time. High microwave radiation powers used for the excitation pulse could potentially damage sensitive elements of the detection circuit, hence recording of the free induction decay is only possible after ending the excitation pulse and its duration should be short relative to the relaxation time, typically a few microseconds. Finally, the frequencydependent spectrum is obtained by a Fourier transformation of the time-dependent free induction decay signal.

In chirped pulse Fourier transform microwave spectroscopy ${ }^{132,133}$ however, a broadband excitation pulse causes a simultaneous polarisation of all rotational transitions 


\section{Experimental and Theoretical Methods}

within the probed spectral region. ${ }^{128}$ This results in corresponding broadband free induction decays, allowing for a simultaneous recording of a large frequency range. This measurement range increase from about $1 \mathrm{MHz}$ in cavity-based spectrometers to sometimes more than $10 \mathrm{GHz}$ in chirped pulse spectrometers results in a 1000-fold improvement in data acquisition rates. ${ }^{128}$ Since cavity-based instruments provide significantly larger absolute sensitivities and better spectral resolutions than chirped pulse set-ups, most studies ${ }^{134-136}$ use a combination of both techniques, adding cavity-based measurements to spectra obtained at chirped pulse instruments for a better characterisation of regions of interest discovered in the broadband spectra.

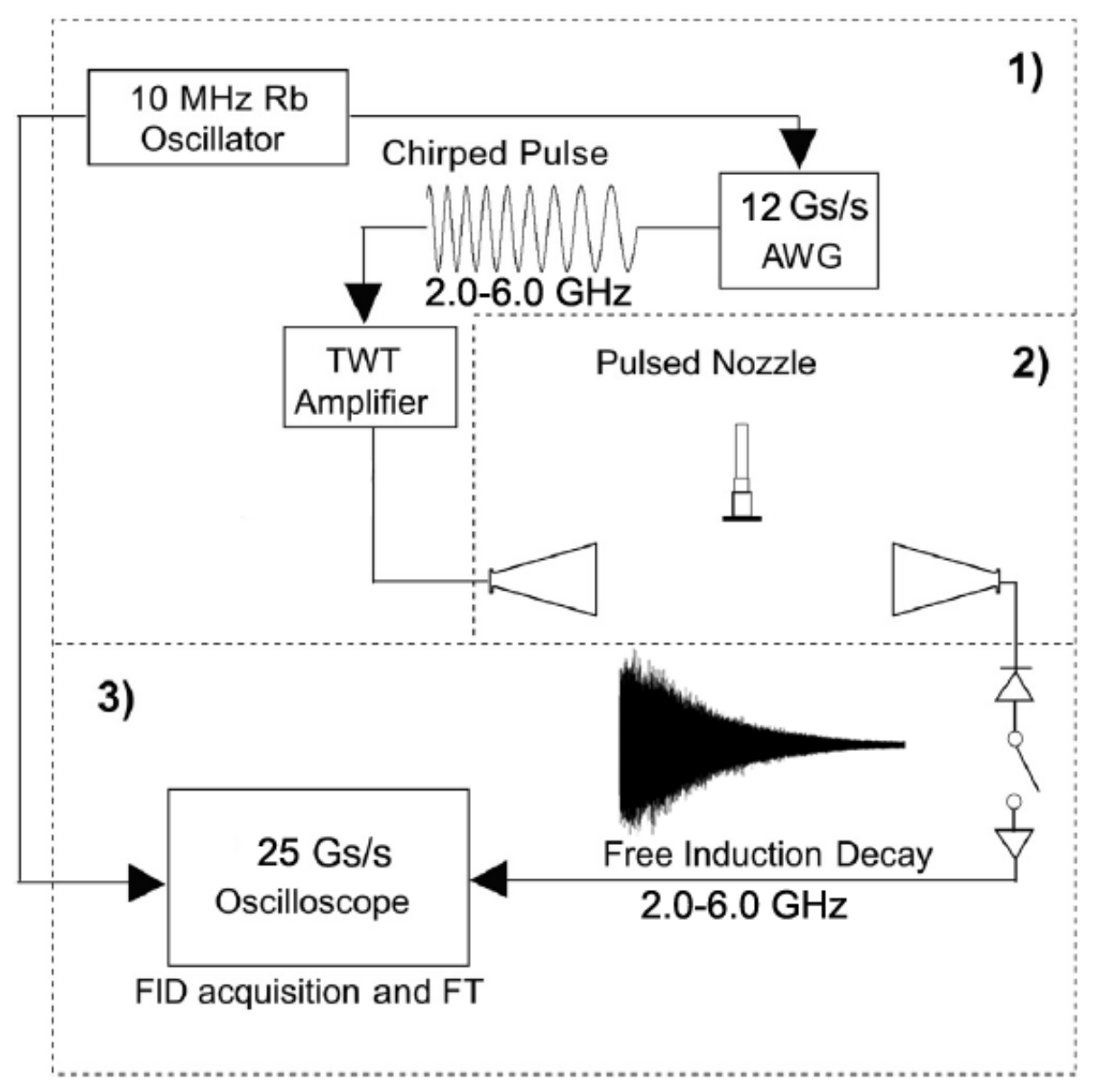

Figure 1.4.: Scheme of the set-up for chirped pulse Fourier transform microwave spectroscopy (reprinted with adaptations by Dr. N. A. Seifert from reference 137 with permission from Elsevier and Dr. N. A. Seifert). The scheme consists of separate parts for pulse generation and amplification (1), the supersonic expansion (2) and the detection and amplification of the free induction decay (3). Abbreviations: AWG: arbitrary waveform generator, TWT: travelling wave tube, FID: free induction decay, FT: Fourier transformation. 


\section{Experimental and Theoretical Methods}

\subsubsection{Chirped Pulse Fourier Transform Microwave Spectroscopy}

The set-up ${ }^{138,139}$ located at the University of Alberta is depicted in Figure 1.4. A microwave pulse with a frequency range from 2 to $6 \mathrm{GHz}$ was generated by a $12 \mathrm{Gs} \mathrm{s}^{-1}$ arbitrary waveform generator during a $4 \mu$ s linear frequency sweep chirp and amplified by a $400 \mathrm{~W}$ travelling wave tube. This excitation pulse was broadcast onto the perpendicularly propagating pulsed supersonic expansion from a circular nozzle $(d=1.25 \mathrm{~mm})$ by a high gain and directionality $62 \mathrm{~cm}$ horn antenna. Pre-blended gas mixtures of analyte molecules diluted in helium or neon were expanded at a frequency of $1 \mathrm{~Hz}$ into the $0.9 \mathrm{~m}^{3}$ vacuum chamber evacuated by a rotary vane (Leybold, Trivac D65B, $65 \mathrm{~m}^{3} \mathrm{~h}^{-1}$ ) and diffusion pump (Leybold, DIP8000, $28000 \mathrm{~m}^{3} \mathrm{~h}^{-1}$ ), keeping the background pressure during expansions between 0.02 and $0.10 \mathrm{mbar}$. An identical second antenna placed $50 \mathrm{~cm}$ from the first served as receiver of the coherent molecular emission. The signal processing circuit contained a low-noise $+56 \mathrm{dBm}$ amplifier, which was protected from the excitation pulse by a high power $p$ - $i$-n diode limiter and a $p$ - $i$ - $n$ diode switch with $500 \mathrm{~ns}$ rise and fall times, enabling recording of six free induction decays per gas pulse. After amplification, the free induction decay signal was digitised by a $25 \mathrm{Gs} \mathrm{s}^{-1}$ oscilloscope. A $10 \mathrm{MHz}$ Rb oscillator synchronising the arbitrary waveform generator and oscilloscope allowed for synchronous pulse-to-pulse operation and signal averaging in the time domain before producing the rotational spectrum in the frequency domain by fast Fourier transformation with a frequency precision of about $10 \mathrm{kHz}$.

\subsubsection{Cavity-enhanced Fourier Transform Microwave Spectroscopy}

For additional microwave spectroscopic measurements at higher resolution than achievable with the chirped pulse spectrometer, a cavity-based Fourier transform microwave instrument $^{140}$ in the $7-18 \mathrm{GHz}$ spectral range based on the design by Balle and Flygare $^{130,131}$ was used. The linear microwave cavity consisted of two spherical aluminium mirrors $(d=260 \mathrm{~mm}, R=380 \mathrm{~mm}$ ) placed inside a vacuum chamber, which was evacuated by a diffusion pump at a speed of $7200 \mathrm{~m}^{3} \mathrm{~h}^{-1}$, resulting in a background pressure of about 0.05 mbar during the expansion. The first mirror was fixed on one of the vacuum flanges of the chamber, while the second one could be linearly moved by a computer controlled dc motor drive. Therefore, the mirror separation could be varied from 200$400 \mathrm{~mm}$ until resonance with the external microwave radiation was achieved. After passing through a solid state power amplifier, microwave radiation from a Hewlett-Packard synthesizer source was coupled into and out of the cavity through a wire hook antenna. Control of the excitation pulse length, typically about $10 \mu \mathrm{s}$, as well as protection of the detection circuit from this pulse were realised with $p$ - $i$-n diode switches. The detected free induction decay signal was recovered by a low noise microwave amplifier and downconverted to intermediate frequencies around $15 \mathrm{MHz}$. After analog-to-digital conversion, the rotational spectrum in the frequency domain was obtained by a Fourier transformation of the sampled time domain signal. The pulsed, supersonic expansion of premixed gas compositions through a circular nozzle $(d=0.8 \mathrm{~mm})$ placed in the center of the first mirror propagated parallel to the cavity axis. Hence, all recorded molecular 


\section{Experimental and Theoretical Methods}

transitions were split into Doppler doublets. The frequency uncertainty of measured transitions was estimated to be less than $2 \mathrm{kHz}$.

\subsection{Theoretical Methods}

Three different program packages were used for quantum chemical calculations, Gaussian09 ${ }^{141}$ (Revision E01), ORCA ${ }^{142}$ (Version 4.0.1) and Molpro ${ }^{143}$ (Version 2012.1). For all compounds investigated in this work, an initial search for local minima of the conformational space of all aggregates of interest was performed at the B3LYP level of approximation including Grimme's dispersion correction and Becke-Johnson damping (abbreviated D3(BJ) ${ }^{144,145}$ with varying basis sets. At a later stage, the initial conformational search was performed at the faster PBEh-3c level ${ }^{146}$ in addition to B3LYP-D3(BJ) optimisations. Harmonic frequency calculations were always performed for all local minima at the exploratory levels of theory. In most cases, starting structures for conformational searches were generated manually, while an additional search for 1,1,1,3,3,3-hexafluoro2-propanol trimer structures was independently performed by Prof. S. Grimme using the GFN-xTB software package. ${ }^{147}$

Especially for the benchmarking studies presented in this work, a wide range of approximations for electronic structure calculations was tested. Employed levels of theory range from semi-empirical methods like AM1, PM6, PBEh-3c and B97-3c over dispersion-corrected hybrid density functionals such as B3LYP-D3(BJ), B2PLYPD3(BJ), PBE0-D3(BJ), $\omega B$ 97-XD, BLYP-D3(BJ), BP86-D3(BJ), M06-2X and B97D3(BJ) to wavefunction-based ab initio methods like HF, MP2, SCS-MP2 and CCSD(T) with local and explicit correlation. Typically, the aug-cc-pVTZ (aVTZ) basis set was used for most density functional theory calculations, while at later stages of this work the def2-QZVP basis set was employed in combination with the B3LYP-D3(BJ) method. A full list of employed keywords for all calculations in the different program packages is given in Tables A.3, A.4 and A.5 in appendix A.1.

Predictions from calculations used in benchmarking studies were consistently carried out at the same level of approximation from structure optimisation over harmonic frequency calculations to anharmonic frequency calculations in selected cases using second order vibrational perturbation theory (VPT2). ${ }^{148}$ For compounds where the calculations primarily served as an aid for assigning experimental vibrational bands, electronic single point energies were calculated at more elaborate levels of theory like $\operatorname{CCSD}(\mathrm{T})$ while using structures and zero-point vibrational energies obtained from calculations with lower computational cost such as B3LYP-D3(BJ).

Quantum chemical calculations for complexes containing molecular oxygen, a diradical, would require methods which use more than a single Slater determinant reference wavefunction. ${ }^{149}$ Such calculations, like complete active space self consistent field ${ }^{150}$ (CASSCF) or multiconfiguration reference configuration-interaction ${ }^{151}$ (MRCI) are very demanding with regard to expertise and computation time, especially if gradients for structure optimisations and frequency calculations are required. Therefore, they are considered to be well beyond the scope of this work and quantum chemical calculations 


\section{Experimental and Theoretical Methods}

will be restricted to aggregates containing molecular nitrogen.

\subsubsection{Benchmarking Theoretical Predictions}

Performance evaluation in benchmarking studies requires some form of quantification for prediction quality of tested theoretical approaches in comparison to chosen reference values. ${ }^{11,152,153}$ Typically, molecular test sets ${ }^{28,41}$ from data bases such as S22 $2^{16-18}$ or GMTKN30 ${ }^{19-21}$ are used and theoretical methods are judged based on some variation of the difference between prediction and reference for several $(n)$ quantities like the mean relative deviation (MRD), ${ }^{41}$ mean absolute error (MAE) ${ }^{28,154}$ or the root-mean-square deviation (RMSD) ${ }^{28,154}$ (see Equations A.1, ${ }^{155}$ A.2 ${ }^{154}$ and A.3 ${ }^{154}$ in appendix A.1). All these measures lack an assessment of the inherent error of the reference value. ${ }^{152}$ Therefore, in this work the deviation $\delta^{2}(x)$ as defined in Equation 1.1 is employed as a comprehensive quantity for performance evaluation of different quantum chemical methods tested against experimental observables $x$.

$$
\delta^{2}(x)=\left(\frac{x_{\text {theo }}-x_{\exp }}{\Delta x_{\exp }}\right)^{2}
$$

The difference of theoretically predicted $\left(x_{\text {theo }}\right)$ and experimentally obtained $\left(x_{\exp }\right)$ reference values scaled by the experimental error $\Delta x_{\exp }$ is squared to minimise contributions from predictions within the experimental error margin. Normalised by the total number of evaluated constraints $(n)$, the sum of all $\delta^{2}(x)$ gives a summarised impression of electronic structure method quality for the particular benchmark sample. Increasing quantity and variety of experimental observables in the test data set minimises the risk of systematic error cancellation.

Considering a fictional test data set (Table 1.1) of theoretical predictions $\left(x_{\text {theo }}\right)$ and experimental reference values $\left(x_{\exp }\right)$ with their respective error bounds $\left(\Delta x_{\exp }\right)$ demonstrates advantages of the proposed method. Error assessment through $\delta^{2}(x)$ yields similar and comparable values if differences between experiment and theory and experimental error bounds are of similar magnitude (Table 1.1, rows 1-3), revealing data in the first row of Table 1.1 as the worst agreement between experiment and theory of the four test cases in the chosen fictional set. The MAE and especially RMSD assessments are both dominated by large differences between prediction and reference, ignoring large experimental errors (row 3). Since both MAE and RMSD are not dimensionless, quantities in the data set are required to carry the same dimension which makes them unsuitable for a multi-observable benchmarking as proposed in this work. The MRD is dimensionless and can in principle be used for such an assessment, although it possibly displays the drawback of being dominated by a single quantity if a large difference between prediction and a small reference value occurs (row 2). Additionally, several different experimental observables will be added to the test data set. While a bad method might underestimate vibrational band centre positions, it could potentially overestimate rotational constants or spectral shifts, leaving the possibility of fortuitous error cancellation if the sign of deviations is not removed by using absolute or squared values. However, interpretational care is needed for $\delta^{2}(x)$ if a certain quantity has a particularly small experimental 


\section{Experimental and Theoretical Methods}

compared to the theoretical prediction error. It may thus dominate the benchmark assessment, impeding a broader evaluation of a method. In the discussed fictional data set, this would occur if the quantity in row 4 could hypothetically be measured with an accuracy of $\Delta x_{\exp }=0.001$, resulting in $\sqrt{\delta^{2}(x)}=100$. If such a situation occurs in the following, it will be highlighted.

On the experimental side, conservative and thus large uncertainty margins $\Delta x_{\exp }$ are selected to establish correct experimental error bounds. Following this philosophy, the maximum error is preferentially chosen over a Gaussian error propagation for derived quantities.

Table 1.1.: Different error assessments for a fictional test data set of theoretical predictions $\left(x_{\text {theo }}\right)$ and experimental reference values $\left(x_{\exp }\right)$ with their respective error bounds $\left(\Delta x_{\exp }\right)$. The proposed $\delta^{2}(x)$ (Equation 1.1) is compared to the mean relative deviation (MRD), mean absolute error (MAE) and root-mean-square deviation (RMSD). Vertical lines indicate separation between values which do not $\left(\sqrt{\delta^{2}(x)}, \mathrm{MRD}\right)$ or do (MAE, RMSD) carry dimensions.

\begin{tabular}{ccc|cc|cc}
\hline$x_{\text {theo }}$ & $x_{\exp }$ & $\Delta x_{\exp }$ & $\sqrt{\delta^{2}(x)}$ & MRD & MAE & RMSD \\
\hline 1.5 & 1.0 & 0.1 & 5.0 & 0.5 & 0.5 & 0.25 \\
-0.5 & 0.2 & 0.3 & 2.3 & -3.5 & 0.7 & 0.49 \\
11.3 & 10.4 & 0.4 & 2.3 & 0.1 & 0.9 & 0.81 \\
-0.2 & -0.1 & 0.2 & 0.5 & 1.0 & 0.1 & 0.01 \\
\hline \multicolumn{3}{c}{ Mean } & 2.5 & -0.5 & 0.6 & 0.62 \\
\hline
\end{tabular}





\section{Carboxylic Acids}

\section{Carboxylic Acids}

Aggregates of formic and acetic acid and their mono-deuterated isotopologues with nitrogen and oxygen were studied with filet-jet FTIR spectroscopy in the OH and OD stretching region. Experimental observables suitable for benchmarking of quantum chemical calculations include $\mathrm{OH}$ stretching band centre positions, hydrogen bond induced spectral downshifts as well as the absolute dissociation energy of the formic acid dimer. Most of the discussed experiments and quantum chemical calculations were carried out as part of Enno Meyer's bachelor thesis ${ }^{156}$ and the results obtained for nitrogen containing aggregates are published in reference 157 . The occasional verbatim reproduction of small sections from that reference is not explicitly marked or cited.

\subsection{Introduction}

As trace gases with a significant abundance in earth's atmosphere, ${ }^{158}$ interactions of the most simple carboxylic acids with the most abundant atmospheric molecules motivate an investigation at the level of binary complexes. ${ }^{159,160}$ The small size of those aggregates allows for broad exploration of highly accurate electronic structure and anharmonic vibrational frequency calculations. Especially the binary aggregate of formic acid with nitrogen is a valuable addition to the vibrational spectroscopy data pool used for benchmarking theoretical predictions.

A wide range of spectroscopic studies on both acids and their aggregates have been performed previously. The photo-induced trans-cis-isomerisation of the monomer ${ }^{161-164}$ and subsequent tunnelling relaxation ${ }^{164-168}$ observed in rare gas matrices is difficult to study in the gas phase, since the cis equilibrium population is low even at room temperature ${ }^{169,170}$ due to the $16.3(4) \mathrm{kJ} \mathrm{mol}^{-1}$ energy difference. ${ }^{171}$ However, isomerism in the aggregates of several trans monomers can be observed more readily and subtly manipulated by nitrogen addition.

The most stable, cyclic dimer isomers of both acids with their two cooperative hydrogen bonds have been the subject of various studies using photoacoustic ${ }^{172}$, nuclear magnetic resonance ${ }^{173}$, cavity ring-down, ${ }^{174-176}$ direct laser absorption, ${ }^{177}$ broadband infrared $^{97,102,178-184}$ and Raman ${ }^{98,185-189}$ spectroscopy. The double hydrogen bond induces a significant downshift of the dimer $\mathrm{OH}$ stretching frequencies into the wavenumber region between 3200 and $2550 \mathrm{~cm}^{-1}$, where anharmonic couplings become possible ${ }^{190}$ and heavy perturbations ${ }^{191}$ result in broad and highly structured $\mathrm{OH}$ stretching spectra. ${ }^{181,183,187}$ Open dimer isomers are of particular interest as intermediate steps in hydrogen bond dissociation. ${ }^{172,192-194}$ Since their direct gas phase detection has only been reported once ${ }^{195}$ and remains unconfirmed, indirect methods such as chemical blocking 


\section{Carboxylic Acids}

of one $\mathrm{OH}$ group by an added methyl group ${ }^{183}$ or matrix isolation techniques ${ }^{188,196-199}$ have been employed. Here, selective nitrogen or oxygen complexation of open dimers and a subsequent depletion of the vibrational signal serve as an alternative approach.

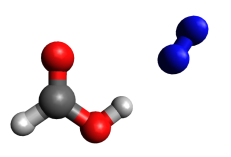

$\mathrm{FN}_{\mathrm{H}}\left(C_{\mathrm{s}}\right)$

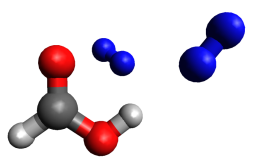

$\mathrm{FN}_{\mathrm{H}} \mathrm{N}_{\mathrm{H}}\left(C_{1}\right)$

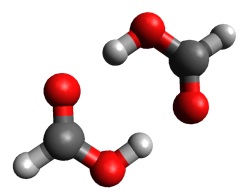

$(\mathrm{FF})\left(C_{2 \mathrm{~h}}\right)$

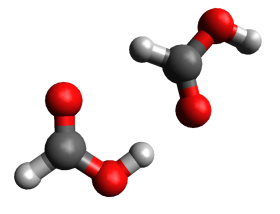

FF $\left(C_{\mathrm{s}}\right)$

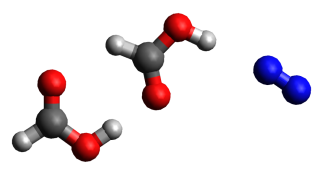

$\operatorname{FFN}_{\mathrm{H}}\left(C_{\mathrm{s}}\right)$

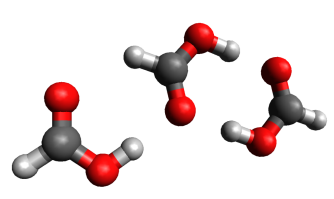

$\mathrm{F}(\mathrm{FF})\left(C_{\mathrm{s}}\right)$

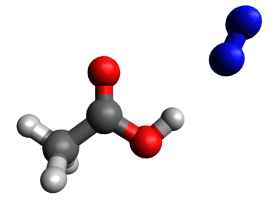

$\mathrm{AN}_{\mathrm{H}}\left(C_{\mathrm{s}}\right)$

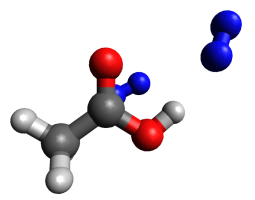

$\mathrm{AN}_{\mathrm{H}} \mathrm{N}_{\mathrm{C}^{\prime}}\left(C_{1}\right)$

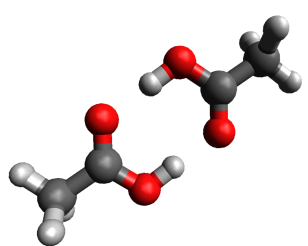

(AA) $\left(C_{2 h}\right)$

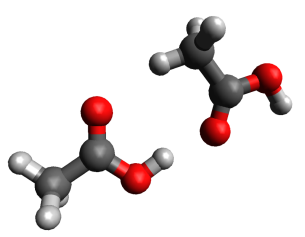

$\mathrm{AA}\left(C_{1}\right)$

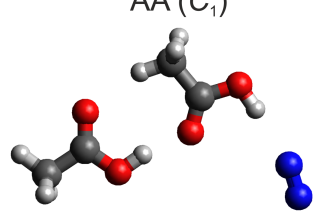

$\operatorname{AAN}_{\mathrm{H}}\left(C_{1}\right)$

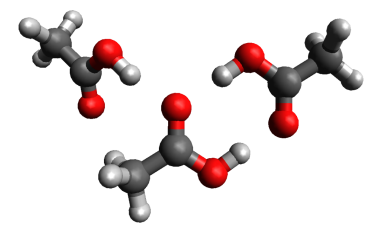

$\mathrm{A}(\mathrm{AA})\left(C_{1}\right)$

Figure 2.1.: Most stable structures of $\mathrm{F}$ and A clusters alongside mixed aggregates with $\mathrm{N}$ (reprinted with adaptations from reference 157 licensed under CC BY 4.0) optimised at the B3LYP-D3(BJ)/def2-QZVP level with their respective symmetry point groups given in parentheses. Metastable open acid dimers FF and AA and their most stable $\mathrm{N}$ aggregates are included. 


\section{Carboxylic Acids}

Table 2.1.: Theoretically predicted spectral properties of $\mathrm{F}, \mathrm{A}$ and $\mathrm{N}$ aggregates obtained at the B3LYP-D3(BJ)/def2-QZVP level, including harmonic $\left(\omega_{\mathrm{OH}}\right)$ and anharmonic $\left(\tilde{\nu}_{\mathrm{OH}}\right)$ $\mathrm{OH}$ stretching band center positions in $\mathrm{cm}^{-1}$, harmonic $\left(S_{\omega}\right)$ and anharmonic $\left(S_{\nu}\right)$ integrated infrared band strengths in $\mathrm{km} \mathrm{mol}^{-1}$, harmonic $\left(\Delta \omega_{\mathrm{OH}}\right)$ and anharmonic $\left(\Delta \tilde{\nu}_{\mathrm{OH}}\right)$ spectral downshifts relative to the isolated monomer in $\mathrm{cm}^{-1}$ as well as lowest predicted harmonic $\left(\omega_{\mathrm{l}}\right)$ and anharmonic $\left(\tilde{\nu}_{1}\right)$ wavenumbers in $\mathrm{cm}^{-1}$, indicating limitations of numerical differentiation or of VPT2 which may also affect $\mathrm{OH}$ stretching coupling constants for all systems but $\mathrm{F}$ and pure $\mathrm{F}$ aggregates in italic font.

\begin{tabular}{|c|c|c|c|c|c|c|c|c|}
\hline Structure & $\omega_{\mathrm{OH}}$ & $S_{\omega}$ & $\Delta \omega_{\mathrm{OH}}$ & $\tilde{\nu}_{\mathrm{OH}}$ & $S_{\nu}$ & $\Delta \tilde{\nu}_{\mathrm{OH}}$ & $\omega_{1}$ & $\tilde{\nu}_{1}$ \\
\hline $\mathrm{F}$ & 3727 & 60 & - & 3537 & 50 & - & 631 & 625 \\
\hline $\mathrm{FN}_{\mathrm{H}}$ & 3690 & 267 & 37 & 3511 & 185 & 26 & 25 & -11 \\
\hline $\mathrm{FN}_{\mathrm{H}} \mathrm{N}_{\mathrm{H}}$ & 3688 & 259 & 39 & 3512 & 120 & 25 & 16 & 228 \\
\hline $\mathrm{A}$ & 3750 & 61 & - & 3561 & 49 & - & 71 & 112 \\
\hline $\mathrm{AN}_{\mathrm{H}}$ & 3719 & 258 & 31 & 3538 & 244 & 23 & 26 & 59 \\
\hline $\mathrm{AN}_{\mathrm{H}} \mathrm{N}_{\mathrm{C}^{\prime}}$ & 3718 & 230 & 32 & 3527 & 111 & 34 & 12 & -749 \\
\hline$(\mathrm{FF})$ & $\begin{array}{l}3156 \\
3030 \\
\end{array}$ & $\begin{array}{c}2166 \\
0 \\
\end{array}$ & $\begin{array}{l}571 \\
697 \\
\end{array}$ & $\begin{array}{l}2835 \\
2627 \\
\end{array}$ & $\begin{array}{c}717 \\
0 \\
\end{array}$ & $\begin{array}{l}702 \\
910 \\
\end{array}$ & 75 & 39 \\
\hline (AA) & $\begin{array}{l}3139 \\
3025 \\
\end{array}$ & $\begin{array}{c}3440 \\
0 \\
\end{array}$ & $\begin{array}{l}611 \\
725 \\
\end{array}$ & $\begin{array}{l}2797 \\
2648 \\
\end{array}$ & $\begin{array}{c}2330 \\
0\end{array}$ & $\begin{array}{l}764 \\
913 \\
\end{array}$ & 42 & 338 \\
\hline $\mathrm{FF}$ & $\begin{array}{l}3720 \\
3333\end{array}$ & $\begin{array}{c}69 \\
987\end{array}$ & $\begin{array}{c}7 \\
394\end{array}$ & $\begin{array}{l}3523 \\
3129\end{array}$ & $\begin{array}{c}34 \\
779\end{array}$ & $\begin{array}{c}14 \\
408\end{array}$ & 63 & 10 \\
\hline $\mathrm{FFN}_{\mathrm{H}}$ & $\begin{array}{l}3672 \\
3304 \\
\end{array}$ & $\begin{array}{c}326 \\
1104 \\
\end{array}$ & $\begin{array}{c}55 \\
423 \\
\end{array}$ & $\begin{array}{l}3490 \\
3103 \\
\end{array}$ & $\begin{array}{l}291 \\
842 \\
\end{array}$ & $\begin{array}{c}47 \\
434 \\
\end{array}$ & 15 & -432 \\
\hline AA & $\begin{array}{l}3742 \\
3403 \\
\end{array}$ & $\begin{array}{c}67 \\
1158 \\
\end{array}$ & $\begin{array}{c}8 \\
347 \\
\end{array}$ & $\begin{array}{l}3552 \\
3197 \\
\end{array}$ & $\begin{array}{l}73 \\
91 \\
\end{array}$ & $\begin{array}{c}9 \\
364 \\
\end{array}$ & 5 & 347 \\
\hline $\mathrm{AAN}_{\mathrm{H}}$ & $\begin{array}{l}3702 \\
3381\end{array}$ & $\begin{array}{c}302 \\
1236 \\
\end{array}$ & $\begin{array}{c}48 \\
369 \\
\end{array}$ & - & - & - & 4 & - \\
\hline $\mathrm{F}(\mathrm{FF})$ & $\begin{array}{l}3369 \\
3207 \\
3031\end{array}$ & $\begin{array}{c}653 \\
2361 \\
763\end{array}$ & $\begin{array}{l}358 \\
520 \\
696\end{array}$ & $\begin{array}{l}3163 \\
2925 \\
2689\end{array}$ & $\begin{array}{c}592 \\
206 \\
12034\end{array}$ & $\begin{array}{l}374 \\
612 \\
848\end{array}$ & 32 & 13 \\
\hline $\mathrm{A}(\mathrm{AA})$ & $\begin{array}{l}3398 \\
3199 \\
3007\end{array}$ & $\begin{array}{c}930 \\
2688 \\
940\end{array}$ & $\begin{array}{l}352 \\
551 \\
743\end{array}$ & $\begin{array}{l}- \\
- \\
-\end{array}$ & $\begin{array}{l}- \\
- \\
-\end{array}$ & $\begin{array}{l}- \\
- \\
-\end{array}$ & 14 & - \\
\hline
\end{tabular}

\subsection{Quantum Chemical Calculations and Nomenclature}

The studied molecules are abbreviated as F (formic acid), A (acetic acid), N (nitrogen), and $\mathrm{O}$ (oxygen). Cluster compositions (Figure 2.1) are described by repeating the number of letters according to the cluster size in the direction from hydrogen bond donors to acceptors. Following reference 102, cyclic units or subunits are enclosed in parentheses. 


\section{Carboxylic Acids}

A formic acid trimer with the third acid docking to the cyclic dimer is therefore abbreviated as $\mathrm{F}(\mathrm{FF})$. The docking site of nitrogen and oxygen is identified by a subscript, labelling binding to the acidic $\mathrm{OH}$ proton $(\mathrm{H})$ or the carbonyl $(\mathrm{C})$ or alcohol $(\mathrm{O})$ oxygen of the respective acid. For binding at the carbonyl oxygen, weak secondary $\mathrm{CH} \cdots \mathrm{N}$ $\left(\mathrm{N}_{\mathrm{C}}\right)$ or $\mathrm{OH} \cdots \mathrm{N}\left(\mathrm{N}_{\mathrm{C}^{\prime}}\right)$ interactions are possible (Figures A.1 and A.2 in appendix A.2).

The global minimum aggregate structures of each composition and the most stable open $\mathrm{F}$ and $\mathrm{A}$ dimers are depicted in Figure 2.1. Additional higher energy conformations are shown in Figures A.1 and A.2 in appendix A.2. N with a large $\left(-5.0(2) \times 10^{-40} \mathrm{C} \mathrm{m}^{2}\right)$ quadrupole moment consisting of negative partial charges at the molecule ends and positive ones at its centre ${ }^{45}$ strongly prefers binding at the acidic $\mathrm{OH}$ proton of both acids as found in $\mathrm{FN}_{\mathrm{H}}$ and $\mathrm{AN}_{\mathrm{H}}$. This structural motif will be found consistently throughout this work for other donor molecules as well. The most stable acid homotrimers $\mathrm{F}(\mathrm{FF})$ and $\mathrm{A}(\mathrm{AA})$ contain a cyclic dimer subunit with the third acid attached via an $\mathrm{OH} \cdots \mathrm{O}$ and a weaker $\mathrm{CH} \cdots \mathrm{O}$ hydrogen bond, a docking motif also found in the open dimers $\mathrm{FF}$ and AA.

Calculations from this work (see Tables 2.1, 2.2 and 2.3) indicate that formation of a weak $\mathrm{OH} \cdots \mathrm{N}$ hydrogen bond causes spectral downshifts of the $\mathrm{OH}$ stretching vibration in both mixed binary $\mathrm{N}$ aggregates with significant anharmonic contributions. Low frequency large amplitude vibrations introduced by $\mathrm{N}$ addition or a methyl rotor in A cause a certain unreliability in VPT2 predictions, since the lowest anharmonic wavenumber predictions for the softest vibrations in all clusters except pure $\mathrm{F}$ aggregates are unphysical. Typically, this problem barely occurs for localised high frequency modes such as hydride stretching vibrations, but they might still be affected through coupling to low frequency modes.

In open homodimers and homotrimers of both acids, the docking molecule $\mathrm{OH}$ stretching vibration is predicted to be significantly less downshifted from the monomer than corresponding vibrations of the double hydrogen bonded cyclic motif. Hence, complex Fermi resonance interaction ${ }^{190}$ is avoided, facilitating assignments of docking $\mathrm{OH}$ stretching vibrations.

\subsection{FTIR Spectra}

Formic acid- $d_{0}$ ( $\geq 99 \%$, Acros Organics), formic acid- $d_{1}\left(95 \%\right.$ in $\mathrm{D}_{2} \mathrm{O}, 98 \% \mathrm{D}$, abcr), acetic acid- $d_{0}\left(\geq 99 \%\right.$, Fluka Chemika; 99.8\%, Acros Organics) and acetic acid- $d_{1}$ (99\% D, abcr) were mixed without further purification with helium (99.996\%, Linde) and optionally nitrogen (99.999\%, Air Liquide) or oxygen (99.998\%, Air Liquide). For the $\mathrm{OH}$ and $\mathrm{OD}$ stretching measurements from $4100-2450 \mathrm{~cm}^{-1}$, the filet-jet set-up was equipped with the $150 \mathrm{~W}$ tungsten lamp, $\mathrm{CaF}_{2}$ beam splitter and optics as well as the liquid nitrogen cooled $3 \mathrm{~mm}^{2} \mathrm{InSb}$ detector.

The following spectral analysis of pure $\mathrm{F}$ and $\mathrm{A}$ expansions will focus on disentangling monomer and trimer from cyclic dimer contributions. Difference spectra between expansion conditions with high and low analyte concentrations which remove $\mathrm{OH}$ stretching bands previously assigned to the cyclic dimers ${ }^{175,183,190}$ serve as a useful tool, as they 


\section{Carboxylic Acids}

Table 2.2.: Theoretically predicted dissociation energies in $\mathrm{kJ} \mathrm{mol}^{-1}$ of $\mathrm{F}, \mathrm{A}$ and $\mathrm{N}$ aggregates into the most stable monomers obtained at the B3LYP-D3(BJ)/def2-QZVP level with $\left(D_{0}\right)$ and without $\left(D_{\mathrm{e}}\right)$ harmonic zero-point vibrational energy.

\begin{tabular}{ccc}
\hline Structure & $D_{\mathrm{e}}$ & $D_{0}$ \\
\hline $\mathrm{FN}_{\mathrm{H}}$ & 9.6 & 6.5 \\
\hline $\mathrm{FN}_{\mathrm{H}} \mathrm{N}_{\mathrm{H}}$ & 15.5 & 11.1 \\
\hline $\mathrm{AN}_{\mathrm{H}}$ & 9.2 & 6.5 \\
\hline $\mathrm{AN}_{\mathrm{H}} \mathrm{C}_{\mathrm{C}^{\prime}}$ & 15.7 & 11.8 \\
\hline$(\mathrm{FF})$ & 73.8 & 66.2 \\
\hline$(\mathrm{AA})$ & 76.1 & 70.7 \\
\hline $\mathrm{FF}$ & 43.9 & 37.6 \\
\hline $\mathrm{FFN}$ & 54.6 & 45.4 \\
\hline $\mathrm{AA}$ & 42.1 & 37.5 \\
\hline $\mathrm{AAN}$ & 66.7 & 44.9 \\
\hline $\mathrm{F}(\mathrm{FF})$ & 113.9 & 100.8 \\
\hline $\mathrm{A}(\mathrm{AA})$ & 115.1 & 105.1 \\
\hline
\end{tabular}

display negative monomer and positive trimer bands, respectively. In a next step, the spectral data of expansions containing $\mathrm{N}$ can be analysed with difference spectra corrected for acid monomer and dimer contributions, respectively, revealing vibrational bands which can be assigned to various $\mathrm{N}$ clusters of both acids.

\subsubsection{Formic Acid}

Spectra of expansions containing only $\mathrm{F}$ and helium are shown in Figure 2.2. At low F concentration and stagnation pressure (trace b) the spectrum mostly contains monomer contributions, namely $\mathrm{CH}$ and $\mathrm{OH}$ stretching fundamentals at 2942 and $3570 \mathrm{~cm}^{-1}$, respectively, both significantly broadened by rovibrational transitions. Two weak bands near the $\mathrm{OH}$ stretching fundamental at $3538 / 3533 \mathrm{~cm}^{-1}$ are assigned to the first CO stretching overtone. ${ }^{200}$ With increasing stagnation pressure (trace d), the broad and highly structured $\mathrm{OH}$ stretching spectrum ${ }^{175}$ of (FF) grows significantly between 3200 and $2550 \mathrm{~cm}^{-1}$. The previous assignment of monomer bands is confirmed by the room temperature gas phase spectrum (trace a) and negative contributions in the (FF)corrected difference spectrum (trace c). At high stagnation pressure, increased F concentration (trace $\mathrm{f}$ ) and with a positive sign in the (FF)-corrected difference spectrum (trace e), the $\mathrm{OH}$ stretching fundamental of the docking acid of $\underline{\mathrm{F}}(\mathrm{FF})$ appears at $3258 \mathrm{~cm}^{-1}$. 


\section{Carboxylic Acids}

Table 2.3.: Comparison of theoretical predictions of spectral properties and binding energies of $\mathrm{F}, \mathrm{A}$ and $\mathrm{N}$ aggregates obtained at the B3LYP-D3(BJ)/def2-QZVP and $\operatorname{LCCSD}\left(\mathrm{T}^{*}\right)-\mathrm{F} 12 / \mathrm{VDZ}-\mathrm{F} 12$ (int) levels. Listed properties include harmonic OH stretching band centre positions $\left(\omega_{\mathrm{OH}}\right)$, harmonic spectral downshifts relative to the isolated monomer $\left(\Delta \omega_{\mathrm{OH}}\right)$ and the lowest predicted harmonic wavenumber $\left(\omega_{1}\right) \mathrm{in} \mathrm{cm}^{-1}$, as well as dissociation energies into the most stable monomers in $\mathrm{kJ} \mathrm{mol}^{-1}$ with $\left(D_{0}\right)$ and without $\left(D_{\mathrm{e}}\right)$ harmonic vibrational zero-point energy.

\begin{tabular}{|c|c|c|c|c|c|c|}
\hline Structure & Level of theory & $\omega_{\mathrm{OH}}$ & $\Delta \omega_{\mathrm{OH}}$ & $D_{\mathrm{e}}$ & $D_{0}$ & $\omega_{l}$ \\
\hline \multirow{2}{*}{$\mathrm{F}$} & B3LYP-D3 & 3727 & - & - & - & 631 \\
\hline & $\operatorname{LCCSD}\left(\mathrm{T}^{*}\right)-\mathrm{F} 12$ & 3754 & - & - & - & 630 \\
\hline \multirow{2}{*}{$\mathrm{FN}_{\mathrm{H}}$} & B3LYP-D3 & 3690 & 37 & 9.6 & 6.5 & 25 \\
\hline & $\operatorname{LCCSD}\left(\mathrm{T}^{*}\right)-\mathrm{F} 12$ & 3730 & 24 & 7.9 & 5.0 & 27 \\
\hline \multirow{2}{*}{ A } & B3LYP-D3 & 3750 & - & - & - & 71 \\
\hline & $\operatorname{LCCSD}\left(\mathrm{T}^{*}\right)-\mathrm{F} 12$ & 3767 & - & - & - & 78 \\
\hline \multirow{3}{*}{$(\mathrm{FF})$} & B3LYP-D3 & 3156 & 571 & 738 & 662 & 75 \\
\hline & & 3030 & 697 & & & \\
\hline & $\operatorname{LCCSD}\left(\mathrm{T}^{*}\right)-\mathrm{F} 12$ & $\begin{array}{l}3318 \\
3219\end{array}$ & $\begin{array}{l}436 \\
535\end{array}$ & 65.0 & 56.7 & 71 \\
\hline \multirow{4}{*}{$\mathrm{FF}$} & B3LYP-D3 & 3720 & 7 & 43.9 & 37.6 & 63 \\
\hline & & 3333 & 394 & & & \\
\hline & $\operatorname{LCCSD}\left(\mathrm{T}^{*}\right)-\mathrm{F} 12$ & 3747 & 7 & 40.2 & 33.8 & 61 \\
\hline & & 3440 & 314 & & & \\
\hline
\end{tabular}

A weak band at $3393 \mathrm{~cm}^{-1}$ could be caused by $(\mathrm{FF})$ or $\mathrm{F}(\mathrm{FF})$, but a more rigorous assignment requires further measurements at higher $\mathrm{F}$ concentration.

\subsubsection{Formic Acid Nitrogen}

FTIR spectra with an excess of $\mathrm{N}$ compared to $\mathrm{F}$ added to the expanded gas mixture are depicted in Figure 2.3. After addition of $2.5 \% \mathrm{~N}$ (trace $\mathrm{c}$ ), the mixed $\mathrm{FN}_{\mathrm{H}}$ dimer band emerges at $3553 \mathrm{~cm}^{-1}$. The (FF)-corrected difference spectrum (trace b) shows this band as a positive contribution, while monomer bands are negative due to $\mathrm{N}$ complexation induced depletion of isolated monomer. Adding $15 \% \mathrm{~N}$ (trace e) increases the intensity of the $\mathrm{FN}_{\mathrm{H}}$ band. The stronger monomer depletion also leads to decreased intensity of the $\mathrm{F}(\mathrm{FF})$ band at $3258 \mathrm{~cm}^{-1}$ (trace d) which is also observed after addition of $2.5 \%$ $\mathrm{N}$ to a higher $\mathrm{F}$ concentration (traces $\mathrm{f}, \mathrm{g}$ and $\mathrm{h}$ ). Since the band at $3393 \mathrm{~cm}^{-1}$ is not downshifted upon $\mathrm{N}$ addition, an assignment to the open $\mathrm{FF}$ dimer is unlikely. In contrast to $(\mathrm{FF})$ or $\mathrm{F}(\mathrm{FF})$, the $\mathrm{FF}$ isomer offers a free $\mathrm{OH}$ group and therefore an attractive docking site for $\mathrm{N}$ and the corresponding $\mathrm{OH}$ stretching vibration should 
2. Carboxylic Acids

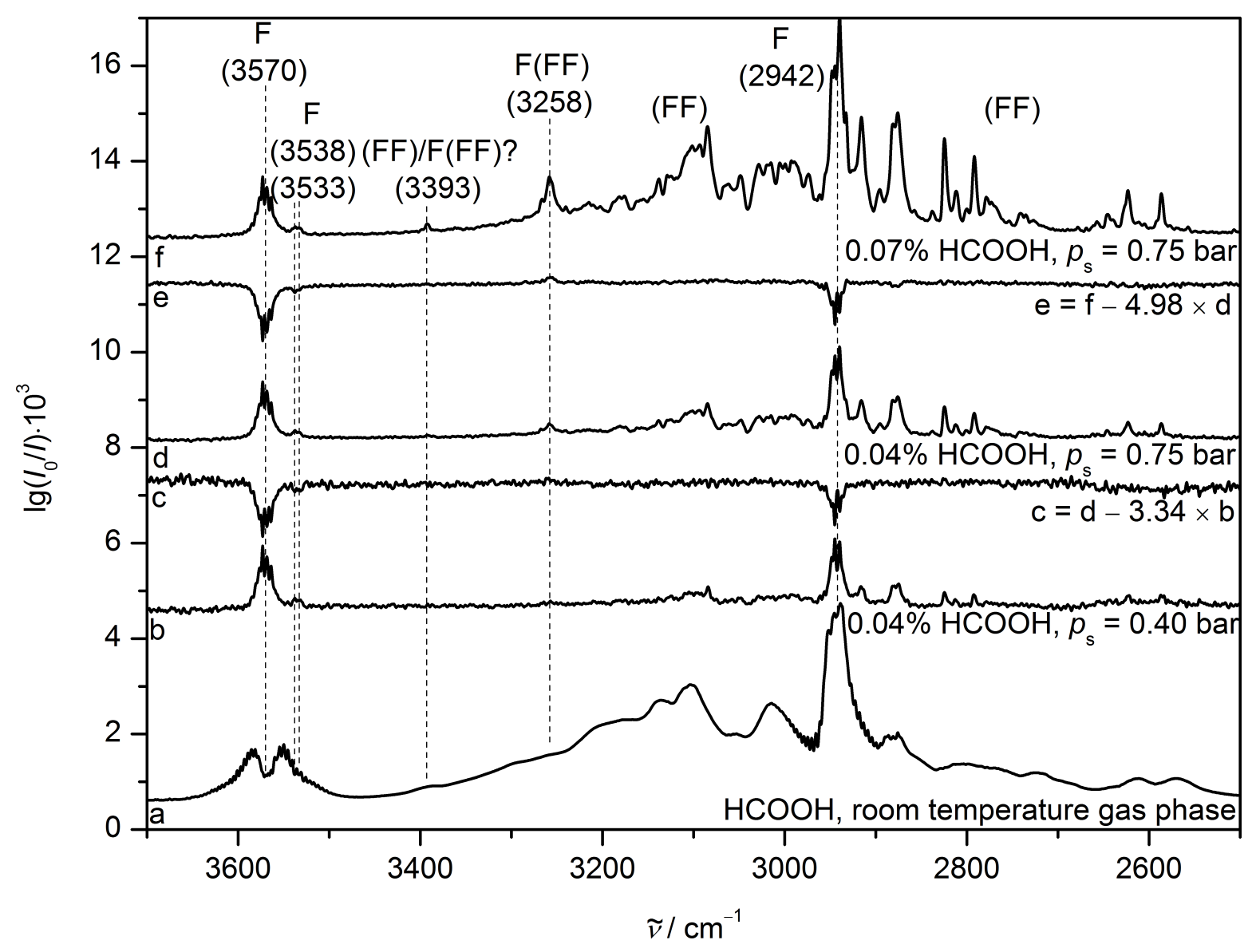

Figure 2.2.: OH stretching FTIR spectra of F (reprinted with adaptations from reference 157 licensed under CC BY 4.0) expanded in helium with increasing concentration and stagnation pressure $p_{\mathrm{s}}$ from bottom to top (traces $\mathrm{b}$, d and $\mathrm{f}$ ). (FF)-corrected difference spectra (traces $\mathrm{c}$ and e) and a room temperature gas phase spectrum (trace a) are included. All spectra were scaled to matching height of the monomer band at $3570 \mathrm{~cm}^{-1}$. Wavenumbers and (tentative) labels are provided.

display a significant downshift after $\mathrm{N}$ complexation.

More detailed spectra of $\mathrm{F}$ and its binary $\mathrm{N}$ complex as well as the mono-deuterated HCOOD isotopologue are shown in Figure 2.4. A suspected strong Fermi resonance of $\mathrm{OH}$ stretching fundamental and CO stretching overtone at $3538 / 3533 \mathrm{~cm}^{-1}$ is unlikely due to the similar intensity of the feature in the HCOOD spectrum (trace a). Hence, the $\mathrm{F}$ monomer band centre position of $3570(2) \mathrm{cm}^{-1}$ in good agreement with literature values in Ne matrices $\left(3570.0 \mathrm{~cm}^{-1}\right)^{201}$ and He nanodroplets $\left(3569.1 \mathrm{~cm}^{-1}\right)^{200}$ is found by calculating the centre of mass of the band integral, excluding the $\mathrm{CO}$ stretching overtone, according to Equation 2.1. Further details about the integration procedure are listed in Table A.6 in appendix A.2. 


\section{Carboxylic Acids}

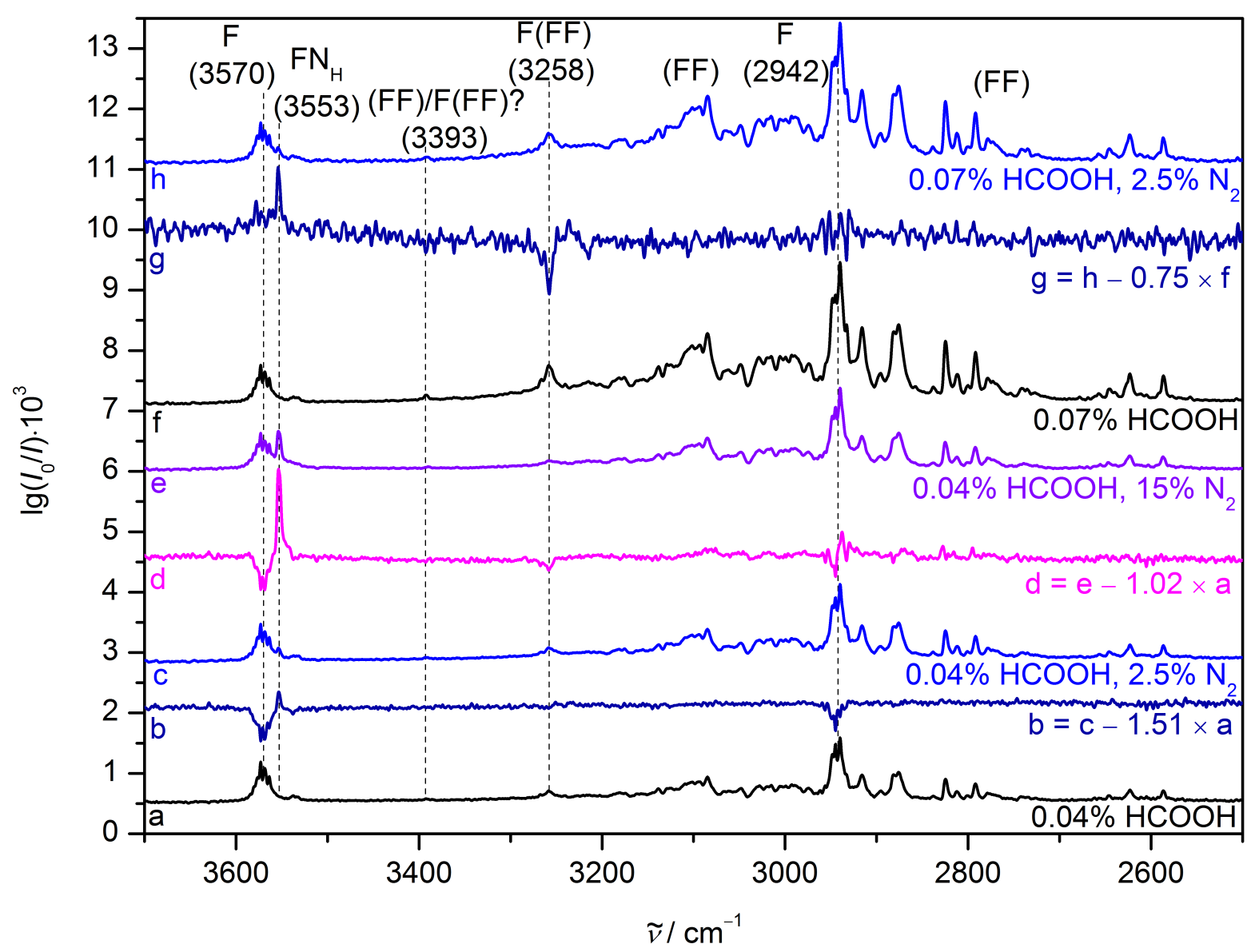

Figure 2.3.: OH stretching FTIR spectra of $\mathrm{F}$ (reprinted with adaptations from reference 157 licensed under CC BY 4.0) expanded in helium (traces a and f), $2.5 \% \mathrm{~N}$ in helium (traces $\mathrm{c}$ and $\mathrm{h}$ ) and $15 \% \mathrm{~N}$ in helium (trace e). A stagnation pressure of $p_{\mathrm{s}}=0.75 \mathrm{bar}$ was used for all shown spectra. (FF)-corrected difference spectra are included (traces b, $\mathrm{d}$ and $\mathrm{g}$ ). All spectra were scaled to matching height of the monomer band at $3570 \mathrm{~cm}^{-1}$. Wavenumbers and (tentative) labels are provided.

$$
\tilde{\nu}=\frac{\int I(\tilde{\nu}) \times \tilde{\nu} \mathrm{d} \tilde{\nu}}{\int I(\tilde{\nu}) \mathrm{d} \tilde{\nu}}
$$

A conservative estimate of experimental errors is the half-width at half-maximum (HWHM) of Q-branches for any vibrational band, even though high resolution gas phase measurements $\left(3570.5 \mathrm{~cm}^{-1}\right)^{202,203}$ would allow for a more accurate evaluation of the vibrational band centre position. By maintaining the larger error bar, consistency of the error evaluation for cluster bands recorded at lower resolution during this work is achieved and potential unidentified coupling partners and their effects on band positions are accounted for. Most $\mathrm{OH}$ stretching band centres from the literature are in excellent agreement with the determined value, only ultrafast two-colour transient laser 


\section{Carboxylic Acids}

absorption measurements ${ }^{192}$ give a deviating gas phase wavenumber of $3585 \mathrm{~cm}^{-1}$.

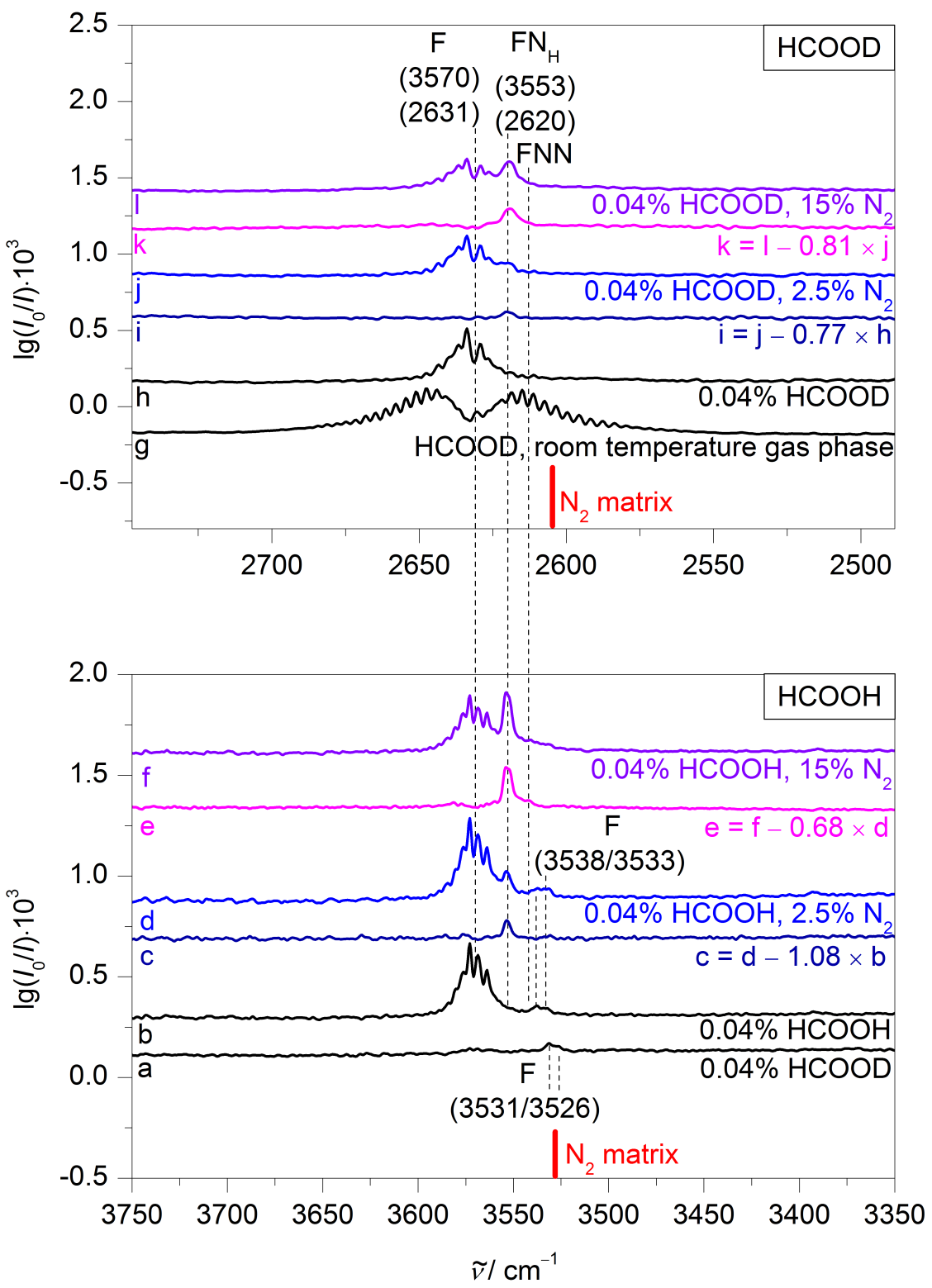

Figure 2.4.: $\mathrm{OH}$ and OD stretching FTIR spectra (reprinted with adaptations from reference 157 licensed under CC BY 4.0) of formic acid- $d_{1}$ (upper box) and formic acid- $d_{0}$ (lower box except for trace a) expanded in helium (traces a, b and h), $2.5 \% \mathrm{~N}$ in helium (traces $\mathrm{d}$ and $\mathrm{j}$ ) and $15 \% \mathrm{~N}$ in helium (traces $\mathrm{f}$ and $\mathrm{l}$ ). A stagnation pressure of $p_{\mathrm{s}}=0.75$ bar was employed for all shown spectra. Monomer-corrected difference spectra (traces c, e, i and k) and a room temperature gas phase spectrum (trace g, scaled by a factor of 0.3) are included. The OD wavenumber scale width is compressed by a factor of 0.65 and shifted by $496 \mathrm{~cm}^{-1}$ such that the $\mathrm{F}$ and $\mathrm{FN}_{\mathrm{H}}$ signals match for the two isotopologues. Wavenumbers and (tentative) labels are provided. Red bars at the bottom indicate the band position of $\mathrm{F}$ in a bulk nitrogen matrix. ${ }^{160}$ 


\section{Carboxylic Acids}

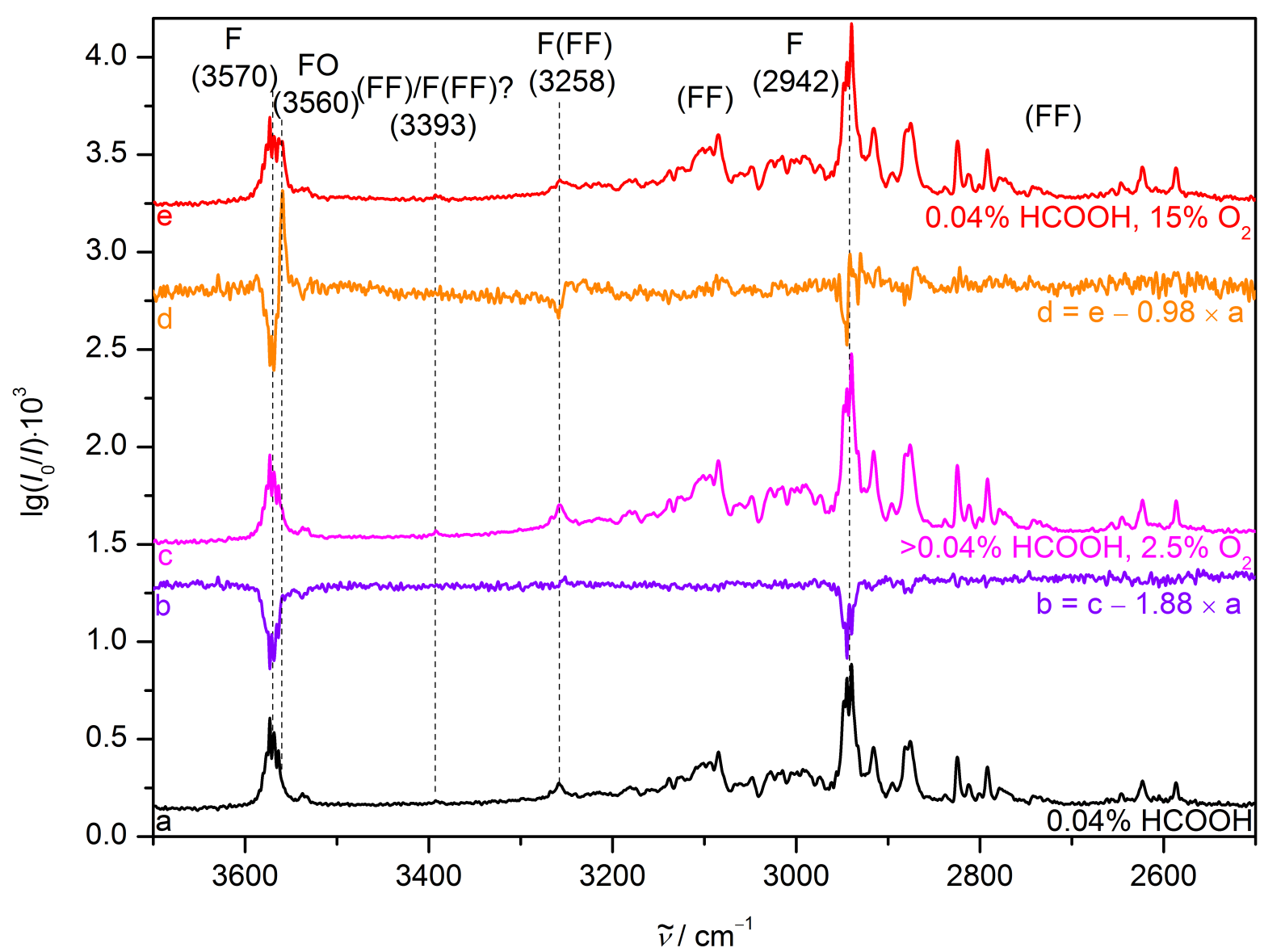

Figure 2.5.: $\mathrm{OH}$ stretching FTIR spectra of $\mathrm{F}$ expanded in helium (trace a), $2.5 \% \mathrm{O}$ in helium (trace c) and $15 \% \mathrm{O}$ in helium (trace e). A stagnation pressure of $p_{\mathrm{s}}=0.75 \mathrm{bar}$ was used for all shown spectra. (FF)-corrected difference spectra are included (traces b and d). All spectra were scaled to matching height of the monomer band at $3570 \mathrm{~cm}^{-1}$. Wavenumbers and (tentative) labels are provided.

The $\mathrm{FN}_{\mathrm{H}}$ band at $3553(2) \mathrm{cm}^{-1}$ is observed after addition of $2.5 \% \mathrm{~N}$ (traces $\mathrm{c}$ and d). Higher $\mathrm{N}$ admixture (15\%, traces e and $\mathrm{f}$ ) leads to broadening of the band towards lower wavenumbers and a small shoulder, indicative of complexation by multiple $\mathrm{N}$ molecules and subsequent cooperativity-induced enhanced downshifts. This is supported by experiments in bulk $\mathrm{N}$ matrix ${ }^{160}$ where the monomer band at $3528 \mathrm{~cm}^{-1}$ yields a gasto-matrix shift of $42(2) \mathrm{cm}^{-1}$, clearly exceeding the $17(4) \mathrm{cm}^{-1}$ shift induced by single $\mathrm{N}$ coordination.

Similarly, the OD stretching vibration of HCOOD is shifted from $2631(2) \mathrm{cm}^{-1}$ to $2620(2) \mathrm{cm}^{-1}$ (traces g to $\mathrm{l}$ ). The monomer band centre is in excellent agreement with the $2631.37 \mathrm{~cm}^{-1}$ found in He nanodroplets. ${ }^{204}$ OD stretching in a $\mathrm{N}$ matrix ${ }^{160}$ of $2603 \mathrm{~cm}^{-1}$ results in a gas-to-matrix shift of $31(2) \mathrm{cm}^{-1}$, again surpassing the $11(4) \mathrm{cm}^{-1}$ downshift found for single $\mathrm{N}$ coordination. 


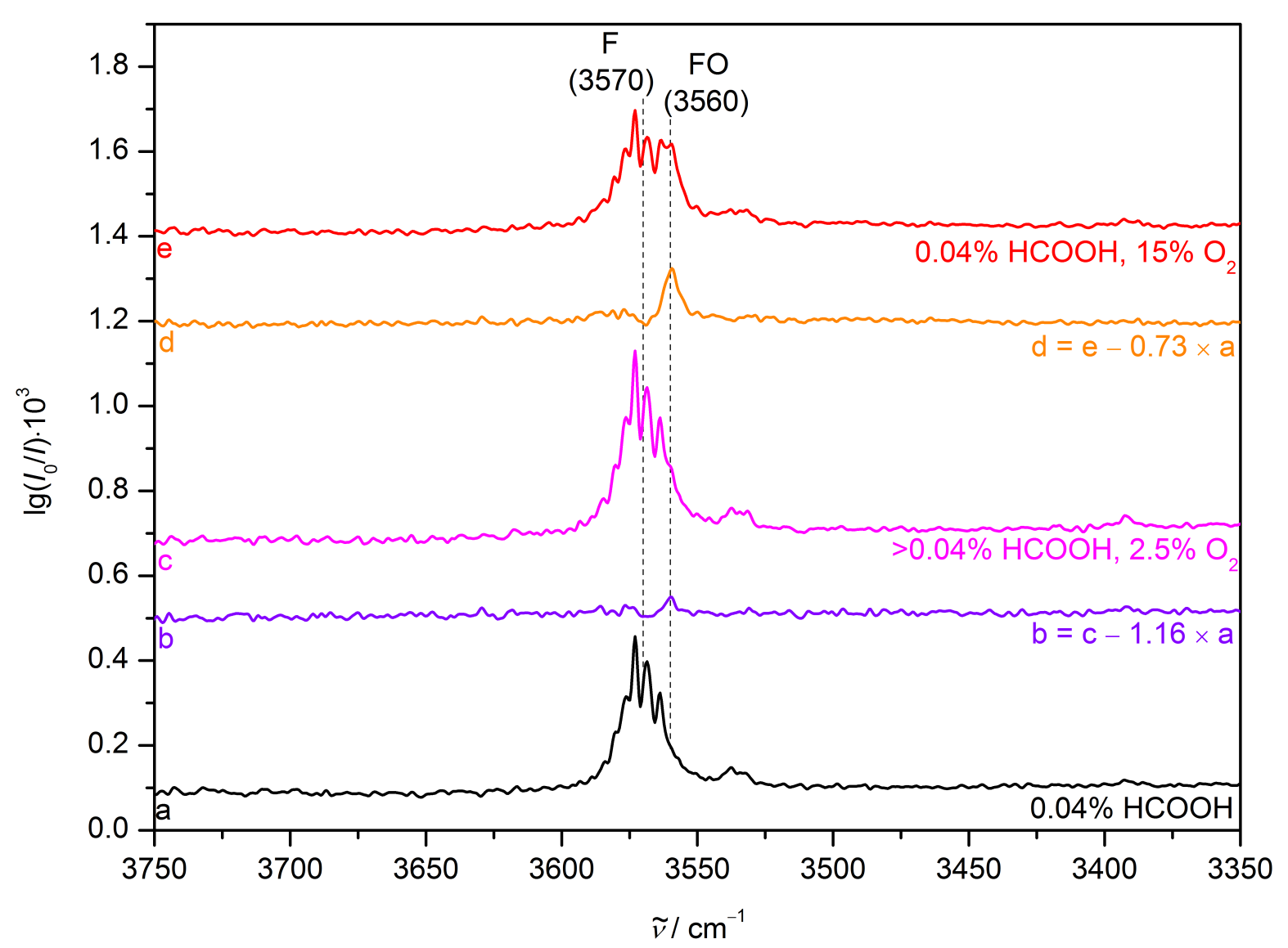

Figure 2.6.: $\mathrm{OH}$ stretching FTIR spectra of $\mathrm{F}$ expanded in helium (trace a), $2.5 \% \mathrm{O}$ in helium (trace c) and $15 \% \mathrm{O}$ in helium (trace e). A stagnation pressure of $p_{\mathrm{s}}=0.75$ bar was used for all shown spectra. Monomer-corrected difference spectra are included (traces $\mathrm{b}$ and $\mathrm{d}$ ). Wavenumbers and (tentative) labels are provided.

\subsubsection{Formic Acid Oxygen}

FTIR spectra with $\mathrm{O}$ instead of $\mathrm{N}$ added to expanded gas mixtures are shown in Figure 2.5. Observed effects closely mirror the findings after $\mathrm{N}$ addition. The mixed FO dimer band appears at $3560(2) \mathrm{cm}^{-1}\left(\mathrm{FN}_{\mathrm{H}}: 3553(2) \mathrm{cm}^{-1}\right)$ with $15 \% \mathrm{O}$ (trace e) and as positive contribution in the $(\mathrm{FF})$-corrected difference spectrum (trace $\mathrm{d}$ ). Monomer depletion induced by $\mathrm{O}$ complexation results in somewhat lower intensities of the $\mathrm{F}(\mathrm{FF})$ band at $3258 \mathrm{~cm}^{-1}$ (trace $\mathrm{d}$ ). In the spectrum with $2.5 \% \mathrm{O}$ (trace c), monomer depletion is compensated by a slightly larger F concentration, resulting in similar homotrimer band intensities in traces $\mathrm{c}$ and $\mathrm{a}$. This accidental increase in $\mathrm{F}$ concentration is also indicated by the factor of 1.88 required for calculating the (FF)-corrected difference spectrum (trace b), significantly exceeding the expected value of about 1 when assuming that the concentration is about the same for measurements a and c.

More detailed spectra of monomer and binary dimer $\mathrm{OH}$ stretching vibrations are depicted in Figure 2.6. Similar to the N case, the FO band broadens to lower wavenumbers 


\section{Carboxylic Acids}

upon increased $\mathrm{O}$ addition of $15 \%$ (traces $\mathrm{d}$ and e) compared to lower $\mathrm{O}$ admixture of $2.5 \%$ (traces $\mathrm{b}$ and $\mathrm{c}$ ), indicating multiple $\mathrm{O}$ coordination. The O-induced downshift of $10(4) \mathrm{cm}^{-1}$ is lower than the corresponding value of $17(4) \mathrm{cm}^{-1}$ for $\mathrm{N}$ complexation. Without complementary quantum chemical calculations, an assignment of the vibrational band to a specific structure is not possible, although the significant $\mathrm{OH}$ stretching downshift indicates $\mathrm{O}$ binding at the hydroxyl proton.

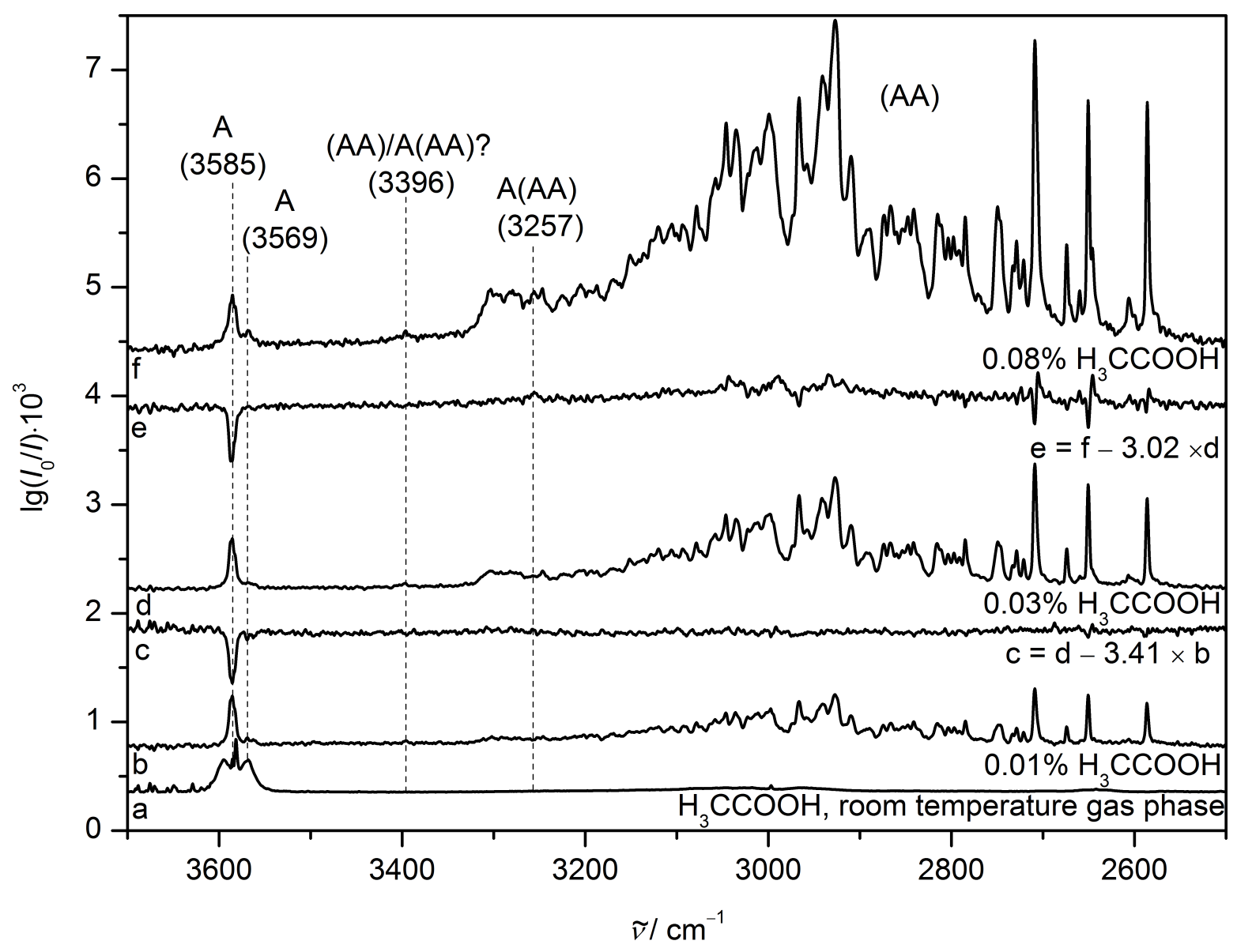

Figure 2.7.: OH stretching FTIR spectra of A (reprinted with adaptations from reference 157 licensed under CC BY 4.0) expanded in helium with increasing concentration from bottom to top (traces $\mathrm{b}, \mathrm{d}$ and $\mathrm{f}$ ). A stagnation pressure of $p_{\mathrm{s}}=0.75$ bar was used for all shown spectra. (AA)-corrected difference spectra (traces c and e) and a room temperature gas phase spectrum (trace a) are included. All spectra were scaled to matching height of the monomer band at $3585 \mathrm{~cm}^{-1}$. Wavenumbers and (tentative) labels are provided.

\subsubsection{Acetic Acid}

$\mathrm{OH}$ stretching spectra of A expanded in helium show growing cluster contributions in the wavenumber region from 3400 to $2500 \mathrm{~cm}^{-1}$ with increasing A concentrations (Figure 


\section{Carboxylic Acids}

2.7, from trace b over d to f) in excellent agreement with previous work. ${ }^{181,183}$ Analogous to $\mathrm{F}$ spectra, the monomer $\mathrm{OH}$ stretching vibration is found at $3585 \mathrm{~cm}^{-1}$ as negative feature in (AA)-corrected difference spectra (traces c and e), while the very weak positive band in the difference spectrum between the highest and intermediate concentrations (trace e) at $3257 \mathrm{~cm}^{-1}$ is assigned to the $\mathrm{A}(\mathrm{AA})$ trimer. The band is much smaller than the corresponding $\mathrm{F}(\mathrm{FF})$ contribution and embedded in the broad $\mathrm{OH}$ stretching spectrum of (AA). An assignment of the additional weak feature at $3396 \mathrm{~cm}^{-1}$ to either $(\mathrm{AA})$ or $\mathrm{A}(\mathrm{AA})$ requires further measurements at higher $\mathrm{A}$ concentrations.

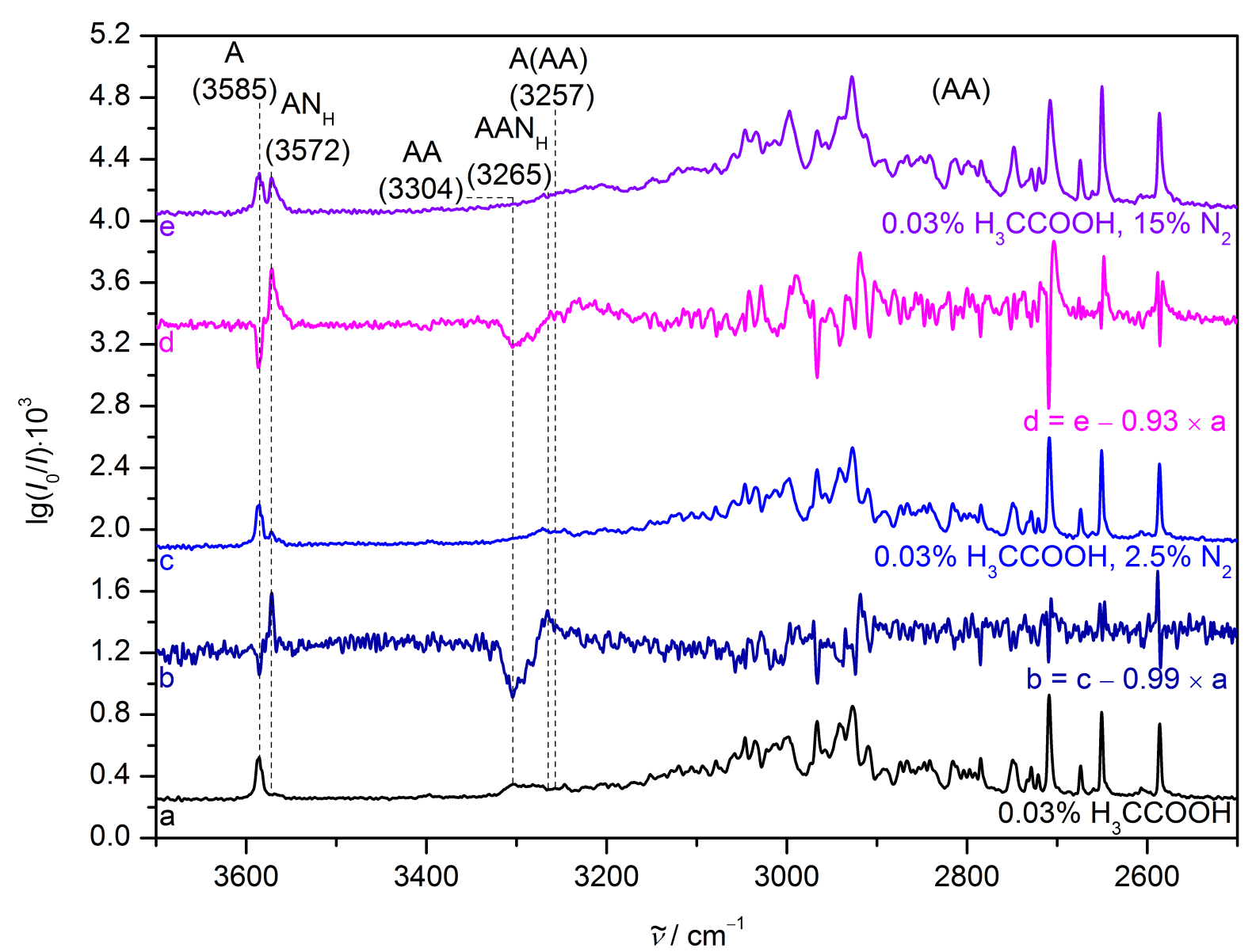

Figure 2.8.: OH stretching FTIR spectra of A (reprinted with adaptations from reference 157 licensed under CC BY 4.0) expanded in helium (trace a), $2.5 \% \mathrm{~N}$ in helium (trace c) and $15 \% \mathrm{~N}$ in helium (trace e). A stagnation pressure of $p_{\mathrm{s}}=0.75$ bar was used for all shown spectra. (AA)-corrected difference spectra are included (traces b and d). All spectra were scaled to matching height of the monomer band at $3585 \mathrm{~cm}^{-1}$. Wavenumbers and (tentative) labels are provided. 


\section{Carboxylic Acids}

\subsubsection{Acetic Acid Nitrogen}

The influence of $\mathrm{N}$ addition on $\mathrm{A}$ is shown in Figure 2.8. Monomer complexation by $\mathrm{N}$ is observed at $3572 \mathrm{~cm}^{-1}$ as positive contribution in (AA)-corrected difference spectra (traces b and d). A vibration around $3304 \mathrm{~cm}^{-1}$ is shifted to about $3265 \mathrm{~cm}^{-1}$, as indicated by negative and corresponding positive features in difference spectra. The dimer origin proven by absence of this band in (AA)-corrected difference spectra of expansions of $\mathrm{A}$ in helium (Figure 2.7, traces $\mathrm{c}$ and e) allows for the assignment of this band to the open AA dimer, as the cyclic (AA) isomer does not provide an attractive binding site for $\mathrm{N}$ and its known vibrational features ${ }^{183}$ are not affected by $\mathrm{N}$ addition. The absence of a corresponding open dimer band in $\mathrm{F}$ is difficult to rationalise. While a FTIR imaging investigation ${ }^{102}$ found a much lower abundance of FF than $\mathrm{F}(\mathrm{FF})$ under expansion conditions similar to this work, kinetic trapping of the open dimer appears to occur more readily for A. This experimental evidence of the persistent band at $3304 \mathrm{~cm}^{-1}$ contradicts indications from calculations and matrix isolation studies of a lower barrier for conversion from open to cyclic dimers for A than F. ${ }^{198}$

$15 \% \mathrm{~N}$ in the expansion (trace e) leads to $\mathrm{N}$ coating of (AA), as evidenced by some broadening and small wavenumber shifts (trace d), similarly observed after Ar coating. ${ }^{181}$ Additionally, the AAN band at $3265 \mathrm{~cm}^{-1}$ is further downshifted, hinting at multiple $\mathrm{N}$ coordination of the dangling $\mathrm{OH}$ group, while the wavenumber in a $\mathrm{N}$ matrix ${ }^{205}$ between 3207 and $3187 \mathrm{~cm}^{-1}$ is again not reached by gas phase coordination with the matrix host.

More detailed spectra of $\mathrm{A}$ and its binary $\mathrm{N}$ aggregate are shown in Figure 2.9. Similar to the $\mathrm{F}$ case, a weak feature at lower wavenumber than the monomer fundamental is found at $3569 \mathrm{~cm}^{-1}$ (trace b), possibly the CO stretching overtone. This feature is also observed with similar intensity at $3541 \mathrm{~cm}^{-1}$ for $\mathrm{H}_{3} \mathrm{CCOOD}$ (trace a), ruling out a strong Fermi resonance with the $\mathrm{OH}$ stretching fundamental. Calculating the monomer band centre position according to Equation 2.1 (see Table A.6 in appendix A.2 for further details) therefore yields a value of $3585(4) \mathrm{cm}^{-1}$ in excellent agreement with $3585.34 \mathrm{~cm}^{-1}$ obtained by high resolution gas phase measurements. ${ }^{206}$

The $\mathrm{AN}_{\mathrm{H}}$ band centre is observed at $3572(2) \mathrm{cm}^{-1}$ (traces c and $\mathrm{d}$ ), while admixture of $15 \% \mathrm{~N}$ results in multiple $\mathrm{N}$ coordination again, broadening the band to lower wavenumbers (traces e and $\mathrm{f}$ ). The $\mathrm{N}$ matrix ${ }^{160}$ band position of $3548(4) \mathrm{cm}^{-1}$ is again further downshifted from the free monomer than any complex formed in gas phase or supersonic expansion.

The OD stretching vibration of mono-deuterated A (traces $\mathrm{g}$ to $\mathrm{l}$ ) is downshifted from $2644(2) \mathrm{cm}^{-1}$ to $2634(2) \mathrm{cm}^{-1}$ when $\mathrm{N}$ is added. The jet monomer band position again agrees with the high resolution gas phase value ${ }^{206}$ of $2644.24 \mathrm{~cm}^{-1}$, while being surrounded by $\mathrm{OH}$ stretching bands of the mixed cyclic dimer containing a deuterated and non-deuterated acid, denoted as $(\mathrm{AA})^{\mathrm{m}}$ at $2784 \mathrm{~cm}^{-1}, 2705 \mathrm{~cm}^{-1}, 2653 \mathrm{~cm}^{-1}, 2583 \mathrm{~cm}^{-1}$ and $2500 \mathrm{~cm}^{-1}$, respectively. Confirmation of this assignment was achieved by adding $10 \%$ of non-deuterated A to an expansion of deuterated A as shown in Figure 2.10. The similar magnitudes of hydrogen bond induced downshift enhanced by Fermi resonances in the dimer and deuteration mass effect in the monomer result in this atypical OH/OD 


\section{Carboxylic Acids}

spectral overlap.
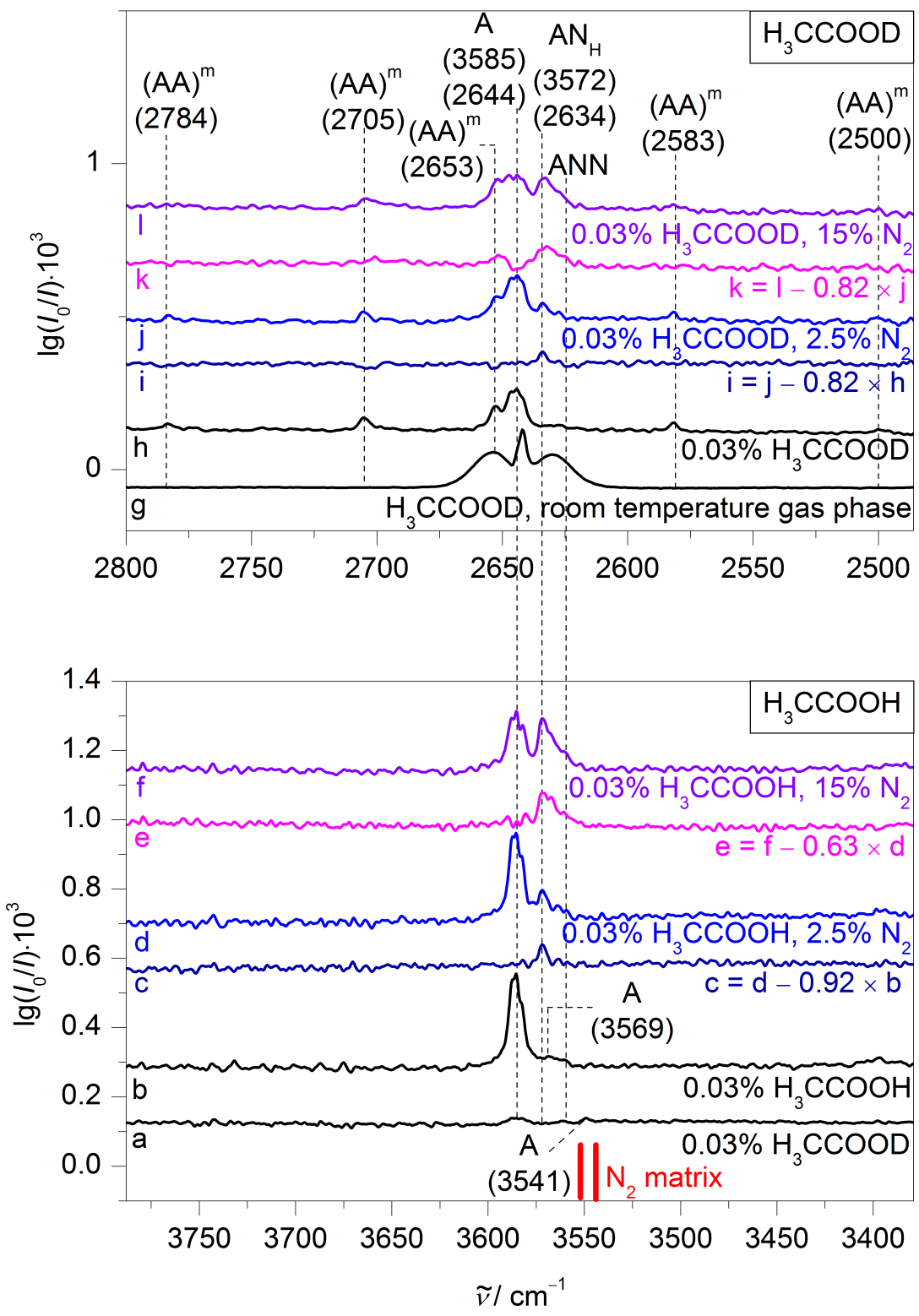

Figure 2.9.: $\mathrm{OH}$ and OD stretching FTIR spectra (reprinted from reference 157 licensed under CC BY 4.0) of acetic acid- $d_{1}$ (upper box) and acetic acid- $d_{0}$ (lower box except for trace a) expanded in helium (traces a, b and h), $2.5 \% \mathrm{~N}$ in helium (traces $\mathrm{d}$ and $\mathrm{j}$ ) and $15 \% \mathrm{~N}$ in helium (traces $\mathrm{f}$ and $\mathrm{l}$ ). A stagnation pressure of $p_{\mathrm{s}}=0.75$ bar was employed for all shown spectra. Monomer-corrected difference spectra (traces c, e, i and k) and a room temperature gas phase spectrum (trace g, scaled by a factor of 0.1 ) are included. The OD wavenumber scale is compressed by a factor of 0.77 and shifted by $148 \mathrm{~cm}^{-1}$ such that the $\mathrm{A}$ and $\mathrm{AN}_{\mathrm{H}}$ signals match for the two isotopologues. Wavenumbers and (tentative) labels are provided. The mixed cyclic dimer of $\mathrm{H}_{3} \mathrm{CCOOH}$ and $\mathrm{H}_{3} \mathrm{CCOOD}$ is denoted as $(\mathrm{AA})^{\mathrm{m}}$. Red bars at the bottom indicate the band positions of $\mathrm{A}$ in a bulk nitrogen matrix. ${ }^{160}$ 


\section{Carboxylic Acids}

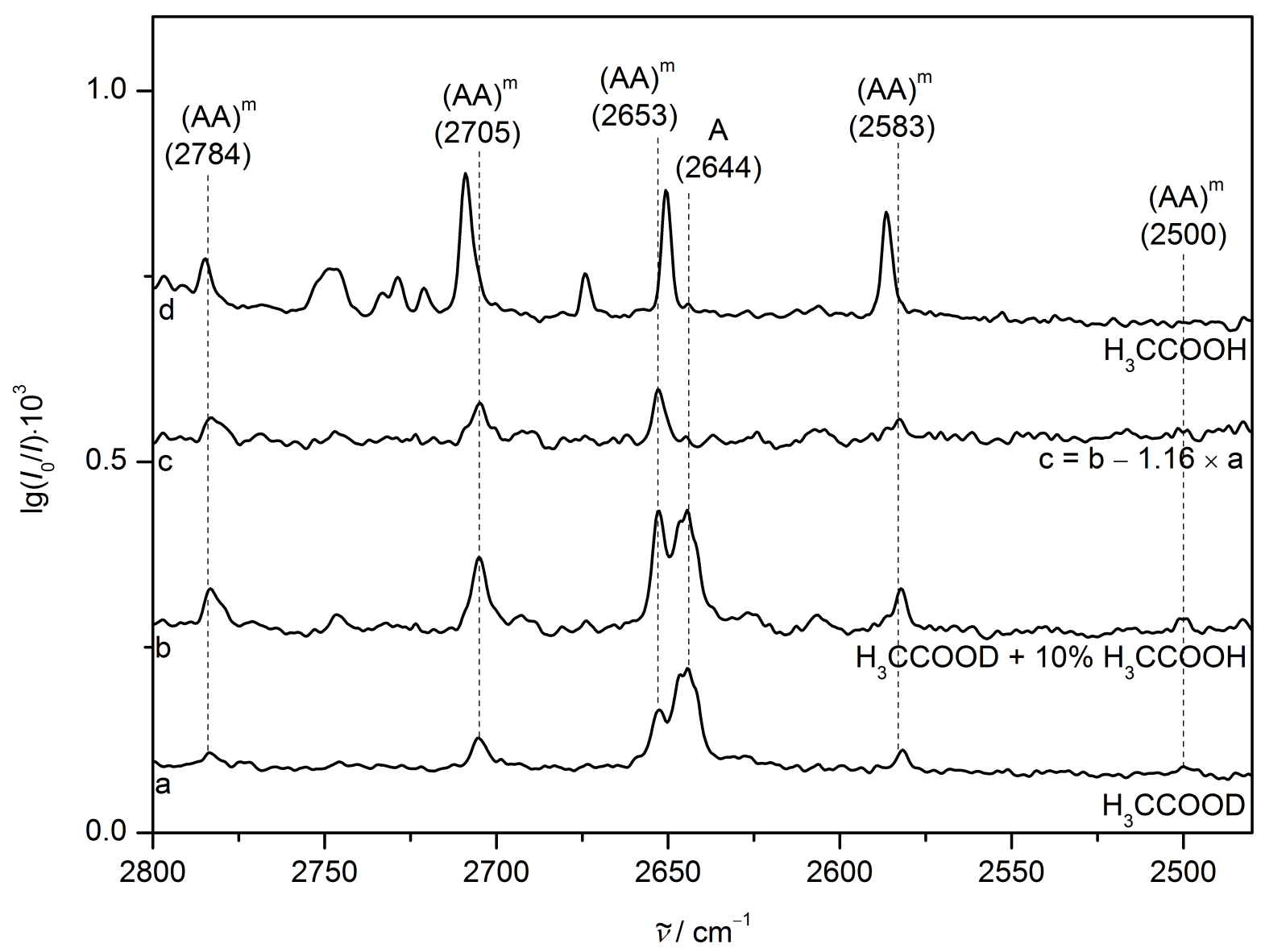

Figure 2.10.: $\mathrm{OH}$ and OD stretching FTIR spectra (reprinted from reference 157 licensed under CC BY 4.0) of $\mathrm{H}_{3} \mathrm{CCOOD}$ (trace a), $\mathrm{H}_{3} \mathrm{CCOOD}$ with addition of $10 \% \mathrm{H}_{3} \mathrm{CCOOH}$ (trace $\mathrm{b}$ ) and $\mathrm{H}_{3} \mathrm{CCOOH}$ (trace $\mathrm{d}$ ). A stagnation pressure of $p_{\mathrm{s}}=0.75$ bar was employed for all shown spectra. A difference spectrum corrected for the $\mathrm{H}_{3} \mathrm{CCOOD}$ monomer is included (trace $\mathrm{c}$ ). Wavenumbers and (tentative) labels are provided. The mixed cyclic dimer of $\mathrm{H}_{3} \mathrm{CCOOH}$ and $\mathrm{H}_{3} \mathrm{CCOOD}$ is denoted as $(\mathrm{AA})^{\mathrm{m}}$. The analysis shows that the extra bands marked $(\mathrm{AA})^{\mathrm{m}}$ in the $\mathrm{H}_{3}$ CCOOD spectrum (a) are due to mixed dimers in which one of the acidic OD groups is exchanged by an $\mathrm{OH}$ group.

\subsubsection{Acetic Acid Oxygen}

Spectra of $\mathrm{A}$ with an addition of $\mathrm{O}$ are shown in Figure 2.11. Generally, larger $\mathrm{O}$ than $\mathrm{N}$ admixtures are necessary to achieve spectral effects of similar magnitude, therefore only spectra with $15 \% \mathrm{O}$ were recorded. Paralleling the findings for $\mathrm{N}$ addition, a mixed dimer is found at $3578 \mathrm{~cm}^{-1}$ as positive contribution in the (AA)-corrected difference spectrum (trace b). O coating of (AA) is observed as well as $\mathrm{O}$ complexation of AA, shifting the band at $3304 \mathrm{~cm}^{-1}$ to $3269 \mathrm{~cm}^{-1}$, suggesting coordination of $\mathrm{O}$ at the free $\mathrm{OH}$ groups of $\mathrm{A}$ and AA, respectively. Again, rigorous assignments require complementary quantum 


\section{Carboxylic Acids}

chemical calculations. More detailed spectra of the monomer and mixed AO dimer are depicted in Figure 2.12, showing that multiple $\mathrm{O}$ coordination again broadens the mixed dimer band to lower wavenumbers (trace b). Observed O-induced downshifts for both acids are lower than corresponding $\mathrm{N}$-induced effects, hinting at weaker binding of $\mathrm{O}$ compared to $\mathrm{N}$, which might be linked to the weaker $\left(-1.4(2) \times 10^{-40} \mathrm{C} \mathrm{m}^{2}\right)$ quadrupole moment ${ }^{46}$ which is reduced to $30 \%$ compared to N. ${ }^{45}$

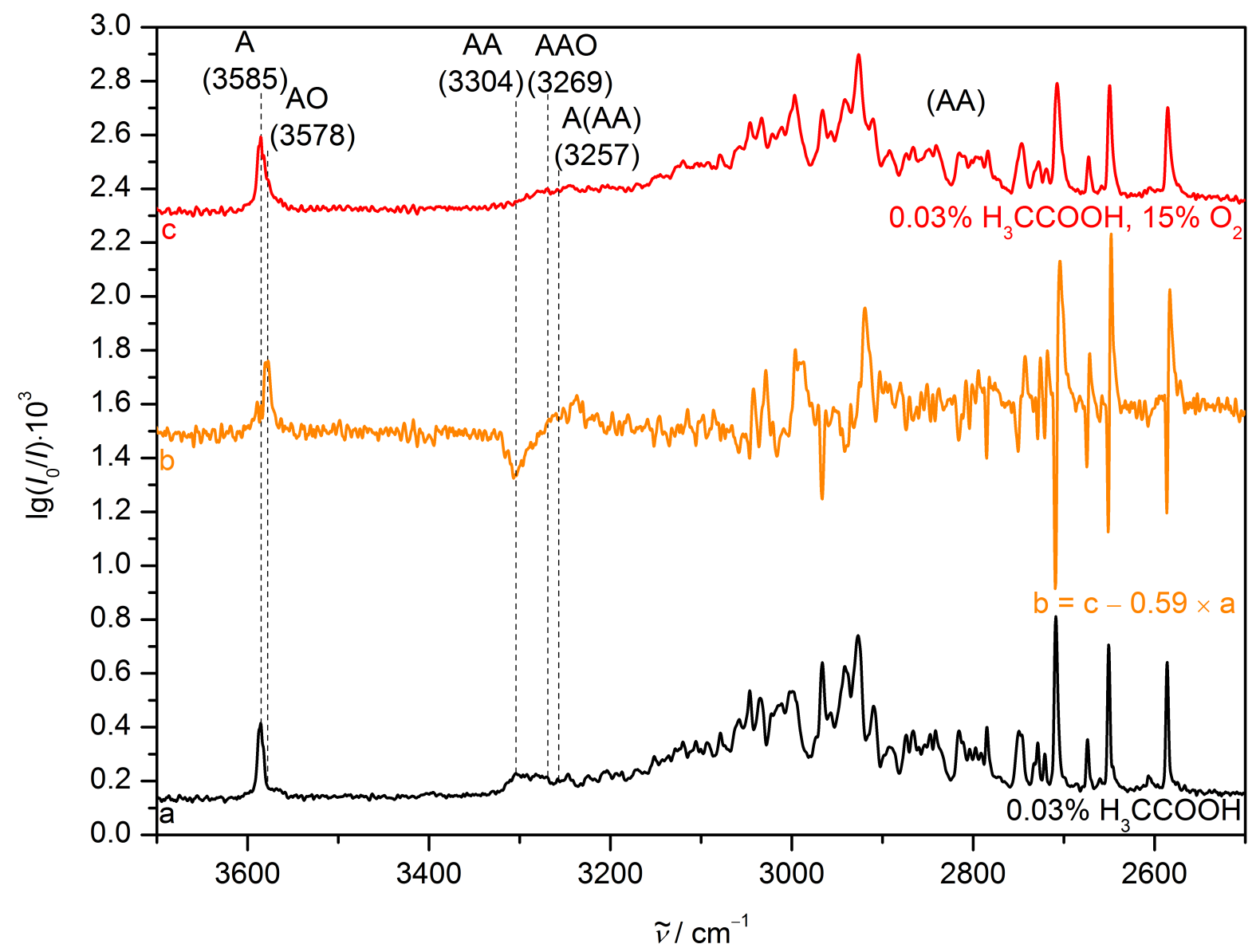

Figure 2.11.: OH stretching FTIR spectra of A expanded in helium (trace a) and $15 \% \mathrm{O}$ in helium (trace c). A stagnation pressure of $p_{\mathrm{s}}=0.75$ bar was used for all shown spectra. An (AA)-corrected difference spectrum is included (trace b). All spectra were scaled to matching height of the monomer band at $3585 \mathrm{~cm}^{-1}$. Wavenumbers and (tentative) labels are provided.

\subsection{Benchmarking Quantum Chemical Calculations}

One of the largest obstacles for direct comparison of theoretically predicted vibrational wavenumbers and experimental band positions is vibrational anharmonicity. ${ }^{207}$ Rou- 


\section{Carboxylic Acids}

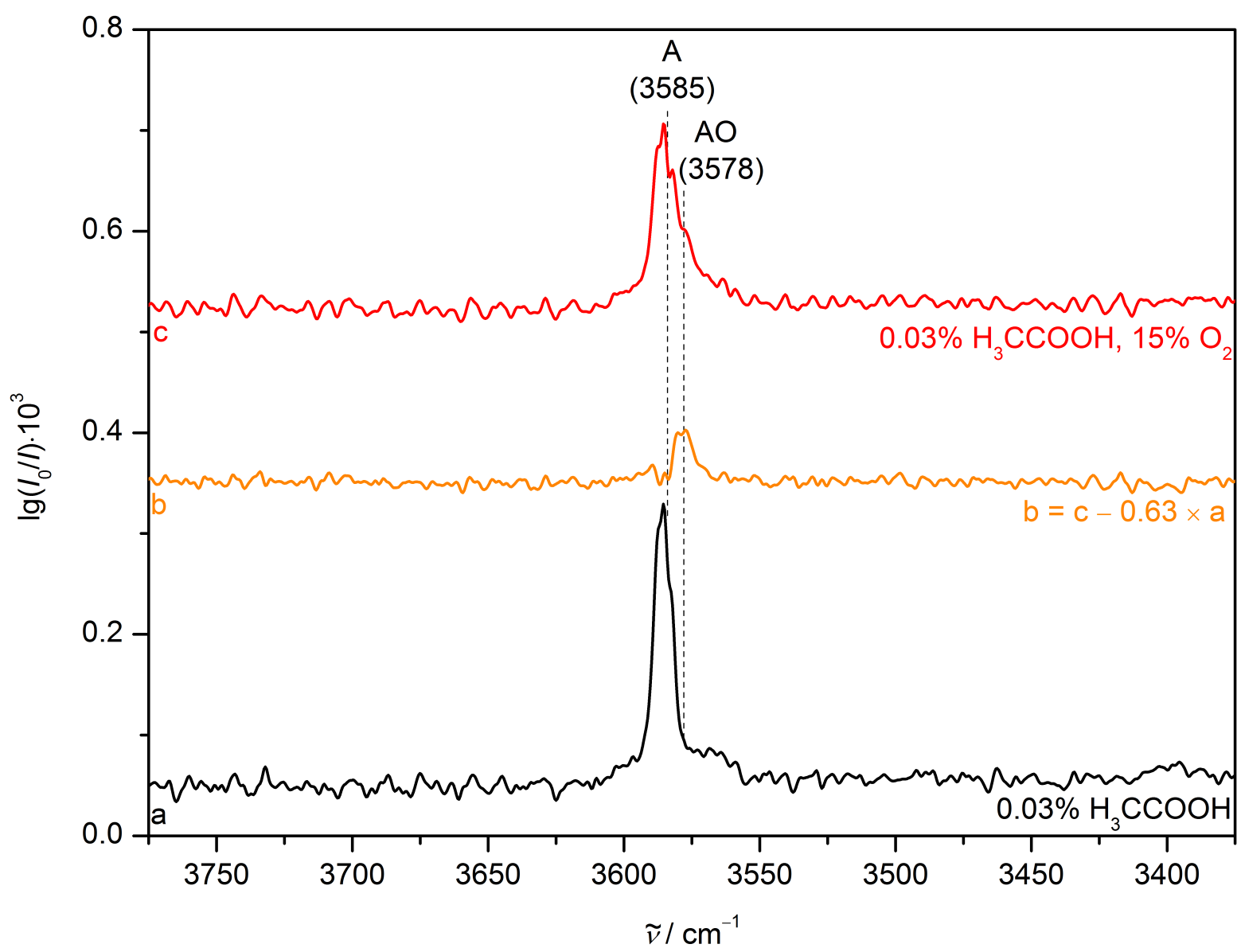

Figure 2.12.: $\mathrm{OH}$ stretching FTIR spectra of A expanded in helium (trace a) and $15 \%$ $\mathrm{O}$ in helium (trace c). A stagnation pressure of $p_{\mathrm{s}}=0.75$ bar was used for all shown spectra. A monomer-corrected difference spectrum is included (trace b). Wavenumbers and (tentative) labels are provided.

tinely, quantum chemical calculations are performed within the double harmonic approximation. Apart from electric harmonicity, which assumes a linear course of the dipole moment function along the vibrational coordinate, mechanical harmonicity leads to a description of vibrational modes of a polyatomic system by completely decoupled quadratic potential functions. The most simple method which goes beyond this approximation is to include linear two-mode and quadratic one-mode contributions in secondorder perturbation theory with inclusion of up to quartic vibrational potentials. ${ }^{208}$ Then, the sum of vibrational term energies $G\left(v_{i}\right)$ in a system with $N$ normal modes is given by Equation 2.2. ${ }^{99}$

$$
G\left(\mathrm{v}_{1}, \mathrm{v}_{2}, \ldots, \mathrm{v}_{N}\right)=\sum_{i}^{N} \omega_{i}\left(\mathrm{v}_{i}+\frac{1}{2}\right)+\sum_{i \leq j}^{N, N} x_{i, j}\left(\mathrm{v}_{i}+\frac{1}{2}\right)\left(\mathrm{v}_{j}+\frac{1}{2}\right)
$$




\section{Carboxylic Acids}

The harmonic contribution is linearly dependent on $\omega_{i}$, the harmonic wavenumber of mode $i$, and the corresponding vibrational quantum number $\mathrm{v}_{i}$. The non-quadratic part of the vibrational potential is approximately accounted for by the diagonal anharmonicity constants obtained for $i=j$ and the off-diagonal constants $x_{i, j}$ if $i \neq j$ describe pairwise couplings between potentials of different normal modes. The terms diagonal and off-diagonal refer to the positions of the anharmonicity constants after arrangement in a matrix. The wavenumber $(n \tilde{\nu})_{i}$ for a transition of mode $i$ from the vibrational ground state $\mathrm{v}_{i}=0$ to any given excited state $\mathrm{v}_{i}=n$ is therefore described by Equation 2.3 .

$$
(n \tilde{\nu})_{i}=n \omega_{i}+\left(n^{2}+n\right) x_{i, i}+\frac{n}{2} \sum_{j \neq i} x_{i, j}
$$

For fundamental transitions from $\mathrm{v}_{i}=0$ to $\mathrm{v}_{i}=1$ this results in Equation 2.4, while first overtone transitions from $\mathrm{v}_{i}=0$ to $\mathrm{v}_{i}=2$ are given by Equation 2.5.

$$
\begin{gathered}
\tilde{\nu}_{i}=\omega_{i}+2 x_{i, i}+\frac{1}{2} \sum_{j \neq i} x_{i, j} \\
(2 \tilde{\nu})_{i}=2 \omega_{i}+6 x_{i, i}+\sum_{j \neq i} x_{i, j}
\end{gathered}
$$

Wavenumbers for combination bands $\tilde{\nu}^{(1,1) \leftarrow(0,0)}$ with simultaneous excitation of two modes $i$ and $j$ and resulting quantum number changes from $\mathrm{v}_{i}=0$ to $\mathrm{v}_{i}=1$ and $\mathrm{v}_{j}=0$ to $\mathrm{v}_{j}=1$ are obtained according to Equation 2.6.

$$
\tilde{\nu}^{(1,1) \leftarrow(0,0)}=\tilde{\nu}_{i}+\tilde{\nu}_{j}+x_{i, j}
$$

Therefore, diagonal anharmonicity constants can be derived from the experimental wavenumbers of the fundamental and first overtone transitions, while the off-diagonal constants require both fundamental transitions and their respective combination bands.

Two fundamentally different approaches for applying anharmonic vibrational spectra as benchmarks for electronic structure predictions were used in this work. The first option where the full burden is put on the theory side and anharmonic vibrational frequencies are directly calculated may be labelled as 'theory meets experiment'. Of the various available methods for theoretical anharmonicity treatment, second order vibrational perturbation theory ${ }^{148}$ (VPT2) as implemented in Gaussian09 is operable with an acceptable amount of computational effort. A major drawback of calculating local quartic force constants based on a single minimum energy structure is a poor description of large amplitude motions. An acceptable performance is therefore expected for stiff and rigid molecules such as the F monomer, while $\mathrm{N}$ complexation or the mobile methyl rotor in A might cause a sharp decline in the quality of VPT2 predictions (see Table 2.1). Overcoming this drawback by using an analytical representation of the BornOppenheimer potential energy surface ${ }^{209-212}$ and variational approaches for calculating anharmonic vibrational energy levels is beyond the scope of this work because of the 


\section{Carboxylic Acids}

required computational costs, but has been explored by research groups which focus on theoretical chemistry for $\mathrm{F}^{209,213}$ and $(\mathrm{FF}) .{ }^{210,214}$

The second approach follows an 'experiment meets theory' philosophy and involves the estimation of experimental harmonic vibrational wavenumbers, which can be directly compared to standard harmonic calculations. This requires experimental measurements and assignments of several transitions which may be difficult to obtain because of low intensities such as overtones and combination bands or conservative estimates with large error bars where experimental data is incomplete. Not putting the full burden on the theory side therefore leads to larger experimental error bounds, but this approach allows for testing of more elaborate levels of electronic structure theory.

\subsubsection{Binding Energy of (FF)}

(FF) has been used as a model system in numerous quantum chemical studies, ${ }^{191,215-224}$ especially since its dissociation energy of $D_{0}=59.5(5) \mathrm{kJ} \mathrm{mol}^{-1}$ has been experimentally determined. ${ }^{97}$ This experimental value can serve as a direct benchmark for high level approaches from the literature and this work (see Tables 2.2 and 2.3). Energies are a particularly suitable experimental observable for benchmarking purposes and corresponding predictions are readily available from standard harmonic calculations, even though they have to include zero-point vibrational corrections. Anharmonic effects on these corrections tend to display a favourable error cancellation and are therefore usually negligible. ${ }^{51}$

Calculations from this work at the B3LYP-D3(BJ)/def2-QZVP level show that the dissociation energy is overestimated by $6.7(5) \mathrm{kJ} \mathrm{mol}^{-1}$, which is further increased by $1.1 \mathrm{~kJ} \mathrm{~mol}^{-1}$ after application of anharmonic VPT2 corrections. However, at the harmonic LCCSD( $\left.\mathrm{T}^{*}\right)$-F12/VDZ-F12(int) level, $D_{0}$ is underestimated by a margin of $2.8(5) \mathrm{kJ} \mathrm{mol}^{-1}$. A combination of this harmonic contribution and the B3LYP-D3 anharmonicity correction therefore only misses the experimental reference by $1.7(5) \mathrm{kJ} \mathrm{mol}^{-1}$. A similar approach from the literature ${ }^{225}$ which combines harmonic canonical CCSD(T) predictions extrapolated to the basis set limit as well as anharmonic corrections at the MP2/aVDZ level reaches an even better agreement of $0.3(5) \mathrm{kJ} \mathrm{mol}^{-1}$.

\subsubsection{Harmonic Vibrational Wavenumbers of $F$ and $F N_{H}$}

For the extensively studied F, ${ }^{102,186,201,203,226-230}$ all fundamental band centre positions have been determined experimentally as well as the first $\mathrm{OH}$ stretching overtone and all binary combination bands with the $\mathrm{OH}$ stretching fundamental (see Table A.7 in appendix A.2). Thus, the complete set of anharmonic coupling constants $x_{1, j}$ can be extracted from experiment. The conservatively estimated experimental error bar of each anharmonictiy constant amounts to about $1 \mathrm{~cm}^{-1}$, which is then doubled to account for more complex anharmonic effects not considered in the perturbation theory approach such as possible unresolved Fermi resonances. Consequently, the anharmonic fundamental band position of $\tilde{\nu}_{\mathrm{OH}}=3570(2) \mathrm{cm}^{-1}$ needs to be corrected by twice the diagonal 


\section{Carboxylic Acids}

anharmonicity of $2 x_{1,1}=-172(4) \mathrm{cm}^{-1}$ and half of the sum over all off-diagonal constants of $0.5 \sum_{j \neq i} x_{i, j}=22(8) \mathrm{cm}^{-1}$, resulting in a total anharmonicity correction of $194(12) \mathrm{cm}^{-1}$ and an estimated harmonic band position of $\omega_{\mathrm{OH}}(\mathrm{F})=3764(14) \mathrm{cm}^{-1}$. The fraction of off-diagonal contributions amounts to approximately $10 \%$ of the total anharmonicity. In more typical cases of less well-studied molecules, $10 \%$ of the relatively easily accessible diagonal anharmonicity will therefore be assumed as an appropriate error margin to account for usually unknown off-diagonal anharmonicity constants.

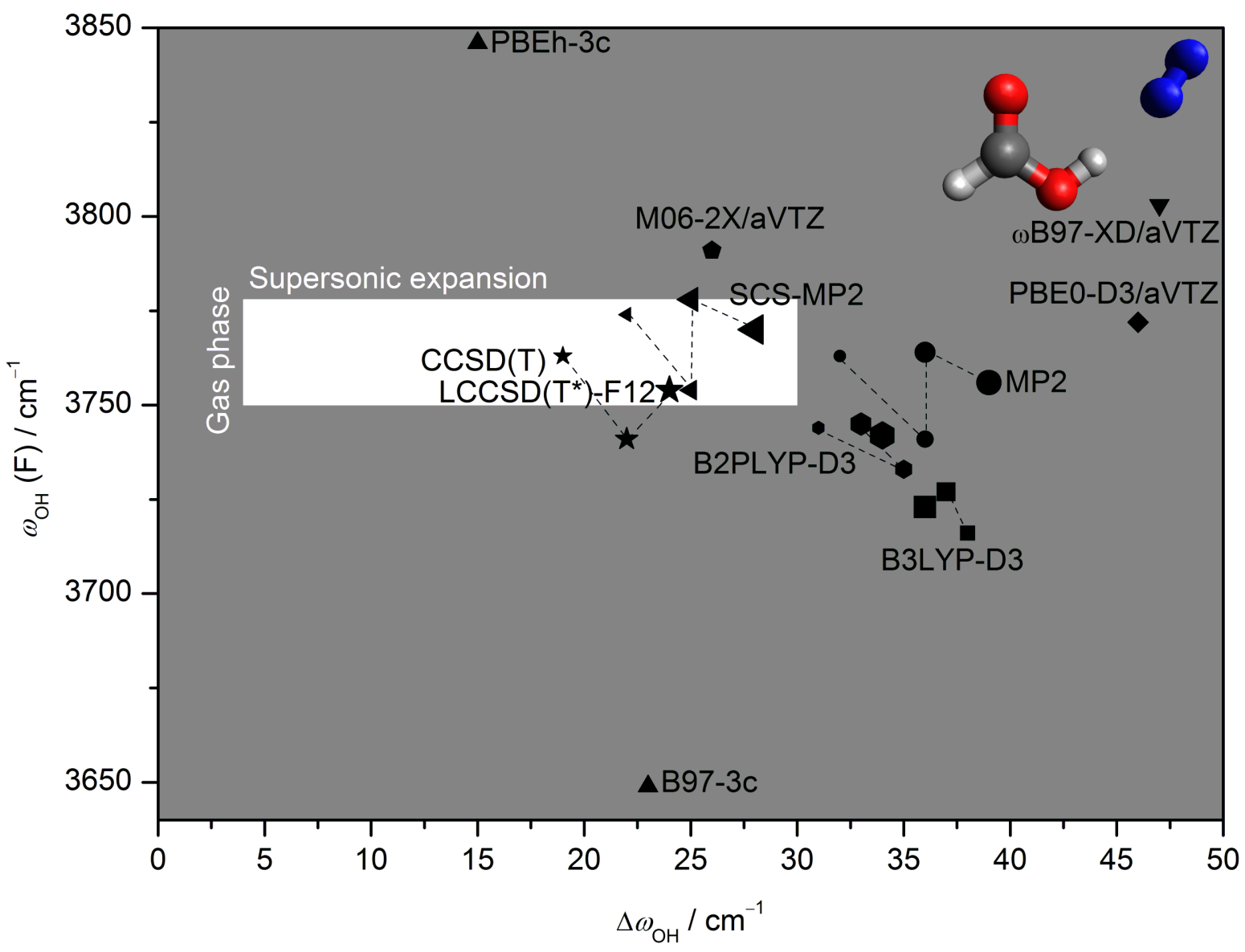

Figure 2.13.: Theoretically predicted harmonic $\mathrm{OH}$ stretching band position of $\mathrm{F}$ $\left(\omega_{\mathrm{OH}}(\mathrm{F})\right)$ plotted against the harmonic downshift $\left(\Delta \omega_{\mathrm{OH}}\right)$ from $\mathrm{F}$ to $\mathrm{FN}_{\mathrm{H}}$ for different computational methods implemented in Gaussian09 and ORCA 4.0 (reprinted from reference 157 licensed under CC BY 4.0). Symbol size qualitatively encodes the size of the basis set (see Table A.8 in appendix A.2). The experimental harmonic monomer band position was approximately derived from the fundamental transitions, the first $\mathrm{OH}$ stretching overtone and various combination bands (see Table A.7 in appendix A.2). Experimental wavenumber downshifts are anharmonic. Harmonic values are assumed to deviate from them by up to $50 \%$ due to anharmonic effects for hydrogen bond-induced shifts. The zone of incompatibility with experiment is dark grey, predictions within the white area are compatible with experiment. 


\section{Carboxylic Acids}

Theoretical VPT2 predictions for the $\mathrm{OH}$ stretching anharmonicity constants of $\mathrm{F}$ at various levels of electronic structure theory are listed in Table 2.4. With the exception of M06-2X, all tested methods predict the total anharmonicity and the fraction of diagonal anharmonicity very well. This is partly due to fortuitous error cancellation, as systematic underestimation of couplings to $\tilde{\nu}_{3,4,6,8}$ compensates for overestimation of coupling to the $\mathrm{OH}$ bending mode $\tilde{\nu}_{5}$, but generally VPT2 seems to perform well for a localised hydride stretching mode in a rigid molecule like $\mathrm{F}$.

Estimating the harmonic N-induced downshift from the experimental anharmonic value of $\Delta \tilde{\nu}_{\mathrm{OH}}=17(4) \mathrm{cm}^{-1}$ is more challenging, as neither the first overtone nor any combination bands of $\mathrm{FN}_{\mathrm{H}}$ are known. For example, the $110 \mathrm{~cm}^{-1}$ anharmonic downshift from methanol monomer to dimer differs from the estimated harmonic value ${ }^{231}$ of about $121 \mathrm{~cm}^{-1}$ by $10 \%$. In this case, the diagonal anharmonicity contribution ${ }^{30}$ to the total downshift of $+13 \mathrm{~cm}^{-1}$ or about $12 \%$ is overcompensated by large off-diagonal additions amounting to about $-25 \mathrm{~cm}^{-1}$ or $23 \%$. However, directions and magnitudes of these anharmonic contributions to the downshift may differ significantly from this example in other cases. ${ }^{34}$ To establish correct bounds and account for possible worst case scenarios in both directions if no anharmonicity constants are experimentally known, a conservatively estimated error bar of $50 \%$ of the total downshift is therefore added to the experimental error, resulting in a harmonic downshift of $\Delta \omega_{\mathrm{OH}}=17(13) \mathrm{cm}^{-1}$.

Table 2.4.: Experimental values ${ }^{102,186,201,203,226-230}$ for anharmonic couplings of the $\mathrm{OH}$ stretching vibration (1) to modes $i$ of $F$ compared to theoretically (VPT2) predicted values calculated at the MP2/aVQZ, B2PLYP-D3/aVQZ, B3LYP-D3(BJ)/def2-QZVP, PBE0D3(BJ)/aVTZ, M06-2X/aVTZ and $\omega$ B97-XD/aVTZ levels of approximation. The bottom rows show the total anharmonic correction to mode 1 as well as the fraction of diagonal anharmonicity correction.

\begin{tabular}{|c|c|c|c|c|c|c|c|}
\hline$i$ & MP2 & B2PLYP-D3 & B3LYP-D3 & $\begin{array}{l}x_{1, i} / \mathrm{cm}^{-1} \\
\text { PBE0-D3 }\end{array}$ & M06-2X & $\omega \mathrm{B} 97-\mathrm{XD}$ & Experiment \\
\hline 1 & -84 & -85 & -86 & -83 & -73 & -87 & $-86(2)$ \\
\hline 2 & -4 & -3 & -4 & -3 & -3 & -3 & $-5(2)$ \\
\hline 3 & -1 & -1 & -1 & -1 & -1 & -1 & $-4(2)$ \\
\hline 4 & -5 & -5 & -4 & -4 & -3 & -4 & $-8(2)$ \\
\hline 5 & -20 & -20 & -20 & -19 & -16 & -18 & $-13(2)$ \\
\hline 6 & -3 & -2 & 0 & -2 & -1 & -1 & $-5(2)$ \\
\hline 7 & -5 & -4 & -4 & -4 & -2 & -4 & $-4(2)$ \\
\hline 8 & -1 & -1 & -1 & -1 & -1 & -1 & $-3(2)$ \\
\hline 9 & -3 & -2 & -2 & -3 & -1 & -7 & $-2(2)$ \\
\hline $2 x_{1,1}+0.5 \sum_{i \neq 1} x_{1, i}$ & -190 & -189 & -190 & -185 & -160 & -192 & $-194(12)$ \\
\hline$\frac{2 x_{1,1}}{2 x_{1,1}+0.5 \sum_{i \neq 1} x_{1, i}}$ & 0.89 & 0.90 & 0.90 & 0.90 & 0.91 & 0.90 & 0.89 \\
\hline
\end{tabular}

Harmonic predictions for the $\mathrm{OH}$ stretching frequency of $\mathrm{F}$ and the $\mathrm{N}$-induced spectroscopic downshift in $\mathrm{FN}_{\mathrm{H}}$ at different levels of electronic structure theory are depicted in Figure 2.13 and listed in Table A.8 in appendix A.2. Canonic CCSD(T) predictions are accurate for the monomer wavenumber as well as the downshift with the VTZ basis set. With the larger aVTZ basis set, the monomer wavenumber is underestimated, but 


\section{Carboxylic Acids}

extrapolation to the basis set limit $\left(3761 \mathrm{~cm}^{-1}\right)^{225}$ yields a prediction within the experimental error bars. This is equally true for LCCSD( $\left.\mathrm{T}^{*}\right)$-F12/VDZ-F12(int) predictions and nonlocal explicitly correlated CCSD(T)-F12/VTZ-F12 ${ }^{209}\left(3766 \mathrm{~cm}^{-1}\right)$. SCS-MP2 predictions fulfil both experimental constraints as well with all tested basis set sizes. While PBEh-3c, M06-2X and B97-3c all give correct downshift predictions, the first two overestimate the monomer wavenumber whereas the B97-3c prediction is too low. MP2, B2PLYP-D3, B3LYP-D3, PBE0-D3 and $\omega$ B97-XD predict correct monomer band positions in some cases, but systematically overestimate the hydrogen bond induced downshift.

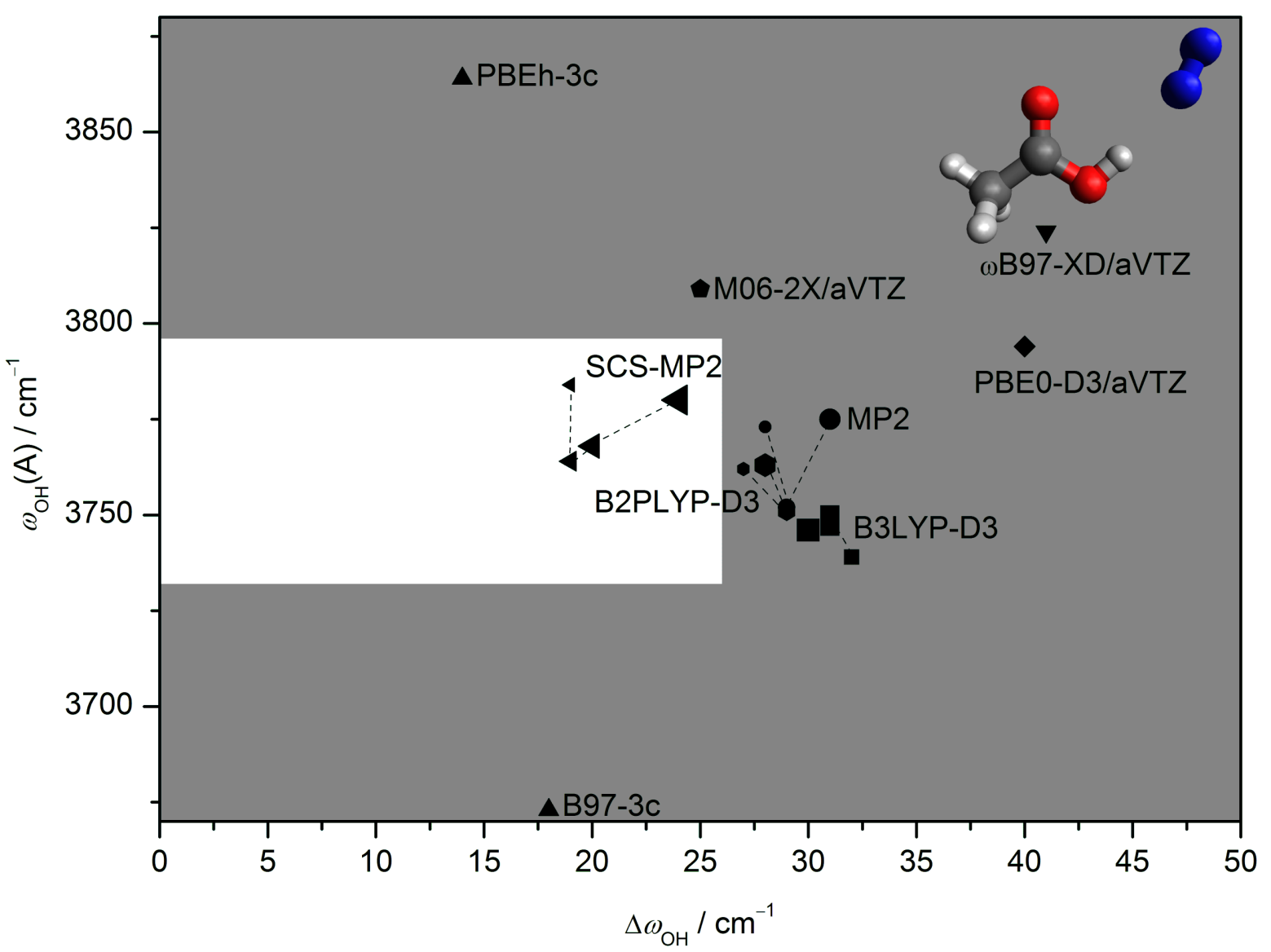

Figure 2.14.: Theoretically predicted harmonic $\mathrm{OH}$ stretching band position of $\mathrm{A}$ $\left(\omega_{\mathrm{OH}}(\mathrm{A})\right)$ plotted against the harmonic downshift $\left(\Delta \omega_{\mathrm{OH}}\right)$ from $\mathrm{A}$ to $\mathrm{AN}_{\mathrm{H}}$ for different computational methods implemented in Gaussian09 and ORCA 4.0 (reprinted from reference 157 licensed under CC BY 4.0). Symbol size qualitatively encodes the size of the basis set (see Table A.8 in appendix A.2). The experimental harmonic monomer band position was approximately derived from the fundamental and first overtone ${ }^{232}$ transitions. Experimental wavenumber differences are anharmonic. Harmonic values are assumed to deviate from them by up to $50 \%$ due to anharmonic effects for hydrogen bond-induced shifts. The zone of incompatibility with experiment is dark grey, predictions within the white area are compatible with experiment. 


\section{Carboxylic Acids}

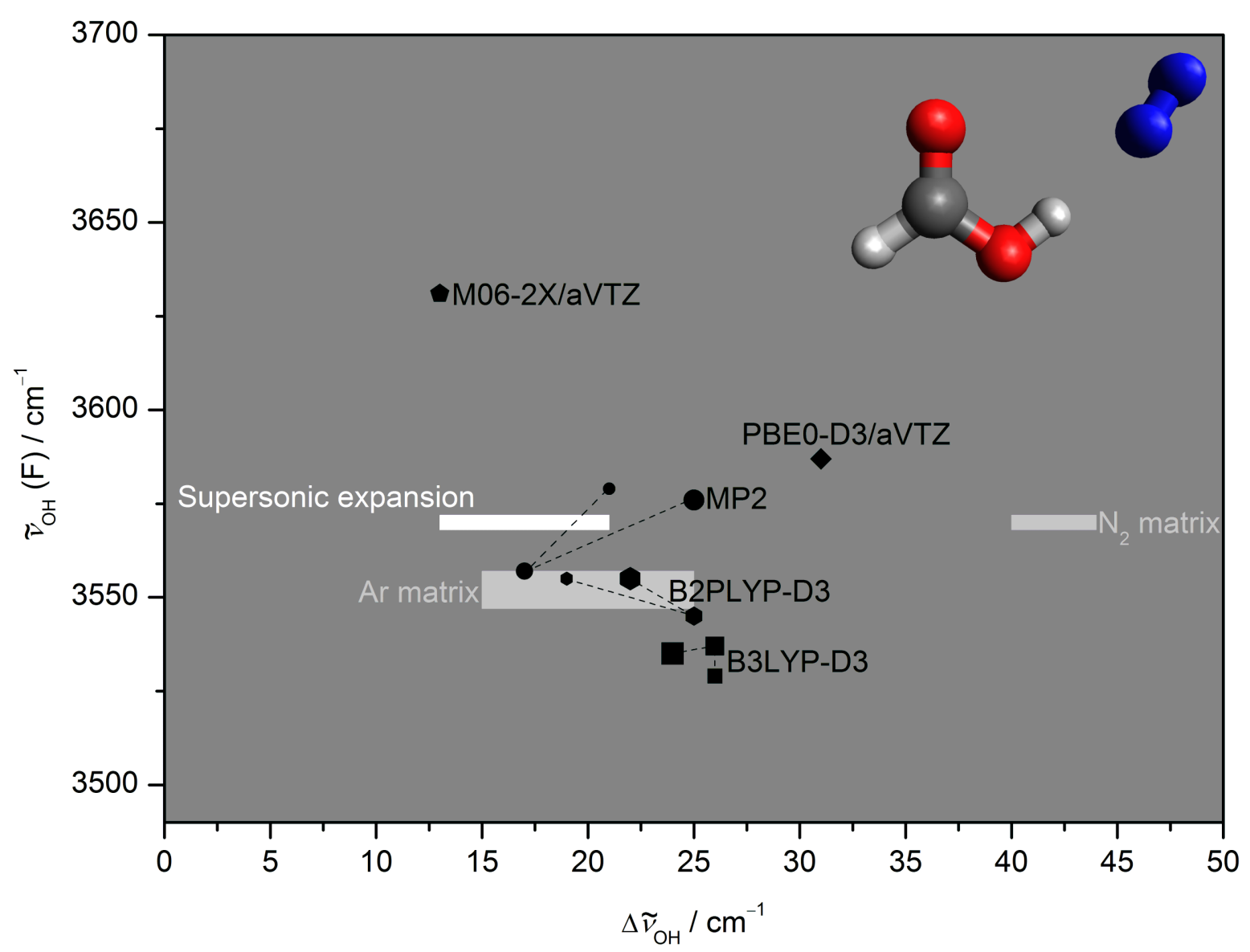

Figure 2.15.: Theoretically predicted anharmonic (VPT2) OH stretching band position of $\mathrm{F}\left(\tilde{\nu}_{\mathrm{OH}}(\mathrm{F})\right)$ plotted against the anharmonic downshift $\left(\Delta \tilde{\nu}_{\mathrm{OH}}\right)$ from $\mathrm{F}$ to $\mathrm{FN}_{\mathrm{H}}$ for different computational methods implemented in Gaussian09 (reprinted from reference 157 licensed under CC BY 4.0). Symbol size qualitatively encodes the size of the basis set (see Table A.8 in appendix A.2). The zone of incompatibility with experiment is in dark grey, predictions within the white area are compatible with experiment. The light grey areas encode the band positions of $\mathrm{F}$ and $\mathrm{FN}_{\mathrm{H}}$ in Ar matrix ${ }^{159}$ and the shift from $\mathrm{F}$ in supersonic expansion to $\mathrm{F}$ in a bulk $\mathrm{N}$ matrix. ${ }^{160}$

In summary, high level wave function based methods such as CCSD(T) or SCS-MP2 predict quantities within the experimental error bars. The large error margins introduced when moving from anharmonic to harmonic experimental vibrational band positions prevent a sharper discrimination between those high level ab initio approaches, but are still sufficient to rule out $75 \%$ of the tested methods.

\subsubsection{Harmonic Vibrational Wavenumbers of $A$ and $A N_{H}$}

For A, only the first $\mathrm{OH}$ stretching overtone ${ }^{232}\left(6991 \mathrm{~cm}^{-1}\right)$ is experimentally known. Therefore, the anharmonic band centre of $\tilde{\nu}_{\mathrm{OH}}(\mathrm{A})=3585(4) \mathrm{cm}^{-1}$ is corrected by twice 


\section{Carboxylic Acids}

the diagonal anharmonicity of $2 x_{1,1}=-180(10) \mathrm{cm}^{-1}$ and the missing off-diagonal constants are replaced by an additional error margin of $10 \%$ of the diagonal anharmonicity correction, $18 \mathrm{~cm}^{-1}$, resulting in a total correction of $180(28) \mathrm{cm}^{-1}$ and an estimated harmonic band position of $\omega_{\mathrm{OH}}(\mathrm{A})=3765(32) \mathrm{cm}^{-1}$ with a significantly larger uncertainty than in the $\mathrm{F}$ case. Adding a $50 \%$ error to the anharmonic downshift from $\mathrm{A}$ to $\mathrm{AN}_{\mathrm{H}}$ of $\Delta \tilde{\nu}_{\mathrm{OH}}=13(6) \mathrm{cm}^{-1}$ results in an estimated harmonic downshift of $\Delta \omega_{\mathrm{OH}}=13(13) \mathrm{cm}^{-1}$.

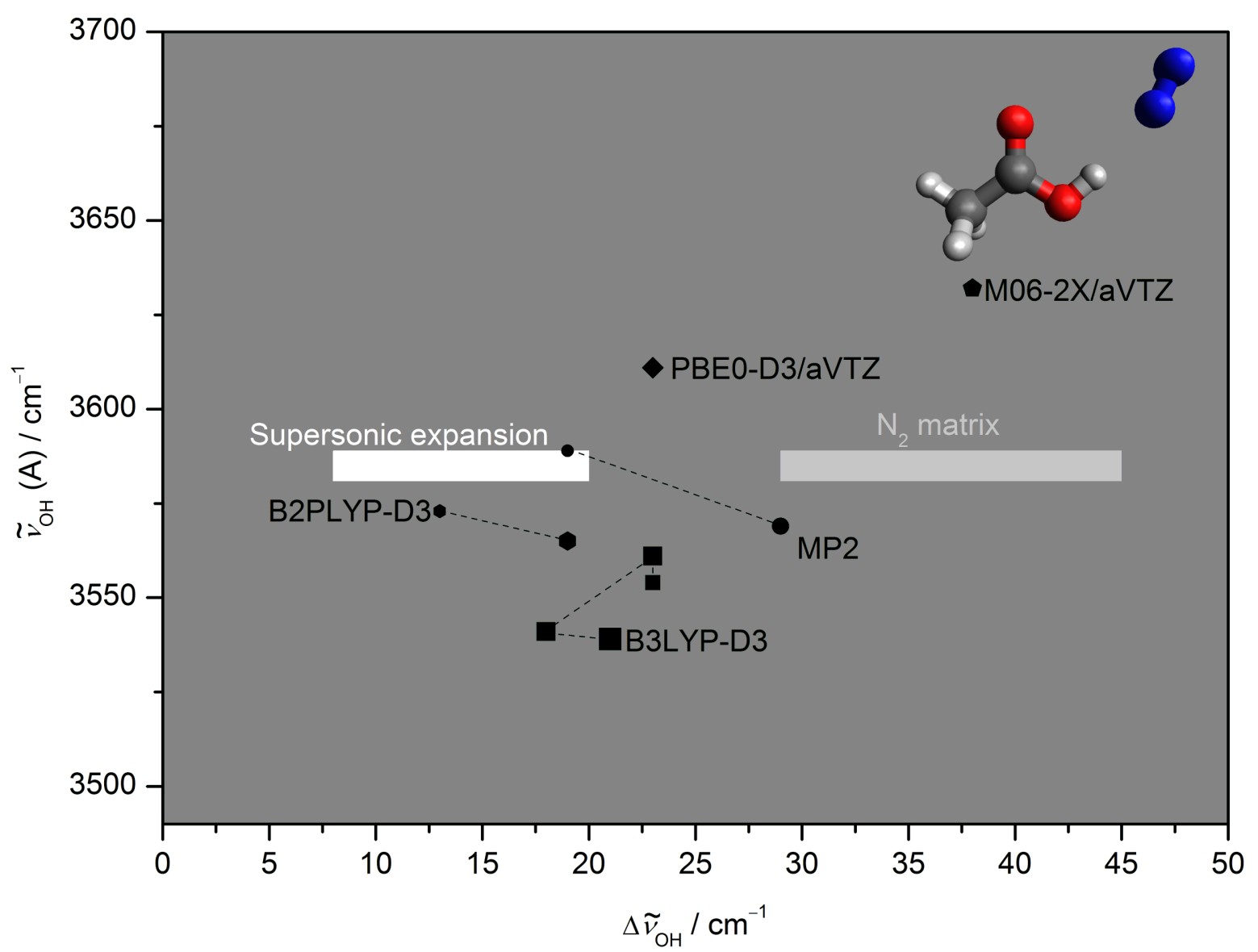

Figure 2.16.: Theoretically predicted anharmonic (VPT2) OH stretching band position of A $\left(\tilde{\nu}_{\mathrm{OH}}(\mathrm{A})\right)$ plotted against the anharmonic downshift $\left(\Delta \tilde{\nu}_{\mathrm{OH}}\right)$ from $\mathrm{A}$ to $\mathrm{AN}_{\mathrm{H}}$ for different computational methods implemented in Gaussian09 (reprinted from reference 157 licensed under CC BY 4.0). Symbol size qualitatively encodes the size of the basis set (see Table A.8 in appendix A.2). The zone of incompatibility with experiment is grey, predictions within the white area are compatible with experiment. The light grey area encodes the shift from A in supersonic expansion to $\mathrm{A}$ in a bulk nitrogen matrix. ${ }^{160}$

Predicted harmonic monomer wavenumbers and downshifts are depicted in Figure 2.14 and listed in Table A.8 in appendix A.2. Expensive CCSD(T) calculations were not performed for the large A system, but predictions from all other methods confirm the trends found for the F case. 


\section{Carboxylic Acids}

\subsubsection{Anharmonic Vibrational Wavenumbers of $F$ and $F N_{H}$}

High level anharmonic calculations are available from literature for $\mathrm{F}$, but not $\mathrm{FN}_{\mathrm{H}}$. VCI calculations using CCSD(T) potential energy surfaces yield predictions of $3575 \mathrm{~cm}^{-1209}$ and $3567 \mathrm{~cm}^{-1213}$ for the $\mathrm{OH}$ stretching vibration, respectively, while a combination ${ }^{225}$ of harmonic $\operatorname{CCSD}(\mathrm{T})$ and anharmonicity from a VPT2 calculation at MP2/aVDZ level predicts $3563 \mathrm{~cm}^{-1}$. Although spectroscopic accuracy with an error of less than $1 \mathrm{~cm}^{-1}$ is not quite reached, these elaborate approaches almost reproduce the experimental value of $3570(2) \mathrm{cm}^{-1}$. The best theory combination carried out in this work are harmonic LCCSD(T*)-F12/VDZ-F12 predictions complemented by anharmonic VPT2 corrections at the B3LYP-D3(BJ)/def2-QZVP level, which predict a monomer band centre position of $3564 \mathrm{~cm}^{-1}$ and a $13 \mathrm{~cm}^{-1}$ downshift.

Anharmonic VPT2 predictions at lower levels of theory are depicted in Figure 2.15, listed in Table A.8 in appendix A.2 and directly compared to experiment. In addition to the experimental values in supersonic expansion (white area), light grey areas in Figure 2.15 show the wavenumbers of $\mathrm{F}$ and $\mathrm{FN}_{\mathrm{H}}$ in an $\mathrm{Ar}$ matrix ${ }^{159}$ and the gas-to-matrix shift in a N matrix. ${ }^{160}$ Site splitting in Ar ${ }^{159,162}$ causes five different F bands at 3556.7, $3553.7,3550.4,3548.3$ and $3546.8 \mathrm{~cm}^{-1}$, respectively, resulting in a band position of $\mathrm{F}$ in Ar matrix of $3552(5) \mathrm{cm}^{-1}$. The $\mathrm{FN}_{\mathrm{H}}$ band centre at $3531.7 \mathrm{~cm}^{-1}$ yields a downshift of $20(5) \mathrm{cm}^{-1}$, very similar to the value in supersonic expansion, although this might not apply to other molecular systems due to possible stronger interactions of analyte and matrix environment. The gas-to-matrix shift in a $\mathrm{N}$ matrix of $42(2) \mathrm{cm}^{-1}$ is clearly larger because cooperative and packing forces of the bulk environment influence this value.

The gas phase complexation shift is only predicted correctly by M06-2X, MP2 and B2PLYP-D3 calculations, which fail to predict accurate monomer wavenumbers. The deviations of MP2 and B2PLYP-D3 also increase with basis set size, a clear hint at error cancellation in calculations with smaller basis sets. B3LYP-D3 and PBE0-D3 predict incorrect monomer band centres as well as an overestimated downshift. None of the explored methods delivers correct predictions for both experimental constraints, but since all of them already failed in the harmonic approximation, a final judgement on a VPT2 treatment of $\mathrm{F}$ and $\mathrm{FN}_{\mathrm{H}}$ would require a CCSD(T) or at least SCS-MP2 approach.

\subsubsection{Anharmonic Vibrational Wavenumbers of $A$ and $A N_{H}$}

Anharmonic predictions for $\mathrm{A}$ and $\mathrm{AN}_{\mathrm{H}}$ are shown in Figure 2.16. Since $\mathrm{AN}_{\mathrm{H}}$ has not been investigated in an Ar matrix, the light grey area only encodes the gas-to-matrix shift for a N matrix. Two monomer bands ${ }^{160}$ at 3551.9 and $3544.1 \mathrm{~cm}^{-1}$ result in a band position of $3548(4) \mathrm{cm}^{-1}$ and a shift of $37(8) \mathrm{cm}^{-1}$. Again, no explored method meets both experimental constraints. The difference between the $\mathrm{F}$ case and $\mathrm{A}$ is the presence of a methyl rotor. The corresponding large amplitude motions are poorly described by the VPT2 treatment which impedes disentanglement of different error sources. Incorrect predictions could be blamed on electronic structure, harmonic force field or anharmonic effects. However, once a theoretical approach is fully validated for F, A could serve as discriminator for anharmonic treatments. 


\section{Carboxylic Acids}

\subsubsection{Theory Performance Evaluation}

Since anharmonic VPT2 frequency predictions for A are mostly unreliable, the following analysis focuses on $\mathrm{F}$ and $\mathrm{FN}_{\mathrm{H}}$. Squared deviations of predictions from all employed theoretical methods with their largest tested basis set, respectively, calculated according to Equation 1.1 are listed in Table 2.5. Separate analysis of harmonic and anharmonic predictions reveals significantly larger errors in the anharmonic meeting point than for corresponding harmonic predictions, mostly due to lower experimental error bars for anharmonic references. The lowest $\delta^{2}$ values for harmonic predictions are achieved by $\operatorname{LCCSD}\left(\mathrm{T}^{*}\right)$, while MP2/VQZ delivers the best results of all tested methods with fully anharmonic VPT2 calculations (Table 2.6). Therefore, anharmonicity corrections from MP2/VQZ $\left(-188 \mathrm{~cm}^{-1}\right.$ for $\omega_{\mathrm{OH}, \mathrm{corr}},-11 \mathrm{~cm}^{-1}$ for $\left.\Delta \omega_{\mathrm{OH}, \mathrm{corr}}\right)$ are applied to all tested levels of theory. This induces a large spread in the $\delta^{2}$ values for the monomer band centre, but in most cases no significant changes in the performance of different calculations for the predicted shifts. Anharmonicity fractions of the total shift obtained when applying anharmonic MP2/VQZ corrections to harmonic shifts range from 23 ( $\omega$ B97-XD/aVTZ) to $50 \%$ (CCSD(T)/aVTZ), with only one outlier (PBEh-3c with $73 \%$ ), further justifying the assumed error margin of $50 \%$ when comparing predicted harmonic and experimental anharmonic shifts that will be used throughout this work.

Table 2.5.: Squared deviations $\delta^{2}(x)$ of theoretical predictions from experimental reference values derived from $\mathrm{OH}$ stretching IR spectra of $\mathrm{F}$ and $\mathrm{FN}_{\mathrm{H}}$ calculated according to Equation 1.1. Separate analysis for harmonic $\left(\omega_{\mathrm{OH}}, \Delta \omega_{\mathrm{OH}}\right)$ and fully anharmonic $\left(\tilde{\nu}_{\mathrm{OH}}, \Delta \tilde{\nu}_{\mathrm{OH}}\right)$ predictions is provided. Harmonic values from all levels of theory are additionally corrected by anharmonicity from MP2/VQZ (VPT2) calculations and compared to anharmonic experimental references $\left(\omega_{\mathrm{OH}, \mathrm{corr}}, \Delta \omega_{\mathrm{OH}, \mathrm{corr}}\right)$.

\begin{tabular}{|c|c|c|c|c|c|c|}
\hline Level of theory & $\delta^{2}\left(\omega_{\mathrm{OH}}\right)$ & $\delta^{2}\left(\Delta \omega_{\mathrm{OH}}\right)$ & $\delta^{2}\left(\tilde{\nu}_{\mathrm{OH}}\right)$ & $\delta^{2}\left(\Delta \tilde{\nu}_{\mathrm{OH}}\right)$ & $\delta^{2}\left(\omega_{\mathrm{OH}, \mathrm{corr}}\right)$ & $\delta^{2}\left(\Delta \omega_{\mathrm{OH}, \mathrm{corr}}\right)$ \\
\hline LCCSD(T*)-F12/VDZ-F12 & 0.5 & 0.3 & - & - & 4.0 & 1.0 \\
\hline SCS-MP2/aVQZ & 0.2 & 0.7 & - & - & 36.0 & 0.0 \\
\hline $\operatorname{CCSD}(\mathrm{T}) / \mathrm{aVTZ}$ & 2.7 & 0.1 & - & - & 72.3 & 2.3 \\
\hline MP2/aVQZ & 0.3 & 2.9 & - & - & 1.0 & 7.6 \\
\hline B2PLYP-D3(BJ)/VQZ & 1.8 & 1.5 & 56.3 & 1.6 & 42.3 & 1.6 \\
\hline M06-2X/aVTZ & 3.7 & 0.5 & 930.3 & 1.0 & 272.3 & 0.3 \\
\hline PBE0-D3(BJ)/aVTZ & 0.3 & 5.0 & 72.3 & 12.3 & 49.0 & 20.3 \\
\hline B3LYP-D3(BJ)/def2-QZVP & 7.0 & 2.4 & 272.3 & 5.1 & 240.3 & 5.1 \\
\hline$\omega \mathrm{B} 97-\mathrm{XD} / \mathrm{aVTZ}$ & 7.8 & 5.3 & 420.3 & 650.3 & 506.3 & 22.6 \\
\hline PBEh-3c & 34.3 & 0.0 & - & - & 1936.0 & 10.6 \\
\hline B97-3c & 67.5 & 0.2 & - & - & 2970.3 & 1.6 \\
\hline
\end{tabular}

Ranking methods according to the lowest mean $\delta^{2}(x)$ (Table 2.6) reveals no significant changes in the sequence when switching from harmonic to fully anharmonic method evaluations with the exception of M06-2X/aVTZ. Some minor differences occur between harmonic and anharmonicity-corrected rankings, although the best (LCCSD $\left(\mathrm{T}^{*}\right)$ F12/VDZ-F12) and worst ( $\omega$ B97-XD, PBEh-3c and B97-3c) ranking methods remain the same in both cases. Some approaches which show very similar harmonic performances 


\section{Carboxylic Acids}

benefit differently from the MP2-anharmonicity with MP2 and SCS-MP2 improving their ranking and $\operatorname{CCSD}(\mathrm{T})$ dropping from fourth to eighth position. This may hint at slight errors in the MP2-anharmoncity, but an experimental confirmation would require currently unfeasible measurements of various weak transitions such as overtones and combination bands for $\mathrm{FN}_{\mathrm{H}}$. Generally, the small size of the test data set increases the possibility of good method performances due to fortuitous error cancellation. While bad rankings are always a sign of systematic errors in a theoretical approach, corroborating good rankings requires expansion of the experimental data base, as will be shown below.

Table 2.6.: Method rankings based on squared deviations $\delta^{2}(x)$ of theoretical predictions from two experimental reference values derived from $\mathrm{OH}$ stretching IR spectra of $\mathrm{F}$ and $\mathrm{FN}_{\mathrm{H}}$ (Table 2.5).

\begin{tabular}{cccc}
\hline Level of theory & Ranking $\left(\omega_{\mathrm{OH}}, \Delta \omega_{\mathrm{OH}}\right)$ & Ranking $\left(\tilde{\nu}_{\mathrm{OH}}, \Delta \tilde{\nu}_{\mathrm{OH}}\right)$ & Ranking $\left(\omega_{\mathrm{OH}, \text { corr }}, \Delta \omega_{\mathrm{OH}, \mathrm{corr}}\right)$ \\
\hline LCCSD(T*)-F12/VDZ-F12 & 1 & - & 1 \\
SCS-MP2/aVQZ & 2 & - & 4 \\
MP2/VQZ & 3 & 1 & 3 \\
CCSD(T)/aVTZ & 4 & - & 8 \\
MP2/aVQZ & 5 & - & 2 \\
B2PLYP-D3(BJ)/VQZ & 6 & 2 & 5 \\
B2PLYP-D3(BJ)/aVQZ & 7 & - & 6 \\
M06-2X/aVTZ & 8 & 5 & 10 \\
PBE0-D3(BJ)/aVTZ & 9 & 3 & 8 \\
B3LYP-D3(BJ)/def2-QZVP & 10 & 4 & 9 \\
$\omega$ B97-XD/aVTZ & 11 & 6 & 11 \\
PBEh-3c & 12 & - & 12 \\
B97-3c & 13 & - & 13 \\
\hline
\end{tabular}

\subsection{Summary}

Adding molecular nitrogen and oxygen to formic and acetic acid leads to the formation of binary aggregates with subsequent $\mathrm{OH}$ stretching wavenumber downshifts between 10 and $20 \mathrm{~cm}^{-1}$. These subtle effects caused by weak hydrogen bonds allow for quantitative testing of electronic structure and vibrational dynamics methods. Experiments performed in supersonic expansions serve this purpose much more effectively than corresponding bulk matrix isolation findings and should therefore be preferably used for benchmarking of theoretical predictions which are commonly modelling isolated molecules or aggregates. ${ }^{160,225}$

Even though the weak hydrogen bonds between dipolar $\mathrm{OH}$ and quadrupolar nitrogen may be rationalised in large part by electrostatics, tested density functional theory methods systematically overestimate resulting frequency shifts. Harmonic CCSD(T) predictions with local and explicit correlation as well as anharmonic VPT2 corrections from lower levels of theory almost reproduce experimental values, but verification of this success requires complementing VPT2 calculations at the same level.

Carefully disentangling underlying electronic structure methods and nuclear motion effects is crucial, but greatly increases experimental complexity by requiring large data 


\section{Carboxylic Acids}

sets of vibrational overtones and combination bands. Formic acid and its binary nitrogen complex are promising candidates and electronic structure methods with accurate predictions for this system could potentially be used to judge the quality of different vibrational anharmonicity treatments, as they are extended to the more challenging acetic acid case. The tested VPT2 approach performs well for the rigid formic acid monomer, but exhibits problems once large amplitude motions are introduced to the studied molecular system via nitrogen aggregate formation or methyl group rotors in acetic acid.

Additionally, the open dimer isomer in acetic acid is identified by selective nitrogen and oxygen complexation. Rationalising the lower abundance of open dimers in formic acid requires investigations by different experimental techniques. ${ }^{102}$

As the molecular systems used for benchmarking purposes in the following chapters are much larger than formic and acetic acid, only the set of theoretical methods listed in Table 2.7 could be applied to all tested aggregates throughout this work. Their mean squared deviations $\delta^{2}(x)$ for the four benchmarks including harmonic monomer wavenumbers and nitrogen induced spectral downshifts of both carboxylic acids are included for comparison with other benchmarks presented below alongside their respective ranking for the isolated analysis of the carboxylic acids. Since the VTP2 scheme cannot be reliably applied to any other structure apart from the formic acid monomer, anharmonic frequency calculations are excluded from the comparative analysis of benchmarking quantities throughout this work.

Table 2.7.: Method rankings based on squared deviations $\delta^{2}(x)$ of theoretical predictions from four experimental reference values calculated according to Equation 1.1 derived from presented harmonic monomer wavenumber and nitrogen induced downshift benchmarks of formic and acetic acid.

\begin{tabular}{ccc}
\hline Level of theory & $\delta^{2}(x)$ & Ranking \\
\hline SCS-MP2/VTZ & 0.3 & 1 \\
MP2/VTZ & 0.7 & 2 \\
B2PLYP-D3(BJ)/VTZ & 1.1 & 3 \\
M06-2X/aVTZ & 1.7 & 4 \\
PBE0-D3(BJ)/aVTZ & 2.6 & 5 \\
B3LYP-D3(BJ)/def2-QZVP & 2.9 & 6 \\
$\omega B 97-X D / a V T Z ~$ & 5.3 & 7 \\
PBEh-3c & 11.0 & 8 \\
B97-3c & 19.0 & 9 \\
\hline
\end{tabular}





\section{1,1,1,3,3,3-Hexafluoro-2-propanol}

1,1,1,3,3,3-hexafluoro-2-propanol and its aggregates were extensively studied with filetjet FTIR, curry-jet Raman, chirped pulse Fourier transform microwave and cavityenhanced Fourier transform microwave spectroscopy. Microwave spectra were recorded at the University of Alberta together with Dr. Nathan A. Seifert during a guest stay in the groups of Prof. Dr. Yunjie Xu and Prof. Dr. Wolfgang Jäger. Raman spectra were obtained by Maxim Gawrilow, while Prof. Dr. Stefan Grimme performed an additional, independent theoretical search for possible trimer structures with the GFN-xTB ${ }^{147}$ and GFN2-xTB ${ }^{233}$ methods. MP2/aVTZ calculations for the most stable homoaggregates were carried out by Fabian Bohle at the Mulliken Center for Theoretical Chemistry of Bonn University. FTIR spectra of the dimer and its nitrogen aggregates are published in reference 234, while combined trimer FTIR, Raman and microwave spectra are published in reference 235. The occasional verbatim reproduction of small sections from those references is not explicitly marked or cited.

\subsection{Introduction}

Fluorinated alcohols display unusual solvent properties ${ }^{236-238}$ such as accelerated rates of catalytic reactions ${ }^{239-242}$ and profound influence on peptide folding ${ }^{243-245}$ or supramolecular assemblies. ${ }^{246}$ These effects are rooted in the subtle interplay of strong intermolecular hydrogen bonding ${ }^{247,248}$ and fluorous interactions, ${ }^{249-251}$ which establishes fluorinated alcohols as interesting benchmarking objects for quantum chemistry. ${ }^{252}$

Fluorous interactions even seem to facilitate chirality induction and recognition ${ }^{253}$ of flexible achiral molecules in crystals ${ }^{254-258}$ or chiral liquids ${ }^{259,260}$ which justifies investigations on the level of small complexes such as the homochiral dimer of 2,2,2trifluoroethanol ${ }^{261,262}$ and its less stable heterochiral counterpart ${ }^{263}$ with further chirality synchronisation in the trimer. ${ }^{139}$ Additional studies of fluorinated alcohols in the gas phase include microwave spectroscopy of 2 -fluoroethanol tetramers ${ }^{264}$ or Raman and infrared spectra of 1,1,1-trifluoro-2-propanol. ${ }^{265,266}$

1,1,1,3,3,3-hexafluoro-2-propanol has been studied by infrared spectroscopy in cryogenic argon, nitrogen and carbon monoxide matrices, ${ }^{267}$ crystal structure analysis, ${ }^{255,268}$ infrared and Raman spectroscopy in solid, liquid and gaseous state, ${ }^{269,270}$ as well as infrared ${ }^{101,266,271}$ and microwave ${ }^{272,273}$ spectroscopy in supersonic expansions. 


\subsection{Nomenclature and Experimental Details}

Employed molecule abbreviations for 1,1,1,3,3,3-hexafluoro-2-propanol (H), nitrogen (N) and oxygen $(\mathrm{O})$ are listed in hydrogen bond donor-acceptor sequence to represent cluster compositions. The respective nitrogen and oxygen docking sites are marked by subscripts and include the acidic $\mathrm{OH}(\mathrm{H})$ and $\mathrm{CH}(\mathrm{C})$ protons, the oxygen $(\mathrm{O})$ and the $\mathrm{CF}_{3}$ moieties (F). Monomer conformations of 1,1,1,3,3,3-hexafluoro-2-propanol are distinguished by their varying $\mathrm{HOCH}$ dihedral angles and marked by subscripts at the respective letter, including trans $\left(t, 180^{\circ} \mathrm{HOCH}\right.$ dihedral angle $)$, gauche $\left(g, \approx \pm 60^{\circ} \mathrm{HOCH}\right.$ dihedral angle $)$ and cis $\left(c, 0^{\circ} \mathrm{HOCH}\right.$ dihedral angle) monomers. Differing signs of two gauche $\mathrm{HOCH}$ dihedral angles in a cluster are denoted by using $g$ ', while cyclic structures or subunits in a cluster are indicated by parentheses.

OH stretching FTIR spectra from $4100-2450 \mathrm{~cm}^{-1}$ were obtained with the filet-jet setup equipped with the $150 \mathrm{~W}$ tungsten lamp, $\mathrm{CaF}_{2}$ beam splitter and optics as well as the liquid nitrogen cooled $3 \mathrm{~mm}^{2}$ InSb detector. For both infrared and Raman spectra 1,1,1,3,3,3-hexafluoro-2-propanol (99\%, FluoroChem) was mixed with helium $(99.996 \%$, Linde) and optionally argon (99.999\%, Linde), nitrogen $(99.999 \%$, Linde and Air Liquide) or oxygen (99.998\%, Air Liquide). For the microwave measurements 1,1,1,3,3,3hexafluoro-2-propanol (99.9\%, Sigma Aldrich, purified from dissolved gases through multiple freeze-pump-thaw cycles under primary vacuum), neon (99.999\%, Praxair), helium (99.995\%, Praxair) and nitrogen (99.995\%, Praxair) were used without further purification or chemical modification.

Variation of $\mathrm{H}$ concentrations in the carrier gas mixture (Figures A.3, A.4 and A.5 in appendix A.3) was used to assign the different vibrational transitions of homoclusters to their respective aggregate size.

\subsection{Monomer}

Two $\mathrm{H}$ monomer conformations, $\mathrm{H}_{t}$ and $\mathrm{H}_{g}$, are stable minima in the gas phase (Figure 3.1). ${ }^{101,271}$ The most stable $\mathrm{H}_{t}$ conformer has been structurally characterised by microwave spectroscopy alongside its binary aggregate with water, while rotational transitions assigned to $\mathrm{H}_{g}$ proved elusive. ${ }^{272,273}$ Detection of $\mathrm{H}_{g}$ by microwave spectroscopy is difficult due to the large energy difference of about $5 \mathrm{~kJ} \mathrm{~mol}^{-1}$, a very efficient relaxation to $\mathrm{H}_{t}$ in the jet expansion ${ }^{101,271,274}$ despite relatively large interconversion barriers ${ }^{275}$ and an expected rapid proton tunnelling between $\mathrm{H}_{g}$ and $\mathrm{H}_{g^{\prime}}$ (Figure 3.1).

Predicted dipole moments increase from $\mathrm{H}_{t}$ over $\mathrm{H}_{g}$ to $\mathrm{H}_{c}$ (Table 3.1), which leads to a relative stabilisation of $\mathrm{H}_{g}$ and $\mathrm{H}_{c}$ in more polar environments, as indicated by calculations including a polarisable continuum model (PCM, Figure 3.1, see Table A.3 in appendix A.1 for employed keywords). Consequently, the crystal structure of $\mathrm{H}$ exclusively contains helices of $\mathrm{H}_{g}$ monomers with some of their $\mathrm{HOCH}$ dihedral angles approaching the $0^{\circ}$ limit found in $\mathrm{H}_{c} \cdot{ }^{255}$ 


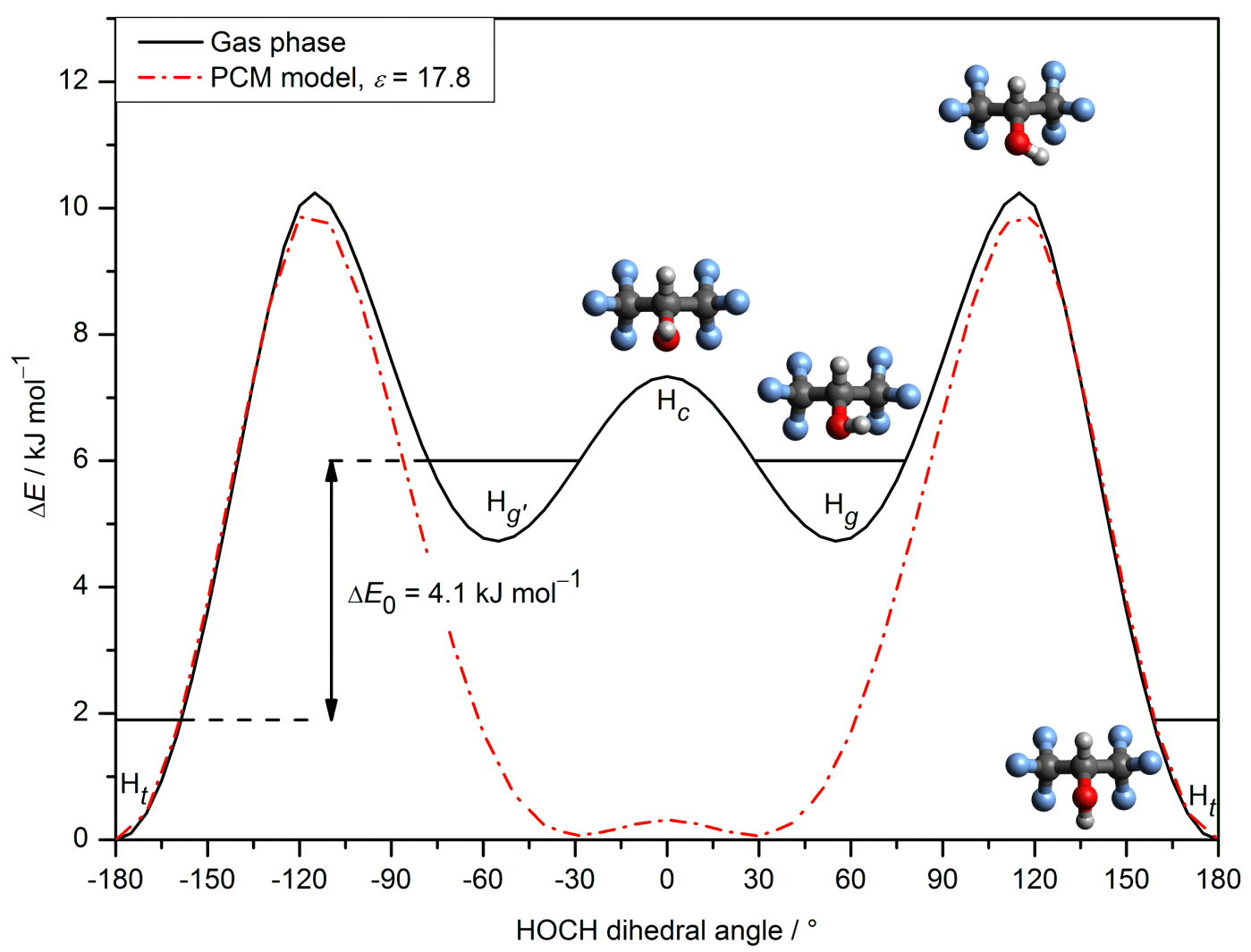

Figure 3.1.: Fully relaxed B3LYP-D3(BJ)/def2-QZVP OH torsional scans for the H monomer in the gas phase (solid black trace) and with polarisable continuum model (PCM) solvation (red dash-dotted trace; $\varepsilon=17.8$, the dielectric constant for $\mathrm{H}$ at room temperature) ${ }^{276,277}$ with structural snapshots (reprinted with adaptations from reference 235 with permission from John Wiley and Sons). The zero-point corrected energy levels in each well are indicated with solid lines.

Table 3.1.: Theoretically predicted harmonic $\left(\omega_{\mathrm{OH}}\right) \mathrm{OH}$ stretching wavenumber and lowest predicted wavenumber $\left(\omega_{\mathrm{l}}\right)$, in $\mathrm{cm}^{-1}$, IR intensity $\left(S_{\mathrm{OH}}\right)$ in $\mathrm{km} \mathrm{mol}^{-1}$, Raman scattering cross section $\left(\sigma_{\mathrm{OH}}\right)$ assuming $T_{\mathrm{vib}}=100 \mathrm{~K}$ in $10^{-36} \mathrm{~m}^{2} \mathrm{sr}^{-1}$, relative electronic and harmonic vibrational zero-point corrected energies $\left(\Delta E_{\mathrm{e}}\right.$ and $\left.\Delta E_{0}\right)$ compared to the most stable $\mathrm{H}_{t}$ conformation in $\mathrm{kJ} \mathrm{mol}^{-1}$, as well as equilibrium rotational constants $\left(A_{\mathrm{e}}, B_{\mathrm{e}}\right.$, $\left.C_{\mathrm{e}}\right)$ in $\mathrm{MHz}$ and magnitudes of the electric dipole moment components $\left(\left|\mu_{a}\right|,\left|\mu_{b}\right|,\left|\mu_{c}\right|\right)$ in D calculated at the B3LYP-D3(BJ)/def2-QZVP level of approximation.

\begin{tabular}{ccccccccccccc}
\hline Structure & $\omega_{\mathrm{OH}}$ & $S_{\mathrm{OH}}$ & $\sigma_{\mathrm{OH}}$ & $\Delta E_{\mathrm{e}}$ & $\Delta E_{0}$ & $\omega_{\mathrm{l}}$ & $A_{\mathrm{e}}$ & $B_{\mathrm{e}}$ & $C_{\mathrm{e}}$ & $\left|\mu_{a}\right|$ & $\left|\mu_{b}\right|$ & $\left|\mu_{c}\right|$ \\
\hline $\mathrm{H}_{t}$ & 3789 & 63 & 42 & 0.0 & 0.0 & 25 & 2089.8 & 1042.2 & 922.9 & 0.0 & 0.6 & 0.1 \\
$\mathrm{H}_{g}$ & 3827 & 75 & 63 & 4.7 & 4.1 & 37 & 2093.2 & 1043.8 & 923.8 & 1.1 & 1.3 & 1.9 \\
$\mathrm{H}_{c}$ & 3864 & 91 & 61 & 7.3 & 5.4 & -201 & 2085.3 & 1041.6 & 921.7 & 0.0 & 1.6 & 2.5 \\
\hline
\end{tabular}




\subsection{Binary Complex with Nitrogen}

The most stable predicted binary $\mathrm{N}$ aggregates for both monomers feature linear coordination of the acidic $\mathrm{OH}$ proton (Figure 3.2 and Table 3.2, higher energy conformers in Figure A.6 and Table A.9 in appendix A.3).

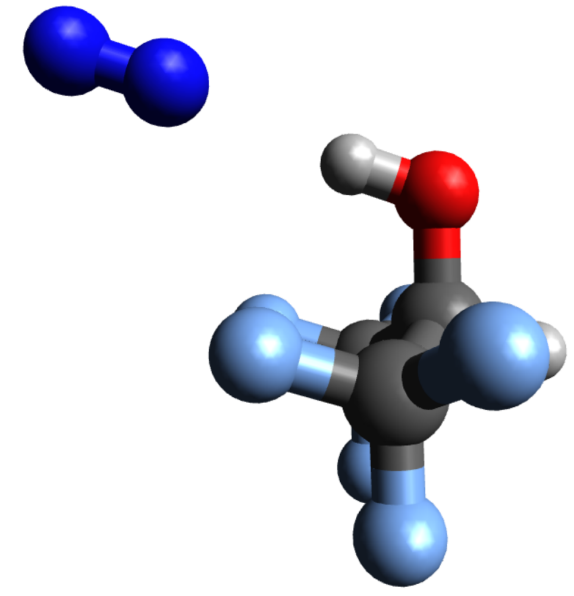

$\mathrm{H}_{t} \mathrm{~N}_{\mathrm{H}}(8.4)$

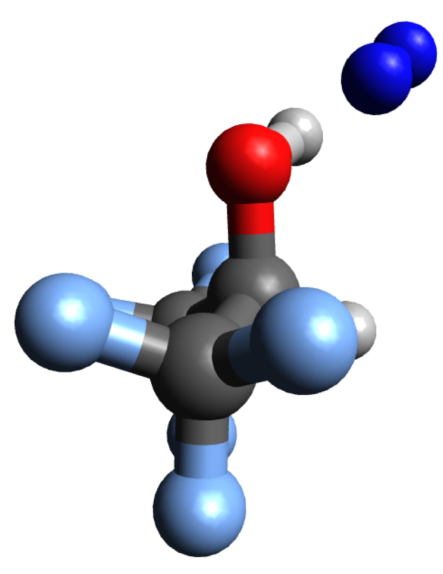

$\mathrm{H}_{g} \mathrm{~N}_{\mathrm{H}}(8.0)$

Figure 3.2.: Most stable structures of binary HN aggregates for both $\mathrm{H}$ monomer conformations optimised at the B3LYP-D3(BJ)/def2-QZVP level. Zero-point corrected dissociation energies into the realised and locally optimised monomer conformations in $\mathrm{kJ} \mathrm{mol}^{-1}$ are given in parentheses.

Table 3.2.: Theoretically predicted harmonic $\left(\omega_{\mathrm{OH}}\right) \mathrm{OH}$ stretching wavenumber, spectral downshift from the respective monomer $\left(\Delta \omega_{\mathrm{OH}}\right)$ and lowest predicted wavenumber $\left(\omega_{1}\right)$ in $\mathrm{cm}^{-1}$, IR intensity $\left(S_{\mathrm{OH}}\right)$ in $\mathrm{km} \mathrm{mol}^{-1}$, relative electronic and harmonic vibrational zeropoint corrected energies $\left(\Delta E_{\mathrm{e}}\right.$ and $\left.\Delta E_{0}\right)$ compared to the most stable $\mathrm{H}_{t} \mathrm{~N}_{\mathrm{H}}$ conformation in $\mathrm{kJ} \mathrm{mol}^{-1}$, as well as equilibrium rotational constants $\left(A_{\mathrm{e}}, B_{\mathrm{e}}, C_{\mathrm{e}}\right)$ in $\mathrm{MHz}$ and magnitudes of the electric dipole moment components $\left(\left|\mu_{a}\right|,\left|\mu_{b}\right|,\left|\mu_{c}\right|\right)$ in D calculated at the B3LYP-D3(BJ)/def2-QZVP level of approximation.

\begin{tabular}{ccccccccccccc}
\hline Structure & $\omega_{\mathrm{OH}}$ & $S_{\mathrm{OH}}$ & $\Delta \omega_{\mathrm{OH}}$ & $\Delta E_{\mathrm{e}}$ & $\Delta E_{0}$ & $\omega_{1}$ & $A_{\mathrm{e}}$ & $B_{\mathrm{e}}$ & $C_{\mathrm{e}}$ & $\left|\mu_{a}\right|$ & $\left|\mu_{b}\right|$ & $\left|\mu_{c}\right|$ \\
\hline $\mathrm{H}_{t} \mathrm{~N}_{\mathrm{H}}$ & 3753 & 281 & 36 & 0.0 & 0.0 & 18 & 958.2 & 743.0 & 535.7 & 0.8 & 0.0 & 0.4 \\
$\mathrm{H}_{g} \mathrm{~N}_{\mathrm{H}}$ & 3797 & 326 & 30 & 5.2 & 4.5 & 13 & 1224.7 & 544.9 & 442.4 & 2.7 & 0.6 & 1.4 \\
\hline
\end{tabular}

In the chirped pulse Fourier transform microwave (CP-FTMW) spectrum of an expansion of $0.1 \% \mathrm{H}$ and $0.5 \% \mathrm{~N}$ in helium (Figure 3.3) weak $\mu_{a^{-}}$and $\mu_{c^{-}}$-type transitions consistent with the predicted rotational constants for $\mathrm{H}_{t} \mathrm{~N}_{\mathrm{H}}$ were observed (Table 3.3, 


\section{1,1,1,3,3,3-Hexafluoro-2-propanol}

full list of assigned transitions in Table A.10 in appendix A.3). These transitions can be easily separated from $\mathrm{H}$ homoaggregates due to the hyperfine splittings caused by the double ${ }^{14} \mathrm{~N}$ nuclear quadrupoles. Consistent with the low abundance of $\mathrm{H}_{g}$ in supersonic expansions, ${ }^{101,271}$ no transitions from $\mathrm{H}_{g} \mathrm{~N}_{\mathrm{H}}$ or any other binary $\mathrm{HN}$ aggregate were observed.
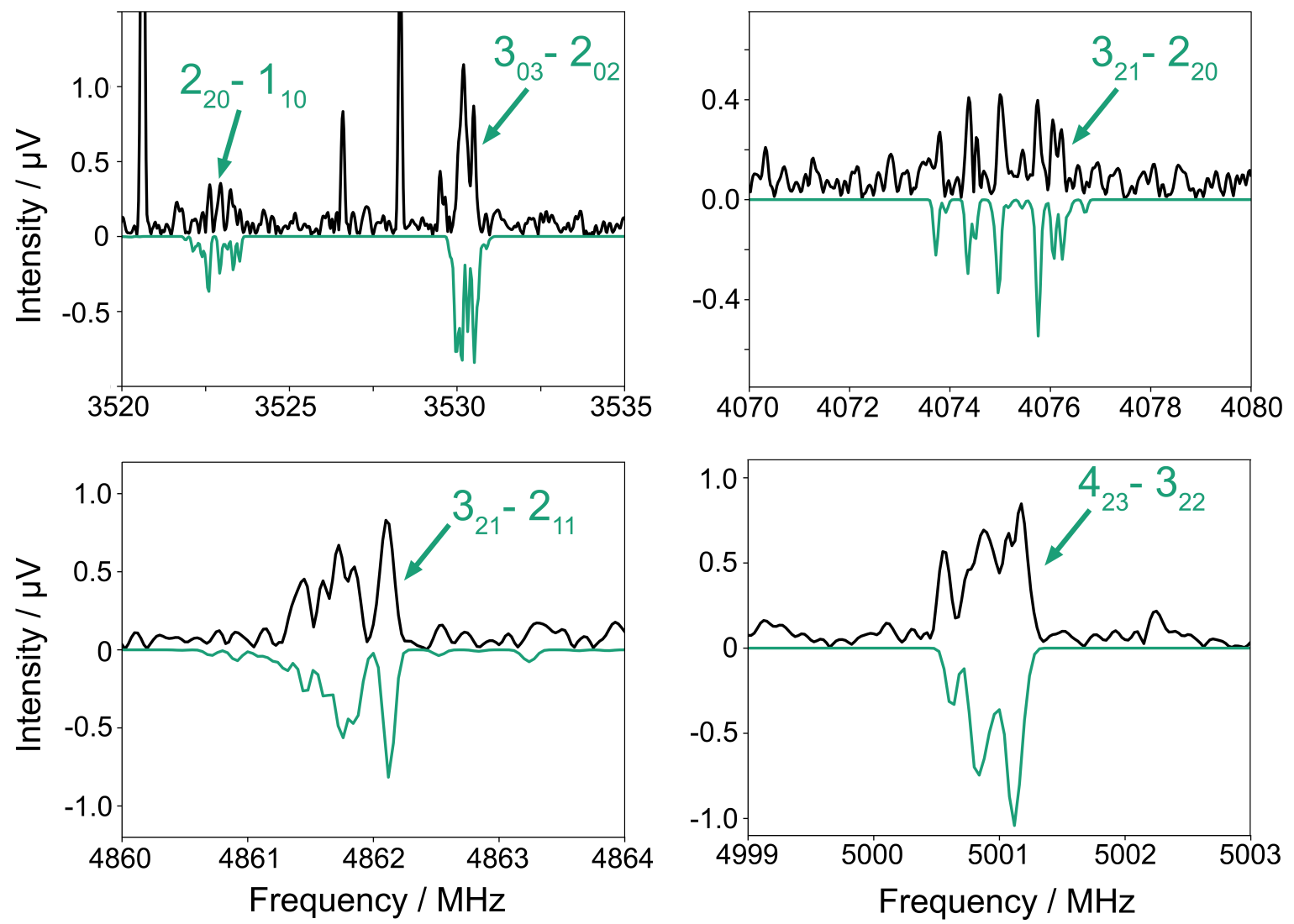

Figure 3.3.: Representative portions of the chirped pulse Fourier transform microwave spectrum of $0.1 \% \mathrm{H}$ and $0.5 \% \mathrm{~N}$ expanded in helium (black traces). Predicted lineshapes assuming a full-width at half maximum of $120 \mathrm{kHz}$ using the fitted rotational and quadrupole parameters listed in Table are added for comparison (green traces).

The relatively Doppler broadened linewidths of $120 \mathrm{kHz}$ prevent a full experimental resolution of the hyperfine structure caused by the two N nuclear quadrupoles. Statistically convergent fits of both rotational constants and hyperfine parameters would therefore require further measurements at higher spectral resolution. However, an initial fit with a satisfactory standard deviation of about $23 \mathrm{kHz}$ is achieved by fixing the $\mathrm{N}$ nuclear quadrupole coupling constants to values predicted theoretically at the B3LYPD3(BJ)/6-311++g(2d,p) level (Table 3.3). The rigid rotor parameters can therefore be determined with error bars of less than $0.1 \mathrm{MHz}$.

Possibilities for further experiments aiming for a fully experimental resolution of the 


\section{1,1,1,3,3,3-Hexafluoro-2-propanol}

hyperfine splittings include repetition of the CP-FTMW experiment with a longer acquisition time of the free induction decay switching from 20 to $40 \mu \mathrm{s}$. Using the cavityenhanced Fourier transform microwave spectrometer is another option which was tested briefly, although only transitions between 7 and $18 \mathrm{GHz}$ can be observed with this instrument, where the hyperfine splitting collapses due to the high $\mathrm{J}$ quantum numbers associated with rotational transitions in this particular spectral region. The higher resolution of the cavity-based set-up is also accompanied by doubling of all observed transitions due to the Doppler effect, an artifact of the parallel directions of expansion and radiation propagation. The last possible experiment includes using isotopically labelled ${ }^{14} \mathrm{~N}^{15} \mathrm{~N}$, which would allow for a separate assignment of the two sets of quadrupole coupling constants.

Table 3.3.: Experimental spectroscopic parameters of the binary $\mathrm{H}_{t} \mathrm{~N}_{\mathrm{H}}$ complex including rotational constants $(A, B, C)$ in $\mathrm{MHz}$, quartic centrifugal distortion constants $\left(\Delta_{\mathrm{J}}, \Delta_{\mathrm{JK}}\right.$, $\left.\Delta_{\mathrm{K}}, \delta_{\mathrm{J}}, \delta_{\mathrm{K}}\right)$ in $\mathrm{kHz}$, quadrupole coupling constants $\left(\chi_{a a},\left(\chi_{b b}-\chi_{c c}\right)\right)$ in $\mathrm{MHz}$, number of distinct frequencies $(N)$ assigned to transitions included in the fit and standard deviation $(\sigma)$ of the fit in $\mathrm{kHz}$. Quantities in brackets are fixed to the specified values, parentheses indicate values fixed to B3LYP-D3(BJ) $/ 6-311++\mathrm{g}(2 \mathrm{~d}, \mathrm{p})$ predictions.

\begin{tabular}{cc}
\hline$A$ & $968.1004(28)$ \\
$B$ & $734.3211(29)$ \\
$C$ & $533.2677(22)$ \\
$\Delta_{\mathrm{J}}$ & $0.068(95)$ \\
$\Delta_{\mathrm{JK}}$ & $0.040(16)$ \\
$\Delta_{\mathrm{K}}$ & {$[0]$} \\
$\delta_{\mathrm{J}}$ & $0.091(40)$ \\
$\delta_{\mathrm{K}}$ & {$[0]$} \\
$\chi_{a a}($ acceptor $\mathrm{N})$ & $(-2.385)$ \\
$\left(\chi_{b b}-\chi_{c c}\right)($ acceptor $\mathrm{N})$ & $(3.123)$ \\
$\chi_{a a}($ free N) & $(-2.527)$ \\
$\left(\chi_{b b}-\chi_{c c}\right)($ free N) & $(3.335)$ \\
$N$ & 52 \\
$\sigma$ & 22.6 \\
\hline
\end{tabular}

The $\mathrm{H}_{t} \mathrm{~N}_{\mathrm{H}}$ signal is also observed in $\mathrm{OH}$ stretching FTIR spectra after addition of $2.5 \%$ $\mathrm{N}$ to a helium expansion with very low $\mathrm{H}$ concentration (Figure 3.4), which prevents $\mathrm{HH}$ homodimer formation. The hydrogen bonded dimer $\mathrm{OH}$ stretching band is downshifted from the free monomer by $18 \mathrm{~cm}^{-1}$, a similar magnitude to shifts found for carboxylic acids upon $\mathrm{N}$ complexation. This finding further corroborates the assignment of $\mathrm{H}_{t} \mathrm{~N}_{\mathrm{H}}$ as the most stable binary aggregate, since substantial downshifts are only expected for linear $\mathrm{OH} \cdots \mathrm{N}$ coordination (Table A.9 in appendix A.3). 


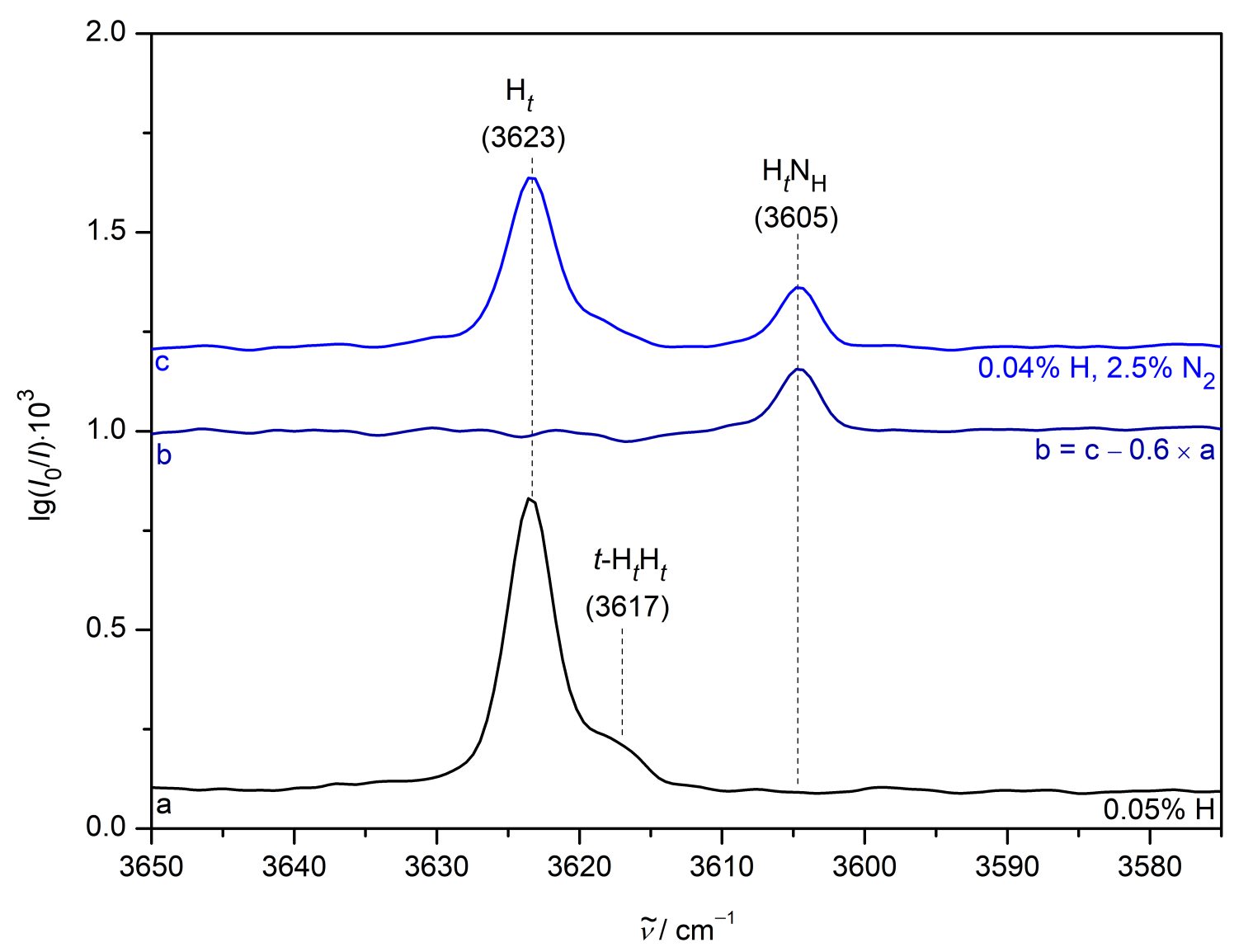

Figure 3.4.: $\mathrm{OH}$ stretching FTIR jet spectra of $\mathrm{H}$ expanded in helium (trace a) and helium mixed with $2.5 \% \mathrm{~N}$ (trace c). A monomer-corrected difference spectrum is included (trace b). Wavenumbers and assignments are provided.

\subsection{Homodimer}

The three most stable predicted dimers of $\mathrm{H}$ are depicted in Figure 3.5 (higher energy conformers in Figure A.7 in appendix A.3) and their predicted properties are summarised in Table 3.4 (higher energy conformers in Table A.11 in appendix A.3). The two most stable structures exclusively feature $\mathrm{H}_{t}$ monomers and only differ in the position of acceptor $\mathrm{OH}$ and donor $\mathrm{CO}$ bond with respect to the $\mathrm{OH} \cdots \mathrm{O}$ hydrogen bond axis. The trans conformer is therefore denoted as $t-\mathrm{H}_{t} \mathrm{H}_{t}$, the cis conformer as $c-\mathrm{H}_{t} \mathrm{H}_{t}$.

A CP-FTMW spectrum of $0.1 \% \mathrm{H}$ expanded in neon contains two sets of rotational transitions associated with HH dimers (Table 3.5, complete lists of all assigned transitions in Tables A.12 and A.13 in appendix A.3). The most intense set of lines contains strong $\mu_{c^{-}}$with additional weak $\mu_{a^{-}}$and no $\mu_{b^{-}}$type transitions. As the rotational constants and the relative intensities of $\mu_{a}, \mu_{b^{-}}$and $\mu_{c}$-type transitions are consistent with predictions for $t-\mathrm{H}_{t} \mathrm{H}_{t}$ (Table 3.4), this structure is unambiguously identified as the most stable HH dimer. 


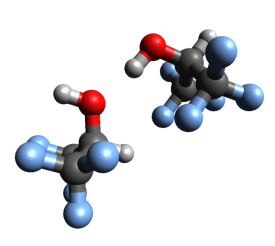

$t-\mathrm{H}_{t} \mathrm{H}_{t}(24.7)$

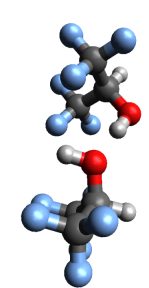

$c-\mathrm{H}_{t} \mathrm{H}_{t}(23.2)$

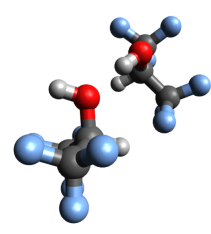

$\mathrm{H}_{g} \mathrm{H}_{t}(26.1)$

Figure 3.5.: Most stable structures of HH dimers optimised at the B3LYP-D3(BJ)/def2QZVP level. Zero-point corrected dissociation energies into the realised and locally optimised monomer conformations in $\mathrm{kJ} \mathrm{mol}^{-1}$ are given in parentheses.

Table 3.4.: Theoretically predicted harmonic $\left(\omega_{\mathrm{OH}}\right) \mathrm{OH}$ stretching wavenumber, spectral downshift from the respective monomer $\left(\Delta \omega_{\mathrm{OH}}\right)$ and lowest predicted wavenumber $\left(\omega_{\mathrm{l}}\right)$ in $\mathrm{cm}^{-1}$, IR intensity $\left(S_{\mathrm{OH}}\right)$ in $\mathrm{km} \mathrm{mol}^{-1}$, Raman scattering cross section $\left(\sigma_{\mathrm{OH}}\right)$ assuming $T_{\mathrm{vib}}=100 \mathrm{~K}$ in $10^{-36} \mathrm{~m}^{2} \mathrm{sr}^{-1}$, relative electronic and harmonic vibrational zero-point corrected energies $\left(\Delta E_{\mathrm{e}}\right.$ and $\left.\Delta E_{0}\right)$ compared to the most stable $\mathrm{H}_{t} \mathrm{~N}_{\mathrm{H}}$ conformation in $\mathrm{kJ} \mathrm{mol}^{-1}$, as well as equilibrium rotational constants $\left(A_{\mathrm{e}}, B_{\mathrm{e}}, C_{\mathrm{e}}\right)$ in $\mathrm{MHz}$ and magnitudes of the electric dipole moment components $\left(\left|\mu_{a}\right|,\left|\mu_{b}\right|,\left|\mu_{c}\right|\right)$ in D calculated at the B3LYPD3(BJ)/def2-QZVP level of approximation.

\begin{tabular}{cccccccccccccc}
\hline Structure & $\omega_{\mathrm{OH}}$ & $S_{\mathrm{OH}}$ & $\sigma_{\mathrm{OH}}$ & $\Delta \omega_{\mathrm{OH}}$ & $\Delta E_{\mathrm{e}}$ & $\Delta E_{0}$ & $\omega_{\mathrm{l}}$ & $A_{\mathrm{e}}$ & $B_{\mathrm{e}}$ & $C_{\mathrm{e}}$ & $\left|\mu_{a}\right|$ & $\left|\mu_{b}\right|$ & $\left|\mu_{c}\right|$ \\
\hline \multirow{2}{*}{$t-\mathrm{H}_{t} \mathrm{H}_{t}$} & 3788 & 79 & 45 & 1 & 0.0 & 0.0 & 7 & 479.7 & 206.1 & 169.8 & 0.4 & 0.0 & 0.9 \\
& 3624 & 612 & 147 & 165 & & & & & & & & & \\
$c-\mathrm{H}_{t} \mathrm{H}_{t}$ & 3774 & 80 & 29 & 5 & 1.2 & 1.5 & 10 & 525.5 & 186.3 & 156.6 & 0.6 & 0.3 & 0.6 \\
& 3611 & 654 & 166 & 178 & 1.2 & & & & & & & & \\
\multirow{2}{*}{$\mathrm{H}_{g} \mathrm{H}_{t}$} & 3783 & 77 & 40 & 6 & 2.6 & 2.7 & 12 & 570.6 & 154.7 & 153.3 & 3.1 & 0.9 & 0.8 \\
\hline
\end{tabular}

Table 3.5.: Experimental spectroscopic parameters of $\mathrm{HH}$ dimers including rotational constants $(A, B, C)$ in $\mathrm{MHz}$, quartic centrifugal distortion constants $\left(\Delta_{\mathrm{J}}, \Delta_{\mathrm{JK}}, \Delta_{\mathrm{K}}, \delta_{\mathrm{J}}\right.$, $\left.\delta_{\mathrm{K}}\right)$ in $\mathrm{kHz}$, number of distinct frequencies $(N)$ assigned to transitions included in the fit and standard deviation $(\sigma)$ of the fit in $\mathrm{kHz}$.

\begin{tabular}{ccc}
\hline & \multicolumn{2}{c}{ Structure } \\
Parameter & $t-\mathrm{H}_{t} \mathrm{H}_{t}$ & $\mathrm{H}_{g} \mathrm{H}_{t}$ \\
\hline$A$ & $484.69908(14)$ & $581.73604(75)$ \\
$B$ & $206.661259(78)$ & $154.26937(11)$ \\
$C$ & $170.59577(10)$ & $152.79671(11)$ \\
$\Delta_{\mathrm{J}}$ & $0.01192(24)$ & $0.01247(15)$ \\
$\Delta_{\mathrm{JK}}$ & $0.0426(11)$ & - \\
$\Delta_{\mathrm{K}}$ & $-0.0498(10)$ & $-0.174(26)$ \\
$\delta_{\mathrm{J}}$ & $0.001683(95)$ & - \\
$\delta_{\mathrm{K}}$ & $0.0233(50)$ & - \\
$N$ & 235 & 84 \\
$\sigma$ & 8.0 & 6.0 \\
\hline
\end{tabular}




\section{1,1,1,3,3,3-Hexafluoro-2-propanol}

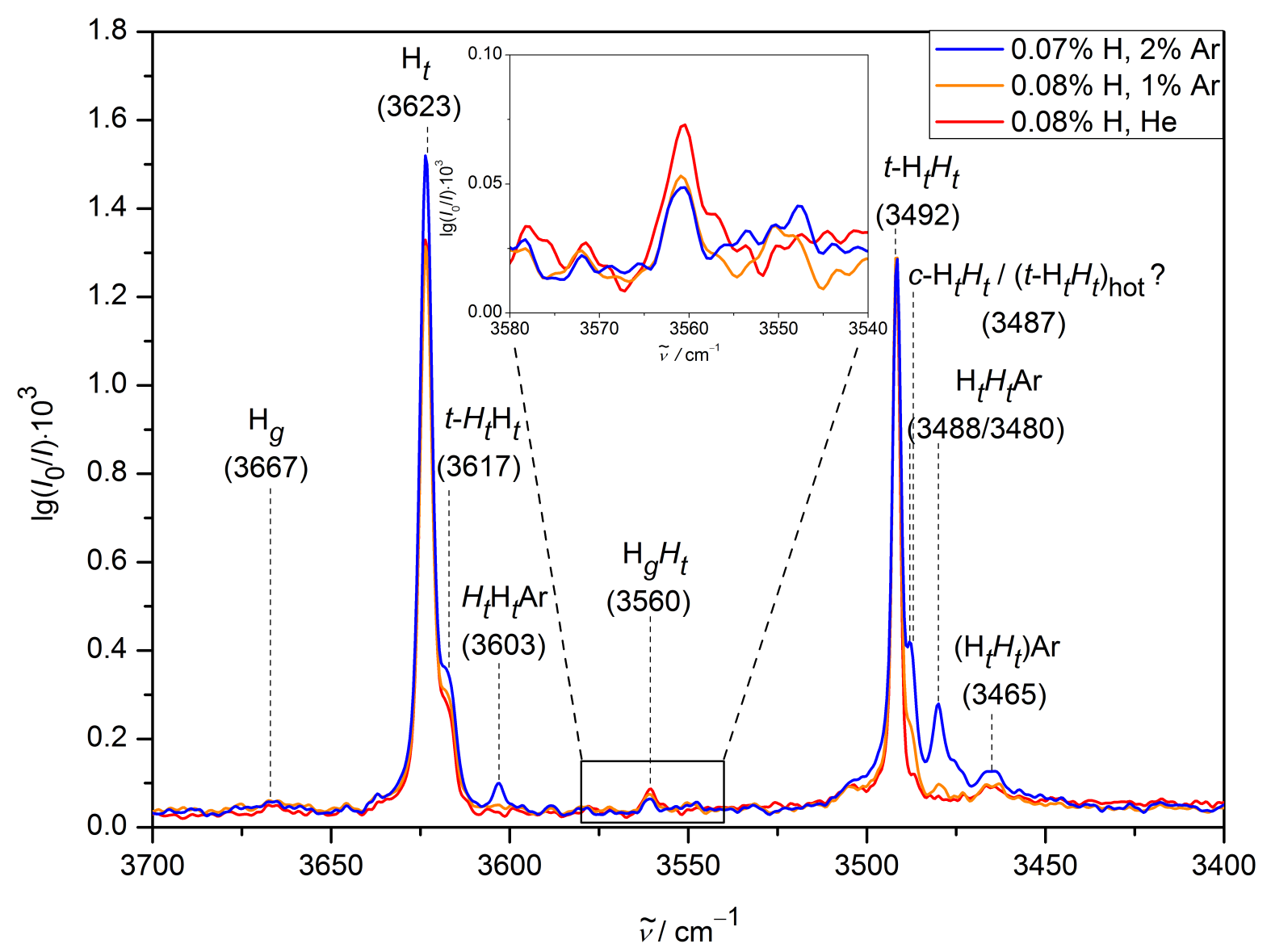

Figure 3.6.: $\mathrm{OH}$ stretching FTIR jet spectra of $\mathrm{H}$ expanded in helium (red trace, reprinted from reference 234 with permission from John Wiley and Sons) and helium mixed with 1 (orange trace) and $2 \%$ (blue trace) argon, respectively. All spectra were scaled to matching height of the $t-\mathrm{H}_{t} \mathrm{H}_{t}$ donor signal at $3492 \mathrm{~cm}^{-1}$. Wavenumbers and (tentative) assignments are provided. Roman symbols denote the alcohol monomer unit involved in the vibration, italic symbols the passive monomer. HH dimers embedded in argon are denoted as (HH)Ar, in this particular case the parentheses do not mark a cyclic structure.

The second set associated with a $\mathrm{HH}$ dimer contains only $\mu_{a}$-type transitions and displays a distinctively small asymmetry $\frac{B-C}{4} \approx 0.37 \mathrm{MHz}$. Both observations as well as the fitted rotational constants are consistent with predictions for the $\mathrm{H}_{g} \mathrm{H}_{t}$ isomer (Table 3.4) and allow for an unambiguous assignment. The $\mathrm{H}_{g} \mathrm{H}_{t}$ transitions show almost equal intensities compared to $t-\mathrm{H}_{t} \mathrm{H}_{t}$ lines, mainly due to the large $3.1 \mathrm{D} \mu_{a}$ dipole moment component. Hence, the relative intensities of $t-\mathrm{H}_{t} \mathrm{H}_{t}$ and $\mathrm{H}_{g} \mathrm{H}_{t}$ are consistent with the calculated energy penalty of about $3 \mathrm{~kJ} \mathrm{~mol}^{-1}$ for $\mathrm{H}_{g} \mathrm{H}_{t}$.

Despite a thorough search, no set of rotational transitions consistent with $c-\mathrm{H}_{t} \mathrm{H}_{t}$ was observed, even when switching the carrier gas from neon to helium, which results in slightly higher conformational temperatures in the expansion.

$\mathrm{OH}$ stretching FTIR spectra confirm these findings (Figure 3.6). Downshifted from the 


\section{1,1,1,3,3,3-Hexafluoro-2-propanol}

monomer transitions at $3667\left(\mathrm{H}_{g}\right)$ and $3623 \mathrm{~cm}^{-1}\left(\mathrm{H}_{t}\right)$ four vibrational bands associated with $\mathrm{HH}$ dimers are observed. The pair with the highest intensity associated with one dominant dimer conformation consists of a dangling hydrogen bond acceptor $\mathrm{H}_{t}$ signal at $3617 \mathrm{~cm}^{-1}$ and a strong hydrogen bond donor $\mathrm{H}_{t}$ signal at $3492 \mathrm{~cm}^{-1}$ and is therefore assigned to the most stable $t-\mathrm{H}_{t} \mathrm{H}_{t}$ dimer identified in the microwave spectra. The third dimer signal is a weak band at $3560 \mathrm{~cm}^{-1}$ assigned to the hydrogen bond donor of $\mathrm{H}_{g} \mathrm{H}_{t}$, while a weak fourth signal at $3487 \mathrm{~cm}^{-1}$ might be caused by small amounts of $c-\mathrm{H}_{t} \mathrm{H}_{t}$ or correspond to a hot transition of $t-\mathrm{H}_{t} \mathrm{H}_{t}$ with a thermally excited and insufficiently cooled low frequency mode.

Small amounts of argon are added to the expansion, resulting in reduced conformational temperatures. Under these conditions the metastable $\mathrm{H}_{g} \mathrm{H}_{t}$ signal loses intensity relative to the most stable $t-\mathrm{H}_{t} \mathrm{H}_{t}$ dimer and vibrational bands associated with argon clusters of the latter emerge, namely combined acceptor vibrations at 3603 and two donor vibrations at 3488 and $3480 \mathrm{~cm}^{-1}$. A broad signal of argon-nanocoated $\mathrm{H}_{t} \mathrm{H}_{t}$ is also observed at $3465 \mathrm{~cm}^{-1}$.

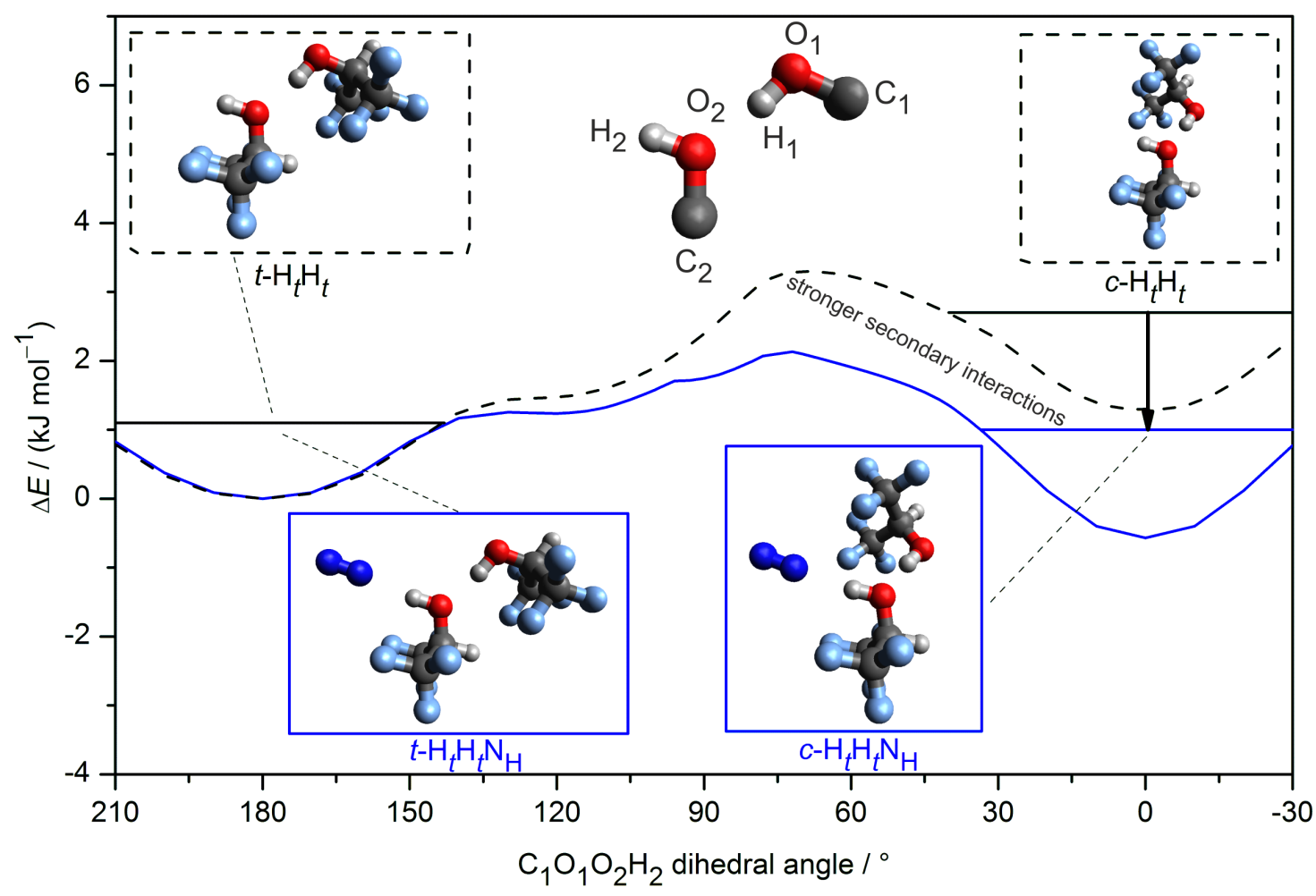

Figure 3.7.: Fully relaxed B3LYP-D3(BJ)/aVTZ OH $\cdots$ O torsional scan for $\mathrm{H}_{t} \mathrm{H}_{t}$ without (dashed black trace) and with (solid blue trace) an acceptor-attached $\mathrm{N}$ unit, varying the dihedral angle of the acceptor $\mathrm{OH}$ bond relative to the donor $\mathrm{OC}$ bond, together with structural snapshots (reprinted with adaptations from reference 234 with permission from John Wiley and Sons). The zero-point corrected energy levels in each well are indicated with solid lines. 


\section{1,1,1,3,3,3-Hexafluoro-2-propanol}

The absence of $c-\mathrm{H}_{t} \mathrm{H}_{t}$ in both microwave and infrared spectra despite simultaneous observation of the less stable $\mathrm{H}_{g} \mathrm{H}_{t}$ isomer can be rationalised by the interconversion potential between $c-\mathrm{H}_{t} \mathrm{H}_{t}$ and the most stable $t-\mathrm{H}_{t} \mathrm{H}_{t}$ through a torsion around the hydrogen bond axis (Figure 3.7, dashed black trace; complete potential with transition states in Figure A.8 in appendix A.3). Relaxation from $c$ - to $t-\mathrm{H}_{t} \mathrm{H}_{t}$ occurs readily over a very shallow barrier of less than $1 \mathrm{~kJ} \mathrm{~mol}^{-1}$, which typically results in conformational temperatures between 50 and $100 \mathrm{~K} .{ }^{101}$ Therefore, the energy difference between both torsional $\mathrm{H}_{t} \mathrm{H}_{t}$ isomers needs to be larger than $1 \mathrm{~kJ} \mathrm{~mol}^{-1}$ to account for the experimentally observed extent of relaxation, consistent with the B3LYP-D3(BJ)/def2-QZVP prediction of $1.5 \mathrm{~kJ} \mathrm{~mol}^{-1}$. The observation of $\mathrm{H}_{g} \mathrm{H}_{t}$ despite an even larger predicted energy penalty suggests some extent of kinetic trapping due to a large barrier for conversion from $\mathrm{H}_{g} \mathrm{H}_{t}$ to any of the two more stable $\mathrm{H}_{t} \mathrm{H}_{t}$ isomers, consistent with the barrier predictions of about $7 \mathrm{~kJ} \mathrm{~mol}^{-1}$ for the conversion to $t-\mathrm{H}_{t} \mathrm{H}_{t}$ and $8 \mathrm{~kJ} \mathrm{~mol}^{-1}$ for the conversion to $c-\mathrm{H}_{t} \mathrm{H}_{t}$ (Figure A.9 in appendix A.3).

\subsection{Homodimer with Nitrogen}

Indirect observation of $c-\mathrm{H}_{t} \mathrm{H}_{t}$ is possible through $\mathrm{N}$ addition (Figure 3.7, solid blue trace). The preferred $\mathrm{N}$ docking site at both $\mathrm{H}_{t} \mathrm{H}_{t}$ isomers is the highly acidic acceptor $\mathrm{OH}$, resulting in $\mathrm{H}_{t} \mathrm{H}_{t} \mathrm{~N}_{\mathrm{H}}$ complexes. Since $c-\mathrm{H}_{t} \mathrm{H}_{t}$ offers the possibility of stronger secondary $\mathrm{H} \cdots \mathrm{N}$ interactions than $t-\mathrm{H}_{t} \mathrm{H}_{t}$, mostly between the donor $\mathrm{CF}_{3}$ moiety and the acceptor $\mathrm{N}$ atom, $\mathrm{N}$ complexation stabilises $c-\mathrm{H}_{t} \mathrm{H}_{t}$ relative to $t-\mathrm{H}_{t} \mathrm{H}_{t}$, while the low barrier allowing for free interconversion between both structures is maintained.

This theoretical prediction is confirmed in the FTIR experiment (Figure 3.8). After $\mathrm{N}$ addition four vibrational signals assigned to HHN complexes (based on their intensity scaling with different $\mathrm{N}$ concentrations, see Figure A.10 in appendix A.3) emerge at $3583,3563,3473$ and $3463 \mathrm{~cm}^{-1}$. Their spectral downshifts from the respective acceptor and donor signals of $t-\mathrm{H}_{t} \mathrm{H}_{t}$ caused by cooperativity of the two $\mathrm{OH} \cdots \mathrm{OH}$ and $\mathrm{OH} \cdots \mathrm{N}$ hydrogen bonds allow for an assignment to $\mathrm{H}_{t} \mathrm{H}_{t} \mathrm{~N}_{\mathrm{H}}$ structures, which proves the presence of both $t-\mathrm{H}_{t} \mathrm{H}_{t} \mathrm{~N}_{\mathrm{H}}$ and $c-\mathrm{H}_{t} \mathrm{H}_{t} \mathrm{~N}_{\mathrm{H}}$.

\subsection{Homotrimer}

Stable structures of the HHH homotrimer (Figure 3.9) were found by two independent search strategies. Prof. Dr. Stefan Grimme used a composite algorithm consisting of normal mode following, 'genetic' structure crossing and 1 to 2 ns molecular dynamics based simulated annealing (GFN-xTB ${ }^{147}$, GFN2-xTB ${ }^{233}$ ) for the initial conformational search. This first step was followed by low-temperature (50 to $100 \mathrm{~K})$ molecular dynamics simulations starting from the most stable conformers with subsequent optimisation of equidistantly taken structural snapshots. Structures identified by this procedure were then optimised at the PBEh-3c and B3LYP-D3(BJ)/def2-QZVP levels.

The second independent search was conducted manually with 40 starting guess struc- 


\section{1,1,1,3,3,3-Hexafluoro-2-propanol}

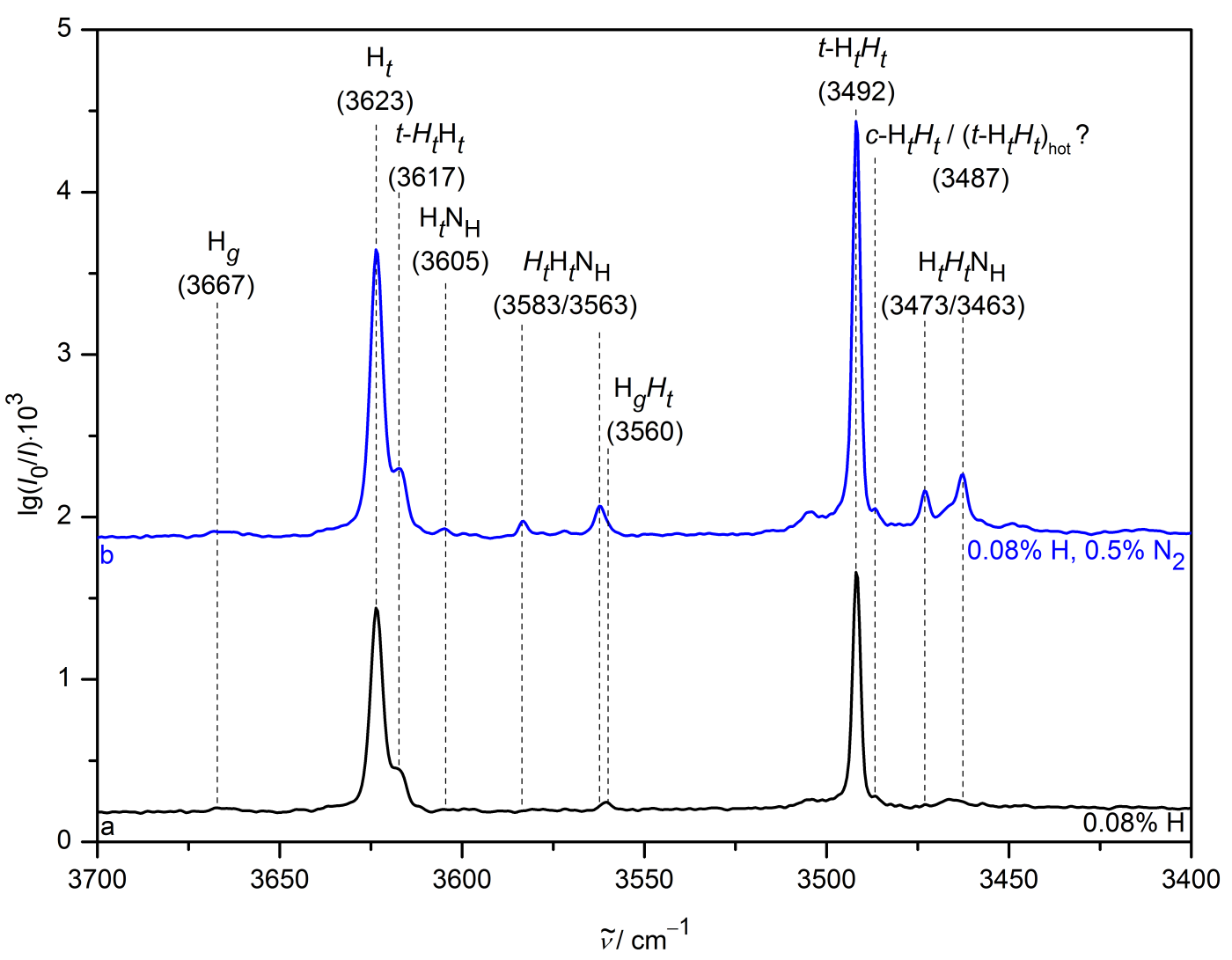

Figure 3.8.: OH stretching FTIR jet spectra of $\mathrm{H}$ expanded in helium (trace a) and helium mixed with $0.5 \% \mathrm{~N}$ (trace b), respectively (reprinted with adaptations from reference 234 with permission from John Wiley and Sons). Wavenumbers and (tentative) assignments are provided. Roman symbols denote the alcohol monomer unit involved in the vibration, italic symbols the passive monomer.

tures comprised of all possible $\mathrm{H}$ monomer combinations optimised at the PBEh-3c level, followed by further consideration at the B3LYP-D3(BJ)/def2-QZVP level of approximation. Predicted properties of the six most stable structures are summarised in Table 3.6 .

Weak progressions of rotational $\mu_{c}$-type transitions associated with a $\mathrm{HHH}$ trimer were identified in the CP-FTMW spectrum and the fitted rotational constants (Table 3.7, full list of assigned transitions in Table A.14 in appendix A.3) match predictions for $\left(\mathrm{H}_{g} \mathrm{H}_{g} \mathrm{H}_{g^{\prime}}\right)$, which corresponds to the observed most stable trimer as will be shown below. The only other possible structure with a predicted rotational spectrum consisting only of $\mu_{c}$-type transitions is $\left(\mathrm{H}_{t} \mathrm{H}_{g} \mathrm{H}_{g^{\prime}}\right)$-II, while structures with very low dipole moments such as $\mathrm{H}_{t} \mathrm{H}_{t} \mathrm{H}_{t}$ and $\left(\mathrm{H}_{t} \mathrm{H}_{t} \mathrm{H}_{t}\right)$ might not be observed due to the low signal-to-noise ratio for $\mathrm{HHH}$ trimers in the microwave spectrum (Figure 3.10) and therefore cannot be ruled out as possible candidates for the most stable HHH trimer structure based on the microwave data. The low signal-to-noise ratio in the CP-FTMW spectrum also explains 


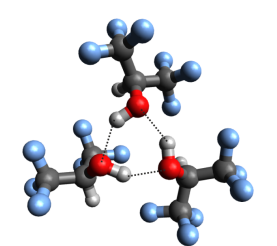

$\left(\mathrm{H}_{g} \mathrm{H}_{g} \mathrm{H}_{g^{\prime}}\right)[73.8]$

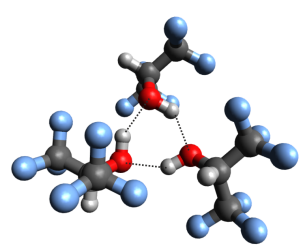

$\left(\mathrm{H}_{t} \mathrm{H}_{t} \mathrm{H}_{\mathrm{g}}\right)[63.2]$

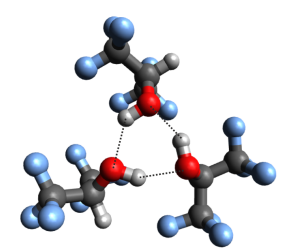

$\left(\mathrm{H}_{t} \mathrm{H}_{g} \mathrm{H}_{g^{\prime}}\right)[68.3]$

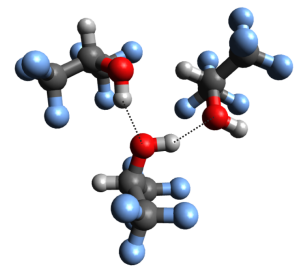

$\mathrm{H}_{t} \mathrm{H}_{t} \mathrm{H}_{t}(59.1)$

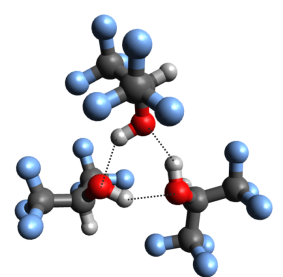

$\left(\mathrm{H}_{t} \mathrm{H}_{g} \mathrm{H}_{g^{\prime}}\right)-\mathrm{II}(67.6)$

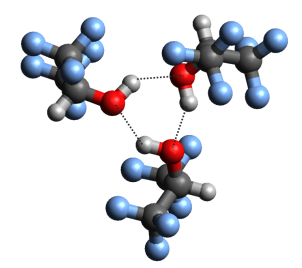

$\left(\mathrm{H}_{t} \mathrm{H}_{t} \mathrm{H}_{t}\right)(58.7)$

Figure 3.9.: Structures of the six most stable HHH trimers optimised at the B3LYPD3(BJ)/def2-QZVP level (reprinted with adaptations from reference 235 with permission from John Wiley and Sons). Zero-point corrected dissociation energies into the realised and locally optimised monomer conformations in $\mathrm{kJ} \mathrm{mol}^{-1}$ are given in brackets.

the non-observation of $\mu_{b}$-type transitions despite a significant predicted $\mu_{b}$ dipole moment component for $\left(\mathrm{H}_{g} \mathrm{H}_{g} \mathrm{H}_{g^{\prime}}\right)$. The Boltzmann-weighted relative intensity distribution for $\mu_{b^{-}}$-type transitions of $\left(\mathrm{H}_{g} \mathrm{H}_{g} \mathrm{H}_{g^{\prime}}\right)$ reaches its maximum around $2 \mathrm{GHz}$ (Figure A.11 in appendix A.3) where the sensitivity of the employed 2 to $6 \mathrm{GHz}$ instrument is worse than around the centre frequencies from 3 to $5 \mathrm{GHz}$, where the strongest $\mu_{c}$-type transitions are observed.

As microwave spectra alone strongly hint at $\left(\mathrm{H}_{g} \mathrm{H}_{g} \mathrm{H}_{g^{\prime}}\right)$ as the global minimum structure, but cannot be used to identify the most stable HHH trimer unambiguously, an experimental multi-messenger approach was chosen with added $\mathrm{OH}$ stretching FTIR and Raman spectra (Figure 3.11), thus simultaneously probing dipoles, dipole derivatives and polarisability derivatives.

Two strong signals are observed in the infrared spectrum at 3505 and $3467 \mathrm{~cm}^{-1}$ with an additional weak band at $3415 \mathrm{~cm}^{-1}$, while the strong band at $3467 \mathrm{~cm}^{-1}$ displays a shoulder at $3458 \mathrm{~cm}^{-1}$. The intensity pattern in the Raman spectrum is complementary with the two high frequency bands showing low intensities and the $3415 \mathrm{~cm}^{-1}$ band gaining enough intensity to identify an additional shoulder at $3408 \mathrm{~cm}^{-1}$. The intensity distribution in both Raman and infrared spectra is typical for a cyclic hydrogen bond topology with three cooperative $\mathrm{XH} \cdots \mathrm{XH}$ hydrogen bonds ${ }^{96}$ which is further corroborated by the lack of $\mathrm{N}$ coordination (Figure A.12 in appendix A.3). Both shoulders at 3458 and $3408 \mathrm{~cm}^{-1}$ most likely correspond to a second higher energy trimer in the expansion. The frequency pattern of the three strongest transitions at 3505, 3467 and $3415 \mathrm{~cm}^{-1}$ assigned to the most stable trimer structure rules out $C_{3}$ symmetry, as no near- 


\section{1,1,1,3,3,3-Hexafluoro-2-propanol}

Table 3.6.: Theoretically predicted harmonic $\left(\omega_{\mathrm{OH}}\right) \mathrm{OH}$ stretching wavenumber, spectral downshift from the respective monomer $\left(\Delta \omega_{\mathrm{OH}}\right)$ and lowest predicted wavenumber $\left(\omega_{\mathrm{l}}\right)$ in $\mathrm{cm}^{-1}$, IR intensity $\left(S_{\mathrm{OH}}\right)$ in $\mathrm{km} \mathrm{mol}^{-1}$, Raman scattering cross section $\left(\sigma_{\mathrm{OH}}\right)$ assuming $T_{\text {vib }}=100 \mathrm{~K}$ in $10^{-36} \mathrm{~m}^{2} \mathrm{sr}^{-1}$, relative electronic and harmonic vibrational zero-point corrected energies $\left(\Delta E_{\mathrm{e}}\right.$ and $\left.\Delta E_{0}\right)$ compared to the most stable $\mathrm{H}_{t} \mathrm{~N}_{\mathrm{H}}$ conformation in $\mathrm{kJ} \mathrm{mol}^{-1}$, as well as equilibrium rotational constants $\left(A_{\mathrm{e}}, B_{\mathrm{e}}, C_{\mathrm{e}}\right)$ in $\mathrm{MHz}$ and magnitudes of the electric dipole moment components $\left(\left|\mu_{a}\right|,\left|\mu_{b}\right|,\left|\mu_{c}\right|\right)$ in D calculated at the B3LYPD3(BJ)/def2-QZVP level of approximation.

\begin{tabular}{|c|c|c|c|c|c|c|c|c|c|c|c|c|c|}
\hline Structure & $\omega_{\mathrm{OH}}$ & $S_{\mathrm{OH}}$ & $\sigma_{\mathrm{OH}}$ & $\Delta \omega_{\mathrm{OH}}$ & $\Delta E_{\mathrm{e}}$ & $\Delta E_{0}$ & $\omega_{1}$ & $A_{\mathrm{e}}$ & $B_{\mathrm{e}}$ & $C_{\mathrm{e}}$ & $\left|\mu_{a}\right|$ & $\left|\mu_{b}\right|$ & $\left|\mu_{c}\right|$ \\
\hline & 3628 & 757 & 66 & 199 & & & & & & & & & \\
\hline \multirow{3}{*}{$\left(\mathrm{H}_{g} \mathrm{H}_{g} \mathrm{H}_{g^{\prime}}\right)$} & 3597 & 870 & 87 & 230 & 0.0 & 0.0 & 10 & 138.3 & 127.9 & 79.1 & 0.1 & 1.3 & 1.5 \\
\hline & 3539 & 209 & 268 & 288 & & & & & & & & & \\
\hline & 3615 & 625 & 56 & 174 & & & & & & & & & \\
\hline \multirow[t]{3}{*}{$\left(\mathrm{H}_{t} \mathrm{H}_{g} \mathrm{H}_{g^{\prime}}\right)$} & 3592 & 888 & 98 & 235 & 1.0 & 1.5 & 10 & 143.5 & 124.5 & 82.3 & 0.8 & 1.1 & 0.1 \\
\hline & 3530 & 257 & 259 & 297 & & & & & & & & & \\
\hline & 3601 & 895 & 82 & 188 & & & & & & & & & \\
\hline \multirow[t]{3}{*}{$\left(\mathrm{H}_{t} \mathrm{H}_{g} \mathrm{H}_{g^{\prime}}\right)-\mathrm{II}$} & 3573 & 872 & 59 & 254 & 1.9 & 2.2 & 8 & 145.8 & 115.5 & 80.2 & 0.9 & 0.4 & 1.0 \\
\hline & 3522 & 81 & 298 & 305 & & & & & & & & & \\
\hline & 3609 & 798 & 81 & 218 & & & & & & & & & \\
\hline \multirow[t]{3}{*}{$\left(\mathrm{H}_{t} \mathrm{H}_{t} \mathrm{H}_{g}\right)$} & 3573 & 599 & 110 & 216 & 2.1 & 2.4 & 8 & 134.2 & 124.5 & 80.6 & 2.2 & 1.1 & 0.4 \\
\hline & 3501 & 323 & 229 & 288 & & & & & & & & & \\
\hline & 3785 & 79 & 40 & 4 & & & & & & & & & \\
\hline \multirow[t]{3}{*}{$\mathrm{H}_{t} \mathrm{H}_{t} \mathrm{H}_{t}$} & 3548 & 869 & 80 & 241 & 3.1 & 2.5 & 11 & 139.9 & 124.6 & 88.6 & 0.4 & 0.4 & 0.6 \\
\hline & 3510 & 583 & 192 & 279 & & & & & & & & & \\
\hline & 3589 & 754 & 62 & 200 & & & & & & & & & \\
\hline \multirow[t]{2}{*}{$\left(\mathrm{H}_{t} \mathrm{H}_{t} \mathrm{H}_{t}\right)$} & 3574 & 795 & 71 & 215 & 2.8 & 2.9 & 8 & 127.0 & 124.6 & 83.2 & 0.2 & 0.1 & 0.2 \\
\hline & 3527 & 83 & 273 & 262 & & & & & & & & & \\
\hline
\end{tabular}

degeneracy is observed. Band positions around the dimer donor at $3492 \mathrm{~cm}^{-1}$ indicate substantial hydrogen bond strain, counteracting the cooperative downshift enhancement expected in a ring topology of three $\mathrm{OH} \cdots \mathrm{OH}$ hydrogen bonds.

The infrared and Raman spectra are only consistent with two of the six calculated structures (Figure A.13 in appendix A.3), $\left(\mathrm{H}_{g} \mathrm{H}_{g} \mathrm{H}_{g^{\prime}}\right)$ and $\left(\mathrm{H}_{t} \mathrm{H}_{g} \mathrm{H}_{g^{\prime}}\right)$. Since $\left(\mathrm{H}_{t} \mathrm{H}_{g} \mathrm{H}_{g^{\prime}}\right)$ has already been ruled out by the microwave spectra, $\left(\mathrm{H}_{g} \mathrm{H}_{g} \mathrm{H}_{g^{\prime}}\right)$ is unambiguously assigned as the most stable $\mathrm{HHH}$ trimer through the employed multi-messenger approach. The fact that both theoretical searches predict this structure as the global minimum trimer also creates a reassuring agreement between experiment and theory. The good match of the observed shoulders at 3458 and $3408 \mathrm{~cm}^{-1}$ in the infrared and Raman spectra with predicted frequencies of the second most stable $\left(\mathrm{H}_{t} \mathrm{H}_{g} \mathrm{H}_{g^{\prime}}\right)$ structure (Figure 3.11) makes a minor contribution of this high energy conformer, which shares the $\mathrm{H}_{g} \mathrm{H}_{g^{\prime}}$ motif with the minimum energy structure, in the helium expansions likely. However, rotational constants consistent with $\left(\mathrm{H}_{t} \mathrm{H}_{g} \mathrm{H}_{g^{\prime}}\right)$ would be required for a firm assignment and could not be observed in the microwave spectra due to the limited signal-to-noise ratio. Additionally, neon expansions used for the microwave spectra with the highest trimer intensities result in lower conformational temperatures and disfavour the formation of high energy conformers. 


\section{1,1,1,3,3,3-Hexafluoro-2-propanol}

Table 3.7.: Experimental spectroscopic parameters of the $\left(\mathrm{H}_{g} \mathrm{H}_{g} \mathrm{H}_{g^{\prime}}\right)$ trimer including rotational constants $(A, B, C)$ in $\mathrm{MHz}$, quartic centrifugal distortion constants $\left(\Delta_{\mathrm{J}}, \Delta_{\mathrm{JK}}\right.$, $\left.\Delta_{\mathrm{K}}, \delta_{\mathrm{J}}, \delta_{\mathrm{K}}\right)$ in $\mathrm{kHz}$, number of distinct frequencies $(N)$ assigned to transitions included in the fit and standard deviation $(\sigma)$ of the fit in $\mathrm{kHz}$.

\begin{tabular}{cc}
\hline Parameter & $\begin{array}{c}\text { Structure } \\
\left(\mathrm{H}_{g} \mathrm{H}_{g} \mathrm{H}_{g^{\prime}}\right)\end{array}$ \\
\hline$A$ & $137.95241(56)$ \\
$B$ & $128.82814(57)$ \\
$C$ & $79.0071(74)$ \\
$\Delta_{\mathrm{J}}$ & $0.242(83)$ \\
$\Delta_{\mathrm{JK}}$ & $-0.72(25)$ \\
$\Delta_{\mathrm{K}}$ & $0.49(16)$ \\
$\delta_{\mathrm{J}}$ & $-0.117(41)$ \\
$\delta_{\mathrm{K}}$ & $0.277(98)$ \\
$N$ & 142 \\
$\sigma$ & 10.8 \\
\hline
\end{tabular}

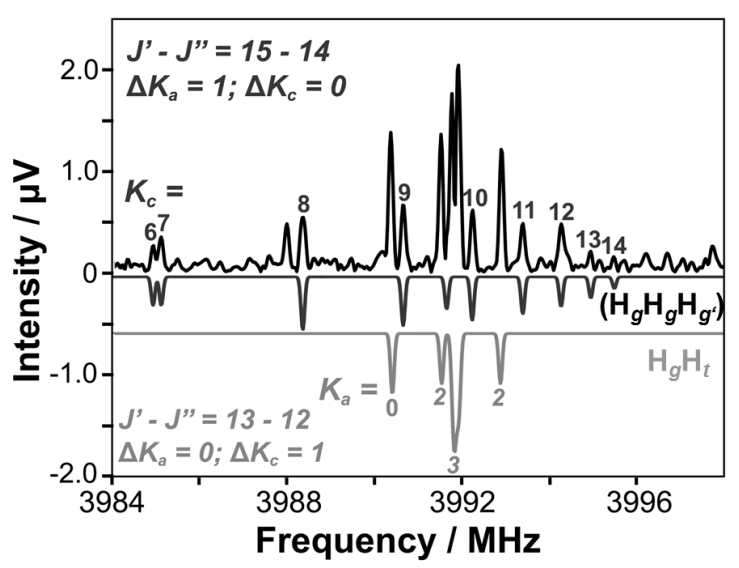

Figure 3.10.: Representative portion of the CP-FTMW spectrum of the HHH trimer (reprinted with adaptations from reference 235 with permission from John Wiley and Sons). Comparisons to predicted spectra from fitted rotational constants of $\left(\mathrm{H}_{g} \mathrm{H}_{g} \mathrm{H}_{g^{\prime}}\right)$ (black negative trace) and $\mathrm{H}_{g} \mathrm{H}_{t}$ (grey negative trace) are included.

The surprising stability of the scalemic $\left(\mathrm{H}_{g} \mathrm{H}_{g} \mathrm{H}_{g^{\prime}}\right)$ which requires a reorganisation energy of about $15 \mathrm{~kJ} \mathrm{~mol}^{-1}$ for the conversion of three achiral $\mathrm{H}_{t}$ monomers to the less stable transiently chiral $\mathrm{H}_{g}$ form (Figure 3.1) can be rationalised in two different ways. The increasing dipole moment from $\mathrm{H}_{t}$ over $\mathrm{H}_{g}$ to $\mathrm{H}_{c}$ generally stabilises the latter monomer conformations in more polar environments such as the solid state, ${ }^{255}$ the liquid phase or in $\mathrm{CCl}_{4}$ solution ${ }^{269,276}$ as they allow for larger intermolecular dipole- 


\section{1,1,1,3,3,3-Hexafluoro-2-propanol}

dipole interactions. Therefore the energetic preference switch from $\mathrm{H}_{t}$ to $\mathrm{H}_{g}$ or $\mathrm{H}_{c}$ is a straightforward consequence of increasing $\mathrm{H}$ aggregation. The $\left(\mathrm{H}_{g} \mathrm{H}_{g} \mathrm{H}_{g^{\prime}}\right)$ structure is further stabilised by a ring of secondary $\mathrm{CH} \cdots \mathrm{FC}$ contacts in addition to the primary $\mathrm{OH} \cdots \mathrm{OH}$ hydrogen bond ring, whereas the influence of minimised CF $\cdots$ FC contacts on the relative energy is less pronounced (Table A.15 in appendix A.3). Symmetryadapted perturbation theory (SAPT) analysis ${ }^{278}$ might be employed for a theoretical corroboration of these findings.

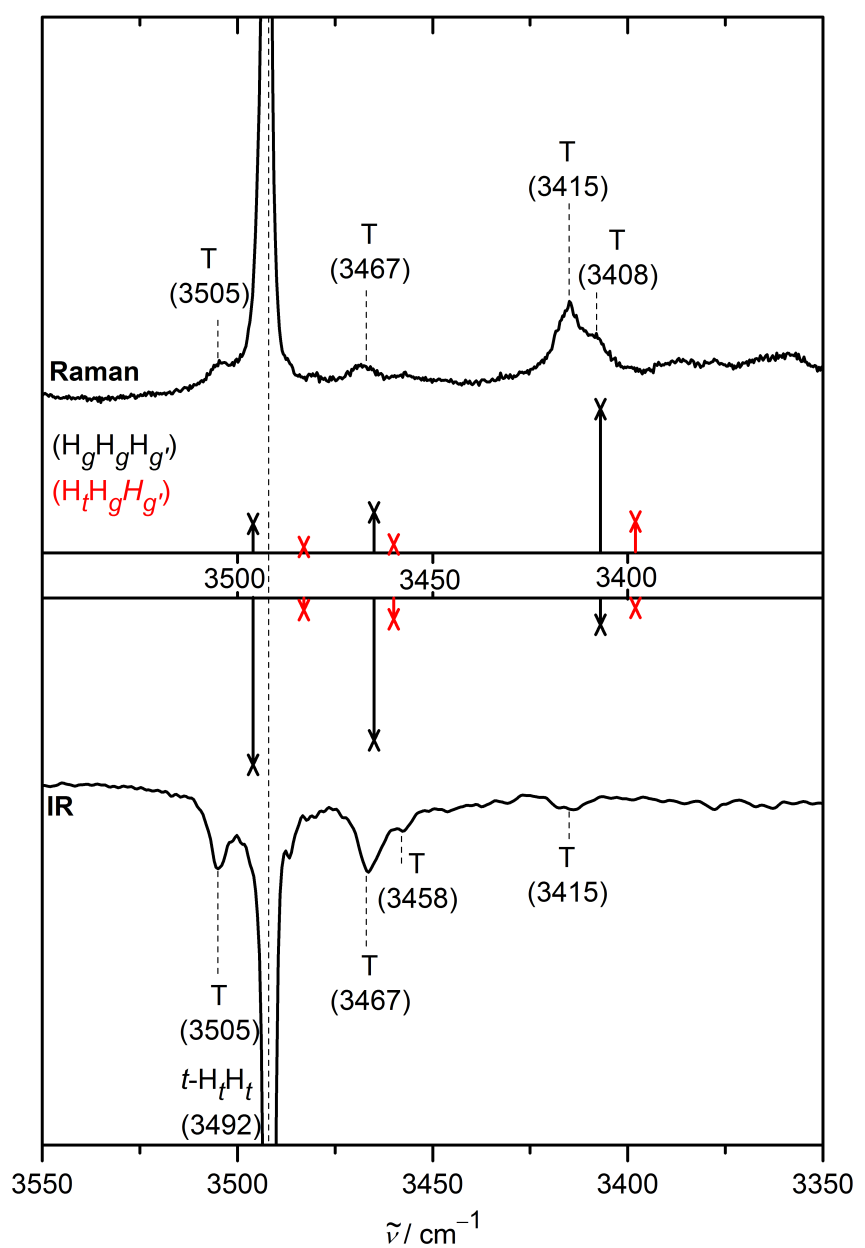

Figure 3.11.: OH-stretching Raman (top) and inverted FTIR (bottom) spectra of jetcooled $\mathrm{H}$ (reprinted with adaptations from reference 235 with permission from John Wiley and Sons). Wavenumbers and labels for proposed assignments (dimer $t-\mathrm{H}_{t} \mathrm{H}_{t}$, trimers $\mathrm{T}$ ) are provided. The harmonic IR and Raman stick spectra of the two most stable trimers at the B3LYP-D3(BJ)/def2-QZVP level are scaled to the experimental $t-\mathrm{H}_{t} \mathrm{H}_{t}$ donor vibration and the $\left(\mathrm{H}_{t} \mathrm{H}_{g} \mathrm{H}_{g^{\prime}}\right)$ intensities are reduced to $20 \%$ to account for the predicted energy penalty of $1.5 \mathrm{~kJ} \mathrm{~mol}^{-1}$. 


\section{1,1,1,3,3,3-Hexafluoro-2-propanol}

\subsection{Oxygen Aggregates}

$\mathrm{OH}$ stretching FTIR spectra of $\mathrm{H}$ with $\mathrm{O}$ addition (Figure 3.12) were obtained. Assignments to specific structures are somewhat difficult due to the lack of reliable accompanying quantum chemical calculations, but the observed $\mathrm{OH}$ stretching downshifts indicate $\mathrm{O}_{\mathrm{H}}$ coordination.

The mixed $\mathrm{H}_{t} \mathrm{O}$ dimer signal is observed at $3614 \mathrm{~cm}^{-1}$ with 2.5 and $10 \% \mathrm{O}$ addition, while three additional bands due to $\mathrm{O}$ coordinated $\mathrm{HH}$ dimers emerge downshifted from the $t-\mathrm{H}_{t} \mathrm{H}_{t}$ acceptor and donor vibrations, respectively. The broad signals at 3594 and $3466 \mathrm{~cm}^{-1}$ are only visible with $10 \% \mathrm{O}$ and are therefore assigned to O-nanocoated $\mathrm{H}_{t} \mathrm{H}_{t}$. The pair of $\mathrm{O}$ coordinated $\mathrm{OH}$ acceptor signals at 3597 and $3855 \mathrm{~cm}^{-1}$ and the corresponding $\mathrm{OH}$ donor bands at 3485 and $3478 \mathrm{~cm}^{-1}$ could either be assigned to a mixture of $t-\mathrm{H}_{t} \mathrm{H}_{t} \mathrm{O}_{\mathrm{H}}$ and $c-\mathrm{H}_{t} \mathrm{H}_{t} \mathrm{O}_{\mathrm{H}}$ similar to the $\mathrm{N}$ case or multiple $\mathrm{O}$ coordination of just one $\mathrm{H}_{t} \mathrm{H}_{t}$ conformer. Further measurements with different $\mathrm{O}$ fractions in the expansion and quantum chemical calculations regarding the influence of $\mathrm{O}$ complexation on the torsional isomerism of $\mathrm{H}_{t} \mathrm{H}_{t}$ would be necessary for an assignment.

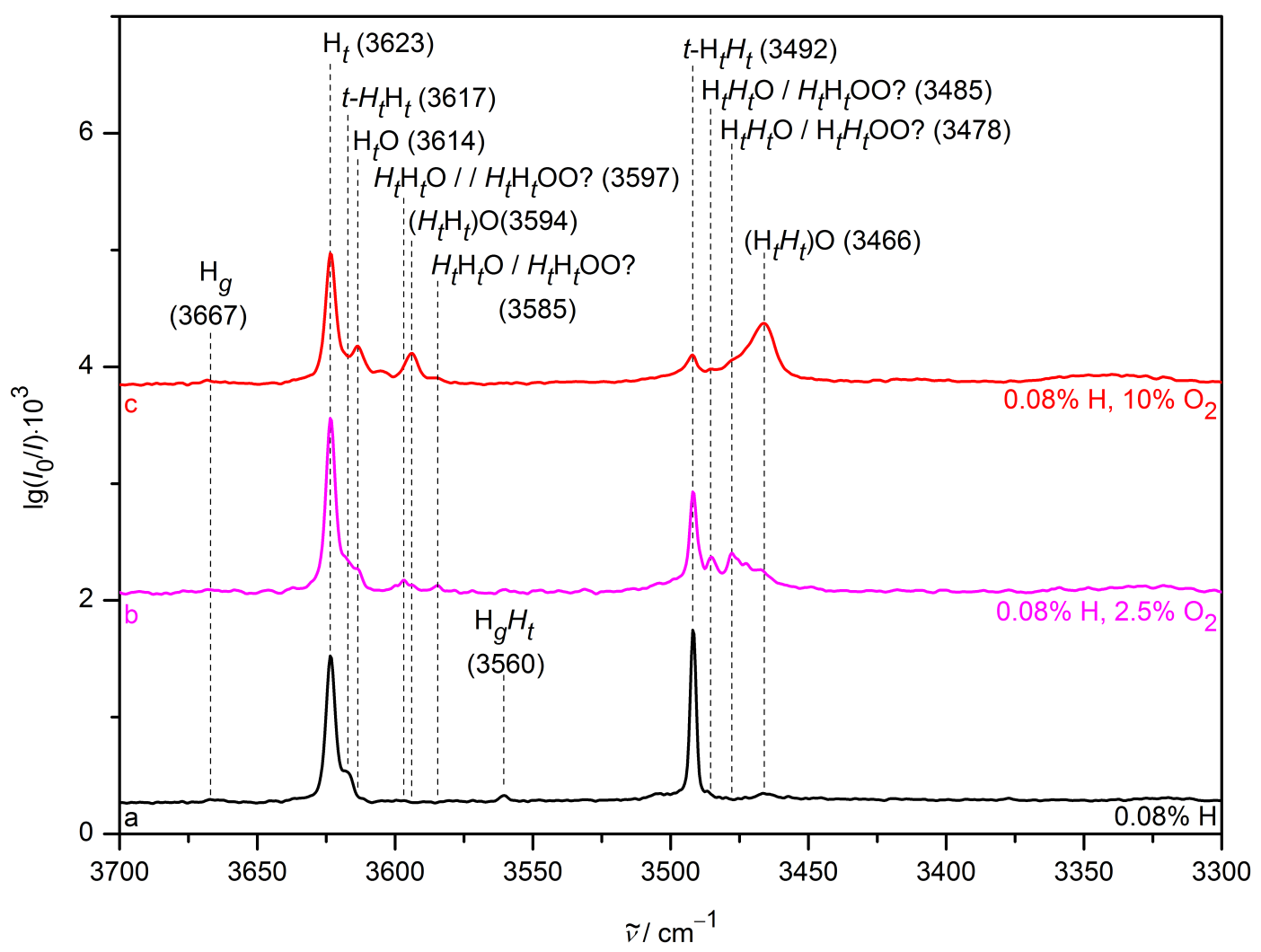

Figure 3.12.: $\mathrm{OH}$ stretching FTIR jet spectra of $\mathrm{H}$ expanded in helium (trace a) and helium mixed with increasing amounts of $\mathrm{O}$ (traces $\mathrm{b}$ and $\mathrm{c}$ ). Wavenumbers and (tentative) assignments are provided. Roman symbols denote the alcohol monomer unit involved in the vibration, italic symbols the passive monomer. $\mathrm{HH}$ dimers embedded in $\mathrm{O}$ are denoted as $(\mathrm{HH}) \mathrm{O}$, in this particular case the parentheses do not mark a cyclic structure. 


\subsection{Benchmarking Quantum Chemical Calculations}

The unambiguous spectral assignments for a wide range of $\mathrm{H}$ complexes allow for an extensive test of different electronic structure calculations against various experimental observables. Structural information encoded in rotational constants is available for the $\mathrm{H}_{t}$ monomer ${ }^{272}$ and its binary $\mathrm{N}$ complex as well as for two different $\mathrm{HH}$ dimers. The vibrational $\mathrm{OH}$ stretching band centre position for the $\mathrm{H}_{t}$ monomer is used alongside spectral shifts induced by $\mathrm{N}$ complexation, which also causes splittings in the $\mathrm{HH}$ dimer hydrogen bond acceptor and donor $\mathrm{OH}$ transitions. Finally, relative conformational energies can be derived from infrared band intensities for the three most stable $\mathrm{HH}$ dimers as well as both torsional $\mathrm{H}_{t} \mathrm{H}_{t} \mathrm{~N}_{\mathrm{H}}$ isomers. Due to large computational costs for the HHH homotrimer caused by its size, only a few theoretical methods were applied to the system and it is not included in the benchmarking analysis.

Since the tested standard calculations (see Table A.16 in appendix A.3 for $\mathrm{H}_{t}$ and $\mathrm{H}_{t} \mathrm{~N}_{\mathrm{H}}$ and Table A.17 in appendix A.3 for $\mathrm{HH}$ dimers and HHN trimers) are restricted to the widely used double harmonic approximation, careful error assessment is required to account for missing anharmonic effects on vibrational band positions and zero-point energies as well as neglected zero-point motion effects on rotational constants. Although numerous levels of electronic structure calculation were tested, the following analysis will be restricted to the method test set already established for carboxylic acids in the previous chapter to allow for a comparison between the two benchmark systems.

\subsubsection{Rotational Constants}

Predictions for rotational constants are typically derived from the static geometry of minimum energy structures, neglecting both harmonic and anharmonic vibrational effects on the equilibrium structure. ${ }^{40,41,279,280}$ Including only the readily available harmonic part would be counterproductive, since its influence on rotational constants is typically of opposite sign and smaller magnitude than corresponding anharmonic effects, as already seen in diatomic molecules. ${ }^{280}$ On average, rotational constants predicted while neglecting vibrational effects should be about $1 \%$ larger than experimental ones with deviations ranging from 0.2 to $1.6 \%{ }^{40,41,279}$ Therefore, differences between theory and experiment of 0.2 and $1.6 \%$ are chosen as approximate error bounds for the predicted $\mathrm{H}_{t}$ rotational constants (Table 3.8). Future work may change the exact numbers of these lower and upper bounds, especially since they are based on analyses in references 279 , 41 and 40 which are restricted to molecular test sets comprised of isolated monomers. These tend to be more rigid than weakly bound complexes. Consequently, larger error bounds from 0 to $1.8 \%$ are chosen for the binary $\mathrm{H}_{t} \mathrm{~N}_{\mathrm{H}}, t-\mathrm{H}_{t} \mathrm{H}_{t}$ and $\mathrm{H}_{g} \mathrm{H}_{t}$ aggregates.

Theoretical predictions tested against the experimental constraints are depicted in Figures $3.13,3.14$ and 3.15 for $\mathrm{H}_{t}$ and $\mathrm{H}_{t} \mathrm{~N}_{\mathrm{H}}$, while corresponding values for $t-\mathrm{H}_{t} \mathrm{H}_{t}$ and $\mathrm{H}_{g} \mathrm{H}_{t}$ dimers are shown in Figures A.14, A.15 and A.16 in appendix A.3. None of the tested theoretical approaches provides accurate rotational constants for all four structures, the range of predictions from different methods is particularly large for the weakly bound clusters. This reveals partly accurate predictions, also for other observ- 


\section{1,1,1,3,3,3-Hexafluoro-2-propanol}

Table 3.8.: Experimental estimates for equilibrium rotational constants $\left(A_{\mathrm{e}}, B_{\mathrm{e}}, C_{\mathrm{e}}\right)$ in $\mathrm{MHz}$ for the $\mathrm{H}_{t}$ monomer ${ }^{272}$ and the binary $\mathrm{H}_{t} \mathrm{~N}_{\mathrm{H}}, t-\mathrm{H}_{t} \mathrm{H}_{t}$ and $\mathrm{H}_{g} \mathrm{H}_{t}$ aggregates. Error bounds ranging from 0.2 to $1.6 \%$ deviation of experimental and predicted values were chosen for the monomer, non-covalently bound dimers are estimated with larger error bounds from 0 to $1.8 \%$.

\begin{tabular}{ccccc}
\hline & \multicolumn{4}{c}{ Structure } \\
Parameter & $\mathrm{H}_{t}{ }^{272}$ & $\mathrm{H}_{t} \mathrm{~N}_{\mathrm{H}}$ & $t-\mathrm{H}_{t} \mathrm{H}_{t}$ & $\mathrm{H}_{g} \mathrm{H}_{t}$ \\
\hline$A_{\mathrm{e}}$ & $2124(15)$ & $977(9)$ & $489(5)$ & $587(6)$ \\
$B_{\mathrm{e}}$ & $1063(8)$ & $741(7)$ & $209(2)$ & $156(2)$ \\
$C_{\mathrm{e}}$ & $941(7)$ & $538(5)$ & $172(2)$ & $154(2)$ \\
\hline
\end{tabular}

ables tested in the following sections, as results of fortuitous error cancellation, as they are based on inaccurate structural information.

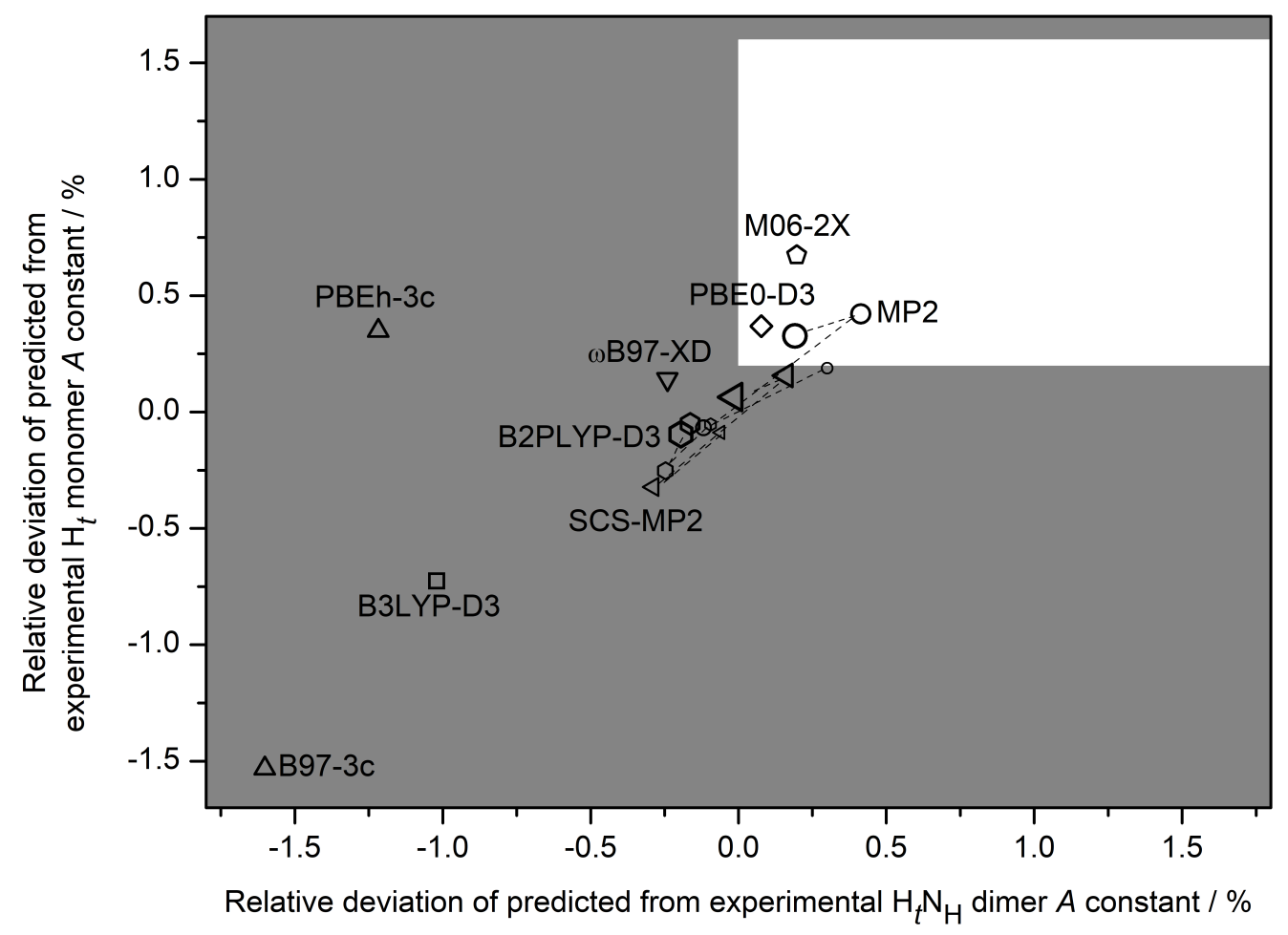

Figure 3.13.: Relative deviation of the theoretically predicted $\left(A_{\mathrm{e}}\right)$ from the experimental rotational constant $(A)$ for the $\mathrm{H}_{t}$ monomer and mixed $\mathrm{H}_{t} \mathrm{~N}_{\mathrm{H}}$ dimer at different levels of electronic structure calculation. For MP2, SCS-MP2 and B2PLYP-D3 increasing symbol size encodes the increasing basis set size from VTZ over aVTZ and VQZ to aVQZ. The zone of estimated incompatibility with experiment is grey. Only predictions within the white area are compatible with experiment for both species. 


\section{1,1,1,3,3,3-Hexafluoro-2-propanol}

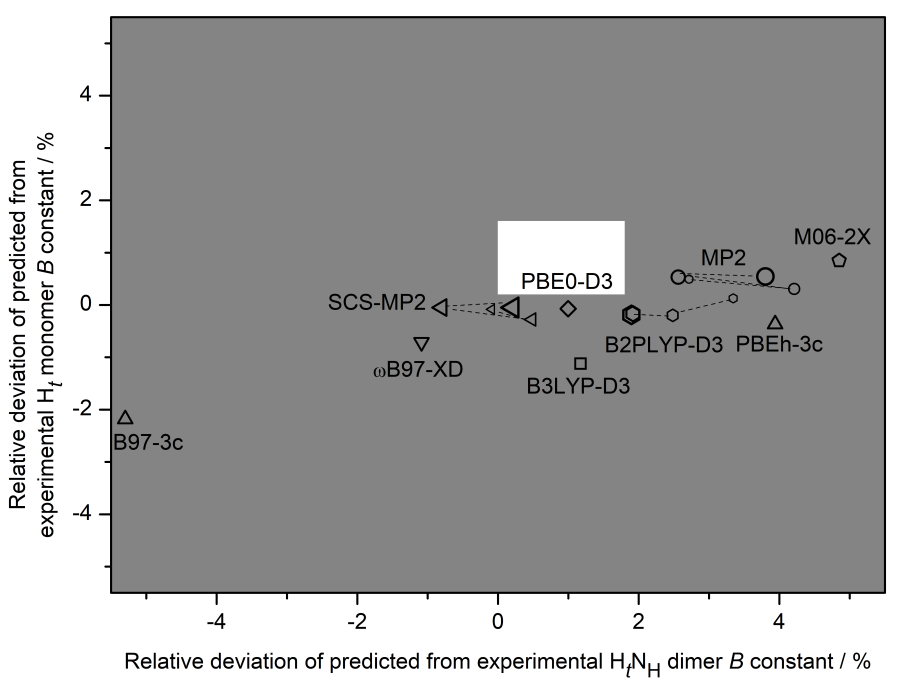

Figure 3.14.: Relative deviation of the theoretically predicted $\left(B_{\mathrm{e}}\right)$ from the experimental rotational constant $(B)$ for the $\mathrm{H}_{t}$ monomer and mixed $\mathrm{H}_{t} \mathrm{~N}_{\mathrm{H}}$ dimer at different levels of electronic structure calculation. For MP2, SCS-MP2 and B2PLYP-D3 increasing symbol size encodes the increasing basis set size from VTZ over aVTZ and VQZ to aVQZ. The zone of estimated incompatibility with experiment is grey. Only predictions within the white area are compatible with experiment for both species.

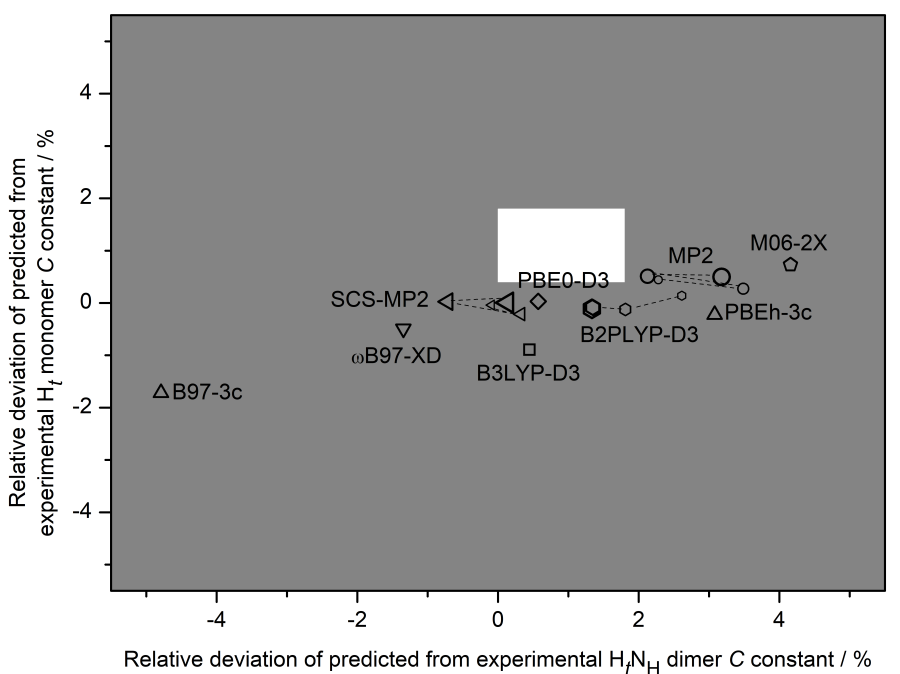

Figure 3.15.: Relative deviation of the theoretically predicted $\left(C_{\mathrm{e}}\right)$ from the experimental rotational constant $(C)$ for the $\mathrm{H}_{t}$ monomer and mixed $\mathrm{H}_{t} \mathrm{~N}_{\mathrm{H}}$ dimer at different levels of electronic structure calculation. For MP2, SCS-MP2 and B2PLYP-D3 increasing symbol size encodes the increasing basis set size from VTZ over aVTZ and VQZ to aVQZ. The zone of estimated incompatibility with experiment is grey. Only predictions within the white area are compatible with experiment for both species. 


\section{1,1,1,3,3,3-Hexafluoro-2-propanol}

Squared deviations calculated according to Equation 1.1 (Table 3.9) reveal that PBE0D3(BJ)/aVTZ provides the most accurate predictions for the tested molecular structures, followed by SCS-MP2/VTZ. The larger aVTZ, VQZ and aVQZ basis sets used for MP2, SCS-MP2 and B2PLYP-D3(BJ) are not considered in the analysis, as those calculations could only be carried out for $\mathrm{H}_{t}$ and $\mathrm{H}_{t} \mathrm{~N}_{\mathrm{H}}$, not for the larger $\mathrm{HH}$ dimer structures. On average, deviations of theory and experiment are significantly higher for molecular complexes with the theoretical description of $\mathrm{HH}$ dimers being worse than corresponding predictions for the monomer and binary $\mathrm{N}$ complex, which might hint at the necessity of larger error bars to account for missing anharmonicity information in weakly bound clusters. However, larger test data sets with corresponding anharmonicity analyses are required for corroboration.

Table 3.9.: Mean squared deviations $\delta^{2}(x)$ of theoretical predictions from three experimental reference values derived from all three rotational constants of $\mathrm{H}_{t}, \mathrm{H}_{t} \mathrm{~N}_{\mathrm{H}}, t-\mathrm{H}_{t} \mathrm{H}_{t}$ and $\mathrm{H}_{g} \mathrm{H}_{t}$ calculated according to Equation 1.1. Separate analysis for all four structures is provided, the mean value for all structures therefore includes 12 experimental observables.

\begin{tabular}{cccccc}
\hline & \multicolumn{5}{c}{$\delta^{2}\left(A_{\mathrm{e}}, B_{\mathrm{e}}, C_{\mathrm{e}}\right)$} \\
Level of theory & $\mathrm{H}_{t}$ & $\mathrm{H}_{t} \mathrm{~N}_{\mathrm{H}}$ & $t-\mathrm{H}_{t} \mathrm{H}_{t}$ & $\mathrm{H}_{g} \mathrm{H}_{t}$ & all structures \\
\hline PBE0-D3(BJ)/aVTZ & 1.3 & 0.3 & 1.6 & 1.8 & 1.3 \\
SCS-MP2/VTZ & 1.9 & 1.2 & 1.4 & 2.0 & 1.6 \\
MP2/VTZ & 0.6 & 2.3 & 1.2 & 7.5 & 2.9 \\
B3LYP-D3(BJ)/def2-QZVP & 7.1 & 1.6 & 2.8 & 3.5 & 3.8 \\
B2PLYP-D3(BJ)VTZ & 1.4 & 4.1 & 2.1 & 7.8 & 3.8 \\
wB97-XD/aVTZ & 3.6 & 4.2 & 3.4 & 7.9 & 4.8 \\
M06-2X/aVTZ & 0.0 & 11.0 & 5.0 & 14.9 & 7.7 \\
PBEh-3c & 2.2 & 7.6 & 24.1 & 48.6 & 20.6 \\
B97-3c & 16.0 & 31.7 & 55.2 & 28.9 & 33.0 \\
\hline Mean & 3.8 & 7.1 & 10.8 & 13.7 & 8.8 \\
\hline
\end{tabular}

\subsubsection{Vibrational Band Centre Positions}

Vibrational $\mathrm{OH}$ stretching band centre positions and hydrogen bond induced downshifts are analysed following the scheme already established for carboxylic acids in the previous chapter. Since anharmonic (VPT2) calculations for the large $\mathrm{H}$ are too demanding with regard to computational resources, only harmonic predictions for vibrational frequencies are available. Consequently, large error bars have to be assumed for the comparison between anharmonic experiment and harmonic theory. For the $\mathrm{H}_{t}$ monomer $\mathrm{OH}$ stretching band centre, an estimated experimental harmonic wavenumber of $3791(17) \mathrm{cm}^{-1}$ is obtained from the $7079 \mathrm{~cm}^{-1}$ overtone ${ }^{101}$ and by adding $10 \%$ of the $84 \mathrm{~cm}^{-1}$ diagonal anharmonicity to the error bar to account for neglected off-diagonal contributions. The harmonic $\mathrm{H}_{g} \mathrm{OH}$ stretching wavenumber is estimated analogously to $3836(17) \mathrm{cm}^{-1}$ from the $3667 \mathrm{~cm}^{-1}$ fundamental transition and the $7165 \mathrm{~cm}^{-1}$ overtone. ${ }^{266}$ 
3. 1,1,1,3,3,3-Hexafluoro-2-propanol

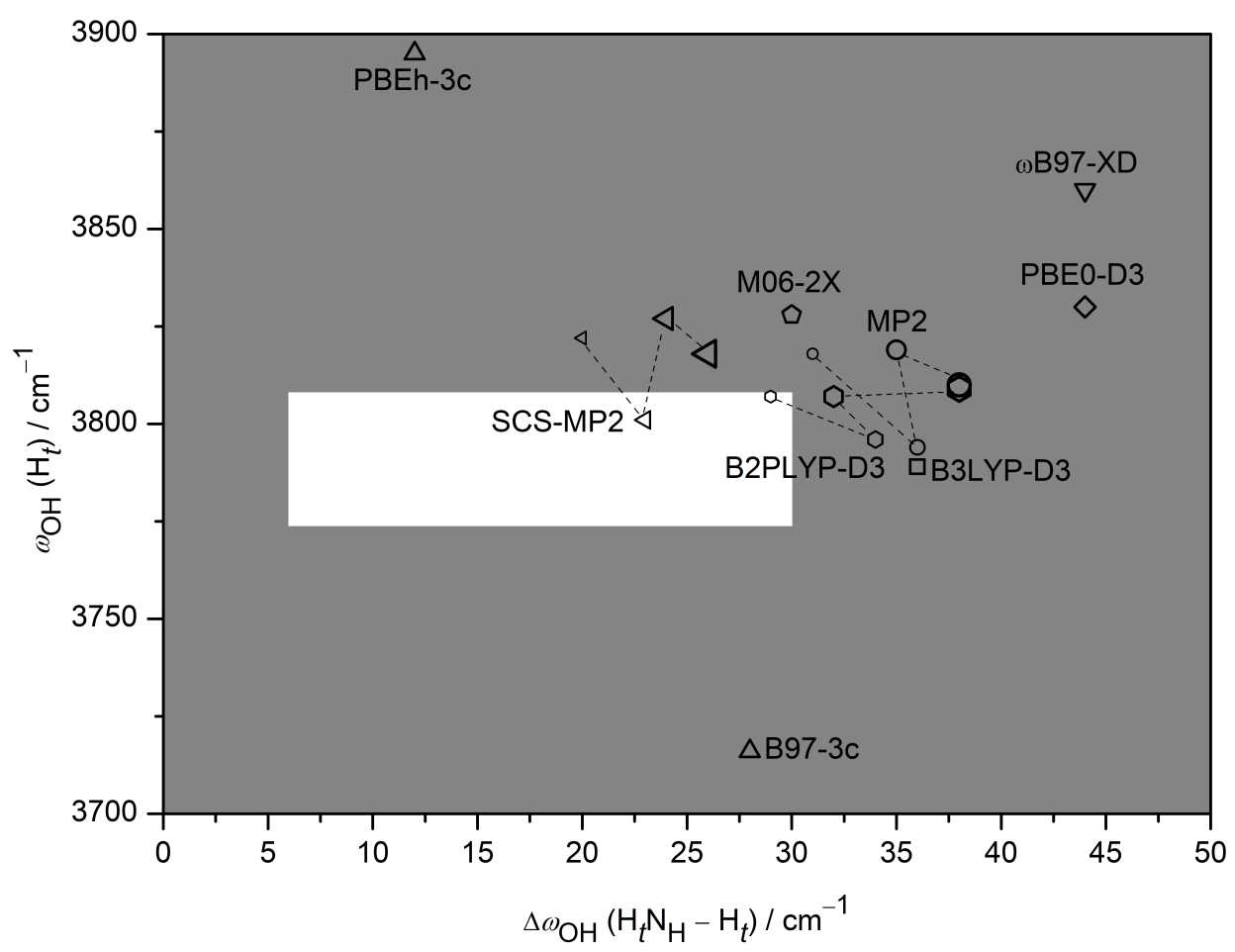

Figure 3.16.: Theoretically predicted harmonic $\mathrm{OH}$ stretching band centre position of $\mathrm{H}_{t}$ $\left(\omega_{\mathrm{OH}}\left(\mathrm{H}_{t}\right)\right)$ plotted against the harmonic downshift $\left(\Delta \omega_{\mathrm{OH}}\left(\mathrm{H}_{t} \mathrm{~N}_{\mathrm{H}}-\mathrm{H}_{t}\right)\right)$ from $\mathrm{H}_{t}$ to $\mathrm{H}_{t} \mathrm{~N}_{\mathrm{H}}$ for different computational methods implemented in Gaussian09 and ORCA 4.0 (reprinted with adaptations from reference 234 with permission from John Wiley and Sons). For MP2, SCS-MP2 and B2PLYP-D3 increasing symbol size encodes the increasing basis set size from VTZ over aVTZ and VQZ to aVQZ. The experimental harmonic monomer band position was approximately derived from the fundamental and first overtone transitions. ${ }^{101}$ Experimental wavenumber downshifts are anharmonic. Harmonic values are assumed to deviate from them by up to $\pm 50 \%$ due to anharmonic effects for hydrogen-bond-induced shifts. The estimated zone of incompatibility with the experiment is grey and predictions within the white area are compatible with experiment for both properties.

For the observed $18 \mathrm{~cm}^{-1}$ downshift induced by $\mathrm{N}$ complexation, error bounds of $50 \%$ corresponding to $9 \mathrm{~cm}^{-1}$ are assumed as neither the dimer overtone nor any off-diagonal anharmonicity contributions are experimentally known (Figure 3.16). The anharmonic experimental spectral splitting between both monomer isomers is $44 \mathrm{~cm}^{-1}$, while the estimated harmonic band centre positions differ by $45 \mathrm{~cm}^{-1}$. Diagonal anharmonicities ${ }^{266}$ for $\mathrm{H}_{t}\left(x_{1,1}=84 \mathrm{~cm}^{-1}\right)$ and $\mathrm{H}_{g}\left(x_{1,1}=85 \mathrm{~cm}^{-1}\right)$ are very similar, which is also likely for the off-diagonal contributions. Therefore, the error bounds for the harmonic spectral splitting are estimated to be very narrow, about twice the difference of harmonic and anharmonic spectral splitting to account for neglected off-diagonal anharmonicity, resulting in $2 \mathrm{~cm}^{-1}$, which are added to the experimental band position uncertainties of $4 \mathrm{~cm}^{-1}$, resulting in a total error bar of $6 \mathrm{~cm}^{-1}$ (Figure 3.17). Spectral splittings of the two observed $\mathrm{H}_{t} \mathrm{H}_{t} \mathrm{~N}_{\mathrm{H}}$ trimers are compared to harmonic predictions and values consis- 


\section{1,1,1,3,3,3-Hexafluoro-2-propanol}

tent with experiment range from $10(5) \mathrm{cm}^{-1}$ in the donor $\mathrm{OH}$ region to $20(10) \mathrm{cm}^{-1}$ in the acceptor region (Figure 3.18), as the $50 \%$ error bounds need to be applied again for these structures.

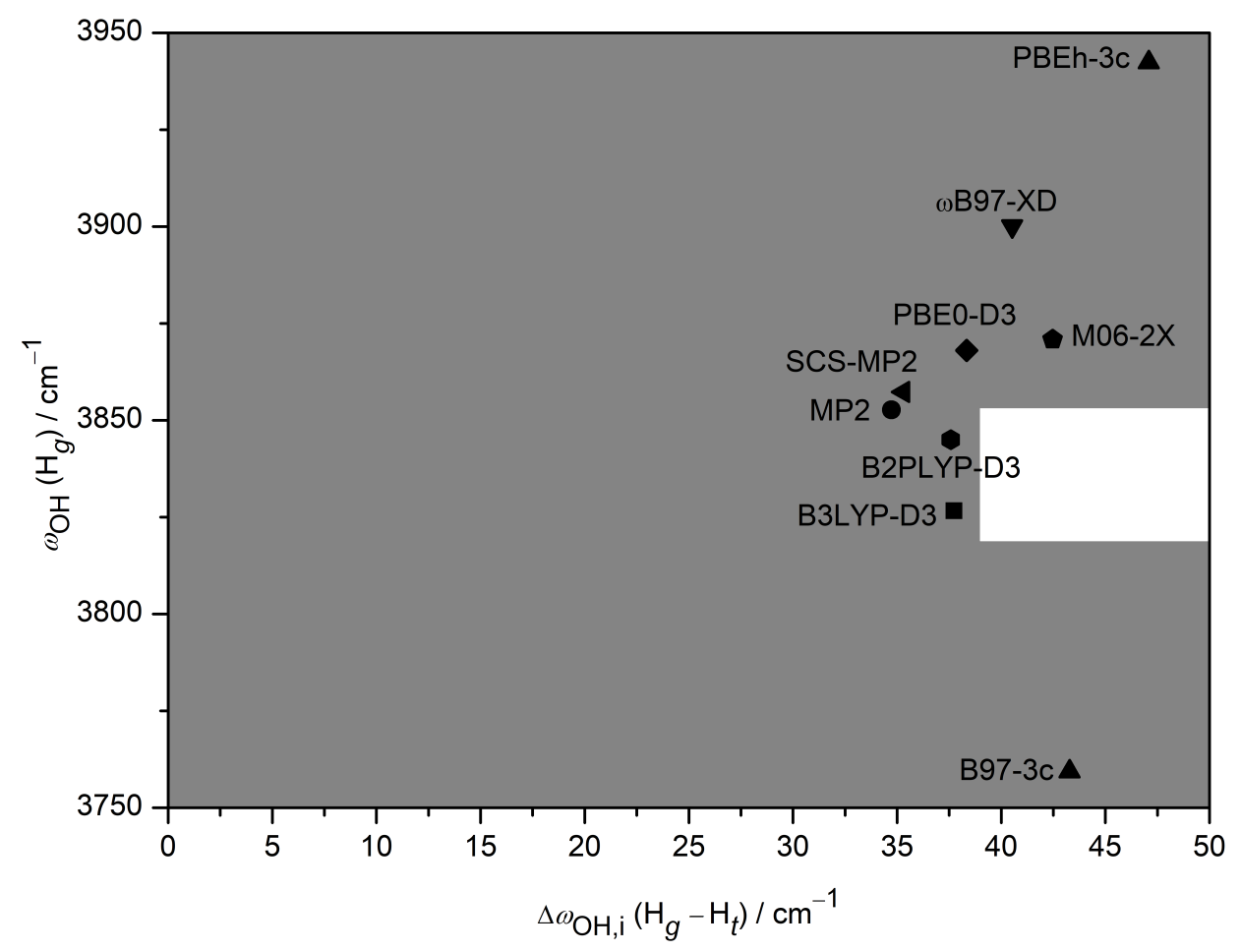

Figure 3.17.: Theoretically predicted harmonic $\mathrm{OH}$ stretching band centre position of $\mathrm{H}_{g}$ $\left(\omega_{\mathrm{OH}}\left(\mathrm{H}_{g}\right)\right)$ plotted against the harmonic spectral splitting $\left(\Delta \omega_{\mathrm{OH}, \mathrm{i}}\left(\mathrm{H}_{g}-\mathrm{H}_{t}\right)\right)$ between the $\mathrm{H}_{g}$ and $\mathrm{H}_{t}$ isomers for different computational methods implemented in Gaussian09 and ORCA 4.0. The experimental harmonic monomer band position was approximately derived from the fundamental and first overtone transitions. ${ }^{266}$ Experimental wavenumber splittings are anharmonic, but due to systematic error cancellation through similar anharmonicities ${ }^{266}$ in both monomer structures, harmonic values are assumed to deviate from them by twice the difference between estimated harmonic and experimental anharmonic splitting to account for neglected off-diagonal anharmonicity, which is added to the experimental half-width at half-maximum of measured vibrational bands. The zone of incompatibility with experiment is grey and predictions within the white area are compatible with experiment for both properties.

Deviations calculated according to Equation 1.1 (Table 3.10) showcase that an accurate reproduction of both monomer $\mathrm{OH}$ stretching wavenumbers provides the most challenging test for current electronic structure methods. Predictions for spectral downhshifts and splittings often benefit from error cancellation and in the current analysis also from the large error bars which are necessary in the comparison of harmonic theory and anharmonic experiment. Particularly the relatively large $\mathrm{H}_{t} \mathrm{H}_{t} \mathrm{~N}_{\mathrm{H}}$ acceptor splitting provides almost no discriminatory function due to these error bounds. Using 


\section{1,1,1,3,3,3-Hexafluoro-2-propanol}

even larger shifts as benchmarks such as those from $\mathrm{H}_{t}$ monomer to $\mathrm{HH}$ dimers without further experimental data like $\mathrm{HH}$ dimer overtones and combination bands would therefore only enlarge this problem.

The combination of rotational and vibrational data reveals electronic structure methods with partly good predictions as the result of fortuitous error cancellation. The best values for rotational constants in the previous section were provided at the PBE0D3(BJ)/aVTZ level, while the corresponding vibrational energy levels predicted at this particular level of theory are significantly worse in comparison with experiment.

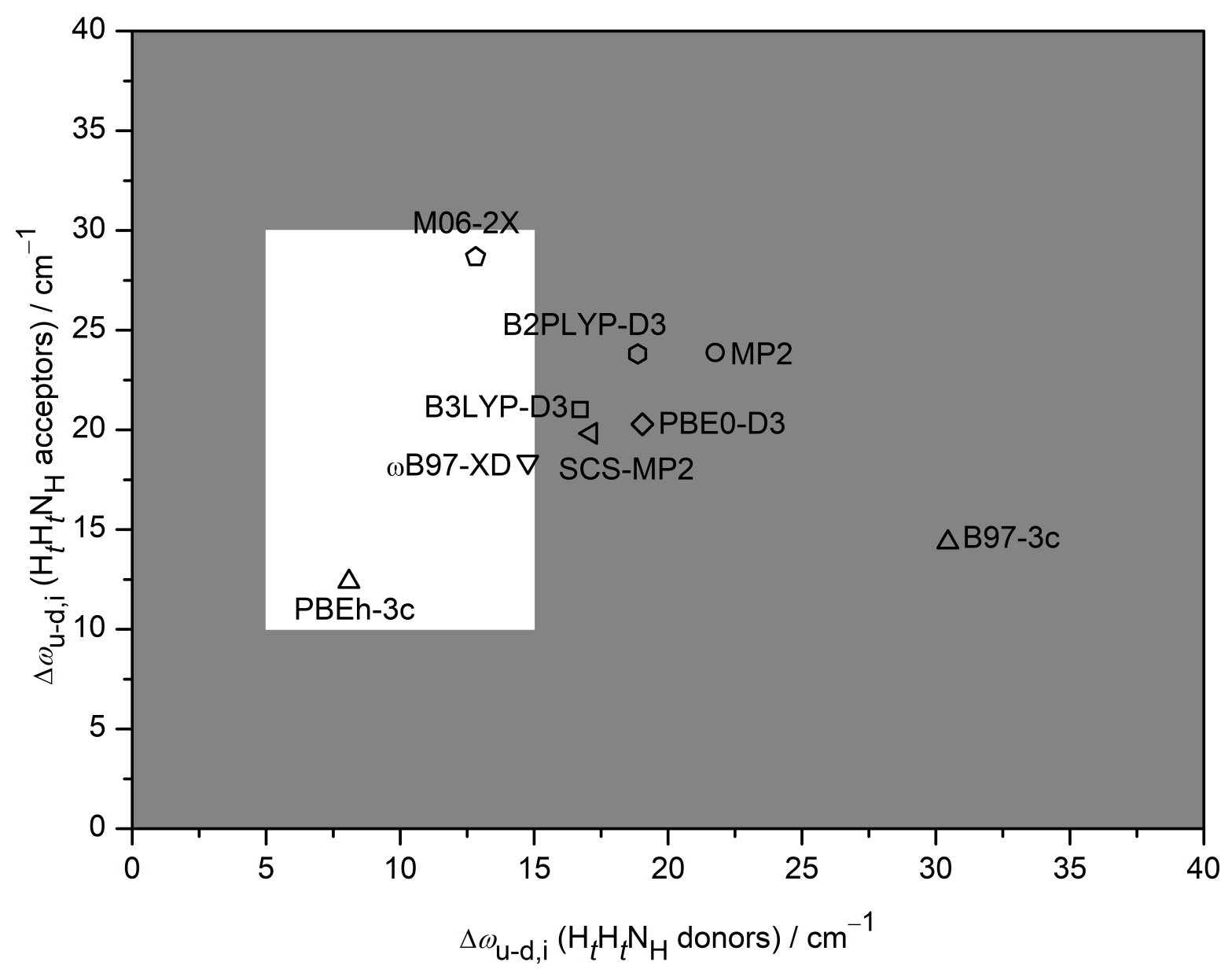

Figure 3.18.: Theoretically predicted harmonic OH stretching wavenumber splittings between both $\mathrm{H}_{t} \mathrm{H}_{t} \mathrm{~N}_{\mathrm{H}}$ isomers $\left(\omega_{\mathrm{u}-\mathrm{d}, \mathrm{i}}\right)$ in the $\mathrm{OH} \cdots \mathrm{OH}$ hydrogen bond donor and acceptor region (reprinted with adaptations from reference 234 with permission from John Wiley and Sons). The conformer with the higher wavenumber prediction is denoted as $u$ (up), the lower frequency predictions as d (down). Different computational methods implemented in Gaussian09 and ORCA 4.0 are included. Experimental wavenumber splittings are anharmonic. Harmonic values are assumed to deviate from them by up to $\pm 50 \%$ due to anharmonic effects. The estimated zone of incompatibility with the experiment is grey and predictions within the white area are compatible with experiment for both properties. 


\section{1,1,1,3,3,3-Hexafluoro-2-propanol}

Table 3.10.: Squared deviations $\delta^{2}(x)$ of theoretical predictions from experimental reference values derived from $\mathrm{OH}$ stretching FTIR spectra of $\mathrm{H}_{t}, \mathrm{H}_{g}, \mathrm{H}_{t} \mathrm{~N}_{\mathrm{H}}, t-\mathrm{H}_{t} \mathrm{H}_{t} \mathrm{~N}_{\mathrm{H}}$ and $c-\mathrm{H}_{t} \mathrm{H}_{t} \mathrm{~N}_{\mathrm{H}}$ calculated according to Equation 1.1. Separate analysis for the monomer band centre positions of both isomers $\left(\omega_{\mathrm{OH}}\left(\mathrm{H}_{t}\right), \omega_{\mathrm{OH}}\left(\mathrm{H}_{g}\right)\right)$, the $\mathrm{N}$ induced downshift in the binary complex $\left(\Delta \omega_{\mathrm{OH}}\left(\mathrm{H}_{t} \mathrm{~N}_{\mathrm{H}}-\mathrm{H}_{t}\right)\right)$ as well as wavenumber splittings between both $\mathrm{H}_{t} \mathrm{H}_{t} \mathrm{~N}_{\mathrm{H}}$ isomers $\left(\omega_{\mathrm{u}-\mathrm{d}, \mathrm{i}}\right)$ is provided. The mean value of all vibrational transitions therefore includes six experimental reference values.

\begin{tabular}{|c|c|c|c|c|c|c|c|}
\hline Level of theory & $\begin{array}{c}\delta^{2}\left(\omega_{\mathrm{OH}}\right) \\
\mathrm{H}_{t}\end{array}$ & $\begin{array}{c}\delta^{2}\left(\omega_{\mathrm{OH}}\right) \\
\mathrm{H}_{g}\end{array}$ & $\begin{array}{c}\delta^{2}\left(\Delta \omega_{\mathrm{OH}, \mathrm{i}}\right) \\
\mathrm{H}_{g}-\mathrm{H}_{t}\end{array}$ & $\begin{array}{c}\delta^{2}\left(\Delta \omega_{\mathrm{OH}}\right) \\
\mathrm{H}_{t} \mathrm{~N}_{\mathrm{H}}-\mathrm{H}_{t}\end{array}$ & $\begin{array}{c}\delta^{2}\left(\Delta \omega_{\mathrm{u}-\mathrm{d}, \mathrm{i}}\right) \\
\mathrm{H}_{t} \mathrm{H}_{t} \mathrm{~N}_{\mathrm{H}} \\
\text { donors }\end{array}$ & $\begin{array}{c}\delta^{2}\left(\Delta \omega_{\mathrm{u}-\mathrm{d}, \mathrm{i}}\right) \\
\mathrm{H}_{t} \mathrm{H}_{t} \mathrm{~N}_{\mathrm{H}} \\
\text { acceptors }\end{array}$ & $\begin{array}{l}\delta^{2}(x) \\
\text { Mean }\end{array}$ \\
\hline B2PLYP-D3(BJ)VTZ & 0.9 & 0.3 & 1.5 & 1.5 & 3.1 & 0.1 & 1.3 \\
\hline B3LYP-D3(BJ)/def2-QZVP & 0.0 & 0.3 & 1.5 & 4.0 & 1.8 & 0.0 & 1.3 \\
\hline SCS-MP2/VTZ & 3.3 & 1.6 & 2.6 & 0.0 & 2.0 & 0.0 & 1.6 \\
\hline M06-2X/aVTZ & 4.8 & 4.2 & 0.2 & 1.8 & 0.3 & 0.8 & 2.0 \\
\hline MP2/VTZ & 2.5 & 1.0 & 2.9 & 2.1 & 5.5 & 0.2 & 2.4 \\
\hline PBE0-D3(BJ)/aVTZ & 5.2 & 3.6 & 1.2 & 8.3 & 3.3 & 0.0 & 3.6 \\
\hline$\omega \mathrm{B} 97-\mathrm{XD} / \mathrm{aVTZ}$ & 16.3 & 14.2 & 0.6 & 8.3 & 0.9 & 0.0 & 6.7 \\
\hline B97-3c & 19.5 & 20.4 & 0.7 & 1.2 & 16.7 & 0.3 & 9.7 \\
\hline PBEh-3c & 37.6 & 39.2 & 0.1 & 0.4 & 0.1 & 0.6 & 13.0 \\
\hline Mean & 10.0 & 9.4 & 1.3 & 3.1 & 3.8 & 0.2 & 5.1 \\
\hline
\end{tabular}

\subsubsection{Relative Conformational Energies}

The combined spectroscopic evidence, especially from FTIR spectra, is used to derive relative experimental energies of different molecular conformations with respect to the respective minimum energy structure, which can be directly compared to harmonic theoretical predictions, ${ }^{48-54}$ because anharmonicity effects on zero-point vibrational energies are smaller than for XH stretching modes and will mostly cancel among similar docking sites. ${ }^{51}$ For the two most stable $\mathrm{H}_{t} \mathrm{H}_{t}$ dimers, the non-detection of $c-\mathrm{H}_{t} \mathrm{H}_{t}$ implies a relative abundance of less than $10 \%$ compared to $t-\mathrm{H}_{t} \mathrm{H}_{t}$ in the expansions, as detection of minor $c-\mathrm{H}_{t} \mathrm{H}_{t}$ contributions might be impeded by spectral overlap with $t-\mathrm{H}_{t} \mathrm{H}_{t}$ or the $\left(\mathrm{H}_{g} \mathrm{H}_{g} \mathrm{H}_{g^{\prime}}\right)$ trimer in the infrared and Raman spectra or the low permanent dipole moment in the microwave spectra. Due to the low interconversion barrier between $c$ - and $t-\mathrm{H}_{t} \mathrm{H}_{t}$ a conformational temperature between of $50 \mathrm{~K}$ can be realistically estimated, ${ }^{101}$ which translates to an energy difference of at least $1.0 \mathrm{~kJ} \mathrm{~mol}^{-1}$ via the Boltzmann distribution.

However, some minor symmetry and entropy effects need to be considered in addition. The $t-\mathrm{H}_{t} \mathrm{H}_{t}$ isomer is $C_{s}$-symmetric, which leads to a configurational Gibbs energy advantage of 0.5 to $1.0 \mathrm{~kJ} \mathrm{~mol}^{-1}$ for the non-symmetric, chiral $c-\mathrm{H}_{t} \mathrm{H}_{t}$. This is counteracted by the shallow torsional well of $t-\mathrm{H}_{t} \mathrm{H}_{t}$ (Figure 3.7) with the predicted normal mode between 5 and $10 \mathrm{~cm}^{-1}$ compared to the 10 to $20 \mathrm{~cm}^{-1}$ for $c-\mathrm{H}_{t} \mathrm{H}_{t}$ (Table A.17 in appendix A.3) translating into a Gibbs energy advantage of 0.3 to $1.0 \mathrm{~kJ} \mathrm{~mol}^{-1}$ for $t-\mathrm{H}_{t} \mathrm{H}_{t}$ at an estimated vibrational temperature between 50 and $100 \mathrm{~K}$, so that both effects roughly cancel. This also applies to the respective $\mathrm{H}_{t} \mathrm{H}_{t} \mathrm{~N}_{\mathrm{H}}$ complexes.

The relative abundance of the less stable $\mathrm{H}_{g} \mathrm{H}_{t}$ isomer is determined from band inte- 


\section{1,1,1,3,3,3-Hexafluoro-2-propanol}

grals of the two $\mathrm{OH}$ donor vibrations in the FTIR spectra (see Table A.18 in appendix A.3 for further details on the integration). The integral ratio implies a $\mathrm{H}_{g} \mathrm{H}_{t}$ fraction of about $5 \%$ in the expansion. Therefore, a relative $\mathrm{H}_{g} \mathrm{H}_{t}$ abundance of about $5(1) \%$ is estimated, the error bar including integration uncertainties as well as predicted relative infrared band strengths of both isomers (Table A.17 in appendix A.3). Since the theoretical band strength ratio of about 1 varies with the level of theory within $\pm 10 \%$, no corrections are applied but $10 \%$ are added to the error bar of the relative $\mathrm{H}_{g} \mathrm{H}_{t}$ abundance. However, $\mathrm{H}_{g} \mathrm{H}_{t}$ benefits from a two-fold degeneracy, which the $C_{s}$-symmetric $t-\mathrm{H}_{t} \mathrm{H}_{t}$ lacks, resulting in a formal statistically corrected fraction of 2.5(5)\%. An accurate estimation of the conformational temperature in the expansion is somewhat difficult, as the large energy barrier for conversion exerts a significant influence over this particular quantity. ${ }^{48,54,101}$ Physically meaningful boundaries are provided by the temperature at which gas mixtures are stored in the reservoir (room temperature, about $300 \mathrm{~K}$ ) and the typically observed rotational temperature at the filet-jet set-up of $10 \mathrm{~K} .{ }^{54}$ These conservatively large error bounds translate to an energy difference of $5.1(4.8) \mathrm{kJ} \mathrm{mol}^{-1}$ (Figure 3.19). These error bounds are too large for an effective discrimination between most theoretical methods. Therefore, this energy difference is excluded from the detailed analysis of squared deviations, but the combined relative dimer energies rule out predictions at the M06-2X/aVTZ, PBEh-3c and B97-3c levels.

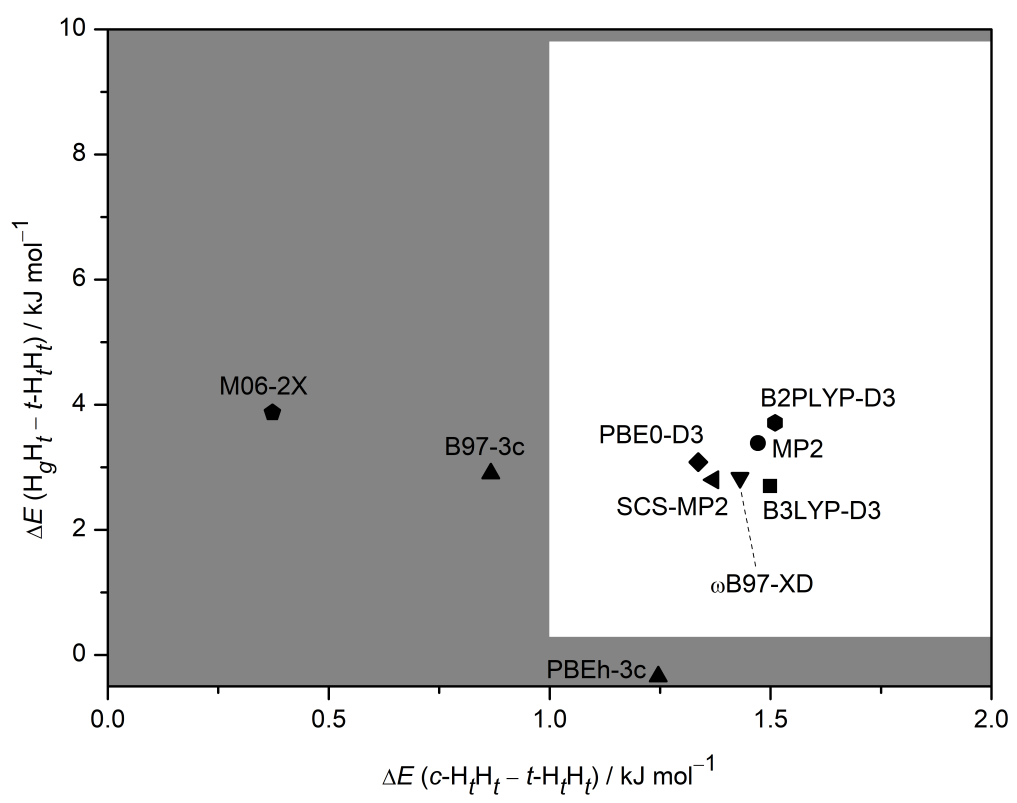

Figure 3.19.: Zero-point-corrected energy differences $\Delta E$ between different $\mathrm{HH}$ dimer isomers for different electronic structure methods (reprinted with adaptations from reference 234 with permission from John Wiley and Sons). White areas are compatible with experiment as they predict depopulation of the less stable $c-\mathrm{H}_{t} \mathrm{H}_{t}$ isomer to less than $10 \%$ abundance with the energy difference exceeding $1 \mathrm{~kJ} \mathrm{~mol}^{-1}$ as well as the correct relative abundance of the $\mathrm{H}_{g} \mathrm{H}_{t}$ isomer for an estimated conformational temperature between 10 and $300 \mathrm{~K}$. 


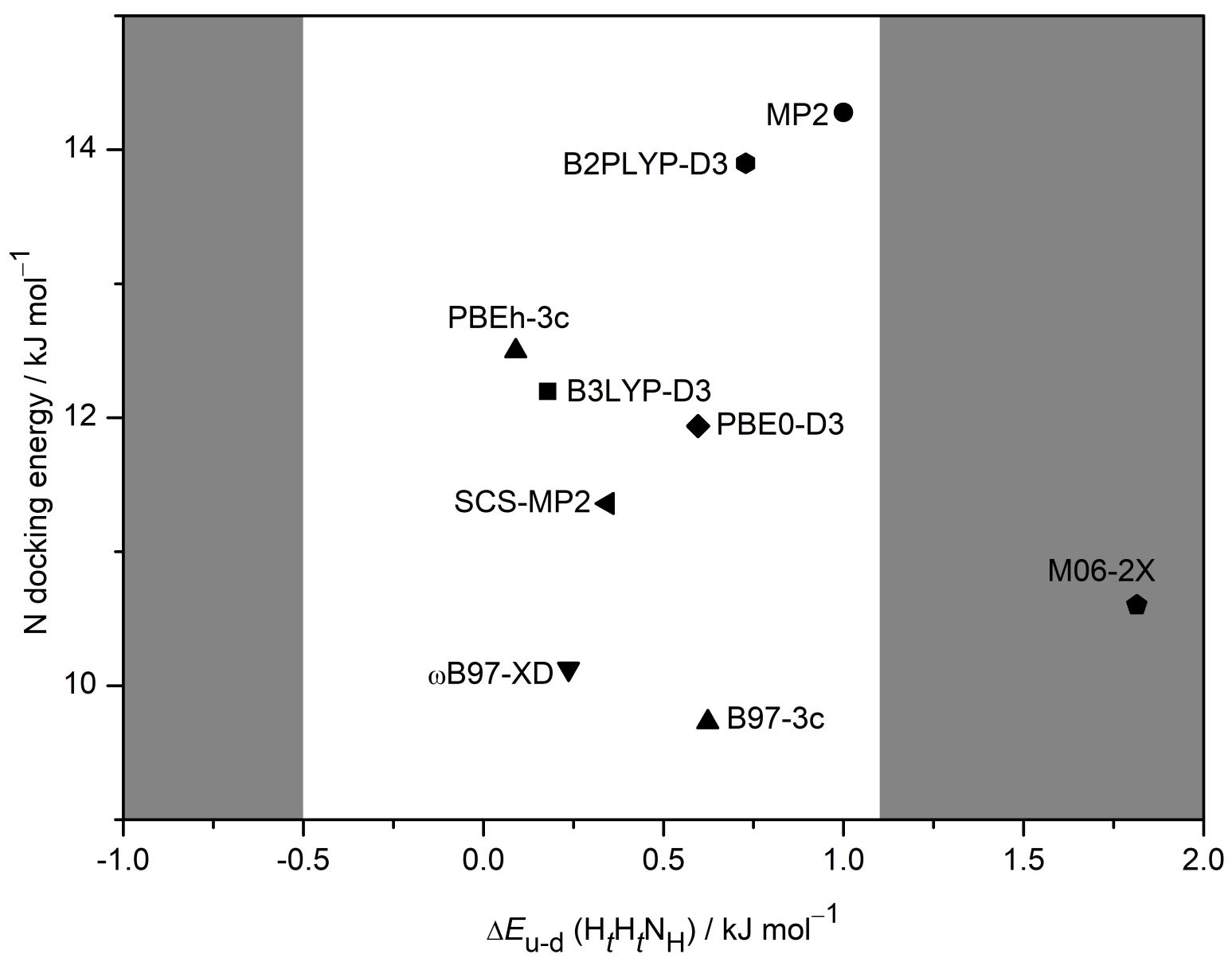

Figure 3.20.: $\mathrm{N}$ dissociation energy for the most stable trimeric HHN complexes plotted against zero-point corrected energy differences $\Delta E_{\mathrm{u}-\mathrm{d}}$ of the two $\mathrm{H}_{t} \mathrm{H}_{t} \mathrm{~N}_{\mathrm{H}}$ isomers (reprinted with adaptations from reference 234 with permission from John Wiley and Sons). The pair of $\mathrm{OH}$ stretching bands at 3583 and $3473 \mathrm{~cm}^{-1}$ is assigned to the less stable isomer $(\mathrm{u})$, the remaining pair at 3563 and $3463 \mathrm{~cm}^{-1}$ to the more stable isomer (d). White areas are compatible with experiment as they predict an energy difference of $0.3(8) \mathrm{kJ} \mathrm{mol}^{-1}$ between the two $\mathrm{H}_{t} \mathrm{H}_{t} \mathrm{~N}_{\mathrm{H}}$ isomers.

Following a similar scheme, the conformational energy difference between the two observed $\mathrm{H}_{t} \mathrm{H}_{t} \mathrm{~N}_{\mathrm{H}}$ isomers is determined (Figure 3.20). The band integrals of the isomer assigned to the further downshifted pair of bands at 3563 and $3463 \mathrm{~cm}^{-1}$ are about 1.4(3) times larger than the corresponding band integrals of the second isomer at 3583 and $3473 \mathrm{~cm}^{-1}$ (see Table A.18 in appendix A.3 for further details on the integration). Theoretical predictions for the infrared cross sections of both isomers are very similar within $\pm 10 \%$ (Table A.17 in appendix A.3) which is added to the uncertainty, resulting in an abundance ratio of 1.4(4). The calculated energy barrier for interconversion between both isomers is well below $5 \mathrm{~kJ} \mathrm{~mol}^{-1}$, which allows for effective conformational cooling ${ }^{54,106}$ and results in an estimated conformational temperature between 50 and $100 \mathrm{~K}$. Therefore, the energy difference between $t$ - and $c-\mathrm{H}_{t} \mathrm{H}_{t} \mathrm{~N}_{\mathrm{H}}$ is determined as 


\section{1,1,1,3,3,3-Hexafluoro-2-propanol}

$0.3(3) \mathrm{kJ} \mathrm{mol}^{-1}$. Due to the entropy and symmetry effects for $t$ - and $c-\mathrm{H}_{t} \mathrm{H}_{t}$ discussed before, an uncertainty of about $\pm 0.5 \mathrm{~kJ} \mathrm{~mol}^{-1}$ is added to the energy difference, resulting in $0.3(8) \mathrm{kJ} \mathrm{mol}^{-1}$.

Similar to the relative conformational energies of $\mathrm{HH}$ dimers, almost all tested electronic structure methods describe the energy balance of $\mathrm{H}_{t} \mathrm{H}_{t} \mathrm{~N}_{\mathrm{H}}$ trimers correctly, with M06-2X/aVTZ being the only outlier. Figures 3.19 and 3.20 further showcase the limited discriminatory function of the employed relative energy benchmarks for electronic structure method quality, which is also reflected in very small deviations (Table 3.11). Most methods seem to benefit from error cancellation in the calculation of relative energies, at least for the studied clusters. As the formic acid dimer example ${ }^{97}$ demonstrates, absolute dissociation energies ${ }^{22,281}$ might be preferable as a benchmarking observable, although they are difficult to obtain experimentally.

Table 3.11.: Squared deviations $\delta^{2}(x)$ of theoretical predictions from experimental reference values derived from relative conformational energies of the two observed $\mathrm{H}_{t} \mathrm{H}_{t} \mathrm{~N}_{\mathrm{H}}$ isomers calculated according to Equation 1.1.

\begin{tabular}{cc}
\hline & $\delta^{2}\left(\Delta E_{\mathrm{u}-\mathrm{d}}\right)$ \\
Level of theory & $\mathrm{H}_{t} \mathrm{H}_{t} \mathrm{~N}_{\mathrm{H}}$ \\
\hline SCS-MP2/VTZ & 0.0 \\
$\omega$ B97-XD/aVTZ & 0.0 \\
B3LYP-D3(BJ)/def2-QZVP & 0.0 \\
PBEh-3c & 0.1 \\
PBE0-D3(BJ)/aVTZ & 0.1 \\
B97-3c & 0.2 \\
B2PLYP-D3(BJ)/VTZ & 0.3 \\
MP2/VTZ & 0.8 \\
M06-2X/aVTZ & 3.6 \\
\hline Mean & 0.6 \\
\hline
\end{tabular}

\subsubsection{Theory Performance Evaluation}

Ranking the tested theoretical methods according to the lowest mean $\delta^{2}(x)$ (Table 3.12) reveals SCS-MP2/VTZ as the best method for the particular test set of molecules and observables. The ranking based on multiple observables is similar to the one obtained for the purely vibrational analysis of formic acid and its nitrogen complex, at least for the best and worst methods. Nonetheless, some approaches such as PBE0-D3(BJ)/aVTZ or B3LYP-D3(BJ)/def2-QZVP rank significantly better for the 1,1,1,3,3,3-hexafluoro2-propanol benchmark than in the carboxylic acid case (Table 2.7). The ranking may change upon inclusion of rotational constants for carboxylic acids, which are only available for the monomers and not the respective $\mathrm{N}$ complexes. ${ }^{282-285}$ 


\section{1,1,1,3,3,3-Hexafluoro-2-propanol}

Table 3.12.: Method rankings based on squared deviations $\delta^{2}(x)$ of theoretical predictions from experimental reference values calculated according to Equation 1.1 derived from all 19 presented benchmarks of 1,1,1,3,3,3-hexafluoro-2-propanol and its aggregates.

\begin{tabular}{ccc}
\hline Level of theory & $\delta^{2}(x)$ & Ranking \\
\hline SCS-MP2/VTZ & 1.5 & 1 \\
PBE0-D3(BJ)/aVTZ & 1.9 & 2 \\
MP2/VTZ & 2.6 & 3 \\
B3LYP-D3(BJ)/def2-QZVP & 2.8 & 4 \\
B2PLYP-D3(BJ)/VTZ & 2.8 & 5 \\
wB97-XD/aVTZ & 5.1 & 6 \\
M06-2X/aVTZ & 5.7 & 7 \\
PBEh-3c & 17.1 & 8 \\
B97-3c & 23.9 & 9 \\
\hline
\end{tabular}

\subsection{Summary}

An experimental multi-messenger approach including infrared, Raman and microwave spectroscopy allows for the assignment of spectral signatures corresponding to multiple structures of 1,1,1,3,3,3-hexafluoro-2-propanol. The most stable monomers, dimers, trimers and several mixed aggregates with nitrogen are unambiguously identified. Intriguing examples of unusual molecular conformations driven by intermolecular interactions are provided by torsional isomerism in the homodimer and chirality synchronisation in the homotrimer.

The wealth of experimental data obtained for this molecule allows for deriving several experimental observables as benchmarking quantities for standard harmonic electronic structure calculations, including rotational constants directly linked to molecular structures, vibrational energy levels and relative conformational energies. The variety of these observables reveals seemingly good theoretical predictions at some levels of approximation in limited areas of the benchmarking set as the result of fortuitous error cancellation. 



\section{Aliphatic Alcohols}

\section{Aliphatic Alcohols}

Dimers of methanol, methanol- $d_{1}$, tert-butyl alcohol and ethanol as well as their nitrogen and oxygen complexes were investigated by recording their respective OH, OD and NN stretching vibrations with filet-jet FTIR and curry-jet Raman spectroscopy. Increasing nitrogen coordination induced progressive spectral downshifts of $\mathrm{OH}$ stretching fundamentals, correlated to matrix isolation shifts. Monomers lack cooperativity-induced $\mathrm{OH}$ hydrogen bond donor strength enhancements, while trimers with a cyclic hydrogen bond topology do not provide dangling $\mathrm{OH}$ bonds as attractive nitrogen docking sites, therefore both are more resistant to nitrogen complexation or coating. Some of the quantum chemical calculations for methanol and tert-butyl alcohol as well as FTIR spectroscopy of methanol were carried out as part of Mareike Wallrabe's bachelor thesis ${ }^{286}$ and Niels Karschin assisted in the recording of Raman spectra during a research project. Results obtained for nitrogen complexes of alcohol dimers are published in reference 287. The occasional verbatim reproduction of small sections from that reference is not explicitly marked or cited.

\subsection{Introduction}

As described in section 1.1.3, matrix isolation FTIR spectroscopy is widely used for stabilisation of transient species as well as weakly bound aggregates and subsequent analysis of their respective structures and dynamics. ${ }^{55,57,89}$ Characteristic spectral shifts and site splittings due to the bulk matrix environment are frequently observed, even for light and weakly perturbating hosts such as para-hydrogen ${ }^{30,288}$ and neon, ${ }^{289}$ similarly for helium nanodroplets. ${ }^{290}$ Rigorous comparison of theoretical predictions and vibrational data obtained in cryomatrices is therefore problematic ${ }^{157,160,225}$ and requires better understanding of matrix effects, which can be offered by stepwise clustering approaches. $^{291,292}$

A strong perturbation even compared to $\operatorname{argon}^{292}$ is found in bulk nitrogen matrices (see Tables A.19 and A.20 in appendix A.4) due to specific and directional interactions of dopant molecules with nitrogen caused by its significant quadrupole moment. Therefore, molecular nitrogen can also be used to determine zeolite acidity strength by measurements of $\mathrm{OH}$ stretching shifts induced by nitrogen adsorption, ${ }^{293}$ although direct correlations between binding energy and spectral shifts are somewhat equivocal. ${ }^{47}$ Hence, systematically studying stepwise nitrogen coating of hydrogen bonded aggregates can assist in rationalising gas-to-matrix shifts and address whether they are caused by cooperative or additive effects. ${ }^{61,94}$ Simple aliphatic alcohols are chosen for their tunable size, donor quality ${ }^{294}$ and conformational flexibility, ${ }^{60,91}$ thus enabling analysis of 


\section{Aliphatic Alcohols}

systematic trends over isolated findings.

Matrix induced $\mathrm{OH}$ stretching downshifts observed in bulk nitrogen (N) matrices $^{56,58-60,62,117-119,122,123}$ compared to findings in supersonic expansions ${ }^{30,31,92,103,295-297}$ for methanol (M), tert-butyl alcohol (B) and ethanol (E) are given in Table 4.1 and more detailed in Table A.19 in appendix A.4. Wide wavenumber ranges are caused by site splittings and tentative best estimates narrowing those ranges are obtained by focussing on dominant sites and literature assignments for methanol and tert-butyl alcohol. In the ethanol case, this procedure is impeded by additional conformational isomerism. Matrix isolation shifts substantially increase upon switching from alcohol monomers to dimer donor vibrations, indicating significant cooperativity between $\mathrm{OH} \cdots \mathrm{O}$ and $\mathrm{OH} \cdots \mathrm{N}$ hydrogen bonds, especially for methanol.

Ethanol band centre positions in nitrogen matrix obtained with a grating instrument ${ }^{121}$ differ significantly from references 60 and 62 . Since the guest-to-host ratio is too high and the signal-to-noise ratio too low for rigorous dimer assignments, values from reference 121 are excluded from entries in Tables 4.1 and A.19.

Table 4.1.: Nitrogen matrix induced downshifts ${ }^{56,58-60,62,117-119,122,123}\left(\Delta \tilde{\nu}_{\text {mi }}\right)$ relative to band centres obtained in supersonic expansions ${ }^{30,31,92,103,295-297}$ for the $\mathrm{OH}$ stretching fundamental of the investigated alcohol aggregates. Molecules are abbreviated with $\mathrm{M}$ (methanol), B (tert-butyl alcohol) and E (ethanol). These single letters are repeated according to the number of units present in the donor-acceptor sequence for a description of cluster compositions. Roman structure symbols label the monomer unit involved in the vibration, italic symbols the passive monomer. Tentative best estimates for the actual downshift by determining dominant sites or following literature assignments are added for M and B. See Table A.19 in appendix A.4 for details.

\begin{tabular}{ccc}
\hline & \multicolumn{2}{c}{$\Delta \tilde{\nu}_{\mathrm{mi}} / \mathrm{cm}^{-1}$} \\
Structure & Range & Best estimate \\
\hline $\mathrm{M}$ & $20-25$ & 21 \\
$M \mathrm{M}$ & $16-33$ & 24 \\
$\mathrm{M} M$ & $55-86$ & 71 \\
$\mathrm{~B}$ & $13-14$ & 13 \\
$\mathrm{~B} B$ & $-4-72$ & $\approx 27$ \\
$\mathrm{E}$ & $10-25$ & - \\
$E \mathrm{E}$ & $0-37$ & - \\
$\mathrm{E} E$ & $14-78$ & - \\
\hline
\end{tabular}

\subsection{Quantum Chemical Calculations and Nomenclature}

Abbreviations used for studied alcohols include M (methanol), B (tert-butyl alcohol) and $\mathrm{E}$ (ethanol), which are listed in hydrogen bond donor-acceptor sequence according 


\section{Aliphatic Alcohols}

to the number of monomer units present to describe cluster composition, as in previous chapters. As before, nitrogen $(\mathrm{N})$ and oxygen $(\mathrm{O})$ docking sites at specific alcohol backbone atoms are marked by subscripts ( $\mathrm{C}, \mathrm{O}$ and $\mathrm{H})$. Completely and unspecifically nanosolvated species are enclosed in parentheses, so the methanol dimer surrounded by a large number of nitrogen molecules is abbreviated as (MM)N. Ethanol monomer trans $\left(180^{\circ} \mathrm{HOCH}\right.$ dihedral angle) and gauche $\left(\approx \pm 60^{\circ} \mathrm{HOCH}\right.$ dihedral angle) conformations are denoted by subscripts $t$ and $g$. The label $g$ ' indicates differing signs of two gauche $\mathrm{HOCH}$ dihedral angles in a cluster.

Geometry optimisations and harmonic frequency calculations for all clusters were carried out at the B3LYP-D3(BJ)/aVTZ level, complemented by CCSD(T)/aVTZ single point energies. Additionally, anharmonic (VPT2) frequencies at the B3LYP level as well as harmonic $\operatorname{CCSD}(\mathrm{T})$ frequencies with local and explicit correlation were calculated for $\mathrm{N}, \mathrm{M}$ and $\mathrm{MM}$ as displayed in Table 4.2. $\operatorname{CCSD}(\mathrm{T})$ predictions closely match all experimental reference values. For the harmonic NN stretching wavenumber, canonical CCSD(T) improves with increasing basis set size to $2358 \mathrm{~cm}^{-1}$ for aVQZ and $2357 \mathrm{~cm}^{-1}$ for aV5Z, respectively, while extrapolation to the basis set limit ${ }^{298}$ yields $2363 \mathrm{~cm}^{-1}$. The MP2 and B3LYP approximations show deficiencies of similar magnitude for these smallest studied molecules and complexes. The MP2 level is thus neglected for larger aggregates due to its higher computational cost.

Table 4.2.: Experimentally obtained and theoretically predicted (VPT2) anharmonic ( $\tilde{\nu})$ and harmonic $\left(\omega_{e}\right) \mathrm{OH}$ and NN stretching band centre positions as well as diagonal anharmonicity constants $\left(x_{1,1}\right)$ in $\mathrm{cm}^{-1}$ for $\mathrm{N}, \mathrm{M}$ and $\mathrm{M} M$. Harmonic wavenumbers in square brackets were obtained within a formal one-dimensional Morse model from experimental $\mathrm{OH}$ stretching overtones. Employed levels of theory include LCCSD( $\left.\mathrm{T}^{*}\right)-\mathrm{F} 12 / \mathrm{VDZ}-$ F12(int) (LCCSD(T)), CCSD(T)/aVTZ (CCSD(T)), B3LYP-D3(BJ)/aVTZ (B3LYP) and MP2/aVTZ (MP2).

\begin{tabular}{c|ccc|ccc|ccc}
\hline & \multicolumn{3}{|c}{$\mathrm{N}$} & \multicolumn{3}{c}{$\mathrm{M}$} & \multicolumn{2}{c}{$\mathrm{M} M$} \\
& $\tilde{\nu}$ & $\omega_{e}$ & $x_{1,1}$ & $\tilde{\nu}$ & $\omega_{e}$ & $x_{1,1}$ & $\tilde{\nu}$ & $\omega_{e}$ & $x_{1,1}$ \\
\hline Reference & $2329.91^{299}$ & $2358.57^{300}$ & $14.33^{300}$ & $3685^{30}$ & {$[3857(18)]$} & $86^{30}$ & $3575^{30}$ & {$[3773(20)] / 3742^{231}$} & $99^{30}$ \\
& & & & & $3683^{120}$ & $3863^{231}$ & & & \\
LCCSD(T) & - & 2354 & - & - & 3862 & - & - & 3740 & - \\
CCSD(T) & - & 2343 & - & - & 3844 & - & - & - & - \\
B3LYP & 2422 & 2447 & 13 & 3654 & 3829 & 85 & 3483 & 3653 & 105 \\
MP2 & 2149 & 2187 & 19 & 3687 & 3860 & 82 & 3553 & 3695 & 108 \\
\hline
\end{tabular}

The most stable structures of mixed $\mathrm{M}$ and $\mathrm{B}$ complexes with $\mathrm{N}$ are depicted in Figure 4.1. Higher energy conformers are shown in Figures A.17 and A.18 and corresponding spectral predictions are listed in Tables A.21 and A.22 in appendix A.4. As observed for other hydrogen bond donor molecules, $\mathrm{N}$ prefers binding at the $\mathrm{OH}$ proton of either the isolated monomer or the dangling acceptor $\mathrm{OH}$ of a dimer, where $\mathrm{OH} \cdots \mathrm{N}$ hydrogen bonds are slightly bent from the typically preferred linearity to allow for interactions of the central positive partial $\mathrm{N}$ quadrupole charges with the donor molecule oxygen lone pairs. 


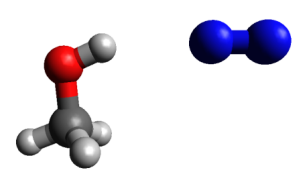

$\mathrm{MN}_{\mathrm{H}}$

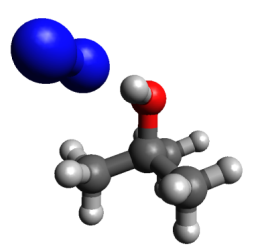

$\mathrm{BN}_{\mathrm{H}}$

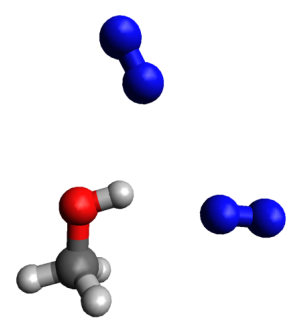

$\mathrm{MN}_{\mathrm{H}} \mathrm{N}_{\mathrm{H}}$

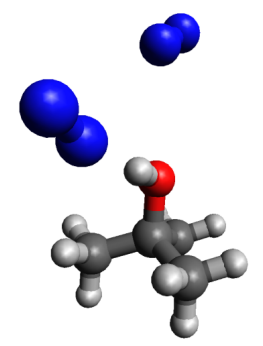

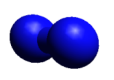

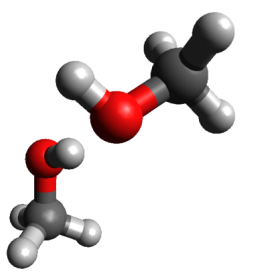

$\mathrm{MMN}_{\mathrm{H}}$

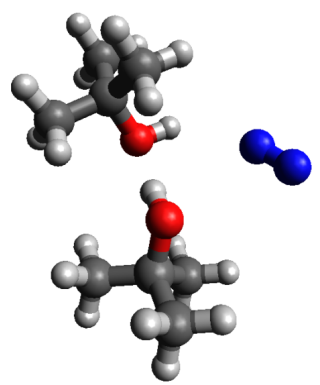

$\mathrm{BBN}_{\mathrm{H}}$

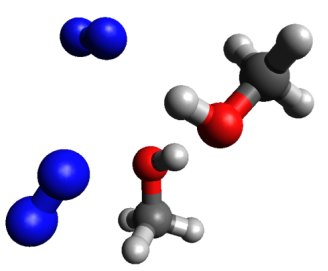

$\mathrm{MMN}_{\mathrm{H}} \mathrm{N}_{\mathrm{H}}$

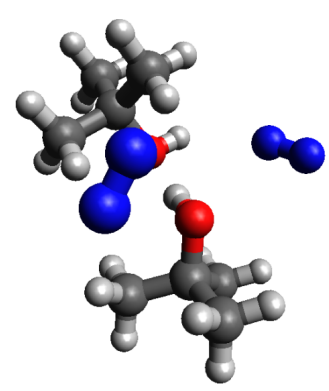

$\mathrm{N}_{\mathrm{O}} \mathrm{BBN}_{\mathrm{H}}$

Figure 4.1.: Most stable structures of mixed MN and BN aggregates optimised at the B3LYP-D3(BJ)/aVTZ level (reprinted with adaptations from reference 287 licensed under CC BY 4.0).

Single N coordination of M or B is predicted to be spectrally unimpressive with little to no $\mathrm{OH}$ stretching shifts and infrared intensity enhancements (Table 4.3). However, predicted downshifts for dimer donor $\mathrm{OH}$ vibrations upon single $\mathrm{N}_{\mathrm{H}}$ coordination at the acceptor alcohol vary between 20 and $25 \mathrm{~cm}^{-1}$ for both MM and $\mathrm{BB}$, further increased by cooperative double $\mathrm{N}_{\mathrm{H}}$ or push-pull coordination like in $\mathrm{N}_{\mathrm{O}} \mathrm{BBN}_{\mathrm{H}}$. Acceptor $\mathrm{OH}$ vibration downshifts are also predicted to be quite substantial for MM between 15 and $20 \mathrm{~cm}^{-1}$, while being less pronounced in BB.

\subsection{FTIR Spectra with Nitrogen}

Methanol- $d_{0}$ (99.98\%, Sigma-Aldrich), methanol- $d_{1}$ (99.0\% D, euriso-top), tert-butyl alcohol (99\%, Roth) and ethanol (99.8\%, Sigma-Aldrich) were used without further purification and mixed with helium $(99.996 \%$, Linde) as well as optionally nitrogen (99.999\%, Linde) or argon (99.999\%, Linde). For the OH and OD stretching measurements from $4100-2450 \mathrm{~cm}^{-1}$, the filet-jet set-up was equipped with the $150 \mathrm{~W}$ tungsten lamp, $\mathrm{CaF}_{2}$ beam splitter and optics as well as the liquid nitrogen cooled $3 \mathrm{~mm}^{2} \mathrm{InSb}$ detector. 


\section{Aliphatic Alcohols}

Table 4.3.: Theoretically predicted harmonic $\left(\omega_{\mathrm{OH}}\right) \mathrm{OH}$ stretching band centre positions and integrated infrared band strengths $S_{\omega}$ of the most stable M, MN, B and BN aggregates as well as spectral harmonic downshifts relative to the corresponding vibration in the pure alcohol fragments $\left(\Delta \omega_{\mathrm{OH}}\right)$ obtained at the B3LYP-D3(BJ)/aVTZ level. Roman symbols denote the alcohol monomer unit involved in the vibration, italic symbols the passive monomer.

\begin{tabular}{cccc}
\hline Structure & $\omega_{\mathrm{OH}} / \mathrm{cm}^{-1}$ & $S_{\omega} / \mathrm{km} \mathrm{mol}^{-1}$ & $\Delta \omega_{\mathrm{OH}} / \mathrm{cm}^{-1}$ \\
\hline $\mathrm{M}$ & 3829 & 31 & - \\
$\mathrm{MN}_{\mathrm{H}}$ & 3831 & 103 & -2 \\
$\mathrm{MN}_{\mathrm{H}} \mathrm{N}_{\mathrm{H}}$ & 3829 & 98 & 0 \\
$M \mathrm{M}$ & 3825 & 42 & - \\
$M \mathrm{MN}_{\mathrm{H}}$ & 3807 & 129 & 18 \\
$M \mathrm{MN}_{\mathrm{H}} \mathrm{N}_{\mathrm{H}}$ & 3810 & 78 & 15 \\
\hline $\mathrm{M} M$ & 3653 & 551 & - \\
$\mathrm{M} M \mathrm{~N}_{\mathrm{H}}$ & 3629 & 552 & 24 \\
$\mathrm{M} M \mathrm{~N}_{\mathrm{H}} \mathrm{N}_{\mathrm{H}}$ & 3618 & 534 & 35 \\
\hline $\mathrm{B}$ & 3801 & 14 & - \\
$\mathrm{BN}$ & 3810 & 87 & -9 \\
$\mathrm{BN} \mathrm{N}_{\mathrm{H}}$ & 3805 & 94 & -4 \\
$B \mathrm{~B}$ & 3795 & 19 & - \\
$B B N_{\mathrm{H}}$ & 3792 & 57 & 3 \\
$\mathrm{~N}_{\mathrm{O}} B \mathrm{BN}_{\mathrm{H}}$ & 3792 & 55 & 3 \\
\hline $\mathrm{B} B$ & 3614 & 525 & - \\
$\mathrm{B} B \mathrm{~N}_{\mathrm{H}}$ & 3593 & 543 & 21 \\
$\mathrm{~N}_{\mathrm{O}} \mathrm{B} B \mathrm{~N}_{\mathrm{H}}$ & 3590 & 530 & 24 \\
\hline
\end{tabular}




\section{Aliphatic Alcohols}

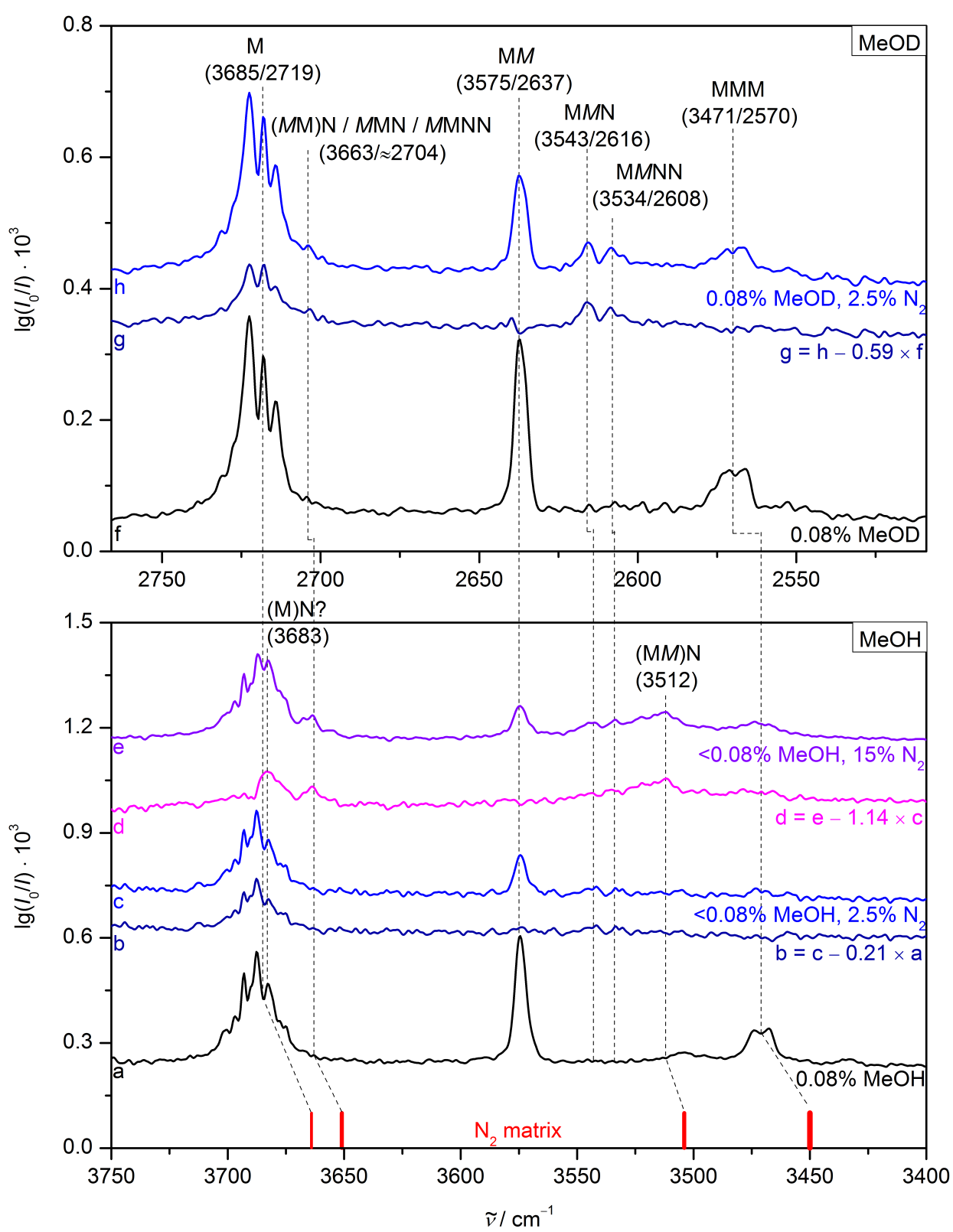

Figure 4.2.: $\mathrm{OH}$ and OD stretching FTIR spectra (reprinted with adaptations from reference 287 licensed under CC BY 4.0) of methanol- $d_{1}$ (upper box) and methanol- $d_{0}$ (lower box) expanded in helium (traces a and f), $2.5 \% \mathrm{~N}$ in helium (traces $\mathrm{c}$ and $\mathrm{h}$ ) and $15 \% \mathrm{~N}$ in helium (trace e). A stagnation pressure of $p_{\mathrm{s}}=0.75 \mathrm{bar}$ was employed for all shown spectra. MM-corrected difference spectra (traces $\mathrm{b}, \mathrm{d}$ and $\mathrm{g}$ ) are included. The OD wavenumber scale width is compressed by a factor of 0.73 and shifted by $13 \mathrm{~cm}^{-1}$ such that the M and MM signals match for the two isotopologues. Wavenumbers and (tentative) labels are provided. Red bars at the bottom indicate band positions of M, MM and MMM in a bulk nitrogen matrix. ${ }^{58,59,118}$ 


\section{Aliphatic Alcohols}

\subsubsection{Methanol}

Methanol- $d_{0}$ expanded in helium as depicted in Figure 4.2 (trace a) displays the wellknown ${ }^{92,301} \mathrm{OH}$ stretching vibrations of $\mathrm{M}, \mathrm{MM}$ and MMM at 3685, 3575 and $3471 \mathrm{~cm}^{-1}$, respectively, with the corresponding OD stretching vibrations of methanol- $d_{1}$ at 2719 , 2637 and $2570 \mathrm{~cm}^{-1}$ (trace f). After adding $2.5 \% \mathrm{~N}$ (trace c), two weak bands grow in downshifted from $\mathrm{MM}$ at 3543 and $3534 \mathrm{~cm}^{-1}$, also visible as positive contributions in the MM-corrected difference spectrum (trace b) and indicative of N-coordinated MM. Both signals appear in the OD stretching spectra of methanol- $d_{1}$ as well (traces $g$ and h) at 2616 and $2608 \mathrm{~cm}^{-1}$, ruling out resonances with dark states as possible reasons for their appearance and strongly hinting at the formation of MMN and MMNN aggregates. Spectral OD downshifts are about $30 \%$ lower than corresponding effects in OH stretching vibrations. $\mathrm{M}$ and $\mathrm{MMM}$ signals are not visibly influenced by $\mathrm{N}$ addition. A large excess of $15 \% \mathrm{~N}$ (traces $\mathrm{d}$ and e) induces an additional, broad new signal centred at $3512 \mathrm{~cm}^{-1}$, assigned to fully $\mathrm{N}$ nanocoated MM. A shoulder of the monomer band at $3663 \mathrm{~cm}^{-1}$ most likely originates from combined, downshifted acceptor $\mathrm{OH}$ of $\mathrm{MM}$ at various degrees of $\mathrm{N}$ complexation or nanosolvation. Predicted spectral shifts of this band after the first $\mathrm{N}_{\mathrm{H}}$ coordination are small, and therefore superposition of different cluster acceptor $\mathrm{OH}$ vibrations possibly results in the large observed intensity of the otherwise weak signal. Contributions from N nanocoated $\mathrm{M}$ to this band are less likely, since significantly smaller spectral shifts are predicted. However, traces of N-coated $\mathrm{M}$ might be visible in the monomer band broadening to lower wavenumbers around $3683 \mathrm{~cm}^{-1}$ (traces d and e).

These spectral findings of preferred $\mathrm{N}$ docking at MM help rationalise the anomalously large gas-to-matrix shift of MM compared to M and MMM. A strong interaction of $\mathrm{N}$ with the $\mathrm{OH}$ group requires cooperative polarisation due to dimer formation, which $\mathrm{M}$ lacks. In MMM, all three $\mathrm{OH}$ groups are engaged in a cyclic hydrogen bonding topology and therefore not available for $\mathrm{N}$ complexation.

Harmonic downshift predictions from M to MM provided by density functionals between 150 and $180 \mathrm{~cm}^{-1}$ agree better with the shift observed in a $\mathrm{N}$ matrix ${ }^{58,59,118}$ of about $160 \mathrm{~cm}^{-1}$ than with experimental gas phase data ${ }^{30}$ of $110 \mathrm{~cm}^{-1}$. However, $\operatorname{CCSD}(\mathrm{T})$ predictions of about $120 \mathrm{~cm}^{-1}$ for the harmonic shift ${ }^{231}$ reveal this agreement as a result of fortuitous error cancellation, as expected for theoretical modelling neglecting the matrix environment. The discrepancy between prediction and gas phase experiment is possibly further enhanced by off-diagonal anharmonic contributions from the floppy methyl rotors. Their motions are likely quenched through nanocoating or matrix isolation, although this assumption needs to be corroborated by comparison to more rigid $\mathrm{B}$ complexes.

\subsection{2. tert-Butyl Alcohol}

OH stretching spectra of B (Figure 4.3, trace a) show the previously assigned ${ }^{296}$ monomer band at $3642 \mathrm{~cm}^{-1}$ as well as the dimer acceptor and donor vibrations ${ }^{296}$ at 3630 and $3497 \mathrm{~cm}^{-1}$, respectively. Weak BBB signals are visible near 3453 and $3411 \mathrm{~cm}^{-1}$. Two 


\section{Aliphatic Alcohols}

new bands appear downshifted from the dimer donor vibration at 3480 and $3474 \mathrm{~cm}^{-1}$, respectively, upon addition of $1 \% \mathrm{~N}$ and as positive signals in the respective BB-corrected difference spectrum (trace b). Both signals grow in intensity after increased $\mathrm{N}$ addition of $2.5 \%$ (trace e) and the difference spectrum corrected for the signal at $3480 \mathrm{~cm}^{-1}$ (trace $\mathrm{d}$ ) reveals a positive contribution and thus larger intensity gain of the band at $3474 \mathrm{~cm}^{-1}$, which allows for an assignment of the two signals to BBN and BBNN, respectively. Addition of $15 \% \mathrm{~N}$ (traces $\mathrm{f}$ and $\mathrm{g}$ ) again results in unspecific $\mathrm{N}$ coating. The corresponding band centred around $3469 \mathrm{~cm}^{-1}$ closely matches the band positions of the two dominant BB sites in a N matrix. ${ }^{122}$ Similar to the M case, the combined acceptor vibrations of various $\mathrm{N}$ coordinated $\mathrm{BB}$ clusters are found at $3627 \mathrm{~cm}^{-1}$. Qualitative spectral findings are very similar for $\mathrm{M}$ and $\mathrm{B}$, while the peak separation in $\mathrm{B}$ is much smaller between selectively $\mathrm{N}$ coordinated and globally solvated dimers. Possibly, the bulky alkyl group in B does not allow for the extent of cooperativity between different $\mathrm{N}$ docking sites found in $\mathrm{M}$.

\subsubsection{Ethanol}

The E monomer displays two conformations, the more stable $\mathrm{E}_{t}$ at $3678 \mathrm{~cm}^{-1}$ and the chiral $\mathrm{E}_{g}$ at $3660 \mathrm{~cm}^{-1}$ (Figure 4.4, trace a). ${ }^{60,295}$ This induces larger conformational variety in the dimer compared to $\mathrm{M}$ and $\mathrm{B}$. Six different conformers were theoretically predicted $^{302}$ to be stable with regard to rearrangements and their structures were subsequently confirmed by microwave spectroscopy. ${ }^{32,303}$ Low resolution vibrational spectra typically only show up to four different conformers due to spectral overlap, ${ }^{31}$ while matrix isolation enables photo-induced interconversion between various conformations. ${ }^{62}$

Addition of $10 \%$ argon (Figure 4.4, trace c) to the helium carrier gas results in a more effective cooling and lower expansion temperatures. Due to the decreased conformational temperature, metastable dimer conformations such as $\mathrm{E}_{t} \mathrm{E}_{g}$ are depopulated relative to the most stable $\mathrm{E}_{g} \mathrm{E}_{g}$ conformer ${ }^{295}$ with its $\mathrm{OH}$ stretching vibrations at 3654 and $3531 \mathrm{~cm}^{-1}$, respectively. Alongside the metastable dimer hydrogen bond donor $\mathrm{OH}$ stretching signals at 3547 and $3539 \mathrm{~cm}^{-1}$, a weak feature at $3572 \mathrm{~cm}^{-1}$ also displays decreasing intensity with lower temperature. The spectral separation of 25 or $33 \mathrm{~cm}^{-1}$ from the corresponding dimer donor vibrations hints at thermal co-excitation of a low frequency bending mode of the two ethyl chains, similar to MMM. ${ }^{301}$ An assignment of the band to other metastable dimer conformations is unlikely, because none of the stable dimers displays predicted $\mathrm{OH}$ stretching frequencies (see Table A.23 in appendix A.4) in this particular region. ${ }^{295}$

Some structure can be found in the EEE vibrational band with shoulders at 3462, 3454, 3446 and $3440 \mathrm{~cm}^{-1}$, respectively. This structure is lost after argon addition and only the broad band at $3446 \mathrm{~cm}^{-1}$ remains. Similarly, $\mathrm{OH}$ stretching vibrations of tetramers and larger clusters show distinct shoulders at $3311,3288,3268$ and $3212 \mathrm{~cm}^{-1}$ with the latter undergoing an upshift after argon addition. Tentative assignments of this conformational structure first reported in this work for EEE and EEEE is rather difficult due to the large homogeneous and residual inhomogeneous broadening compared to the shift differences of all involved vibrational signals. 


\section{Aliphatic Alcohols}

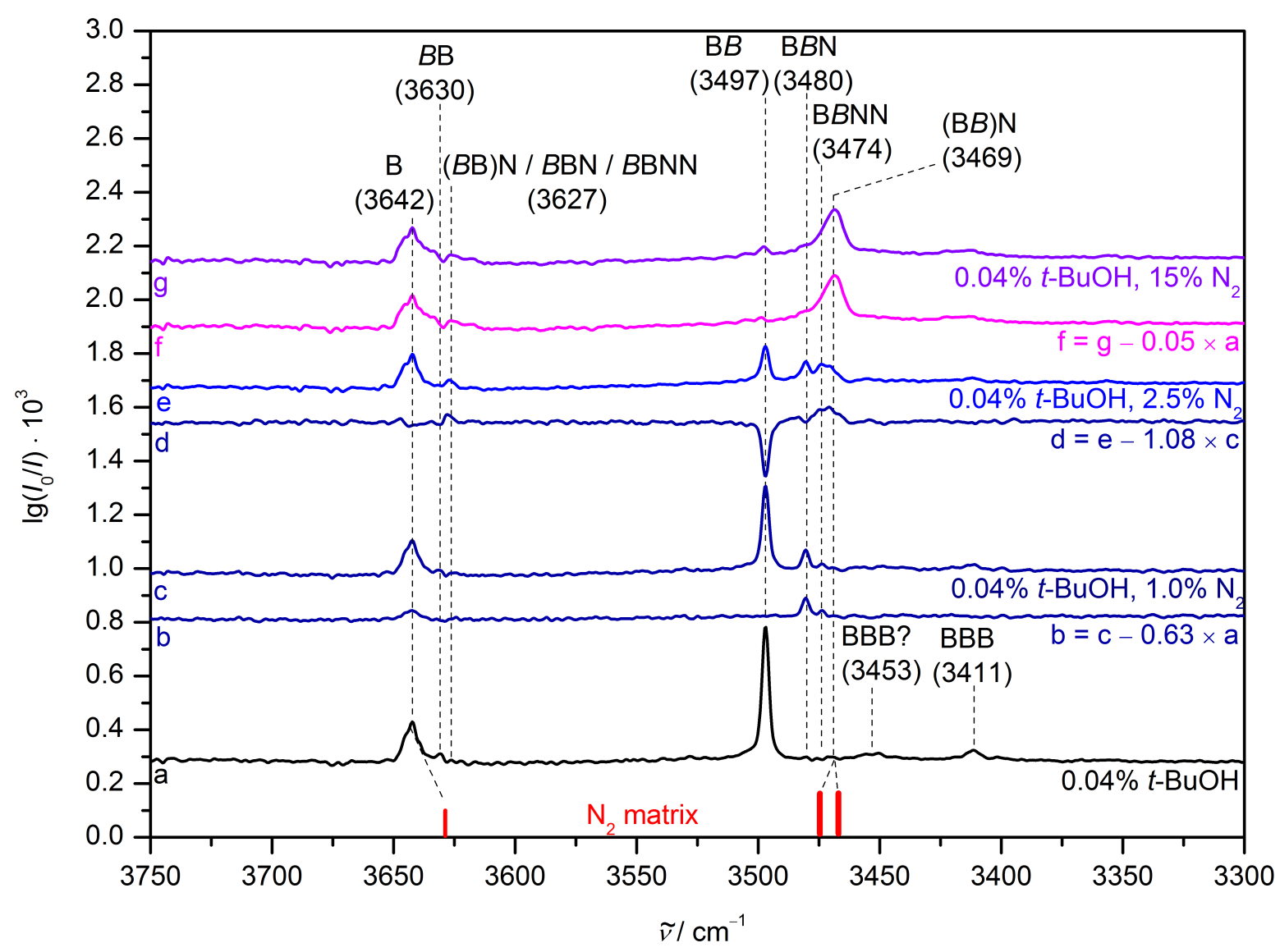

Figure 4.3.: OH stretching FTIR spectra of B (reprinted with adaptations from reference 287 licensed under CC BY 4.0) expanded in helium (trace a), 1\%, 2.5\% and $15 \% \mathrm{~N}$ in helium (traces $\mathrm{c}$, e and $\mathrm{g}$ ). A stagnation pressure of $p_{\mathrm{s}}=0.75 \mathrm{bar}$ was employed for all shown spectra. BB- (traces $\mathrm{b}$ and $\mathrm{f}$ ) and BBN-corrected (trace d) difference spectra are included. Wavenumbers and (tentative) labels are provided. Red bars at the bottom indicate the band positions of $\mathrm{B}$ and $\mathrm{BB}$ in a bulk nitrogen matrix. ${ }^{122}$

Expanding $\mathrm{E}$ in a mixture of helium and $2.7 \% \mathrm{~N}$ (Figure 4.5, traces b and c) results in two additional features at 3520 and $3508 \mathrm{~cm}^{-1}$. An assignment to EEN and EENN appears reasonable because of analogue findings in the M and B cases. However, spectral splittings attributed to conformational isomerism in $\mathrm{E}$ are of a similar order of magnitude and therefore cannot be ruled out completely. Conformational temperatures in the expansion drop after $\mathrm{N}$ addition, since the metastable dimer signals at 3547 and $3539 \mathrm{~cm}^{-1}$ lose intensity relative to $\mathrm{E}_{g} \mathrm{E}_{g}$ and appear as negative signals in the $\mathrm{E}_{g} \mathrm{E}_{g}$-corrected difference spectrum (trace $\mathrm{b}$ ). Hence, spectral findings could be due to removal of conformational flexibility through $\mathrm{N}$ addition or band overlap between different conformational and $\mathrm{N}$ coordinated EE species. Nanocoated EE is obtained after addition of $15 \% \mathrm{~N}$ (traces $\mathrm{d}$ and e) with a broad donor band centred at $3491 \mathrm{~cm}^{-1}$ and the combined acceptor vibrations of various $\mathrm{N}$ coordinated and solvated EE appear at 


\section{Aliphatic Alcohols}

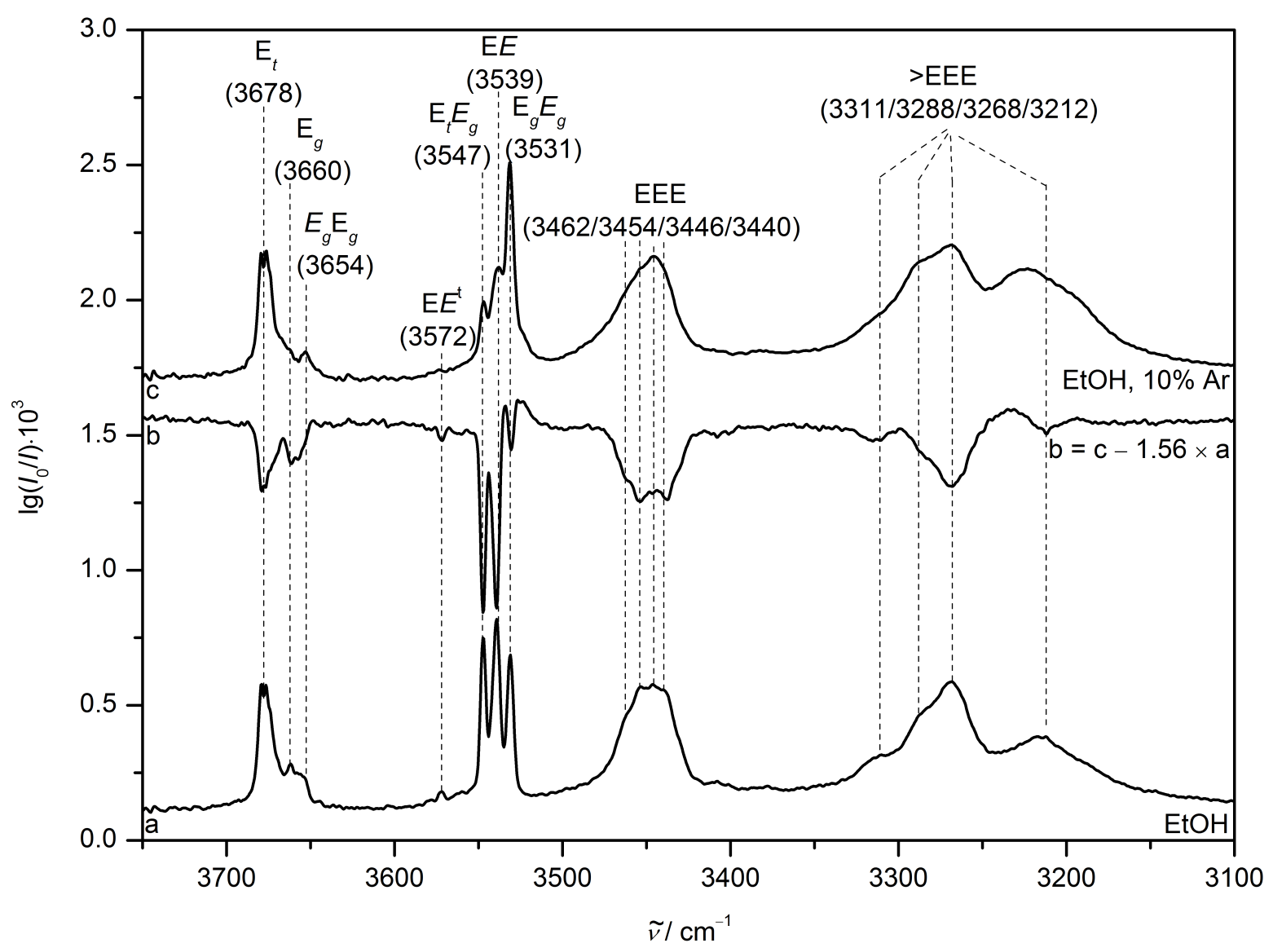

Figure 4.4.: OH stretching FTIR spectra of E (reprinted with adaptations from reference 287 licensed under CC BY 4.0) expanded in helium (trace a) and $10 \%$ argon in helium (trace c). A stagnation pressure of $p_{\mathrm{s}}=0.75$ bar was employed for all shown spectra. An $\mathrm{E}_{g} \mathrm{E}_{g}$-corrected difference spectrum (trace $\mathrm{b}$ ) is included. Wavenumbers and (tentative) labels are provided. The depletion of a low-frequency combination band $\mathrm{E} E^{\mathrm{t}}$ alongside its parent $\mathrm{OH}$ stretching vibration is evident after argon addition.

$3658 \mathrm{~cm}^{-1}$. Comparison of the $(\mathrm{EE}) \mathrm{N}$ donor peak position to the upshifted bulk matrix value $^{62}$ of $3517 \mathrm{~cm}^{-1}$ reveals a difference between $\mathrm{E}$ and the other two alcohols. In $\mathrm{M}$, the bulk matrix signal is downshifted from $(\mathrm{MM}) \mathrm{N}$ and in $\mathrm{B}$ almost no difference between bulk matrix and (BB)N is found. The most likely cause for this discrepancy is conformational isomerism in $\mathrm{E}$, since the $\mathrm{E}_{t} \mathrm{E}_{g}$ conformer dominant in $\mathrm{N}$ matrix environment differs from the preferred $\mathrm{E}_{g} \mathrm{E}_{g}$ gas phase structure. This unusually small gas-to-matrix shift of EE is also observed in argon matrices. ${ }^{31,62} \mathrm{~N}$ coating in supersonic expansions also seems to favour other EE conformations than bulk matrix deposition, possibly the strongly downshifted $\mathrm{E}_{g} \mathrm{E}_{g}$ structure. 


\section{Aliphatic Alcohols}

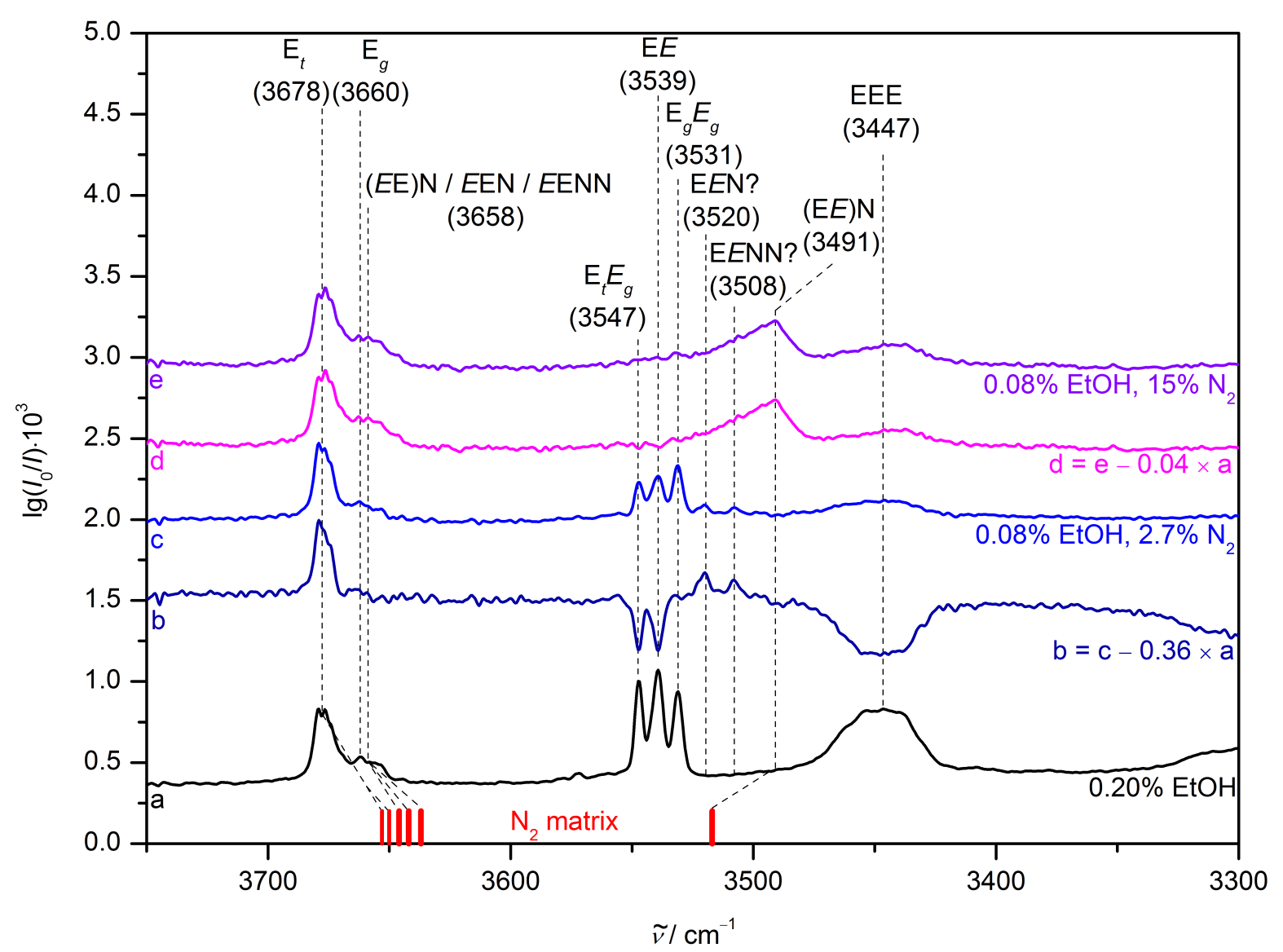

Figure 4.5.: OH stretching FTIR spectra of $\mathrm{E}$ (reprinted with adaptations from reference 287 licensed under CC BY 4.0) expanded in helium (trace a), $2.7 \%$ and $15 \% \mathrm{~N}$ in helium (traces $\mathrm{c}$ and $\mathrm{e}$ ). A stagnation pressure of $p_{\mathrm{s}}=0.75$ bar was employed for all shown spectra. $\mathrm{E}_{g} \mathrm{E}_{g}$-corrected (traces $\mathrm{b}$ and $\mathrm{d}$ ) difference spectra are included. All spectra were scaled to matching height of the $\mathrm{E}_{t}$ monomer band at $3678 \mathrm{~cm}^{-1}$. Wavenumbers and (tentative) labels are provided. Red bars at the bottom indicate the dominant band positions of $\mathrm{E}$ and $\mathrm{EE}$ in a bulk nitrogen matrix. ${ }^{60,62}$ 


\section{Aliphatic Alcohols}

In the absence of microwave spectra, ${ }^{304}$ assisting quantum chemical calculations (see Table A.23 in appendix A.4) of various EEN clusters are unable to disentangle this interplay of conformational isomerism and $\mathrm{N}$ coordination effects on the vibrational band positions and therefore do not allow for assignments of the two peaks at 3520 and $3508 \mathrm{~cm}^{-1}$. Calculated EEN clusters show the same $\mathrm{N}$ docking sites already found for $\mathrm{M}$ and $\mathrm{B}$. The most stable EEN trimer likely features an $\mathrm{EEN}_{\mathrm{H}}$ motif, which corresponds to larger predicted downshifts and $\mathrm{N}$ docking energies (Figure 4.6) than acceptor or donor $\mathrm{O}$ coordination. However, the experimentally observed EE conformation remains unclear. In addition to the predicted minimum energy structure, $\mathrm{E}_{g} \mathrm{E}_{g^{\prime}} \mathrm{N}_{\mathrm{H}}$, seven similar structures with different EE conformers and a relative energy difference of less than $1 \mathrm{~kJ} \mathrm{~mol}^{-1}$ are found. Similar conformational variety is found for EENN with ten conformers less than $2 \mathrm{~kJ} \mathrm{~mol}^{-1}$ higher in energy than the $\mathrm{E}_{t} \mathrm{E}_{t} \mathrm{~N}_{\mathrm{H}} \mathrm{N}_{\mathrm{H}}$ minimum.

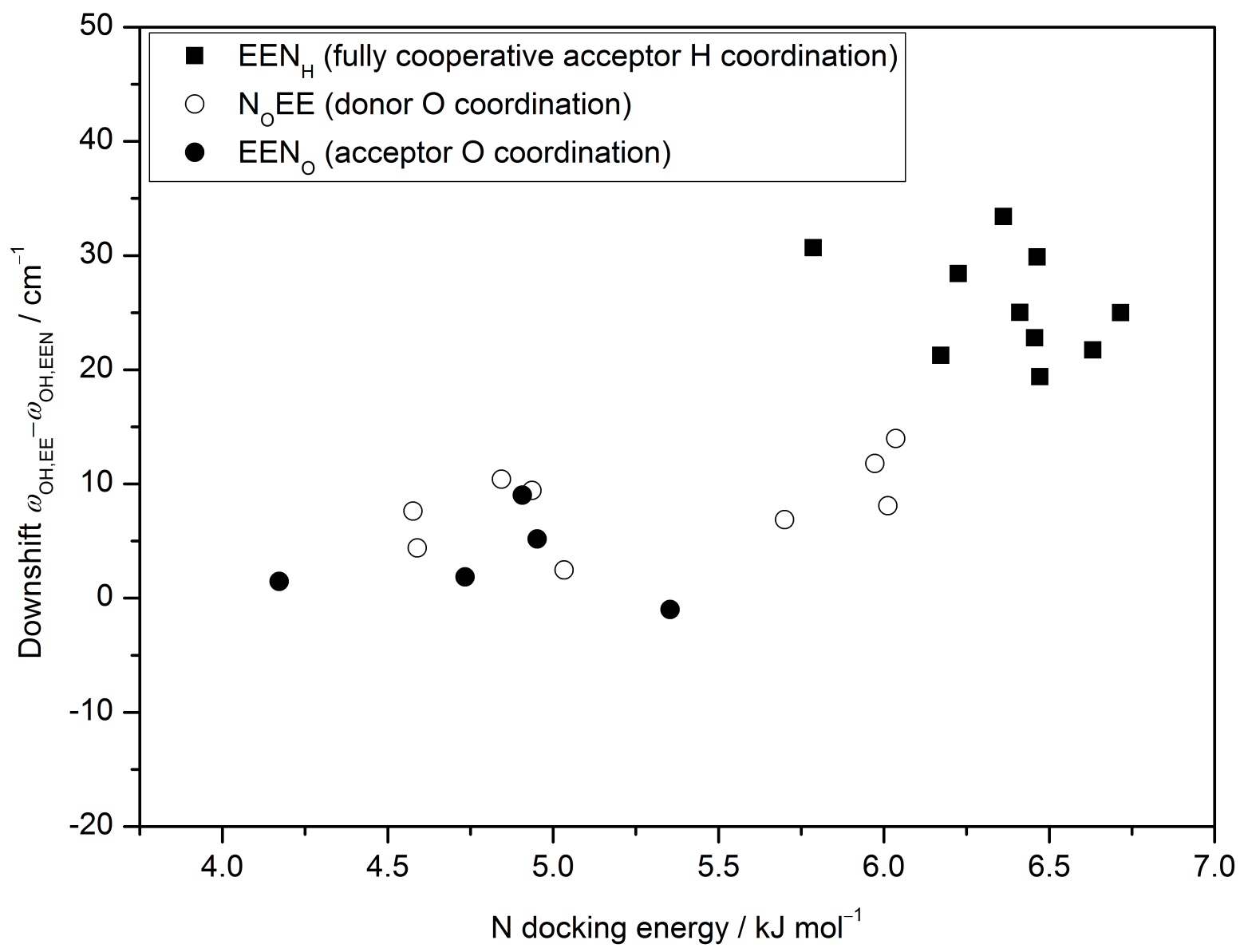

Figure 4.6.: Theoretically predicted incremental spectral OH stretching downshift from EE to EEN and zero-point corrected dissociation energy of EEN into the corresponding EE conformation and free $\mathrm{N}$ (docking energy) at the B3LYP-D3(BJ)/aVTZ level (reprinted with adaptations from reference 287 licensed under CC BY 4.0). See Table A.23 in appendix A.4 for further details. 


\section{Aliphatic Alcohols}

\subsubsection{Alkylation Trends}

N-induced spectral $\mathrm{OH}$ stretching shifts for all three alcohols are summarised in Table 4.4. Downshifts observed in the homodimers with respect to the donor conformation increase from $\mathrm{M}$ over $\mathrm{E}_{g}$ to $\mathrm{B}$. Harmonic predictions are almost constant, while overestimating the experimental values significantly. The latter is a known problem of the B3LYP level. ${ }^{157,231}$

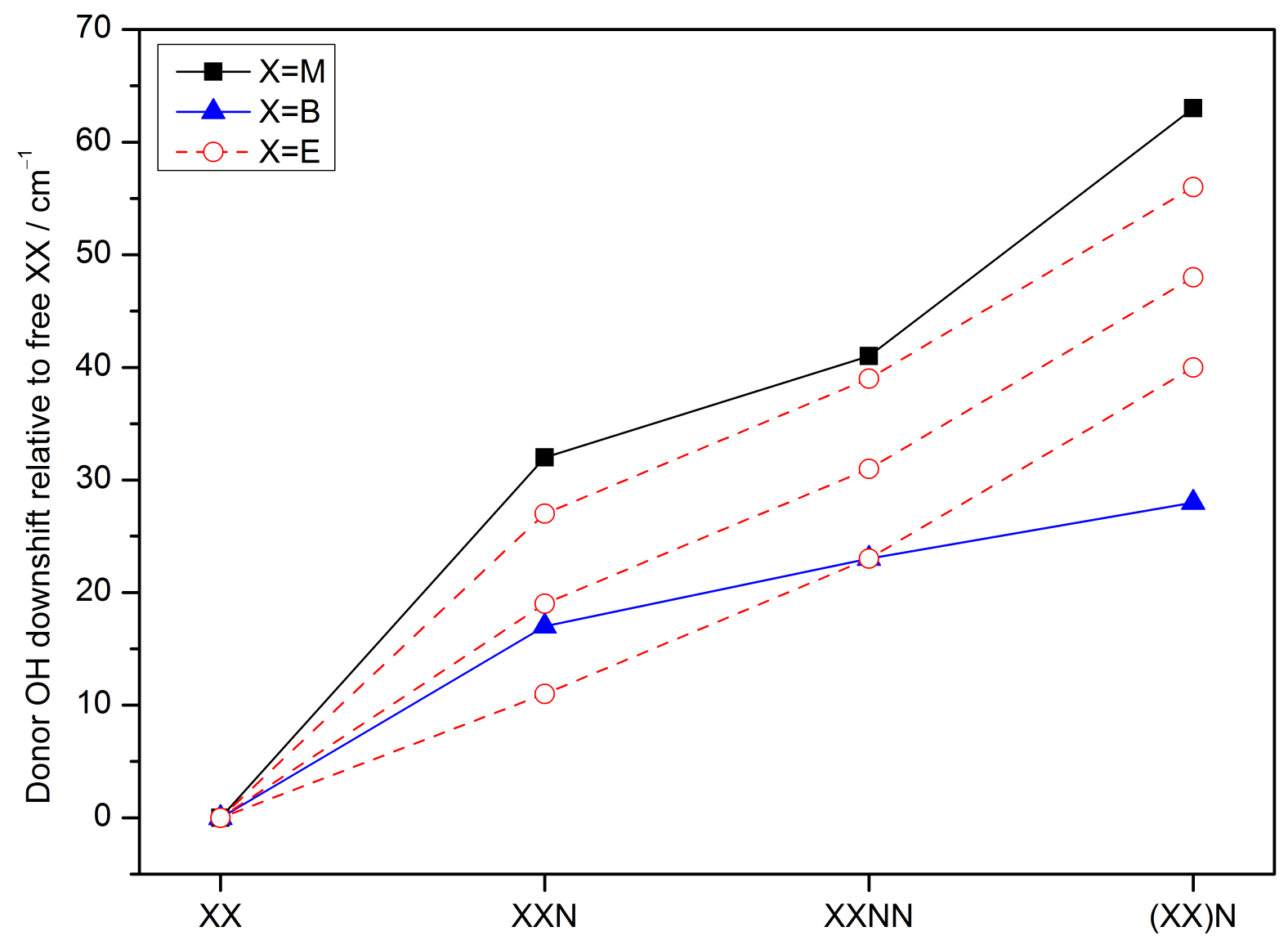

Figure 4.7.: Experimental $\mathrm{OH}$ stretching downshift trends found for aliphatic alcohol dimers as a function of $\mathrm{N}$ coordination (reprinted with adaptations from reference 287 licensed under CC BY 4.0). Quick saturation is observed for the bulky B, but not for $\mathrm{M}$. The three dashed lines for the intermediate alcohol E correspond to different uniform conformational EE assignments along the nanocoating coordinate, each assigned to one of the three experimentally resolved dimer donor bands. The lowest trend corresponds to an $\mathrm{E}_{g} \mathrm{E}_{g}$ assignment, the highest to $\mathrm{E}_{t} \mathrm{E}_{g}$, while assignment to $\mathrm{E}_{g} \mathrm{E}_{g}$ (lowest trend) upon $\mathrm{N}$ docking is inconsistent with $\mathrm{B}$ and $\mathrm{M}$ due to the XXN data point.

$\mathrm{N}$ docking energies at the different alcohol aggregates are summarised in Table 4.5. Binding at all monomers with a dissociation energy of 3 to $4 \mathrm{~kJ} \mathrm{~mol}^{-1}$ is significantly less stable than $\mathrm{N}$ addition to alcohol dimers ranging from 6 to $8 \mathrm{~kJ} \mathrm{~mol}^{-1}$. In com- 


\section{Aliphatic Alcohols}

bination with small predicted spectral shifts and infrared intensity enhancements, the low visibility of the binary aggregates in competition with the cooperative complexes is thus explained. The uniform binding energy predictions for all aggregates also indicate a largely electrostatic interaction, as polarisation or dispersion effects should grow with increasing alkyl group size.

Single N coordination causes a large spectral shift in MM. The corresponding EE value is much smaller, even lower than in BB, despite having the largest predicted shift. This again hints at conformational changes in EE upon $\mathrm{N}$ coordination, which can only be fully resolved by microwave spectroscopy. ${ }^{32,303,304}$ Secondary N coordination and nanosolvation follows the same trend, though slightly attenuated as shown in Figure 4.7. The downshift as a function of $\mathrm{N}$ coordination for $\mathrm{BB}$ is fairly straightforward with a quickly saturating shift due to limited access of $\mathrm{N}$ and resulting cooperativity in case of the bulky alcohol dimer. With the better accessibility of MM, substantial shift enhancements for every $\mathrm{N}$ coordination step are observed, which might also be partly attributed to quenching of large amplitude motions in MM. The intermediate case of EE is highly dependent on conformational assignments. Dashed lines in Figure 4.7 assume uniform conformations along the nanocoating coordinate, each assigned to one of the three experimentally resolved dimer donor bands. The lowest trend corresponding to an $\mathrm{E}_{g} \mathrm{E}_{g}$ assignment is rather unlikely due to the low EEN shift, while the highest one with an $\mathrm{E}_{t} \mathrm{E}_{g}$ assignment contradicts the bulk matrix findings. ${ }^{62}$ Even the intermediate trend rests on the possibly incorrect assumption that the two observed bands are caused by EEN and EENN rather than EEN with different EE conformations.

Table 4.4.: Experimental $\left(\Delta \tilde{\nu}_{\mathrm{OH}, \exp }\right)$ and harmonic $\left(\Delta \omega_{\mathrm{OH}, \text { theo }}\right)$ donor $\mathrm{OH}$ stretching downshift predicted at the B3LYP-D3(BJ)/aVTZ level between the structures given in the first column.

\begin{tabular}{ccc}
\hline Structures & $\Delta \tilde{\nu}_{\mathrm{OH}, \exp } / \mathrm{cm}^{-1}$ & $\Delta \omega_{\mathrm{OH}, \text { theo }} / \mathrm{cm}^{-1}$ \\
\hline $\mathrm{M}-\mathrm{M} M$ & 110 & 176 \\
$\mathrm{M} M-\mathrm{M} M \mathrm{~N}$ & 32 & 24 \\
$\mathrm{M} M \mathrm{NN}-\mathrm{M} M$ & 41 & 35 \\
$\mathrm{M} M-(\mathrm{M} M) \mathrm{N}$ & 63 & - \\
\hline $\mathrm{B}-\mathrm{B} B$ & 145 & 188 \\
$\mathrm{~B} B-\mathrm{B} B \mathrm{~N}$ & 17 & 21 \\
$\mathrm{~B} B-\mathrm{B} B \mathrm{NN}$ & 23 & 24 \\
$\mathrm{~B} B-(\mathrm{B} B) \mathrm{N}$ & 28 & - \\
\hline $\mathrm{E}_{g}-\mathrm{E}_{g} \mathrm{E}_{g}$ & 129 & 176 \\
$\mathrm{E}_{g} \mathrm{E}_{g}-\mathrm{E} E \mathrm{~N}$ & 11 & 28 \\
$\mathrm{E}_{g} \mathrm{E}_{g}-\mathrm{E} E \mathrm{NN}$ & 23 & 33 \\
$\mathrm{E}_{g} \mathrm{E}_{g}-(\mathrm{E} E) \mathrm{N}$ & 40 & - \\
\hline
\end{tabular}

For the conformationally unambiguous cases of $\mathrm{M}$ and $\mathrm{B}, \mathrm{N}$ coating can also help in 


\section{Aliphatic Alcohols}

Table 4.5.: Incremental electronic $\left(N_{\mathrm{e}}\right)$ and harmonic vibrational zero-point corrected $\left(N_{0}\right)$ energies required for abstraction of an isolated $\mathrm{N}$ for the different alcohol-nitrogen heteroaggregates. $\operatorname{CCSD}(\mathrm{T})$ single point energies were obtained by using optimised structures and zero-point energies from B3LYP-D3(BJ)/aVTZ calculations.

\begin{tabular}{cccrc}
\hline & \multicolumn{2}{c}{ B3LYP-D3(BJ)/aVTZ } & \multicolumn{2}{c}{ CCSD(T)/aVTZ } \\
Structures & $N_{\mathrm{e}} / \mathrm{kJ} \mathrm{mol}^{-1}$ & $N_{0} / \mathrm{kJ} \mathrm{mol}^{-1}$ & $N_{\mathrm{e}} / \mathrm{kJ} \mathrm{mol}^{-1}$ & $N_{0} / \mathrm{kJ} \mathrm{mol}^{-1}$ \\
\hline $\mathrm{MN}_{\mathrm{H}} \rightarrow \mathrm{M}+\mathrm{N}$ & 6.0 & 3.5 & 6.9 & 4.5 \\
$\mathrm{MMN}_{\mathrm{H}} \rightarrow \mathrm{MM}+\mathrm{N}$ & 8.8 & 6.0 & 10.1 & 7.3 \\
$\mathrm{MMN}_{\mathrm{H}} \mathrm{N}_{\mathrm{H}} \rightarrow \mathrm{MMN}_{\mathrm{H}}+\mathrm{N}$ & 8.1 & 6.1 & 10.3 & 8.4 \\
\hline $\mathrm{BN}_{\mathrm{H}} \rightarrow \mathrm{B}+\mathrm{N}$ & 6.3 & 4.2 & - & - \\
$\mathrm{BBN}_{\mathrm{H}} \rightarrow \mathrm{BB}+\mathrm{N}$ & 10.2 & 7.8 & - & - \\
$\mathrm{N}_{\mathrm{O}} \mathrm{BBN}_{\mathrm{H}} \rightarrow \mathrm{BBN}_{\mathrm{H}}+\mathrm{N}$ & 7.6 & 6.1 & - & - \\
$\mathrm{BBN}_{\mathrm{H}} \mathrm{N}_{\mathrm{H}} \rightarrow \mathrm{BBN}_{\mathrm{H}}+\mathrm{N}$ & 7.2 & 5.5 & - & - \\
\hline $\mathrm{E}_{t} \mathrm{~N}_{\mathrm{H}} \rightarrow \mathrm{E}_{t}+\mathrm{N}$ & 5.9 & 3.7 & 7.1 & 4.8 \\
$\mathrm{E}_{g} \mathrm{E}_{g^{\prime}} \mathrm{N}_{\mathrm{H}} \rightarrow \mathrm{E}_{g} \mathrm{E}_{g^{\prime}}+\mathrm{N}$ & 9.4 & 6.7 & 11.3 & 8.6 \\
$\mathrm{E}_{t} \mathrm{E}_{t} \mathrm{~N}_{\mathrm{H}} \mathrm{N}_{\mathrm{H}} \rightarrow \mathrm{E}_{t} \mathrm{E}_{t} \mathrm{~N}_{\mathrm{H}}+\mathrm{N}$ & 8.4 & 6.9 & - & - \\
\hline
\end{tabular}

rationalising the unusually small downshift from $\mathrm{M}$ to $\mathrm{MM}$ in the gas phase. In Figure 4.8 the difference between $\mathrm{B}$ and $\mathrm{M}$ donor $\mathrm{OH}$ stretching downshifts, $\Delta_{\mathrm{B}-\mathrm{M}}$, calculated according to Equation 4.1 is shown as a function of progressive $\mathrm{N}$ coordination.

$$
\Delta_{\mathrm{B}-\mathrm{M}}=\tilde{\nu}_{\mathrm{B}}(\text { monomer })-\tilde{\nu}_{\mathrm{B}}(\text { dimer donor })-\left(\tilde{\nu}_{\mathrm{M}}(\text { monomer })-\tilde{\nu}_{\mathrm{M}}(\text { dimer donor })\right)
$$

For the free dimers in supersonic expansion the BB downshift is larger by $35 \mathrm{~cm}^{-1}$. Calculating downshifts attributed to single, double and multiple $\mathrm{N}$ coordination requires the assumption that similar $\mathrm{N}$-induced shifts occur for the respective $\mathrm{M}$ and $\mathrm{B}$ monomers, since these band positions are not observed experimentally. According to theoretical predictions, this assumption appears reasonable, nonetheless a conservative error bar of $5 \mathrm{~cm}^{-1}$ is subsequently introduced. Site effects in $\mathrm{N}$ matrix add an error of $5 \mathrm{~cm}^{-1}$ in $\mathrm{BB}$ and $15 \mathrm{~cm}^{-1}$ in $\mathrm{MM}$, respectively. With progressive $\mathrm{N}$ coating $\Delta_{\mathrm{B}-\mathrm{M}}$ drops steadily to zero. This supports the assumption, that intrinsic harmonic downshifts may be quite similar for MM and BB. ${ }^{120}$ The unusually small downshift in MM is mostly caused by large amplitude motion weakening the hydrogen bond. ${ }^{120,305}$ These effects are attenuated in the heavier and bulkier $\mathrm{B}$ system and can be quenched in $\mathrm{M}$ through $\mathrm{N}$ coating. Similarly, in argon matrix ${ }^{58,62}$ the downshifts are equal within their site splitting induced error bars with values of $135(15) \mathrm{cm}^{-1}$ for $\mathrm{B}$ and $140(12) \mathrm{cm}^{-1}$ for $\mathrm{M}$, respectively. A matrix host with weaker interactions such as neon should cause a divergence of the shifts towards gas phase values again, but only the value of $125(4) \mathrm{cm}^{-1}$ for $\mathrm{MM}^{30}$ is available from the literature so far. 


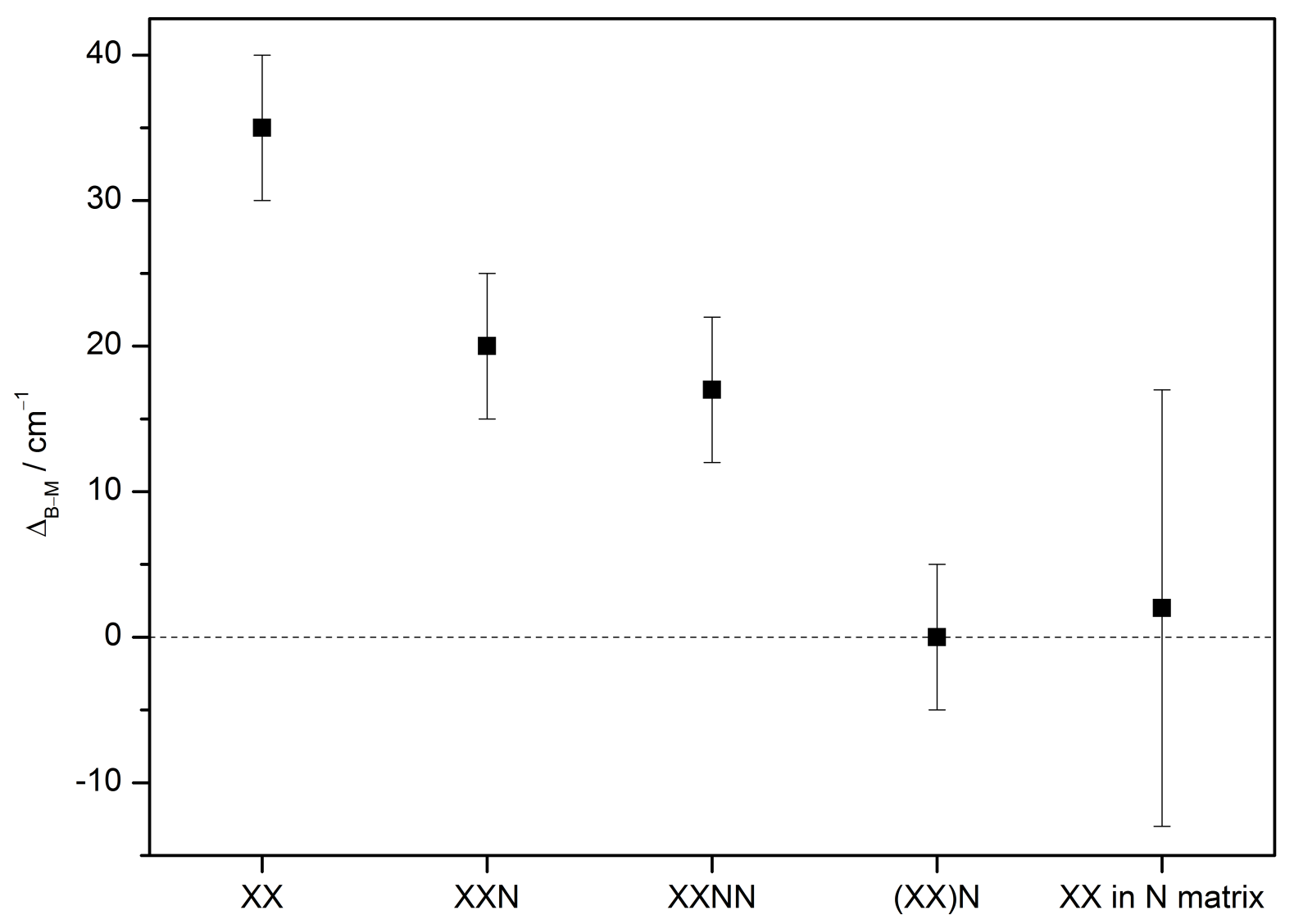

Figure 4.8.: Experimental difference between $\mathrm{B}$ and $\mathrm{M}$ donor $\mathrm{OH}$ stretching downshifts (reprinted from reference 157 licensed under CC BY 4.0) from monomer to dimer $\left(\Delta_{\mathrm{B}-\mathrm{M}}\right)$ as a function of progressive $\mathrm{N}$ coordination of $\mathrm{XX}(\mathrm{X}=\mathrm{M}, \mathrm{B})$.

\subsection{FTIR Spectra with Oxygen}

OH stretching FTIR spectra with oxygen (99.998\%, Air Liquide) instead of nitrogen added to the expansion were obtained for methanol and ethanol. The lack of quantum chemical calculations impedes assignments of vibrational bands to specific structures, but general spectral trends can be extracted from the data and compared to nitrogen complexation.

\subsubsection{Methanol}

Similar to $\mathrm{N}$ addition, $2.5 \% \mathrm{O}$ (Figure 4.9, trace c) induces a new band downshifted from the MM donor vibration at $3560 \mathrm{~cm}^{-1}$, also visible as positive feature in the MM-corrected difference spectrum (trace b). As previously observed in this work with other hydrogen bond donor molecules such as carboxylic acids and 1,1,1,3,3,3hexafluoroisopropanol, the $\mathrm{O}$ induced downshift is much weaker than corresponding $\mathrm{N}$ induced values and the band intensity is lower. Therefore, an additional MMOO signal 


\section{Aliphatic Alcohols}

similar to $\mathrm{N}$ complexation is not observed. However, addition of $15 \% \mathrm{O}$ again induces a broad band roughly centred at $3543 \mathrm{~cm}^{-1}$, which indicates $\mathrm{O}$ coating of MM. The combined acceptor vibrations of all $\mathrm{O}$ coordinated or coated MM clusters appear at $3679 \mathrm{~cm}^{-1}$. Parallel to findings with N, M and MMM bands do not display significant changes.

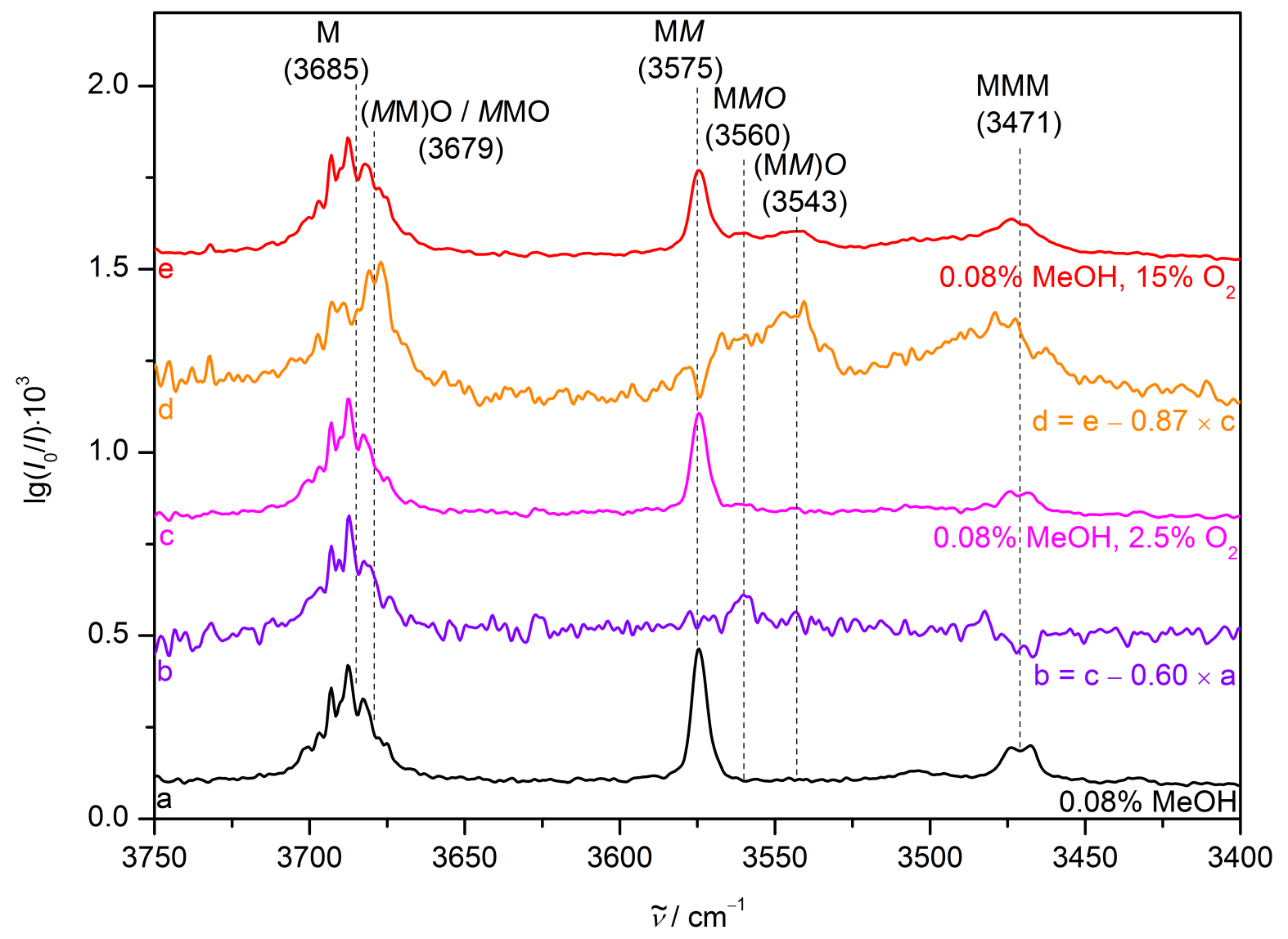

Figure 4.9.: $\mathrm{OH}$ stretching FTIR spectra of $\mathrm{M}$ expanded in helium (trace a), $2.5 \%$ and $15 \% \mathrm{O}$ in helium (traces c and e). A stagnation pressure of $p_{\mathrm{s}}=0.75$ bar was employed for all shown spectra. MM-corrected difference spectra (traces b and d) are included. Wavenumbers and (tentative) labels are provided. All spectra were scaled to matching height of the monomer band at $3685 \mathrm{~cm}^{-1}$.

\subsubsection{Ethanol}

Adding $2.5 \% \mathrm{O}$ to $\mathrm{E}$ (Figure 4.10, trace c) again yields results very similar to the ones observed for $\mathrm{N}$ addition. The conformational temperature in the expansion drops, as vibrational bands associated with metastable EE dimers lose intensity relative to the most stable $\mathrm{E}_{g} \mathrm{E}_{g}$ dimer band at $3531 \mathrm{~cm}^{-1}$ (trace b) and the EEO donor band appears at $3525 \mathrm{~cm}^{-1}$. Unspecific $\mathrm{O}$ coating of $\mathrm{EE}$ after addition of $15 \%$ is found with the broad 


\section{Aliphatic Alcohols}

donor band centred at $3520 \mathrm{~cm}^{-1}$. Again, observed spectral shifts and infrared intensities for $\mathrm{O}$ complexes are lower than corresponding quantities found after $\mathrm{N}$ addition. The acceptor region is difficult to judge because of band overlaps between both monomer conformations and various cluster acceptor vibrations.

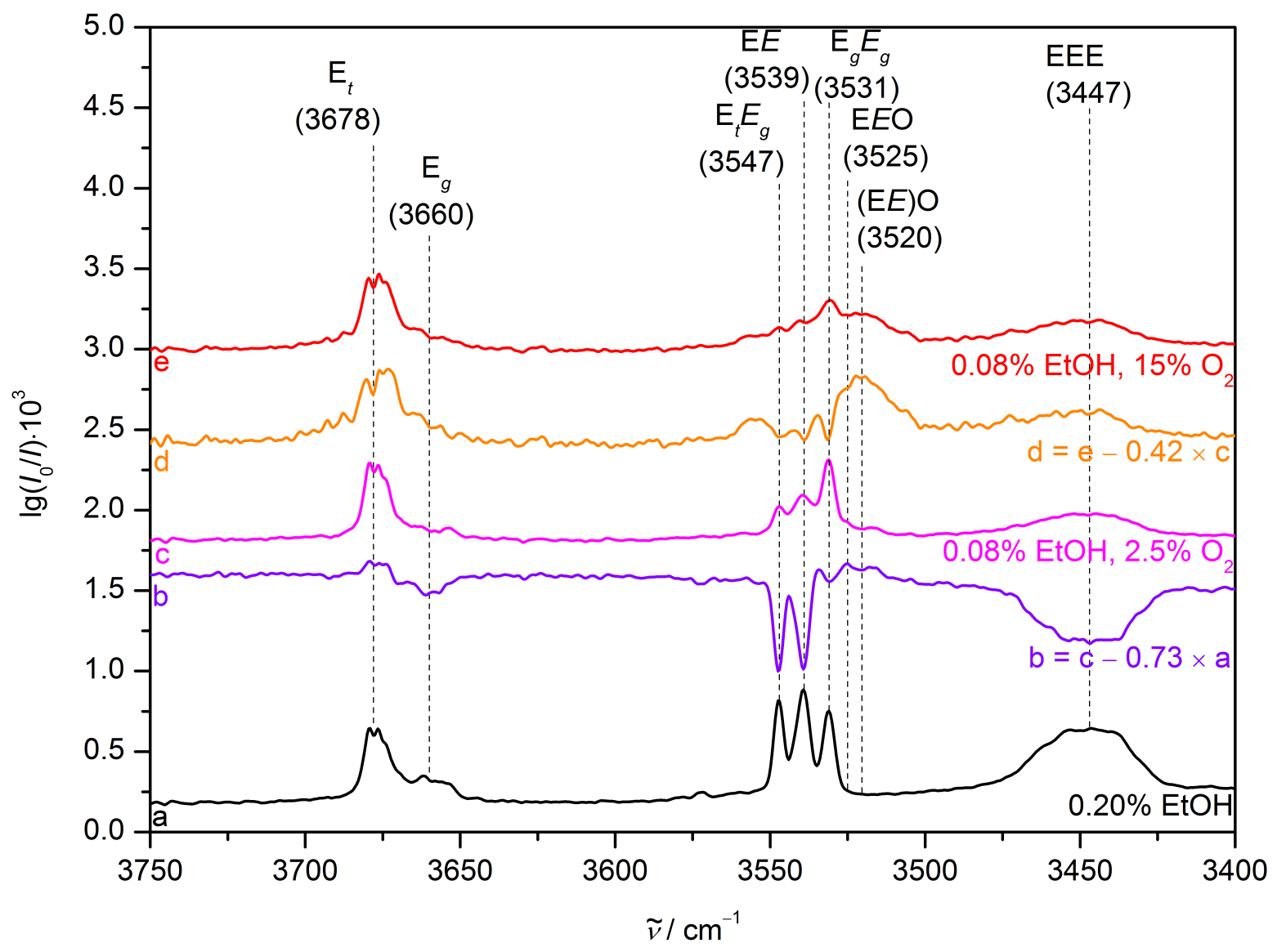

Figure 4.10.: $\mathrm{OH}$ stretching FTIR spectra of E expanded in helium (trace a), $2.5 \%$ and $15 \% \mathrm{O}$ in helium (traces $\mathrm{c}$ and e). A stagnation pressure of $p_{\mathrm{s}}=0.75$ bar was employed for all shown spectra. $\mathrm{E}_{g} \mathrm{E}_{g}$-corrected difference spectra (traces $\mathrm{b}$ and $\mathrm{d}$ ) are included. Wavenumbers and (tentative) labels are provided. All spectra were scaled to matching height of the $\mathrm{E}_{t}$ monomer band at $3678 \mathrm{~cm}^{-1}$.

\subsection{Raman Spectra}

Raman spectra of $\mathrm{E}$ and $\mathrm{N}$ mixtures in helium were recorded in the spectral region of the $\mathrm{OH}$ and NN stretching vibrations. No mixed EN clusters were observed under the employed expansion conditions, but the spectra allow for the determination of rotational temperatures in the expansion by analysing intensities of $\mathrm{N}$ rovibrational transitions. 


\section{Aliphatic Alcohols}

The $\mathrm{E}_{t} / \mathrm{E}_{g}$ ratio derived from $\mathrm{OH}$ stretching spectra is used for simultaneous evaluation of conformational temperatures.

\subsubsection{Rotational Temperature}

Rovibrational transitions of $\mathrm{N}$ are shown in Figure 4.11. Corresponding spectra recorded with the laser probing the expansion at different distances from the nozzle are depicted in Figures A.19 and A.20 in appendix A.4.

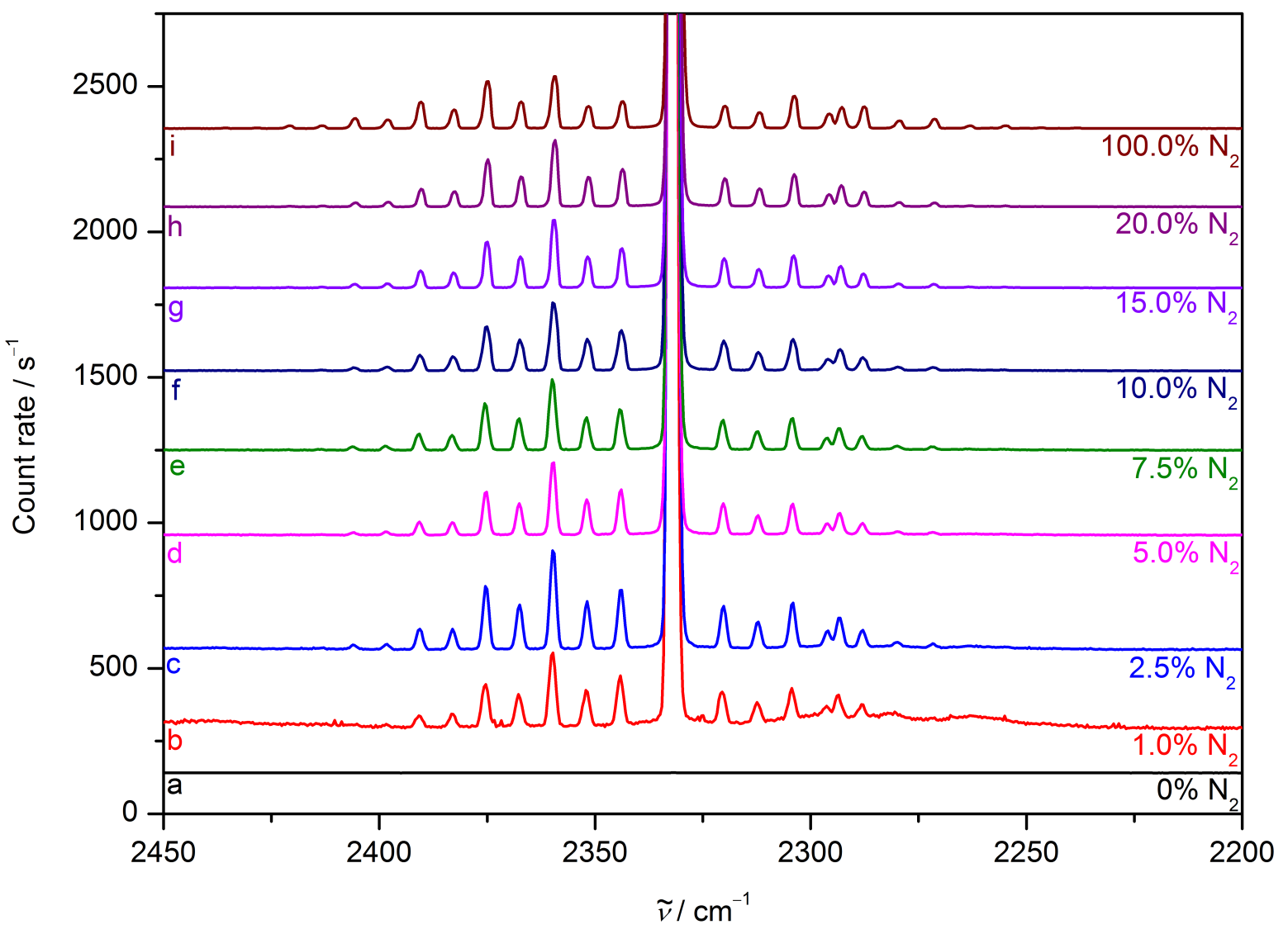

Figure 4.11.: NN stretching Raman spectra of E expanded in helium (trace a), N (trace i), $1.0 \%, 2.5 \%, 5.0 \%, 7.5 \%, 10.0 \%, 15.0 \%$ and $20.0 \% \mathrm{~N}$ in helium (traces b to h). E was kept at a temperature of $273 \mathrm{~K}$, the carrier gas pressure in the gas line at 1.5 bar and the stagnation pressure in the reservoir at 0.75 bar, while the laser probed the expansion at a $1 \mathrm{~mm}$ nozzle distance. All spectra were scaled to matching height of the NN stretching Q-branch at $2330 \mathrm{~cm}^{-1}$.

The intensity $I$ of rovibrational Raman transitions of a linear rotor such as $\mathrm{N}$ can be calculated according to Equation $4.2^{306,307}$ from the lower state rotational quantum number $\mathrm{J}^{\prime \prime}$, the degeneracy due to nuclear spin statistics $g_{I}$, the rotational partition function $Z_{\mathrm{rot}}$, the Planck constant $h$, the Boltzmann constant $k_{\mathrm{B}}$ and the rotational temperature $T_{\text {rot }}$. The factor $G$ contains experimental parameters such as the wavelength 


\section{Aliphatic Alcohols}

and radiation density of the excitation laser, the scattering geometry and the anisotropy of the polarisability and can be approximately assumed as constant for all considered transitions. ${ }^{107}$ The rotational constant $\tilde{B}^{\prime \prime}$ for the first excited vibrational state $(\mathrm{v}=1)$ can be calculated from the equilibrium rotational constant ${ }^{308} \tilde{B}_{\mathrm{e}}=1.99824 \mathrm{~cm}^{-1}$ and the vibration-rotation coupling constant ${ }^{309} \alpha_{\mathrm{e}}=-0.017318 \mathrm{~cm}^{-1}$ (Equation 4.3).

$$
\begin{gathered}
I=G \frac{\left(\mathrm{J}^{\prime \prime}+1\right)\left(\mathrm{J}^{\prime \prime}+2\right)}{\left(2 \mathrm{~J}^{\prime \prime}+1\right)\left(2 \mathrm{~J}^{\prime \prime}+3\right)} \frac{g_{I}\left(2 \mathrm{~J}^{\prime \prime}+1\right)}{Z_{\mathrm{rot}}} \exp \left[-\frac{h c \tilde{B}^{\prime \prime} \mathrm{J}^{\prime \prime}\left(\mathrm{J}^{\prime \prime}+1\right)}{k_{\mathrm{B}} T_{\mathrm{rot}}}\right] \\
\tilde{B}_{\mathrm{v}}=\tilde{B}_{\mathrm{e}}+\alpha\left(\mathrm{v}+\frac{1}{2}\right)
\end{gathered}
$$

From Equation 4.2 it can be derived that a plot of $\frac{\left(\mathrm{J}^{\prime \prime}+1\right)\left(\mathrm{J}^{\prime \prime}+2\right)}{\left(2 \mathrm{~J}^{\prime \prime}+1\right)\left(2 \mathrm{~J}^{\prime \prime}+3\right)} I$ against $\mathrm{J}^{\prime \prime}\left(\mathrm{J}^{\prime \prime}+1\right)$ yields a linear function with a slope of $-\frac{h c \tilde{B^{\prime \prime}}}{k_{\mathrm{B}} T_{\text {rot }}}$. Intensities $I$ are obtained by integration of the $S$-branch transitions $(\Delta \mathrm{J}=2)$, which are chosen for their higher intensity compared to corresponding $O$-branch $(\Delta \mathrm{J}=-2)$ lines and separately analysed for even and odd $\mathrm{J}$ quantum numbers due to the different nuclear spin statistics. Hence, two rotational temperatures $T_{\text {rot,even }}, T_{\text {rot,odd }}$ as well as respective uncertainties $\Delta T_{\text {rot,even }}$ and $\Delta T_{\text {rot,odd }}$ are obtained from least-squares linear regressions of intensities from every spectrum and combined to the final rotational temperature by their error-weighted average according to Equation 4.4 with the uncertainty $\Delta T_{\text {rot }}$ (Equation 4.5 ). Details of the integration method and results can be found in Table A.24 in appendix A.4.

$$
\begin{gathered}
T_{\text {rot }}=\frac{\left(\frac{T_{\text {rot,even }}}{\Delta T_{\text {rot,even }}}\right)+\left(\frac{T_{\text {rot,odd }}}{\Delta T_{\text {rot,odd }}}\right)}{\left(\frac{1}{\Delta T_{\text {rot,even }}}\right)+\left(\frac{1}{\Delta T_{\text {rot }, \text { odd }}}\right)} \\
\Delta T_{\text {rot }}=\frac{\left(\frac{\left(T_{\text {rot }}-T_{\text {rot,even }}\right)^{2}}{\Delta T_{\text {rot,even }}}\right)+\left(\frac{\left(T_{\text {rot }}-T_{\text {rot }, \text { odd }}\right)^{2}}{\Delta T_{\text {rot }, \text { odd }}}\right)}{\left(\frac{1}{\Delta T_{\text {rot,even }}}\right)+\left(\frac{1}{\Delta T_{\text {rot }, \text { odd }}}\right)}
\end{gathered}
$$

Rotational temperatures (Figure 4.12) drop sharply upon N addition. Starting from about 50 to $70 \mathrm{~K}$, temperatures quickly converge to values well below $10 \mathrm{~K}$. From previous work ${ }^{105,125}$, a decline of expansion temperatures with increasing nozzle distance due to the larger number of collisions would be expected. For small N molar fractions this is difficult to verify, since the low signal-to-noise ratio only allows for a small number of rovibrational lines to be analysed resulting in large error bars. Larger $\mathrm{N}$ molar fractions lead to a very effective rotational cooling and rather uniform values are obtained for all three studied nozzle distances. 


\section{Aliphatic Alcohols}

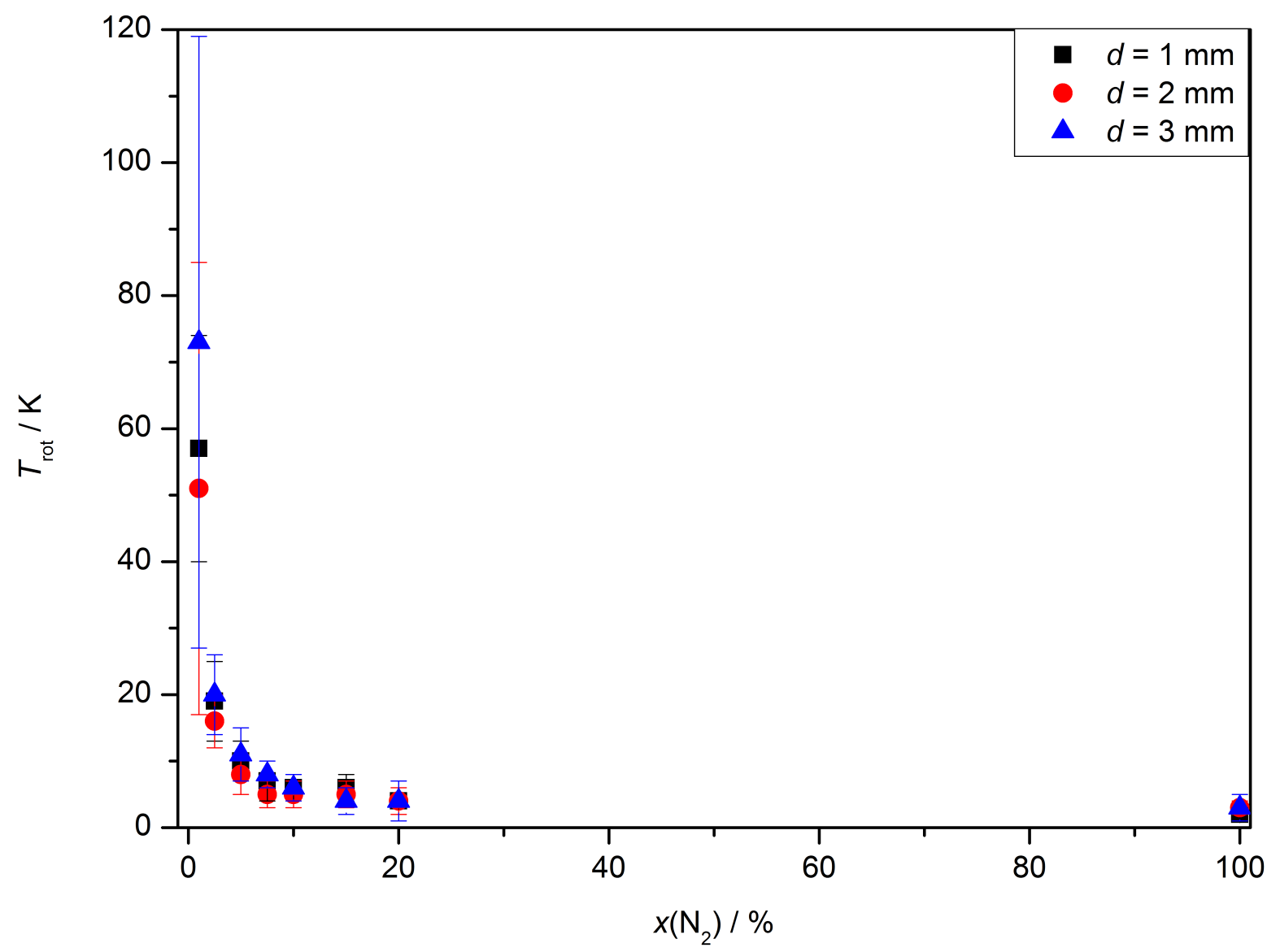

Figure 4.12.: Rotational temperatures $T_{\text {rot }}$ derived from NN stretching Raman spectra of $\mathrm{E}$ expanded in mixtures of $\mathrm{N}$ and helium as a function of the molar $\mathrm{N}$ fraction $x$ with the laser probing the expansion at different nozzle distances $d$.

\subsubsection{Conformational Temperature}

$\mathrm{OH}$ stretching Raman spectra of $\mathrm{E}_{t}$ and $\mathrm{E}_{g}$ are shown in Figure 4.13. Corresponding spectra at different nozzle distances are depicted in Figures A.21 and A.22 in appendix A.4. Conformational temperatures $T_{\text {conf }}$ are derived from the experimental band intensity ratio $I_{t} / I_{g}$ and the known energy difference $295,310-312$ of $\Delta E=0.49 \mathrm{~kJ} \mathrm{~mol}^{-1}$ between both conformers according to Equation 4.6, ${ }^{295}$ including the degeneracy ratio of $g_{g} / g_{t}=2$ and the ratio of Raman scattering cross sections ${ }^{295} \sigma_{\text {rel }}=\sigma_{t} / \sigma_{g}=1.5(1)$. The band intensities were obtained by integration with linear baseline corrections interpolated from the respective integral bounds in the OriginPro (Version 8.5) program package, while error bars were estimated by adding and subtracting the spectral peak-to-peak noise of about 0.1 Counts s $^{-1}{ }^{295}$

$$
T_{\text {conf }}=\frac{\Delta E}{k_{\mathrm{B}}} \ln \left(\frac{g_{g} I_{t}}{g_{t} \sigma_{\mathrm{rel}} I_{g}}\right)^{-1}
$$




\section{Aliphatic Alcohols}

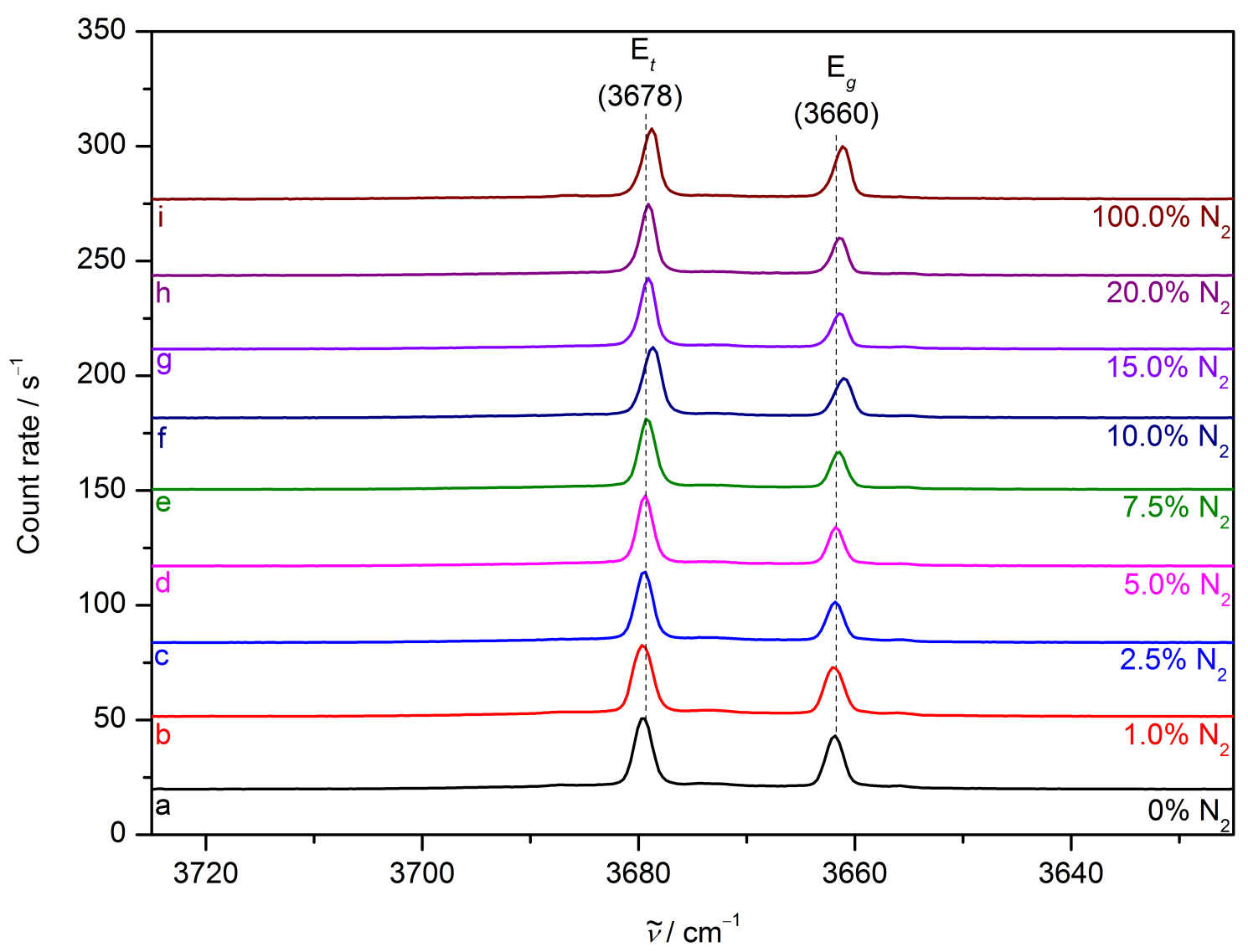

Figure 4.13.: $\mathrm{OH}$ stretching Raman spectra of E expanded in helium (trace a), $\mathrm{N}$ (trace i), $1.0 \%, 2.5 \%, 5.0 \%, 7.5 \%, 10.0 \%, 15.0 \%$ and $20.0 \% \mathrm{~N}$ in helium (traces b to h). E was kept at a temperature of $273 \mathrm{~K}$, the carrier gas pressure in the gas line at $1.5 \mathrm{bar}$ and the stagnation pressure in the reservoir at $0.75 \mathrm{bar}$, while the laser probed the expansion at a $1 \mathrm{~mm}$ nozzle distance. All spectra were scaled to matching height of the $\mathrm{E}_{t}$ band at $3678 \mathrm{~cm}^{-1}$.

Conformational temperatures (Figure 4.14) of E display a cooling behaviour different from the barrier-less relaxation of rotational states of $\mathrm{N}$. Using only $\mathrm{N}$ as carrier gas results in similar or slightly higher conformational temperatures than helium expansions. As expected, ${ }^{105,125}$ lower values are found with increasing nozzle distance. For all three distances, the conformational temperature reaches a distinct local minimum with $\mathrm{N}$ molar fractions of about $10 \%$ before increasing again. Moderate admixture of the heavier $\mathrm{N}$ induces more effective cooling, but larger molar fractions possibly need to be cooled by a sufficient number of collisions with helium before being able to provide effective cooling for E. 


\section{Aliphatic Alcohols}

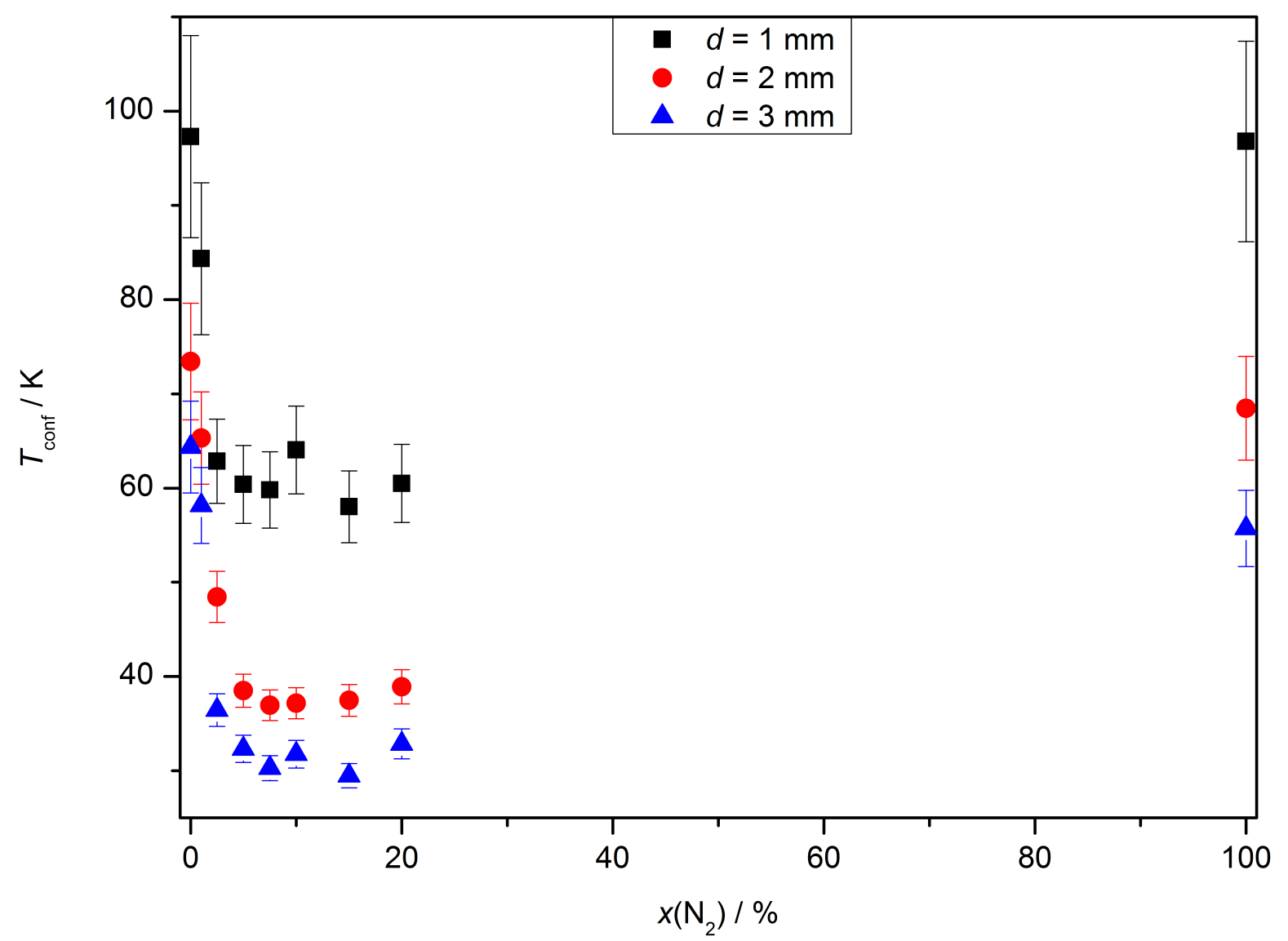

Figure 4.14.: Conformational temperatures $T_{\text {conf }}$ derived from $\mathrm{OH}$ stretching Raman spectra of $\mathrm{E}$ expanded in mixtures of $\mathrm{N}$ and helium as a function of the molar $\mathrm{N}$ fraction $x$ with the laser probing the expansion at different nozzle distances $d$.

\subsection{Summary}

Nitrogen and oxygen complexation of alcohol dimers was observed with FTIR jet spectroscopy. Activation and increased polarisation of the $\mathrm{OH}$ bond through a second alcoholic $\mathrm{OH}$ hydrogen bond donor is necessary for effective cluster formation. The cooperative effects ${ }^{88}$ of single and even secondary nitrogen addition on the $\mathrm{OH} \cdots \mathrm{OH}$ hydrogen bond lead to significant spectral changes such as $\mathrm{OH}$ stretching downshifts and infrared intensity enhancements. Unspecific nitrogen coating results in band positions in reasonable agreement with bulk matrix experiment, while effects on the NN stretching vibration may be detected via infrared activation through complexation ${ }^{84}$ or Raman spectroscopy in future work.

Alcohol monomers without cooperative donor strength enhancement show little to no visible spectral effects, while trimers with their cyclic hydrogen bond topology lack a free acceptor $\mathrm{OH}$ as attractive binding site. Nitrogen complexation could thus serve as an indicator of linear chain hydrogen bond arrangements in trimers or larger clusters. ${ }^{67}$ 


\section{Aliphatic Alcohols}

Spectral effects observed after oxygen aggregation are very similar to nitrogen addition, but remain difficult to judge due to the lack of accessible predictions from quantum chemistry.

Furthermore, the effect of a nitrogen additive to the typically used helium carrier gas on conformational and rotational temperatures could be analysed. The heavier molecules in the carrier gas provide more efficient cooling, though observed trends differ significantly for both degrees of freedom in a non-equilibrium system such as a supersonic expansion. 


\section{Pyrrole}

\section{Pyrrole}

The influence of nitrogen aggregation on the NH stretching mode of pyrrole was studied in supersonic expansions with filet-jet FTIR spectroscopy. Additionally, NH stretching FTIR spectra of pyrrole in neon, argon and nitrogen cryomatrices as well as in various mixtures of neon and argon with nitrogen were obtained together with Dr. Stéphane Coussan at Aix-Marseille University. Similar to alcohols, increasing nitrogen coordination induced progressive spectral downshifts of NH stretching fundamentals. The obtained results are published in reference 313. The occasional verbatim reproduction of small sections from that reference is not explicitly marked or cited.

\subsection{Introduction}

The stepwise nitrogen decoration previously performed with aliphatic alcohol dimers in supersonic expansion ${ }^{287}$ to gain insight into gas-to-matrix wavenumber shifts is also executed with the aromatic heterocycle pyrrole $\left(\mathrm{C}_{4} \mathrm{H}_{4} \mathrm{NH}\right)$ and extended to cryogenic matrices. Hence, pyrrole and its nitrogen aggregates are studied by NH stretching FTIR spectroscopy in both helium jet expansions and cryogenic matrices with various hosts such as the weakly interacting neon, ${ }^{314}$ the more strongly perturbing argon ${ }^{315}$ and the quadrupolar nitrogen. ${ }^{316,317}$

Pyrrole offers an intriguing competition between more directional $\mathrm{NH} \cdots \mathrm{N}_{2}$ hydrogen bonds and less directional interactions of $\mathrm{N}_{2}$ with the aromatic $\pi$ electron density upon nitrogen solvation. ${ }^{318}$ A sensitive probe of such interactions is the NH stretching mode, while spectral effects such as wavenumber shifts and infrared intensity enhancements induced by complexation are somewhat less pronounced in most other fundamental modes with the exception of $\mathrm{NH}$ bending vibrations. In some cases ${ }^{84-86}$ the formation of nitrogen complexes allows for infrared detection of the otherwise inactive NN stretching mode, which was prevented by spectral overlap with incompletely compensated atmospheric carbon dioxide in this work.

Previous experimental studies of pyrrole include optothermal molecular beam, ${ }^{319} \mathrm{mi}$ crowave, ${ }^{320,321}$ cavity ring-down, ${ }^{322}$ infrared photodissociation ${ }^{323}$ and aromatically labelled UV/IR double resonance ${ }^{324}$ spectroscopy as well as size selection by scattering. ${ }^{325}$ Matrix isolation FTIR spectroscopy studies were performed in para-hydrogen, ${ }^{316}$ nitrogen $^{316,317}$ and argon hosts ${ }^{315}$ alongside infrared spectroscopy in solution ${ }^{326,327}$ and supersonic expansions. ${ }^{328,329}$

Additional interest in pyrrole was induced by reports of an unusual 'improper' $\mathrm{NH} \cdots \mathrm{N}_{2}$ hydrogen bond with a corresponding $\mathrm{NH}$ stretching upshift in reference 317 based on infrared spectra in nitrogen matrices and partly anharmonic MP2 calculations 


\section{Pyrrole}

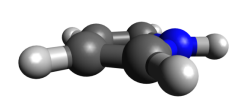

$P$

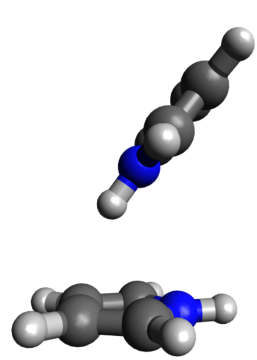

PP (23.7)

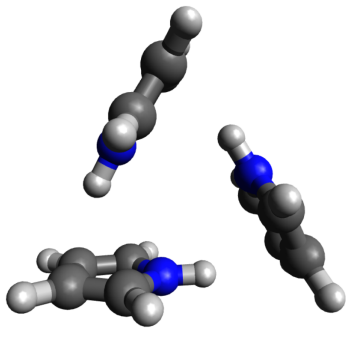

PPP (80.6)

Figure 5.1.: Stable structures of the pyrrole monomer $(\mathrm{P})$, dimer $(\mathrm{PP})$ and trimer $(\mathrm{PPP})$ optimised at the B3LYP-D3(BJ)/aVTZ level (reprinted from reference 313 licensed under CC BY 3.0). Harmonically zero-point corrected dissociation energies in $\mathrm{kJ} \mathrm{mol}^{-1}$ are provided in parentheses.

with small basis sets. The study received considerable attention, ${ }^{316,330-332}$ although more reliable calculations ${ }^{323}$ with dispersion-corrected density functionals already challenged the spectral interpretation given in reference 317. The incremental NH stretching downshift induced by stepwise nitrogen addition in both supersonic expansions and cryogenic matrices explored in this work clearly corrects the false proposals from reference 317 and the findings themselves provide an interesting and challenging benchmarking object for quantum chemistry. Neural network potentials ${ }^{64,65}$ including accurate nitrogen pair potentials ${ }^{333}$ might be a promising candidate for future modelling of bulk nitrogen environments.

\subsection{Quantum Chemical Calculations and Nomenclature}

The studied molecules are abbreviated as $\mathrm{P}$ (pyrrole) and $\mathrm{N}$ (nitrogen). Cluster compositions are denoted by repetition of the respective number of single letters in donoracceptor direction. Possible binding sites of nitrogen at the pyrrole molecule which are marked by according subscripts to the $\mathrm{N}$ include the $\mathrm{NH}$ group $(\mathrm{H})$, the $\pi$ electron cloud $(\pi)$ and the $\mathrm{CH}$ backbone $(\mathrm{C})$.

Stable structures of pyrrole homoaggregates are depicted in Figure 5.1. Both $\mathrm{P}$ monomer ${ }^{320}$ and PP dimer ${ }^{321}$ structures were experimentally studied by microwave spectroscopy, while the cyclic hydrogen bond topology in the PPP trimer was confirmed by complementary infrared and Raman spectra. ${ }^{329}$ The $\mathrm{NH} \cdots \pi$ hydrogen bond in PP is tilted from the linear arrangement with a $90^{\circ}$ angle to about $55^{\circ}$, a motif often found in dimers of aromatic systems such as P-benzene. ${ }^{334}$ The substantial cooperativity in PPP more than triples the dissociation energy compared to PP, which is still predicted to be significantly more stable than heteroaggregates with $\mathrm{N}$. Their predicted dissociation energies of about $6 \mathrm{~kJ} \mathrm{~mol}^{-1}$, in reasonable agreement with the experimental value of $6.67(8) \mathrm{kJ} \mathrm{mol}^{-1}$ found for the dimer of 1-naphtol with $\mathrm{N},{ }^{22}$ showcase that competition 


\section{Pyrrole}

between homo- and heteroaggregation should be minimised by appropriate experimental conditions such as low P concentrations in supersonic expansions and cryogenic matrices.

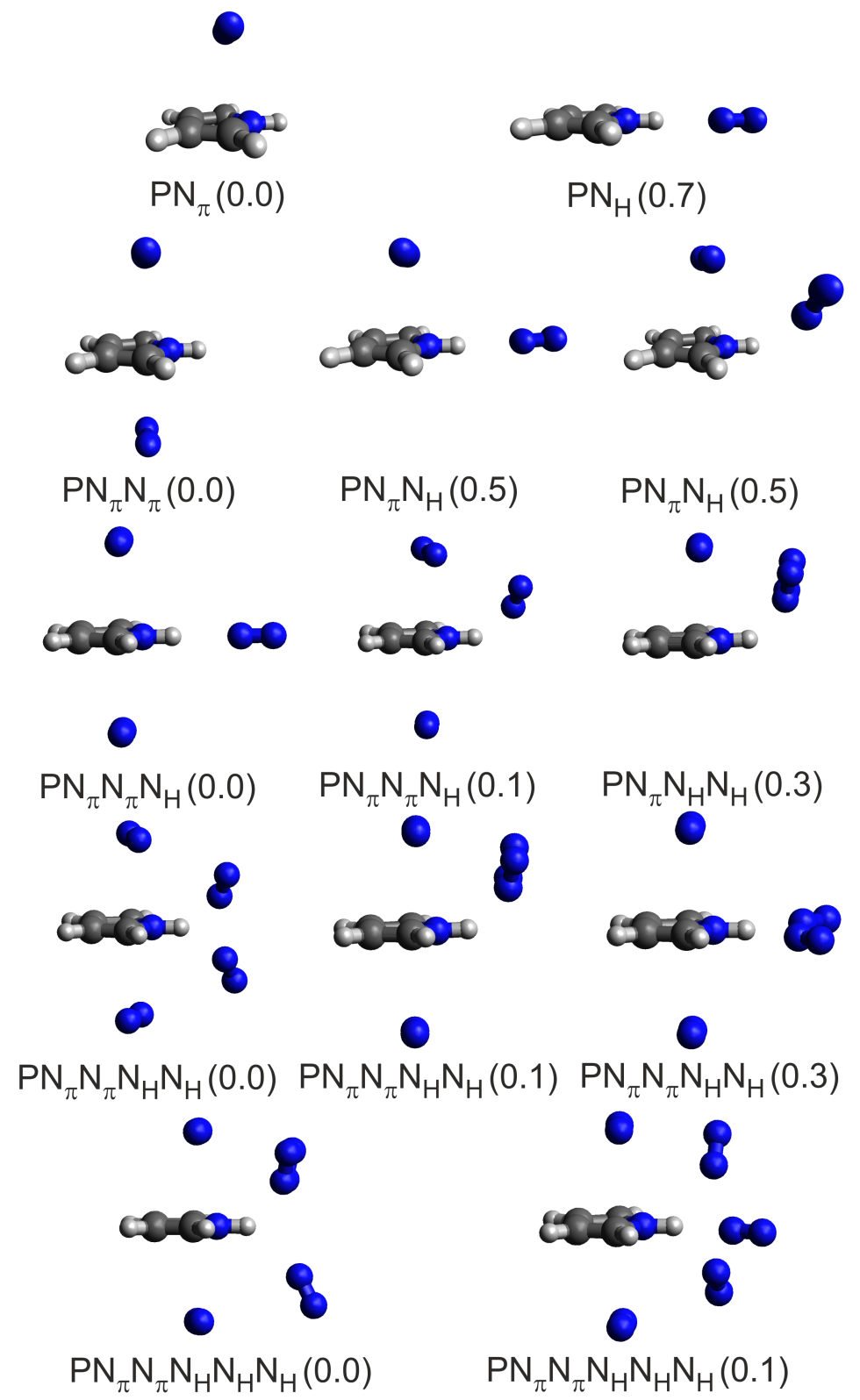

Figure 5.2.: Most stable structures found for heteroaggregates of the pyrrole monomer (P) with increasing N coordination optimised at the B3LYP-D3(BJ)/aVTZ level (reprinted from reference 313 licensed under CC BY 3.0). Relative energies in $\mathrm{kJ} \mathrm{mol}^{-1}$ calculated from $\operatorname{CCSD}(\mathrm{T}) / \mathrm{aVTZ}$ single-point calculations and harmonic zero-point vibrational energy from B3LYP-D3(BJ)/aVTZ are given in parentheses (bottom row exclusively B3LYPD3(BJ)/aVTZ energies). 


\section{Pyrrole}

Table 5.1.: Theoretically predicted properties of the most stable $\mathrm{P}$ and $\mathrm{N}$ aggregates such as harmonic $\left(\omega_{\mathrm{NH}}\right)$ and anharmonic $\left(\tilde{\nu}_{\mathrm{NH}}\right) \mathrm{NH}$ stretching wavenumbers, lowest predicted harmonic $\left(\omega_{1}\right)$ and anharmonic $\left(\tilde{\nu}_{1}\right)$ wavenumbers in $\mathrm{cm}^{-1}$, indicating limitations of numerical differentiation or of VPT2 which may also affect NH stretching coupling constants for all systems but $\mathrm{P}$ in italic font, harmonic $\left(S_{\omega}\right)$ and anharmonic $\left(S_{\nu}\right)$ infrared band strengths in $\mathrm{km} \mathrm{mol}^{-1}$, spectroscopic downshifts $\left(\Delta \omega_{\mathrm{NH}}, \Delta \tilde{\nu}_{\mathrm{NH}}\right)$ relative to $\mathrm{P}$ in $\mathrm{cm}^{-1}$ as well as relative electronic and harmonically zero-point corrected energies $\Delta E_{\mathrm{e}}$ and $\Delta E_{0}$ compared to the most stable cluster conformation in $\mathrm{kJ} \mathrm{mol}^{-1}$. All properties were calculated at the B3LYP-D3(BJ)/aVTZ level of approximation. Only the electronic energy was taken from $\operatorname{CCSD}(\mathrm{T}) / \mathrm{aVTZ}$ single-point calculations for all clusters except hexamers.

\begin{tabular}{ccccccccccc}
\hline Structure & $\omega_{\mathrm{NH}}$ & $S_{\omega}$ & $\Delta \omega_{\mathrm{NH}}$ & $\omega_{\mathrm{l}}$ & $\tilde{\nu}_{\mathrm{NH}}$ & $S_{\nu}$ & $\Delta \tilde{\nu}_{\mathrm{NH}}$ & $\tilde{\nu}_{1}$ & $\Delta E_{\mathrm{e}}$ & $\Delta E_{0}$ \\
\hline $\mathrm{P}$ & 3674 & 65 & - & 496 & 3508 & 51 & - & 492 & - & - \\
\hline $\mathrm{PP}$ & 3669 & 72 & 5 & 17 & - & - & - & - & - & - \\
& 3598 & 324 & 76 & & & & & & & \\
\hline \multirow{2}{*}{$\mathrm{PPP}$} & 3553 & 631 & 121 & & & & & & & \\
& 3553 & 631 & 121 & 22 & - & - & - & - & - & - \\
\hline $\mathrm{PN}_{\pi}$ & 3536 & 0 & 138 & & & & & & & \\
$\mathrm{PN}_{\mathrm{H}}$ & 3674 & 65 & 0 & 21 & 3507 & 51 & 1 & 18810 & 0.0 & 0.0 \\
$\mathrm{PN}_{\pi} \mathrm{N}_{\pi}$ & 3673 & 65 & 1 & 18 & 3517 & 52 & -9 & 132 & 0.0 & 0.0 \\
$\mathrm{PN}_{\pi} \mathrm{N}_{\mathrm{H}}$ & 3668 & 210 & 6 & 10 & 3505 & 131 & 3 & -22 & 0.4 & 0.5 \\
$\mathrm{PN}_{\pi} \mathrm{N}_{\mathrm{H}}$ & 3675 & 94 & -1 & 8 & 3508 & 69 & 0 & 23 & 0.7 & 0.5 \\
\hline $\mathrm{PN}_{\pi} \mathrm{N}_{\pi} \mathrm{N}_{\mathrm{H}}$ & 3667 & 214 & 7 & 9 & - & - & - & - & 0.0 & 0.0 \\
$\mathrm{PN}_{\pi} \mathrm{N}_{\pi} \mathrm{N}_{\mathrm{H}}$ & 3674 & 98 & 0 & 12 & - & - & - & - & 0.5 & 0.1 \\
$\mathrm{PN}_{\pi} \mathrm{N}_{\mathrm{H}} \mathrm{N}_{\mathrm{H}}$ & 3677 & 100 & -3 & 11 & - & - & - & - & 0.8 & 0.3 \\
\hline $\mathrm{PN}_{\pi} \mathrm{N}_{\pi} \mathrm{N}_{\mathrm{H}} \mathrm{N}_{\mathrm{H}}$ & 3676 & 122 & -2 & 10 & - & - & - & - & 0.0 & 0.0 \\
$\mathrm{PN}_{\pi} \mathrm{N}_{\pi} \mathrm{N}_{\mathrm{H}} \mathrm{N}_{\mathrm{H}}$ & 3676 & 104 & -2 & 9 & - & - & - & - & 0.0 & 0.1 \\
$\mathrm{PN}_{\pi} \mathrm{N}_{\pi} \mathrm{N}_{\mathrm{H}} \mathrm{N}_{\mathrm{H}}$ & 3678 & 168 & -4 & 4 & - & - & - & - & 0.1 & 0.3 \\
\hline $\mathrm{PN}_{\pi} \mathrm{N}_{\pi} \mathrm{N}_{\mathrm{H}} \mathrm{N}_{\mathrm{H}} \mathrm{N}_{\mathrm{H}}$ & 3674 & 142 & 0 & 3 & - & - & - & - & 0.0 & 0.0 \\
$\mathrm{PN}_{\pi} \mathrm{N}_{\pi} \mathrm{N}_{\mathrm{H}} \mathrm{N}_{\mathrm{H}} \mathrm{N}_{\mathrm{H}}$ & 3674 & 174 & 0 & 8 & - & - & - & - & 0.1 & 0.1 \\
\hline
\end{tabular}

Structures of the most stable PN heteroaggregates within a $1.0 \mathrm{~kJ} \mathrm{~mol}^{-1}$ energy window from the respective minimum energy structure are shown in Figure 5.2. The most stable binding sites are the $\pi$ electron density above the aromatic ring plane as found in the binary $\mathrm{P}$-argon aggregate ${ }^{335}$ and the $\mathrm{NH}$ proton, which is favoured in the positively charged $\mathrm{PN}^{+}$complex. ${ }^{323}$ Secondary $\mathrm{N}$ attachment is possible at both binding sites and predicted for increasing $\mathrm{N}$ solvation, while significant $\mathrm{NH}$ stretching downshifts within the double harmonic approximation are only predicted for hydrogen bonded $\mathrm{N}_{\mathrm{H}}$ aggregates (Table 5.1). Downshifts are only predicted up to the third N attachment and predictions for solvation complexes such as pentamers and hexamers switch to small up- 


\section{Pyrrole}

or no shifts at all. As comparison to experimental data below will demonstrate, these predictions show difficulties of the tested theoretical approach in the accurate modelling of $\mathrm{N}$ embedding.

Additional conformers with an energy difference larger than $1.0 \mathrm{~kJ} \mathrm{~mol}^{-1}$ from the minimum energy structure found during the conformational search are depicted in Figures A.23, A.24, A.25, A.26 and A.27 and their predicted spectral properties are listed in Table A.25 in appendix A.5. Without detailed interconversion barrier analyses, it remains unclear whether any of the higher energy structures contribute to the kinetically controlled jet experiments. Significantly $\left(>5 \mathrm{~cm}^{-1}\right)$ downshifting structure representatives with linear $\mathrm{N}_{\mathrm{H}}$ coordination tend to move higher in relative energy with increasing coordination number. Evident in theoretical predictions is that directed $\mathrm{NH} \cdots \mathrm{N}$ contacts often result in $\mathrm{NH}$ stretching downshifts, while structures with $\mathrm{NH}$ pointing between two $\mathrm{N}$ units tend to show slight upshifts.

\subsection{FTIR Spectra}

Reduced competition between energetically favoured $\mathrm{P}$ homo- and disfavoured $\mathrm{N}$ heteroaggregation requires high dilution of $\mathrm{P}$ in the matrix host or expansion carrier gas, respectively. In cryogenic matrices $0.025 \%$ pyrrole ( $>98 \%$, purified by multiple freezepump-thaw cycles under primary vacuum) in the respective matrix host gases neon ( $\geq 99.999 \%$, Air Liquide), argon ( $\geq 99.9999 \%$, Air Liquide) or nitrogen $(\geq 99.9999 \%$, Air Liquide) were deposited onto the sample carrier. Unless stated otherwise, spectra in cryogenic matrices were recorded at a temperature of $4.7 \mathrm{~K}$. Spectra in supersonic expansion with the filet-jet set-up were recorded with gas mixtures generated by guiding helium (99.996\%, Linde) optionally containing nitrogen (99.996\%, Air Liquide) at a pressure of 1.6 bar through the thermostatted glass saturator containing liquid pyrrole $\left(99.8 \%\right.$, abcr, no further purification) at a temperature of $-20{ }^{\circ} \mathrm{C}$, resulting in estimated P molar fractions of about $0.1 \%$. For NH stretching measurements from $4100-2450 \mathrm{~cm}^{-1}$, the set-up was equipped with the $150 \mathrm{~W}$ tungsten lamp, $\mathrm{CaF}_{2}$ beam splitter and optics as well as the liquid nitrogen cooled $3 \mathrm{~mm}^{2} \mathrm{InSb}$ detector.

\subsubsection{Neon, Argon and Nitrogen Matrices}

The $\mathrm{P}$ monomer gives rise to a broad and highly structured NH stretching band in a neon matrix (Figure 5.3) between 3540 and $3525 \mathrm{~cm}^{-1}$, further highlighting its sensitivity to different lattice environments even for weakly interacting neon. Annealing to $9 \mathrm{~K}$ induces progressive homoaggregation as indicated by the increasing intensity of the PP donor vibration at $3433.8 \mathrm{~cm}^{-1}$. Additionally, the PPP trimer signal emerges at $3386.0 \mathrm{~cm}^{-1}$. The monomer NH stretching band in an argon matrix (Figure 5.4) displays substantial site splitting from 3523.1 to $3519.9 \mathrm{~cm}^{-1}$ with a dominant site at $3521.1 \mathrm{~cm}^{-1}$. Similar to the neon matrix, the PP and PPP signals emerge after annealing to $30 \mathrm{~K}$ with the PP acceptor bands at $3517.9,3514.1,3511.5$ and $3509.1 \mathrm{~cm}^{-1}$ and the PP donor vibrations at $3420.2,3417.6$ and $3416.6 \mathrm{~cm}^{-1}$, respectively. The band at $3378.6 \mathrm{~cm}^{-1}$ was tentatively 


\section{Pyrrole}

assigned to PPPP in reference 315, but spectral trends in supersonic expansions and cryogenic matrices (Table 5.2) complemented by neon matrix band position from this work allow for an assignment to PPP. Even larger monomer site splittings than in argon matrix are observed in a $\mathrm{N}$ matrix (Figure 5.5), ranging from 3519.5 to $3509.1 \mathrm{~cm}^{-1}$ with the dominant site at $3514.2 \mathrm{~cm}^{-1}$. Annealing to $30 \mathrm{~K}$ induces depopulation of unstable sites with only small fractions at 3509.1 and $3519.5 \mathrm{~cm}^{-1}$ remaining. The latter is falsely seen as a sign of an upshifted $\mathrm{NH}$ stretching vibration due to 'improper' $\mathrm{NH} \cdots \mathrm{N}$ hydrogen bonding in the binary PN complex in reference 317. PP shows substantial site splitting as well with acceptor vibrations at 3503.5 and $3501.1 \mathrm{~cm}^{-1}$ and donor signals at 3423.8, 3417.5 and $3415.1 \mathrm{~cm}^{-1}$, respectively. Even the cyclic PPP displays two signals at 3385.0 and $3379.6 \mathrm{~cm}^{-1}$. Accurately modelling the observed site splittings in an annealed $\mathrm{N}$ matrix with neural network potentials ${ }^{64,65}$ could provide an interesting starting point for theoretical descriptions of the bulk $\mathrm{N}$ matrix environment.

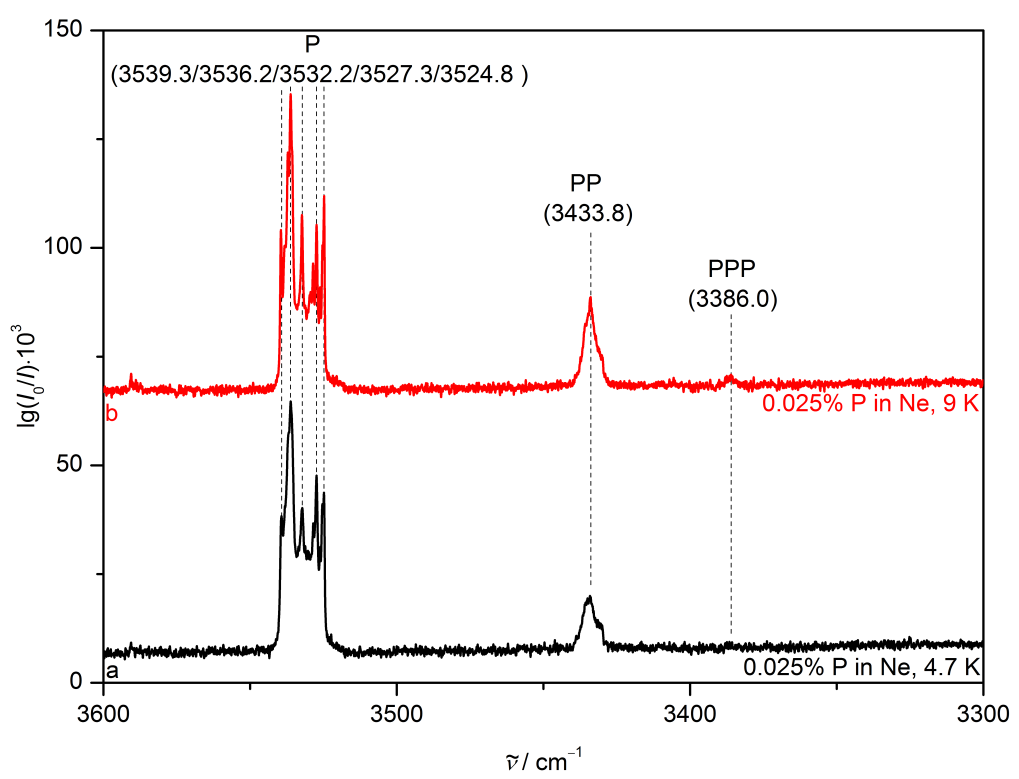

Figure 5.3.: NH stretching FTIR spectra (reprinted from reference 313 licensed under CC BY 3.0) of $0.025 \% \mathrm{P}$ in a neon matrix after deposition at $4.7 \mathrm{~K}$ (trace a) and after annealing to $9 \mathrm{~K}$ and recooling to $4.7 \mathrm{~K}$ (trace $\mathrm{b}$ ). Wavenumbers and assignments are provided.

Careful analysis of gas-to-matrix shifts (Table 5.2) corroborates trends already found with aliphatic alcohols such as an increasing shift when switching from weakly interacting neon to para-hydrogen, the more polarisable argon or quadrupolar $\mathrm{N}$ hosts. These effects are significantly more pronounced for PP than $\mathrm{P}$ due to cooperative effects, especially in the PP donor vibration. The PPP trimer without a free NH group as attractive binding site shows very small gas-to-matrix shifts, uniform across all matrix hosts, further confirming its cyclic structure which is retained even in strongly perturbating matrix environments. 


\section{Pyrrole}

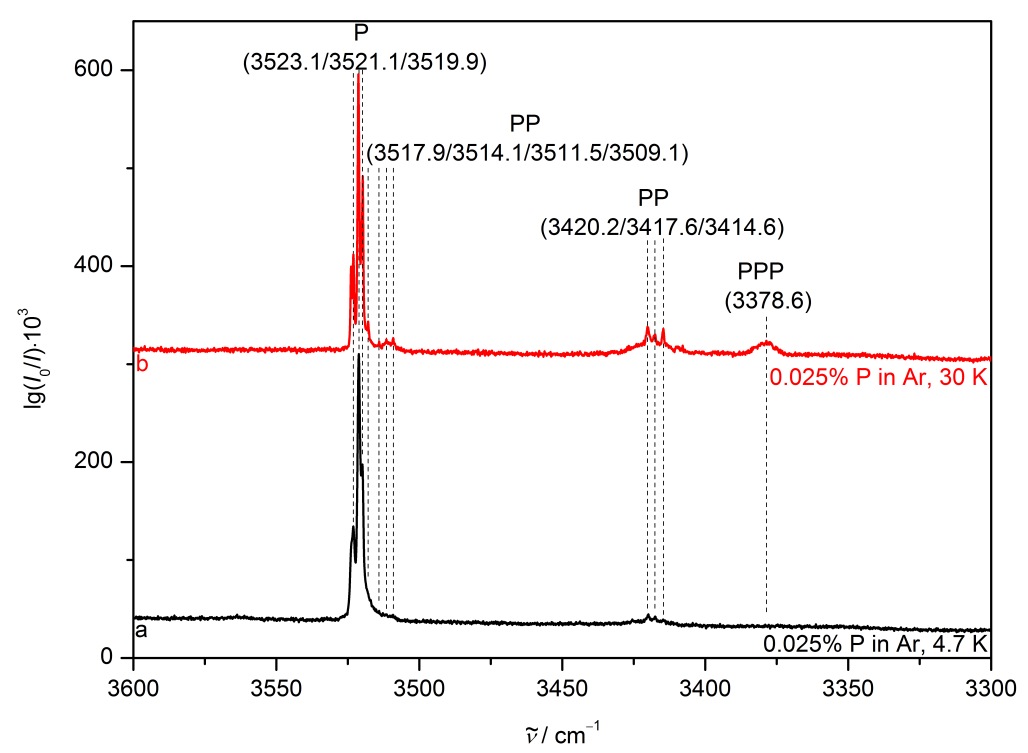

Figure 5.4.: NH stretching FTIR spectra (reprinted from reference 313 licensed under CC BY 3.0) of $0.025 \% \mathrm{P}$ in an argon matrix after deposition at $20 \mathrm{~K}$ and subsequent cooling to $4.7 \mathrm{~K}$ (trace a) as well as after annealing to $30 \mathrm{~K}$ and recooling to $4.7 \mathrm{~K}$ (trace b). Wavenumbers and (tentative) assignments are provided.

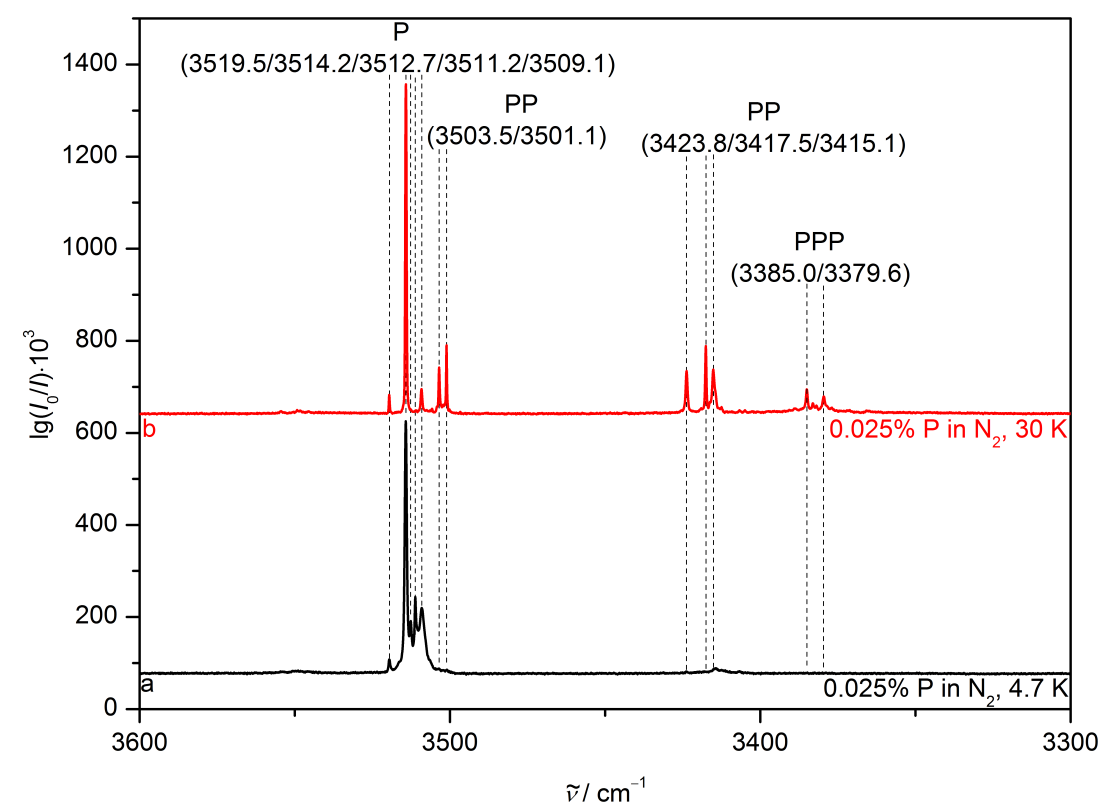

Figure 5.5.: NH stretching FTIR spectra (reprinted from reference 313 licensed under CC BY 3.0) of $0.025 \% \mathrm{P}$ in a N matrix after deposition at $20 \mathrm{~K}$ and subsequent cooling to $4.7 \mathrm{~K}$ (trace a) as well as after annealing to $30 \mathrm{~K}$ and recooling to $4.7 \mathrm{~K}$ (trace b). Wavenumbers and (tentative) assignments are provided. 


\section{Pyrrole}

\subsubsection{Nitrogen Added to Neon and Argon Matrices}

The NH stretching downshift of the $\mathrm{P}$ band position upon switching from neon to $\mathrm{N}$ matrices is incrementally reproduced by adding small amounts of $\mathrm{N}$ to neon matrices (Figure 5.6). With these N-enriched matrices annealing to higher temperatures such as $15 \mathrm{~K}$ is possible for several seconds without immediate matrix evaporation. The high temperature annealing yields new vibrational signals at 3524.9, 3524.0, 3518.6, 3516.2 and $3514.7 \mathrm{~cm}^{-1}$, caused by PN complexes in a neon environment (traces b to e). Assignments to specific calculated structures is somewhat difficult in a bulk matrix environment, although the relatively broad range of observed wavenumbers indicates the abundance of different $\mathrm{N}_{\pi}$ and $\mathrm{N}_{\mathrm{H}}$ clusters with various degrees of $\mathrm{N}$ solvation.

Table 5.2.: NH stretching band centre positions $(\tilde{\nu})$ in $\mathrm{cm}^{-1}$ of the pyrrole monomer $(\mathrm{P})$, dimer (PP) and trimer (PPP) measured in supersonic expansion and different cryomatrices as well as the gas-to-matrix wavenumber downshift induced by matrix isolation $\left(\Delta \tilde{\nu}_{\mathrm{mi}}\right)$ in $\mathrm{cm}^{-1}$. Separate analysis for PP acceptor (upper row) and donor (lower row) vibrations is included.

\begin{tabular}{|c|c|c|c|c|c|c|}
\hline \multirow[t]{2}{*}{ Structure } & \multicolumn{2}{|l|}{$\mathrm{P}$} & \multicolumn{2}{|l|}{$\mathrm{PP}$} & \multicolumn{2}{|c|}{ PPP } \\
\hline & $\tilde{\nu}$ & $\Delta \tilde{\nu}_{\mathrm{mi}}$ & $\tilde{\nu}$ & $\Delta \tilde{\nu}_{\mathrm{mi}}$ & $\tilde{\nu}$ & $\Delta \tilde{\nu}_{\mathrm{mi}}$ \\
\hline \multirow{2}{*}{ Supersonic expansion ${ }^{329}$} & \multirow{2}{*}{3531} & \multirow{2}{*}{ - } & 3524 & - & \multirow{2}{*}{3393} & \multirow{2}{*}{ - } \\
\hline & & & 3444 & - & & \\
\hline Neon & 3540,3525 & $-9,6$ & 3434 & 10 & 3386 & 7 \\
\hline para-Hydrogen ${ }^{316}$ & 3520 & 11 & 3515,3512 & 9,12 & 3385 & 8 \\
\hline \multirow{2}{*}{ Argon $^{315}$} & \multirow[b]{2}{*}{3524,3520} & \multirow[b]{2}{*}{7,11} & \multirow[b]{2}{*}{3418,3409} & \multirow[b]{2}{*}{26,35} & & \\
\hline & & & & & $\begin{array}{c}3396^{315} \\
{[3378]^{329}}\end{array}$ & $\begin{array}{l}-3 \\
15\end{array}$ \\
\hline \multirow{2}{*}{ Nitrogen $^{316,317}$} & \multirow{2}{*}{3520,3509} & \multirow{2}{*}{11,22} & 3504,3501 & 20,23 & \multirow{2}{*}{3385,3380} & \multirow{2}{*}{8,13} \\
\hline & & & 3424,3415 & 20,29 & & \\
\hline
\end{tabular}

The aggregate formation occurs readily upon annealing, since the single components are initially isolated in close proximity to each other in the matrix during deposition and create the complexes after diffusion induced by annealing. Larger $\mathrm{N}$ molar fractions such as $5 \%$ lead to a broad band centred at $3520.2 \mathrm{~cm}^{-1}$, further downshifted to $3515.5 \mathrm{~cm}^{-1}$ after annealing to $9 \mathrm{~K}$ (traces $\mathrm{f}$ and $\mathrm{g}$ ), approaching the dominant band position in pure $\mathrm{N}$ matrix (trace $\mathrm{h}$ ). The inhomogeneous broadening of the band is likely caused by an incomplete and amorphous $\mathrm{N}$ embedding of $\mathrm{P}$ in $\mathrm{N}$ enriched neon matrix grains, which results in a more dispersed distribution of nearly equivalent $\mathrm{NH}$ oscillators. 


\section{Pyrrole}

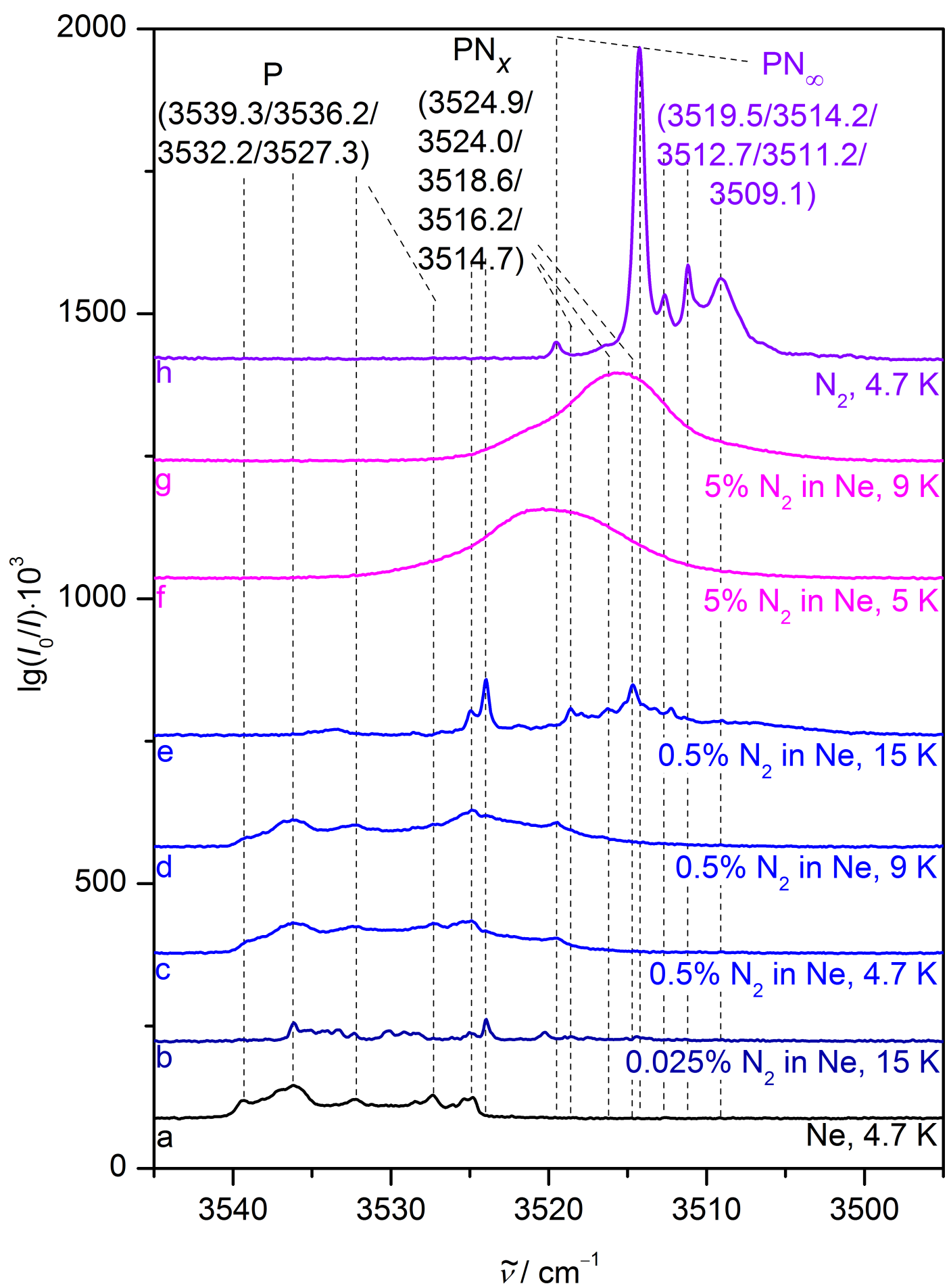

Figure 5.6.: NH stretching FTIR spectra (reprinted from reference 313 licensed under CC BY 3.0) of $0.025 \% \mathrm{P}$ in a neon matrix after deposition at $4.7 \mathrm{~K}$ (trace a). Spectra of $\mathrm{P}$ in neon matrices with increasing $\mathrm{N}$ addition (traces $\mathrm{b}$ to $\mathrm{g}$ ) before and after annealing to the stated temperature and subsequent recooling to $4.7 \mathrm{~K}$ as well as a $\mathrm{N}$ matrix deposited at $20 \mathrm{~K}$ and subsequently cooled to $4.7 \mathrm{~K}$ (trace $\mathrm{h}$ ) are also included. Wavenumbers and (tentative) assignments are provided. 


\section{Pyrrole}

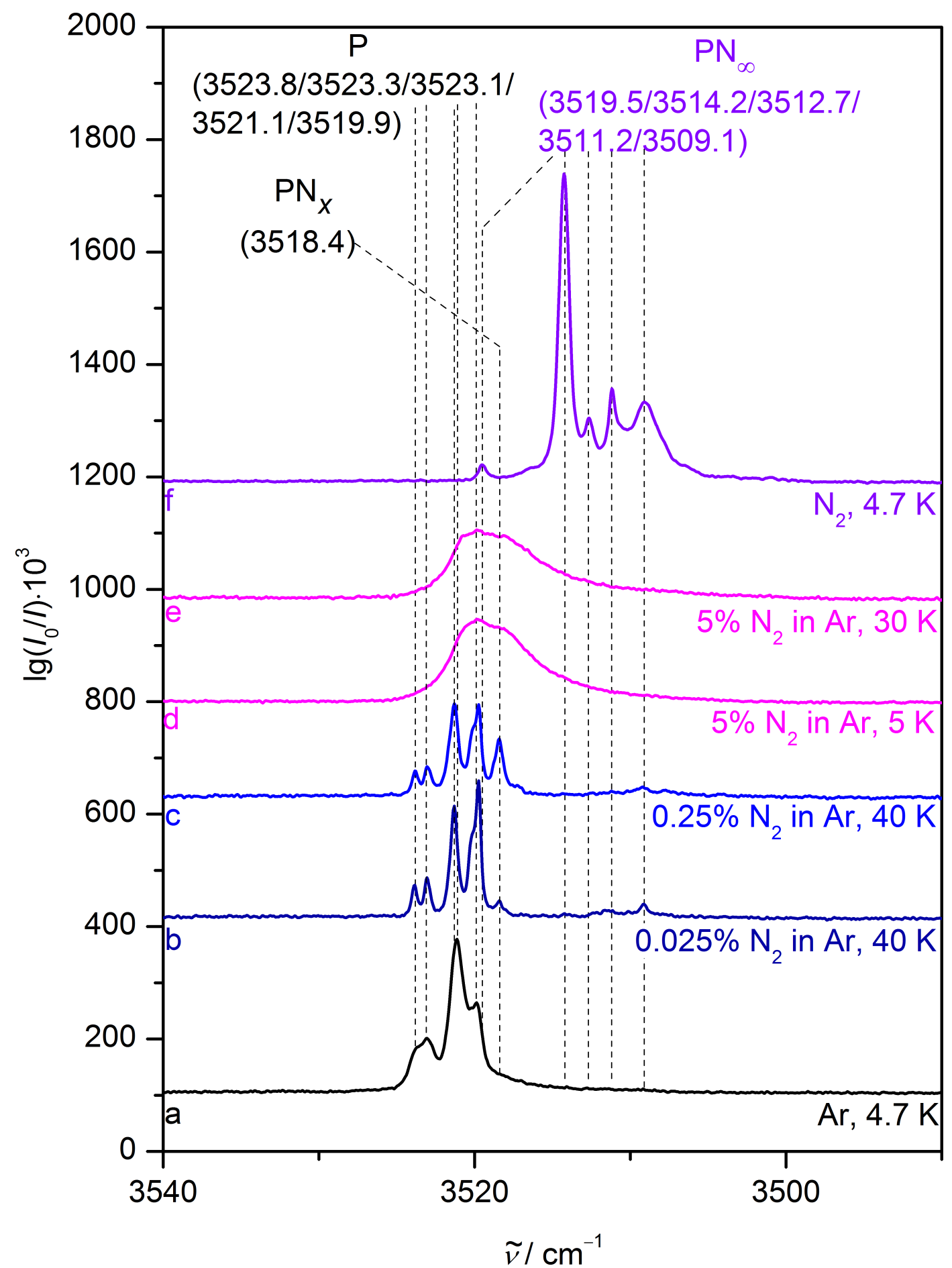

Figure 5.7.: NH stretching FTIR spectra (reprinted from reference 313 licensed under CC BY 3.0) of $0.025 \% \mathrm{P}$ in an argon matrix after deposition at $20 \mathrm{~K}$ and subsequent cooling to $4.7 \mathrm{~K}$ (trace a). Spectra of $\mathrm{P}$ in argon matrices with increasing $\mathrm{N}$ addition (traces $\mathrm{b}$ to e) before and after annealing to the stated temperature and subsequent recooling to $4.7 \mathrm{~K}$ as well as a $\mathrm{N}$ matrix deposited at $20 \mathrm{~K}$ and subsequently cooled to $4.7 \mathrm{~K}$ (trace f) are also included. Wavenumbers and (tentative) assignments are provided. 


\section{Pyrrole}

Observations in argon matrices (Figure 5.7) closely mirror the effects found in neon. New features due to PN complexes appear after addition of $\mathrm{N}$ fractions as small as $0.025 \%$ and annealing to $40 \mathrm{~K}$ at 3518.4 and $3509.1 \mathrm{~cm}^{-1}$ (traces b and c). The broad band after addition of $5 \% \mathrm{~N}$ is again shifted towards the bulk $\mathrm{N}$ band position with an increasing downshift after annealing. Interestingly, the approach towards pure $\mathrm{N}$ is spectrally less complete for argon $\left(3520 \mathrm{~cm}^{-1}\right)$ than for neon, particularly after annealing $\left(3515 \mathrm{~cm}^{-1}\right)$. The softness of the neon matrix allows for a closer arrangement of the $\mathrm{N}$ dopant around $\mathrm{P}$, still sufficiently diluted by the matrix host to prevent sharp spectral features due to long range order. Neon is indeed one of the softest matrices with a crystal force constant ${ }^{336}$ of 3.6 compared to $5.4(\mathrm{~N})$ and $8.2 \mathrm{mdyn}^{-1}$ (argon), respectively.

\subsubsection{Supersonic Expansions}

Free from environmental influences in a helium expansion (Figure 5.8) the $\mathrm{P}$ band centre ${ }^{322,328,329}$ is found at $3531 \mathrm{~cm}^{-1}$ alongside a hot vibrational transition $\left(\mathrm{P}_{\text {hot }}\right)$ at $3507 \mathrm{~cm}^{-1}$, which originates from incomplete vibrational cooling of a low frequency mode, the thermally excited out-of-plane bending mode $\nu_{16}$ at $474.61 \mathrm{~cm}^{-1}{ }^{329}$

A new feature appears at $3527 \mathrm{~cm}^{-1}$ after addition of 2.5 and $5 \% \mathrm{~N}$ (traces c and e), respectively, visible as a positive contribution in the P-corrected difference spectra (traces $\mathrm{b}$ and $\mathrm{d}$ ). The feature grows in intensity with a larger $\mathrm{N}$ addition of $15 \%$ (traces $\mathrm{f}$ and $\mathrm{g}$ ) and a new band at $3518 \mathrm{~cm}^{-1}$ emerges. Similar to the findings in neon and argon matrices, the $\mathrm{P}$ band centre in helium expansion is downshifted towards the bulk $\mathrm{N}$ matrix position (trace $\mathrm{h}$ ) after $\mathrm{N}$ embedding.

\subsection{Summary}

Experimentally observed ranges of the pyrrole monomer $\mathrm{NH}$ stretching band centre position in neon and argon cryomatrices are summarised in Figure 5.9. The total gasto-nitrogen-matrix downshift of $16.6 \mathrm{~cm}^{-1}$ derived from the high resolution gas phase value $^{328}\left(3530.811343(82) \mathrm{cm}^{-1}\right)$ and the dominant nitrogen matrix site at $3514.2 \mathrm{~cm}^{-1}$ is incrementally reproduced by stepwise nitrogen complexation, beginning at the aromatic $\pi$ electron density and the NH proton. Harmonic frequency predictions from density functional theory are able to reproduce this effect qualitatively to a satisfactory degree. The final downshift towards the bulk nitrogen matrix position is observed after embedding of pyrrole in large amounts of nitrogen. Harmonic calculations for these solvation complexes clearly fail to describe the experimental findings by predicting little to no spectral NH stretching downshifts, possibly due to a combination of their finite size, amorphous character and an electronic structure or anharmonicity deficiency. The pyrrole-nitrogen model system provides challenging experimental benchmarks for quantum chemistry, since modelling the packing effects in bulk matrices presumably requires a combination of accurate nitrogen pair ${ }^{333}$ with neural network potentials. ${ }^{64,65}$ Pyrrole homoclusters which display unambiguous and well-defined structures in the gas phase show distinctive site splittings in an annealed nitrogen matrix. These provide 


\section{Pyrrole}

a good starting point for theoretical studies, before attempting a quantitative analysis of the nitrogen complexation downshifts. The reported NH stretching upshift ${ }^{317}$ due to 'improper' $\mathrm{NH} \cdots \mathrm{N}_{2}$ hydrogen bonding is clearly falsified by the multi-experimental approach chosen in this work.

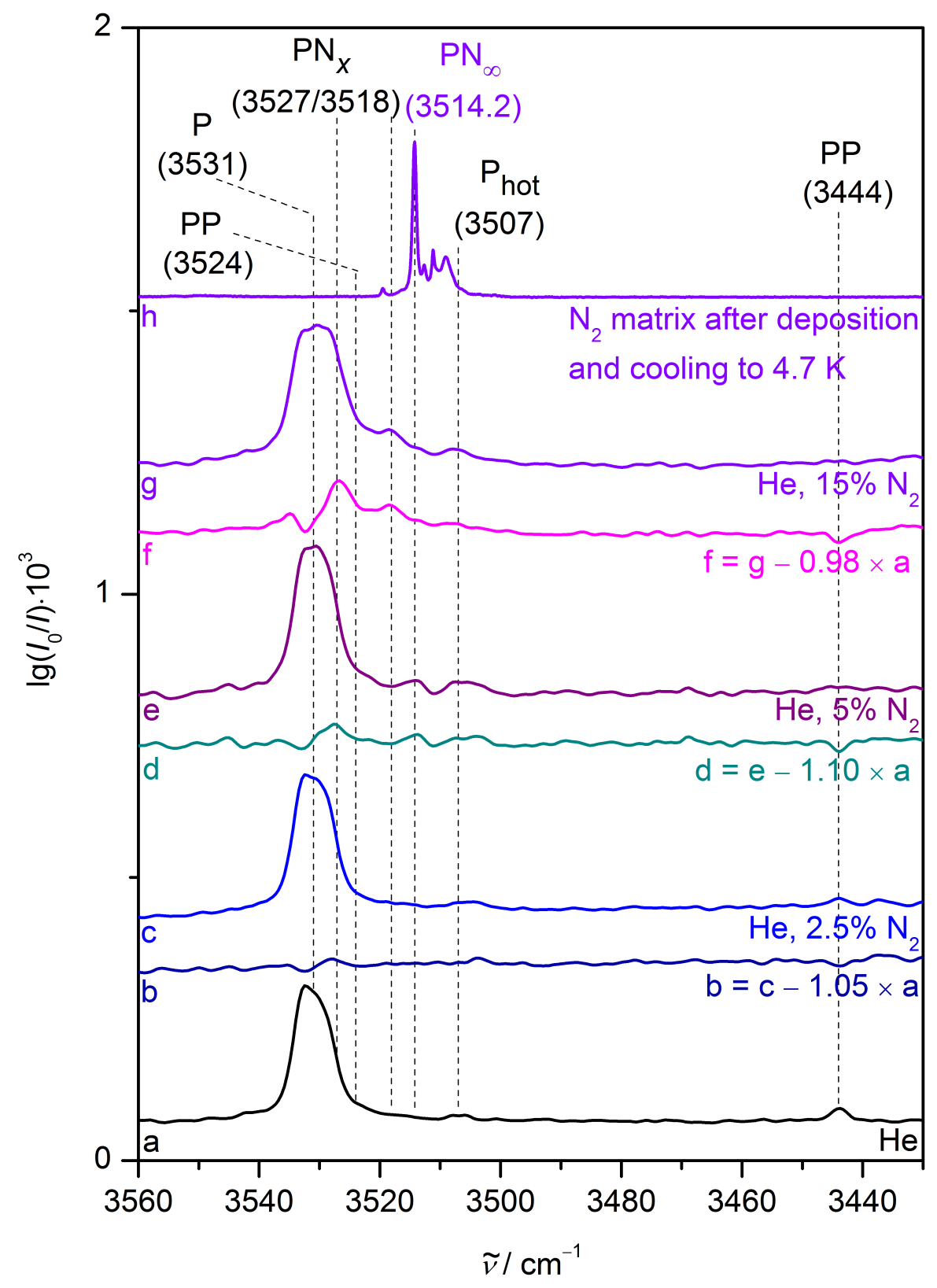

Figure 5.8.: NH stretching FTIR jet spectra (reprinted from reference 313 licensed under CC BY 3.0) of about $0.1 \% \mathrm{P}$ expanded in different mixtures of $\mathrm{N}$ and helium (traces a, c, e and $g$ ) as well as monomer-subtracting difference spectra (traces b, $d$ and f). A spectrum in $\mathrm{N}$ matrix scaled by 0.0005 (trace $\mathrm{h}$ ) is included. Wavenumbers and (tentative) assignments are provided. 


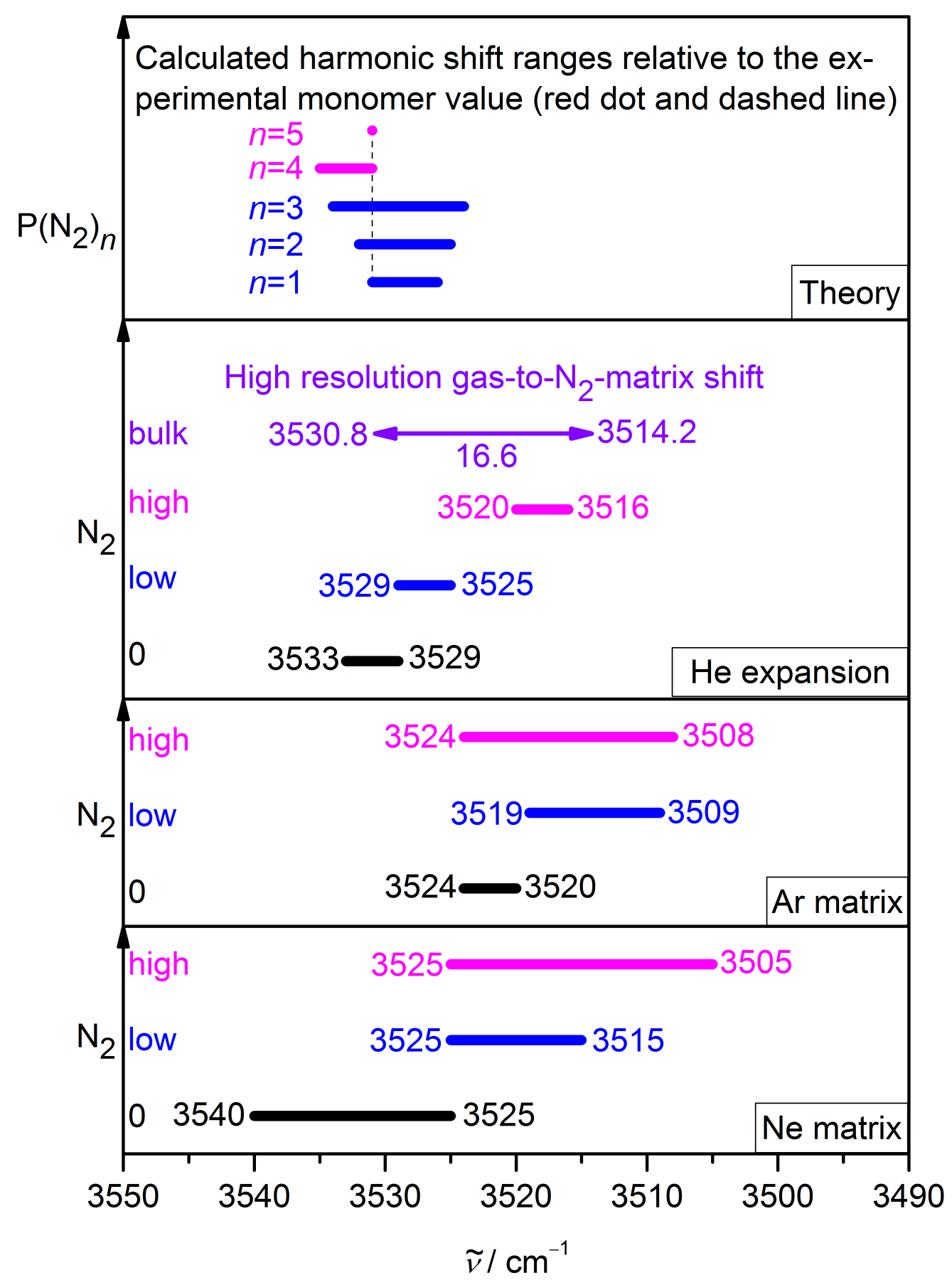

Figure 5.9.: NH stretching band positions of the pyrrole monomer in neon and argon cryomatrices as well as in supersonic helium expansions (reprinted from reference 313 licensed under CC BY 3.0). Increasing $\mathrm{N}$ admixture causes progressive downshifts of the band position in all three environments, approaching the bulk nitrogen matrix limit. High resolution gas phase ${ }^{328}$ and nitrogen matrix spectra allow for an accurate determination of the total gas-to-matrix downshift to $16.6 \mathrm{~cm}^{-1}$. The range of wavenumber shifts predicted for the most stable pyrrole-nitrogen complexes by harmonic B3LYP-D3(BJ)/aVTZ calculations nicely reproduces experimental findings with reasonable accuracy for the first three added $\mathrm{N}$ molecules, but the tested approach fails to describe continuous downshifts in more $\mathrm{N}$-enriched environments and the bulk limit. 



\section{Chloroform}

\section{Chloroform}

The influence of nitrogen aggregation on the $\mathrm{CH}$ and $\mathrm{CCl}$ stretching modes of chloroform was studied in neon, argon and nitrogen cryogenic matrices together with Dr. Stéphane Coussan at Aix-Marseille University as well as in supersonic expansions with filet-jet FTIR spectroscopy. Increasing nitrogen coordination induced progressive spectral upshifts of the $\mathrm{CH}$ and downshifts of the $\mathrm{CCl}$ stretching fundamental. The obtained results have been published in reference 337. The occasional verbatim reproduction of small sections from that reference is not explicitly marked or cited.

\subsection{Introduction}

Similar to pyrrole, stepwise nitrogen decoration in neon and argon matrices as well as helium expansions is performed with chloroform as the hydrogen bond donor. Typically, the formation of XH . . Y hydrogen bonds causes an elongation of the covalent XH bond and spectral downshifts of the corresponding XH stretching vibration. ${ }^{47,338,339}$ However, electron deficient $\mathrm{CH}$ bonds ${ }^{340-342}$ often found in haloforms ${ }^{343,344}$ offer the possibility of 'improper' hydrogen bonding associated with spectral $\mathrm{CH}$ stretching upshifts. ${ }^{345-348} \mathrm{Ag}-$ gregates of haloforms have therefore been extensively studied in cryogenic matrices ${ }^{343,344}$ and the gas phase. ${ }^{349,350}$

Chloroform $\left(\mathrm{CHCl}_{3}\right)$ has been experimentally investigated by infrared, ${ }^{349-355} \mathrm{mi}-$ crowave $^{356}$ and cavity ring-down ${ }^{357}$ spectroscopy as well as vibrationally assisted dissociation and photofragment ionization. ${ }^{358}$ Additional infrared spectroscopic studies were performed in liquid krypton ${ }^{359}$ and argon matrices. ${ }^{344,360}$ Depending on the hydrogen bond acceptor, both spectral downshifts in the binary complex with ammonia ${ }^{350,361}$ and upshifts upon $\mathrm{SO}_{2}$ aggregation ${ }^{349,362}$ have been observed. Similarly, complexation with $\mathrm{N}_{2}, \mathrm{CO}, \mathrm{H}_{2} \mathrm{O}$ and $\mathrm{CH}_{3} \mathrm{CN}$ in argon matrix leads to a seamless transition from up- to downshifts. ${ }^{344}$

In this work, the spectral $\mathrm{CH}$ stretching upshift upon nitrogen complexation of chloroform also reflected in a substantial gas-to-nitrogen matrix upshift is confirmed and difficulties in the theoretical modelling of the weak perturbation leading to the observed spectral effects are highlighted. ${ }^{363,364}$ Additionally the doubly degenerate $E$-symmetric $\mathrm{CCl}$ stretching mode providing high infrared intensity is studied in cryogenic matrices and shows progressive spectral downshifts upon stepwise nitrogen aggregation. 


\section{Chloroform}

\subsection{Quantum Chemical Calculations and Nomenclature}

Molecule abbreviations (C, chloroform, N, nitrogen) are repeated in hydrogen bond donor-acceptor sequence as cluster composition descriptor. Possible docking sites of the acceptor molecule at chloroform such as the $\mathrm{CH}$ proton $(\mathrm{H})$, the carbon $(\mathrm{C})$ or one of the chlorine atoms $(\mathrm{Cl})$ are marked by subscripts at the letter marking the respective acceptor. The most stable aggregates of chloroform and nitrogen are depicted in Figure 6.1, while spectral predictions for the $\mathrm{CH}$ stretching mode are summarised in Table 6.1. Higher energy conformers found during the conformational search are shown in Figures A.28, A.29, A.30, A.31, A.32, A.33 and A.34 with spectral $\mathrm{CH}$ and $\mathrm{CCl}$ predictions listed in Tables A.26 and A.27 in appendix A.6, respectively.
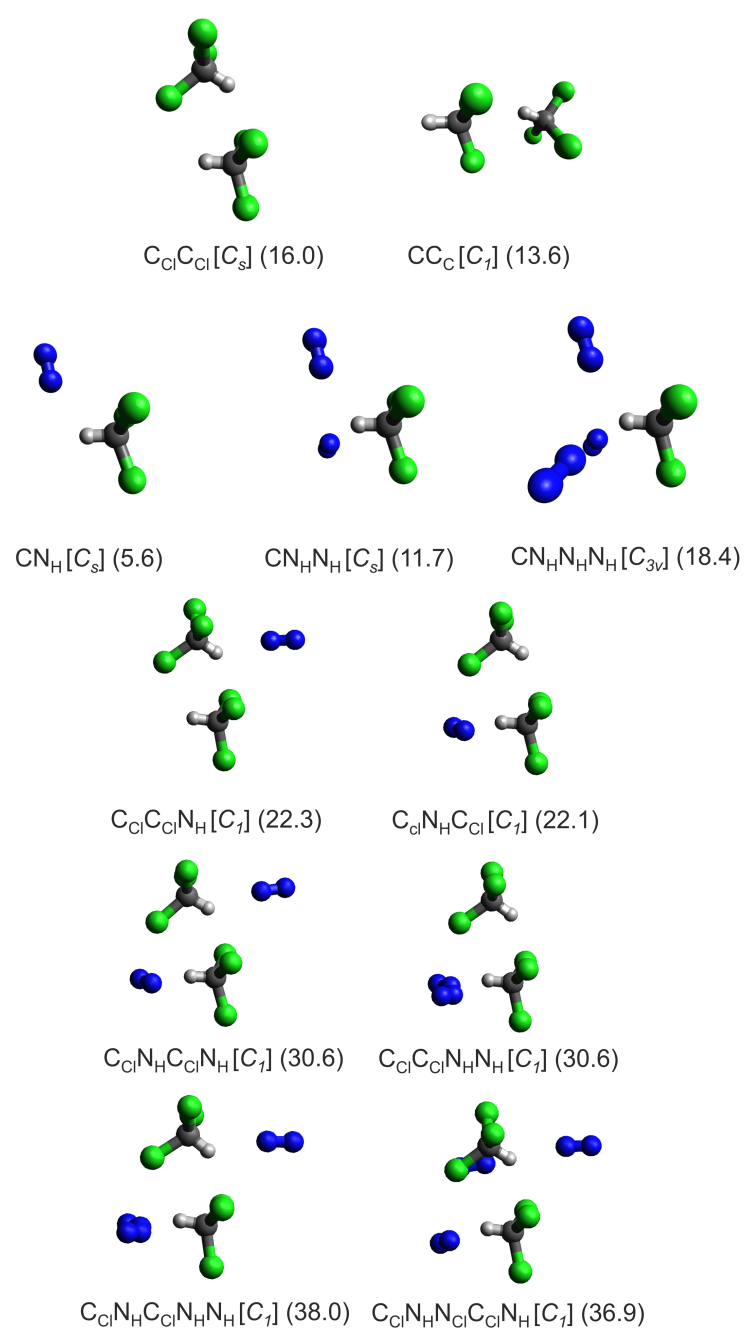

Figure 6.1.: Most stable structures of chloroform homo- and heteroaggregates with nitrogen optimised at the B3LYP-D3(BJ)/def2-QZVP level (reprinted from reference 337). Molecular symmetry point groups are given in brackets, dissociation energies into stable monomers including harmonic vibrational zero-point correction in $\mathrm{kJ} \mathrm{mol}^{-1}$ in parentheses. 


\section{Chloroform}

Table 6.1.: Theoretically predicted harmonic $\left(\omega_{\mathrm{CH}}\right)$ and anharmonic $\left(\tilde{\nu}_{\mathrm{CH}}\right) \mathrm{CH}$ stretching wavenumber in $\mathrm{cm}^{-1}$, IR intensity $(S)$ in $\mathrm{km} \mathrm{mol}^{-1}$, spectroscopic downshifts $\left(\Delta \omega_{\mathrm{CH}}\right.$, $\left.\Delta \tilde{\nu}_{\mathrm{CH}}\right)$ relative to the corresponding vibration in the pure $\mathrm{C}$ monomers or $\mathrm{CC}$ dimers in $\mathrm{cm}^{-1}$, lowest predicted harmonic $\left(\omega_{\mathrm{l}}\right)$ and anharmonic $\left(\tilde{\nu}_{1}\right)$ wavenumbers, dissociation energy into the most stable monomers without $\left(D_{\mathrm{e}}\right)$ and with $\left(D_{0}\right)$ harmonic vibrational zeropoint energy in $\mathrm{kJ} \mathrm{mol}^{-1}$. All properties were calculated at the B3LYP-D3(BJ)/def2QZVP level of theory. Anharmonic vibrational frequencies calculated with second order vibrational perturbation theory (Gaussian09 Rev. E01, ${ }^{141}$ VPT2 ${ }^{148}$ ) are unreliable due to unphysical predictions for low frequency large amplitude motions (indicated by parentheses) which affect localised high frequency modes like the $\mathrm{CH}$ stretching vibration through their respective coupling constants.

\begin{tabular}{|c|c|c|c|c|c|c|c|c|c|c|}
\hline Structure & $\omega_{\mathrm{CH}}$ & $S_{\omega}$ & $\tilde{\nu}_{\mathrm{CH}}$ & $S_{\nu}$ & $\Delta \omega_{\mathrm{CH}}$ & $\Delta \tilde{\nu}_{\mathrm{CH}}$ & $\omega_{1}$ & $\tilde{\nu}_{1}$ & $D_{\mathrm{e}}$ & $D_{0}$ \\
\hline $\mathrm{C}$ & 3169 & 1 & 3030 & 0 & - & - & 258 & 249 & - & - \\
\hline \multirow{2}{*}{$\mathrm{C}_{\mathrm{Cl}} \mathrm{C}_{\mathrm{Cl}}$} & 3183 & 13 & 3039 & 19 & -14 & -9 & \multirow{2}{*}{2} & \multirow{2}{*}{$(-2876)$} & \multirow{2}{*}{17.6} & \multirow{2}{*}{16.0} \\
\hline & 3174 & 5 & 3034 & 4 & -5 & -4 & & & & \\
\hline \multirow{2}{*}{$\mathrm{CC}_{\mathrm{C}}$} & 3185 & 13 & 3044 & 9 & -16 & -14 & \multirow{2}{*}{12} & \multirow{2}{*}{$(-54)$} & \multirow{2}{*}{15.0} & \multirow{2}{*}{13.6} \\
\hline & 3170 & 2 & 3036 & 3 & -1 & -6 & & & & \\
\hline $\mathrm{CN}_{\mathrm{H}}$ & 3185 & 4 & 3038 & 2 & -16 & -8 & 21 & $(38)$ & 7.2 & 5.6 \\
\hline $\mathrm{CN}_{\mathrm{H}} \mathrm{N}_{\mathrm{H}}$ & 3199 & 9 & 3048 & 2 & -30 & -18 & 11 & (633) & 14.8 & 11.7 \\
\hline $\mathrm{CN}_{\mathrm{H}} \mathrm{N}_{\mathrm{H}} \mathrm{N}_{\mathrm{H}}$ & 3216 & 17 & 3078 & $(726540)$ & -47 & -48 & 10 & $(-3326)$ & 22.8 & 18.4 \\
\hline \multirow{2}{*}{$\mathrm{C}_{\mathrm{Cl}} \mathrm{C}_{\mathrm{Cl}} \mathrm{N}_{\mathrm{H}}$} & 3201 & 19 & 3056 & 19 & -18 & -17 & \multirow{2}{*}{10} & \multirow{2}{*}{ (138) } & \multirow{2}{*}{26.6} & \multirow{2}{*}{23.3} \\
\hline & 3174 & 5 & 3036 & 5 & 0 & -2 & & & & \\
\hline \multirow{2}{*}{$\mathrm{C}_{\mathrm{Cl}} \mathrm{N}_{\mathrm{H}} \mathrm{C}_{\mathrm{Cl}}$} & 3189 & 9 & 3045 & 9 & -6 & -11 & \multirow{2}{*}{9} & \multirow{2}{*}{$(-199)$} & \multirow{2}{*}{26.3} & \multirow{2}{*}{23.1} \\
\hline & 3180 & 13 & 3043 & 11 & -6 & -9 & & & & \\
\hline \multirow{2}{*}{$\mathrm{C}_{\mathrm{Cl}} \mathrm{N}_{\mathrm{H}} \mathrm{C}_{\mathrm{Cl}} \mathrm{N}_{\mathrm{H}}$} & 3199 & 18 & - & - & -16 & - & \multirow{2}{*}{9} & \multirow{2}{*}{-} & \multirow{2}{*}{35.4} & \multirow{2}{*}{30.6} \\
\hline & 3189 & 10 & - & - & -15 & - & & & & \\
\hline \multirow{2}{*}{$\mathrm{C}_{\mathrm{Cl}} \mathrm{C}_{\mathrm{Cl}} \mathrm{N}_{\mathrm{H}} \mathrm{N}_{\mathrm{H}}$} & 3206 & 16 & - & - & -23 & - & \multirow{2}{*}{4} & \multirow[b]{2}{*}{ - } & \multirow{2}{*}{35.3} & \multirow{2}{*}{30.6} \\
\hline & 3182 & 13 & - & - & -8 & - & & & & \\
\hline \multirow{2}{*}{$\mathrm{C}_{\mathrm{Cl}} \mathrm{N}_{\mathrm{H}} \mathrm{C}_{\mathrm{Cl}} \mathrm{N}_{\mathrm{H}} \mathrm{N}_{\mathrm{H}}$} & 3206 & 17 & - & - & -23 & - & \multirow{2}{*}{7} & \multirow{2}{*}{ - } & 444 & 380 \\
\hline & 3200 & 18 & - & - & -26 & - & & & 44.4 & 30.0 \\
\hline 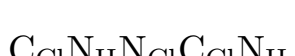 & 3198 & 18 & - & - & -15 & - & 11 & & 31 & 60 \\
\hline $\mathrm{Cl}_{\mathrm{Cl}} \mathrm{N}_{\mathrm{H}} \mathrm{N}_{\mathrm{Cl}} \mathrm{N}_{\mathrm{H}}$ & 3189 & 10 & - & - & -15 & - & 11 & - & 43.1 & 30.9 \\
\hline
\end{tabular}

The most stable $\mathrm{C}_{\mathrm{Cl}} \mathrm{C}_{\mathrm{Cl}}$ homodimer predicted at the B3LYP-D3(BJ)/def2-QZVP level with a double $\mathrm{CH} \cdots \mathrm{Cl}$ hydrogen bond differs from the $\mathrm{CC}_{\mathrm{C}}$ minimum energy structure predicted at the MP2/aVQZ level, ${ }^{364}$ while the most stable mixed complexes with nitrogen feature a progressive coordination of the chloroform $\mathrm{CH}$. Correspondingly, increasing 


\section{Chloroform}

spectral upshifts and significant infrared intensity enhancements for progressing nitrogen solvation are predicted. Shift predictions for the doubly degenerate $\mathrm{CCl}$ stretching mode are somewhat ambiguous with small shifts in both directions. Similar to other hydrogen bond donors studied in this work, dissociation energies for homoaggregates are significantly higher than for heteroaggregates with nitrogen. Therefore, competition between homo- and heteroaggregation should be avoided experimentally by employing low chloroform concentrations in both cryomatrices and supersonic expansions.

Anharmonic frequencies obtained by second order vibrational perturbation theory (VPT2) show the previously noted difficulties occuring for low frequency large amplitude motions and resulting unphysical predictions affect the localised high frequency modes through computed coupling constants.

\subsection{FTIR Spectra}

Highly diluted mixtures of chloroform (between 0.1 and $0.2 \%$ ) in the respective matrix host or expansion carrier gas were used for all experiments to reduce competition between homo- and heterocluster formation. With broadband FTIR spectroscopy from 5000 to $600 \mathrm{~cm}^{-1}$, both the $\mathrm{CH}$ and $\mathrm{CCl}$ stretching modes in cryogenic matrices were recorded simultaneously, while the filet-jet set-up used for the measurements in supersonic expansions was equipped with the $150 \mathrm{~W}$ tungsten lamp, $\mathrm{CaF}_{2}$ beam splitter and optics as well as the liquid nitrogen cooled $3 \mathrm{~mm}^{2} \mathrm{InSb}$ detector, limiting the spectral range to $4100-2450 \mathrm{~cm}^{-1}$ and allowing only for the observation of $\mathrm{CH}$ stretching signals.

\subsubsection{Neon, Argon and Nitrogen Matrices}

Typically, sample mixtures for matrix deposition contained $0.13 \%$ chloroform $(99.9 \%$, stabilised with 0.6-1\% ethanol, Carlo Erba) purified from solved gases by multiple freezepump-thaw cycles and mixed with neon ( $\geq 99.999 \%$, Air Liquide), argon $(\geq 99.9999 \%$, Air Liquide) or nitrogen ( $\geq 99.9999 \%$, Air Liquide). Unless stated otherwise, spectra were recorded at a sample carrier temperature of $4.7 \mathrm{~K}$.

\section{CH Stretching Vibration $\nu_{1}$}

In neon matrix (Figure 6.2) a weak CH stretching signal peaking at $3039.8 \mathrm{~cm}^{-1}$ is observed. After annealing, the band gains intensity and broadens to higher wavenumbers, indicative of $\mathrm{C}$ homocluster formation and corresponding upshifted $\mathrm{CH}$ stretching modes with increased infrared intensities. Assignment of a second band at $3017.5 \mathrm{~cm}^{-1}$ is difficult, as several sources are possible, such as the second $\mathrm{CH}$ stretching mode of the most stable $\mathrm{C}_{\mathrm{Cl}} \mathrm{C}_{\mathrm{Cl}}$ dimer, a different dimer conformation like $\mathrm{CC}_{\mathrm{C}}$ or even a $\mathrm{CCC}$ trimer. Difficulties in theoretical modelling are highlighted by wrongly predicted shift magnitudes for $\mathrm{C}$ homoaggregates and have previously been observed for weakly perturbated $\mathrm{CH}$ bonds. ${ }^{363}$ 


\section{Chloroform}

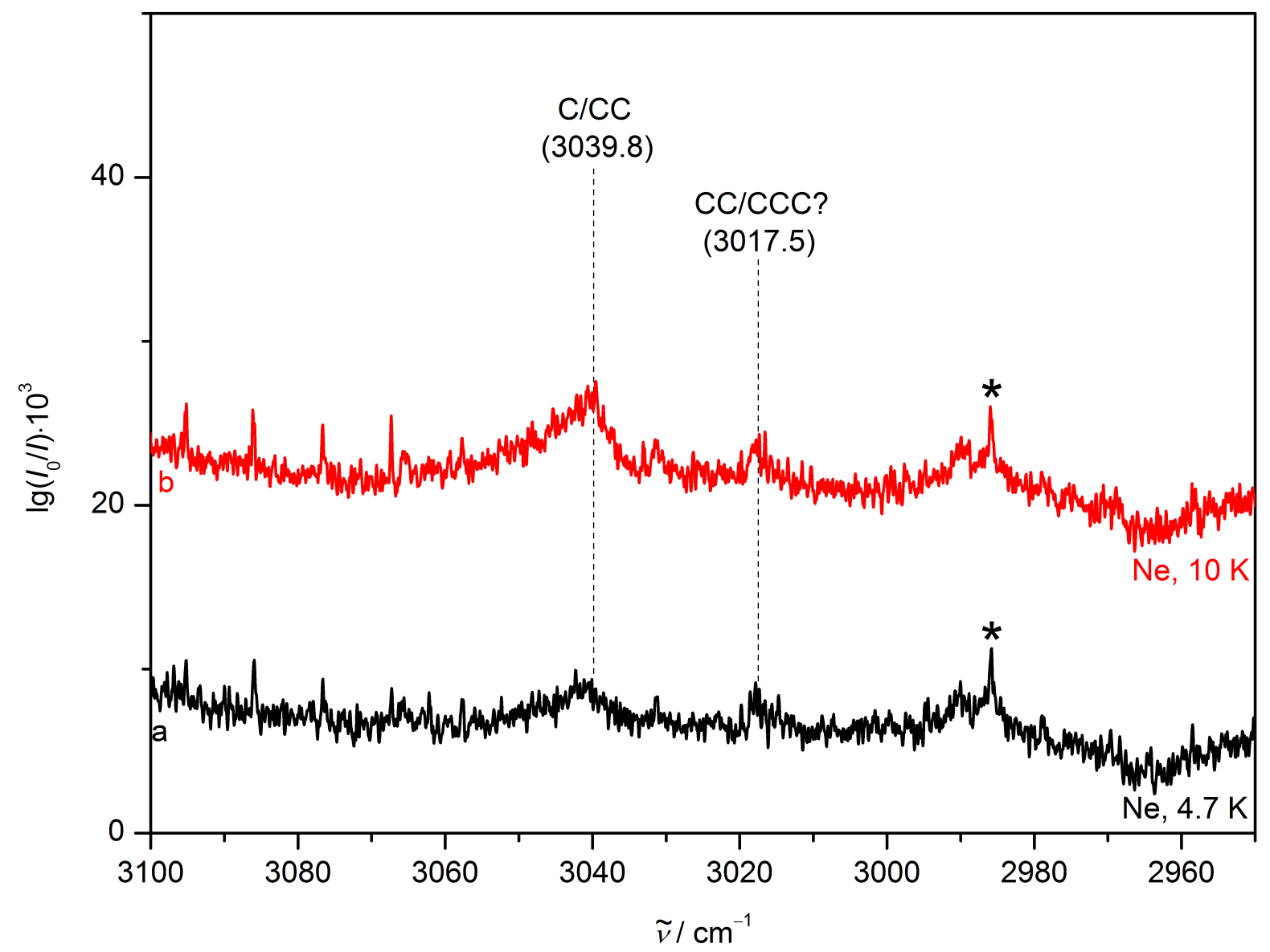

Figure 6.2.: CH stretching FTIR spectra (reprinted with adaptations from reference 337) of $0.13 \% \mathrm{C}$ in neon matrix after deposition at $4.7 \mathrm{~K}$ (trace a) and after annealing to $10 \mathrm{~K}$ and recooling to $4.7 \mathrm{~K}$ (trace $\mathrm{b}$ ). Wavenumbers and (tentative) assignments are provided. Vibrational signals assigned to the ethanol stabiliser are marked by asterisks.

The monomer band centre in argon matrix at $3054.1 \mathrm{~cm}^{-1}$ (Figure 6.3) is in excellent agreement with a previous study at lower spectral resolution. ${ }^{344}$ Annealing results in two downshifted new signals at 3052.1 and $3038.9 \mathrm{~cm}^{-1}$, respectively, caused by the $\mathrm{CC}$ dimer. The direction of the wavenumber shift from monomer to dimer is therefore reversed when comparing neon and argon matrices.

Significant site splitting is observed in N matrix (Figure 6.4) with the dominant site at $3066.6 \mathrm{~cm}^{-1}$. The unstable sites at at 3053.4 and $3039.2 \mathrm{~cm}^{-1}$, respectively, are depopulated through annealing and one of the two expected dimer signals appears at $3030.0 \mathrm{~cm}^{-1}$. Similar to the argon case, a downshift from C to CC is observed in N matrix. With the high resolution gas phase band centre position ${ }^{355}$ of $3032.92642(25) \mathrm{cm}^{-1}$ gas-to-matrix shifts can be determined for $\mathrm{N}\left(33.7 \mathrm{~cm}^{-1}\right)$, argon $\left(21.1 \mathrm{~cm}^{-1}\right)$ and neon $\left(6.9 \mathrm{~cm}^{-1}\right)$. 


\section{Chloroform}

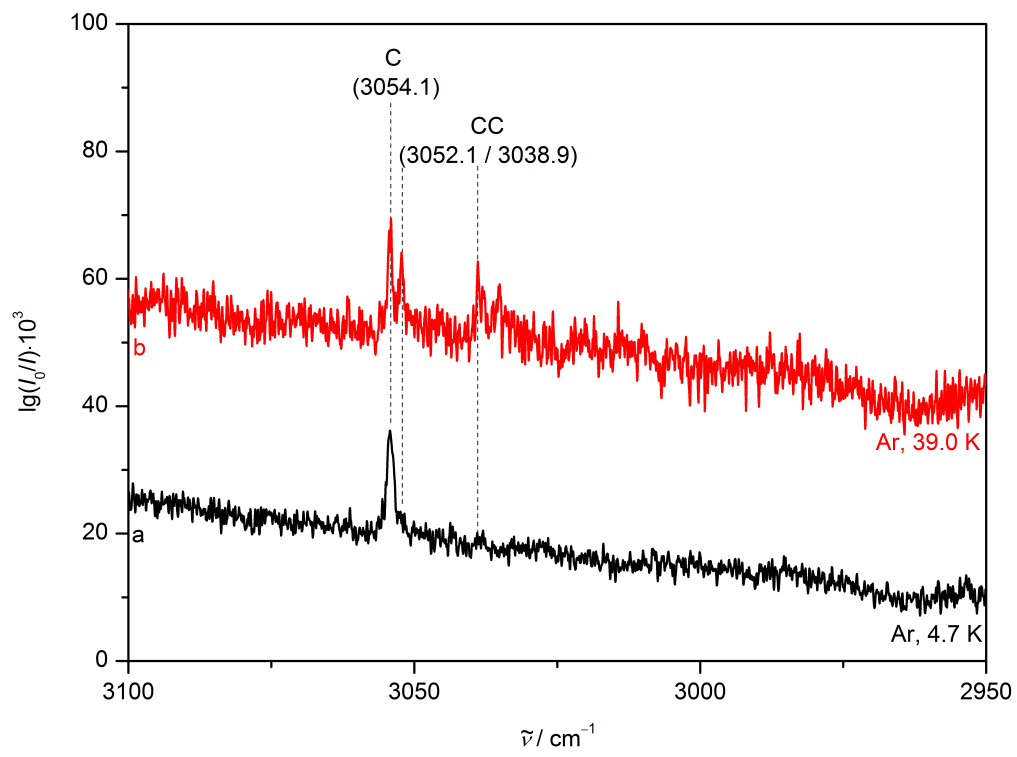

Figure 6.3.: CH stretching FTIR spectra (reprinted with adaptations from reference 337 ) of $0.13 \% \mathrm{C}$ in argon matrix after deposition at $20 \mathrm{~K}$ and subsequent cooling to $4.7 \mathrm{~K}$ (trace a) and after annealing to $39 \mathrm{~K}$ and recooling to $4.7 \mathrm{~K}$ (trace b). Wavenumbers and (tentative) assignments are provided.

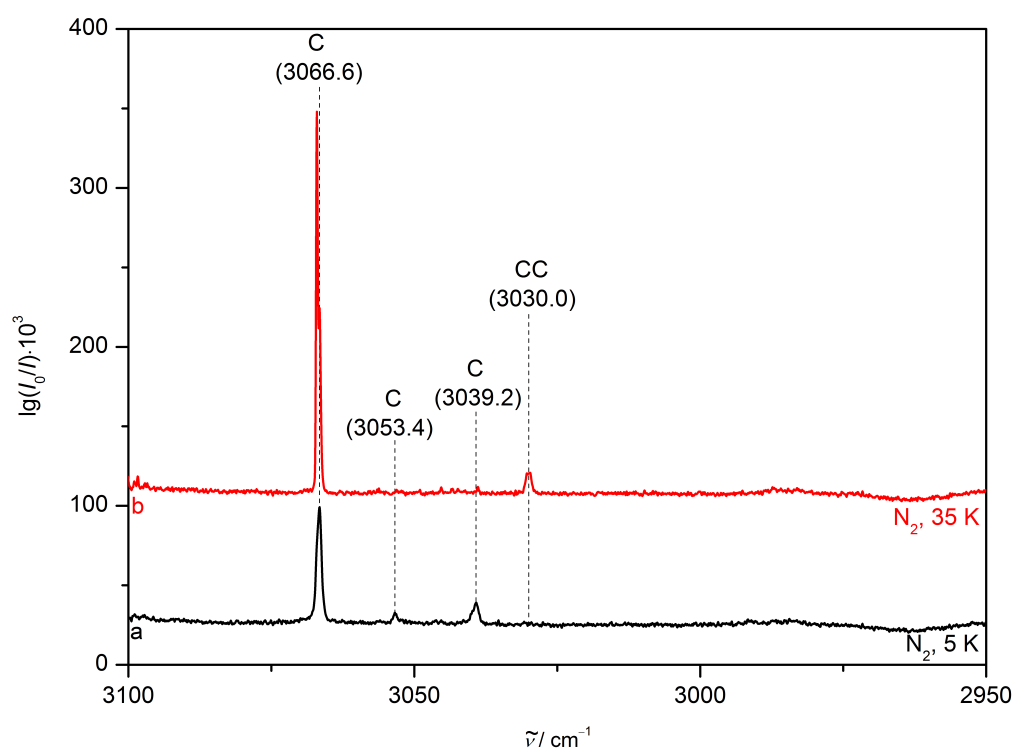

Figure 6.4.: CH stretching FTIR spectra (reprinted with adaptations from reference 337) of $0.13 \% \mathrm{C}$ in $\mathrm{N}$ matrix after deposition at $20 \mathrm{~K}$ and subsequent cooling to $5 \mathrm{~K}$ (trace a) and after annealing to $35 \mathrm{~K}$ and recooling to $5 \mathrm{~K}$ (trace b). Wavenumbers and (tentative) assignments are provided. 


\section{Chloroform}

$\mathrm{CCI}$ Stretching Vibration $\nu_{5}$

The $\mathrm{CCl}$ stretching band in neon matrix (Figure 6.5) shows a broad and highly structured spectrum from 776.4 to $768.4 \mathrm{~cm}^{-1}$. After annealing a weak signal at $765.3 \mathrm{~cm}^{-1}$ assigned to the $\mathrm{CC}$ dimer emerges. The corresponding $\mathrm{CCl}$ stretching band in argon matrix (Figure 6.6) is similarly broad, ranging from 769.9 to $763.7 \mathrm{~cm}^{-1}$. CC dimer signals arise after annealing between 761.9 and $756.0 \mathrm{~cm}^{-1}$, downshifted from the monomer bands. In $\mathrm{N}$ matrix (Figure 6.7) the band ranges from 770.2 to $761.6 \mathrm{~cm}^{-1}$ and annealing results in relative intensity changes of some peaks within the highly structured signal. Since no new features with wavenumber shifts directly assigned to the $\mathrm{CC}$ dimer are observed, these changes likely reflect both dimer formation and depopulation of unstable sites.

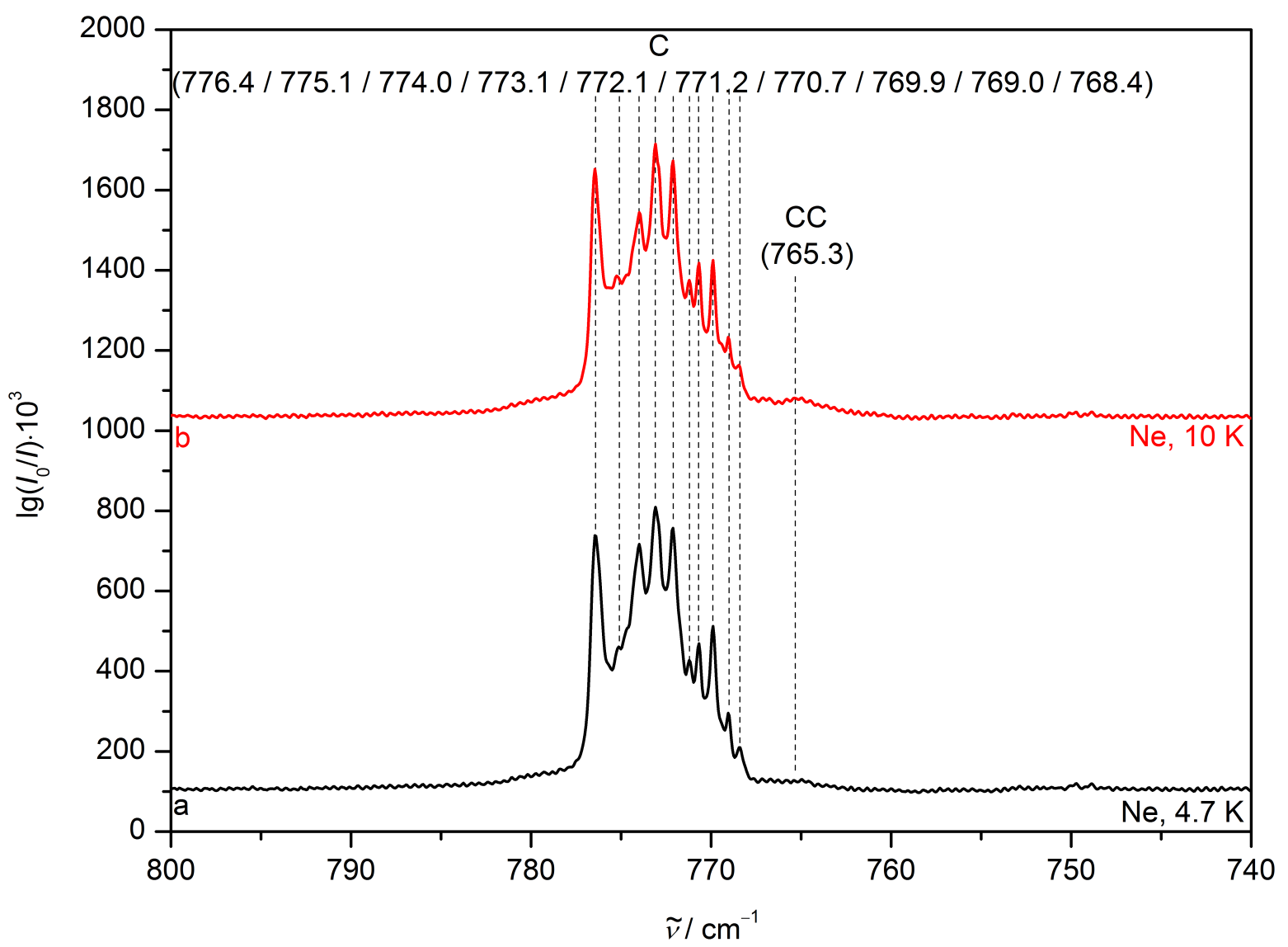

Figure 6.5.: $\mathrm{CCl}$ stretching FTIR spectra (reprinted with adaptations from reference 337 ) of $0.13 \% \mathrm{C}$ in neon matrix after deposition at $4.7 \mathrm{~K}$ (trace a) and after annealing to $10 \mathrm{~K}$ and recooling to $4.7 \mathrm{~K}$ (trace $\mathrm{b}$ ). Wavenumbers and (tentative) assignments are provided. Vibrational signals assigned to the ethanol stabiliser are marked by asterisks. 


\section{Chloroform}

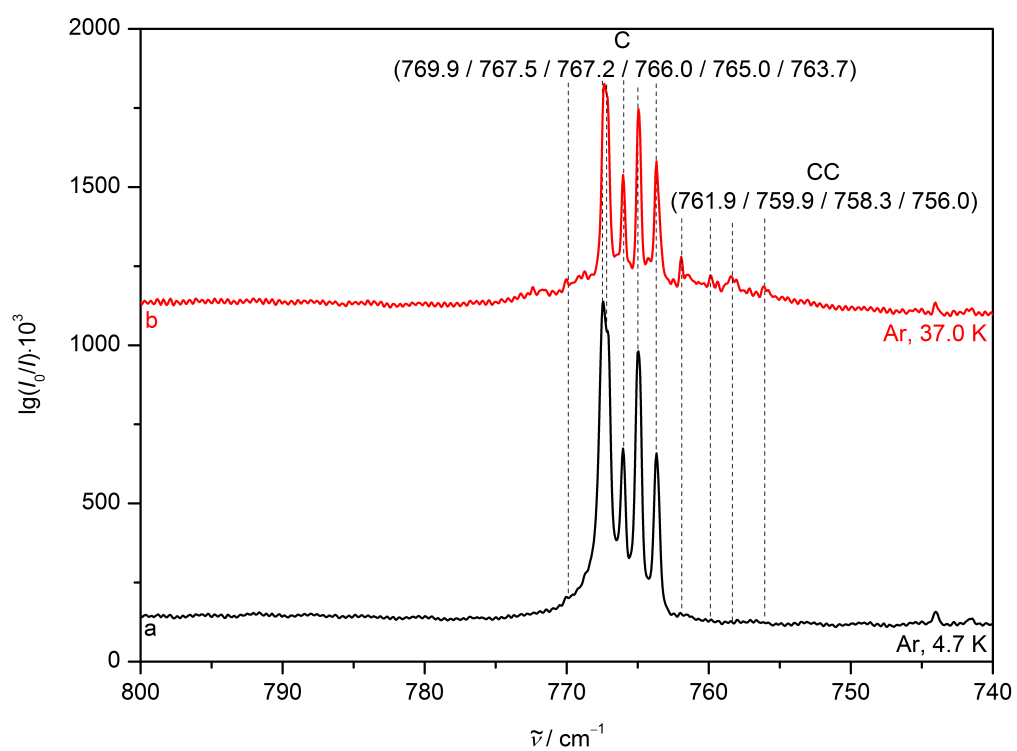

Figure 6.6.: CCl stretching FTIR spectra (reprinted with adaptations from reference 337 ) of $0.13 \% \mathrm{C}$ in argon matrix after deposition at $20 \mathrm{~K}$ and subsequent cooling to $4.7 \mathrm{~K}$ (trace a) and after annealing to $39 \mathrm{~K}$ and recooling to $4.7 \mathrm{~K}$ (trace b). Wavenumbers and (tentative) assignments are provided.

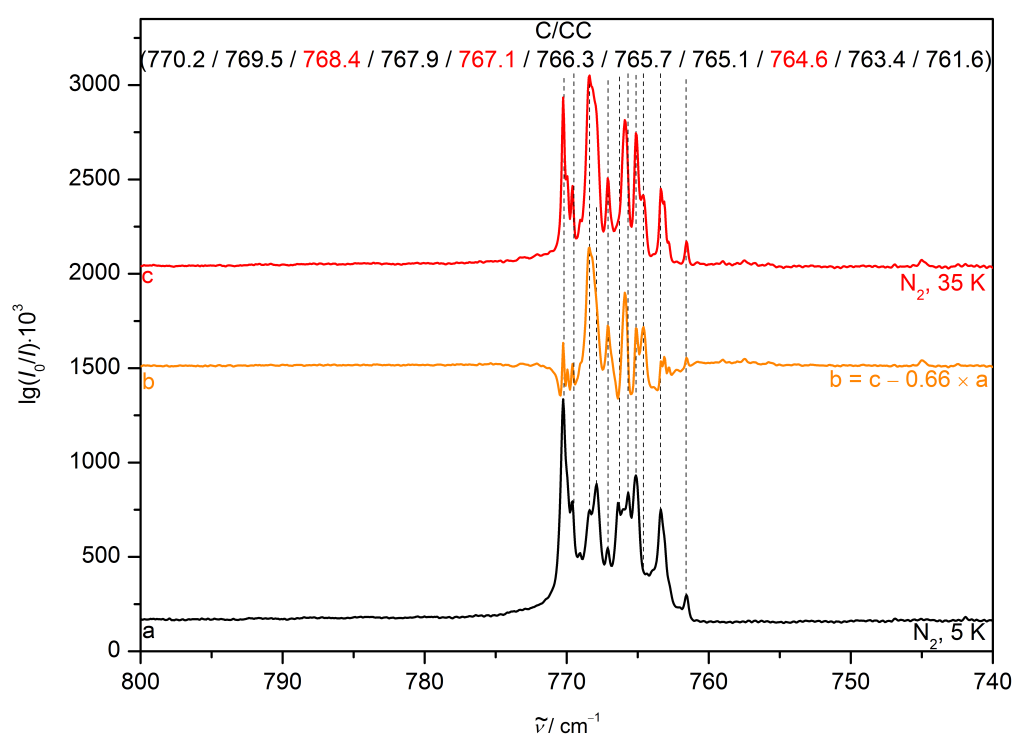

Figure 6.7.: $\mathrm{CCl}$ stretching FTIR spectra (reprinted with adaptations from reference 337 ) of $0.13 \% \mathrm{C}$ in $\mathrm{N}$ matrix after deposition at $20 \mathrm{~K}$ and subsequent cooling to $5 \mathrm{~K}$ (trace a) and after annealing to $35 \mathrm{~K}$ and recooling to $5 \mathrm{~K}$ (trace c). A difference spectrum is included. Wavenumbers and (tentative) assignments are provided. Vibrational bands which gain relative intensity after annealing are marked in red font. 


\section{Chloroform}

The intensity ratio of the $\mathrm{CH}$ and $\mathrm{CCl}$ stretching bands varies significantly when switching between different matrix hosts (Figure 6.8). While the $\mathrm{CCl}$ intensity $\left(S_{\mathrm{CCl}}\right)$ is relatively unaffected by intermolecular interactions (Table A.27), the $\mathrm{CH}$ stretching mode gains intensity $\left(S_{\mathrm{CH}}\right)$ when switching from neon to argon and the strongly interacting N. This effect is typically observed for vibrational modes which are highly sensitive to intermolecular interactions such as $\mathrm{OH}$ stretching vibrations. ${ }^{62,68}$ The roughly 4 -fold intensity enhancement observed in $\mathrm{C}$ upon switching from neon to $\mathrm{N}$ matrices is rather similar to the 3-fold increase of pyrrole NH stretching infrared band strength.

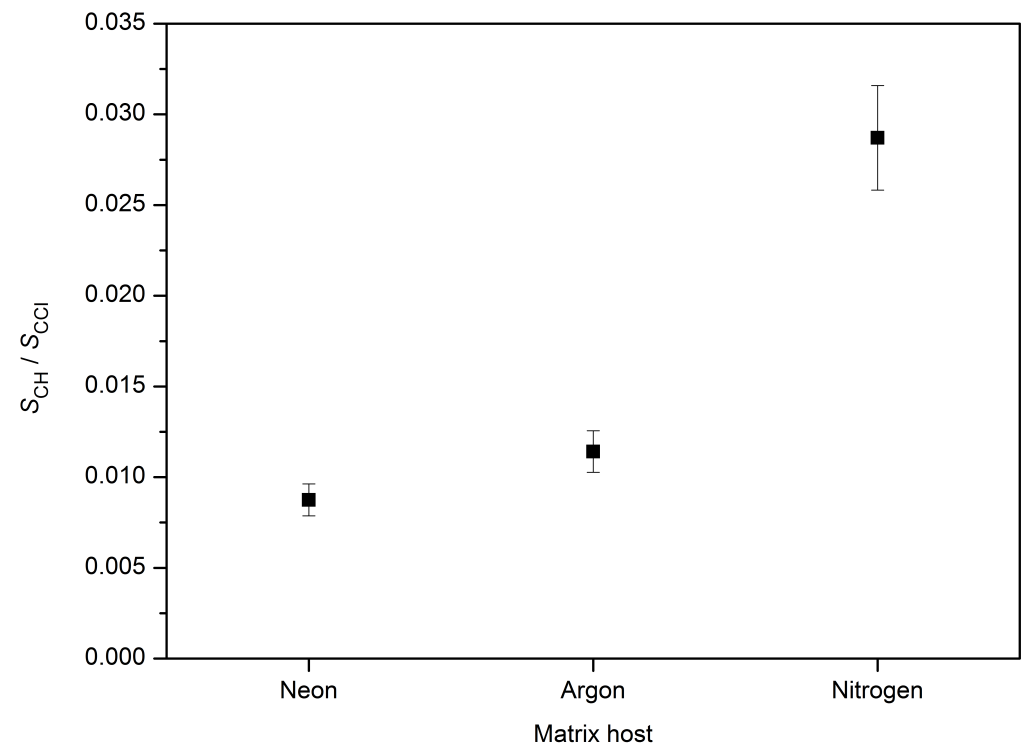

Figure 6.8.: Ratio of experimental $\mathrm{CH}\left(S_{\mathrm{CH}}\right)$ and $\mathrm{CCl}\left(S_{\mathrm{CCl}}\right)$ band integrals depending on the matrix host. Relative integral errors of $1 \%(\mathrm{CCl})$ and $10 \%(\mathrm{CH})$, respectively, were estimated based on the signal-to-noise ratio. While $S_{\mathrm{CCl}}$ is relatively independent of the matrix host, $S_{\mathrm{CH}}$ increases significantly from neon over argon to the strongly interacting $\mathrm{N}$.

\subsubsection{Nitrogen Added to Neon and Argon Matrices}

In analogy to previous experiments with pyrrole, increasing $\mathrm{N}$ fractions were added to neon and argon matrices to induce stepwise $\mathrm{N}$ coordination of $\mathrm{C}$.

\section{CH Stretching Vibration $\nu_{1}$}

In neon matrix (Figure 6.9) the $\mathrm{CH}$ stretching signal is broadened, incrementally upshifted towards the dominant $\mathrm{N}$ matrix band position at $3066.6 \mathrm{~cm}^{-1}$ and gains intensity through stepwise $\mathrm{N}$ addition. All of the described effects are enhanced by annealing. The observations are very similar to the NH stretching mode of pyrrole, again indicating an incomplete and amorphous $\mathrm{C}$ embedding in $\mathrm{N}$ enriched neon matrix grains. The result- 


\section{Chloroform}

ing more dispersed distribution of nearly equivalent $\mathrm{CH}$ oscillators leads to the observed band broadening.

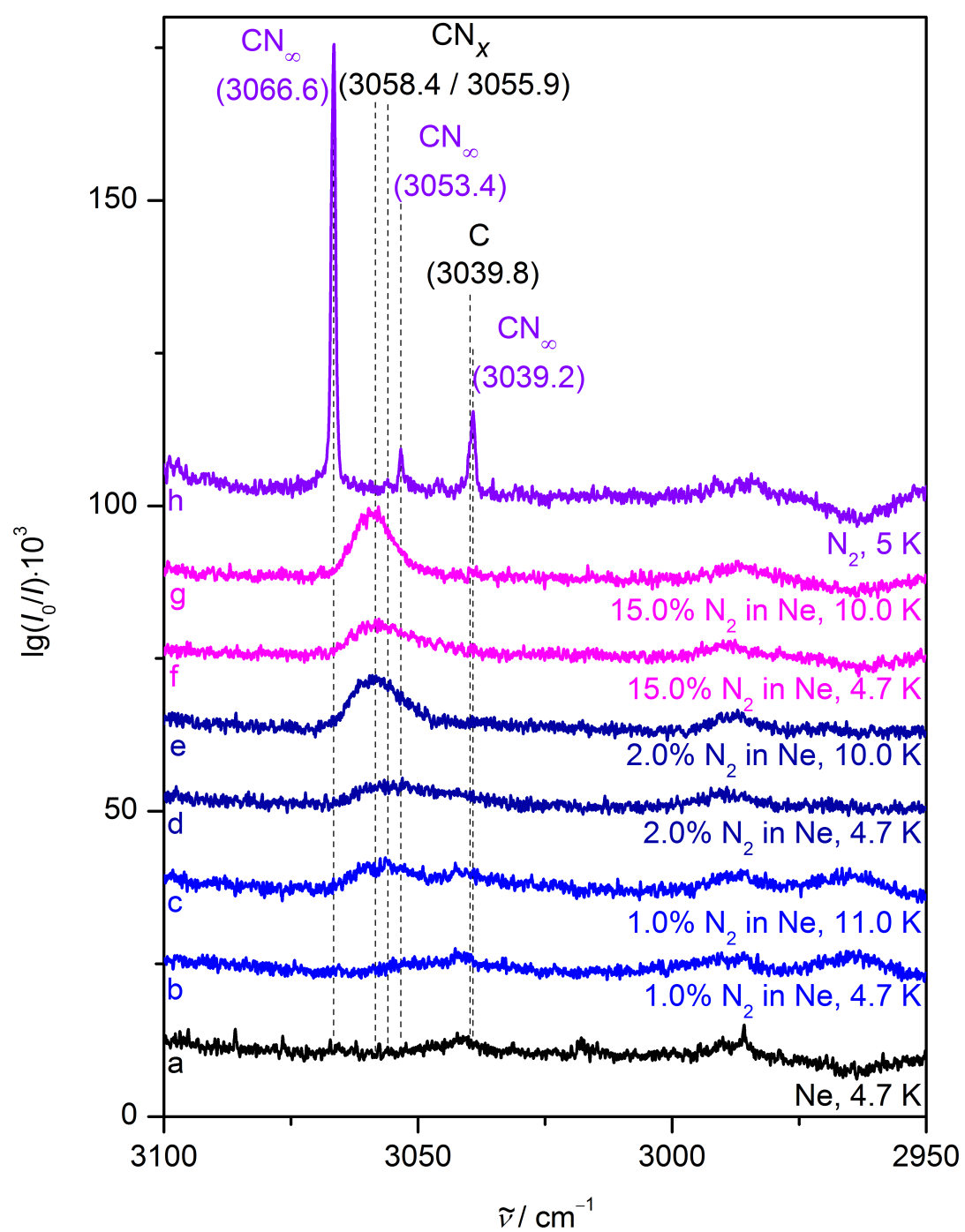

Figure 6.9.: CH stretching FTIR spectra (reprinted with adaptations from reference 337) of $0.13 \% \mathrm{C}$ in neon matrix after deposition at $4.7 \mathrm{~K}$ (trace a). Spectra of neon matrices with increasing $\mathrm{N}$ addition (traces $\mathrm{b}$ to $\mathrm{g}$ ) before and after annealing to the stated temperature and subsequent recooling to $5 \mathrm{~K}$ as well as a $\mathrm{N}$ matrix deposited at $20 \mathrm{~K}$ and subsequently cooled to $4.7 \mathrm{~K}$ (trace $\mathrm{h}$ ) are also included. Wavenumbers and (tentative) assignments are provided.

Very similar observations are made in argon matrix (Figure 6.10). The $\mathrm{N}$ induced upshift of $8.2 \mathrm{~cm}^{-1}$ is in reasonable agreement with the $11 \mathrm{~cm}^{-1}$ upshift found by a previous study at lower spectral resolution. ${ }^{344}$ 


\section{Chloroform}

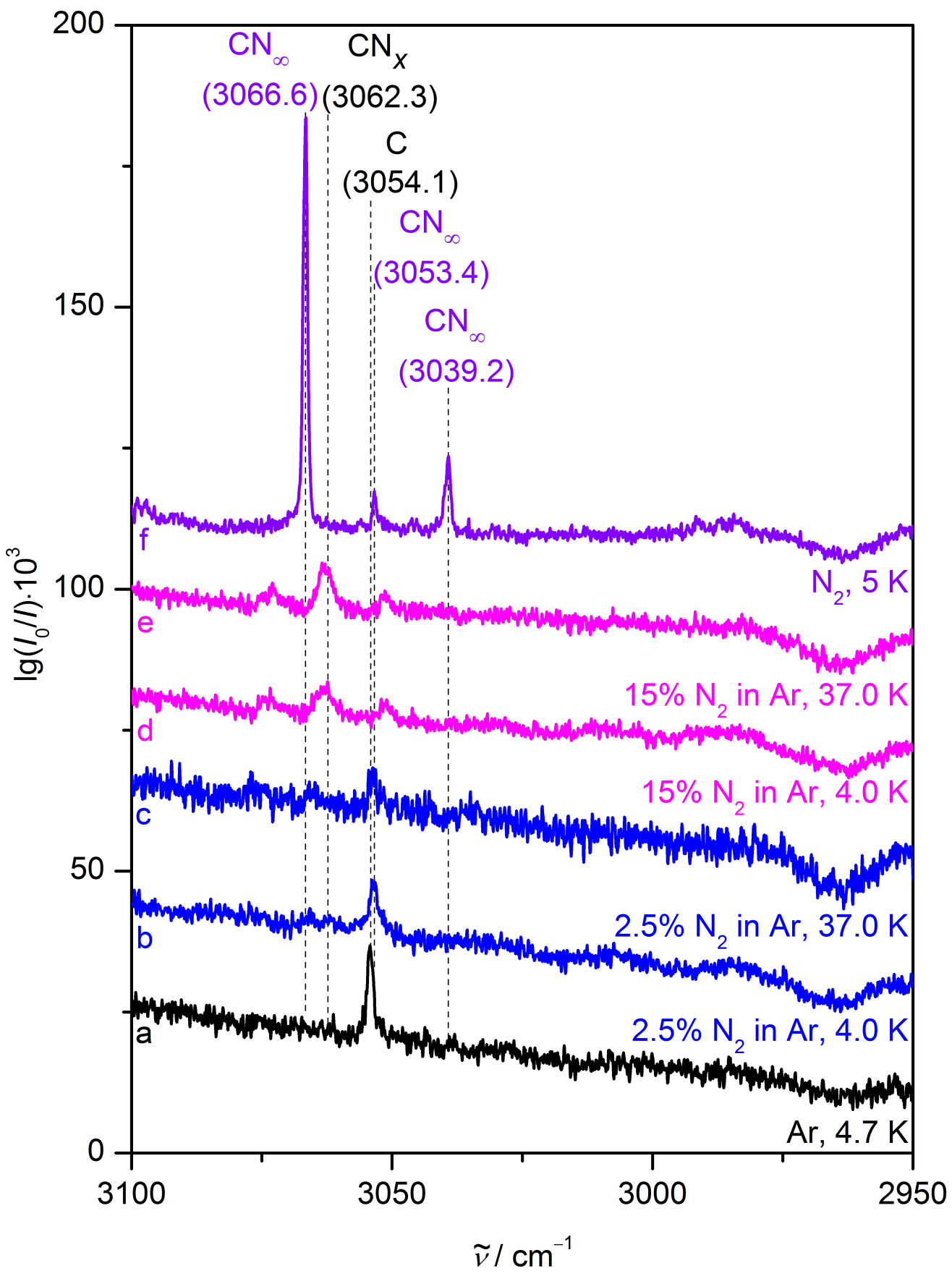

Figure 6.10.: CH stretching FTIR spectra (reprinted with adaptations from reference 337 ) of $0.13 \% \mathrm{C}$ in argon matrix after deposition at $20 \mathrm{~K}$ and subsequent cooling to $4.7 \mathrm{~K}$ (trace a). Spectra of argon matrices with increasing $\mathrm{N}$ addition (traces $\mathrm{b}$ to $\mathrm{g}$ ) before and after annealing to the stated temperature and subsequent recooling to $4.7 \mathrm{~K}$ as well as a $\mathrm{N}$ matrix deposited at $20 \mathrm{~K}$ and subsequently cooled to $4.7 \mathrm{~K}$ (trace $\mathrm{h}$ ) are also included. Wavenumbers and (tentative) assignments are provided. 


\section{Chloroform}

\section{Stretching Vibration $\nu_{5}$}

Stepwise $\mathrm{N}$ addition also shifts the $\mathrm{CCl}$ stretching signal towards the pure $\mathrm{N}$ matrix position. In neon matrix (Figure 6.11) this results in a broad, downshifted signal. Both broadening and wavenumber shift increase upon annealing. The argon matrix band position is already very similar to the pure $\mathrm{N}$ matrix (Figure 6.12), therefore only a band broadening due to unspecific $\mathrm{CN}$ interactions is observed without significant spectral shifts after $\mathrm{N}$ addition.

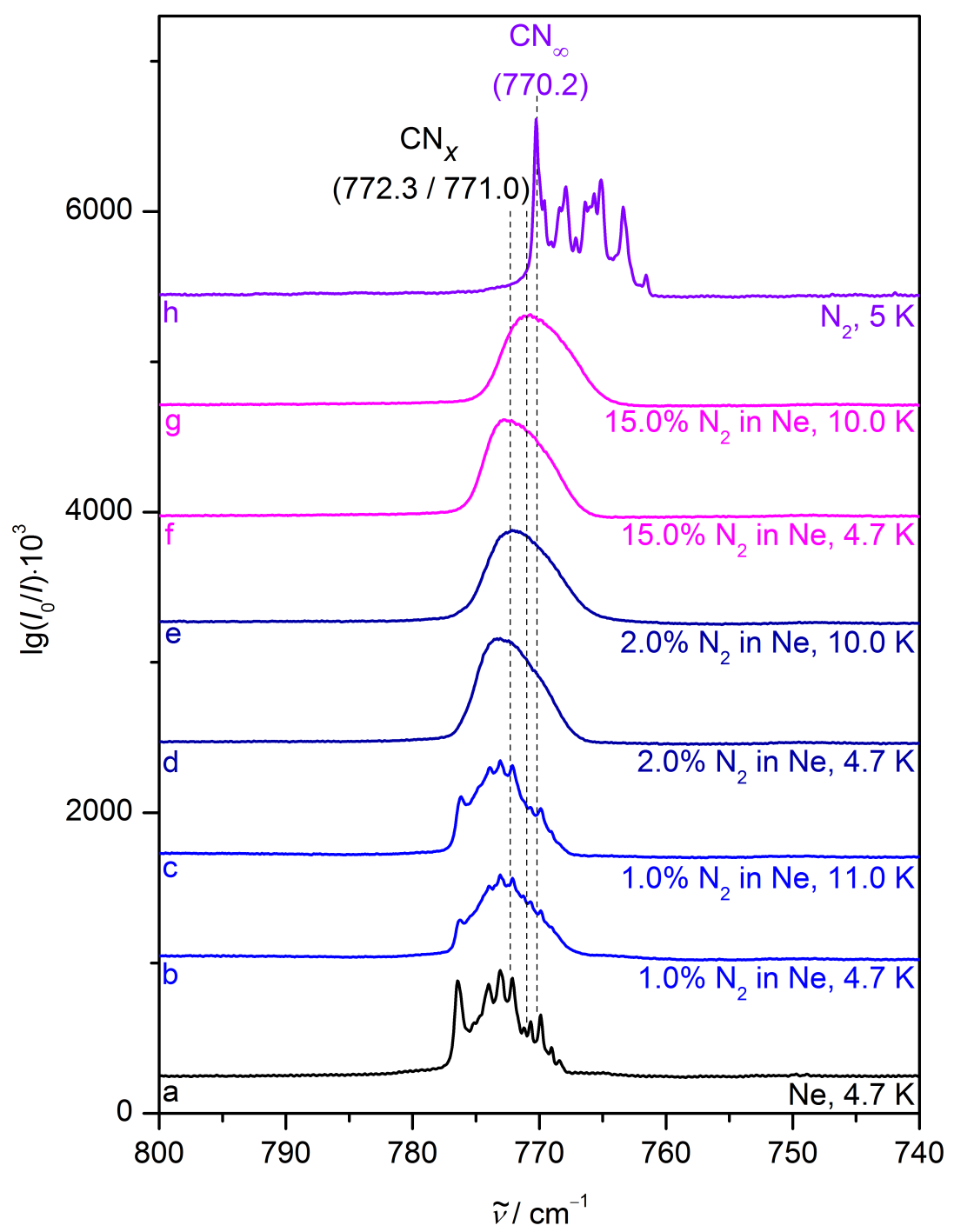

Figure 6.11.: CCl stretching FTIR spectra (reprinted with adaptations from reference 337 ) of $0.13 \% \mathrm{C}$ in neon matrix after deposition at $4.7 \mathrm{~K}$ (trace a). Spectra of neon matrices with increasing $\mathrm{N}$ addition (traces $\mathrm{b}$ to $\mathrm{g}$ ) before and after annealing to the stated temperature and subsequent recooling to $5 \mathrm{~K}$ as well as a $\mathrm{N}$ matrix deposited at $20 \mathrm{~K}$ and subsequently cooled to $4.7 \mathrm{~K}$ (trace $\mathrm{h}$ ) are also included. Wavenumbers and (tentative) assignments are provided. 


\section{Chloroform}

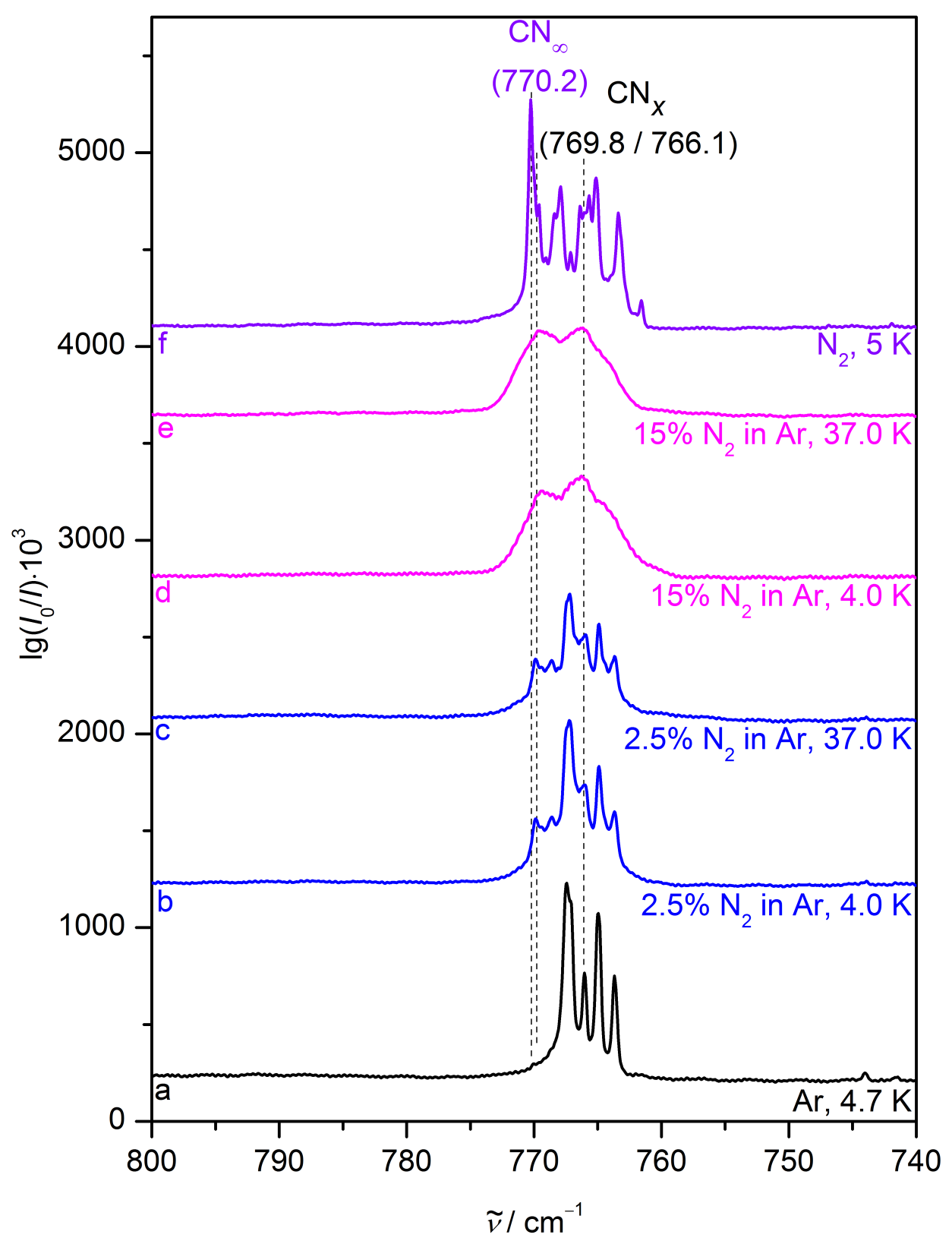

Figure 6.12.: $\mathrm{CCl}$ stretching FTIR spectra (reprinted with adaptations from reference 337 ) of $0.13 \% \mathrm{C}$ in argon matrix after deposition at $20 \mathrm{~K}$ and subsequent cooling to $4.7 \mathrm{~K}$ (trace a). Spectra of argon matrices with increasing $\mathrm{N}$ addition (traces $\mathrm{b}$ to $\mathrm{g}$ ) before and after annealing to the stated temperature and subsequent recooling to $4.7 \mathrm{~K}$ as well as a $\mathrm{N}$ matrix deposited at $20 \mathrm{~K}$ and subsequently cooled to $4.7 \mathrm{~K}$ (trace $\mathrm{h}$ ) are also included. Wavenumbers and (tentative) assignments are provided. 


\section{Chloroform}

\subsubsection{Supersonic Expansion}

For measurements in supersonic expansions, 0.1 and $0.2 \%$ chloroform (>99 \%, stabilised with ethanol, TCI) were mixed with helium $(99.996 \%$, Linde) and optionally nitrogen (99.999\%, Air Liquide) without further purification. Typically, averaged spectra from 1000 gas pulses were used as shown in Figures 6.13 and 6.14.

The $\mathrm{C}$ band centre in supersonic expansion is observed at $3033 \mathrm{~cm}^{-1}$ in agreement with the previously reported high resolution gas phase band position. ${ }^{349,355}$ A larger $\mathrm{C}$ fraction results in a growing band at $3037 \mathrm{~cm}^{-1}$ assigned to the $\mathrm{CC}$ dimer (Figure 6.13, traces $\mathrm{b}$ and $\mathrm{c}$ ), confirming previous unpublished work at higher resolution. ${ }^{365} \mathrm{~A}$ second $\mathrm{CH}$ stretching band assigned to the $\mathrm{CC}$ dimer would be expected, according to the cluster calculations (Table 6.1) with lower spectral upshift and intensity enhancement. However, at the employed spectral resolution and due to the limited signal-to-noise ratio this band is not observed.

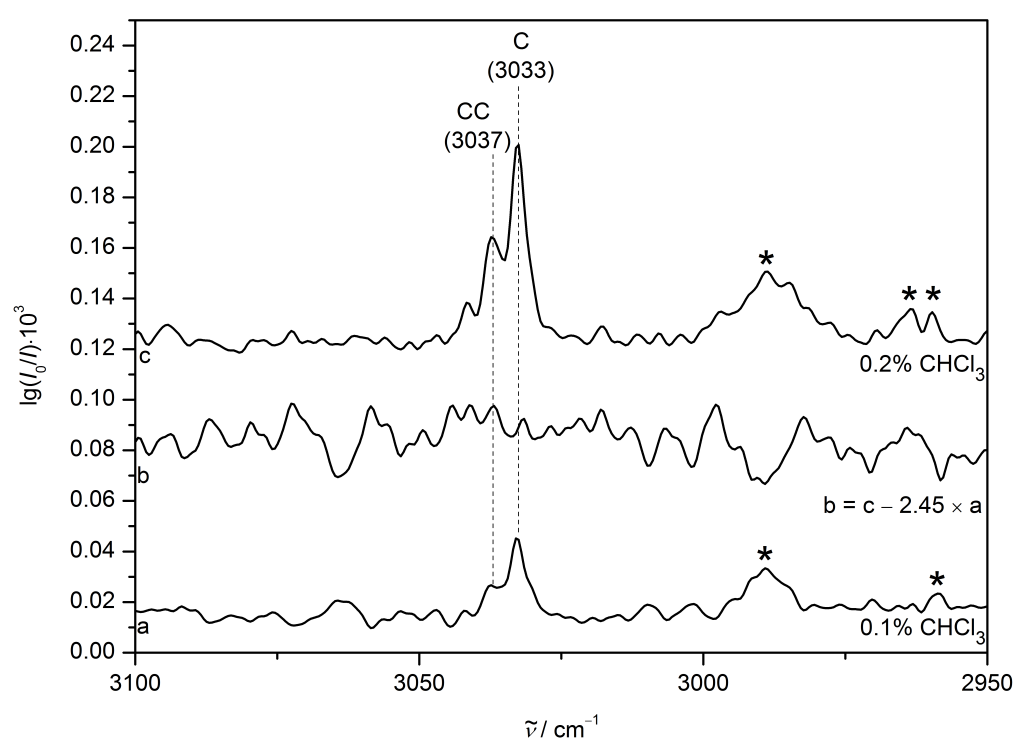

Figure 6.13.: FTIR jet spectra of C expanded in helium as well as a monomer-corrected difference spectrum (reprinted with adaptations from reference 337). Wavenumbers and (tentative) assignments are provided. Vibrational signals assigned to the ethanol stabiliser are marked by asterisks.

The addition of $15 \% \mathrm{~N}$ induces two new signals at 3051 and $3043 \mathrm{~cm}^{-1}$, respectively (Figure 6.14, traces b and c). Both are upshifted from the monomer and likely correspond to $\mathrm{C}$ with different degrees of $\mathrm{N}$ coordination or nanocoating. The bulk matrix band position (Figure 6.14, trace d) is approached, but not fully reached. 


\section{Chloroform}

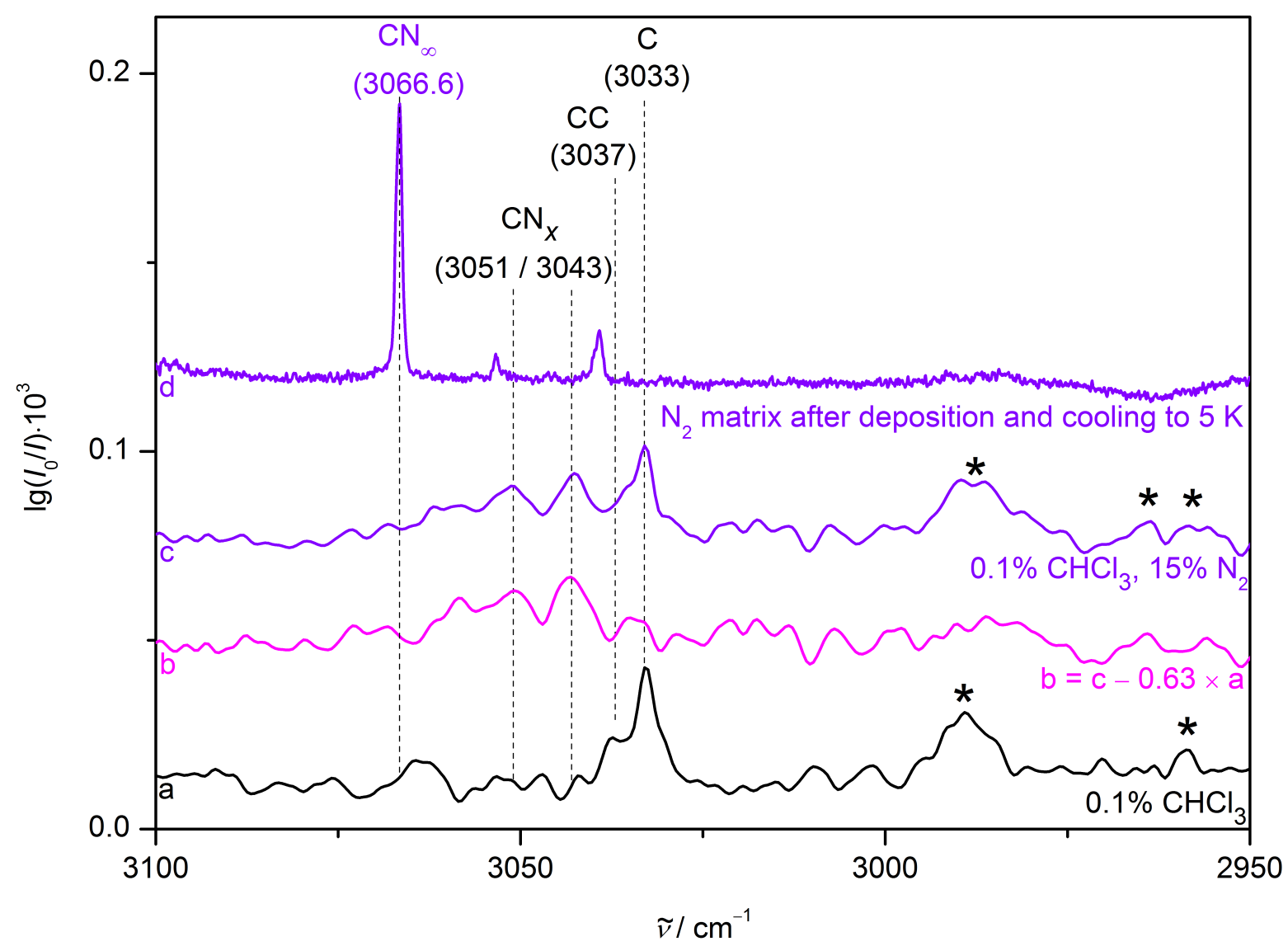

Figure 6.14.: FTIR jet spectra of C (reprinted with adaptations from reference 337) expanded in helium (trace a) and a mixture of $\mathrm{N}$ and helium (trace c) as well as a monomercorrected difference spectrum (trace b). Wavenumbers, (tentative) assignments and a bulk $\mathrm{N}$ matrix spectrum scaled by 0.001 (trace d) are provided. Vibrational signals assigned to the ethanol stabiliser are marked by asterisks.

\subsection{Summary}

The $\mathrm{CH}$ and $\mathrm{CCl}$ stretching modes of chloroform have been studied in cryogenic matrices and supersonic helium expansions. The total gas-to-nitrogen matrix upshift of the $\mathrm{CH}$ stretching vibration amounts to $33.7 \mathrm{~cm}^{-1}$, which can be incrementally approached by stepwise nitrogen addition to jet expansions as well as neon and argon matrices (Figure 6.15). Density functional wavenumber predictions within the double harmonic approximation are able to reproduce this effect with reasonable accuracy, although overshooting of the gas-to-matrix shift already occurs for triple nitrogen coordination. The shortcomings in theoretical modelling are also highlighted by inaccurate predictions for spectral shifts from chloroform monomer to dimer, which differ in direction between weakly perturbating neon matrices, the isolated gas phase and strongly interacting argon and nitrogen matrices. Possible weaknesses of theoretical modelling include electronic struc- 


\section{Chloroform}

ture and anharmonicity deficiencies and the finite size of the calculated clusters not accounting for the bulk matrix environment.

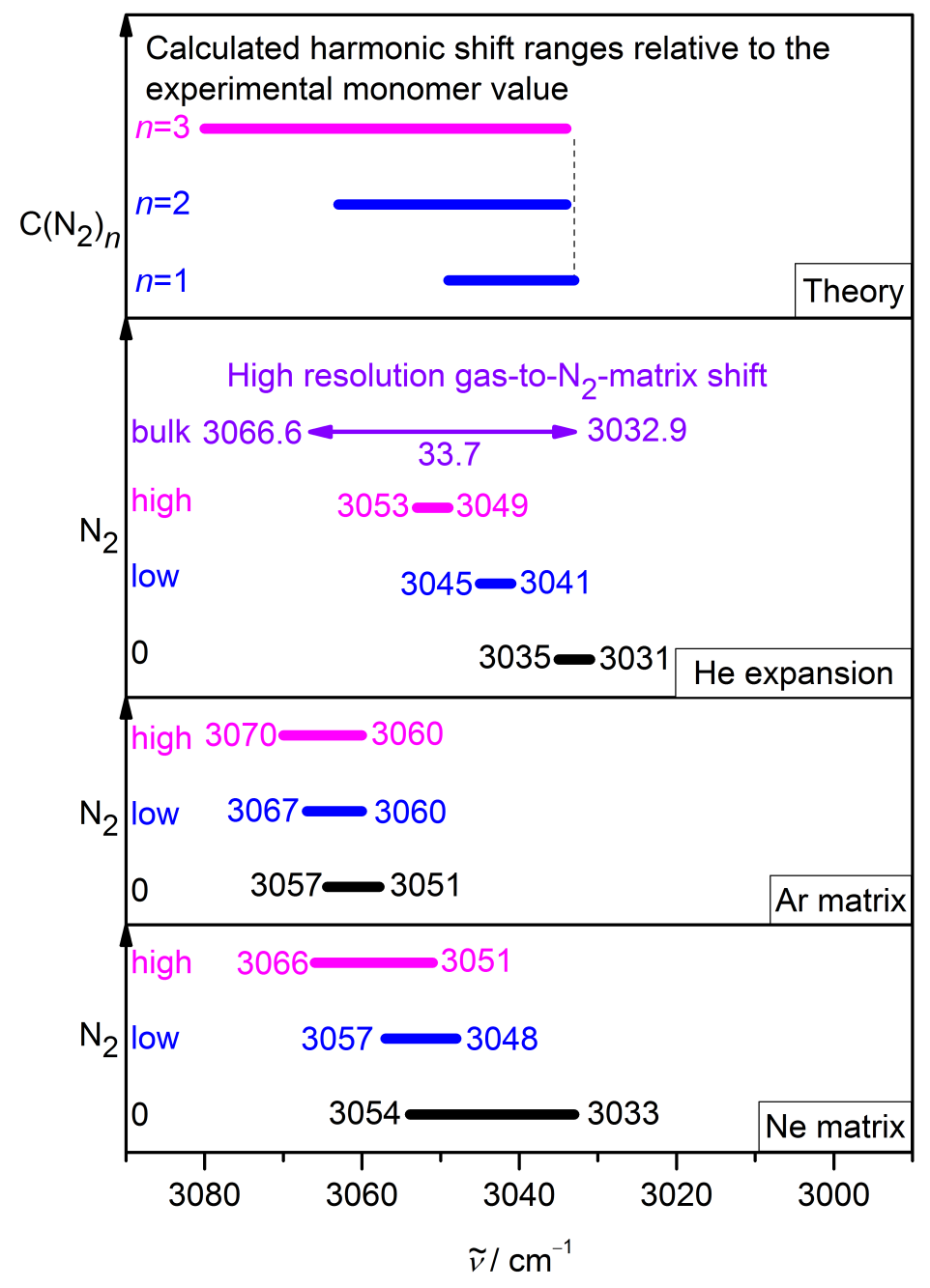

Figure 6.15.: $\mathrm{CH}$ stretching band positions of $\mathrm{C}$ in neon and argon cryomatrices as well as in supersonic helium expansions (reprinted from reference 337). Increasing $\mathrm{N}$ admixture causes progressive upshifts of the band position in all three environments, approaching the bulk N matrix limit. High resolution gas phase ${ }^{349,355}$ and $\mathrm{N}$ matrix spectra allow for an accurate determination of the total gas-to-matrix upshift to $33.7 \mathrm{~cm}^{-1}$. The range of wavenumber shifts predicted for CN complexes by harmonic B3LYP-D3(BJ)/def2-QZVP calculations reproduces experimental findings with reasonable accuracy for the first two added $\mathrm{N}$ molecules, but the tested approach overestimates the upshift in more N-enriched environments, overshooting the bulk limit.

Nitrogen cluster formation additionally results in spectral downshifts of the very intense doubly degenerate $E$-symmetric $\mathrm{CCl}$ stretching mode. The degeneracy and the small observed wavenumber shifts render theoretical predictions for this mode somewhat ambiguous and difficult to judge. 


\section{Cavity-enhanced Raman Spectroscopy}

Linear cavities for laser radiation consisting of two highly reflective mirrors were constructed and the power build-up inside the resonator was evaluated for potential applications as excitation light source in Raman spectroscopy. Resonances of cavity and laser with subsequent light intensity enhancements were successfully realised with single frequency $635 \mathrm{~nm}$ diode and $532 \mathrm{~nm}$ frequency-doubled Nd:YAG lasers together with Dr. Matthias Heger and partly during a research project of Fabian Hecker and Elsa Lang's bachelor thesis. ${ }^{366}$ A malfunction of the $532 \mathrm{~nm}$ laser and degradation of the $635 \mathrm{~nm}$ cavity mirrors prevented successful recording of cavity-enhanced Raman spectra.

\subsection{Introduction}

Spontaneous Raman scattering experiments inherently suffer from low quantum efficiency and resulting low intensities, ${ }^{367}$ especially for samples with small molecular density such as analytes in gas phase or supersonic expansions. Surface-enhanced, ${ }^{368}$ photoacoustic stimulated, ${ }^{369,370}$ non-linear ${ }^{371}$ or coherent anti-Stokes ${ }^{372,373}$ Raman spectroscopy offer possible sensitivity improvements, but mostly require several high output power laser light sources. Cavity-enhanced Raman spectroscopy ${ }^{374-377}$ exploits the linear scaling of spontaneous Raman scattering with intensity of the excitation laser, since incident laser power can be enhanced by several orders of magnitude inside a resonant external cavity. ${ }^{378-382}$ Efficiency of the power build-up is increased by active matching of cavity length and laser wavelength ${ }^{378,381}$ as well as passive optical feedback from resonating cavity to the laser. ${ }^{375,376,379,380,382}$

\subsection{Experimental Set-ups}

Optical set-ups were changed frequently throughout the project for intensity enhancement evaluation at two different laser wavelengths, $635 \mathrm{~nm}$ and $532 \mathrm{~nm}$, as well as detection of spontaneous Raman scattering.

\subsubsection{Power Enhancement Evaluation Set-ups}

The $635 \mathrm{~nm}$ set-up for initial testing of power build-up is depicted in Figure 7.1. The laser diode (Hitachi, HL6322G, $\lambda=635 \mathrm{~nm}$ ) was mounted on a temperature-stabilised 


\section{Cavity-enhanced Raman Spectroscopy}

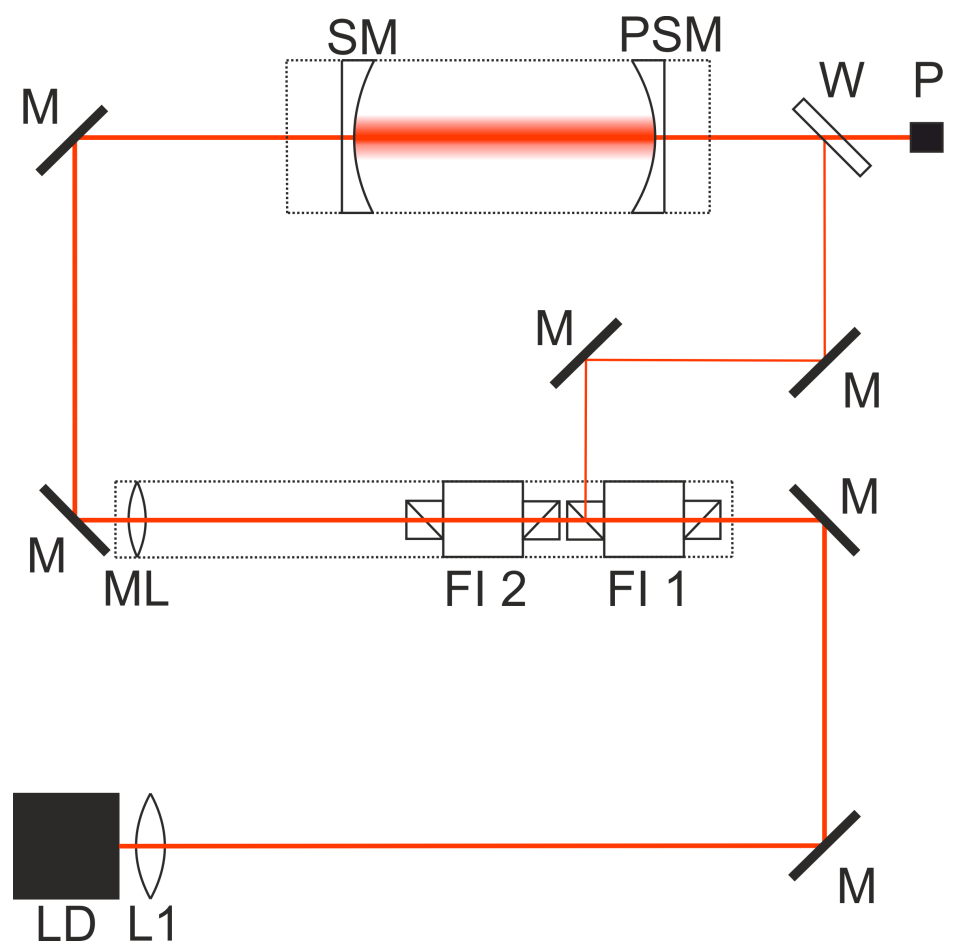

Figure 7.1.: Scheme of the set-up for cavity-enhancement of a $635 \mathrm{~nm}$ diode laser based on references 374-376 and 377. Abbreviations: LD: laser diode, L1: collimating lens with $f=4.51 \mathrm{~mm}, \mathrm{M}$ : broad band mirrors, FI: Faraday isolator, ML: mode matching lens with $f=500 \mathrm{~mm}$, SM: high reflectivity mirror, PSM: piezo-actuated high reflectivity mirror, W: wedged window, P: photodiode, dashed lines indicate optical cage systems used for alignment.

thermoelectric cooler (Thorlabs, TCLDM9) regulated by OEM diode current and temperature controllers (Thorlabs, LDC200C and TED200C) with an optical output power of $13 \mathrm{~mW}$ at $12{ }^{\circ} \mathrm{C}$ and $65 \mathrm{~mA}$. After collimation with an aspheric lens (Thorlabs, C230TME-A, $d=4.95 \mathrm{~mm}, f=4.51 \mathrm{~mm}$ ), the laser beam was guided through a $30 \mathrm{~mm}$ optical cage system by two broadband mirrors (Thorlabs, BB1-E02, $d=25.4 \mathrm{~mm}$ ), ensuring central beam positioning in two Faraday isolators (Thorlabs, IO3D-633-PBS, $88 \%$ transmission, $36 \mathrm{~dB}$ isolation) with polarising beam splitters at both ends, preventing direct back-reflections from the first cavity mirror to the laser diode, as well as an achromatic mode matching lens (Thorlabs, AC254-500-A, $d=25.4 \mathrm{~mm}, f=500 \mathrm{~mm}$ ). Two further broadband mirrors were used for central alignment of the laser beam into the linear cavity comprised of two high reflectivity mirrors (Newport, 10CV00SR.30F, $d=25.4 \mathrm{~mm}, R=1000 \mathrm{~mm}, \rho \geq 99.97 \% @ 583-663 \mathrm{~nm}$ ) at a distance of about $15 \mathrm{~cm}$ in a second $30 \mathrm{~mm}$ cage system. The rear cavity mirror was placed in a kinematic mount with piezo-electric actuators (Thorlabs, KC1-PZ, controlled by MDT693B). Light leaking out of the cavity was directed onto a wedged window (Thorlabs, WW11050, $d=25.4 \mathrm{~mm}$ ) and the propagating part of the beam was detected by a Si photodiode (Thorlabs, 


\section{Cavity-enhanced Raman Spectroscopy}

PDA36A). The reflected part was directed into the first Faraday isolator via two broadband mirrors and the exit polarising beam splitter. By providing controlled optical feedback of radiation with the cavity resonance wavelength to the laser diode, emitted frequencies were optically locked to the cavity during resonance.

In addition to passive optical feedback, the piezo-actuated mirror was used for active, mechanical matching of cavity length and emitted laser wavelength. A sinusoidal modulation with $750 \mathrm{~Hz}$ frequency and $6 \mathrm{~V}$ amplitude was applied from a function generator (GW Instek, SFG - 2004), resulting in a linear piezo travel of $\pm 320 \mathrm{~nm}$ along the central cavity axis, tuning the mirror distance in and out of resonance length. Power buildup during resonance was detected by the photodiode and a signed error voltage was generated by an attached lock-in amplifier (Stanford Research Systems 5105, $316 \mathrm{mV}$ sensitivity, $100 \mathrm{kHz}$ high pass filter, $5 \mathrm{kHz}$ low pass filter, $12 \mathrm{~dB}$ oct $^{-1}$ slope, $1 \mathrm{~s}$ time constant) set to the reference frequency of the sinusoidal modulation. Error and modulation voltages were mixed via homebuilt electronics and applied to the piezo-actuator, thus keeping the occurence of cavity resonance in the center of each modulation cycle and therefore accounting for thermal and vibrational deviations in average cavity length.

The set-up used for the $532 \mathrm{~nm}$ laser (Coherent, Verdi V5, $\lambda=532 \mathrm{~nm}, P_{\max }=5 \mathrm{~W}$ ) was mostly identical with the $635 \mathrm{~nm}$ configuration described previously. Apart from the laser, the two Faraday isolators were replaced by one designed for the changed wavelength (Thorlabs, IO-5-532-HP, $89 \%$ transmission, 38-44 dB isolation) and a different mode matching lens was employed (uncoated, $d=50.8 \mathrm{~mm}, f=1330 \mathrm{~mm}$ ). Both high reflectivity mirrors were replaced as well (Layertec, Batch R0708035, $d=7.75 \mathrm{~mm}$, $R=1000 \mathrm{~mm}, \rho \geq 99.99 \% @ 532 \mathrm{~nm}$ ). Since the $532 \mathrm{~nm}$ laser is a frequency-doubled Nd:YAG system, no optical feedback from the cavity was provided to the laser.

\subsubsection{Raman Spectroscopy Set-up}

Spectral analysis of spontaneous Raman scattering required a monochromator (McPherson Inc., Model 205f, $0.5 \mathrm{~m}$ focal length, $f / 3.2$, ruled grating with 1800 grooves $\mathrm{mm}^{-1}$ ) with a vertical entrance slit and a liquid nitrogen cooled CCD camera (Princeton Instruments Digital Spectroscopy, Spec-10, 400B/LN, $1340 \times 400$ pixel of size $20 \mu \mathrm{m} \times 20 \mu \mathrm{m}$ ). Since the horizontal cavity placement in previous set-ups prevented effective light projection onto the vertical monochromator entrance slit, several changes depicted in Figure 7.2 were made. After passing through the Faraday isolators, the laser beam was directed upwards in a $30 \mathrm{~mm}$ vertical optical cage, through the mode matching lens in a connecting horizontal cage and then reflected down into the cavity aligned in a second vertical optical cage. Light leaking out of the cavity was redirected to horizontal propagation direction by a broad band mirror and guided onto the wedged window, partly detected by the photodiode, partly diverted into the first Faraday isolator for optical feedback to the laser diode.

Raman scattered light from the cavity centre was collimated with a lens $(d=40 \mathrm{~mm}$, $f=50 \mathrm{~mm}$ ), directed to the Czerny-Turner monochromator via three broadband mirrors and focussed onto the $50 \mu \mathrm{m}$ entrance slit by a second lens $(d=50.8 \mathrm{~mm}, f=15 \mathrm{~mm}$, $f / 3.4$ ), before detection by the CCD camera. 


\section{Cavity-enhanced Raman Spectroscopy}

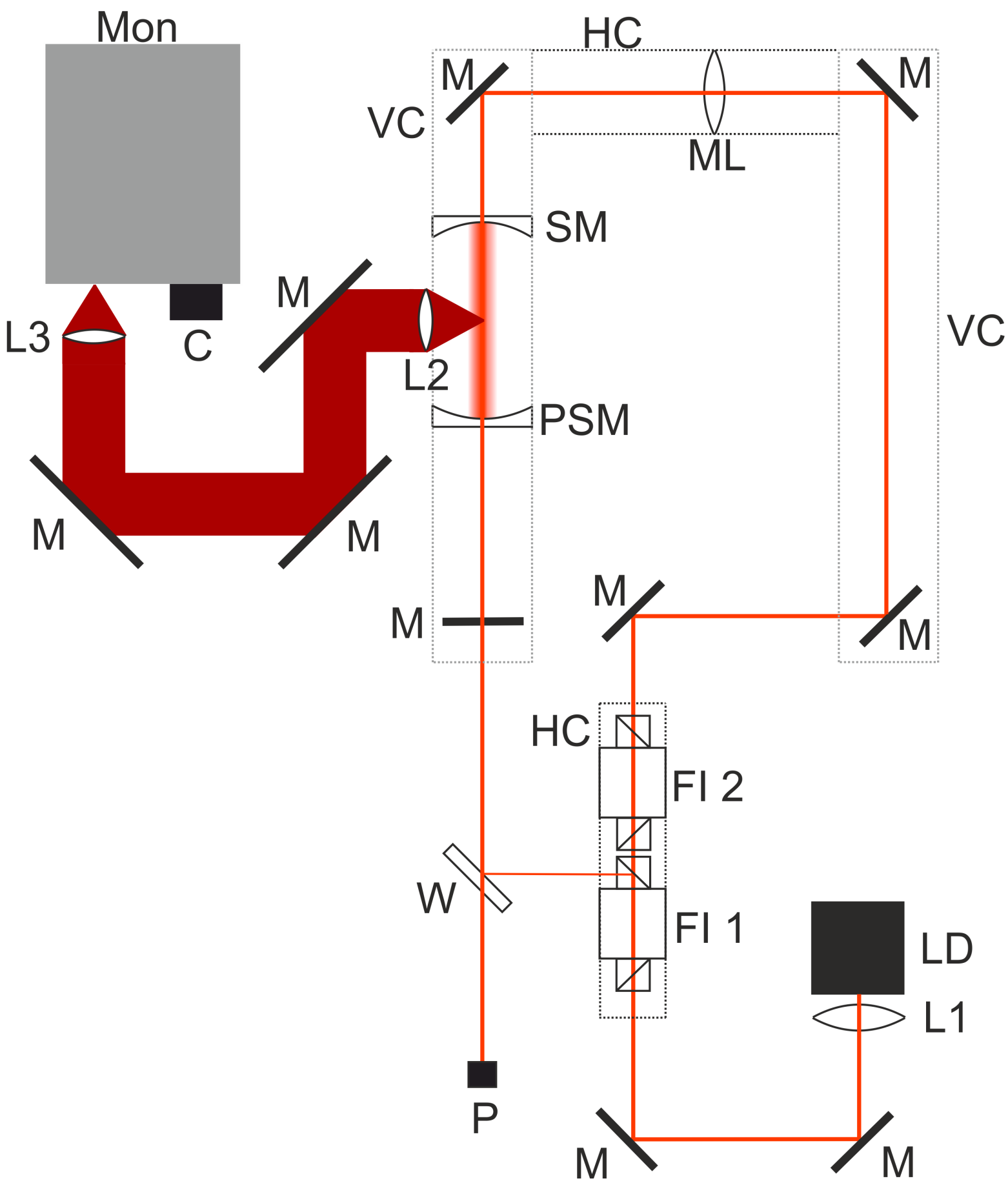

Figure 7.2.: Scheme of the set-up for cavity-enhanced Raman spectroscopy with a $635 \mathrm{~nm}$ diode laser (adapted from reference 366). Abbreviations: LD: laser diode, L1: collimating lens with $f=4.51 \mathrm{~mm}, \mathrm{M}$ : broad band mirrors, FI: Faraday isolator, ML: mode matching lens with $f=500 \mathrm{~mm}$, SM: high reflectivity mirror, PSM: piezo-actuated high reflectivity mirror, W: wedged window, P: photodiode, L2: lens with $f=50 \mathrm{~mm}$ collimating Raman scattered light, L3: lens with $f=15 \mathrm{~mm}$ focussing Raman scattered light onto monochromator slit, Mon: Czerny-Turner monochromator, C: CCD camera, black dashed lines indicate horizontal (HC), grey dashed lines vertical (VC) optical cage systems used for alignment. 


\section{Cavity-enhanced Raman Spectroscopy}

\subsection{Results and Discussion}

Two different red diode lasers with output powers of 13 and $100 \mathrm{~mW}$ (Oclaro, HL63163DG, $\lambda=633 \mathrm{~nm}$ ), respectively, are tested and characterised. Intensity enhancements inside the cavity with optical feedback are determined for the $13 \mathrm{~mW}$ diode using a photodiode. Power build-up without optical feedback is measured for both the red $13 \mathrm{~mW}$ diode and the $532 \mathrm{~nm} \mathrm{Nd:YAG} \mathrm{laser.}$

\subsubsection{Laser Diode Characterisation}

Both red diode lasers yielded efficient power build-up in only small parts of their respective operating range. At a constant temperature of $12^{\circ} \mathrm{C}$, the injection current was therefore varied in steps of $1 \mathrm{~mA}$ over the entire recommended operating range, while the laser emission spectrum was determined by analysing Rayleigh scattered light from ambient air with a high resolution monochromator (HORIBA Jobin-Yvon, THR 1500, $1.5 \mathrm{~m}$ focal length, $f / 14$, ruled grating with 2400 grooves $\mathrm{mm}^{-1}$ ) and a thermoelectrically cooled CCD camera (Andor Technology, Model DU420A-OE, $1024 \times 265$ pixel). Spectral lines from a Ne emission light source (L.O.T.-Oriel, LSP032) were employed for wavelength calibration. As shown in Figure 7.3, single frequency emission of the diode laser is possible (black trace), but can change to multi frequency emission depending on the injection current (red trace). As Figure 7.4 shows, multi frequency emission of the $13 \mathrm{~mW}$ diode usually occurs near mode hops and two areas of stable single frequency emission exist around $65 \mathrm{~mA}$ and $78.5 \mathrm{~mA}$. The $100 \mathrm{~mW}$ diode only displays one single frequency emission area at low injection currents of about $91 \mathrm{~mA}$ corresponding to $25 \mathrm{~mW}$ output and additional power after current increase is diverted into other laser modes. Single frequency emission by the employed laser diodes coincides with strong cavity resonances and large intensity enhancements and therefore appears to be essential for effective optical feedback and subsequent power build-up in the cavity, which also rationalises previous unsuccessful attempts of cavity-enhancement with a multi frequency $405 \mathrm{~nm}$ diode laser. ${ }^{383}$

\subsubsection{Power Enhancement Evaluations}

Magnitudes of achieved cavity-enhancements with respect to the incident laser output power are determined with the set-up depicted in Figure 7.1. Peak voltages of the photodiode observed during cavity resonances are converted to optical powers and corrected by measured transmissions of the rear cavity mirror and wedged window listed in Table A.28 in appendix A.7 to estimate intracavity optical power. Calibration of the voltages provided by the photodiode is achieved with a power meter (Coherent, Labmaster E with detector B057) and conversion factors are shown in Table A.29 in appendix A.7. The resulting estimated enhancements are summarised in Table 7.1.

The red diode laser shows a significant increase in intracavity power to about $3 \mathrm{~W}$ compared to the initial laser output of $13 \mathrm{~mW}$. After blocking the optical feedback cycle, power inside the cavity is diminished to about $8 \mathrm{~mW}$, corresponding to a $36 \%$ 


\section{Cavity-enhanced Raman Spectroscopy}

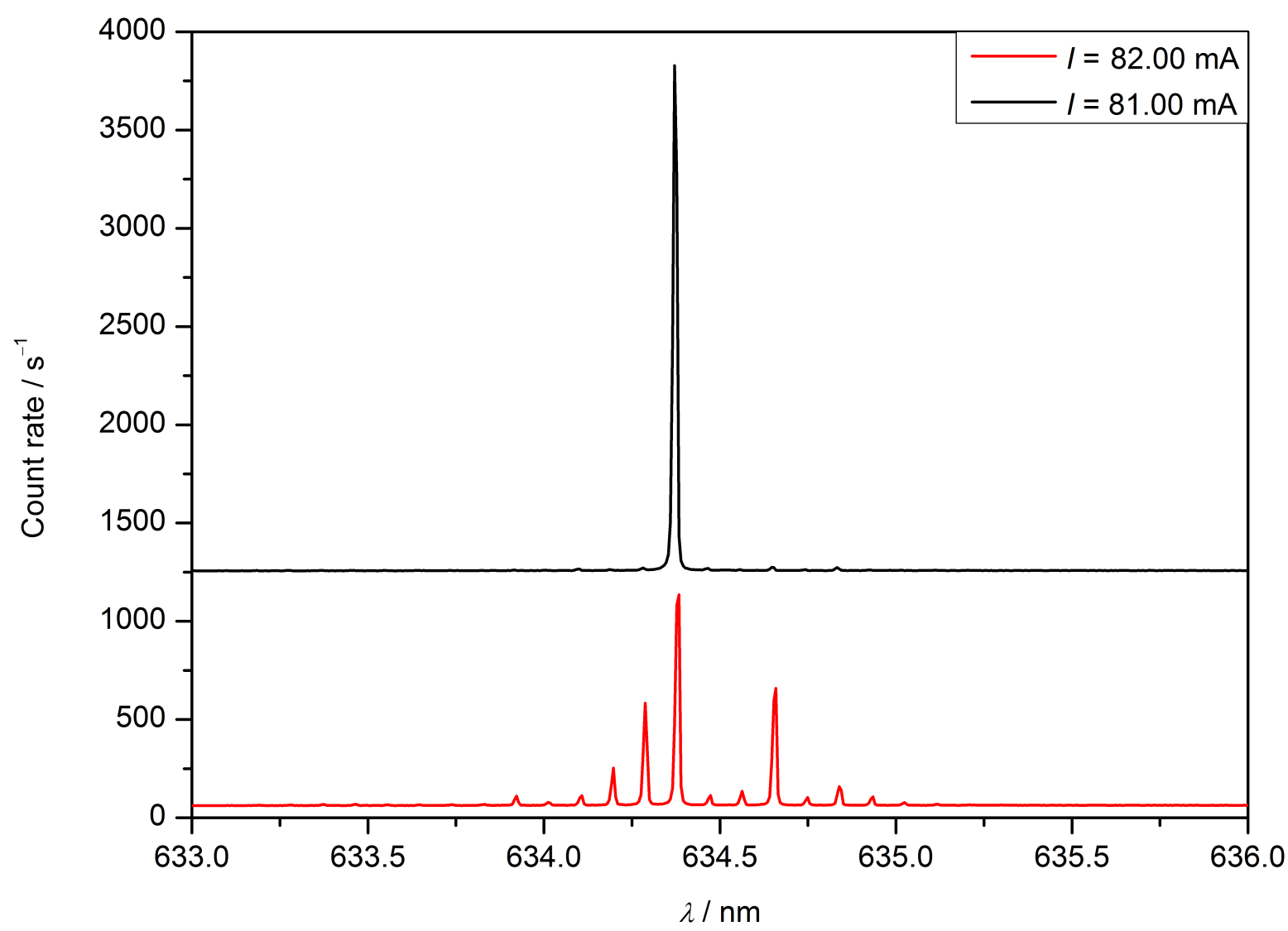

Figure 7.3.: Rayleigh scattered light from ambient air corresponding to the emission spectrum of a $13 \mathrm{~mW}, 635 \mathrm{~nm}$ laser diode at a temperature of $12{ }^{\circ} \mathrm{C}$ with two different injection currents $(I)$. Slight current variations of $1 \mathrm{~mA}$ change the emission behaviour from single (black trace) to multi frequency (red trace) emission.

net power loss and an enhancement factor lower than one. While passing through all optical elements before the front cavity mirror, $42 \%$ of incident laser power is lost on mirror surfaces and in the Faraday isolators or lenses, which is not compensated by cavity-enhancement without optical feedback. The green laser beam displays a lower power loss of $34 \%$ before entering the cavity, mostly because only one Faraday isolator is used instead of two in the $635 \mathrm{~nm}$ set-up. Nonetheless, cavity-enhancement is even lower for the green laser than for the red diode with a net enhancement factor of 0.5 and no possibility to increase power build-up by optical feedback.

Improvements to the set-up would be possible by employing different methods for matching cavity length and laser wavelength such as the Pound-Drever technique, ${ }^{384}$ which has been successfully utilised to achieve enhancement factors of 250 with the green Verdi V5 laser system. ${ }^{378}$ Since the set-up is operated in ambient air, repeated scattering from dust particles, nitrogen and oxygen occurring as the laser beam undergoes multiple passes inside the cavity also limits achievable enhancements. Encapsulation of the cavity mirrors inside a vacuum vessel therefore constitutes another potential improvement. 
7. Cavity-enhanced Raman Spectroscopy
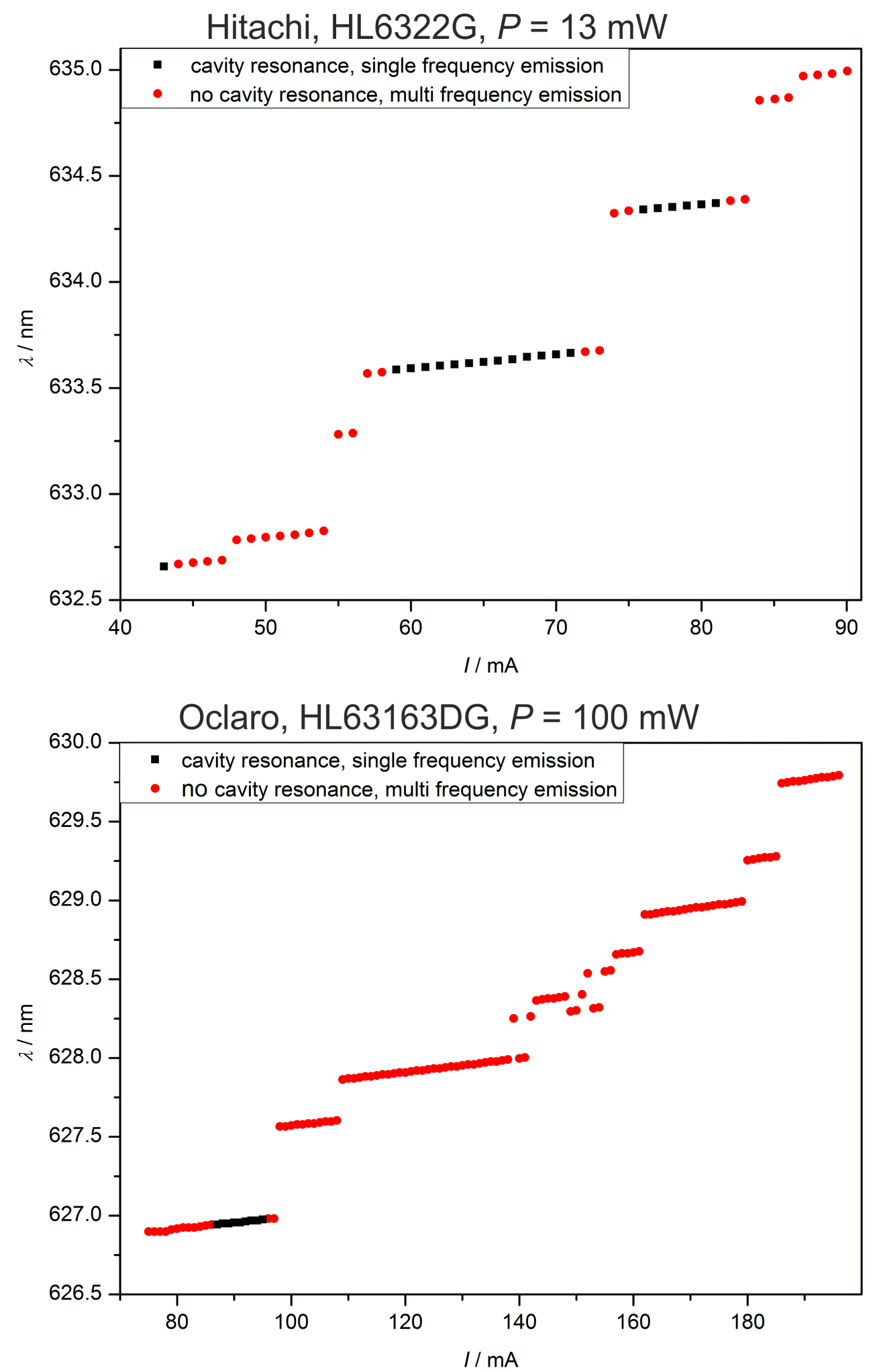

Figure 7.4.: Wavelength $\lambda$ of $13 \mathrm{~mW} 635 \mathrm{~nm}$ (top) and $100 \mathrm{~mW} 633 \mathrm{~nm}$ (bottom) laser diodes at a temperature of $12{ }^{\circ} \mathrm{C}$ with different injection currents $(I)$. Single frequency emission enabling cavity resonances is marked by black squares, multi frequency emission preventing resonances by red dots. 


\section{Cavity-enhanced Raman Spectroscopy}

Table 7.1.: Incident laser output power $\left(P_{\text {in }}\right)$, intracavity power $\left(P_{\text {cav }}\right)$ estimated from photodiode measurements behind the cavity and resulting cavity-enhancement for both tested wavelengths.

\begin{tabular}{cccc}
\hline & & \multicolumn{2}{c}{ Wavelength } \\
& & $635 \mathrm{~nm}$ & $532 \mathrm{~nm}$ \\
\hline$P_{\text {in }} / \mathrm{mW}$ & 13 & 200 \\
\hline \multirow{2}{*}{$P_{\text {cav }} / \mathrm{W}$} & optical feedback & 3 & - \\
& no optical feedback & $8 \times 10^{-3}$ & $1 \times 10^{-1}$ \\
\hline \multirow{2}{*}{ Cavity-enhancement } & optical feedback & 230 & - \\
& no optical feedback & 0.6 & 0.5 \\
\hline
\end{tabular}

\subsubsection{Raman Spectroscopy}

Final judgement of the performance displayed by the cavity-enhancement set-ups is not possible from simple estimates of intracavity power, as this method ignores the duty cycle of the system. Peak powers estimated in the previous section are only reached for a short period of time, before being significantly lowered by the sinusoidally modulated rear cavity mirror movement necessary for cavity length stabilisation over time. Since CCD cameras employed in Raman spectroscopy typically average the intensity of scattered light over several seconds up to a few minutes, the diminishing intracavity power at the edges of each modulation cycle is expected to have a large impact on Raman intensities. Thus, the best possible evaluation can be achieved through recording of Raman spectra and comparison with results obtained without cavity-enhancement as enabled by the set-up depicted in Figure 7.2.

For recording a test spectrum of ambient air without cavity-enhancement, the cavity mirrors were removed from the set-up and resulting rotational Raman spectra of oxygen and nitrogen are depicted in Figure 7.5 (red trace). A previously recorded reference spectrum using the Verdi V5 $532 \mathrm{~nm}$ laser with $1 \mathrm{~W}$ output power and the high resolution $1.5 \mathrm{~m}$ focal length monochromator used for the laser diode characterisation described in section 7.3.1 is included (green trace). Comparison of both spectra reveals shortcomings of the diode laser spectrum in both signal-to-noise ratio and spectral resolution. Lower intensities are expected from the red laser due to the significantly lower output power and the cubic increase of CCD camera detected Raman scattering intensity with higher excitation frequency. ${ }^{367}$ The variation in spectral resolution is mostly caused by the different monochromators. Incorporation of the Verdi V5 laser into the set-up in Figure 7.2 failed because of a laser system malfunction.

While attempting to record cavity-enhanced Raman spectra with the red laser diode, cavity resonances were noticeably weaker than the ones observed during evaluations with the photodiode. Re-examination of the cavity mirror transmissions listed in Table A.28 showed higher values for both mirrors. Rear mirror transmission increased 


\section{Cavity-enhanced Raman Spectroscopy}

from $7 \times 10^{-3} \%$ to $1.3 \times 10^{-2} \%$, while the corresponding value of the front mirror only changed slightly from $6 \times 10^{-3} \%$ to $7 \times 10^{-3} \%$. Increased transmission most likely corresponds to decreased reflectivity caused by degradation of the mirror coating, severely reducing possible cavity-enhancements. The increased transmission was found on the entire mirror surface, ruling out local radiation damage, which should be confined to a single spot. Presumably, repeated cleaning of the mirror surface with ethanol caused a continuous decrease of reflectivity by slowly diminishing the coating. Therefore, no cavity-enhanced Raman spectra using the red diode laser could be obtained.

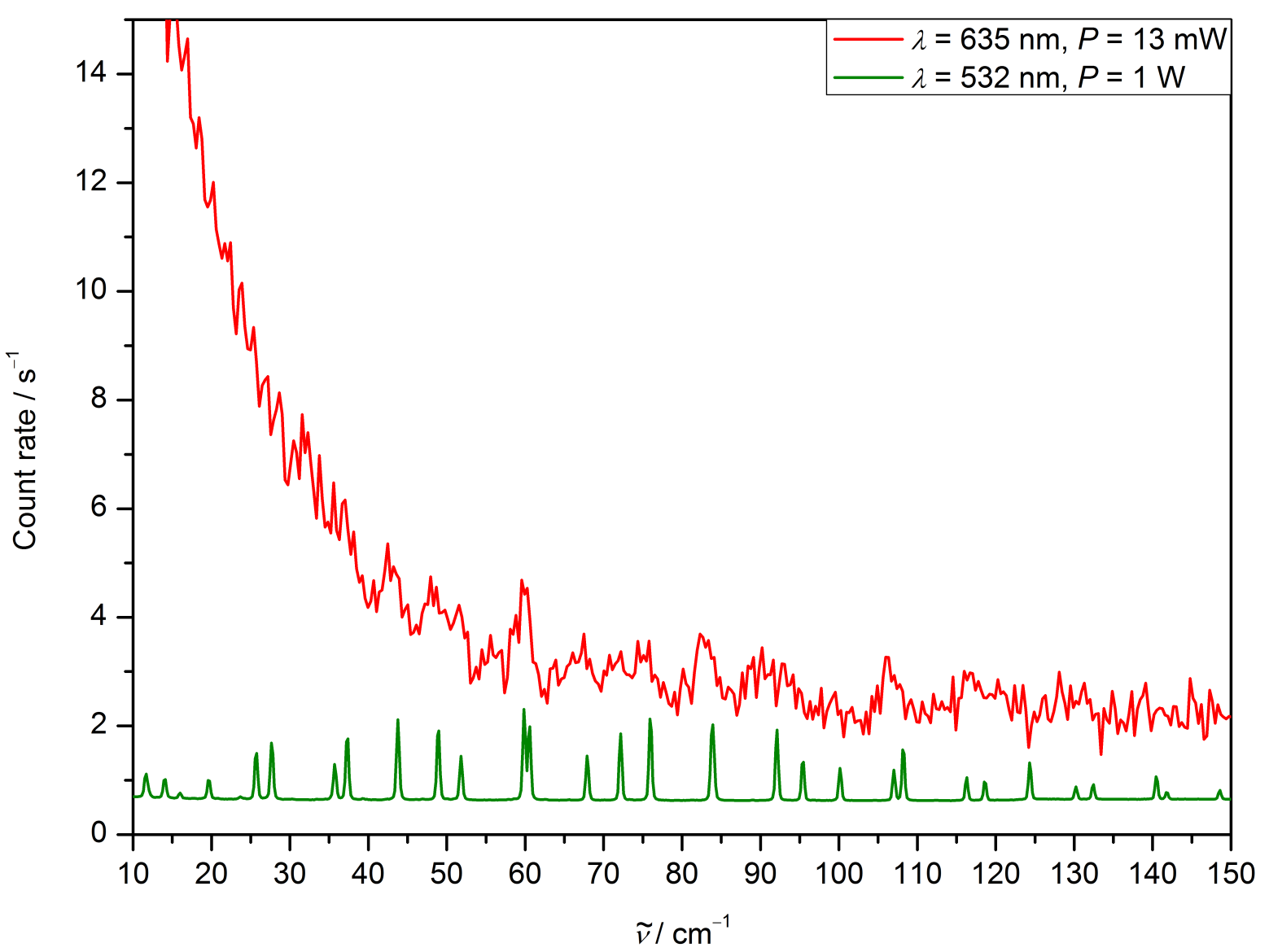

Figure 7.5.: Rotational Raman spectrum of nitrogen and oxygen in ambient air recorded with the $13 \mathrm{~mW} 635 \mathrm{~nm}$ laser diode (red trace) and the set-up depicted in Figure 7.2 with removed cavity mirrors. A reference spectrum recorded with the Verdi V5 $532 \mathrm{~nm}$ laser set to $1 \mathrm{~W}$ output power and the high resolution $1.5 \mathrm{~m}$ focal length monochromator used for the previous laser diode characterisation scaled by a factor of 0.01 is included (green trace). 


\section{Cavity-enhanced Raman Spectroscopy}

\subsection{Summary}

External linear cavities for laser power build-up are successfully constructed for two different wavelengths and partially characterised. Stabilisation of cavity resonances is achieved via active, mechanical matching of mirror distance to laser wavelength and in the case of diode laser sources passive optical feedback from the resonant cavity. Single frequency laser emission appears to be a crucial condition for effective optical feedback, which is unfortunately not found in laser diodes with high incident output powers. Estimated intracavity peak intensities during resonances show that a relatively simple set-up like the tested one requires optical feedback to achieve net power gains. Full performance evaluations of cavity-enhanced compared to linear Raman spectroscopy could not be carried out because recording of cavity-enhanced Raman spectra was prevented by a malfunction of the employed $532 \mathrm{~nm}$ laser and degradation of the $635 \mathrm{~nm}$ cavity mirrors. 


\section{Conclusions and Outlook}

Molecular aggregates of the most abundant components of earth's atmosphere, nitrogen and oxygen, weakly bound at various molecules by hydrogen bonding have been studied in cold environments such as supersonic expansions and cryogenic matrices by multiple spectroscopic techniques. Experimental observables for nitrogen complexes used as benchmarks for the quality of different quantum chemical electronic structure methods include rotational constants linked to molecular structures obtained by microwave spectroscopy as well as vibrational transitions and relative conformational energy differences derived from infrared and Raman spectroscopy (Figure 8.1 and Table A.30 in appendix A.8). Combining data on all these different observables and molecules identifies partial matches between experiment and theory as results of fortuitous error cancellation, since an accurate electronic structure description must result in precise predictions for all experimental observations.

Table 8.1.: Method rankings based on squared deviations $\delta^{2}(x)$ of theoretical predictions from experimental reference values calculated according to Equation 1.1 derived from all 23 benchmarks schematically depicted in Figure 8.1 and listed in Table A.30 in appendix A.8.

\begin{tabular}{ccc}
\hline Level of theory & $\delta^{2}(x)$ & Ranking \\
\hline SCS-MP2/VTZ & 1.3 & 1 \\
PBE0-D3(BJ)/aVTZ & 2.1 & 2 \\
MP2/VTZ & 2.3 & 3 \\
B2PLYP-D3(BJ)/VTZ & 2.5 & 4 \\
B3LYP-D3(BJ)/def2-QZVP & 2.8 & 5 \\
M06-2X/aVTZ & 5.0 & 6 \\
$\omega$ B97-XD/aVTZ & 5.2 & 7 \\
PBEh-3c & 16.1 & 8 \\
B97-3c & 23.0 & 9 \\
\hline
\end{tabular}

A set of popular methods currently used routinely is tested against benchmarks derived in this work for isolated clusters in the gas phase and the overall performance quality is rated as listed in Table 8.1. None of the tested approaches provides accurate predictions for all experimental observations, even though large error bounds are employed to account for the harmonic approximation used in most calculations. Future work may include additional experimental efforts to close the gap between harmonic theory and anharmonic experiment, for instance by measurements of vibrational overtones, combination bands and low frequency large amplitude motions. From the theory side 


\section{Conclusions and Outlook}

a reliable and cost-efficient description of vibrational anharmonicity and its effects on both molecular structures and vibrational spectra would be desirable. The benchmark values established in this work could then be used with the significantly lower purely experimental error bounds.
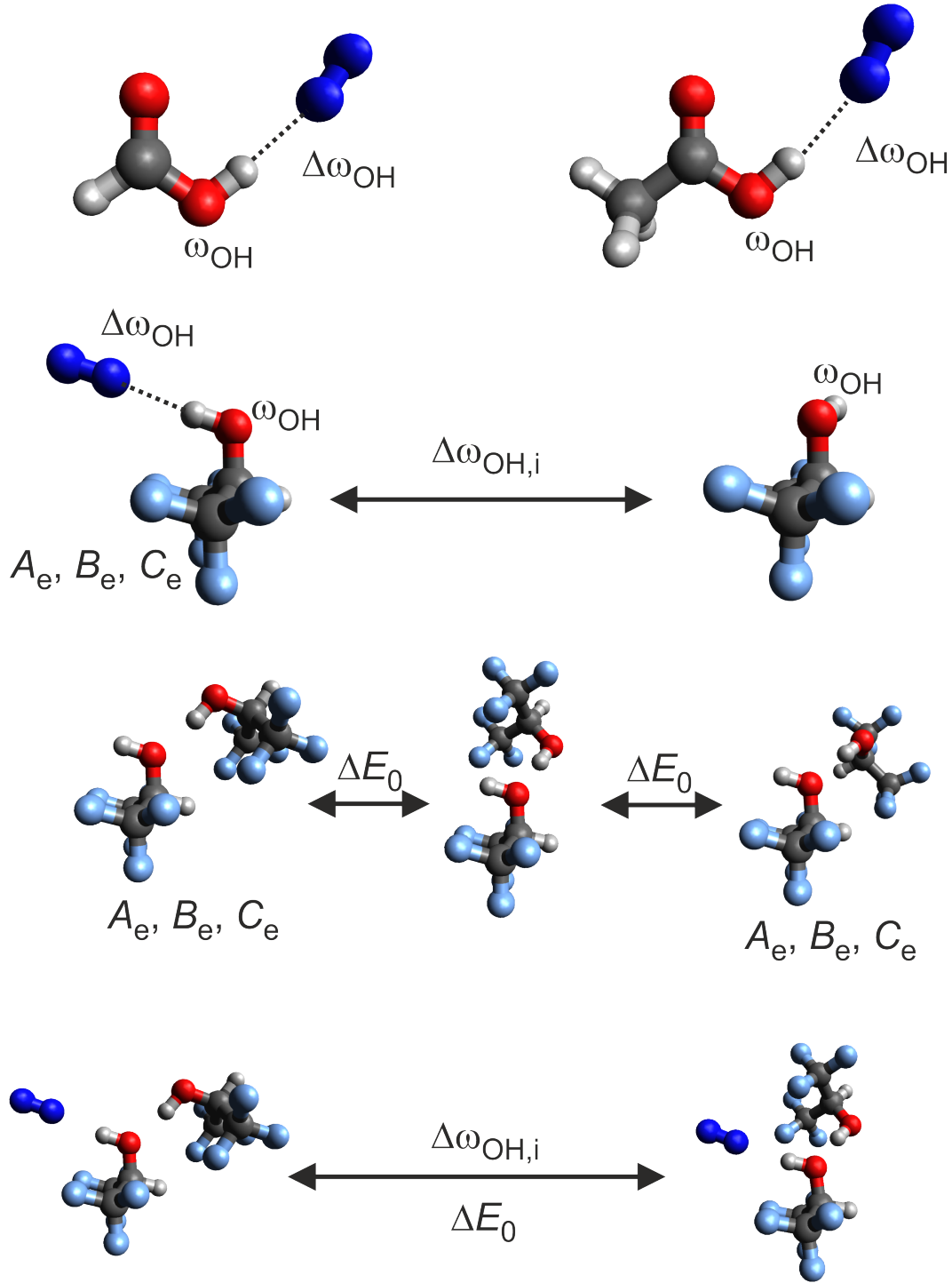

Figure 8.1.: Schematic summary of the experimental benchmarks developed throughout this work (see Table A.30 in appenix A.8 for details). The molecular test set is comprised of four isolated monomers and 8 complexes, while employed observables include harmonic monomer $\mathrm{OH}$ stretching band centre positions $\left(\omega_{\mathrm{OH}}\right)$, hydrogen bond induced spectral downshifts $\left(\Delta \omega_{\mathrm{OH}}\right)$, wavenumber splittings between different conformational isomers $\left(\Delta \omega_{\mathrm{OH}, \mathrm{i}}\right)$, equilibrium rotational constants $\left(A_{\mathrm{e}}, B_{\mathrm{e}}, C_{\mathrm{e}}\right)$ as well as zero-point-corrected relative conformational energies $\left(\Delta E_{0}\right)$. 


\section{Conclusions and Outlook}

A first step towards experimental benchmarks for theoretical descriptions of the chemically more relevant condensed phase is taken using cryogenic nitrogen matrices. Rigid molecules like pyrrole with only one stable ground state conformation show spectral splittings not observed in the gas phase due to population of different sites in the solid bulk matrix. Neural network potentials constitute a promising candidate for modelling such complex environments and their interactions with embedded molecules.

Experimental data on oxygen complexes are provided as well, although accurate quantum chemical calculations of the open-shell diradical are very demanding with regard to expertise and computation time. Therefore, future collaborations with interested research groups specialised in theoretical descriptions of open-shell systems may be required. Especially the binary formic acid oxygen dimer provides an interesting first step towards experimental benchmarks for diradicals due to its small size and the resulting comparatively modest demands on computational resources. 



\section{Bibliography}

\section{Bibliography}

[1] Moore, G. E., Cramming more Components onto Integrated Circuits, Electron., 1965, 38, 114-117.

[2] Thiel, W., Theoretical Chemistry - Quo Vadis?, Angew. Chem. Int. Ed., 2011, 50, 9216-9217.

[3] Vogiatzis, K. D., Ma, D., Olsen, J., Gagliardi, L., de Jong, W. A., Pushing Configuration-interaction to the Limit: Towards Massively Parallel MCSCF Calculations, J. Chem. Phys., 2017, 147, 184111.

[4] Riplinger, C., Sandhoefer, B., Hansen, A., Neese, F., Natural Triple Excitations in Local Coupled Cluster Calculations with Pair Natural Orbitals, J. Chem. Phys., 2013, 139, 134101.

[5] Dirac, P. A. M., Quantum Mechanics of Many-Electron Systems, Proc. R. Soc. London Ser. A, 1929, 123, 714-733.

[6] Quack, M., How Important is Parity Violation for Molecular and Biomolecular Chirality?, Angew. Chem. Int. Ed., 2002, 41, 4618-4630.

[7] Kolos, W., Wolniewicz, L., Improved Theoretical Ground-State Energy of the Hydrogen Molecule, J. Chem. Phys., 1968, 49, 404-410.

[8] Herzberg, G., Monfils, A., The Dissociation Energies of the $\mathrm{H}_{2}, \mathrm{HD}$, and $\mathrm{D}_{2}$ Molecules, J. Mol. Spectrosc., 1961, 5, 482-498.

[9] Herzberg, G., The Dissociation Energy of the Hydrogen Molecule, J. Mol. Spectrosc., 1970, 33, 147-168.

[10] Liu, J., Salumbides, E. J., Hollenstein, U., Koelemeij, J. C. J., Eikema, K. S. E., Ubachs, W., Merkt, F., Determination of the Ionization and Dissociation Energies of the Hydrogen Molecule, J. Chem. Phys., 2009, 130, 174306.

[11] Mata, R. A., Suhm, M. A., Benchmarking Quantum Chemical Methods: Are We Heading in the Right Direction?, Angew. Chem. Int. Ed., 2017, 56, 11011-11018.

[12] Čížek, J., On the Correlation Problem in Atomic and Molecular Systems. Calculation of Wavefunction Components in Ursell-Type Expansion Using QuantumField Theoretical Methods, J. Chem. Phys., 1966, 45, 4256-4266. 


\section{Bibliography}

[13] Stillinger, F. H., Møller-Plesset Convergence Issues in Computational Quantum Chemistry, J. Chem. Phys., 2000, 112, 9711-9715.

[14] Perdew, J. P., Schmidt, K., Jacob's Ladder of Density Functional Approximations for the Exchange-correlation Energy, in van Doren, V. E., van Alseoy, K., Geerlings, P. (editors), Density Functional Theory and its Application to Materials, AIP, New York, 2001, 1-20.

[15] Sousa, S. F., Fernandes, P. A., Ramos, M. J., General Performance of Density Functionals, J. Phys. Chem. A, 2007, 111, 10439-10452.

[16] Jurecka, P., Sponer, J., Cerný, J., Hobza, P., Benchmark Database of Accurate (MP2 and CCSD(T) Complete Basis Set Limit) Interaction Energies of Small Model Complexes, DNA Base Pairs, and Amino Acid Pairs, Phys. Chem. Chem. Phys., 2006, 8, 1985-1993.

[17] Takatani, T., Hohenstein, E. G., Malagoli, M., Marshall, M. S., Sherrill, C. D., Basis Set Consistent Revision of the S22 Test Set of Noncovalent Interaction Energies, J. Chem. Phys., 2010, 132, 144104.

[18] Podeszwa, R., Patkowski, K., Szalewicz, K., Improved Interaction Energy Benchmarks for Dimers of Biological Relevance, Phys. Chem. Chem. Phys., 2010, 12, 5974-5979.

[19] Hujo, W., Grimme, S., Comparison of the Performance of Dispersion-corrected Density Functional Theory for Weak Hydrogen Bonds, Phys. Chem. Chem. Phys., 2011, 13, 13942-13950.

[20] Goerigk, L., Grimme, S., A Thorough Benchmark of Density Functional Methods for General Main Group Thermochemistry, Kinetics, and Noncovalent Interactions, Phys. Chem. Chem. Phys., 2011, 13, 6670-6688.

[21] Goerigk, L., Grimme, S., Efficient and Accurate Double-Hybrid-Meta-GGA Density Functionals-Evaluation with the Extended GMTKN30 Database for General Main Group Thermochemistry, Kinetics, and Noncovalent Interactions, J. Chem. Theory Comput., 2011, 7, 291-309.

[22] Knochenmuss, R., Sinha, R. K., Leutwyler, S., Intermolecular Dissociation Energies of Dispersively Bound Complexes of Aromatics with Noble Gases and Nitrogen, J. Chem. Phys., 2018, 148, 134302.

[23] Ramabhadran, R. O., Raghavachari, K., Extrapolation to the Gold-Standard in Quantum Chemistry: Computationally Efficient and Accurate CCSD(T) Energies for Large Molecules Using an Automated Thermochemical Hierarchy, J. Chem. Theory Comput., 2013, 9, 3986-3994. 


\section{Bibliography}

[24] Yamada, Y., Hongo, K., Egashira, K., Kita, Y., Nagashima, U., Tachikawa, M., Gold-standard Coupled-cluster Study of the Ground-state Chromium Dimer Cation, Chem. Phys. Lett., 2013, 555, 84-86.

[25] Witte, J., Goldey, M., Neaton, J. B., Head-Gordon, M., Beyond Energies: Geometries of Nonbonded Molecular Complexes as Metrics for Assessing Electronic Structure Approaches, J. Chem. Theory Comput., 2015, 11, 1481-1492.

[26] Anacker, T., Friedrich, J., New Accurate Benchmark Energies for Large Water Clusters: DFT is Better than Expected, J. Comput. Chem., 2014, 35, 634-643.

[27] Lejaeghere, K., Bihlmayer, G., Björkman, T., Blaha, P., Blügel, S., Blum, V., Caliste, D., Castelli, I. E., Clark, S. J., Dal Corso, A., de Gironcoli, S., Deutsch, T., Dewhurst, J. K., Di Marco, I., Draxl, C., Dułak, M., Eriksson, O., Flores-Livas, J. A., Garrity, K. F., Genovese, L., Giannozzi, P., Giantomassi, M., Goedecker, S., Gonze, X., Grånäs, O., Gross, E. K. U., Gulans, A., Gygi, F., Hamann, D. R., Hasnip, P. J., Holzwarth, N. A. W., Iuşan, D., Jochym, D. B., Jollet, F., Jones, D., Kresse, G., Koepernik, K., Küçükbenli, E., Kvashnin, Y. O., Locht, I. L. M., Lubeck, S., Marsman, M., Marzari, N., Nitzsche, U., Nordström, L., Ozaki, T., Paulatto, L., Pickard, C. J., Poelmans, W., Probert, M. I. J., Refson, K., Richter, M., Rignanese, G.-M., Saha, S., Scheffler, M., Schlipf, M., Schwarz, K., Sharma, S., Tavazza, F., Thunström, P., Tkatchenko, A., Torrent, M., Vanderbilt, D., van Setten, M. J., van Speybroeck, V., Wills, J. M., Yates, J. R., Zhang, G.-X., Cottenier, S., Reproducibility in Density Functional Theory Calculations of Solids, Science, 2016, 351, 1415.

[28] Hickey, A. L., Rowley, C. N., Benchmarking Quantum Chemical Methods for the Calculation of Molecular Dipole Moments and Polarizabilities, J. Phys. Chem. A, 2014, 118, 3678-3687.

[29] Howard, J. C., Tschumper, G. S., Benchmark Structures and Harmonic Vibrational Frequencies Near the CCSD(T) Complete Basis Set Limit for Small Water Clusters: $\left(\mathrm{H}_{2} \mathrm{O}\right)_{n}=2,3,4,5,6$, J. Chem. Theory Comput., 2015, 11, 2126-2136.

[30] Kollipost, F., Papendorf, K., Lee, Y.-F., Lee, Y.-P., Suhm, M. A., Alcohol Dimers - How Much Diagonal OH Anharmonicity?, Phys. Chem. Chem. Phys., 2014, 16, 15948-15956.

[31] Emmeluth, C., Dyczmons, V., Kinzel, T., Botschwina, P., Suhm, M. A., Yáñez, M., Combined Jet Relaxation and Quantum Chemical Study of the Pairing Preferences of Ethanol, Phys. Chem. Chem. Phys., 2005, 7, 991-997.

[32] Hearn, J. P. I., Cobley, R. V., Howard, B. J., High-resolution Spectroscopy of Induced Chiral Dimers: A Study of the Dimers of Ethanol by Fourier Transform Microwave Spectroscopy, J. Chem. Phys., 2005, 123, 134324. 


\section{Bibliography}

[33] Umer, M., Kopp, W. A., Leonhard, K., Efficient yet Accurate Approximations for Ab Initio Calculations of Alcohol Cluster Thermochemistry, J. Chem. Phys., 2015, 143, 214306.

[34] Heger, M., Mata, R. A., Suhm, M. A., Soft Hydrogen Bonds to Alkenes: the Methanol-ethene Prototype under Experimental and Theoretical Scrutiny, Chem. Sci., 2015, 6, 3738-3745.

[35] Herman, M., Georges, R., Hepp, M., Hurtmans, D., High Resolution Fourier Transform Spectroscopy of Jet-cooled Molecules, Int. Rev. Phys. Chem., 2000, 19, 277-325.

[36] Hagena, O. F., Obert, W., Cluster Formation in Expanding Supersonic Jets: Effect of Pressure, Temperature, Nozzle Size, and Test Gas, J. Chem. Phys., 1972, 56, 1793-1802.

[37] Fabricant, B., Krieger, D., Muenter, J. S., Molecular Beam Electric Resonance Study of Formaldehyde, Thioformaldehyde, and Ketene, J. Chem. Phys., 1977, 67, 1576-1586.

[38] Puzzarini, C., Stanton, J. F., Gauss, J., Quantum-chemical Calculation of Spectroscopic Parameters for Rotational Spectroscopy, Int. Rev. Phys. Chem., 2010, 29, 273-367.

[39] Kesharwani, M. K., Brauer, B., Martin, J. M. L., Frequency and Zero-point Vibrational Energy Scale Factors for Double-hybrid Density Functionals (and other Selected Methods): Can Anharmonic Force Fields be Avoided?, J. Phys. Chem. A, 2015, 119, 1701-1714.

[40] Risthaus, T., Steinmetz, M., Grimme, S., Implementation of Nuclear Gradients of Range-separated Hybrid Density Functionals and Benchmarking on Rotational Constants for Organic Molecules, J. Comput. Chem., 2014, 35, 1509-1516.

[41] Grimme, S., Steinmetz, M., Effects of London Dispersion Correction in Density Functional Theory on the Structures of Organic Molecules in the Gas Phase, Phys. Chem. Chem. Phys., 2013, 15, 16031-16042.

[42] Eisenschitz, R., London, F., Über das Verhältnis der van der Waalsschen Kräfte zu den homöopolaren Bindungskräften, Z. Physik, 1930, 60, 491-527.

[43] Huggins, M. L., 50 Years of Hydrogen Bond Theory, Angew. Chem. Int. Ed., 1971, 10, 147-152.

[44] Gray, C. G., On the Theory of Multipole Interactions, Can. J. Phys., 1968, 46, $135-139$. 


\section{Bibliography}

[45] Ritchie, G. L., Watson, J. N., Keir, R. I., Temperature Dependence of Electric Field-Gradient Induced Birefringence (Buckingham Effect) and Molecular Quadrupole Moment of $\mathrm{N}_{2}$. Comparison of Experiment and Theory, Chem. Phys. Lett., 2003, 370, 376-380.

[46] Buckingham, A. D., Disch, R. L., Dunmur, D. A., Quadrupole Moments of Some Simple Molecules, J. Am. Chem. Soc., 1968, 90, 3104-3107.

[47] Iogansen, A. V., Direct Proportionality of the Hydrogen Bonding Energy and the Intensification of the Stretching $\vee(\mathrm{XH})$ Vibration in Infrared Spectra, Spectrochim. Acta A, 1999, 55, 1585-1612.

[48] Poblotzki, A., Gottschalk, H. C., Suhm, M. A., Tipping the Scales: Spectroscopic Tools for Intermolecular Energy Balances, J. Phys. Chem. Lett., 2017, 8, 56565665 .

[49] Bocklitz, S., Suhm, M. A., Polymer Segments at the Folding Limit: Raman Scattering for the Diglyme Benchmark, ChemPhysChem, 2017, 18, 3570-3575.

[50] Poblotzki, A., Altnöder, J., Suhm, M. A., Subtle Solvation Behaviour of a Biofuel Additive: The Methanol Complex with 2,5-dimethylfuran, Phys. Chem. Chem. Phys., 2016, 18, 27265-27271.

[51] Gottschalk, H. C., Altnöder, J., Heger, M., Suhm, M. A., Control over the Hydrogen-bond Docking Site in Anisole by Ring Methylation, Angew. Chem. Int. Ed., 2016, 55, 1921-1924.

[52] Heger, M., Altnöder, J., Poblotzki, A., Suhm, M. A., To $\pi$ or not to $\pi-$ How Does Methanol Dock onto Anisole?, Phys. Chem. Chem. Phys., 2015, 17, 13045-13052.

[53] Lüttschwager, N. O. B., Wassermann, T. N., Mata, R. A., Suhm, M. A., The Last Globally Stable Extended Alkane, Angew. Chem. Int. Ed., 2013, 52, 463-466.

[54] Gottschalk, H. C., Poblotzki, A., Suhm, M. A., Al-Mogren, M. M., Antony, J., Auer, A. A., Baptista, L., Benoit, D. M., Bistoni, G., Bohle, F., Dahmani, R., Firaha, D., Grimme, S., Hansen, A., Harding, M. E., Hochlaf, M., Holzer, C., Jansen, G., Klopper, W., Kopp, W. A., Kröger, L. C., Leonhard, K., Mouhib, H., Neese, F., Pereira, M. N., Ulusoy, I. S., Wuttke, A., Mata, R. A., The Furan Microsolvation Blind Challenge for Quantum Chemical Methods: First Steps, J. Chem. Phys., 2018, 148, 014301.

[55] Whittle, E., Dows, D. A., Pimentel, G. C., Matrix Isolation Method for the Experimental Study of Unstable Species, J. Chem. Phys., 1954, 22, 1943-1944.

[56] van Thiel, M., Becker, E. D., Pimentel, G. C., Infrared Studies of Hydrogen Bonding of Methanol by the Matrix Isolation Technique, J. Chem. Phys., 1957, 27, 95-99. 


\section{Bibliography}

[57] Nelander, B., The Peroxy Radical as Hydrogen Bond Donor and Hydrogen Bond Acceptor. A Matrix Isolation Study, J. Phys. Chem. A, 1997, 101, 9092-9096.

[58] Perchard, J. P., Mielke, Z., Anharmonicity and Hydrogen Bonding: I. A Nearinfrared Study of Methanol Trapped in Nitrogen and Argon Matrices, Chem. Phys., 2001, 264, 221-234.

[59] Coussan, S., Loutellier, A., Perchard, J. P., Racine, S., Peremans, A., Tadjeddine, A., Zheng, W. Q., IR-induced Interconversions Between Five Conformers of Methanol Dimers Trapped in Nitrogen Matrix, Chem. Phys., 1997, 223, 279-292.

[60] Coussan, S., Bouteiller, Y., Perchard, J. P., Zheng, W. Q., Rotational Isomerism of Ethanol and Matrix Isolation Infrared Spectroscopy, J. Phys. Chem. A, 1998, 102, 5789-5793.

[61] Coussan, S., Loutellier, A., Perchard, J. P., Racine, S., Bouteiller, Y., Matrix Isolation Infrared Spectroscopy and DFT Calculations of Complexes Between Water and Nitrogen, J. Mol. Struct., 1998, 471, 37-47.

[62] Coussan, S., Alikhani, M. E., Perchard, J. P., Zheng, W. Q., Infrared-induced Isomerization of Ethanol Dimers Trapped in Argon and Nitrogen Matrices: Monochromatic Irradiation Experiments and DFT Calculations, J. Phys. Chem. A, 2000, 104, 5475-5483.

[63] Gastegger, M., Behler, J., Marquetand, P., Machine Learning Molecular Dynamics for the Simulation of Infrared Spectra, Chem. Sci., 2017, 8, 6924-6935.

[64] Behler, J., First Principles Neural Network Potentials for Reactive Simulations of Large Molecular and Condensed Systems, Angew. Chem. Int. Ed., 2017, 56, $12828-12840$.

[65] Behler, J., Constructing High-dimensional Neural Network Potentials: A Tutorial Review, Int. J. Quantum Chem., 2015, 115, 1032-1050.

[66] Hart, M. H., The Evolution of the Atmosphere of the Earth, Icarus, 1978, 33, 23-39.

[67] Rowland, B., Fisher, M., Devlin, J. P., Probing Icy Surfaces with the DanglingOH-mode Absorption: Large Ice Clusters and Microporous Amorphous Ice, $J$. Chem. Phys., 1991, 95, 1378-1384.

[68] Hujo, W., Gaus, M., Schultze, M., Kubař, T., Grunenberg, J., Elstner, M., Bauerecker, S., Effect of Nitrogen Adsorption on the Mid-infrared Spectrum of Water Clusters, J. Phys. Chem. A, 2011, 115, 6218-6225.

[69] Svishchev, I. M., Boyd, R. J., Van der Waals Complexes of Water with Oxygen and Nitrogen: Infrared Spectra and Atmospheric Implications, J. Phys. Chem. A, 1998, 102, 7294-7296. 


\section{Bibliography}

[70] Kjaergaard, H. G., Robinson, T. W., Howard, D. L., Daniel, J. S., Headrick, J. E., Vaida, V., Complexes of Importance to the Absorption of Solar Radiation, J. Phys. Chem. A, 2003, 107, 10680-10686.

[71] Baranov, Y. I., The Continuum Absorption in $\mathrm{H}_{2} \mathrm{O}+\mathrm{N}_{2}$ Mixtures in the 2000$3250 \mathrm{~cm}^{-1}$ Spectral Region at Temperatures from 326 to 363 K, J. Quant. Spectrosc. Radiat. Transfer, 2011, 112, 2281-2286.

[72] Baranov, Y. I., Lafferty, W. J., The Water Vapour Self- and Water-nitrogen Continuum Absorption in the 1000 and $2500 \mathrm{~cm}^{-1}$ Atmospheric Windows, Phil. Trans. R. Soc. A, 2012, 370, 2578-2589.

[73] Gomes, J. A. G., Gossage, J. L., Balu, H., Kesmez, M., Bowen, F., Lumpkin, R. S., Cocke, D. L., Experimental and Theoretical Study of the Atmospherically Important $\mathrm{O}_{2}-\mathrm{H}_{2} \mathrm{O}$ Complex, Spectrochim. Acta A, 2005, 61, 3082-3086.

[74] Baranov, Y. I., Lafferty, W. J., Fraser, G. T., Investigation of Collision-induced Absorption in the Vibrational Fundamental Bands of $\mathrm{O}_{2}$ and $\mathrm{N}_{2}$ at Elevated Temperatures, J. Mol. Spectrosc., 2005, 233, 160-163.

[75] Baranov, Y. I., Buryak, I. A., Lokshtanov, S. E., Lukyanchenko, V. A., Vigasin, A. A., $\mathrm{H}_{2} \mathrm{O}-\mathrm{N}_{2}$ Collision-induced Absorption Band Intensity in the Region of the $\mathrm{N}_{2}$ Fundamental: Ab Initio Investigation of its Temperature Dependence and Comparison with Laboratory Data, Phil. Trans. R. Soc. A, 2012, 370, 2691-2709.

[76] Kuma, S., Slipchenko, M. N., Kuyanov, K. E., Momose, T., Vilesov, A. F., Infrared Spectra and Intensities of the $\mathrm{H}_{2} \mathrm{O}$ and $\mathrm{N}_{2}$ Complexes in the Range of the $\nu_{1}$ - and $\nu_{3}$-bands of Water, J. Phys. Chem. A, 2006, 110, 10046-10052.

[77] Kuma, S., Slipchenko, M. N., Momose, T., Vilesov, A. F., Infrared Spectra and Intensities of $\mathrm{Ar}-\mathrm{H}_{2} \mathrm{O}$ and $\mathrm{O}_{2}-\mathrm{H}_{2} \mathrm{O}$ Complexes in the range of the $\nu_{3}$ Band of $\mathrm{H}_{2} \mathrm{O}$, J. Phys. Chem. A, 2010, 114, 9022-9027.

[78] Gutberlet, A., Schwaab, G., Havenith, M., High Resolution IR Spectroscopy of $\mathrm{HDO}$ and $\operatorname{HDO}\left(\mathrm{N}_{2}\right)_{n}$ in Helium Nanodroplets, J. Chem. Phys., 2010, 133, 154313.

[79] Springer, S. D., McElmurry, B. A., Wang, Z., Leonov, I. I., Lucchese, R. R., Bevan, J. W., Coudert, L. H., Rovibrational Analysis of the Water Bending Vibration in the Mid-infrared Spectrum of Atmospherically Significant $\mathrm{N}_{2}-\mathrm{H}_{2} \mathrm{O}$ Complex, Chem. Phys. Lett., 2015, 633, 229-233.

[80] Leung, H. O., Marshall, M. D., Suenram, R. D., Lovas, F. J., Microwave Spectrum and Molecular Structure of the $\mathrm{N}_{2}-\mathrm{H}_{2} \mathrm{O}$ Complex, J. Chem. Phys., 1989, 90, 700712 .

[81] Zhu, Y., Zheng, R., Li, S., Yang, Y., Duan, C., Infrared Spectra and Tunneling Dynamics of the $\mathrm{N}_{2}-\mathrm{D}_{2} \mathrm{O}$ and OC- $\mathrm{D}_{2} \mathrm{O}$ Complexes in the $\nu_{2}$ Bend Region of $\mathrm{D}_{2} \mathrm{O}$, J. Chem. Phys., 2013, 139, 214309. 


\section{Bibliography}

[82] Cappelletti, D., Candori, P., Roncaratti, L. F., Pirani, F., A Molecular Beam Scattering Study of the Weakly Bound Complexes of Water and Hydrogen Sulphide with the Main Components of Air, Mol. Phys., 2010, 108, 2179-2185.

[83] Cooper, P. D., Kjaergaard, H. G., Langford, V. S., McKinley, A. J., Quickenden, T. I., Robinson, T. W., Schofield, D. P., Infrared Identification of Matrix Isolated $\mathrm{H}_{2} \mathrm{O}_{-} \mathrm{O}_{2}$, J. Phys. Chem. A, 2005, 109, 4274-4279.

[84] Andrews, L., Davis, S. R., FTIR Observation of N三N Stretching Fundamentals in Hydrogen-bonded Complexes in Solid Argon, J. Chem. Phys., 1985, 83, 49834989.

[85] Sadlej, J., Rowland, B., Devlin, J. P., Buch, V., Vibrational Spectra of Water Complexes with $\mathrm{H}_{2}, \mathrm{~N}_{2}$, and CO, J. Chem. Phys., 1995, 102, 4804-4818.

[86] Fredin, L., Nelander, B., Ribbegård, G., On the Dimerization of Carbon Dioxide in Nitrogen and Argon Matrices, J. Mol. Spectrosc., 1974, 53, 410-416.

[87] MacLeod, K. C., McWilliams, S. F., Mercado, B. Q., Holland, P. L., Stepwise N-H Bond Formation From $\mathrm{N}_{2}$-Derived Iron Nitride, Imide and Amide Intermediates to Ammonia, Chem. Sci., 2016, 7, 5736-5746.

[88] Himmel, H.-J., Reiher, M., Intrinsic Dinitrogen Activation at Bare Metal Atoms, Angew. Chem. Int. Ed., 2006, 45, 6264-6288.

[89] Jose, K. V. J., Gadre, S. R., Sundararajan, K., Viswanathan, K. S., Effect of Matrix on IR Frequencies of Acetylene and Acetylene-Methanol Complex: Infrared Matrix Isolation and Ab Initio Study, J. Chem. Phys., 2007, 127, 104501.

[90] Zischang, J., Suhm, M. A., The OH Stretching Spectrum of Warm Water Clusters, J. Chem. Phys., 2014, 140, 064312.

[91] Suhm, M. A., Hydrogen Bond Dynamics in Alcohol Clusters, in Rice, S. A. (editor), Hydrogen Bond Dynamics in Alcohol Clusters, Advances in Chemical Physics, volume 142, John Wiley \& Sons, Inc, Hoboken, NJ, USA, 2009, 1-57.

[92] Provencal, R. A., Paul, J. B., Roth, K., Chapo, C., Casaes, R. N., Saykally, R. J., Tschumper, G. S., Schaefer, H. F., III., Infrared Cavity Ringdown Spectroscopy of Methanol Clusters: Single Donor Hydrogen Bonding, J. Chem. Phys., 1999, 110, 4258-4267.

[93] Reimann, B., Buchhold, K., Vaupel, S., Brutschy, B., Havlas, Z., Špirko, V., Hobza, P., Improper, Blue-Shifting Hydrogen Bond between Fluorobenzene and Fluoroform, J. Phys. Chem. A, 2001, 105, 5560-5566.

[94] Mahadevi, A. S., Sastry, G. N., Cooperativity in Noncovalent Interactions, Chem. Rev., 2016, 116, 2775-2825. 


\section{Bibliography}

[95] Zielke, P., Suhm, M. A., Concerted Proton Motion in Hydrogen-bonded Trimers: A Spontaneous Raman Scattering Perspective, Phys. Chem. Chem. Phys., 2006, $8,2826-2830$.

[96] Forsting, T., Gottschalk, H. C., Hartwig, B., Mons, M., Suhm, M. A., Correcting the Record: the Dimers and Trimers of trans-N-methylacetamide, Phys. Chem. Chem. Phys., 2017, 19, 10727-10737.

[97] Kollipost, F., Wugt Larsen, R., Domanskaya, A. V., Nörenberg, M., Suhm, M. A., Communication: The Highest Frequency Hydrogen Bond Vibration and an Experimental Value for the Dissociation Energy of Formic Acid Dimer, J. Chem. Phys., 2012, 136, 151101.

[98] Xue, Z., Suhm, M. A., Adding More Weight to a Molecular Recognition Unit: The Low-Frequency Modes of Carboxylic Acid Dimers, Mol. Phys., 2010, 108, 2279-2288.

[99] Heger, M., Diagonal and Off-Diagonal Anharmonicity in Hydrogen-Bonded Systems, PhD thesis, Georg-August-University, Göttingen, 2016.

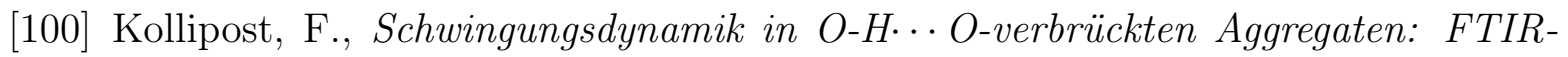
Spektroskopie vom Nah- bis zum Ferninfraroten, PhD thesis, Georg-AugustUniversity, Göttingen, 2015.

[101] Suhm, M. A., Kollipost, F., Femtisecond Single-mole Infrared Spectroscopy of Molecular Clusters, Phys. Chem. Chem. Phys., 2013, 15, 10702-10721.

[102] Meyer, K. A. E., Suhm, M. A., Formic Acid Aggregation in 2D Supersonic Expansions Probed by FTIR Imaging, J. Chem. Phys., 2017, 147, 144305.

[103] Han, H.-L., Camacho, C., Witek, H. A., Lee, Y.-P., Infrared Absorption of Methanol Clusters $\left(\mathrm{CH}_{3} \mathrm{OH}\right)_{n}$ with $n=2-6$ Recorded with a Time-of-flight Mass Spectrometer Using Infrared Depletion and Vacuum-Ultraviolet Ionization, $J$. Chem. Phys., 2011, 134, 144309.

[104] Omi, T., Shitomi, H., Sekiya, N., Takazawa, K., Fujii, M., Nonresonant Ionization Detected IR Spectroscopy for the Vibrational Study in a Supersonic Jet, Chem. Phys. Lett., 1996, 252, 287-293.

[105] Otto, K., Raman-Spektroskopie kleiner Moleküle und Molekülaggregate im Überschallstrahl nach thermischer Anregung, $\mathrm{PhD}$ thesis, Georg-AugustUniversity, Göttingen, 2015.

[106] Bocklitz, S., Conformational Spectroscopy of Flexible Chain Molecules Near the Folding Limit, PhD thesis, Georg-August-University, Göttingen, 2018.

[107] Zielke, P., Ramanstreuung am Überschallstrahl: Wasserstoffbrückendynamik aus neuer Perspektive, Cuvillier, Göttingen, 1st edition, 2007. 


\section{Bibliography}

[108] Lüttschwager, N. O. B., Raman Spectroscopy of Conformational Rearrangements at Low Temperatures: Folding and Stretching of Alkanes in Supersonic Jets, Springer theses, Springer, Cham, 2014.

[109] Forsting, T., Suhm, M. A., Curry-Jet SETUP, 2019, DOI:10.6084/m9.figshare.6395840.v1.

[110] Knözinger, E., Babka, E., Hallamasek, D., Cage Structure and Long-Range Order in Solid Rare Gas Matrixes: A Combined FTIR and XRD Study, J. Phys. Chem. A, 2001, 105, 8176-8182.

[111] Lorenz, M., Kraus, D., Räsänen, M., Bondybey, V. E., Photodissociation of Hydrogen Halides in Rare Gas Matrices, and the Effect of Hydrogen Bonding, $J$. Chem. Phys., 2000, 112, 3803-3811.

[112] Jacox, M. E., The Spectroscopy of Molecular Reaction Intermediates Trapped in the Solid Rare Gases, Chem. Soc. Rev., 2002, 31, 108-115.

[113] Perutz, R. N., Matrix Isolation, Annu. Rep. Prog. Chem., Sect. C, 1985, 82, 157191.

[114] Almond, M. J., Orrin, R. H., Matrix Isolation, Annu. Rep. Prog. Chem., Sect. C, 1991, 88, 3-44.

[115] Almond, M. J., Matrix Isolation, Annu. Rep. Prog. Chem., Sect. C, 1996, 93, $3-55$.

[116] Almond, M. J., Wiltshire, K. S., Matrix Isolation, Annu. Rep. Prog. Chem., Sect. $C, \mathbf{2 0 0 1}, 97,3-60$.

[117] Perchard, J. P., Romain, F., Bouteiller, Y., Determination of Vibrational Parameters of Methanol from Matrix-isolation Infrared Spectroscopy and Ab Initio Calculations. Part 1 - Spectral Analysis in the Domain 11000-200 $\mathrm{cm}^{-1}$, Chem. Phys., 2008, 343, 35-46.

[118] Schriver, L., Burneau, A., Perchard, J. P., Infrared Spectrum of the Methanol Dimer in Matrices. Temperature and Irradiation Effects in Solid Nitrogen, $J$. Chem. Phys., 1982, 77, 4926-4932.

[119] Murto, J., Ovaska, M., Matrix Infrared Study of the Specific Interactions Between Methanol and Nitrogen, and Methanol and Carbon Monoxide, Spectrochim. Acta A, 1983, 39, 149-152.

[120] Heger, M., Andersen, J., Suhm, M. A., Wugt Larsen, R., The Donor OH Stretching-libration Dynamics of Hydrogen-bonded Methanol Dimers in Cryogenic Matrices, Phys. Chem. Chem. Phys., 2016, 18, 3739-3745. 


\section{Bibliography}

[121] Aldiyarov, A., Aryutkina, M., Drobyshev, A., Kurnosov, V., IR Spectroscopy of Ethanol in Nitrogen Cryomatrices with Different Concentration Ratios, Low. Temp. Phys., 2011, 37, 524-531.

[122] Korppi-Tommola, J., Association of tert-butyl Alcohol: A Matrix Infrared Study, J. Mol. Struct., 1977, 40, 13-23.

[123] Korppi-Tommola, J., Tert-butyl Alcohol - Matrix I.R. Spectra and Vibrational Assignment, Spectrochim. Acta A, 1978, 34, 1077-1085.

[124] Coussan, S., Technical drawing, SDM, Lab. PIIM, Université de Provence, Marseille, 2000.

[125] Wassermann, T., Umgebungseinflüsse auf die $C$-C-und $C$-O-Torsionsdynamik in Molekülen und Molekülaggregaten: Schwingungsspektroskopie bei tiefen Temperaturen, Logos, Berlin, 2010.

[126] Caminati, W., Grabow, J.-U., Advancements in Microwave Spectroscopy, in Frontiers and Advances in Molecular Spectroscopy, Elsevier, 2018, 569-598.

[127] Rosenberg, A., Ozier, I., Kudian, A. K., Pure Rotational Spectrum of $\mathrm{CH}_{4}, J$. Chem. Phys., 1972, 57, 568-569.

[128] Park, G. B., Field, R. W., Perspective: The First Ten Years of Broadband Chirped Pulse Fourier Transform Microwave Spectroscopy, J. Chem. Phys., 2016, 144 , 200901.

[129] Patterson, D., Schnell, M., Doyle, J. M., Enantiomer-specific Detection of Chiral Molecules via Microwave Spectroscopy, Nature, 2013, 497, 475-477.

[130] Balle, T. J., Campbell, E. J., Keenan, M. R., Flygare, W. H., A New Method for Observing the Rotational Spectra of Weak Molecular Complexes: $\mathrm{KrHCl}, J$. Chem. Phys., 1979, 71, 2723-2724.

[131] Balle, T. J., Flygare, W. H., Fabry-Perot Cavity Pulsed Fourier Transform Microwave Spectrometer with a Pulsed Nozzle Particle Source, Rev. Sci. Instrum., 1981, 52, 33-45.

[132] Brown, G. G., Dian, B. C., Douglass, K. O., Geyer, S. M., Pate, B. H., The Rotational Spectrum of Epifluorohydrin Measured by Chirped-pulse Fourier Transform Microwave Spectroscopy, J. Mol. Spectrosc., 2006, 238, 200-212.

[133] Brown, G. G., Dian, B. C., Douglass, K. O., Geyer, S. M., Shipman, S. T., Pate, B. H., A Broadband Fourier Transform Microwave Spectrometer Based on Chirped Pulse Excitation, Rev. Sci. Instrum., 2008, 79, 053103.

[134] Dempster, S. P., Sukhorukov, O., Lei, Q.-Y., Jäger, W., Rotational Spectroscopic Study of Hydrogen Cyanide Embedded in Small ${ }^{4}$ He Clusters, J. Chem. Phys., 2012, 137, 174303. 


\section{Bibliography}

[135] Thomas, J., Yiu, J., Rebling, J., Jäger, W., Xu, Y., Chirped-pulse and Cavitybased Fourier Transform Microwave Spectroscopy of a Chiral Epoxy Ester: Methyl Glycidate, J. Phys. Chem. A, 2013, 117, 13249-13254.

[136] Huang, W., Thomas, J., Jäger, W., Xu, Y., Tunnelling and Barrier-less Motions in the 2-fluoroethanol-water Complex: A Rotational Spectroscopic and Ab Initio Study, Phys. Chem. Chem. Phys., 2017, 19, 12221-12228.

[137] Pérez, C., Lobsiger, S., Seifert, N. A., Zaleski, D. P., Temelso, B., Shields, G. C., Kisiel, Z., Pate, B. H., Broadband Fourier Transform Rotational Spectroscopy for Structure Determination: The Water Heptamer, Chem. Phys. Lett., 2013, 571, $1-15$.

[138] Carlson, C. D., Seifert, N. A., Heger, M., Xie, F., Thomas, J., Xu, Y., Conformational Dynamics of 1-phenyl-2,2,2-trifluoroethanol by Rotational Spectroscopy and Ab Initio Calculations, J. Mol. Spectrosc., 2018, 351, 62-67.

[139] Thomas, J., Seifert, N. A., Jäger, W., Xu, Y., A Direct Link from the Gas to the Condensed Phase: A Rotational Spectroscopic Study of 2,2,2-Trifluoroethanol Trimers, Angew. Chem. Int. Ed., 2017, 56, 6289-6293.

[140] Xu, Y., Jäger, W., Evidence for Heavy Atom Large Amplitude Motions in RGcyclopropane van der Waals Complexes $(\mathrm{RG}=\mathrm{Ne}, \mathrm{Ar}, \mathrm{Kr})$ from Rotation-tunneling Spectroscopy, J. Chem. Phys., 1997, 106, 7968-7980.

[141] Frisch, M. J., Trucks, G. W., Schlegel, H. B., Scuseria, G. E., Robb, M. A., Cheeseman, J. R., Scalmani, G., Barone, V., Mennucci, B., Petersson, G. A., Nakatsuji, H., Caricato, M., Li, X., Hratchian, H. P., Izmaylov, A. F., Bloino, J., Zheng, G., Sonnenberg, J. L., Hada, M., Ehara, M., Toyota, K., Fukuda, R., Hasegawa, J., Ishida, M., Nakajima, T., Honda, Y., Kitao, O., Nakai, H., Vreven, T., Montgomery, J. A., Peralta, J. E., Ogliaro, F., Bearpark, M., Heyd, J. J., Brothers, E., Kudin, K. N., Staroverov, V. N., Kobayashi, R., Normand, J., Raghavachari, K., Rendell, A., Burant, J. C., Iyengar, S. S., Tomasi, J., Cossi, M., Rega, N., Millam, J. M., Klene, M., Knox, J. E., Cross, J. B., Bakken, V., Adamo, C., Jaramillo, J., Gomperts, R., Stratmann, R. E., Yazyev, O., Austin, A. J., Cammi, R., Pomelli, C., Ochterski, J. W., Martin, R. L., Morokuma, K., Zakrzewski, V. G., Voth, G. A., Salvador, P., Dannenberg, J. J., Dapprich, S., Daniels, A. D., Farkas, ., Foresman, J. B., Ortiz, J. V., Cioslowski, J., Fox, D. J., Gaussian 09 Revision E.01, gaussian Inc. Wallingford CT 2009.

[142] Neese, F., The ORCA Program System, WIREs Comput. Mol. Sci., 2012, 2, $73-78$.

[143] Werner, H.-J., Knowles, P. J., Knizia, G., Manby, F. R., Schütz, M., Molpro: A General-Purpose Quantum Chemistry Program Package, WIREs Comput. Mol. Sci., 2012, 2, 242-253. 


\section{Bibliography}

[144] Grimme, S., Antony, J., Ehrlich, S., Krieg, H., A Consistent and Accurate Ab Initio Parametrization of Density Functional Dispersion Correction (DFT-D) for the 94 Elements H-Pu, J. Chem. Phys., 2010, 132, 154104.

[145] Grimme, S., Ehrlich, S., Goerigk, L., Effect of the Damping Function in Dispersion Corrected Density Functional Theory, J. Comput. Chem., 2011, 32, 1456-1465.

[146] Grimme, S., Brandenburg, J. G., Bannwarth, C., Hansen, A., Consistent Structures and Interactions by Density Functional Theory with Small Atomic Orbital Basis Sets, J. Chem. Phys., 2015, 143, 054107.

[147] Grimme, S., Bannwarth, C., Shushkov, P., A Robust and Accurate Tight-Binding Quantum Chemical Method for Structures, Vibrational Frequencies, and Noncovalent Interactions of Large Molecular Systems Parametrized for All spd-Block Elements $(\mathrm{Z}=1-86)$, J. Chem. Theory Comput., 2017, 13, 1989-2009.

[148] Bloino, J., Barone, V., A Second-order Perturbation Theory Route to Vibrational Averages and Transition Properties of Molecules: General Formulation and Application to Infrared and Vibrational Circular Dichroism Spectroscopies, J. Chem. Phys., 2012, 136, 124108.

[149] Bally, T., Borden, W. T., Calculations on Open-Shell Molecules: A Beginner's Guide, in Lipkowitz, K. B., Boyd, D. B. (editors), Reviews in Computational Chemistry, Wiley-VCH, New York, N.Y., Reviews in Computational Chemistry, 1999, 1-97.

[150] Hegarty, D., Robb, M. A., Application of Unitary Group Methods to Configuration Interaction Calculations, Mol. Phys., 1979, 38, 1795-1812.

[151] Werner, H.-J., Matrix-formulated Direct Multiconfiguration Self-consistent Field and Multiconfiguration Interaction Methods Reference Configuration, in Lawley, K. P. (editor), Ab Initio Methods in Quantum Chemistry Part 2, Wiley, Chichester and New York, Advances in Chemical Physics, 2007, 1-62.

[152] Simm, G. N., Proppe, J., Reiher, M., Error Assessment of Computational Models in Chemistry, Chimia, 2017, 71, 202-208.

[153] Ruscic, B., Uncertainty Quantification in Thermochemistry, Benchmarking Electronic Structure Computations, and Active Thermochemical Tables, Int. J. Quantum Chem., 2014, 114, 1097-1101.

[154] Chai, T., Draxler, R. R., Root Mean Square Error (RMSE) or Mean Absolute Error (MAE)? Arguments Against Avoiding RMSE in the Literature, Geosci. Model Dev., 2014, 7, 1247-1250.

[155] Bronstein, I. N., Semendjajew, K. A., Taschenbuch der Mathematik, BSB B. G. Teubner Verlagsgesellschaft, Leipzig, 22nd edition, 1985. 


\section{Bibliography}

[156] Meyer, E., Schwingungsspektroskopie von Aggregaten aus Carbonsäuren mit Luftbestandteilen, B.Sc. thesis, Georg-August-University, Göttingen, 2017.

[157] Oswald, S., Meyer, E., Suhm, M. A., Dinitrogen as a Sensor for Metastable Carboxylic Acid Dimers and a Weak Hydrogen Bond Benchmarking Tool, J. Phys. Chem. A, 2018, 122, 2933-2946.

[158] Yu, S., Role of Organic Acids (Formic, Acetic, Pyruvic and Oxalic) in the Formation of Cloud Condensation Nuclei (CCN): A Review, Atmos. Res., 2000, 53, $185-217$.

[159] Lundell, J., Räsänen, M., Latajka, Z., Matrix Isolation FTIR and Ab Initio Study of Complexes Between Formic Acid and Nitrogen, Chem. Phys., 1994, 189, 245260 .

[160] Lopes, S., Domanskaya, A. V., Fausto, R., Räsänen, M., Khriachtchev, L., Formic and Acetic Acids in a Nitrogen Matrix: Enhanced Stability of the Higher-Energy Conformer, J. Chem. Phys., 2010, 133, 144507.

[161] Pettersson, M., Lundell, J., Khriachtchev, L., Räsänen, M., IR Spectrum of the Other Rotamer of Formic Acid, cis-HCOOH, J. Am. Chem. Soc., 1997, 119, 11715-11716.

[162] Maçôas, E. M., Lundell, J., Pettersson, M., Khriachtchev, L., Fausto, R., Räsänen, M., Vibrational Spectroscopy of cis- and trans-Formic Acid in Solid Argon, J. Mol. Spectrosc., 2003, 219, 70-80.

[163] Khriachtchev, L., Rotational Isomers of Small Molecules in Noble-Gas Solids: From Monomers to Hydrogen-Bonded Complexes, J. Mol. Struct., 2008, 880, $14-22$.

[164] Domanskaya, A., Marushkevich, K., Khriachtchev, L., Räsänen, M., Spectroscopic Study of cis-to-trans Tunneling Reaction of HCOOD in Rare Gas Matrices, $J$. Chem. Phys., 2009, 130, 154509.

[165] Pettersson, M., Maçôas, E. M. S., Khriachtchev, L., Lundell, J., Fausto, R., Räsänen, M., Cis $\rightarrow$ trans Conversion of Formic Acid by Dissipative Tunneling in Solid Rare Gases: Influence of Environment on the Tunneling Rate, J. Chem. Phys., 2002, 117, 9095-9098.

[166] Pettersson, M., Maçôas, E. M. S., Khriachtchev, L., Fausto, R., Räsänen, M., Conformational Isomerization of Formic Acid by Vibrational Excitation at Energies Below the Torsional Barrier, J. Am. Chem. Soc., 2003, 125, 4058-4059.

[167] Maçôas, E. M. S., Khriachtchev, L., Pettersson, M., Fausto, R., Räsänen, M., Rotational Isomerism of Acetic Acid Isolated in Rare-Gas Matrices: Effect of Medium and Isotopic Substitution on IR-Induced Isomerization Quantum Yield and cis $\rightarrow$ trans Tunneling Rate, J. Chem. Phys., 2004, 121, 1331-1338. 


\section{Bibliography}

[168] Maçôas, E. M. S., Khriachtchev, L., Pettersson, M., Fausto, R., Räsänen, M., Rotational Isomerization of Small Carboxylic Acids Isolated in Argon Matrices: Tunnelling and Quantum Yields for the Photoinduced Processes, Phys. Chem. Chem. Phys., 2005, 7, 743-749.

[169] Baskakov, O. I., Horneman, V.-M., Lohilahti, J., Alanko, S., High Resolution FTIR Spectra of the $\nu_{9}$ Vibrational Band of cis-rotamers $\mathrm{HCOOH}$ and $\mathrm{H}^{13} \mathrm{COOH}$, J. Mol. Struct., 2006, 795, 49-53.

[170] Meyer, K. A. E., Suhm, M. A., Vibrational Exciton Coupling in Homo and Hetero Dimers of Carboxylic Acids Studied by Linear Infrared and Raman Jet Spectroscopy, J. Chem. Phys., 2018, 149, 104307.

[171] Hocking, W. H., The Other Rotamer of Formic Acid, cis-HCOOH, Z. Naturforsch. A, 1976, 31, 1113-1121.

[172] Winkler, A., Hess, P., Study of the Energetics and Dynamics of Hydrogen Bond Formation in Aliphatic Carboxylic Acid Vapors by Resonant Photoacoustic Spectroscopy, J. Am. Chem. Soc., 1994, 116, 9233-9240.

[173] Hippler, M., Proton Relaxation and Intermolecular Structure of Liquid Formic Acid: A Nuclear Magnetic Resonance Study Dedicated to the Memory of the Late Hermann Gerhard Hertz, Phys. Chem. Chem. Phys., 2002, 4, 1457-1463.

[174] Ito, F., Nakanaga, T., A Jet-Cooled Infrared Spectrum of the Formic Acid Dimer by Cavity Ring-Down Spectroscopy, Chem. Phys. Lett., 2000, 318, 571-577.

[175] Ito, F., Nakanaga, T., Jet-Cooled Infrared Spectra of the Formic Acid Dimer by Cavity Ring-Down Spectroscopy: Observation of the O-H Stretching Region, Chem. Phys., 2002, 277, 163-169.

[176] Ito, F., Jet-Cooled Infrared Spectra of the Formic Acid Dimer by Cavity RingDown Spectroscopy: Observation of the C-O Stretching Region and Vibrational Analysis of the Fermi-Triad System, Chem. Phys. Lett., 2007, 447, 202-207.

[177] Birer, Ö., Havenith, M., High-Resolution Infrared Spectroscopy of the Formic Acid Dimer, Annu. Rev. Phys. Chem., 2009, 60, 263-275.

[178] Millikan, R. C., Pitzer, K. S., The Infrared Spectra of Dimeric and Crystalline Formic Acid, J. Am. Chem. Soc., 1958, 80, 3515-3521.

[179] Maréchal, Y., IR Spectra of Carboxylic Acids in the Gas Phase: A Quantitative Reinvestigation, J. Chem. Phys., 1987, 87, 6344-6353.

[180] Halupka, M., Sander, W., A Simple Method for the Matrix Isolation of Monomeric and Dimeric Carboxylic Acids, Spectrochim. Acta A, 1998, 54, 495-500. 


\section{Bibliography}

[181] Häber, T., Schmitt, U., Emmeluth, C., Suhm, M. A., Ragout-Jet FTIR Spectroscopy of Cluster Isomerism and Cluster Dynamics: From Carboxylic Acid Dimers to $\mathrm{N}_{2} \mathrm{O}$ Nanoparticles, Faraday Disc., 2001, 118, 331-359.

[182] Georges, R., Freytes, M., Hurtmans, D., Kleiner, I., Vander Auwera, J., Herman, M., Jet-Cooled and Room Temperature FTIR Spectra of the Dimer of Formic Acid in the Gas Phase, Chem. Phys., 2004, 305, 187-196.

[183] Emmeluth, C., Suhm, M. A., A Chemical Approach Towards the Spectroscopy of Carboxylic Acid Dimer Isomerism, Phys. Chem. Chem. Phys., 2003, 5, 3094-3099.

[184] Ito, F., Infrared Spectra of Formic Acid Clusters in Noble Gas Matrices, J. Mol. Struct., 2015, 1091, 203-209.

[185] Bertie, J. E., Michaelian, K. H., The Raman Spectrum of Gaseous Acetic Acid at $21{ }^{\circ} \mathrm{C}$, J. Chem. Phys., 1982, 77, 5267-5271.

[186] Bertie, J. E., Michaelian, K. H., The Raman Spectra of Gaseous Formic Acid $-h_{2}$ and $-d_{2}$, J. Chem. Phys., 1982, 76, 886-894.

[187] Zielke, P., Suhm, M. A., Raman Jet Spectroscopy of Formic Acid Dimers: Low Frequency Vibrational Dynamics and Beyond, Phys. Chem. Chem. Phys., 2007, 9, 4528-4534.

[188] Olbert-Majkut, A., Ahokas, J., Lundell, J., Pettersson, M., Raman Spectroscopy of Formic Acid and its Dimers Isolated in low Temperature Argon Matrices, Chem. Phys. Lett., 2009, 468, 176-183.

[189] Xue, Z., Suhm, M. A., Probing the Stiffness of the Simplest Double Hydrogen Bond: the Symmetric Hydrogen Bond Modes of Jet-Cooled Formic Acid Dimer, J. Chem. Phys., 2009, 131, 054301.

[190] Emmeluth, C., Suhm, M. A., Luckhaus, D., A Monomers-in-Dimers Model for Carboxylic Acid Dimers, J. Chem. Phys., 2003, 118, 2242-2255.

[191] Florio, G. M., Zwier, T. S., Myshakin, E. M., Jordan, K. D., Sibert, E. L., Theoretical Modeling of the OH Stretch Infrared Spectrum of Carboxylic Acid Dimers Based on First-Principles Anharmonic Couplings, J. Chem. Phys., 2003, 118, 1735-1746.

[192] Shipman, S. T., Douglass, P. C., Yoo, H. S., Hinkle, C. E., Mierzejewski, E. L., Pate, B. H., Vibrational Dynamics of Carboxylic Acid Dimers in Gas and Dilute Solution, Phys. Chem. Chem. Phys., 2007, 9, 4572-4586.

[193] Sablinskas, V., Pucetaite, M., Ceponkus, J., Kimtys, L., Structure of Propanoic Acid Dimers as Studied by Means of MIR and FIR Spectroscopy, J. Mol. Struct., 2010, 976, 263-269. 


\section{Bibliography}

[194] Gantenberg, M., Halupka, M., Sander, W., Dimerization of Formic Acid-An Example of a "Noncovalent" Reaction Mechanism, Chem. Eur. J., 2000, 6, 1865-1869.

[195] Balabin, R. M., Polar (Acyclic) Isomer of Formic Acid Dimer: Gas-Phase Raman Spectroscopy Study and Thermodynamic Parameters, J. Phys. Chem. A, 2009, 113, 4910-4918.

[196] Marushkevich, K., Khriachtchev, L., Lundell, J., Domanskaya, A., Räsänen, M., Matrix Isolation and Ab Initio Study of Trans-Trans and Trans-Cis Dimers of Formic Acid, J. Phys. Chem. A, 2010, 114, 3495-3502.

[197] Marushkevich, K., Siltanen, M., Räsänen, M., Halonen, L., Khriachtchev, L., Identification of New Dimers of Formic Acid: The Use of a Continuous-Wave Optical Parametric Oscillator in Matrix Isolation Experiments, J. Phys. Chem. Lett., 2011, 2, 695-699.

[198] Sander, W., Gantenberg, M., Aggregation of Acetic and Propionic Acid in Argon Matrices - a Matrix Isolation and Computational Study, Spectrochim. Acta A, 2005, 62, 902-909.

[199] Madeja, F., Havenith, M., Nauta, K., Miller, R. E., Chocholousová, J., Hobza, P., Polar Isomer of Formic Acid Dimers Formed in Helium Nanodroplets, J. Chem. Phys., 2004, 120, 10554-10560.

[200] Madeja, F., Markwick, P., Havenith, M., Nauta, K., Miller, R. E., Rotationally Resolved Infrared Spectroscopy of $h_{2^{-}}$and $d_{1}$-Formic Acid Monomer in Liquid He Droplets, J. Chem. Phys., 2002, 116, 2870-2878.

[201] Redington, R. L., Vibrational Spectra and Normal Coordinate Analysis of Isotopically Labeled Formic Acid Monomers, J. Mol. Spectrosc., 1977, 65, 171-189.

[202] Hurtmans, D., Herregodts, F., Herman, M., Liévin, J., Campargue, A., Garnache, A., Kachanov, A. A., Spectroscopic and Ab Initio Investigation of the $\nu_{\mathrm{OH}}$ Overtone Excitation in trans-formic Acid, J. Chem. Phys., 2000, 113, 1535-1545.

[203] Freytes, M., Hurtmans, D., Kassi, S., Liévin, J., Vander Auwera, J., Campargue, A., Herman, M., Overtone Spectroscopy of Formic Acid, Chem. Phys., 2002, 283, $47-61$.

[204] Das, P., Knapp, C. J., Jäger, W., Ro-Vibrational Spectroscopy of the Formic Acid$d_{1}$ Monomer Embedded in Helium Nanodroplets, J. Mol. Spectrosc., 2017, 341, $17-22$.

[205] Lopes, S., Domanskaya, A. V., Räsänen, M., Khriachtchev, L., Fausto, R., Acetic Acid Dimers in a Nitrogen Matrix: Observation of Structures Containing the Higher-Energy Conformer, J. Chem. Phys., 2015, 143, 104307. 


\section{Bibliography}

[206] Jaffe, D., FT-I.R. Spectra of Acetic Acid and Deuterated Analogs in the Monomer O-H and O-D Regions, Spectrochim. Acta A, 1987, 43, 1393-1396.

[207] Herzberg, G., Molecular Spectra and Molecular Structure II: Infrared and Raman Spectra of Polyatomic Molecules, Van Nostrand, Princeton, 1939.

[208] Bonner, L. G., The Vibrational Spectrum of Water Vapor, Phys. Rev., 1934, 46, 458-464.

[209] Tew, D. P., Mizukami, W., Ab Initio Vibrational Spectroscopy of cis- and transFormic Acid from a Global Potential Energy Surface, J. Phys. Chem. A, 2016, 120, 9815-9828.

[210] Qu, C., Bowman, J. M., An Ab Initio Potential Energy Surface for the Formic Acid Dimer: Zero-Point Energy, Selected Anharmonic Fundamental Energies, and Ground-State Tunneling Splitting Calculated in Relaxed 1-4-Mode Subspaces, Phys. Chem. Chem. Phys., 2016, 18, 24835-24840.

[211] Richardson, J. O., Full- and Reduced-dimensionality Instanton Calculations of the Tunnelling Splitting in the Formic Acid Dimer, Phys. Chem. Chem. Phys., 2017, 19, 966-970.

[212] Sibaev, M., Crittenden, D. L., An Efficient and Numerically Stable Procedure for Generating Sextic Force Fields in Normal Mode Coordinates, J. Chem. Phys., 2016, 144, 214107.

[213] Richter, F., Carbonnière, P., Vibrational Treatment of the Formic Acid Double Minimum Case in Valence Coordinates, J. Chem. Phys., 2018, 148, 064303.

[214] Qu, C., Bowman, J. M., High-dimensional Fitting of Sparse Datasets of CCSD(T) Electronic Energies and MP2 Dipole Moments, Illustrated for the Formic Acid Dimer and its Complex IR Spectrum, J. Chem. Phys., 2018, 148, 241713.

[215] Mackeprang, K., Xu, Z.-H., Maroun, Z., Meuwly, M., Kjaergaard, H. G., Spectroscopy and Dynamics of Double Proton Transfer in Formic Acid Dimer, Phys. Chem. Chem. Phys., 2016, 18, 24654-24662.

[216] Shida, N., Barbara, P. F., Almlöf, J., A Reaction Surface Hamiltonian Treatment of the Double Proton Transfer of Formic Acid Dimer, J. Chem. Phys., 1991, 94, 3633-3643.

[217] Miura, S., Tuckerman, M. E., Klein, M. L., An Ab Initio Path Integral Molecular Dynamics Study of Double Proton Transfer in the Formic Acid Dimer, J. Chem. Phys., 1998, 109, 5290-5299.

[218] Ushiyama, H., Takatsuka, K., Successive Mechanism of Double-Proton Transfer in Formic Acid Dimer: A Classical Study, J. Chem. Phys., 2001, 115, 5903-5912. 


\section{Bibliography}

[219] Chocholoušová, J., Vacek, J., Hobza, P., Potential Energy and Free Energy Surfaces of the Formic Acid Dimer: Correlated Ab Initio Calculations and Molecular Dynamics Simulations, Phys. Chem. Chem. Phys., 2002, 4, 2119-2122.

[220] Luckhaus, D., Concerted Hydrogen Exchange Tunneling in Formic Acid Dimer, J. Phys. Chem. A, 2006, 110, 3151-3158.

[221] Jain, A., Sibert, E. L., Tunneling Splittings in Formic Acid Dimer: an Adiabatic Approximation to the Herring Formula, J. Chem. Phys., 2015, 142, 084115.

[222] Vener, M. V., Kühn, O., Bowman, J. M., Vibrational Spectrum of the Formic Acid Dimer in the $\mathrm{OH}$ Stretch Region. A Model 3D Study, Chem. Phys. Lett., 2001, $349,562-570$.

[223] Luckhaus, D., Hydrogen Exchange in Formic Acid Dimer: Tunnelling Above the Barrier, Phys. Chem. Chem. Phys., 2010, 12, 8357-8361.

[224] Dreyer, J., Hydrogen-bonded Acetic Acid Dimers: Anharmonic Coupling and Linear Infrared Spectra Studied with Density-functional Theory, J. Chem. Phys., 2005, 122, 184306.

[225] Miliordos, E., Xantheas, S. S., On the Validity of the Basis Set Superposition Error and Complete Basis Set Limit Extrapolations for the Binding Energy of the Formic Acid Dimer, J. Chem. Phys., 2015, 142, 094311.

[226] Takami, M., Shimoda, K., Microwave Spectrum of $\mathrm{HCOOH}$ in the $\nu_{\mathrm{CH}}=1$ Vibrational State Observed by Laser-Microwave Double and Triple Resonance, Jpn. J. Appl. Phys., 1974, 13, 1699-1706.

[227] Golebiowski, D., Földes, T., Vanfleteren, T., Herman, M., Perrin, A., Complementary Cavity-Enhanced Spectrometers to Investigate the $\mathrm{OH}+\mathrm{CH}$ Combination Band in trans-Formic Acid, J. Chem. Phys., 2015, 143, 014201.

[228] Hisatsune, I. C., Heicklen, J., Are There 2 Structural Isomers of Formic-Acid, Can. J. Spectrosc., 1973, 18, 135-142.

[229] Millikan, R. C., Pitzer, K. S., Infrared Spectra and Vibrational Assignment of Monomeric Formic Acid, J. Chem. Phys., 1957, 27, 1305-1308.

[230] Deroche, J.-C., Kauppinen, J., Kyrö, E., $\nu_{7}$ and $\nu_{9}$ Bands of Formic Acid near 16 um, J. Mol. Spectrosc., 1979, 78, 379-394.

[231] Heger, M., Suhm, M. A., Mata, R. A., Communication: Towards the Binding Energy and Vibrational Red Shift of the Simplest Organic Hydrogen Bond: Harmonic Constraints for Methanol Dimer, J. Chem. Phys., 2014, 141, 101105.

[232] Lange, K. R., Wells, N. P., Plegge, K. S., Phillips, J. A., Integrated Intensities of $\mathrm{O}-\mathrm{H}$ Stretching Bands: Fundamentals and Overtones in Vapor-Phase Alcohols and Acids, J. Phys. Chem. A, 2001, 105, 3481-3486. 


\section{Bibliography}

[233] Bannwarth, C., Ehlert, S., Grimme, S., GFN2-xTB - an Accurate and Broadly Parametrized Self-Consistent Tight-Binding Quantum Chemical Method with Multipole Electrostatics and Density-Dependent Dispersion Contributions, DOI:10.26434/chemrxiv.7246238, 2018.

[234] Oswald, S., Suhm, M. A., Experimental Reference Data for Hexafluorinated Propanol by Exploring an Unusual Intermolecular Torsional Balance, Angew. Chem. Int. Ed., 2017, 56, 12672-12676.

[235] Oswald, S., Seifert, N. A., Bohle, F., Gawrilow, M., Grimme, S., Jäger, W., Xu, Y., Suhm, M. A., The Chiral Trimer and a Metastable Chiral Dimer of Achiral Hexafluoroisopropanol: A Multi-messenger Study, Angew. Chem. Int. Ed., 2019, accepted, DOI:10.1002/anie.201813881.

[236] Narita, M., Honda, S., Umeyama, H., Obana, S., The Solubility of Peptide Intermediates in Organic Solvents. Solubilizing Potential of Hexafluoro-2-propanol, Bull. Chem. Soc. Jpn., 1988, 61, 281-284.

[237] Bégué, J.-P., Bonnet-Delpon, D., Crousse, B., Fluorinated Alcohols: A New Medium for Selective and Clean Reaction, Synlett, 2004, 18-29.

[238] Manchineella, S., Prathyusha, V., Priyakumar, U. D., Govindaraju, T., Solventinduced Helical Assembly and Reversible Chiroptical Switching of Chiral Cyclicdipeptide-functionalized Naphthalenediimides, Chemistry, 2013, 19, 16615-16624.

[239] Berkessel, A., Adrio, J. A., Dramatic Acceleration of Olefin Epoxidation in Fluorinated Alcohols: Activation of Hydrogen Peroxide by Multiple H-Bond Networks, J. Am. Chem. Soc., 2006, 128, 13412-13420.

[240] Takita, R., Ohshima, T., Shibasaki, M., Highly Enantioselective Catalytic Michael Reaction of $\alpha$-substituted Malonates Using La-linked-BINOL Complex in the Presence of HFIP (1,1,1,3,3,3-hexafluoroisopropanol), Tetrahedron Lett., 2002, 43, $4661-4665$.

[241] Dherbassy, Q., Schwertz, G., Chessé, M., Hazra, C. K., Wencel-Delord, J., Colobert, F., 1,1,1,3,3,3-Hexafluoroisopropanol as a Remarkable Medium for Atroposelective Sulfoxide-Directed Fujiwara-Moritani Reaction with Acrylates and Styrenes, Chemistry, 2016, 22, 1735-1743.

[242] Colomer, I., Batchelor-McAuley, C., Odell, B., Donohoe, T. J., Compton, R. G., Hydrogen Bonding to Hexafluoroisopropanol Controls the Oxidative Strength of Hypervalent Iodine Reagents, J. Am. Chem. Soc., 2016, 138, 8855-8861.

[243] Reddy, S. M. M., Shanmugam, G., Mandal, A. B., 1,1,1,3,3,3-Hexafluoro-2propanol and 2,2,2-Trifluoroethanol Solvents Induce Self-assembly with Different Surface Morphology in an Aromatic Dipeptide, Org. Biomol. Chem., 2014, 12, 6181-6189. 


\section{Bibliography}

[244] Lin, Y., Lee, Y.-H., Yoshimura, Y., Yagi, H., Goto, Y., Solubility and Supersaturation-dependent Protein Misfolding Revealed by Ultrasonication, Langmuir, 2014, 30, 1845-1854.

[245] Gerling, U. I. M., Salwiczek, M., Cadicamo, C. D., Erdbrink, H., Czekelius, C., Grage, S. L., Wadhwani, P., Ulrich, A. S., Behrends, M., Haufe, G., Koksch, B., Fluorinated Amino Acids in Amyloid Formation: A Symphony of Size, Hydrophobicity and $\alpha$-helix Propensity, Chem. Sci., 2014, 5, 819-830.

[246] Omorodion, H., Twamley, B., Platts, J. A., Baker, R. J., Further Evidence on the Importance of Fluorous-Fluorous Interactions in Supramolecular Chemistry: A Combined Structural and Computational Study, Cryst. Growth Des., 2015, 15, $2835-2841$.

[247] Purcell, K. F., Stikeleather, J. A., Brunk, S. D., Linear Enthalpy-Spectral Shift Correlations for 1,1,1,3,3,3-Hexafluoro-2-propanol, J. Am. Chem. Soc., 1969, 91, 4019-4027.

[248] Rochester, C. H., Symonds, J. R., Thermodynamic Studies of Fluoroalcohols. Part 1. - Vapour Pressures and Enthalpies of Vaporization, J. Chem. Soc., Faraday Trans. 1, 1973, 69, 1267-1273.

[249] Schneider, H.-J., Hydrogen Bonds with Fluorine. Studies in Solution, in Gas Phase and by Computations, Conflicting Conclusions from Crystallographic Analyses, Chem. Sci., 2012, 3, 1381-1394.

[250] Barbarich, T. J., Rithner, C. D., Miller, S. M., Anderson, O. P., Strauss, S. H., Significant Inter- and Intramolecular O-H.FFC Hydrogen Bonding, J. Am. Chem. Soc., 1999, 121, 4280-4281.

[251] Kozuch, S., Martin, J. M. L., Halogen Bonds: Benchmarks and Theoretical Analysis, J. Chem. Theory Comput., 2013, 9, 1918-1931.

[252] Řezáč, J., Riley, K. E., Hobza, P., Benchmark Calculations of Noncovalent Interactions of Halogenated Molecules, J. Chem. Theory Comput., 2012, 8, 4285-4292.

[253] Zehnacker, A., Suhm, M. A., Chirality Recognition Between Neutral Molecules in the Gas Phase, Angew. Chem. Int. Ed., 2008, 47, 6970-6992.

[254] Pérez-García, L., Amabilino, D. B., Spontaneous Resolution, Whence and Whither: From Enantiomorphic Solids to Chiral Liquid Crystals, Monolayers and Macro- and Supra-Molecular Polymers and Assemblies, Chem. Soc. Rev., 2007, 36, 941-967.

[255] Berkessel, A., Adrio, J. A., Hüttenhain, D., Neudörfl, J. M., Unveiling the "Booster Effect" of Fluorinated Alcohol Solvents: Aggregation-induced Conformational Changes and Cooperatively Enhanced H-bonding, J. Am. Chem. Soc., 2006, 128, 8421-8426. 


\section{Bibliography}

[256] Viedma, C., Chiral Symmetry Breaking During Crystallization: Complete Chiral Purity Induced by Nonlinear Autocatalysis and Recycling, Phys. Rev. Lett., 2005, 94, 065504 .

[257] Liu, M., Zhang, L., Wang, T., Supramolecular Chirality in Self-Assembled Systems, Chem. Rev., 2015, 115, 7304-7397.

[258] Flack, H. D., Chiral and Achiral Crystal Structures, Helv. Chim. Acta, 2003, 86, 905-921.

[259] Tschierske, C., Ungar, G., Mirror Symmetry Breaking by Chirality Synchronisation in Liquids and Liquid Crystals of Achiral Molecules, ChemPhysChem, 2016, $17,9-26$.

[260] Alaasar, M., Prehm, M., Tschierske, C., Helical Nano-crystallite (HNC) Phases: Chirality Synchronization of Achiral Bent-Core Mesogens in a New Type of Dark Conglomerates, Chemistry, 2016, 22, 6583-6597.

[261] Scharge, T., Häber, T., Suhm, M. A., Quantitative Chirality Synchronization in Trifluoroethanol Dimers, Phys. Chem. Chem. Phys., 2006, 8, 4664-4667.

[262] Scharge, T., Cézard, C., Zielke, P., Schütz, A., Emmeluth, C., Suhm, M. A., A Peptide Co-solvent under Scrutiny: Self-aggregation of 2,2,2-trifluoroethanol, Phys. Chem. Chem. Phys., 2007, 9, 4472-4490.

[263] Thomas, J., Xu, Y., Chirality Synchronization in Trifluoroethanol Dimer Revisited: The Missing Heterochiral Dimer, J. Phys. Chem. Lett., 2014, 5, 1850-1855.

[264] Seifert, N. A., Thomas, J., Jäger, W., Xu, Y., Rotational Spectra and Theoretical Study of Tetramers and Trimers of 2-Fluoroethanol: Dramatic Intermolecular Compensation for Intramolecular Instability, Phys. Chem. Chem. Phys., 2018, 20, 27630-27637.

[265] Durig, J. R., Cox, F. O., Groner, P., Van der Veken, B. J., Far-infrared and Raman spectra of Gaseous 1,1,1-trifluoro-2-propanol: Conformational Stability and Barriers to Internal Rotation, J. Phys. Chem., 1987, 91, 3211-3218.

[266] Scharge, T., Schwache Bindungen mit starker Wirkung: Konformation und Aggregation von halogenierten Alkoholen, Sierke Verlag, Göttingen, 2007.

[267] Barnes, A. J., Murto, J., Infra-red Cryogenic Studies. Part 10.-Conformational Isomerism of 1,1,1,3,3,3-Hexafluoropropan-2-ol, J. Chem. Soc., Faraday Trans. 2, 1972, 68, 1642-1651.

[268] Lipinski, G., Mallik, B., Merz, K., In situ Crystallization Technique - an Approach to Circumvent Crystal Structures with high Z'-Crystallization Behavior of $\left(\mathrm{CF}_{3}\right)_{2} \mathrm{C}-\mathrm{OH}, \mathrm{Z}$. Anorg. Allg. Chem., 2017, 76, 1455. 


\section{Bibliography}

[269] Murto, J., Kivinen, A., Viitala, R., Hyömäki, J., Fluoroalcohols - XX. Infrared and Raman Spectra of Hexafluoro-2-propanol and its Deuterated Analogues, Spectrochim. Acta A, 1973, 29, 1121-1137.

[270] Durig, J. R., Larsen, R. A., Cox, F. O., Van der Veken, B. J., Raman and Far-infrared Spectra of Gaseous 1,1,1,3,3,3-Hexafluoro-2-propanol, Conformational Stability and Barriers to Internal Rotation, J. Mol. Struct., 1988, 172, 183-201.

[271] Schaal, H., Häber, T., Suhm, M. A., Hydrogen Bonding in 2-Propanol. The Effect of Fluorination, J. Phys. Chem. A, 2000, 104, 265-274.

[272] Shahi, A., Arunan, E., Microwave Spectrum of Hexafluoroisopropanol and Torsional Behavior of Molecules with a $\mathrm{CF}_{3}-\mathrm{C}_{-} \mathrm{CF}_{3}$ Group, J. Phys. Chem. A, 2015, $119,5650-5657$.

[273] Shahi, A., Arunan, E., Microwave Spectroscopic and Theoretical Investigations of the Strongly Hydrogen Bonded Hexafluoroisopropanol...Water Complex, Phys. Chem. Chem. Phys., 2015, 17, 24774-24782.

[274] Quack, M., General discussion, Faraday Disc., 1995, 102, 104-107.

[275] Ruoff, R. S., Klots, T. D., Emilsson, T., Gutowsky, H. S., Relaxation of Conformers and Isomers in Seeded Supersonic Jets of Inert Gases, J. Chem. Phys., 1990, 93, $3142-3150$.

[276] Fioroni, M., Burger, K., Mark, A. E., Roccatano, D., Model of 1,1,1,3,3,3Hexafluoro-propan-2-ol for Molecular Dynamics Simulations, J. Phys. Chem. B, 2001, 105, 10967-10975.

[277] Hong, D.-P., Hoshino, M., Kuboi, R., Goto, Y., Clustering of Fluorine-Substituted Alcohols as a Factor Responsible for Their Marked Effects on Proteins and Peptides, J. Am. Chem. Soc., 1999, 121, 8427-8433.

[278] Jansen, G., Symmetry-adapted Perturbation Theory Based on Density Functional Theory for Noncovalent Interactions, WIREs Comput. Mol. Sci., 2014, 4, 127-144.

[279] Puzzarini, C., Heckert, M., Gauss, J., The Accuracy of Rotational Constants Predicted by High-level Quantum-chemical Calculations. I. Molecules Containing First-row Atoms, J. Chem. Phys., 2008, 128, 194108.

[280] Herzberg, G., Molecular Spectra and Molecular Structure: Spectra of Diatomic Molecules, Van Nostrand, Princeton, 2nd edition, 1950.

[281] Rocher-Casterline, B. E., Ch'ng, L. C., Mollner, A. K., Reisler, H., Communication: Determination of the Bond Dissociation Energy $\left(D_{0}\right)$ of the Water Dimer, $\left(\mathrm{H}_{2} \mathrm{O}\right)_{2}$, by Velocity Map Imaging, J. Chem. Phys., 2011, 134, 211101. 


\section{Bibliography}

[282] Lerner, R. G., Dailey, B. P., Friend, J. P., Microwave Spectrum and Structure of Formic Acid, J. Chem. Phys., 1957, 26, 680-683.

[283] Kwei, G. H., Curl, R. F., Microwave Spectrum of $\mathrm{O}^{18}$ Formic Acid and Structure of Formic Acid, J. Chem. Phys., 1960, 32, 1592-1594.

[284] van Eijck, B. P., van Opheusden, J., van Schaik, M., van Zoeren, E., Acetic Acid: Microwave Spectra, Internal Rotation and Substitution Structure, J. Mol. Spectrosc., 1981, 86, 465-479.

[285] Krisher, L. C., Saegebarth, E., Microwave Spectrum of Acetic Acid, $\mathrm{CH}_{3} \mathrm{COOH}$ and $\mathrm{CD}_{3} \mathrm{COOH}$, J. Chem. Phys., 1971, 54, 4553-4558.

[286] Wallrabe, M., Stickstoff- und Sauerstoffkomplexierung über OH-Brücken, B.Sc. thesis, Georg-August-University, Göttingen, 2016.

[287] Oswald, S., Wallrabe, M., Suhm, M. A., Cooperativity in Alcohol-Nitrogen Complexes: Understanding Cryomatrices through Slit Jet Expansions, J. Phys. Chem. A, 2017, 121, 3411-3422.

[288] Yoshioka, K., Raston, P. L., Anderson, D. T., Infrared Spectroscopy of Chemically Doped Solid Parahydrogen, Int. Rev. Phys. Chem., 2006, 25, 469-496.

[289] Andersen, J., Heimdal, J., Mahler, D. W., Nelander, B., Larsen, R. W., Communication: $\mathrm{THz}$ Absorption Spectrum of the $\mathrm{CO}_{2}-\mathrm{H}_{2} \mathrm{O}$ Complex: Observation and Assignment of Intermolecular van der Waals Vibrations, J. Chem. Phys., 2014, $140,091103$.

[290] Toennies, J. P., Vilesov, A. F., Superfluid Helium Droplets: A Uniquely Cold Nanomatrix for Molecules and Molecular Complexes, Angew. Chem. Int. Ed., 2004, 43, 2622-2648.

[291] Crépin, C., Tramer, A., Rare-gas Matrix as an Infinite Rare-gas Cluster: A Spectroscopic Study of 9,10-dichloroanthracene in Argon Matrices, Chem. Phys. Lett., 1990, 170, 446-450.

[292] Lee, J. J., Höfener, S., Klopper, W., Wassermann, T. N., Suhm, M. A., Origin of the Argon Nanocoating Shift in the OH Stretching Fundamental of $n$-propanol: A Combined Experimental and Quantum Chemical Study, J. Phys. Chem. C, 2009, 113, 10929-10938.

[293] Arean, C. O., Delgado, M. R., Nachtigall, P., Thang, H. V., Rubeš, M., Bulánek, R., Chlubná-Eliášová, P., Measuring the Brønsted Acid Strength of Zeolites-Does it Correlate with the O-H Frequency Shift Probed by a Weak Base?, Phys. Chem. Chem. Phys., 2014, 16, 10129-10141. 


\section{Bibliography}

[294] Emmeluth, C., Dyczmons, V., Suhm, M. A., Tuning the Hydrogen Bond Donor/Acceptor Isomerism in Jet-cooled Mixed Dimers of Aliphatic Alcohols, $J$. Phys. Chem. A, 2006, 110, 2906-2915.

[295] Wassermann, T. N., Suhm, M. A., Ethanol Monomers and Dimers Revisited: A Raman Study of Conformational Preferences and Argon Nanocoating Effects, $J$. Phys. Chem. A, 2010, 114, 8223-8233.

[296] Zimmermann, D., Häber, T., Schaal, H., Suhm, M. A., Hydrogen Bonded Rings, Chains and Lassos: The Case of t-butyl Alcohol Clusters, Mol. Phys., 2001, 99, 413-425.

[297] Provencal, R. A., Casaes, R. N., Roth, K., Paul, J. B., Chapo, C. N., Saykally, R. J., Tschumper, G. S., Schaefer, H. F., III., Hydrogen Bonding in Alcohol Clusters: A Comparative Study by Infrared Cavity Ringdown Laser Absorption Spectroscopy, J. Phys. Chem. A, 2000, 104, 1423-1429.

[298] Tew, D. P., Klopper, W., Heckert, M., Gauss, J., Basis Set Limit CCSD(T) Harmonic Vibrational Frequencies, J. Phys. Chem. A, 2007, 111, 11242-11248.

[299] Bendtsen, J., Rasmussen, F., High-resolution Incoherent Fourier Transform Raman Spectrum of the Fundamental Band of ${ }^{14} \mathrm{~N}_{2}$, J. Raman Spectrosc., 2000, 31, $433-438$.

[300] Martínez, R. Z., Bermejo, D., High-resolution Stimulated Raman Study of the First Vibrational Hot Band of ${ }^{14} \mathrm{~N}_{2}$. Separate Observation of the Spectra of the ortho and para Species, J. Raman Spectrosc., 2013, 44, 727-730.

[301] Larsen, R. W., Zielke, P., Suhm, M. A., Hydrogen-bonded OH Stretching Modes of Methanol Clusters: A Combined IR and Raman Isotopomer Study, J. Chem. Phys., 2007, 126, 194307.

[302] Dyczmons, V., Dimers of Ethanol, J. Phys. Chem. A, 2004, 108, 2080-2086.

[303] Loru, D., Peña, I., Sanz, M. E., Ethanol Dimer: Observation of Three New Conformers by Broadband Rotational Spectroscopy, J. Mol. Spectrosc., 2017, 335, 93-101.

[304] Maris, A., Caminati, W., Velino, B., Andrews, C. M., Howard, B. J., Free and Pulsed Jet Rotational Spectra and van der Waals Motions of Ethanol...Argon, Chem. Phys. Lett., 2004, 399, 39-46.

[305] Kollipost, F., Andersen, J., Mahler, D. W., Heimdal, J., Heger, M., Suhm, M. A., Larsen, R. W., The Effect of Hydrogen Bonding on Torsional Dynamics: A Combined Far-infrared Jet and Matrix Isolation Study of Methanol Dimer, J. Chem. Phys., 2014, 141, 174314. 


\section{Bibliography}

[306] Manneback, C., Die Intensität und Polarisation der von zweiatomigen Molekülen gestreuten kohärenten und inkohärenten Strahlung, Z. Physik, 1930, 62, 224-252.

[307] Manneback, C., Berichtigung zu meiner Arbeit "Die Intensität und Polarisation der von zweiatomigen Molekülen gestreuten kohärenten und inkohärenten Strahlung", Z. Physik, 1930, 65, 574.

[308] Bendtsen, J., The Rotational and Rotation-vibrational Raman Spectra of ${ }^{14} \mathrm{~N}_{2}$, ${ }^{14} \mathrm{~N}^{15} \mathrm{~N}$ and ${ }^{15} \mathrm{~N}_{2}$, J. Raman Spectrosc., 1974, 2, 133-145.

[309] Lofthus, A., Krupenie, P. H., The Spectrum of Molecular Nitrogen, J. Phys. Chem. Ref. Data, 1977, 6, 113-307.

[310] Pearson, J. C., Brauer, C. S., Drouin, B. J., The Asymmetric Top - Asymmetric Frame Internal Rotation Spectrum of Ethyl Alcohol, J. Mol. Spectrosc., 2008, 251, 394-409.

[311] Quade, A Note on Internal Rotation-Rotation Interactions in Ethyl Alcohol, J. Mol. Spectrosc., 2000, 203, 200-202.

[312] Kahn, K., Bruice, T. C., Focal-point Conformational Analysis of Ethanol, Propanol, and Isopropanol, ChemPhysChem, 2005, 6, 487-495.

[313] Oswald, S., Suhm, M. A., Coussan, S., Incremental NH Stretching Downshift through Stepwise Nitrogen Complexation of Pyrrole: A Combined Jet Expansion and Matrix Isolation Study, Phys. Chem. Chem. Phys., 2019, 21, 1277-1284.

[314] Pirim, C., Krim, L., A Neon-matrix Isolation Study of the Reaction of Nonenergetic H-atoms with CO Molecules at $3 \mathrm{~K}$, Phys. Chem. Chem. Phys., 2011, 13, 19454-19459.

[315] Gómez-Zavaglia, A., Fausto, R., Self-Aggregation in Pyrrole: Matrix Isolation, Solid State Infrared Spectroscopy, and DFT Study, J. Phys. Chem. A, 2004, 108, 6953-6967.

[316] Sarkar, S., Ramanathan, N., Gopi, R., Sundararajan, K., Pyrrole Multimers and Pyrrole-acetylene Hydrogen Bonded Complexes studied in $\mathrm{N}_{2}$ and para- $\mathrm{H}_{2}$ Matrixes using Matrix Isolation Infrared Spectroscopy and Ab Initio Computations, J. Mol. Struct., 2017, 1149, 387-403.

[317] Pandey, P., Evidence of Blue-shifting N-H ‥ N Hydrogen Bonding Despite Elongation of the $\mathrm{N}-\mathrm{H}$ Bond, RSC Adv., 2015, 5, 79661-79664.

[318] Kanakaraju, R., Kolandaivel, P., Post Hartree-Fock and DFT Studies on Pyrrole...Nitrogen and Pyrrole...Carbon Monoxide Molecules, Int. J. Mol. Sci., 2002, 3, 777-789. 


\section{Bibliography}

[319] Douketis, C., Reilly, J. P., The NH Stretch in Pyrrole: A Study of the Fundamental $(\Delta \mathrm{v}=1)$ and Third Overtone $(\Delta \mathrm{v}=4)$ Bands in the Bulk Gas and in a Molecular Beam, J. Chem. Phys., 1992, 96, 3431-3440.

[320] Nygaard, U., Nielsen, J., Kirchheiner, J., Maltesen, G., Rastrup-Andersen, J., Sørensen, G. O., Microwave Spectra of Isotopic Pyrroles. Molecular Structure, Dipole Moment, and ${ }^{14} \mathrm{~N}$ Quadrupole Coupling Constants of Pyrrole, J. Mol. Struct., 1969, 3, 491-506.

[321] Columberg, G., Bauder, A., Pure Rotational Spectrum, Quadrupole Coupling Constants and Structure of the Dimer of Pyrrole, J. Chem. Phys., 1997, 106, $504-510$.

[322] Matsumoto, Y., Honma, K., NH Stretching Vibrations of Pyrrole Clusters Studied by Infrared Cavity Ringdown Spectroscopy, J. Chem. Phys., 2007, 127, 184310.

[323] Schütz, M., Matsumoto, Y., Bouchet, A., Öztürk, M., Dopfer, O., Microsolvation of the Pyrrole Cation $\left(\mathrm{Py}^{+}\right)$with Nonpolar and Polar Ligands: Infrared Spectra of $\mathrm{Py}^{+}-\mathrm{L}_{n}$ with $\mathrm{L}=\mathrm{Ar}, \mathrm{N}_{2}$, and $\mathrm{H}_{2} \mathrm{O}(n \leq 3)$, Phys. Chem. Chem. Phys., 2017, 19, 3970-3986.

[324] Kumar, S., Das, A., Mimicking Trimeric Interactions in the Aromatic Side Chains of the Proteins: A Gas Phase Study of Indole...(Pyrrole) ${ }_{2}$ Heterotrimer, J. Chem. Phys., 2012, 136, 174302.

[325] Profant, V., Poterya, V., Fárník, M., Slavícek, P., Buck, U., Fragmentation Dynamics of Size-selected Pyrrole Clusters Prepared by Electron Impact Ionization: Forming a Solvated Dimer Ion Core, J. Phys. Chem. A, 2007, 111, 12477-12486.

[326] Bernard-Houplain, M.-C., Sandorfy, C., Low Temperature Infrared Study of Hydrogen Bonding in Dissolved Pyrrole and Indole, Can. J. Chem., 1973, 51, 10751082 .

[327] Stefov, V., Pejov, L., Šoptrajanov, B., Experimental and Quantum Chemical

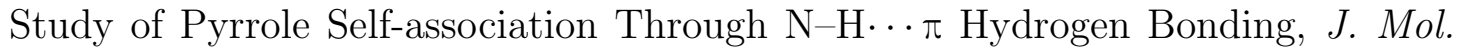
Struct., 2003, 649, 231-243.

[328] Mellouki, A., Georges, R., Herman, M., Snavely, D. L., Leytner, S., Spectroscopic Investigation of Ground State Pyrrole $\left({ }^{12} \mathrm{C}_{4} \mathrm{H}_{5} \mathrm{~N}\right)$ : The N-H Stretch, Chem. Phys., 1997, 220, 311-322.

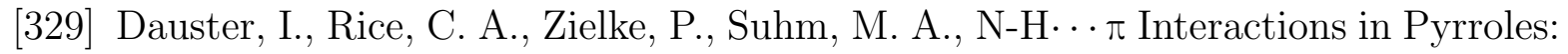
Systematic Trends from the Vibrational Spectroscopy of Clusters, Phys. Chem. Chem. Phys., 2008, 10, 2827-2835.

[330] Moore, K. B., Sadeghian, K., Sherrill, C. D., Ochsenfeld, C., Schaefer, H. F., C-H...O Hydrogen Bonding. The Prototypical Methane-Formaldehyde System: A Critical Assessment, J. Chem. Theory Comput., 2017, 13, 5379-5395. 


\section{Bibliography}

[331] McDowell, S. A., Correlation of the Bond-length Change and Vibrational Frequency Shift in Model Hydrogen-bonded Complexes of Pyrrole, Chem. Phys. Lett., 2017, 674, 146-150.

[332] Jadhav, D. L., Karthick, N. K., Kannan, P. P., Shanmugam, R., Elangovan, A., Arivazhagan, G., Molecular Interaction Forces in Acetone + Ethanol Binary Liquid Solutions: FTIR and Theoretical Studies, J. Mol. Struct., 2017, 1130, 497-502.

[333] van der Avoird, A., Wormer, P. E. S., Jansen, A. P. J., An Improved Intermolecular Potential for Nitrogen, J. Chem. Phys., 1986, 84, 1629-1635.

[334] Pfaffen, C., Infanger, D., Ottiger, P., Frey, H.-M., Leutwyler, S., N-H $\cdots \pi$ Hydrogenbonding and Large-amplitude Tipping Vibrations in Jet-cooled Pyrrole-benzene, Phys. Chem. Chem. Phys., 2011, 13, 14110-14118.

[335] Bohn, R. K., Hillig, K. W., Kuczkowski, R. L., Pyrrole-Argon: Microwave Spectrum, Structure, Dipole Moment, and ${ }^{14} \mathrm{~N}$ Quadrupole Coupling Constants, $J$. Phys. Chem., 1989, 93, 3456-3459.

[336] Jodl, H. J., Solid-State Aspects of Matrices, in Andrews, L., Moskovits, M. (editors), Chemistry and Physics of Matrix-Isolated Species, North-Holland, Amsterdam, 1989, 343-415.

[337] Oswald, S., Coussan, S., Chloroform-Nitrogen Aggregates: Upshifted CH and Downshifted $\mathrm{CCl}$ Stretching Vibrations Observed by Matrix Isolation and Jet Expansion Infrared Spectroscopy, Low. Temp. Phys., 2019, accepted.

[338] Arunan, E., Desiraju, G. R., Klein, R. A., Sadlej, J., Scheiner, S., Alkorta, I., Clary, D. C., Crabtree, R. H., Dannenberg, J. J., Hobza, P., Kjaergaard, H. G., Legon, A. C., Mennucci, B., Nesbitt, D. J., Definition of the Hydrogen Bond (IUPAC Recommendations 2011), Pure Appl. Chem., 2011, 83, 1637-1641.

[339] Arunan, E., Desiraju, G. R., Klein, R. A., Sadlej, J., Scheiner, S., Alkorta, I., Clary, D. C., Crabtree, R. H., Dannenberg, J. J., Hobza, P., Kjaergaard, H. G., Legon, A. C., Mennucci, B., Nesbitt, D. J., Defining the Hydrogen Bond: An Account (IUPAC Technical Report), Pure Appl. Chem., 2011, 83, 1619-1636.

[340] Matsuura, H., Yoshida, H., Hieda, M., Yamanaka, S.-y., Harada, T., Shin-ya, K., Ohno, K., Experimental Evidence for Intramolecular Blue-shifting C-H...O Hydrogen Bonding by Matrix-isolation Infrared Spectroscopy, J. Am. Chem. Soc., 2003, 125, 13910-13911.

[341] Kovács, A., Szabó, A., Nemcsok, D., Hargittai, I., Blue-Shifting C-H $\cdots X$ (X = O, Halogen) Hydrogen Bonds in the Dimers of Formaldehyde Derivatives, J. Phys. Chem. A, 2002, 106, 5671-5678. 


\section{Bibliography}

[342] Hobza, P., Špirko, V., Havlas, Z., Buchhold, K., Reimann, B., Barth, H.-D., Brutschy, B., Anti-hydrogen Bond Between Chloroform and Fluorobenzene, Chem. Phys. Lett., 1999, 299, 180-186.

[343] Barnes, A. J., Molecular Complexes of the Hydrogen Halides Studied by Matrix Isolation Infrared Spectroscopy, J. Mol. Struct., 1983, 100, 259-280.

[344] Ito, F., Matrix-isolation Infrared Studies of 1:1 Molecular Complexes Containing Chloroform $\left(\mathrm{CHCl}_{3}\right)$ and Lewis Bases: Seamless Transition from Blue-shifted to Red-shifted Hydrogen Bonds, J. Chem. Phys., 2012, 137, 014505.

[345] Hobza, P., Havlas, Z., Blue-Shifting Hydrogen Bonds, Chem. Rev., 2000, 100, 4253-4264.

[346] Li, X., Liu, L., Schlegel, H. B., On the Physical Origin of Blue-Shifted Hydrogen Bonds, J. Am. Chem. Soc., 2002, 124, 9639-9647.

[347] Alabugin, I. V., Manoharan, M., Peabody, S., Weinhold, F., Electronic Basis of Improper Hydrogen Bonding: A Subtle Balance of Hyperconjugation and Rehybridization, J. Am. Chem. Soc., 2003, 125, 5973-5987.

[348] Joseph, J., Jemmis, E. D., Red-, blue-, or no-shift in Hydrogen Bonds: A Unified Explanation, J. Am. Chem. Soc., 2007, 129, 4620-4632.

[349] Chung, S., Hippler, M., Infrared Spectroscopy of Hydrogen-bonded $\mathrm{CHCl}_{3}-\mathrm{SO}_{2}$ in the Gas Phase, J. Chem. Phys., 2006, 124, 214316.

[350] Hippler, M., Hesse, S., Suhm, M. A., Quantum-chemical Study and FTIR Jet Spectroscopy of $\mathrm{CHCl}_{3}-\mathrm{NH}_{3}$ Association in the Gas Phase, Phys. Chem. Chem. Phys., 2010, 12, 13555-13565.

[351] Gibian, T. G., McKinney, D. S., Infrared Spectra and Force Constants of Chloroform and Trichlorosilane, J. Am. Chem. Soc., 1951, 73, 1431-1434.

[352] Ruoff, A., Bürger, H., Schwingungsspektren und Kraftkonstanten symmetrischer Kreisel - I. $\mathrm{HCCl}_{3}$ und $\mathrm{DCCl}_{3}$, Spectrochim. Acta A, 1970, 26, 989-997.

[353] Anttila, R., Alanko, S., Horneman, V.-M., The C-H Bending Vibration $\nu_{4}$ of Chloroform $\mathrm{CH}^{35} \mathrm{Cl}_{3}$, Mol. Phys., 2008, 102, 1537-1542.

[354] Lewerenz, M., Quack, M., Vibrational Overtone Intensities of the Isolated CH and CD Chromophores in Fluoroform and Chloroform, Chem. Phys. Lett., 1986, 123, $197-202$.

[355] Pietilä, J., Alanko, S., Horneman, V.-M., Anttila, R., High-Resolution Infrared Studies of $\nu_{1}, 2 \nu_{1}$, and $2 \nu_{4}$ Bands of $\mathrm{CH}^{35} \mathrm{Cl}_{3}$, J. Mol. Spectrosc., 2002, 216, 271283. 


\section{Bibliography}

[356] Ghosh, S. N., Trambarulo, R., Gordy, W., Microwave Spectra and Molecular Structures of Fluoroform, Chloroform, and Methyl Chloroform, J. Chem. Phys., 1952, 20, 605-607.

[357] He, Y., Hippler, M., Quack, M., High-resolution Cavity Ring-down Absorption Spectroscopy of Nitrous Oxide and Chloroform Using a Near-infrared CW Diode Laser, Chem. Phys. Lett., 1998, 289, 527-534.

[358] Hippler, M., Quack, M., Overtone Spectroscopy of Chloroform in a Supersonic Jet by Vibrationally Assisted Dissociation and Photofragment Ionization, Ber. Bunsenges. Phys. Chem., 1995, 99, 417-421.

[359] Delanoye, S. N., Herrebout, W. A., van der Veken, B. J., Blue Shifting Hydrogen Bonding in the Complexes of Chlorofluoro Haloforms with Acetone- $d_{6}$ and Oxirane- $d_{4}$, J. Am. Chem. Soc., 2002, 124, 11854-11855.

[360] Jemmis, E. D., Giju, K. T., Sundararajan, K., Sankaran, K., Vidya, V., Viswanathan, K. S., Leszczynski, J., An Ab Initio and Matrix Isolation Infrared Study of the 1:1 $\mathrm{C}_{2} \mathrm{H}_{2}-\mathrm{CHCl}_{3}$ Adduct, J. Mol. Struct., 1999, 510, 59-68.

[361] Hippler, M., Quantum Chemical Study and Infrared Spectroscopy of Hydrogenbonded $\mathrm{CHCl}_{3}-\mathrm{NH}_{3}$ in the Gas Phase, J. Chem. Phys., 2007, 127, 084306.

[362] Hippler, M., Quantum-chemical Study of $\mathrm{CHCl}_{3}-\mathrm{SO}_{2}$ Association, J. Chem. Phys., 2005, 123, 204311.

[363] Sałdyka, M., Mielke, Z., Mierzwicki, K., Coussan, S., Roubin, P., CH Stretching Vibration of $N$-Methylformamide as a Sensitive Probe of its Complexation: Infrared Matrix Isolation and Computational Study, Phys. Chem. Chem. Phys., 2011, 13, 13992-14002.

[364] Yin, C.-C., Li, A. H.-T., Chao, S. D., Liquid Chloroform Structure from Computer Simulation with a Full $A b$ Initio Intermolecular Interaction Potential, J. Chem. Phys., 2013, 139, 194501.

[365] Mátyus, E., Suhm, M. A., Technical report, 2005.

[366] Lang, E., Schwingungsspektroskopie in der Gasphase mit und ohne Resonanzverstärkung, B.Sc. thesis, Georg-August-University, Göttingen, 2017.

[367] Long, D. A., The Raman Effect: A Unified Treatment of the Theory of Raman Scattering by Molecules, Wiley, New York and Chichester, 2002.

[368] Tian, Z. Q., Surface-enhanced Raman Spectroscopy: Advancements and Applications, J. Raman Spectrosc., 2005, 36, 466-470.

[369] West, G. A., Barrett, J. J., Siebert, D. R., Reddy, K. V., Photoacoustic Spectroscopy, Rev. Sci. Instrum., 1983, 54, 797-817. 


\section{Bibliography}

[370] Spencer, C. L., Watson, V., Hippler, M., Trace Gas Detection of Molecular Hydrogen $\mathrm{H}_{2}$ by Photoacoustic Stimulated Raman Spectroscopy (PARS), Analyst, 2012, 137, 1384-1388.

[371] Lavorel, B., Millot, G., Rotger, M., Rouillé, G., Berger, H., Schrötter, H. W., Non-linear Raman Spectroscopy in Gases, J. Mol. Struct., 1992, 273, 49-59.

[372] Chabay, I., Klauminzer, G. K., Hudson, B. S., Coherent Anti-Stokes Raman Spectroscopy (CARS): Improved Experimental Design and Observation of New Higherorder Processes, Appl. Phys. Lett., 1976, 28, 27-29.

[373] Wrzesinski, P. J., Pestov, D., Lozovoy, V. V., Xu, B., Roy, S., Gord, J. R., Dantus, M., Binary Phase Shaping for Selective Single-beam CARS Spectroscopy and Imaging of Gas-phase Molecules, J. Raman Spectrosc., 2011, 42, 393-398.

[374] Hippler, M., Mohr, C., Keen, K. A., McNaghten, E. D., Cavity-enhanced Resonant Photoacoustic Spectroscopy with Optical Feedback cw Diode Lasers: A Novel Technique for Ultratrace Gas Analysis and High-Resolution Spectroscopy, J. Chem. Phys., 2010, 133, 044308.

[375] Salter, R., Chu, J., Hippler, M., Cavity-enhanced Raman Spectroscopy with Optical Feedback cw Diode Lasers for Gas Phase Analysis and Spectroscopy, Analyst, 2012, 137, 4669-4676.

[376] Hippler, M., Cavity-enhanced Raman Spectroscopy of Natural Gas with Optical Feedback cw-Diode Lasers, Anal. Chem., 2015, 87, 7803-7809.

[377] Smith, T. W., Hippler, M., Cavity-enhanced Raman Spectroscopy in the Biosciences: In Situ, Multicomponent, and Isotope Selective Gas Measurements To Study Hydrogen Production and Consumption by Escherichia coli, Anal. Chem., 2017, 89, 2147-2154.

[378] Taylor, D. J., Glugla, M., Penzhorn, R.-D., Enhanced Raman Sensitivity Using an Actively Stabilized External Resonator, Rev. Sci. Instrum., 2001, 72, 1970-1976.

[379] King, D. A., Pittaro, R. J., Simple Diode Pumping of a Power-buildup Cavity, Opt. Lett., 1998, 23, 774-776.

[380] Ohara, S., Yamaguchi, S., Endo, M., Nanri, K., Fujioka, T., Performance Characteristics of Power Build-Up Cavity for Raman Spectroscopic Measurement, Opt. Rev., 2003, 10, 342-345.

[381] Thorstensen, J., Haugholt, K. H., Ferber, A., Bakke, K. A. H., Tschudi, J., Lowcost Resonant Cavity Raman Gas Probe for Multi-gas Detection, J. Eur. Opt. Soc., 2014, 9, 14054. 


\section{Bibliography}

[382] Keiner, R., Frosch, T., Massad, T., Trumbore, S., Popp, J., Enhanced Raman Multigas Sensing - a Novel Tool for Control and Analysis of ${ }^{13} \mathrm{CO}_{2}$ Labeling Experiments in Environmental Research, Analyst, 2014, 139, 3879-3884.

[383] Oswald, S., Acetone Trace Gas Detection and Aggregation Studies by Linear IR Spectroscopy and Cavity-enhanced Raman Scattering, M.Sc. thesis, Georg-AugustUniversity, Göttingen, 2015.

[384] Drever, R. W. P., Hall, J. L., Kowalski, F. V., Hough, J., Ford, G. M., Munley, A. J., Ward, H., Laser Phase and Frequency Stabilization Using an Optical Resonator, Appl. Phys. B, 1983, 31, 97-105.

[385] Lide, D. R., CRC Handbook of Chemistry and Physics, CRC Press, Boca Raton, 78th edition, 1998.

[386] Coussan, S., Bouteiller, Y., Loutellier, A., Perchard, J. P., Racine, S., Peremans, A., Zheng, W. Q., Tadjeddine, A., Infrared Photoisomerization of the Methanol Dimer Trapped in Argon Matrix: Monochromatic Irradiation Experiments and DFT Calculations, Chem. Phys., 1997, 219, 221-234.

[387] Han, S. W., Kim, K., Infrared Matrix Isolation Study of Acetone and Methanol in Solid Argon, J. Phys. Chem., 1996, 100, 17124-17132. 
A. Appendix

\section{A. Appendix}

\section{A.1. Experimental and Theoretical Methods}

Table A.1.: Experimental parameters of the filet-jet set-up.

\begin{tabular}{cc}
\hline & Optical parameters \\
\hline Resolution & $2 \mathrm{~cm}^{-1}$ \\
Measurement range & $0 \mathrm{~cm}^{-1}-15797.52 \mathrm{~cm}^{-1}$ \\
Radiation source & Globar $(\mathrm{MIR}), 150 \mathrm{~W}$ tungsten lamp (NIR) \\
Lenses & KBr broadband (MIR), CaF $\mathrm{Ca}_{2}(\mathrm{NIR})$ \\
Aperture & $4.0-3.5 \mathrm{~mm}$ \\
Detector & $4 \mathrm{~mm}^{2} \mathrm{HgCdTe}(\mathrm{MIR}) / 3 \mathrm{~mm}^{2} \mathrm{InSb}(\mathrm{NIR})$ sandwich detector element \\
Mirror speed & (internal description: InSb/MCT sandwich neu) \\
Acquisition mode & $80 \mathrm{kHz}$ \\
Pre-amplifier & Single sided fast return \\
Filter & MCT: homemade, $R=1.0 \mathrm{k} \Omega, V_{c}=1.8 \mathrm{~V}$, InSb: $R=2.4-2.2 \mathrm{k} \Omega$ \\
& MCT: none, InSb: $4100-2450 \mathrm{~cm}^{-1}(13 \mathrm{a})$ \\
\hline Phase resolution & Fourier transformation \\
Phase correction mode & $16 \mathrm{~cm}^{-1}$ \\
Apodisation function & Mertz \\
Zerofilling factor & Norton-Beer, Medium \\
\hline
\end{tabular}




\section{A. Appendix}

Table A.2.: Solid state properties of different matrix hosts employed in this work. ${ }^{336}$

\begin{tabular}{cccc}
\hline & $\mathrm{Ne}$ & $\mathrm{Ar}$ & $\mathrm{N}_{2}$ \\
\hline Crystal lattice & cfc & cfc & $\alpha-\mathrm{N}_{2}$ \\
Molecules per unit cell & 1 & 1 & 4 \\
Lattice constant / $\AA$ & 4.47 & 5.31 & 5.66 \\
Substitution gap / $\AA$ & 3.16 & 3.75 & 3.99 \\
Octahedral gap / $\AA$ & 1.31 & 1.56 & - \\
Tetrahedral gap / $\AA$ & 0.71 & 0.85 & - \\
Polarizability / $\AA^{3}$ & 0.39 & 1.63 & 1.76 \\
Lennard-Jones-Parameters: & & & \\
$\varepsilon / \mathrm{cm}^{-1}$ & 24.33 & 83.98 & 66.3 \\
$\sigma / \AA$ & 2.76 & 3.41 & 3.75 \\
Force constant ${ }^{125,336} \kappa / 10^{3}$ mdyn $\AA^{-1}$ & 3.6 & 8.2 & 5.4 \\
Critical temperature $^{385} T_{\mathrm{c}} / \mathrm{K}$ & 44.4 & 150.87 & 126.21 \\
Typical deposition temperature $T_{\mathrm{d}} / \mathrm{K}$ & 4 & $20-22$ & 17 \\
Sublimation temperature under high vacuum conditions $\left(10^{-7}\right.$ mbar $) T_{\mathrm{s}} / \mathrm{K}$ & 11 & 35 & 30 \\
\hline
\end{tabular}

Table A.3.: Gaussian ${ }^{141}$ keywords employed in the different calculations.

\begin{tabular}{cc}
\hline Level of approximation & Employed keywords \\
\hline All & fopt=verytight, output=pickett, freq=raman or freq=anharm \\
../VTZ & cc-pVTZ \\
../aVTZ & aug-cc-pVTZ \\
../VQZ & cc-pVQZ \\
$\ldots$.aVQZ & aug-cc-pVQZ \\
$\ldots$ /aV5Z & aug-cc-pv5z \\
$\ldots$. def2-QZVP & Def2QZVP \\
B3LYP-D3 & b3lyp, int=superfine, empiricaldispersion=gd3bj \\
PBE0-D3 & PBE1PBE, int=superfine, empiricaldispersion=gd3bj \\
M06-2X & M062X, int=superfine \\
WB97-XD & wB97XD, int=superfine \\
B2PLYP-D3 & b2plypd3, int=superfine \\
MP2 & mp2 \\
B3LYP & b3lyp, int=superfine \\
M06-2X-D3 & b97d3, int=superfine \\
B97-D3 & hf \\
HF & am1 \\
AM1 & pm6 6 \\
PM6 & M062X, int=supersion=GD3 \\
BP86-D3 & bm86, int=superfine, empiricaldispersion=gd3bj \\
BLYP-D3 & blyp, int=superfine, empiricaldispersion=gd3bj \\
CCSD(T) & ccsd-t \\
PCM model & scrf=(solvent=generic,read), eps=17.8, atomic radii: UFF, \\
& absolute temperature: $298.15 \mathrm{~K}$ \\
\hline
\end{tabular}




\section{A. Appendix}

Table A.4.: ORCA $^{142}$ keywords employed in the different calculations.

\begin{tabular}{cc}
\hline Level of approximation & Employed keywords \\
\hline All & verytightopt, verytightscf \\
../VTZ & cc-pVTZ, cc-pVTZ/C \\
../aVTZ & aug-cc-pVTZ, aug-cc-pVTZ/C \\
../VQZ & cc-pVQZ, cc-pVQZ/C \\
../aVQZ & aug-cc-pVQZ, aug-cc-pVQZ/C \\
PBEh-3c & pbeh3c, grid5, nofinalgrid, freq \\
B97-3c & b973c, grid5, nofinalgrid, freq \\
SCS-MP2 & RI-SCS-MP2, RHF, numfreq \\
MP2 & RI-MP2, RHF, numfreq \\
B2PLYP-D3 & RI-B2PLYP-D3, RHF, grid5, nofinalgrid \\
\hline
\end{tabular}

Table A.5.: Molpro $^{143}$ keywords employed in the different calculations.

\begin{tabular}{|c|c|}
\hline Level of approximation & Employed keywords \\
\hline All & $\begin{array}{c}\text { gthresh,energy }=1 \mathrm{e}-10, \text { zero }=1 \mathrm{e}-16 \text {; orient,mass; } \\
\text { symmetry,z; } \text { hf,accu, } 16 ; \text { optg,gaussian } \\
\text { gradient }=1.5 \mathrm{E}-5, \text { grms }=1 \mathrm{E}-5, \text { step }=6 \mathrm{E}-5, \text { srms }=4 \mathrm{E}-5 \text {,energy }=1 \mathrm{E}-8 \\
\text { frequencies }\end{array}$ \\
\hline $\operatorname{CCSD}(\mathrm{T})$ & $\operatorname{ccsd}(\mathrm{t})$ \\
\hline .../VTZ & basis $=\mathrm{vtz}$ \\
\hline .../aVTZ & basis $=$ avtz \\
\hline $\begin{array}{l}\mathrm{LCCSD}\left(\mathrm{T}^{*}\right)-\mathrm{F} 12 / \\
\text { VDZ-F12(int) }\end{array}$ & $\begin{array}{c}\text { df-lccsd(t)-f12a; local, loc_method=pipek, npasel=0.03 } \\
\text { interact=1, pipek, delete=2 } \\
\text { basis= vdz-f12 } \\
\text { E_CCSDF12A }=\text { _ENERGC(1), } \\
\text { E_TRIPF12A = _ENERGY }(1)-\text { E_CCSDF12A } \\
\text { E_MP2CORR = EMP2_SING + _EMP2_TRIP } \\
\text { E_MP2F12CORR = E_MP2CORR + _EF12 } \\
\text { E_CHECK = E_CCSDF12A + E_TRIPF12A } \\
\text { E_TARGET =E_CCSDF12A+E_TRIPF12A*E_MP2F12CORR/E_MP2CORR }\end{array}$ \\
\hline
\end{tabular}

$$
\begin{aligned}
\text { MRD } & =\frac{1}{n} \sum_{i=1}^{n} \frac{x_{\text {theo }, i}-x_{\exp , i}}{x_{\exp , i}} \\
\text { MAE } & =\frac{1}{n} \sum_{i=1}^{n}\left|x_{\text {theo }, i}-x_{\exp , i}\right| \\
\text { RMSD } & =\sqrt{\frac{1}{n} \sum_{i=1}^{n}\left(x_{\text {theo }, i}-x_{\text {exp }, i}\right)^{2}}
\end{aligned}
$$


A. Appendix 
A. Appendix

\section{A.2. Carboxylic Acids}
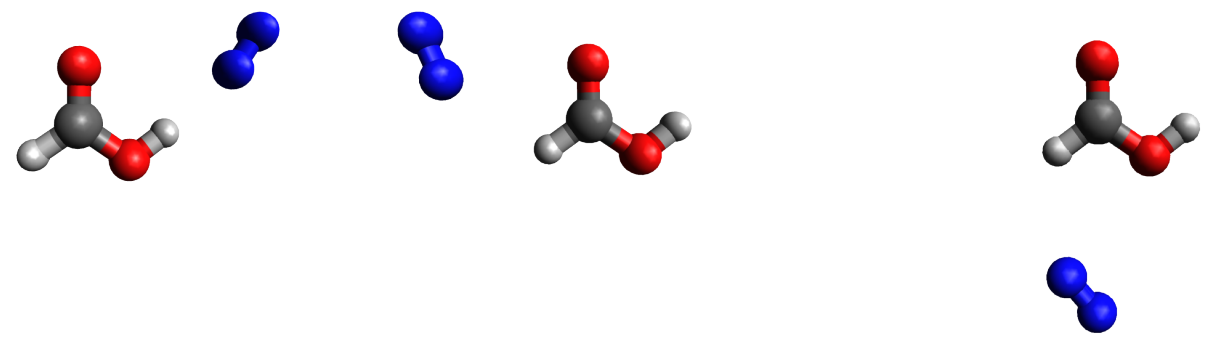

$\mathrm{FN}_{\mathrm{H}}\left(C_{\mathrm{s}}\right)[0.0]$

$\mathrm{FN}_{\mathrm{c}}\left(C_{\mathrm{s}}\right)[3.1]$

$\mathrm{FN}_{\circ}\left(C_{\mathrm{s}}\right)[4.2]$
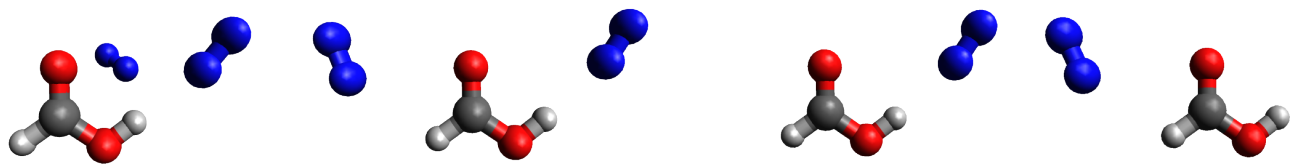

8

8

$\mathrm{FN}_{\mathrm{H}} \mathrm{N}_{\mathrm{H}}\left(C_{1}\right)[0.0] \quad \mathrm{FN}_{\mathrm{H}} \mathrm{N}_{\mathrm{C}}\left(C_{\mathrm{s}}\right)[0.9] \quad \mathrm{FN}_{\mathrm{H}} \mathrm{N}_{\mathrm{O}}\left(C_{\mathrm{s}}\right)[2.0] \quad \mathrm{FN}_{\mathrm{C}} \mathrm{N}_{\mathrm{O}}\left(C_{\mathrm{s}}\right)[5.1]$

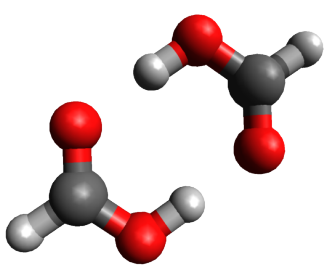

(FF) $\left(C_{2 h}\right)[0.0]$

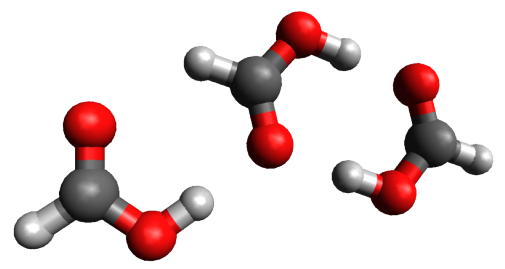

$\mathrm{F}(\mathrm{FF})\left(C_{\mathrm{s}}\right)$

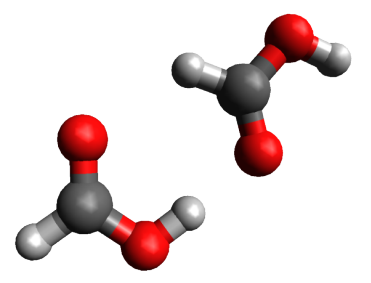

FF $\left(C_{\mathrm{s}}\right)[28.6]$

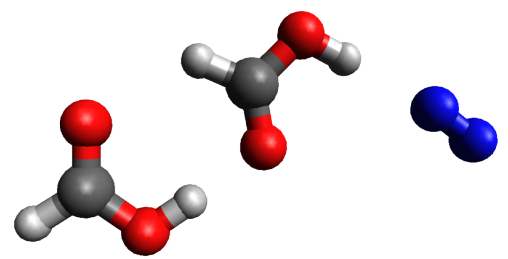

$\operatorname{FFN}_{\mathrm{H}}\left(C_{\mathrm{s}}\right)$

Figure A.1.: Stable structures of pure F and mixed FN aggregates optimised at the B3LYP-D3(BJ)/def2-QZVP level (reprinted from reference 157 licensed under CC BY 4.0). Their respective point groups are given in parentheses, relative harmonically zeropoint-corrected energies in $\mathrm{kJ} \mathrm{mol}^{-1}$ in square brackets. 
A. Appendix

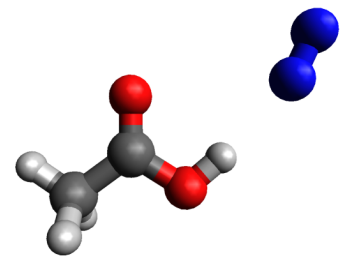

$\operatorname{AN}_{\mathrm{H}}\left(C_{\mathrm{s}}\right)[0.0]$

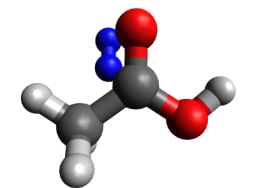

$\operatorname{AN}_{C^{\prime}}\left(C_{1}\right)[2.4]$
8

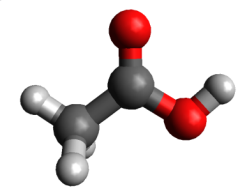

$\operatorname{AN}_{\mathrm{c}}\left(C_{\mathrm{s}}\right)[2.8]$

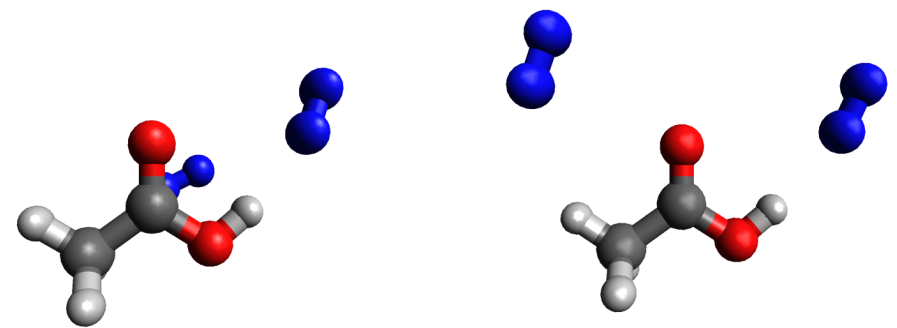

$\mathrm{AN}_{\mathrm{H}} \mathrm{N}_{\mathrm{C}^{\prime}}\left(C_{1}\right)[0.0]$

$\mathrm{AN}_{\mathrm{H}} \mathrm{N}_{\mathrm{C}}\left(C_{\mathrm{s}}\right)[1.5]$

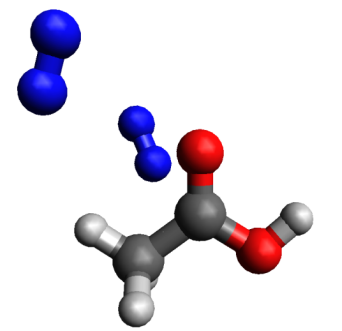

$\mathrm{AN}_{\mathrm{C}} \mathrm{N}_{\mathrm{C}^{\prime}}\left(C_{1}\right)[2.8]$

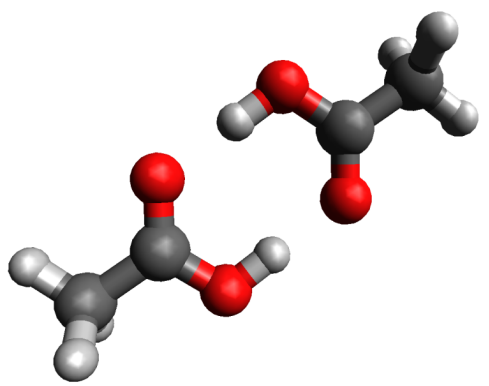

(AA) $\left(C_{2 h}\right)[0.0]$

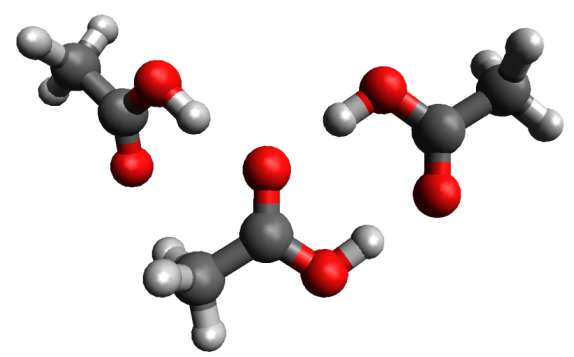

$\mathrm{A}(\mathrm{AA})\left(C_{1}\right)$

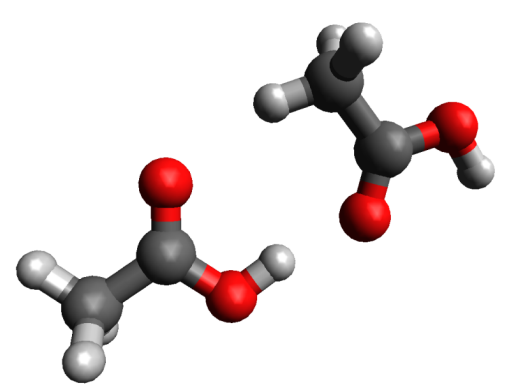

$\mathrm{AA}\left(C_{1}\right)[33.2]$

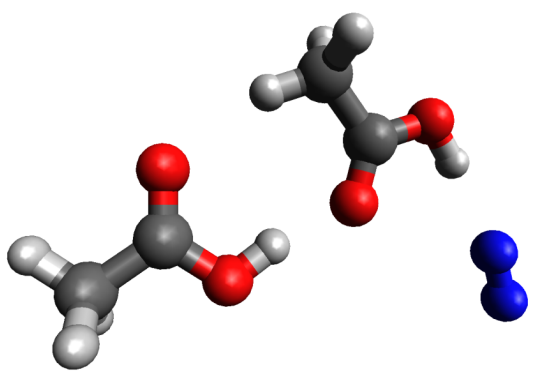

$\operatorname{AAN}_{\mathrm{H}}\left(C_{1}\right)$

Figure A.2.: Stable structures of pure A and mixed AN aggregates optimised at the B3LYP-D3(BJ)/def2-QZVP level (reprinted from reference 157 licensed under CC BY 4.0). Their respective point groups are given in parentheses, relative harmonically zeropoint-corrected energies in $\mathrm{kJ} \mathrm{mol}^{-1}$ in square brackets. 


\section{A. Appendix}

Table A.6.: Integration bounds in $\mathrm{cm}^{-1}$ for evaluation of $\mathrm{F}$ and $\mathrm{A}$ monomer band centre positions. The Bruker OPUS (Version 7.0) program package was used for linear baseline corrections interpolated from the integral bounds and subsequent integrations (OPUS integration method B).

\begin{tabular}{ccc}
\hline & Upper bound & Lower bound \\
\hline $\mathrm{F}$ & 3591 & 3547 \\
$\mathrm{~A}$ & 3599 & 3574 \\
\hline
\end{tabular}

Table A.7.: Experimentally obtained fundamental transitions of F $\left(\tilde{\nu}_{i}\right)$, combination bands with the $\mathrm{OH}$ stretching vibration $\left(\tilde{\nu}_{1}+\tilde{\nu}_{i}\right)$ and resulting anharmonic coupling constants within a perturbation description $\left(x_{1, i}\right)$ in gas phase and supersonic expansion rounded to integer wavenumbers in $\mathrm{cm}^{-1}$. The coupling constant $x_{1,1}$ denotes the diagonal anharmonicity $-\omega_{\mathrm{e}} x_{\mathrm{e}}$. The bottom rows display the total difference between anharmonic and harmonic $\mathrm{OH}$ stretching vibration (mode 1) ${ }^{148}$ and the fraction of the diagonal anharmonicity contribution.

\begin{tabular}{cccc}
\hline $\mathrm{i}$ & $\tilde{\nu_{i}} / \mathrm{cm}^{-1}$ & $\tilde{\nu_{1}}+\tilde{\nu}_{i} / \mathrm{cm}^{-1}$ & $x_{1, i} / \mathrm{cm}^{-1}$ \\
\hline 1 & 3570 & $6968^{203}$ & $-86(2)$ \\
2 & $2942^{186,203,226}$ & $6507^{203,227}$ & $-5(2)$ \\
3 & $1777^{102,186,203}$ & $5343^{203}$ & $-4(2)$ \\
4 & $1380^{201,203}$ & $4942^{203}$ & $-8(2)$ \\
5 & $1223^{203,228}$ & $4780^{203}$ & $-13(2)$ \\
6 & $1105^{203,229}$ & $4670^{203}$ & $-5(2)$ \\
7 & $626^{203,230}$ & $4192^{203}$ & $-4(2)$ \\
8 & $1033^{203,229}$ & $4600^{203}$ & $-3(2)$ \\
9 & $641^{203,230}$ & $4209^{203}$ & $-2(2)$ \\
\hline $2 x_{1,1}+0.5 \sum_{i \neq 1} x_{1, i}$ & & & $-194(12)$ \\
$\frac{2 x_{1,1}}{2 x_{1,1}+0.5 \sum_{i \neq 1} x_{1, i}}$ & & & 0.89 \\
\hline
\end{tabular}

Table A.8.: Theoretically predicted harmonic $\left(\omega_{\mathrm{OH}}\right)$ and anharmonic $\left(\tilde{\nu}_{\mathrm{OH}}\right) \mathrm{OH}$ stretching wavenumber and lowest predicted harmonic $\left(\omega_{1}\right)$ and anharmonic $\left(\tilde{\nu}_{1}\right)$ wavenumber in $\mathrm{cm}^{-1}$, harmonic $\left(S_{\omega}\right)$ and anharmonic $\left(S_{\nu}\right)$ IR intensity in $\mathrm{km} \mathrm{mol}^{-1}$, harmonic $\left(\Delta \omega_{\mathrm{OH}}\right)$ and anharmonic $\left(\Delta \tilde{\nu}_{\mathrm{OH}}\right)$ spectroscopic downshift relative to $\mathrm{F}$ or $\mathrm{A}$ in $\mathrm{cm}^{-1}$, dimer dissociation energies into the most stable monomers without $\left(D_{\mathrm{e}}\right)$ and with $\left(D_{0}\right)$ harmonic vibrational zero-point correction in $\mathrm{kJ} \mathrm{mol}^{-1}$ calculated at different levels of approximation.

\begin{tabular}{cccccccccccc}
\hline Level of theory & Structure & $\omega_{\mathrm{OH}}$ & $S_{\omega}$ & $\Delta \omega_{\mathrm{OH}}$ & $\tilde{\nu}_{\mathrm{OH}}$ & $S_{\nu}$ & $\Delta \tilde{\nu}_{\mathrm{OH}}$ & $D_{\mathrm{e}}$ & $D_{0}$ & $\omega_{1}$ & $\tilde{\nu}_{1}$ \\
\hline & $\mathrm{F}$ & 3716 & 60 & - & 3529 & 51 & - & - & - & 629 & 623 \\
B3LYP-D3/ & $\mathrm{FN}_{\mathrm{H}}$ & 3678 & 267 & 38 & 3503 & 179 & 26 & 8.8 & 5.9 & 23 & 50
\end{tabular}




\section{A. Appendix}

\begin{tabular}{|c|c|c|c|c|c|c|c|c|c|c|c|}
\hline Level of theory & Structure & $\omega_{\mathrm{OH}}$ & $S_{\omega}$ & $\Delta \omega_{\mathrm{OH}}$ & $\tilde{\nu}_{\mathrm{OH}}$ & $S_{\nu}$ & $\Delta \tilde{\nu}_{\mathrm{OH}}$ & $D_{\mathrm{e}}$ & $D_{0}$ & $\omega_{l}$ & $\tilde{\nu}_{1}$ \\
\hline \multirow[t]{2}{*}{ aVTZ } & $\mathrm{A}$ & 3739 & 61 & - & 3554 & 50 & - & - & - & 70 & 60 \\
\hline & $\mathrm{AN}_{\mathrm{H}}$ & 3707 & 253 & 32 & 3531 & 195 & 23 & 8.4 & 5.9 & 24 & 20 \\
\hline \multirow{4}{*}{$\begin{array}{c}\text { B3LYP-D3/ } \\
\text { VQZ }\end{array}$} & $\mathrm{F}$ & 3724 & 59 & - & 3535 & 49 & - & - & - & 632 & 626 \\
\hline & $\mathrm{FN}_{\mathrm{H}}$ & 3688 & 257 & 36 & 3512 & 179 & 23 & 9.9 & 6.9 & 27 & 87 \\
\hline & A & 3747 & 60 & - & 3559 & 46 & - & - & - & 75 & 113 \\
\hline & $\mathrm{AN}_{\mathrm{H}}$ & 3716 & 251 & 31 & 3541 & 189 & 18 & 9.5 & 6.8 & 28 & 42 \\
\hline \multirow{4}{*}{$\begin{array}{c}\text { B3LYP-D3/ } \\
\text { aVQZ }\end{array}$} & $\mathrm{F}$ & 3723 & 61 & - & 3534 & 51 & - & - & - & 631 & 625 \\
\hline & $\mathrm{FN}_{\mathrm{H}}$ & 3687 & 262 & 36 & 3510 & 163 & 24 & 8.8 & 5.8 & 25 & 102 \\
\hline & $\mathrm{A}$ & 3746 & 61 & - & 3560 & 52 & - & - & - & 69 & 67 \\
\hline & $\mathrm{AN}_{\mathrm{H}}$ & 3716 & 252 & 30 & 3539 & 191 & 21 & 8.3 & 5.8 & 26 & 38 \\
\hline \multirow{4}{*}{$\begin{array}{l}\text { B3LYP-D3/ } \\
\text { def2-QZVP }\end{array}$} & $\mathrm{F}$ & 3727 & 60 & - & 3537 & 50 & - & - & - & 631 & 625 \\
\hline & $\mathrm{FN}_{\mathrm{H}}$ & 3690 & 267 & 37 & 3511 & 185 & 26 & 9.6 & 6.5 & 25 & -11 \\
\hline & A & 3750 & 61 & - & 3561 & 49 & - & - & - & 71 & 112 \\
\hline & $\mathrm{AN}_{\mathrm{H}}$ & 3719 & 258 & 31 & 3538 & 244 & 23 & 9.2 & 6.5 & 26 & 59 \\
\hline \multirow{2}{*}{$\begin{array}{c}\operatorname{CCSD}(\mathrm{T}) / \\
\mathrm{VTZ}\end{array}$} & $\mathrm{F}$ & 3763 & - & - & - & - & - & - & - & 629 & - \\
\hline & $\mathrm{FN}_{\mathrm{H}}$ & 3744 & - & 19 & - & - & - & 9.4 & 6.5 & 29 & - \\
\hline \multirow{2}{*}{$\begin{array}{c}\operatorname{CCSD}(\mathrm{T}) / \\
\mathrm{aVTZ} \\
\end{array}$} & $\mathrm{F}$ & 3741 & - & - & - & - & - & - & - & 627 & - \\
\hline & $\mathrm{FN}_{\mathrm{H}}$ & 3719 & - & 22 & - & - & - & 9.8 & 6.9 & 25 & - \\
\hline \multirow{4}{*}{$\begin{array}{c}\operatorname{LCCSD}\left(\mathrm{T}^{*}\right)-\mathrm{F} 12 / \\
\text { VDZ-F12(int) }\end{array}$} & $\mathrm{F}$ & 3754 & - & - & - & - & - & - & - & 630 & - \\
\hline & $\mathrm{FN}_{\mathrm{H}}$ & 3730 & - & 24 & - & - & - & 7.9 & 5.0 & 27 & - \\
\hline & A & 3767 & - & - & - & - & - & - & - & 78 & - \\
\hline & $\mathrm{AN}_{\mathrm{H}}$ & & & & & & & & & & \\
\hline \multirow{4}{*}{$\begin{array}{l}\mathrm{MP2} / \\
\mathrm{VTZ}\end{array}$} & $\mathrm{F}$ & 3763 & 75 & - & 3579 & 64 & - & - & - & 629 & 623 \\
\hline & $\mathrm{FN}_{\mathrm{H}}$ & 3731 & 254 & 32 & 3558 & 179 & 21 & 10.2 & 7.2 & 29 & 39 \\
\hline & A & 3773 & 72 & - & 3589 & 62 & - & - & - & 77 & 88 \\
\hline & $\mathrm{AN}_{\mathrm{H}}$ & 3745 & 247 & 28 & 3570 & 183 & 19 & 9.8 & 7.3 & 28 & 26 \\
\hline \multirow{4}{*}{$\begin{array}{l}\text { MP2/ } \\
\text { aVTZ }\end{array}$} & $\mathrm{F}$ & 3741 & 79 & - & 3557 & 69 & - & - & - & 626 & 620 \\
\hline & $\mathrm{FN}_{\mathrm{H}}$ & 3705 & 279 & 36 & 3540 & 203 & 17 & 10.3 & 7.3 & 23 & 46 \\
\hline & A & 3752 & 75 & - & 3569 & 66 & - & - & - & 78 & 82 \\
\hline & $\mathrm{AN}_{\mathrm{H}}$ & 3723 & 264 & 29 & 3540 & 199 & 29 & 10.1 & 7.5 & 24 & -45 \\
\hline \multirow{4}{*}{$\begin{array}{l}\mathrm{MP} 2 / \\
\mathrm{VQZ}\end{array}$} & $\mathrm{F}$ & 3764 & 80 & - & 3576 & 69 & - & - & - & 630 & 624 \\
\hline & $\mathrm{FN}_{\mathrm{H}}$ & 3728 & 268 & 36 & 3551 & 190 & 25 & 9.6 & 6.6 & 26 & 40 \\
\hline & $\mathrm{A}$ & 3775 & 78 & - & 3588 & 68 & - & - & - & 77 & 89 \\
\hline & $\mathrm{AN}_{\mathrm{H}}$ & 3744 & 258 & 31 & - & - & - & 9.2 & 6.7 & 27 & - \\
\hline \multirow{4}{*}{$\begin{array}{l}\text { MP2/ } \\
\text { aVQZ }\end{array}$} & $\mathrm{F}$ & 3756 & 81 & - & 3566 & 26 & - & - & - & 629 & 623 \\
\hline & $\mathrm{FN}_{\mathrm{H}}$ & 3717 & 275 & 39 & - & - & - & 9.6 & 6.6 & 25 & - \\
\hline & A & 3767 & 78 & - & - & - & - & - & - & 75 & - \\
\hline & $\mathrm{AN}_{\mathrm{H}}$ & - & - & - & - & - & - & - & - & - & - \\
\hline \multirow{4}{*}{$\begin{array}{c}\text { SCS-MP2/ } \\
\text { VTZ }\end{array}$} & $\mathrm{F}$ & 3774 & 70 & - & - & - & - & - & - & 633 & - \\
\hline & $\mathrm{FN}_{\mathrm{H}}$ & 3752 & 224 & 22 & - & - & - & 8.8 & 5.9 & 29 & - \\
\hline & A & 3784 & 67 & - & - & - & - & - & - & 85 & - \\
\hline & $\mathrm{AN}_{\mathrm{H}}$ & 3765 & 216 & 19 & - & - & - & 8.5 & 6.1 & 14 & - \\
\hline \multirow{3}{*}{$\begin{array}{c}\text { SCS-MP2/ } \\
\text { aVTZ }\end{array}$} & $\mathrm{F}$ & 3754 & 74 & - & - & - & - & - & - & 630 & - \\
\hline & $\mathrm{FN}_{\mathrm{H}}$ & 3729 & 250 & 25 & - & - & - & 9.1 & 6.2 & 24 & - \\
\hline & A & 3764 & 71 & - & - & - & - & - & - & 86 & - \\
\hline
\end{tabular}




\section{A. Appendix}

\begin{tabular}{|c|c|c|c|c|c|c|c|c|c|c|c|}
\hline Level of theory & Structure & $\omega_{\mathrm{OH}}$ & $S_{\omega}$ & $\Delta \omega_{\mathrm{OH}}$ & $\tilde{\nu}_{\mathrm{OH}}$ & $S_{\nu}$ & $\Delta \tilde{\nu}_{\mathrm{OH}}$ & $D_{\mathrm{e}}$ & $D_{0}$ & $\omega_{1}$ & $\tilde{\nu}_{1}$ \\
\hline & $\mathrm{AN}_{\mathrm{H}}$ & 3745 & 241 & 19 & - & - & - & 8.8 & 6.3 & 23 & - \\
\hline \multirow{4}{*}{$\begin{array}{l}\text { SCS-MP2/ } \\
\text { VQZ }\end{array}$} & $\mathrm{F}$ & 3778 & 75 & - & - & - & - & - & - & 634 & - \\
\hline & $\mathrm{FN}_{\mathrm{H}}$ & 3753 & 239 & 25 & - & - & - & 8.2 & 5.3 & 25 & - \\
\hline & A & 3788 & 73 & - & - & - & - & - & - & 84 & - \\
\hline & $\mathrm{AN}_{\mathrm{H}}$ & 3768 & 230 & 20 & - & - & - & 7.8 & 5.3 & 23 & - \\
\hline \multirow{4}{*}{$\begin{array}{l}\text { SCS-MP2/ } \\
\text { aVQZ }\end{array}$} & $\mathrm{F}$ & 3770 & 77 & - & - & - & - & - & - & 633 & - \\
\hline & $\mathrm{FN}_{\mathrm{H}}$ & 3742 & 249 & 28 & - & - & - & 8.3 & 5.4 & 27 & - \\
\hline & $\mathrm{A}$ & 3780 & 73 & - & - & - & - & - & - & 74 & - \\
\hline & $\mathrm{AN}_{\mathrm{H}}$ & 3756 & 240 & 24 & - & - & - & 7.9 & 5.5 & 14 & - \\
\hline \multirow{4}{*}{$\begin{array}{c}\text { B2PLYP-D3/ } \\
\text { VTZ }\end{array}$} & $\mathrm{F}$ & 3744 & 63 & - & 3555 & 53 & - & - & - & 630 & 623 \\
\hline & $\mathrm{FN}_{\mathrm{H}}$ & 3713 & 245 & 31 & 3536 & 177 & 19 & 10.4 & 7.3 & 30 & 15 \\
\hline & A & 3762 & 62 & - & 3573 & 51 & - & - & - & 78 & 85 \\
\hline & $\mathrm{AN}_{\mathrm{H}}$ & 3735 & 241 & 27 & 3560 & 174 & 13 & 10.0 & 7.4 & 29 & 20 \\
\hline \multirow{4}{*}{$\begin{array}{c}\text { B2PLYP-D3/ } \\
\text { aVTZ }\end{array}$} & $\mathrm{F}$ & 3733 & 67 & - & 3545 & 57 & - & - & - & 628 & 621 \\
\hline & $\mathrm{FN}_{\mathrm{H}}$ & 3698 & 271 & 35 & 3520 & 196 & 25 & 9.0 & 6.0 & 22 & -15 \\
\hline & A & 3751 & 66 & - & 3565 & 56 & - & - & - & 73 & 70 \\
\hline & $\mathrm{AN}_{\mathrm{H}}$ & 3722 & 258 & 29 & 3546 & 199 & 19 & 8.6 & 6.0 & 22 & 42 \\
\hline \multirow{4}{*}{$\begin{array}{c}\text { B2PLYP-D3/ } \\
\text { VQZ }\end{array}$} & $\mathrm{F}$ & 3745 & 67 & - & 3555 & 57 & - & - & - & 631 & 624 \\
\hline & $\mathrm{FN}_{\mathrm{H}}$ & 3712 & 262 & 33 & 3533 & 184 & 22 & 9.5 & 6.5 & 25 & 23 \\
\hline & A & 3763 & 67 & - & 3575 & 58 & - & - & - & 76 & 68 \\
\hline & $\mathrm{AN}_{\mathrm{H}}$ & 3735 & 254 & 28 & - & - & - & 9.1 & 6.5 & 25 & - \\
\hline \multirow{4}{*}{$\begin{array}{c}\text { B2PLYP-D3/ } \\
\text { aVQZ }\end{array}$} & $\mathrm{F}$ & 3742 & 69 & - & 3553 & 59 & - & - & - & 630 & 623 \\
\hline & $\mathrm{FN}_{\mathrm{H}}$ & 3708 & 266 & 34 & - & - & - & 8.7 & 5.7 & 23 & - \\
\hline & A & 3761 & 68 & - & - & - & - & - & - & 71 & - \\
\hline & $\mathrm{AN}_{\mathrm{H}}$ & - & - & - & - & - & - & - & - & - & - \\
\hline \multirow{4}{*}{$\begin{array}{l}\text { M06-2X/ } \\
\text { aVTZ }\end{array}$} & $\mathrm{F}$ & 3791 & 85 & - & 3631 & 76 & - & - & - & 645 & 638 \\
\hline & $\mathrm{FN}_{\mathrm{H}}$ & 3765 & 248 & 26 & 3618 & 166 & 13 & 7.6 & 4.7 & 32 & 73 \\
\hline & A & 3809 & 86 & - & 3632 & 76 & - & - & - & 88 & -116 \\
\hline & $\mathrm{AN}_{\mathrm{H}}$ & 3784 & 249 & 25 & 3594 & 239 & 38 & 7.4 & 4.9 & 21 & 137 \\
\hline \multirow{4}{*}{$\begin{array}{c}\text { PBE0-D3/ } \\
\text { aVTZ }\end{array}$} & $\mathrm{F}$ & 3772 & 66 & - & 3587 & 56 & - & - & - & 635 & 629 \\
\hline & $\mathrm{FN}_{\mathrm{H}}$ & 3726 & 269 & 46 & 3556 & 190 & 31 & 9.0 & 6.1 & 25 & 40 \\
\hline & A & 3794 & 67 & - & 3611 & 57 & - & - & - & 70 & 78 \\
\hline & $\mathrm{AN}_{\mathrm{H}}$ & 3754 & 258 & 40 & 3588 & 191 & 23 & 8.6 & 6.1 & 26 & -64 \\
\hline \multirow{4}{*}{$\begin{array}{c}\omega \mathrm{B} 97-\mathrm{XD} / \\
\text { aVTZ }\end{array}$} & $\mathrm{F}$ & 3803 & 65 & - & 3611 & 57 & - & - & - & 640 & 633 \\
\hline & $\mathrm{FN}_{\mathrm{H}}$ & 3756 & 254 & 47 & 3492 & 233 & 119 & 7.2 & 4.4 & 25 & 34 \\
\hline & $\mathrm{A}$ & 3824 & 66 & - & 3643 & 57 & - & - & - & 72 & 159 \\
\hline & $\mathrm{AN}_{\mathrm{H}}$ & 3783 & 245 & 41 & 3559 & 268 & 84 & 6.8 & 4.4 & 24 & 18 \\
\hline \multirow{4}{*}{ PBEh-3c } & $\mathrm{F}$ & 3846 & 77 & - & - & - & - & - & - & 658 & - \\
\hline & $\mathrm{FN}_{\mathrm{H}}$ & 3831 & 219 & 15 & - & - & - & 11.2 & 8.0 & 40 & - \\
\hline & A & 3864 & 80 & - & - & - & - & - & - & 72 & - \\
\hline & $\mathrm{AN}_{\mathrm{H}}$ & 3850 & 226 & 14 & - & - & - & 10.9 & 8.1 & 32 & - \\
\hline \multirow{4}{*}{ B97-3c } & $\mathrm{F}$ & 3649 & 34 & - & - & - & - & - & - & 618 & - \\
\hline & $\mathrm{FN}_{\mathrm{H}}$ & 3626 & 147 & 23 & - & - & - & 7.9 & 5.4 & 27 & - \\
\hline & A & 3673 & 34 & - & - & - & - & - & - & 33 & - \\
\hline & $\mathrm{AN}_{\mathrm{H}}$ & 3655 & 138 & 18 & - & - & - & 7.6 & 5.5 & 19 & - \\
\hline
\end{tabular}


A. Appendix 
A. Appendix

\section{A.3. 1,1,1,3,3,3-Hexafluoro-2-propanol}

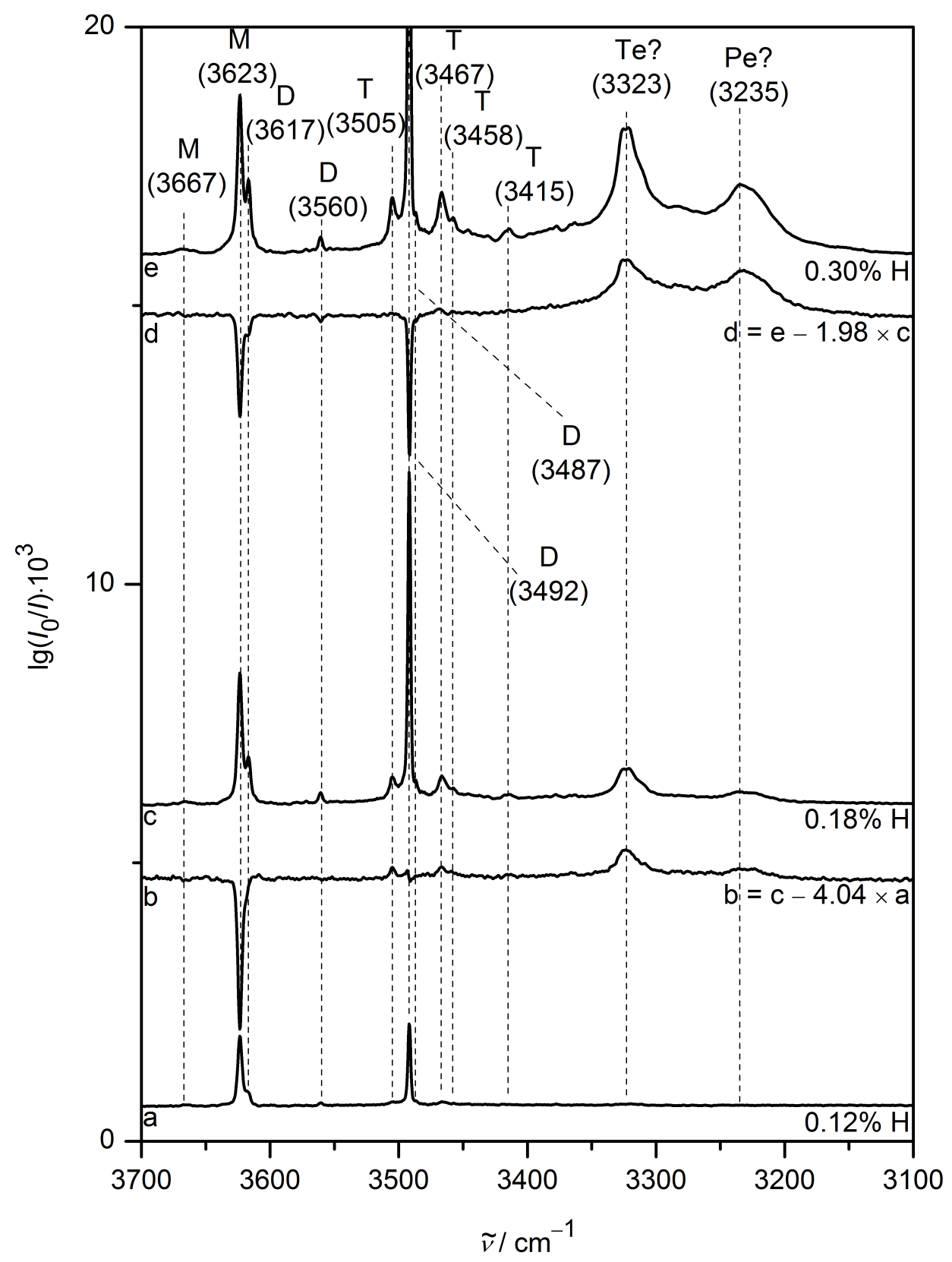

Figure A.3.: $\mathrm{OH}$ stretching FTIR jet spectra of $\mathrm{H}$ (reprinted with adaptations from reference 235 with permission from John Wiley and Sons) with increasing analyte concentration from bottom to top (traces a, c and e). Dimer- (trace b) and trimer- (trace d) corrected difference spectra are included. Wavenumbers and labels are provided. The similar scaling allows for assignments of vibrational signals to common cluster sizes such as monomers $(\mathrm{M})$, dimers $(\mathrm{D})$, trimers $(\mathrm{T})$, tetramers $(\mathrm{Te})$ and pentamers $(\mathrm{Pe})$. 
A. Appendix

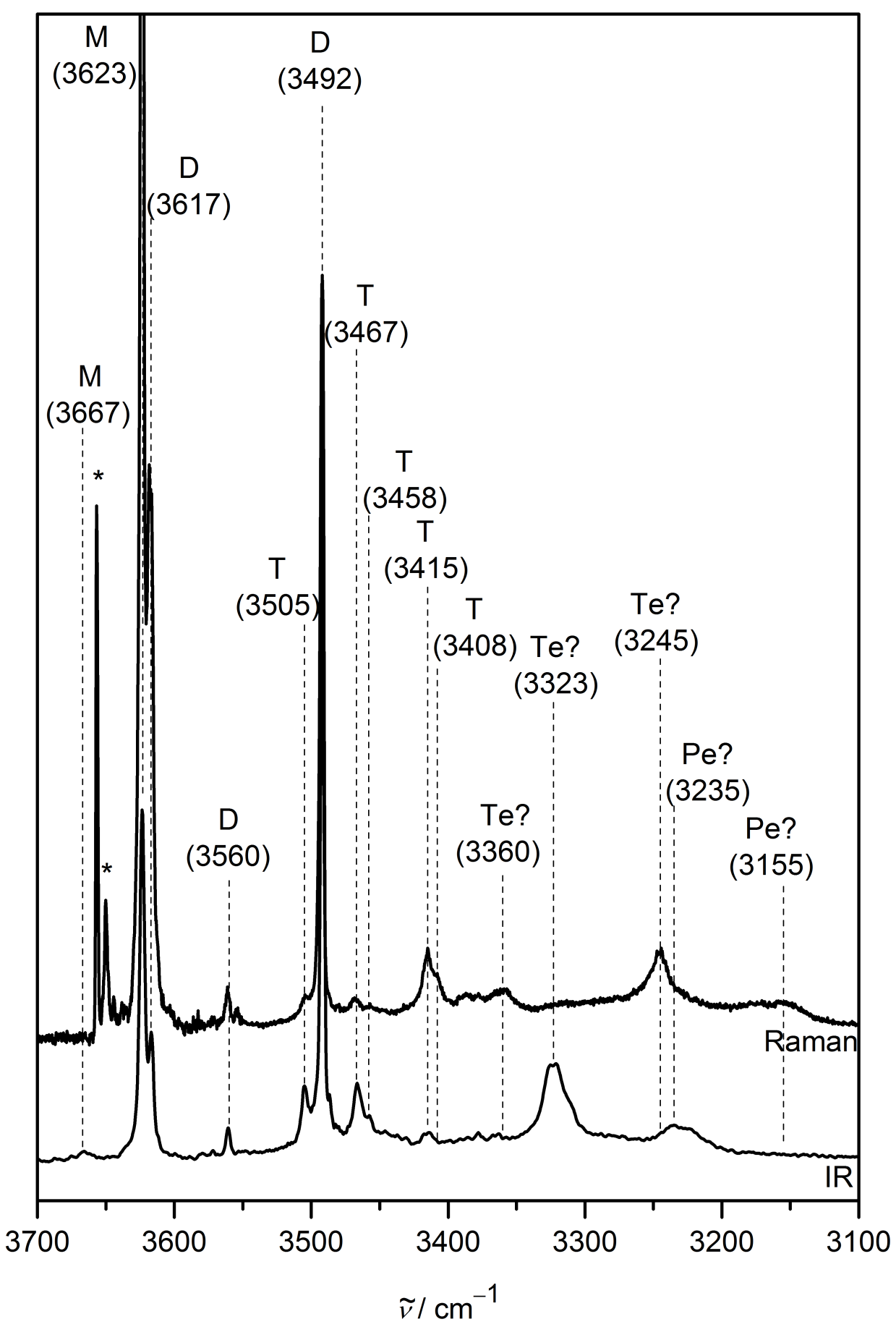

Figure A.4.: OH-stretching FTIR (bottom trace) and Raman (top trace) spectra of H (reprinted with adaptations from reference 235 with permission from John Wiley and Sons). For the Raman spectrum, two different angles of the monochromator grating had to be employed, therefore the signal-to-noise ratio is slightly lower above $3550 \mathrm{~cm}^{-1}$. Water signals are marked by asterisks. Wavenumbers and labels are provided. The similar scaling allows for assignments of vibrational signals to common cluster sizes such as monomers $(\mathrm{M})$, dimers $(\mathrm{D})$, trimers $(\mathrm{T})$, tetramers $(\mathrm{Te})$ and pentamers $(\mathrm{Pe})$. 
A. Appendix

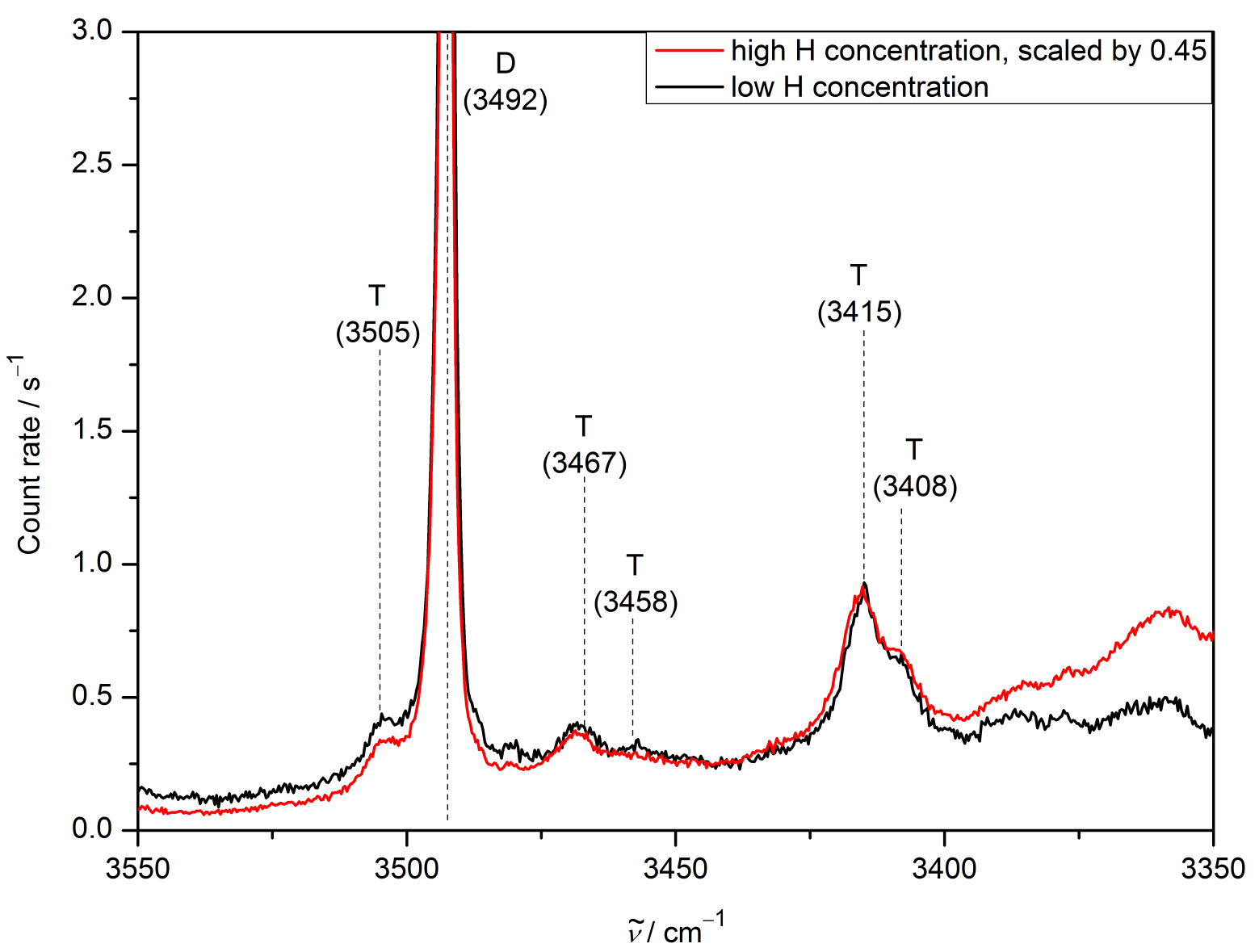

Figure A.5.: $\mathrm{OH}$ stretching Raman jet spectra of $\mathrm{H}$ with high (red trace) and low (black trace) analyte concentration scaled to matching intensity of the strongest trimer ( $\mathrm{T}$ ) signal at $3415 \mathrm{~cm}^{-1}$ (reprinted with adaptations from reference 235 with permission from John Wiley and Sons). Wavenumbers and labels are provided. The similar scaling allows for assignments of vibrational signals to common cluster sizes such as dimers (D) and trimers (T). 
A. Appendix

0
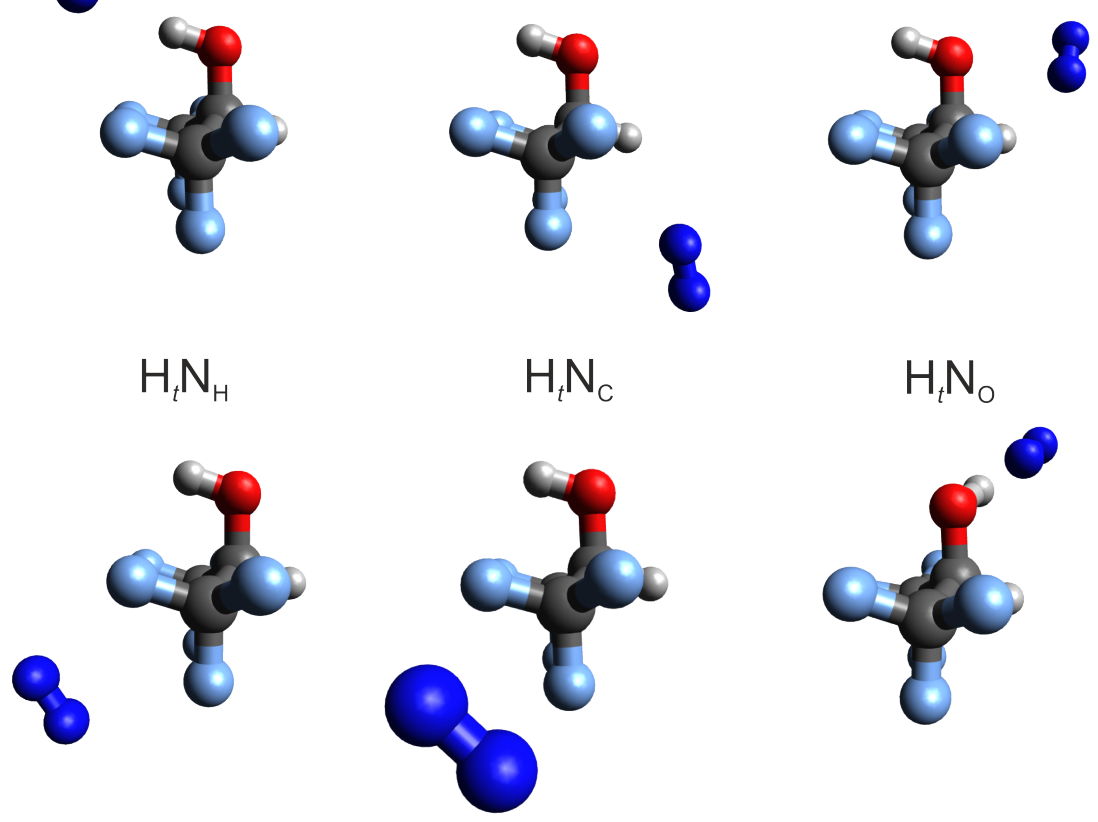

$\mathrm{H}_{t} \mathrm{~N}_{\mathrm{F}}$

$\mathrm{H}_{t} \mathrm{~N}_{\mathrm{F}}$

$\mathrm{H}_{g} \mathrm{~N}_{\mathrm{H}}$
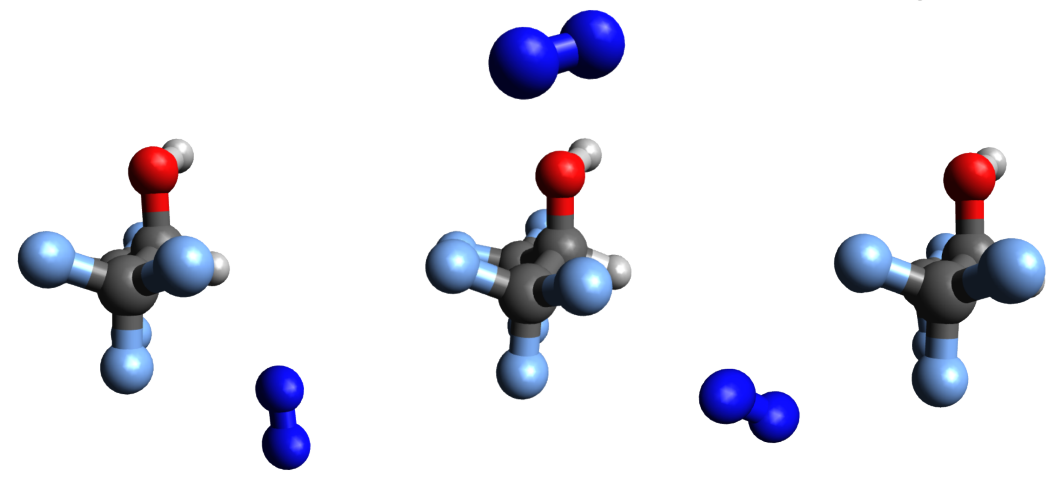

$\mathrm{H}_{g} \mathrm{~N}_{\mathrm{C}}$

$\mathrm{H}_{g} \mathrm{~N}_{\circ}$

$\mathrm{H}_{g} \mathrm{~N}_{\mathrm{F}}$

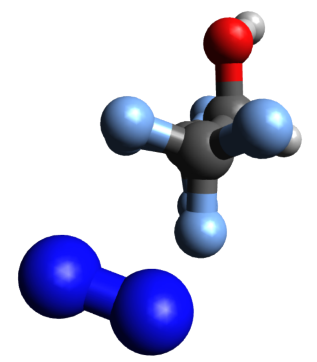

$\mathrm{H}_{g} \mathrm{~N}_{\mathrm{F}}$

$\mathrm{H}_{g} \mathrm{~N}_{F^{\prime \prime}}$

Figure A.6.: Stable structures of binary HN aggregates for both $\mathrm{H}$ monomer conformations optimised at the B3LYP-D3(BJ)/def2-QZVP level. 


\section{A. Appendix}

Table A.9.: Theoretically predicted harmonic $\left(\omega_{\mathrm{OH}}\right) \mathrm{OH}$ stretching wavenumber, spectral downshift from the respective monomer $\left(\Delta \omega_{\mathrm{OH}}\right)$ and lowest predicted wavenumber $\left(\omega_{1}\right)$ in $\mathrm{cm}^{-1}$, IR intensity $\left(S_{\mathrm{OH}}\right)$ in $\mathrm{km} \mathrm{mol}^{-1}$, relative electronic and harmonic vibrational zero-point corrected energies $\left(\Delta E_{\mathrm{e}}\right.$ and $\left.\Delta E_{0}\right)$ compared to the most stable $\mathrm{H}_{t} \mathrm{~N}_{\mathrm{H}}$ conformation in $\mathrm{kJ} \mathrm{mol}^{-1}$, as well as equilibrium rotational constants $\left(A_{\mathrm{e}}, B_{\mathrm{e}}, C_{\mathrm{e}}\right)$ in $\mathrm{MHz}$ and magnitudes of the electric dipole moment components $\left(\left|\mu_{a}\right|,\left|\mu_{b}\right|,\left|\mu_{c}\right|\right)$ in $\mathrm{D}$ calculated at the B3LYP-D3(BJ)/def2-QZVP level of approximation.

\begin{tabular}{ccccccccccccc}
\hline Structure & $\omega_{\mathrm{OH}}$ & $S_{\mathrm{OH}}$ & $\Delta \omega_{\mathrm{OH}}$ & $\Delta E_{\mathrm{e}}$ & $\Delta E_{0}$ & $\omega_{1}$ & $A_{\mathrm{e}}$ & $B_{\mathrm{e}}$ & $C_{\mathrm{e}}$ & $\left|\mu_{a}\right|$ & $\left|\mu_{b}\right|$ & $\left|\mu_{c}\right|$ \\
\hline $\mathrm{H}_{t} \mathrm{~N}_{\mathrm{H}}$ & 3753 & 281 & 36 & 0.0 & 0.0 & 18 & 958.2 & 743.0 & 535.7 & 0.8 & 0.0 & 0.4 \\
$\mathrm{H}_{t} \mathrm{~N}_{\mathrm{C}}$ & 3790 & 64 & -1 & 4.6 & 3.3 & 11 & 947.7 & 766.8 & 551.3 & 0.1 & 0.0 & 0.5 \\
$\mathrm{H}_{t} \mathrm{~N}_{\mathrm{O}}$ & 3789 & 63 & 0 & 4.8 & 3.5 & 14 & 1078.3 & 643.2 & 502.4 & 0.5 & 0.3 & 0.3 \\
$\mathrm{H}_{t} \mathrm{~N}_{\mathrm{F}}$ & 3788 & 61 & 1 & 7.4 & 5.5 & 14 & 979.7 & 830.8 & 572.1 & 0.6 & 0.0 & 0.2 \\
$\mathrm{H}_{t} \mathrm{~N}_{\mathrm{F}^{\prime}}$ & 3789 & 62 & 0 & 8.4 & 6.2 & 5 & 1825.4 & 474.5 & 444.8 & 0.2 & 0.6 & 0.0 \\
$\mathrm{H}_{g} \mathrm{~N}_{\mathrm{H}}$ & 3797 & 326 & 30 & 5.2 & 4.5 & 13 & 1224.7 & 544.9 & 442.4 & 2.7 & 0.6 & 1.4 \\
$\mathrm{H}_{g} \mathrm{~N}_{\mathrm{C}}$ & 3829 & 75 & -2 & 9.6 & 7.6 & 12 & 956.2 & 755.0 & 550.2 & 0.8 & 1.2 & 2.1 \\
$\mathrm{H}_{g} \mathrm{~N}_{\mathrm{O}}$ & 3829 & 76 & -2 & 11.8 & 9.2 & 6 & 1375.2 & 575.0 & 480.3 & 0.4 & 1.4 & 2.0 \\
$\mathrm{H}_{g} \mathrm{~N}_{\mathrm{F}}$ & 3827 & 77 & 0 & 12.2 & 9.6 & 12 & 987.8 & 816.5 & 563.0 & 2.3 & 0.6 & 0.8 \\
$\mathrm{H}_{g} \mathrm{~N}_{\mathrm{F}^{\prime}}$ & 3826 & 75 & 1 & 13.1 & 10.3 & 8 & 1781.6 & 481.1 & 448.0 & 1.7 & 1.2 & 1.5 \\
$\mathrm{H}_{g} \mathrm{~N}_{\mathrm{F}^{\prime \prime}}$ & 3827 & 77 & 0 & 6 & 13.1 & 10.3 & 1585.9 & 505.3 & 451.7 & 0.0 & 2.1 & 1.4 \\
\hline
\end{tabular}

Table A.10.: Experimentally obtained transition frequencies ( $\nu_{\text {exp. }}$ ) of the $\mathrm{H}_{t} \mathrm{~N}_{\mathrm{H}}$ dimer with their respective rotational quantum numbers as well as the differences between the fitted and observed frequencies $\left(\Delta \nu=\nu_{\text {calc. }}-\nu_{\text {exp. }}\right) . \quad \nu_{\text {calc. }}$ is calculated using the fitted experimental spectroscopic constants in Table 3.3. $\quad \mathrm{F}_{1}=\mathrm{J}+\mathrm{I}_{1}$ where $\mathrm{I}_{1}$ is the nuclear quadrupole hyperfine quantum number for the bound $\mathrm{N}$ atom and $\mathrm{F}=\mathrm{F}_{1}+\mathrm{I}_{2}$ where $\mathrm{I}_{2}$ is the nuclear quadrupole hyperfine quantum number for the free $\mathrm{N}$ atom. Transitions which are grouped together were assigned to the same broadened lineshape with an equal weighting for each hyperfine transition in the overall blend assignment.

\begin{tabular}{cccccccccccc}
\hline $\mathrm{J}^{\prime}$ & $\mathrm{K}_{a}^{\prime}$ & $\mathrm{K}_{c}^{\prime}$ & $\mathrm{F}_{1}^{\prime}$ & $\mathrm{F}^{\prime}$ & $\mathrm{J}^{\prime}$ & $\mathrm{K}_{a}^{\prime \prime}$ & $\mathrm{K}_{c}^{\prime \prime}$ & $\mathrm{F}_{1}^{\prime \prime}$ & $\mathrm{F}^{\prime \prime}$ & $\nu_{\text {exp. }} / \mathrm{MHz}$ & $\Delta \nu / \mathrm{MHz}$ \\
\hline 2 & \multirow{2}{*}{0} & 2 & 3 & 4 & 1 & 0 & 1 & 2 & 3 & 2449.9361 & 0.028 \\
& & & 3 & 3 & & & & 2 & 2 & & \\
\hline 2 & 1 & 1 & 3 & 4 & 1 & 1 & 0 & 2 & 3 & 2736.6188 & 0.02 \\
\hline 2 & 1 & 1 & 3 & 4 & 1 & 0 & 1 & 2 & 3 & 3171.2271 & 0.003 \\
\hline & & & 3 & 3 & & & & 2 & 2 & & \\
& & & 4 & 4 & & & & 2 & 3 & 3455.427 & -0.015 \\
3 & \multirow{2}{*}{1} & 3 & 4 & 2 & & & & 2 & 1 & & \\
& & & 2 & 3 & & & 2 & 3 & 4 & & \\
& & & 3 & 2 & & & & 1 & 2 & 3455.6526 & 0.022 \\
\hline 2 & 2 & 0 & 3 & 4 & 1 & 1 & 0 & 2 & 3 & 3522.6103 & 0.023 \\
\hline 2 & 2 & 0 & 3 & 3 & 1 & 1 & 0 & 2 & 2 & 3522.9428 & 0.016 \\
\hline
\end{tabular}




\section{A. Appendix}

\begin{tabular}{|c|c|c|c|c|c|c|c|c|c|c|c|}
\hline $\mathrm{J}^{\prime}$ & $\mathrm{K}_{a}^{\prime}$ & $\mathrm{K}_{c}^{\prime}$ & $\mathrm{F}_{1}^{\prime}$ & $\mathrm{F}^{\prime}$ & J" & $\mathrm{K}_{a}^{\prime \prime}$ & $\mathrm{K}_{c}^{\prime \prime}$ & $\mathrm{F}_{1}^{\prime \prime}$ & F" & $\nu_{\text {exp. }} / \mathrm{MHz}$ & $\Delta \nu / \mathrm{MHz}$ \\
\hline 3 & 0 & 3 & 4 & 5 & 2 & 0 & 2 & 3 & 4 & 3530.1859 & 0.035 \\
\hline 3 & 0 & 3 & 3 & 2 & 2 & 0 & 2 & 2 & 1 & 3530.4992 & -0.006 \\
\hline 2 & 2 & 1 & 3 & 4 & 1 & 1 & 1 & 2 & 3 & 3638.541 & -0.007 \\
\hline 3 & 2 & 2 & 3 & 3 & 2 & 2 & 1 & 2 & 2 & 3801.6365 & -0.037 \\
\hline 3 & 2 & 2 & 3 & 4 & 2 & 2 & 1 & 2 & 3 & 3802.0032 & -0.036 \\
\hline 3 & 2 & 2 & 4 & 4 & 2 & 2 & 1 & 3 & 3 & 3802.5293 & -0.038 \\
\hline 3 & 2 & 2 & 4 & 5 & 2 & 2 & 1 & 3 & 4 & 3803.0842 & -0.009 \\
\hline 3 & 2 & 2 & 2 & 3 & 2 & 2 & 1 & 1 & 2 & 3803.3657 & 0.01 \\
\hline 3 & 2 & 2 & 4 & 3 & 2 & 2 & 1 & 3 & 2 & 3803.5463 & -0.003 \\
\hline 3 & 1 & 2 & $\begin{array}{l}4 \\
2 \\
4 \\
3\end{array}$ & $\begin{array}{l}4 \\
3 \\
5 \\
2\end{array}$ & 2 & 1 & 1 & $\begin{array}{l}3 \\
1 \\
3 \\
2\end{array}$ & $\begin{array}{l}3 \\
2 \\
4 \\
1\end{array}$ & $\begin{array}{l}4033.5892 \\
4033.7201\end{array}$ & $\begin{array}{l}-0.001 \\
0.012\end{array}$ \\
\hline 3 & 2 & 1 & 3 & 3 & 2 & 2 & 0 & 2 & 2 & 4073.7912 & 0.069 \\
\hline 3 & 2 & 1 & 3 & 4 & 2 & 2 & 0 & 2 & 3 & 4074.3769 & 0.021 \\
\hline 3 & 2 & 1 & 3 & 2 & 2 & 2 & 0 & 2 & 1 & 4074.535 & 0.032 \\
\hline 3 & 2 & 1 & 4 & 4 & 2 & 2 & 0 & 3 & 3 & 4075.0123 & 0.036 \\
\hline 3 & 2 & 1 & 4 & 5 & 2 & 2 & 0 & 3 & 4 & 4075.7477 & -0.012 \\
\hline 3 & 2 & 1 & 2 & 3 & 2 & 2 & 0 & 1 & 2 & 4076.0578 & -0.009 \\
\hline 3 & 2 & 1 & 4 & 3 & 2 & 2 & 0 & 3 & 2 & 4076.2161 & -0.026 \\
\hline 4 & 1 & 4 & $\begin{array}{l}3 \\
3 \\
5 \\
5 \\
4 \\
4 \\
5\end{array}$ & $\begin{array}{l}2 \\
4 \\
4 \\
6 \\
3 \\
5 \\
5\end{array}$ & 3 & 1 & 3 & $\begin{array}{l}2 \\
2 \\
4 \\
4 \\
3 \\
3 \\
4\end{array}$ & $\begin{array}{l}1 \\
3 \\
3 \\
5 \\
2 \\
4 \\
4\end{array}$ & 4548.2442 & 0.016 \\
\hline 4 & 0 & 4 & $\begin{array}{l}5 \\
3 \\
5 \\
3 \\
5 \\
3 \\
5 \\
4 \\
4 \\
\end{array}$ & $\begin{array}{l}4 \\
4 \\
4 \\
4 \\
6 \\
3 \\
5 \\
3 \\
5 \\
\end{array}$ & 3 & 0 & 3 & $\begin{array}{l}4 \\
4 \\
2 \\
2 \\
4 \\
2 \\
4 \\
3 \\
3\end{array}$ & $\begin{array}{l}3 \\
3 \\
3 \\
3 \\
5 \\
2 \\
4 \\
2 \\
4\end{array}$ & 4578.3769 & -0.007 \\
\hline 3 & 1 & 2 & 4 & 5 & 2 & 0 & 2 & 3 & 4 & 4754.9776 & -0.03 \\
\hline 3 & 2 & 1 & 4 & 3 & 2 & 1 & 1 & 3 & 2 & 4861.4365 & -0.022 \\
\hline 3 & 2 & 1 & 2 & 3 & 2 & 1 & 1 & 1 & 2 & 4861.613 & 0.001 \\
\hline 3 & 2 & 1 & 4 & 5 & 2 & 1 & 1 & 3 & 4 & 4861.7239 & -0.025 \\
\hline
\end{tabular}


A. Appendix

\begin{tabular}{|c|c|c|c|c|c|c|c|c|c|c|c|}
\hline $\mathrm{J}^{\prime}$ & $\mathrm{K}_{a}^{\prime}$ & $\mathrm{K}_{c}^{\prime}$ & $\mathrm{F}_{1}^{\prime}$ & $\mathrm{F}^{\prime}$ & J" & $\mathrm{K}_{a}^{\prime \prime}$ & $\mathrm{K}_{c}^{\prime \prime}$ & $\mathrm{F}_{1}^{\prime \prime}$ & $F^{\prime \prime}$ & $\nu_{\text {exp. }} / \mathrm{MHz}$ & $\Delta \nu / \mathrm{MHz}$ \\
\hline 3 & 2 & 1 & 4 & 4 & 2 & 1 & 1 & 3 & 3 & 4861.8281 & -0.027 \\
\hline 3 & 2 & 1 & $\begin{array}{l}3 \\
3 \\
3\end{array}$ & $\begin{array}{l}3 \\
2 \\
4\end{array}$ & 2 & 1 & 1 & $\begin{array}{l}2 \\
2 \\
2\end{array}$ & $\begin{array}{l}2 \\
1 \\
3\end{array}$ & 4862.1078 & -0.018 \\
\hline 4 & 2 & 3 & $\begin{array}{l}4 \\
5 \\
5 \\
5 \\
3\end{array}$ & $\begin{array}{l}4 \\
5 \\
6 \\
4 \\
2\end{array}$ & 3 & 2 & 2 & $\begin{array}{l}3 \\
4 \\
4 \\
4 \\
2\end{array}$ & $\begin{array}{l}3 \\
4 \\
5 \\
3 \\
1\end{array}$ & $\begin{array}{c}5000.5612 \\
5000.8819 \\
5001.078 \\
5001.1644\end{array}$ & $\begin{array}{c}-0.061 \\
-0.01 \\
-0.001 \\
-0.006\end{array}$ \\
\hline 3 & 2 & 2 & $\begin{array}{l}3 \\
4 \\
4\end{array}$ & $\begin{array}{l}2 \\
4 \\
5\end{array}$ & 2 & 1 & 2 & $\begin{array}{l}2 \\
3 \\
3\end{array}$ & $\begin{array}{l}1 \\
3 \\
4\end{array}$ & 5107.2227 & 0.007 \\
\hline 4 & 1 & 3 & $\begin{array}{l}5 \\
3 \\
3 \\
5 \\
5\end{array}$ & $\begin{array}{l}4 \\
3 \\
4 \\
6 \\
5\end{array}$ & 3 & 1 & 2 & $\begin{array}{l}4 \\
2 \\
2 \\
4 \\
4\end{array}$ & $\begin{array}{l}3 \\
2 \\
3 \\
5 \\
4\end{array}$ & $\begin{array}{l}5222.9361 \\
5223.1171\end{array}$ & $\begin{array}{l}0.005 \\
0.002\end{array}$ \\
\hline 4 & 1 & 3 & $\begin{array}{l}4 \\
4\end{array}$ & $\begin{array}{l}3 \\
5\end{array}$ & 3 & 1 & 2 & $\begin{array}{l}3 \\
3\end{array}$ & $\begin{array}{l}2 \\
4\end{array}$ & 5223.3352 & -0.006 \\
\hline 3 & 3 & 0 & 4 & 5 & 2 & 2 & 0 & 3 & 4 & 5459.5626 & 0.029 \\
\hline 4 & 2 & 2 & 4 & 4 & 3 & 2 & 1 & 3 & 3 & 5486.2285 & 0.016 \\
\hline 4 & 2 & 2 & 4 & 5 & 3 & 2 & 1 & 3 & 4 & 5486.5423 & -0.005 \\
\hline 5 & 1 & 5 & $\begin{array}{l}4 \\
6 \\
4 \\
6 \\
5 \\
5 \\
4 \\
6 \\
5\end{array}$ & $\begin{array}{l}3 \\
5 \\
5 \\
7 \\
4 \\
6 \\
4 \\
6 \\
5\end{array}$ & 4 & 1 & 4 & $\begin{array}{l}3 \\
5 \\
3 \\
5 \\
4 \\
4 \\
3 \\
5 \\
4\end{array}$ & $\begin{array}{l}2 \\
4 \\
4 \\
6 \\
3 \\
5 \\
3 \\
5 \\
4\end{array}$ & 5624.5249 & 0.003 \\
\hline 5 & 0 & 5 & $\begin{array}{l}4 \\
6 \\
6 \\
4 \\
4 \\
6 \\
6 \\
6 \\
5\end{array}$ & $\begin{array}{l}3 \\
5 \\
5 \\
5 \\
5 \\
7 \\
6 \\
6 \\
5\end{array}$ & 4 & 0 & 4 & $\begin{array}{l}3 \\
3 \\
5 \\
3 \\
5 \\
5 \\
5 \\
4 \\
4\end{array}$ & $\begin{array}{l}2 \\
4 \\
4 \\
4 \\
4 \\
6 \\
5 \\
5 \\
4\end{array}$ & 5634.0921 & -0.008 \\
\hline 5 & 2 & 4 & $\begin{array}{l}5 \\
4 \\
6 \\
5 \\
5\end{array}$ & $\begin{array}{l}5 \\
4 \\
6 \\
6 \\
4\end{array}$ & 4 & 2 & 3 & $\begin{array}{l}4 \\
3 \\
5 \\
4 \\
4\end{array}$ & $\begin{array}{l}4 \\
3 \\
5 \\
5 \\
3\end{array}$ & 6151.3989 & 0.031 \\
\hline
\end{tabular}


A. Appendix

\begin{tabular}{|c|c|c|c|c|c|c|c|c|c|c|c|}
\hline $\mathrm{J}^{\prime}$ & $\mathrm{K}_{a}^{\prime}$ & $\mathrm{K}_{c}^{\prime}$ & $\mathrm{F}_{1}^{\prime}$ & $F^{\prime}$ & J" & $\mathrm{K}_{a}^{\prime \prime}$ & $\mathrm{K}_{c}^{\prime \prime}$ & $\mathrm{F}_{1}^{\prime \prime}$ & F" & $\nu_{\text {exp. }} / \mathrm{MHz}$ & $\Delta \nu / \mathrm{MHz}$ \\
\hline & & & 4 & 3 & & & & 3 & 2 & & \\
\hline & & & 6 & 5 & & & & 5 & 4 & & \\
\hline & & & 4 & 5 & & & & 3 & 4 & & \\
\hline & & & 6 & 7 & & & & 5 & 6 & & \\
\hline \multirow{7}{*}{4} & \multirow{7}{*}{2} & \multirow{7}{*}{2} & 4 & 4 & \multirow{7}{*}{3} & \multirow{7}{*}{1} & \multirow{7}{*}{2} & 3 & 3 & \multirow{7}{*}{6314.9776} & \multirow{7}{*}{0.009} \\
\hline & & & 3 & 2 & & & & 2 & 1 & & \\
\hline & & & 5 & 5 & & & & 4 & 4 & & \\
\hline & & & 5 & 4 & & & & 4 & 3 & & \\
\hline & & & 4 & 5 & & & & 3 & 4 & & \\
\hline & & & 4 & 3 & & & & 3 & 2 & & \\
\hline & & & 3 & 4 & & & & 2 & 3 & & \\
\hline \multirow{8}{*}{6} & \multirow{8}{*}{1} & \multirow{8}{*}{6} & 5 & 4 & \multirow{8}{*}{5} & \multirow{8}{*}{1} & \multirow{8}{*}{5} & 4 & 3 & \multirow{8}{*}{6693.943} & \multirow{8}{*}{0.002} \\
\hline & & & 7 & 6 & & & & 6 & 5 & & \\
\hline & & & 5 & 6 & & & & 4 & 5 & & \\
\hline & & & 7 & 8 & & & & 6 & 7 & & \\
\hline & & & 6 & 5 & & & & 5 & 4 & & \\
\hline & & & 6 & 7 & & & & 5 & 6 & & \\
\hline & & & 5 & 5 & & & & 4 & 4 & & \\
\hline & & & 7 & 7 & & & & 6 & 6 & & \\
\hline \multirow{9}{*}{6} & \multirow{9}{*}{0} & \multirow{9}{*}{6} & 5 & 4 & \multirow{9}{*}{5} & \multirow{9}{*}{0} & \multirow{9}{*}{5} & 4 & 3 & \multirow{9}{*}{6696.5705} & \multirow{9}{*}{-0.003} \\
\hline & & & 7 & 6 & & & & 6 & 5 & & \\
\hline & & & 5 & 6 & & & & 4 & 5 & & \\
\hline & & & 7 & 8 & & & & 6 & 7 & & \\
\hline & & & 6 & 5 & & & & 5 & 4 & & \\
\hline & & & 6 & 7 & & & & 5 & 6 & & \\
\hline & & & 5 & 5 & & & & 4 & 4 & & \\
\hline & & & 7 & 7 & & & & 6 & 6 & & \\
\hline & & & 6 & 6 & & & & 5 & 5 & & \\
\hline
\end{tabular}


A. Appendix
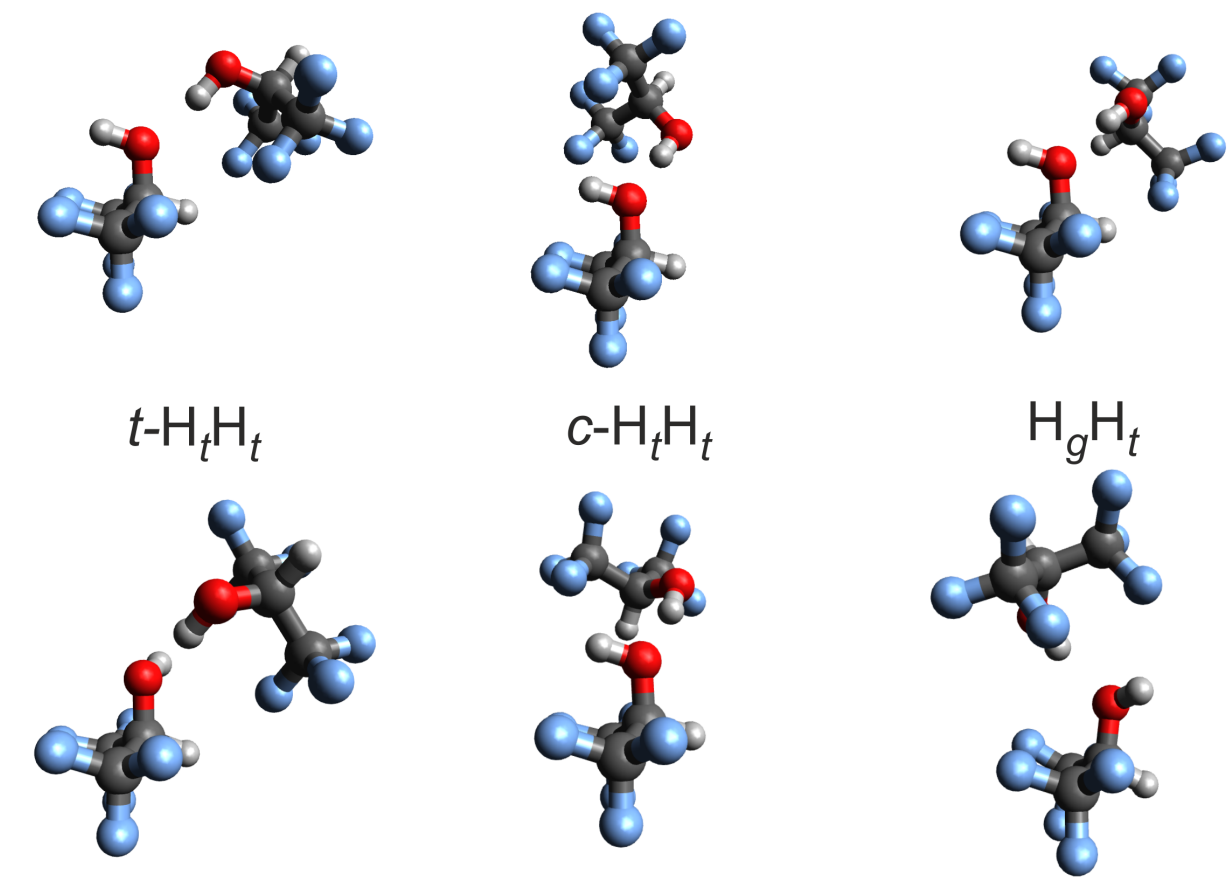

$\mathrm{H}_{t} \mathrm{H}_{g}$

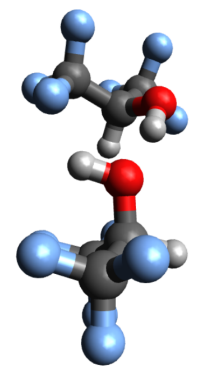

$\mathrm{H}_{g} \mathrm{H}_{t}$

$\mathrm{H}_{t} \mathrm{H}_{g}$
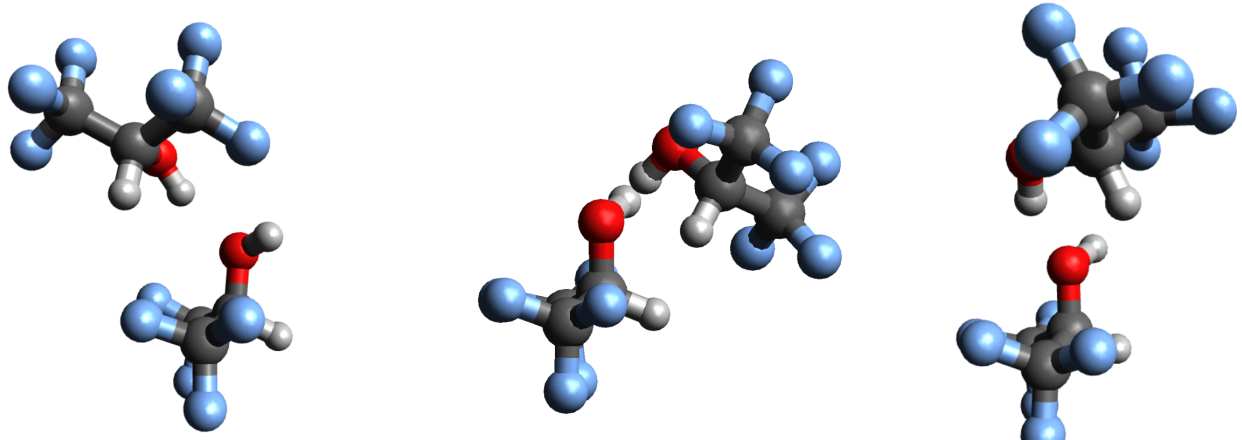

$\mathrm{H}_{g} \mathrm{H}_{g}$

$\mathrm{H}_{g} \mathrm{H}_{g}$

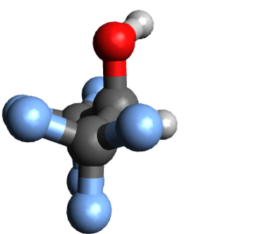

$\mathrm{H}_{g} \mathrm{H}_{g}$.
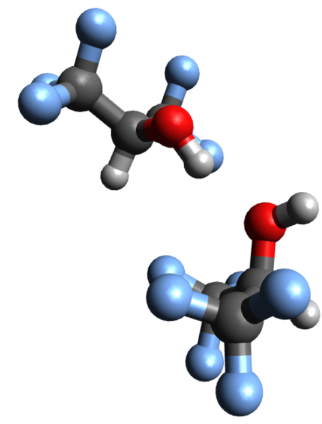

$\mathrm{H}_{g} \mathrm{H}_{g}$

Figure A.7.: Stable structures of HH dimers optimised at the B3LYP-D3(BJ)/def2-QZVP level. 


\section{A. Appendix}

Table A.11.: Theoretically predicted harmonic $\left(\omega_{\mathrm{OH}}\right) \mathrm{OH}$ stretching wavenumber, spectral downshift from the respective monomer $\left(\Delta \omega_{\mathrm{OH}}\right)$ and lowest predicted wavenumber $\left(\omega_{\mathrm{l}}\right)$ in $\mathrm{cm}^{-1}$, IR intensity $\left(S_{\mathrm{OH}}\right)$ in $\mathrm{km} \mathrm{mol}^{-1}$, Raman scattering cross section $\left(\sigma_{\mathrm{OH}}\right)$ assuming $T_{\text {vib }}=100 \mathrm{~K}$ in $10^{-36} \mathrm{~m}^{2} \mathrm{sr}^{-1}$, relative electronic and harmonic vibrational zero-point corrected energies $\left(\Delta E_{\mathrm{e}}\right.$ and $\left.\Delta E_{0}\right)$ compared to the most stable $\mathrm{H}_{t} \mathrm{~N}_{\mathrm{H}}$ conformation in $\mathrm{kJ} \mathrm{mol}^{-1}$, as well as equilibrium rotational constants $\left(A_{\mathrm{e}}, B_{\mathrm{e}}, C_{\mathrm{e}}\right)$ in $\mathrm{MHz}$ and magnitudes of the electric dipole moment components $\left(\left|\mu_{a}\right|,\left|\mu_{b}\right|,\left|\mu_{c}\right|\right)$ in $\mathrm{D}$ calculated at the B3LYP-D3(BJ)/def2-QZVP level of approximation.

\begin{tabular}{cccccccccccccc}
\hline Structure & $\omega_{\mathrm{OH}}$ & $S_{\mathrm{OH}}$ & $\sigma_{\mathrm{OH}}$ & $\Delta \omega_{\mathrm{OH}}$ & $\Delta E_{\mathrm{e}}$ & $\Delta E_{0}$ & $\omega_{1}$ & $A_{\mathrm{e}}$ & $B_{\mathrm{e}}$ & $C_{\mathrm{e}}$ & $\left|\mu_{a}\right|$ & $\left|\mu_{b}\right|$ & $\left|\mu_{c}\right|$ \\
\hline \multirow{2}{*}{$t-\mathrm{H}_{t} \mathrm{H}_{t}$} & 3788 & 79 & 45 & 1 & 0.0 & 0.0 & 7 & 479.7 & 206.1 & 169.8 & 0.4 & 0.0 & 0.9 \\
& 3624 & 612 & 147 & 165 & & & & & & & \\
$c-\mathrm{H}_{t} \mathrm{H}_{t}$ & 3774 & 80 & 29 & 5 & 1.2 & 1.5 & 10 & 525.5 & 186.3 & 156.6 & 0.6 & 0.3 & 0.6 \\
& 3611 & 654 & 166 & 178 & & & & & & & & & \\
$\mathrm{H}_{g} \mathrm{H}_{t}$ & 3783 & 77 & 40 & 6 & 2.6 & 2.7 & 12 & 570.6 & 154.7 & 153.3 & 3.1 & 0.9 & 0.8 \\
& 3688 & 632 & 161 & 139 & & & & & & & & & \\
& 3794 & 130 & 45 & 33 & 2.6 & 2.9 & 10 & 504.3 & 187.7 & 168.2 & 0.9 & 0.5 & 1.0 \\
$\mathrm{H}_{t} \mathrm{H}_{g}$ & 3607 & 598 & 156 & 182 & & & & & & & & & \\
& 3765 & 109 & 29 & 24 & 3.6 & 3.8 & 10 & 588.6 & 154.1 & 146.0 & 3.2 & 0.5 & 0.1 \\
$\mathrm{H}_{g} \mathrm{H}_{t}$ & 3675 & 609 & 180 & 152 & & & & & & & & & \\
& 3811 & 105 & 44 & 16 & 5.0 & 4.7 & 8 & 512.4 & 195.9 & 173.2 & 0.6 & 0.4 & 2.1 \\
$\mathrm{H}_{t} \mathrm{H}_{g}$ & 3631 & 568 & 143 & 158 & & & & & & & & & \\
& 3798 & 137 & 45 & 29 & 6.9 & 6.5 & 7 & 513.4 & 180.0 & 167.4 & 3.6 & 2.6 & 0.8 \\
$\mathrm{H}_{g} \mathrm{H}_{g}$ & 3701 & 493 & 142 & 126 & & & & & & & & & \\
& 3800 & 90 & 41 & 27 & 7.7 & 7.2 & 11 & 597.8 & 154.1 & 144.7 & 2.5 & 1.1 & 0.6 \\
$\mathrm{H}_{g} \mathrm{H}_{g^{\prime}}$ & 3728 & 470 & 140 & 99 & & & & & & & & & \\
& 3824 & 110 & 44 & 3 & 9.1 & 7.8 & 5 & 554.5 & 160.8 & 142.2 & 3.1 & 1.6 & 2.5 \\
$\mathrm{H}_{g} \mathrm{H}_{\mathrm{g}^{\prime}}$ & 3728 & 470 & 140 & 99 & & & & & & & & & \\
$\mathrm{H}_{g} \mathrm{H}_{g^{\prime}}$ & 3817 & 95 & 52 & 10 & 10.8 & 9.8 & 6 & 518.5 & 174.5 & 154.4 & 4.8 & 0.9 & 1.7 \\
\hline
\end{tabular}

Table A.12.: Experimentally obtained transition frequencies $\left(\nu_{\text {exp. }}\right)$ of the $t-\mathrm{H}_{t} \mathrm{H}_{t}$ dimer with their respective rotational quantum numbers as well as the differences between the fitted and observed frequencies $\left(\Delta \nu=\nu_{\text {calc. }}-\nu_{\text {exp. }}\right) . \quad \nu_{\text {calc. }}$ is calculated using the fitted experimental spectroscopic constants.

\begin{tabular}{cccccccc}
\hline $\mathrm{J}^{\prime}$ & $\mathrm{K}_{a}^{\prime}$ & $\mathrm{K}_{c}^{\prime}$ & $\mathrm{J}^{\prime \prime}$ & $\mathrm{K}_{a}^{\prime \prime}$ & $\mathrm{K}_{c}^{\prime \prime}$ & $\nu_{\text {exp. }} / \mathrm{MHz}$ & $\Delta \nu / \mathrm{MHz}$ \\
\hline 10 & 4 & 6 & 10 & 3 & 8 & 2114.0783 & -0.0182 \\
11 & 4 & 7 & 11 & 3 & 9 & 2169.7423 & 0.009 \\
8 & 1 & 7 & 7 & 2 & 5 & 2298.5323 & 0.0019 \\
4 & 2 & 2 & 3 & 1 & 2 & 2336.2138 & 0.0011 \\
5 & 1 & 4 & 4 & 0 & 4 & 2474.6768 & -0.0009 \\
4 & 2 & 3 & 3 & 1 & 3 & 2504.8325 & -0.0051 \\
11 & 5 & 7 & 11 & 4 & 7 & 2570.5977 & 0.0164 \\
10 & 5 & 6 & 10 & 4 & 6 & 2604.7366 & -0.0177 \\
3 & 3 & 0 & 2 & 2 & 0 & 2610.9986 & 0.0063 \\
3 & 3 & 1 & 2 & 2 & 1 & 2614.0911 & 0.001 \\
12 & 5 & 7 & 12 & 4 & 9 & 2623.1011 & 0.0151 \\
11 & 5 & 6 & 11 & 4 & 8 & 2624.7167 & 0.012 \\
9 & 5 & 5 & 9 & 4 & 5 & 2626.7593 & 0.0127 \\
9 & 5 & 4 & 9 & 4 & 6 & 2638.075 & 0.0277 \\
8 & 5 & 4 & 8 & 4 & 4 & 2640.6442 & -0.0114
\end{tabular}




\section{A. Appendix}

\begin{tabular}{cccccccc}
$\mathrm{J}^{\prime}$ & $\mathrm{K}_{a}^{\prime}$ & $\mathrm{K}_{c}^{\prime}$ & $\mathrm{J}^{\prime \prime}$ & $\mathrm{K}_{a}^{\prime \prime}$ & $\mathrm{K}_{c}^{\prime \prime}$ & $\nu_{\text {exp. }} / \mathrm{MHz}$ & $\Delta \nu / \mathrm{MHz}$ \\
\hline 8 & 5 & 3 & 8 & 4 & 5 & 2645.0314 & -0.0154 \\
7 & 5 & 3 & 7 & 4 & 3 & 2649.3283 & -0.0032 \\
7 & 5 & 2 & 7 & 4 & 4 & 2650.8052 & 0.0019 \\
6 & 5 & 2 & 6 & 4 & 2 & 2654.6519 & 0.006 \\
6 & 5 & 1 & 6 & 4 & 3 & 2655.0518 & 0.0038 \\
5 & 5 & 1 & 5 & 4 & 1 & 2657.7893 & -0.0072 \\
5 & 2 & 3 & 4 & 1 & 3 & 2699.9574 & -0.0002 \\
5 & 2 & 4 & 4 & 1 & 4 & 2952.9641 & -0.0045 \\
12 & 3 & 9 & 11 & 4 & 7 & 2949.5407 & 0.008 \\
4 & 3 & 1 & 3 & 2 & 1 & 2982.4444 & -0.0016 \\
6 & 1 & 5 & 5 & 0 & 5 & 2987.2968 & 0.0012 \\
4 & 3 & 2 & 3 & 2 & 2 & 2997.3881 & 0.0006 \\
6 & 2 & 4 & 5 & 1 & 4 & 3081.9443 & -0.0005 \\
15 & 6 & 10 & 15 & 5 & 10 & 3108.7033 & 0.0077 \\
8 & 2 & 6 & 7 & 2 & 5 & 3147.9225 & 0.0124 \\
13 & 6 & 8 & 13 & 5 & 8 & 3182.1828 & -0.0083 \\
9 & 0 & 9 & 8 & 0 & 8 & 3184.1152 & -0.003 \\
14 & 6 & 8 & 14 & 5 & 10 & 3184.6996 & 0.0084 \\
11 & 2 & 9 & 10 & 3 & 7 & 3184.8544 & -0.0064 \\
13 & 6 & 7 & 13 & 5 & 9 & 3198.1393 & -0.0006 \\
12 & 6 & 7 & 12 & 5 & 7 & 3203.5803 & 0.0003 \\
12 & 6 & 6 & 12 & 5 & 8 & 3210.8046 & 0.0041 \\
11 & 6 & 6 & 11 & 5 & 6 & 3218.7787 & 0.0037 \\
11 & 6 & 5 & 11 & 5 & 7 & 3221.7839 & -0.003 \\
10 & 6 & 5 & 10 & 5 & 5 & 3229.6191 & -0.007 \\
10 & 6 & 4 & 10 & 5 & 6 & 3230.7714 & 0.0058 \\
9 & 6 & 4 & 9 & 5 & 4 & 3237.365 & -0.0002 \\
9 & 6 & 3 & 9 & 5 & 5 & 3237.7485 & 0.0014 \\
8 & 6 & 3 & 8 & 5 & 3 & 3242.837 & 0.0137 \\
8 & 6 & 2 & 8 & 5 & 4 & 3242.9091 & -0.0235 \\
7 & 6 & 2 & 7 & 5 & 2 & 3246.5894 & 0.006 \\
7 & 6 & 1 & 7 & 5 & 3 & 3246.5894 & -0.0192 \\
6 & 6 & 1 & 6 & 5 & 1 & 3249.0869 & 0.0092 \\
6 & 6 & 0 & 6 & 5 & 2 & 3249.0869 & 0.005 \\
9 & 2 & 8 & 8 & 2 & 7 & 3338.6911 & -0.0203 \\
5 & 3 & 2 & 4 & 2 & 2 & 3344.2653 & 0.0006 \\
5 & 3 & 3 & 4 & 2 & 3 & 3386.6492 & -0.0025 \\
9 & 3 & 7 & 8 & 3 & 6 & 3415.9214 & -0.0231 \\
9 & 5 & 5 & 8 & 5 & 4 & 3416.8295 & -0.0209 \\
9 & 5 & 4 & 8 & 5 & 3 & 3417.1306 & 0.0131 \\
6 & 2 & 5 & 5 & 1 & 5 & 3418.2262 & 0 \\
9 & 1 & 8 & 8 & 1 & 7 & 3427.0973 & 0.0104 \\
9 & 4 & 5 & 8 & 4 & 4 & 3430.7571 & -0.0023 \\
9 & 3 & 6 & 8 & 3 & 5 & 3485.3325 & -0.0105 \\
7 & 2 & 5 & 6 & 1 & 5 & 3488.4928 & -0.0035 \\
10 & 0 & 10 & 9 & 0 & 9 & 3522.8595 & 0.0002 \\
7 & 1 & 6 & 6 & 0 & 6 & 3529.4932 & 0.0054 \\
9 & 2 & 7 & 8 & 2 & 6 & 3543.2245 & 0.0008 \\
4 & 4 & 0 & 3 & 3 & 0 & 3581.9338 & 0.0011 \\
4 & 4 & 1 & 3 & 3 & 1 & 3582.1106 & -0.0011 \\
6 & 3 & 3 & 5 & 2 & 3 & 3693.4592 & -0.0037
\end{tabular}




\section{A. Appendix}

\begin{tabular}{|c|c|c|c|c|c|c|c|}
\hline $\mathrm{J}^{\prime}$ & $\mathrm{K}_{a}^{\prime}$ & $\mathrm{K}_{c}^{\prime}$ & $\mathrm{J}^{\prime \prime}$ & $\mathrm{K}_{a}^{\prime \prime}$ & $\mathrm{K}_{c}^{\prime \prime}$ & $\nu_{\text {exp. }} / \mathrm{MHz}$ & $\Delta \nu / \mathrm{MHz}$ \\
\hline 10 & 2 & 9 & 9 & 2 & 8 & 3694.9372 & -0.0038 \\
\hline 17 & 7 & 11 & 17 & 6 & 11 & 3728.8272 & -0.0166 \\
\hline 17 & 7 & 10 & 17 & 6 & 12 & 3747.8625 & -0.0006 \\
\hline 16 & 7 & 10 & 16 & 6 & 10 & 3756.8787 & 0.0099 \\
\hline 16 & 7 & 9 & 16 & 6 & 11 & 3766.1955 & -0.0005 \\
\hline 10 & 1 & 9 & 9 & 1 & 8 & 3770.258 & -0.009 \\
\hline 15 & 7 & 9 & 15 & 6 & 9 & 3778.2022 & -0.0028 \\
\hline 15 & 7 & 8 & 15 & 6 & 10 & 3782.5191 & -0.0092 \\
\hline 6 & 3 & 4 & 5 & 2 & 4 & 3784.4734 & 0.0002 \\
\hline 14 & 7 & 8 & 14 & 6 & 8 & 3794.6305 & -0.004 \\
\hline 14 & 7 & 7 & 14 & 6 & 9 & 3796.5228 & 0.0073 \\
\hline 13 & 7 & 7 & 13 & 6 & 7 & 3807.3661 & -0.0014 \\
\hline 13 & 7 & 6 & 13 & 6 & 8 & 3808.1291 & 0.0007 \\
\hline 12 & 7 & 6 & 12 & 6 & 6 & 3817.2473 & 0.0097 \\
\hline 12 & 7 & 5 & 12 & 6 & 7 & 3817.5255 & 0.0053 \\
\hline 11 & 7 & 5 & 11 & 6 & 5 & 3824.9113 & 0.0719 \\
\hline 11 & 7 & 4 & 11 & 6 & 6 & 3824.9113 & -0.0228 \\
\hline 10 & 7 & 4 & 10 & 6 & 4 & 3830.6325 & 0.0137 \\
\hline 10 & 7 & 3 & 10 & 6 & 5 & 3830.6325 & -0.0141 \\
\hline 9 & 7 & 3 & 9 & 6 & 3 & 3834.9268 & -0.0005 \\
\hline 9 & 7 & 2 & 9 & 6 & 4 & 3834.9268 & -0.0075 \\
\hline 8 & 7 & 1 & 8 & 6 & 3 & 3838.0594 & 0.0031 \\
\hline 8 & 7 & 2 & 8 & 6 & 2 & 3838.0594 & 0.0045 \\
\hline 7 & 7 & 1 & 7 & 6 & 1 & 3840.2546 & 0.0072 \\
\hline 7 & 7 & 0 & 7 & 6 & 2 & 3840.2546 & 0.007 \\
\hline 11 & 1 & 11 & 10 & 1 & 10 & 3857.9546 & 0.0067 \\
\hline 13 & 2 & 11 & 12 & 3 & 9 & 3862.1067 & -0.0058 \\
\hline 11 & 0 & 11 & 10 & 0 & 10 & 3862.3957 & 0.005 \\
\hline 10 & 3 & 7 & 9 & 3 & 6 & 3896.8042 & -0.0019 \\
\hline 7 & 2 & 6 & 6 & 1 & 6 & 3899.9935 & -0.0011 \\
\hline 8 & 2 & 6 & 7 & 1 & 6 & 3924.1719 & -0.0011 \\
\hline 10 & 2 & 8 & 9 & 2 & 7 & 3930.6931 & -0.0042 \\
\hline 5 & 4 & 1 & 4 & 3 & 1 & 3959.382 & -0.002 \\
\hline 5 & 4 & 2 & 4 & 3 & 2 & 3960.6129 & -0.0012 \\
\hline 5 & 4 & 1 & 4 & 3 & 1 & 3959.382 & -0.002 \\
\hline 5 & 4 & 2 & 4 & 3 & 2 & 3960.6129 & -0.0012 \\
\hline 7 & 3 & 4 & 6 & 2 & 4 & 4031.1222 & -0.0015 \\
\hline 11 & 2 & 10 & 10 & 2 & 9 & 4047.8299 & -0.0007 \\
\hline 8 & 1 & 7 & 7 & 0 & 7 & 4095.5865 & 0 \\
\hline 11 & 1 & 10 & 10 & 1 & 9 & 4107.2359 & 0.0029 \\
\hline 11 & 3 & 9 & 10 & 3 & 8 & 4164.3182 & -0.0068 \\
\hline 11 & 7 & 5 & 10 & 7 & 4 & 4171.036 & 0.0054 \\
\hline 11 & 7 & 4 & 10 & 7 & 3 & 4171.036 & 0.004 \\
\hline 7 & 3 & 5 & 6 & 2 & 5 & 4193.3475 & 0.0003 \\
\hline 12 & 1 & 12 & 11 & 1 & 11 & 4199.8892 & -0.0187 \\
\hline 12 & 0 & 12 & 11 & 0 & 11 & 4202.5147 & 0.0014 \\
\hline 11 & 3 & 8 & 10 & 3 & 7 & 4309.9335 & 0.0008 \\
\hline 6 & 4 & 2 & 5 & 3 & 2 & 4334.6848 & -0.004 \\
\hline 6 & 4 & 3 & 5 & 3 & 3 & 4339.4822 & -0.0005 \\
\hline 8 & 3 & 5 & 7 & 2 & 5 & 4363.5663 & -0.0009 \\
\hline 17 & 8 & 10 & 17 & 7 & 10 & 4368.779 & -0.007 \\
\hline
\end{tabular}


A. Appendix

\begin{tabular}{|c|c|c|c|c|c|c|c|}
\hline $\mathrm{J}^{\prime}$ & $\mathrm{K}_{a}^{\prime}$ & $\mathrm{K}_{c}^{\prime}$ & $\mathrm{J}^{\prime \prime}$ & $\mathrm{K}_{a}^{\prime \prime}$ & $\mathrm{K}_{c}^{\prime \prime}$ & $\nu_{\text {exp. }} / \mathrm{MHz}$ & $\Delta \nu / \mathrm{MHz}$ \\
\hline 17 & 8 & 9 & 17 & 7 & 11 & 4369.8978 & 0.0004 \\
\hline 16 & 8 & 9 & 16 & 7 & 9 & 4383.1771 & 0.0022 \\
\hline 16 & 8 & 8 & 16 & 7 & 10 & 4383.6383 & -0.0058 \\
\hline 9 & 2 & 7 & 8 & 1 & 7 & 4392.603 & -0.0004 \\
\hline 15 & 8 & 8 & 15 & 7 & 8 & 4394.9231 & 0.013 \\
\hline 15 & 8 & 7 & 15 & 7 & 9 & 4395.0771 & -0.0184 \\
\hline 8 & 2 & 7 & 7 & 1 & 7 & 4397.1651 & -0.002 \\
\hline 12 & 2 & 11 & 11 & 2 & 10 & 4397.7415 & -0.0089 \\
\hline 14 & 8 & 7 & 14 & 7 & 7 & 4404.4844 & 0.0499 \\
\hline 14 & 8 & 6 & 14 & 7 & 8 & 4404.4844 & -0.018 \\
\hline 13 & 8 & 6 & 13 & 7 & 6 & 4412.1081 & 0.0092 \\
\hline 13 & 8 & 5 & 13 & 7 & 7 & 4412.1081 & -0.0135 \\
\hline 12 & 8 & 5 & 12 & 7 & 5 & 4418.1969 & 0.004 \\
\hline 11 & 8 & 4 & 11 & 7 & 4 & 4422.9615 & -0.0024 \\
\hline 10 & 8 & 3 & 10 & 7 & 3 & 4426.6282 & -0.0001 \\
\hline 9 & 8 & 2 & 9 & 7 & 2 & 4429.3759 & -0.0014 \\
\hline 8 & 8 & 0 & 8 & 7 & 2 & 4431.379 & -0.0015 \\
\hline 12 & 1 & 11 & 11 & 1 & 10 & 4441.419 & -0.0088 \\
\hline 5 & 5 & 0 & 4 & 4 & 0 & 4551.4393 & 0.0026 \\
\hline 5 & 5 & 1 & 4 & 4 & 1 & 4551.4393 & -0.0058 \\
\hline 8 & 3 & 6 & 7 & 2 & 6 & 4615.4456 & -0.002 \\
\hline 12 & 4 & 8 & 11 & 4 & 7 & 4623.6721 & -0.0175 \\
\hline 12 & 2 & 10 & 11 & 2 & 9 & 4675.9862 & -0.0036 \\
\hline 9 & 1 & 8 & 8 & 0 & 8 & 4676.4562 & 0 \\
\hline 9 & 3 & 6 & 8 & 2 & 6 & 4700.9987 & -0.0016 \\
\hline 7 & 4 & 3 & 6 & 3 & 3 & 4705.539 & -0.0005 \\
\hline 7 & 4 & 4 & 6 & 3 & 4 & 4719.4124 & -0.0017 \\
\hline 12 & 3 & 9 & 11 & 3 & 8 & 4720.5087 & 0.0077 \\
\hline 13 & 2 & 12 & 12 & 2 & 11 & 4745.1803 & 0.003 \\
\hline 21 & 2 & 19 & 20 & 3 & 17 & 4763.3767 & -0.0154 \\
\hline 13 & 1 & 12 & 12 & 1 & 11 & 4775.4737 & -0.0069 \\
\hline 16 & 3 & 13 & 15 & 4 & 11 & 4799.0332 & 0.0031 \\
\hline 14 & 1 & 14 & 13 & 1 & 13 & 4882.9691 & 0.0163 \\
\hline 14 & 0 & 14 & 13 & 0 & 13 & 4883.8095 & 0.0057 \\
\hline 10 & 2 & 8 & 9 & 1 & 8 & 4896.2164 & 0.0024 \\
\hline 13 & 3 & 11 & 12 & 3 & 10 & 4898.2966 & -0.0032 \\
\hline 9 & 2 & 8 & 8 & 1 & 8 & 4908.1384 & 0.0022 \\
\hline 6 & 5 & 1 & 5 & 4 & 1 & 4929.6027 & 0.0388 \\
\hline 6 & 5 & 2 & 5 & 4 & 2 & 4929.6027 & -0.0369 \\
\hline 13 & 8 & 6 & 12 & 8 & 5 & 4930.5181 & 0.0014 \\
\hline 13 & 7 & 6 & 12 & 7 & 5 & 4936.6066 & -0.0041 \\
\hline 13 & 7 & 7 & 12 & 7 & 6 & 4936.6066 & 0.0114 \\
\hline 13 & 6 & 7 & 12 & 6 & 6 & 4946.4667 & 0.0016 \\
\hline 19 & 9 & 10 & 19 & 8 & 12 & 4957.7819 & -0.0071 \\
\hline 18 & 9 & 9 & 18 & 8 & 11 & 4970.9803 & -0.0158 \\
\hline 16 & 9 & 7 & 16 & 8 & 9 & 4991.488 & -0.0169 \\
\hline 16 & 9 & 8 & 16 & 8 & 8 & 4991.488 & -0.0009 \\
\hline 15 & 9 & 7 & 15 & 8 & 7 & 4999.2555 & 0.0016 \\
\hline 14 & 9 & 6 & 14 & 8 & 6 & 5005.6135 & -0.0068 \\
\hline 13 & 9 & 5 & 13 & 8 & 5 & 5010.7705 & -0.0077 \\
\hline 12 & 9 & 4 & 12 & 8 & 4 & 5014.8969 & -0.001 \\
\hline
\end{tabular}




\section{A. Appendix}

\begin{tabular}{|c|c|c|c|c|c|c|c|}
\hline $\mathrm{J}^{\prime}$ & $\mathrm{K}_{a}^{\prime}$ & $\mathrm{K}_{c}^{\prime}$ & $\mathrm{J}^{\prime \prime}$ & $\mathrm{K}_{a}^{\prime \prime}$ & $\mathrm{K}_{c}^{\prime \prime}$ & $\nu_{\text {exp. }} / \mathrm{MHz}$ & $\Delta \nu / \mathrm{MHz}$ \\
\hline 12 & 9 & 3 & 12 & 8 & 5 & 5014.8969 & -0.0011 \\
\hline 11 & 9 & 2 & 11 & 8 & 4 & 5018.1372 & 0.0035 \\
\hline 11 & 9 & 3 & 11 & 8 & 3 & 5018.1372 & 0.0035 \\
\hline 10 & 9 & 1 & 10 & 8 & 3 & 5020.6235 & -0.001 \\
\hline 10 & 9 & 2 & 10 & 8 & 2 & 5020.6235 & -0.001 \\
\hline 9 & 9 & 0 & 9 & 8 & 2 & 5022.4999 & 0.0032 \\
\hline 9 & 9 & 1 & 9 & 8 & 1 & 5022.4999 & 0.0032 \\
\hline 13 & 2 & 11 & 12 & 2 & 10 & 5031.6995 & 0.0042 \\
\hline 9 & 3 & 7 & 8 & 2 & 7 & 5052.4603 & 0.0012 \\
\hline 10 & 3 & 7 & 9 & 2 & 7 & 5054.5814 & -0.0014 \\
\hline 8 & 4 & 4 & 7 & 3 & 4 & 5068.6772 & -0.0004 \\
\hline 8 & 4 & 5 & 7 & 3 & 5 & 5101.6747 & -0.0005 \\
\hline 14 & 1 & 13 & 13 & 1 & 12 & 5110.7119 & -0.0001 \\
\hline 13 & 3 & 10 & 12 & 3 & 9 & 5124.8897 & -0.0016 \\
\hline 15 & 1 & 15 & 14 & 1 & 14 & 5224.2676 & 0.017 \\
\hline 15 & 0 & 15 & 14 & 0 & 14 & 5224.7119 & -0.0157 \\
\hline 14 & 3 & 12 & 13 & 3 & 11 & 5259.2564 & -0.0043 \\
\hline 10 & 1 & 9 & 9 & 0 & 9 & 5262.6051 & 0 \\
\hline 7 & 5 & 3 & 6 & 4 & 3 & 5307.5117 & -0.0007 \\
\hline 7 & 5 & 2 & 6 & 4 & 2 & 5307.1375 & -0.002 \\
\hline 14 & 4 & 11 & 13 & 4 & 10 & 5340.4754 & 0.0063 \\
\hline 14 & 2 & 12 & 13 & 2 & 11 & 5376.5307 & 0.0172 \\
\hline 9 & 4 & 5 & 8 & 3 & 5 & 5420.3653 & -0.0033 \\
\hline 10 & 2 & 9 & 9 & 1 & 9 & 5430.9056 & 0.002 \\
\hline 18 & 3 & 15 & 17 & 4 & 13 & 5432.2631 & 0.0079 \\
\hline 11 & 3 & 8 & 10 & 2 & 8 & 5433.8152 & -0.0029 \\
\hline 11 & 2 & 9 & 10 & 1 & 9 & 5434.69 & -0.0032 \\
\hline 9 & 4 & 6 & 8 & 3 & 6 & 5488.1054 & -0.0007 \\
\hline 10 & 3 & 8 & 9 & 2 & 8 & 5505.4557 & -0.0024 \\
\hline 14 & 3 & 11 & 13 & 3 & 10 & 5520.5493 & 0.0151 \\
\hline 6 & 6 & 0 & 5 & 5 & 0 & 5520.8425 & -0.002 \\
\hline 16 & 1 & 16 & 15 & 1 & 15 & 5565.4796 & 0.002 \\
\hline 19 & 10 & 10 & 19 & 9 & 10 & 5569.2044 & 0.0218 \\
\hline 19 & 10 & 9 & 19 & 9 & 11 & 5569.2044 & 0.0116 \\
\hline 17 & 10 & 8 & 17 & 9 & 8 & 5586.3394 & -0.0039 \\
\hline 16 & 10 & 7 & 16 & 9 & 7 & 5592.9319 & -0.0094 \\
\hline 16 & 10 & 6 & 16 & 9 & 8 & 5592.9319 & -0.0098 \\
\hline 15 & 10 & 6 & 15 & 9 & 6 & 5598.4151 & -0.0101 \\
\hline 15 & 10 & 5 & 15 & 9 & 7 & 5598.4151 & -0.0102 \\
\hline 14 & 10 & 5 & 14 & 9 & 5 & 5602.9271 & -0.007 \\
\hline 14 & 10 & 4 & 14 & 9 & 6 & 5602.9271 & -0.0071 \\
\hline 13 & 10 & 3 & 13 & 9 & 5 & 5606.5926 & -0.0024 \\
\hline 13 & 10 & 4 & 13 & 9 & 4 & 5606.5926 & -0.0024 \\
\hline 12 & 10 & 2 & 12 & 9 & 4 & 5609.5198 & -0.0044 \\
\hline 12 & 10 & 3 & 12 & 9 & 3 & 5609.5198 & -0.0044 \\
\hline 11 & 10 & 1 & 11 & 9 & 3 & 5611.826 & -0.0024 \\
\hline 11 & 10 & 2 & 11 & 9 & 2 & 5611.826 & -0.0024 \\
\hline 10 & 10 & 0 & 10 & 9 & 2 & 5613.5916 & -0.0132 \\
\hline 10 & 10 & 1 & 10 & 9 & 1 & 5613.5916 & -0.0132 \\
\hline 15 & 3 & 13 & 14 & 3 & 12 & 5616.3017 & 0.0143 \\
\hline 8 & 5 & 3 & 7 & 4 & 3 & 5683.5631 & -0.0025 \\
\hline
\end{tabular}




\section{A. Appendix}

\begin{tabular}{cccccccc}
$\mathrm{J}^{\prime}$ & $\mathrm{K}_{a}^{\prime}$ & $\mathrm{K}_{c}^{\prime}$ & $\mathrm{J}^{\prime \prime}$ & $\mathrm{K}_{a}^{\prime \prime}$ & $\mathrm{K}_{c}^{\prime \prime}$ & $\nu_{\text {exp. }} / \mathrm{MHz}$ & $\Delta \nu / \mathrm{MHz}$ \\
\hline 8 & 5 & 4 & 7 & 4 & 4 & 5684.9017 & -0.0024 \\
10 & 4 & 6 & 9 & 3 & 6 & 5757.7445 & -0.0009 \\
12 & 3 & 9 & 11 & 2 & 9 & 5845.5707 & -0.0018 \\
11 & 1 & 10 & 10 & 0 & 10 & 5846.9854 & 0.0065 \\
10 & 4 & 7 & 9 & 3 & 7 & 5880.9934 & -0.0015 \\
7 & 6 & 1 & 6 & 5 & 1 & 5899.0733 & 0 \\
11 & 2 & 10 & 10 & 1 & 10 & 5963.2775 & 0.0028 \\
11 & 3 & 9 & 10 & 2 & 9 & 5974.8468 & 0.0045 \\
14 & 9 & 5 & 13 & 8 & 5 & 10318.727 & 0.0043 \\
14 & 9 & 6 & 13 & 8 & 6 & 10318.727 & 0.0039 \\
20 & 3 & 17 & 19 & 2 & 17 & 10320.2095 & -0.0041 \\
11 & 11 & 0 & 10 & 10 & 0 & 10367.8149 & 0.0041 \\
16 & 8 & 8 & 15 & 7 & 8 & 10475.5171 & 0.0013 \\
16 & 8 & 9 & 15 & 7 & 9 & 10475.682 & 0.0016 \\
13 & 10 & 3 & 12 & 9 & 3 & 10532.9868 & -0.0055 \\
13 & 10 & 4 & 12 & 9 & 4 & 10532.9868 & -0.0055 \\
\hline
\end{tabular}

Table A.13.: Experimentally obtained transition frequencies $\left(\nu_{\text {exp. }}\right)$ of the $\mathrm{H}_{g} \mathrm{H}_{t}$ dimer with their respective rotational quantum numbers as well as the differences between the fitted and observed frequencies $\left(\Delta \nu=\nu_{\text {calc. }}-\nu_{\text {exp. }}\right) . \quad \nu_{\text {calc. }}$ is calculated using the fitted experimental spectroscopic constants.

\begin{tabular}{cccccccc}
\hline $\mathrm{J}^{\prime}$ & $\mathrm{K}_{a}^{\prime}$ & $\mathrm{K}_{c}^{\prime}$ & $\mathrm{J}^{\prime \prime}$ & $\mathrm{K}_{a}^{\prime \prime}$ & $\mathrm{K}_{c}^{\prime \prime}$ & $\nu_{\text {exp. }} / \mathrm{MHz}$ & $\Delta \nu / \mathrm{MHz}$ \\
\hline 7 & 0 & 7 & 6 & 0 & 6 & 2149.232 & -0.0008 \\
7 & 1 & 6 & 6 & 1 & 5 & 2154.5387 & -0.0108 \\
8 & 1 & 8 & 7 & 1 & 7 & 2450.5388 & 0.0015 \\
8 & 0 & 8 & 7 & 0 & 7 & 2456.1864 & 0.0022 \\
8 & 2 & 6 & 7 & 2 & 5 & 2456.7741 & -0.0014 \\
8 & 1 & 7 & 7 & 1 & 6 & 2462.3202 & 0.0031 \\
9 & 1 & 9 & 8 & 1 & 8 & 2756.8214 & -0.0016 \\
9 & 0 & 9 & 8 & 0 & 8 & 2763.1041 & 0.001 \\
9 & 2 & 7 & 8 & 2 & 6 & 2763.9478 & 0.0024 \\
9 & 1 & 8 & 8 & 1 & 7 & 2770.0774 & 0.0031 \\
10 & 1 & 10 & 9 & 1 & 9 & 3063.0995 & 0.0013 \\
10 & 0 & 10 & 9 & 0 & 9 & 3069.9857 & 0.0005 \\
10 & 2 & 9 & 9 & 2 & 8 & 3070.5184 & 0.0034 \\
10 & 2 & 8 & 9 & 2 & 7 & 3071.139 & -0.0016 \\
10 & 1 & 9 & 9 & 1 & 8 & 3077.8238 & 0.0039 \\
11 & 1 & 11 & 10 & 1 & 10 & 3369.3556 & -0.0054 \\
11 & 0 & 11 & 10 & 0 & 10 & 3376.8273 & 0.0005 \\
11 & 2 & 10 & 10 & 2 & 9 & 3377.5395 & 0.0089 \\
11 & 2 & 9 & 10 & 2 & 8 & 3378.3636 & -0.0005 \\
11 & 1 & 10 & 10 & 1 & 9 & 3385.551 & -0.0014 \\
12 & 1 & 12 & 11 & 1 & 11 & 3675.6145 & 0.0035 \\
12 & 0 & 12 & 11 & 0 & 11 & 3683.6244 & 0.0003 \\
12 & 2 & 11 & 11 & 2 & 10 & 3684.5318 & -0.0041 \\
12 & 2 & 10 & 11 & 2 & 9 & 3685.6133 & -0.0052
\end{tabular}




\section{A. Appendix}

\begin{tabular}{|c|c|c|c|c|c|c|c|}
\hline $\mathrm{J}^{\prime}$ & $\mathrm{K}_{a}^{\prime}$ & $\mathrm{K}_{c}^{\prime}$ & $\mathrm{J}^{\prime \prime}$ & $\mathrm{K}_{a}^{\prime \prime}$ & $\mathrm{K}_{c}^{\prime \prime}$ & $\nu_{\text {exp. }} / \mathrm{MHz}$ & $\Delta \nu / \mathrm{MHz}$ \\
\hline 12 & 1 & 11 & 11 & 1 & 10 & 3693.2705 & -0.0002 \\
\hline 13 & 1 & 13 & 12 & 1 & 12 & 3981.8452 & -0.0016 \\
\hline 13 & 0 & 13 & 12 & 0 & 12 & 3990.3743 & 0.0011 \\
\hline 13 & 2 & 12 & 12 & 2 & 11 & 3991.523 & -0.0069 \\
\hline 13 & 3 & 11 & 12 & 3 & 10 & 3991.9169 & -0.0055 \\
\hline 13 & 3 & 10 & 12 & 3 & 9 & 3991.9169 & -0.0239 \\
\hline 13 & 2 & 11 & 12 & 2 & 10 & 3992.9085 & 0.0022 \\
\hline 13 & 1 & 12 & 12 & 1 & 11 & 4000.9773 & 0.0042 \\
\hline 14 & 1 & 14 & 13 & 1 & 13 & 4288.068 & 0.0006 \\
\hline 14 & 0 & 14 & 13 & 0 & 13 & 4297.0707 & 0.0001 \\
\hline 14 & 2 & 13 & 13 & 2 & 12 & 4298.5082 & -0.0038 \\
\hline 14 & 1 & 13 & 13 & 1 & 12 & 4308.6713 & 0.0133 \\
\hline 15 & 1 & 15 & 14 & 1 & 14 & 4594.2708 & -0.001 \\
\hline 15 & 0 & 15 & 14 & 0 & 14 & 4603.7169 & 0.004 \\
\hline 15 & 2 & 14 & 14 & 2 & 13 & 4605.4764 & -0.0047 \\
\hline 15 & 2 & 13 & 14 & 2 & 12 & 4607.581 & -0.0097 \\
\hline 15 & 1 & 14 & 14 & 1 & 13 & 4616.3262 & 0.0022 \\
\hline 16 & 1 & 16 & 15 & 1 & 15 & 4900.4603 & 0.0009 \\
\hline 16 & 0 & 16 & 15 & 0 & 15 & 4910.2978 & 0.0008 \\
\hline 16 & 2 & 15 & 15 & 2 & 14 & 4912.4285 & -0.0076 \\
\hline 16 & 4 & 13 & 15 & 4 & 12 & 4913.0326 & -0.0032 \\
\hline 16 & 4 & 12 & 15 & 4 & 11 & 4913.0326 & -0.0036 \\
\hline 16 & 3 & 13 & 15 & 3 & 12 & 4913.2342 & 0.0205 \\
\hline 16 & 2 & 14 & 15 & 2 & 13 & 4914.9956 & 0.0038 \\
\hline 16 & 1 & 15 & 15 & 1 & 14 & 4923.97 & 0.0004 \\
\hline 17 & 1 & 17 & 16 & 1 & 16 & 5206.6307 & 0.0018 \\
\hline 17 & 0 & 17 & 16 & 0 & 16 & 5216.8151 & -0.0046 \\
\hline 17 & 2 & 16 & 16 & 2 & 15 & 5219.3726 & -0.0036 \\
\hline 17 & 3 & 15 & 16 & 3 & 14 & 5220.2832 & 0.0404 \\
\hline 17 & 3 & 14 & 16 & 3 & 13 & 5220.2832 & -0.0306 \\
\hline 17 & 2 & 15 & 16 & 2 & 14 & 5222.4397 & 0.0054 \\
\hline 17 & 1 & 16 & 16 & 1 & 15 & 5231.5902 & -0.0029 \\
\hline 18 & 1 & 18 & 17 & 1 & 17 & 5512.7848 & 0.0052 \\
\hline 18 & 0 & 18 & 17 & 0 & 17 & 5523.2775 & -0.0011 \\
\hline 18 & 2 & 17 & 17 & 2 & 16 & 5526.2937 & -0.0069 \\
\hline 18 & 3 & 16 & 17 & 3 & 15 & 5527.3656 & 0.0407 \\
\hline 18 & 3 & 15 & 17 & 3 & 14 & 5527.3656 & -0.0539 \\
\hline 18 & 2 & 16 & 17 & 2 & 15 & 5529.9224 & 0.0028 \\
\hline 18 & 1 & 17 & 17 & 1 & 16 & 5539.1882 & -0.0045 \\
\hline 19 & 1 & 19 & 18 & 1 & 18 & 5818.9168 & 0.0062 \\
\hline 19 & 0 & 19 & 18 & 0 & 18 & 5829.6741 & 0.0025 \\
\hline 19 & 2 & 18 & 18 & 2 & 17 & 5833.2015 & -0.0068 \\
\hline 19 & 3 & 17 & 18 & 3 & 16 & 5834.3991 & -0.0079 \\
\hline 19 & 3 & 16 & 18 & 3 & 15 & 5834.535 & 0.0037 \\
\hline 19 & 2 & 17 & 18 & 2 & 16 & 5837.4563 & 0.0075 \\
\hline 19 & 1 & 18 & 18 & 1 & 17 & 5846.7664 & -0.0005 \\
\hline 6 & 3 & 4 & 5 & 2 & 3 & 3983.3133 & 0.0017 \\
\hline 6 & 3 & 3 & 5 & 2 & 4 & 3983.4547 & 0.0097 \\
\hline 4 & 4 & 0 & 3 & 3 & 1 & 4225.6427 & -0.0095 \\
\hline 7 & 3 & 5 & 6 & 2 & 4 & 4290.2726 & -0.014 \\
\hline 7 & 3 & 4 & 6 & 2 & 5 & 4290.5538 & 0 \\
\hline
\end{tabular}




\section{A. Appendix}

\begin{tabular}{cccccccc}
$\mathrm{J}^{\prime}$ & $\mathrm{K}_{a}^{\prime}$ & $\mathrm{K}_{c}^{\prime}$ & $\mathrm{J}^{\prime \prime}$ & $\mathrm{K}_{a}^{\prime \prime}$ & $\mathrm{K}_{c}^{\prime \prime}$ & $\nu_{\text {exp. }} / \mathrm{MHz}$ & $\Delta \nu / \mathrm{MHz}$ \\
\hline 5 & 4 & 1 & 4 & 3 & 2 & 4532.7224 & 0.0061 \\
6 & 4 & 3 & 5 & 3 & 2 & 4839.7834 & 0.0059 \\
9 & 3 & 7 & 8 & 2 & 6 & 4904.0658 & 0.015 \\
7 & 4 & 4 & 6 & 3 & 3 & 5146.8353 & 0.0002 \\
5 & 5 & 0 & 4 & 4 & 1 & 5389.0866 & -0.0009 \\
5 & 5 & 1 & 4 & 4 & 0 & 5389.0866 & -0.0009 \\
8 & 4 & 5 & 7 & 3 & 4 & 5453.8796 & -0.0078 \\
6 & 5 & 1 & 5 & 4 & 2 & 5696.1564 & 0.0061 \\
9 & 4 & 5 & 8 & 3 & 6 & 5760.9251 & -0.0105 \\
\hline
\end{tabular}

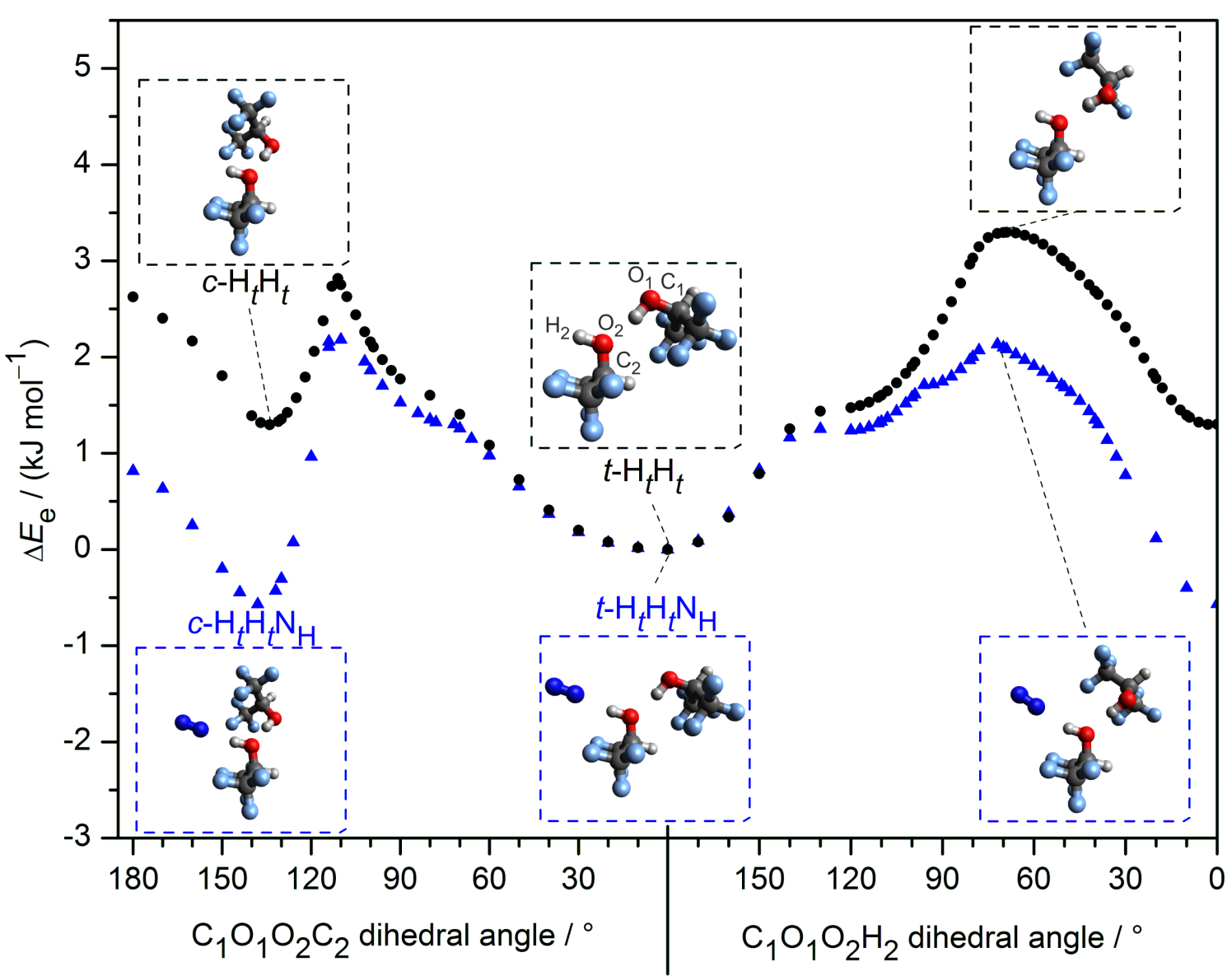

Figure A.8.: Fully relaxed B3LYP-D3(BJ)/aVTZ OH $\cdots \mathrm{O}$ torsional scans for $\mathrm{H}_{t} \mathrm{H}_{t}$ with (blue triangles) and without (black dots) an acceptor-attached $\mathrm{N}$ unit, varying the dihedral angle of the acceptor $\mathrm{OH}$ (to the right) or $\mathrm{OC}$ bond (to the left) relative to the donor OC bond, together with structural snapshots (reprinted with adaptations from reference 234 with permission from John Wiley and Sons). The transition state (right) conserves the dominant $\mathrm{OH} \cdots \mathrm{O}$ hydrogen bond. The non-equivalence of the two torsion angles results from a kinking of the $c-\mathrm{H}_{t} \mathrm{H}_{t}$ structure away from $C_{\mathrm{s}}$ symmetry to maximise $\mathrm{F} \cdots \mathrm{F}$ interactions on one side of the plane. 
A. Appendix

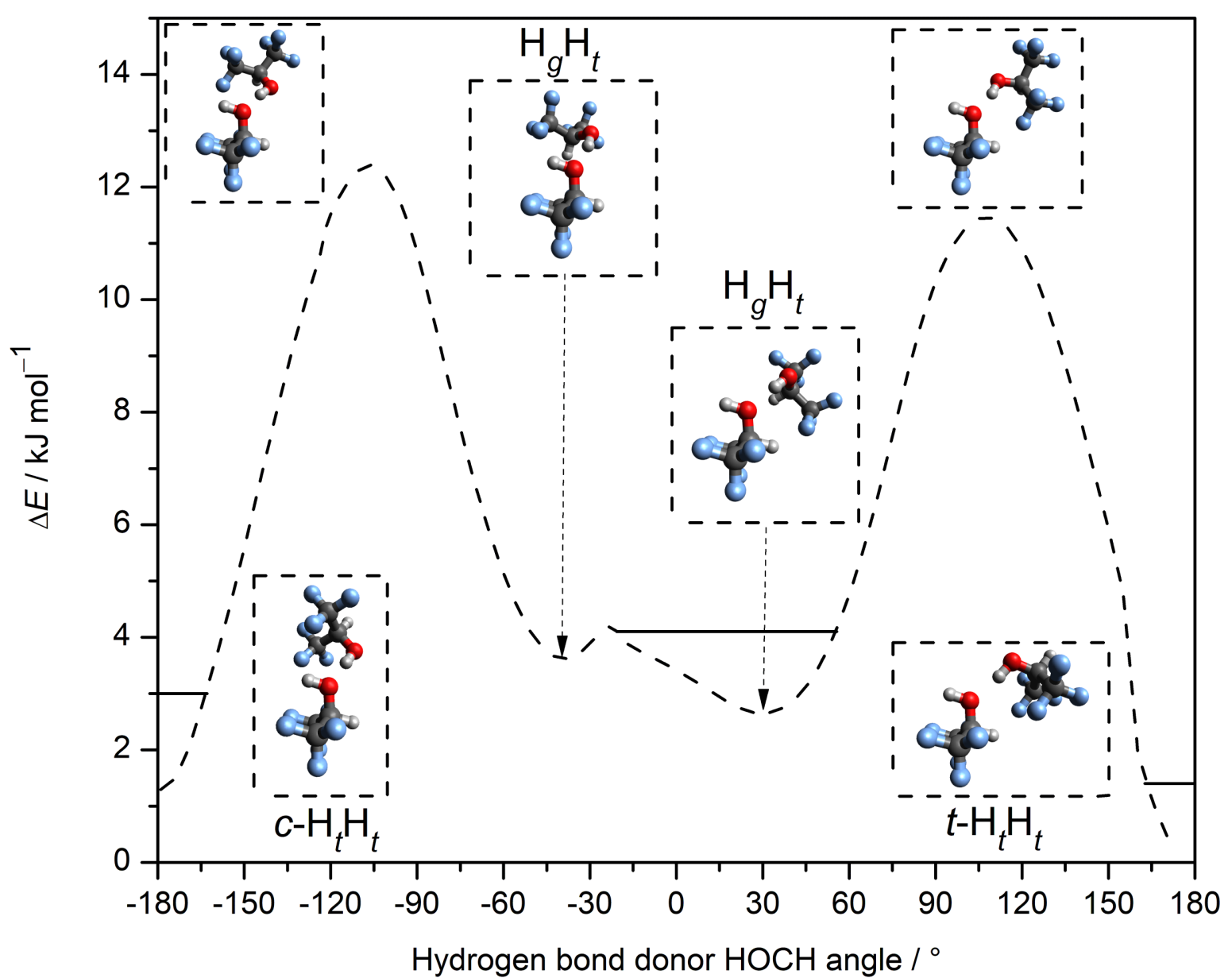

Figure A.9.: Fully relaxed B3LYP-D3(BJ)/aVTZ OH torsional scan for $\mathrm{H}_{g} \mathrm{H}_{t}$, varying the HOCH dihedral angle of the hydrogen bond donor monomer, together with structural snapshots. The zero-point corrected energy levels in each well are indicated with solid lines. The non-equivalence of the two torsion angle signs results from a kinking of the $\mathrm{H}_{g} \mathrm{H}_{t}$ and $c-\mathrm{H}_{t} \mathrm{H}_{t}$ structures away from $C_{\mathrm{s}}$ symmetry to maximise $\mathrm{F} \cdots \mathrm{F}$ interactions on one side of the plane. 


\section{A. Appendix}

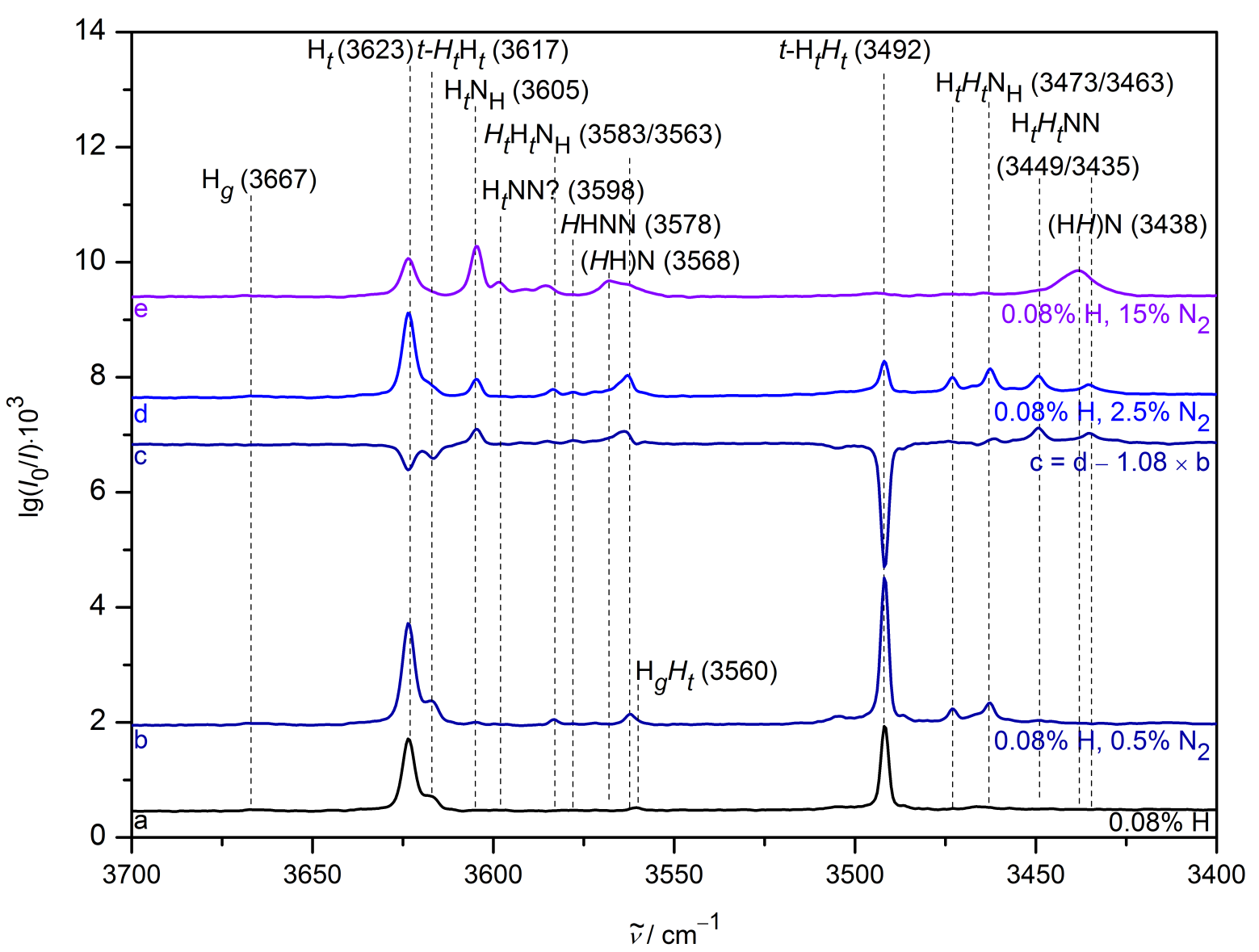

Figure A.10.: $\mathrm{OH}$ stretching FTIR jet spectra of $\mathrm{H}$ expanded in helium (trace a) and helium mixed with increasing amounts of $\mathrm{N}$ (traces $\mathrm{b}, \mathrm{d}$ and e), respectively (reprinted with adaptations from reference 234 with permission from John Wiley and Sons). A HHN-corrected difference spectrum (trace c) is included. Wavenumbers and (tentative) assignments are provided. Roman symbols denote the alcohol monomer unit involved in the vibration, italic symbols the passive monomer. $\mathrm{HH}$ dimers embedded in $\mathrm{N}$ are denoted as $(\mathrm{HH}) \mathrm{N}$, in this particular case the parentheses do not mark a cyclic structure.

Table A.14.: Experimentally obtained transition frequencies $\left(\nu_{\text {exp. }}\right)$ of the $\left(\mathrm{H}_{g} \mathrm{H}_{g} \mathrm{H}_{g^{\prime}}\right)$ trimer with their respective rotational quantum numbers as well as the differences between the fitted and observed frequencies $\left(\Delta \nu=\nu_{\text {calc. }}-\nu_{\text {exp. }}\right) . \nu_{\text {calc. }}$ is calculated using the fitted experimental spectroscopic constants.

\begin{tabular}{cccccccc}
\hline $\mathrm{J}^{\prime}$ & $\mathrm{K}_{a}^{\prime}$ & $\mathrm{K}_{c}^{\prime}$ & $\mathrm{J}^{\prime \prime}$ & $\mathrm{K}_{a}^{\prime \prime}$ & $\mathrm{K}_{c}^{\prime \prime}$ & $\nu_{\text {exp. }} / \mathrm{MHz}$ & $\Delta \nu / \mathrm{MHz}$ \\
\hline 12 & 8 & 4 & 11 & 7 & 4 & 3163.6848 & 0.008 \\
12 & 10 & 2 & 11 & 9 & 2 & 3180.4893 & 0.0095 \\
12 & 7 & 5 & 11 & 6 & 5 & 3182.9256 & -0.0226 \\
12 & 8 & 5 & 11 & 7 & 5 & 3186.3231 & -0.0134 \\
12 & 9 & 4 & 11 & 8 & 4 & 3187.7169 & -0.0042 \\
12 & 6 & 6 & 11 & 5 & 6 & 3189.3425 & 0.0208
\end{tabular}




\section{A. Appendix}

\begin{tabular}{|c|c|c|c|c|c|c|c|}
\hline $\mathrm{J}^{\prime}$ & $\mathrm{K}_{a}^{\prime}$ & $\mathrm{K}_{c}^{\prime}$ & $\mathrm{J}^{\prime \prime}$ & $\mathrm{K}_{a}^{\prime \prime}$ & $\mathrm{K}_{c}^{\prime \prime}$ & $\nu_{\text {exp. }} / \mathrm{MHz}$ & $\Delta \nu / \mathrm{MHz}$ \\
\hline 12 & 7 & 6 & 11 & 6 & 6 & 3189.5442 & 0.0006 \\
\hline 12 & 5 & 7 & 11 & 4 & 7 & 3192.0963 & 0.0025 \\
\hline 12 & 4 & 8 & 11 & 3 & 8 & 3193.7544 & -0.017 \\
\hline 12 & 3 & 9 & 11 & 2 & 9 & 3194.8998 & -0.0009 \\
\hline 12 & 2 & 10 & 11 & 1 & 10 & 3195.6968 & -0.0046 \\
\hline 12 & 11 & 1 & 11 & 10 & 1 & 3238.1773 & -0.0068 \\
\hline 12 & 11 & 2 & 11 & 10 & 2 & 3242.3961 & 0.0112 \\
\hline 13 & 10 & 3 & 12 & 9 & 3 & 3413.9826 & 0.0176 \\
\hline 13 & 9 & 4 & 12 & 8 & 4 & 3419.5651 & -0.0176 \\
\hline 13 & 7 & 6 & 12 & 6 & 6 & 3453.0298 & -0.0072 \\
\hline 13 & 6 & 7 & 12 & 5 & 7 & 3456.7746 & 0.0224 \\
\hline 13 & 11 & 2 & 12 & 10 & 2 & 3457.7451 & -0.0155 \\
\hline 13 & 5 & 8 & 12 & 4 & 8 & 3458.8909 & -0.0195 \\
\hline 13 & 4 & 9 & 12 & 3 & 9 & 3460.3531 & 0.0007 \\
\hline 13 & 3 & 10 & 12 & 2 & 10 & 3461.4029 & 0.0303 \\
\hline 13 & 2 & 11 & 12 & 1 & 11 & 3462.1083 & -0.0147 \\
\hline 13 & 11 & 3 & 12 & 10 & 3 & 3477.4983 & -0.001 \\
\hline 13 & 13 & 0 & 12 & 12 & 0 & 3563.4874 & 0.0341 \\
\hline 13 & 13 & 1 & 12 & 12 & 1 & 3563.4874 & -0.053 \\
\hline 14 & 9 & 5 & 13 & 8 & 5 & 3702.3582 & -0.0143 \\
\hline 14 & 10 & 5 & 13 & 9 & 5 & 3714.5016 & 0.0028 \\
\hline 14 & 8 & 6 & 13 & 7 & 6 & 3716.0345 & -0.006 \\
\hline 14 & 9 & 6 & 13 & 8 & 6 & 3717.2822 & -0.0167 \\
\hline 14 & 8 & 7 & 13 & 7 & 7 & 3721.1281 & 0.001 \\
\hline 14 & 11 & 4 & 13 & 10 & 4 & 3722.465 & 0.0008 \\
\hline 14 & 6 & 8 & 13 & 5 & 8 & 3723.8 & 0.001 \\
\hline 14 & 5 & 9 & 13 & 4 & 9 & 3725.6199 & 0.0089 \\
\hline 14 & 4 & 10 & 13 & 3 & 10 & 3726.8992 & 0.0102 \\
\hline 14 & 3 & 11 & 13 & 2 & 11 & 3727.8332 & 0.0055 \\
\hline 14 & 2 & 12 & 13 & 1 & 12 & 3728.5285 & -0.0103 \\
\hline 14 & 1 & 13 & 13 & 0 & 13 & 3729.0985 & 0.0071 \\
\hline 14 & 12 & 2 & 13 & 11 & 2 & 3736.2472 & -0.0088 \\
\hline 14 & 12 & 3 & 13 & 11 & 3 & 3750.5521 & 0.013 \\
\hline 14 & 13 & 1 & 13 & 12 & 1 & 3791.7288 & -0.0123 \\
\hline 14 & 13 & 2 & 13 & 12 & 2 & 3793.1461 & 0.0042 \\
\hline 15 & 11 & 4 & 14 & 10 & 4 & 3934.6 & -0.0178 \\
\hline 15 & 12 & 3 & 14 & 11 & 3 & 3955.14 & 0.0202 \\
\hline 15 & 10 & 5 & 14 & 9 & 5 & 3959.0181 & 0.0054 \\
\hline 15 & 9 & 6 & 14 & 8 & 6 & 3978.097 & 0.0072 \\
\hline 15 & 10 & 6 & 14 & 9 & 6 & 3980.7334 & -0.0053 \\
\hline 15 & 8 & 7 & 14 & 7 & 7 & 3984.9426 & 0.0034 \\
\hline 15 & 9 & 7 & 14 & 8 & 7 & 3985.1205 & -0.006 \\
\hline 15 & 6 & 9 & 14 & 5 & 9 & 3990.6626 & 0.0032 \\
\hline 15 & 5 & 10 & 14 & 4 & 10 & 3992.2459 & 0.0081 \\
\hline 15 & 4 & 11 & 14 & 3 & 11 & 3993.3935 & -0.0014 \\
\hline 15 & 3 & 12 & 14 & 2 & 12 & 3994.2775 & 0.0069 \\
\hline 15 & 2 & 13 & 14 & 1 & 13 & 3994.9417 & -0.0085 \\
\hline 15 & 1 & 14 & 14 & 0 & 14 & 3995.4751 & -0.0139 \\
\hline 15 & 13 & 2 & 14 & 12 & 2 & 4014.8652 & 0.0001 \\
\hline 15 & 13 & 3 & 14 & 12 & 3 & 4024.4716 & 0.0041 \\
\hline 15 & 14 & 2 & 14 & 13 & 2 & 4068.8605 & 0.0147 \\
\hline
\end{tabular}




\section{A. Appendix}

\begin{tabular}{|c|c|c|c|c|c|c|c|}
\hline $\mathrm{J}^{\prime}$ & $\mathrm{K}_{a}^{\prime}$ & $\mathrm{K}_{c}^{\prime}$ & $\mathrm{J}^{\prime \prime}$ & $\mathrm{K}_{a}^{\prime \prime}$ & $\mathrm{K}_{c}^{\prime \prime}$ & $\nu_{\text {exp. }} / \mathrm{MHz}$ & $\Delta \nu / \mathrm{MHz}_{2}$ \\
\hline 16 & 12 & 4 & 15 & 11 & 4 & 4196.2038 & 0.0184 \\
\hline 1 & 1 & & & & & & \\
\hline & 3 & 3 & & 12 & 3 & & \\
\hline 16 & 10 & 6 & & & ( & & ( 01004 \\
\hline 16 & 11 & $c$ & 15 & 10 & 6 & & \\
\hline 16 & $0_{0}+2$ & 7 & 15 & & ? & & \\
\hline 6 & 10 & 1 & 15 & & 2 & & 035 \\
\hline 16 & 8 & 0 & 1 & 7 & 8 & & \\
\hline 1 & 9 & 8 & 15 & $\varepsilon$ & $\varepsilon$ & & -0.0006 \\
\hline 16 & 7 & 9 & & 6 & s & & \\
\hline 16 & 8 & & & 7 & & & \\
\hline 6 & 6 & 10 & 1 & & 10 & & \\
\hline 1 & 6 & 10 & & & 1 & & \\
\hline 1 & 5 & 1 & 1 & 4 & 1 & & \\
\hline 1 & 6 & 11 & 1 & & 1 & & \\
\hline 1 & 4 & 12 & & 3 & 12 & & \\
\hline 6 & 5 & 12 & & 4 & & & \\
\hline 18 & 8 & 1 & & & & & \\
\hline 1 & 4 & 1 & & & & & \\
\hline 1 & 2 & 14 & & & 1 & & \\
\hline 1 & 3 & & & & 1 & & \\
\hline 1 & 1 & 1 & 1 & ( & 15 & & \\
\hline 1 & 2 & 15 & & & $1-$ & & \\
\hline 1 & 13 & & & & & & \\
\hline 1 & 1 & & & & & & \\
\hline 1 & 15 & 2 & & & & & 131 \\
\hline 1 & 1 & 5 & & & & & \\
\hline 1 & 11 & $c$ & 1 & 1 & 6 & & 22 \\
\hline 1 & 12 & $c_{0} \rightarrow$ & & 1 & & & \\
\hline & +2 & : & & & & & \\
\hline 1 & 1 & & & 1 & & & \\
\hline 1 & 1 & & & & & & \\
\hline $1^{\prime}$ & 1 & & & & & & \\
\hline 17 & 10 & 8 & & 9 & 8 & & \\
\hline 1 & $0_{-1}>$ & & & & & & 17 \\
\hline 1 & 8 & & & & & & \\
\hline 1 & 7 & 1 & 1 & & & & \\
\hline 1 & 8 & & & & & & \\
\hline 17 & 6 & 11 & & 5 & 11 & & \\
\hline 17 & 7 & 11 & 19 & 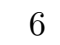 & 11 & & \\
\hline 17 & $-x^{2}$ & & & & & & \\
\hline 1 & 6 & & & & & & \\
\hline 1 & 4 & 1 & 1 & & 1 & & \\
\hline 1 & 5 & & & & & & \\
\hline 17 & 3 & 14 & 16 & 2 & 14 & & -0.0036 \\
\hline 17 & 4 & 14 & 1 & 3 & 14 & & 36 \\
\hline 17 & 2 & 15 & & & & & \\
\hline 1 & 3 & 15 & 1 & & 15 & & \\
\hline 1 & 14 & 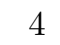 & & & & & \\
\hline 17 & $1:$ & 2 & 16 & & & 570.5259 & -0.0037 \\
\hline 17 & 16 & 1 & 16 & 15 & 1 & 4620.2834 & \\
\hline
\end{tabular}




\section{A. Appendix}

\begin{tabular}{|c|c|c|c|c|c|c|c|}
\hline $\mathrm{J}^{\prime}$ & $\mathrm{K}_{a}^{\prime}$ & $\mathrm{K}_{c}^{\prime}$ & $\mathrm{J}^{\prime \prime}$ & $\mathrm{K}_{a}^{\prime \prime}$ & $\mathrm{K}_{c}^{\prime \prime}$ & $\nu_{\text {exp. }} / \mathrm{MHz}$ & $\Delta \nu / \mathrm{MHz}$ \\
\hline 17 & 16 & 2 & 16 & 15 & 2 & 4620.5295 & 0.0071 \\
\hline 17 & 17 & 0 & 16 & 16 & 0 & 4667.1241 & 0.0114 \\
\hline 17 & 17 & 1 & 16 & 16 & 1 & 4667.1241 & 0.0064 \\
\hline 18 & 13 & 5 & 17 & 12 & 5 & 4723.689 & 0.0118 \\
\hline 18 & 14 & 4 & 17 & 13 & 4 & 4730.7759 & 0.0032 \\
\hline 18 & 13 & 6 & 17 & 12 & 6 & 4770.9746 & -0.0008 \\
\hline 18 & 11 & 7 & 17 & 10 & 7 & 4773.0358 & 0.0146 \\
\hline 18 & 12 & 7 & 17 & 11 & 7 & 4775.0324 & 0.0019 \\
\hline 18 & 14 & 5 & 17 & 13 & 5 & 4778.5391 & 0.0033 \\
\hline 18 & 10 & 8 & 17 & 9 & 8 & 4780.2645 & 0.0103 \\
\hline 18 & 11 & 8 & 17 & 10 & 8 & 4780.3967 & -0.0072 \\
\hline 18 & 9 & 9 & 17 & 8 & 9 & 4784.3496 & -0.002 \\
\hline 18 & 10 & 9 & 17 & 9 & 9 & 4784.3496 & -0.0095 \\
\hline 18 & 8 & 10 & 17 & 7 & 10 & 4787.1417 & 0.0014 \\
\hline 18 & 9 & 10 & 17 & 8 & 10 & 4787.1417 & 0.0011 \\
\hline 18 & 15 & 3 & 17 & 14 & 3 & 4788.966 & -0.0133 \\
\hline 18 & 7 & 11 & 17 & 6 & 11 & 4789.1708 & 0.0064 \\
\hline 18 & 6 & 12 & 17 & 5 & 12 & 4790.683 & -0.005 \\
\hline 18 & 5 & 13 & 17 & 4 & 13 & 4791.8605 & -0.0064 \\
\hline 18 & 4 & 14 & 17 & 3 & 14 & 4792.7987 & -0.0004 \\
\hline 18 & 3 & 15 & 17 & 2 & 15 & 4793.563 & 0.0127 \\
\hline 18 & 2 & 16 & 17 & 1 & 16 & 4794.1723 & 0.0076 \\
\hline 18 & 15 & 4 & 17 & 14 & 4 & 4806.7551 & 0.0108 \\
\hline 18 & 16 & 2 & 17 & 15 & 2 & 4847.5185 & -0.0183 \\
\hline 18 & 16 & 3 & 17 & 15 & 3 & 4849.7265 & 0.0239 \\
\hline 18 & 18 & 0 & 17 & 17 & 0 & 4943.0215 & 0.0093 \\
\hline 19 & 14 & 5 & 18 & 13 & 5 & 4981.5257 & -0.0223 \\
\hline 19 & 12 & 7 & 18 & 11 & 7 & 5033.8677 & -0.0033 \\
\hline 19 & 13 & 7 & 18 & 12 & 7 & 5037.7555 & -0.0255 \\
\hline 19 & 11 & 8 & 18 & 10 & 8 & 5043.3502 & -0.0171 \\
\hline 19 & 15 & 5 & 18 & 14 & 5 & 5047.4923 & 0.012 \\
\hline 19 & 9 & 10 & 18 & 8 & 10 & 5051.6762 & 0.0001 \\
\hline 19 & 8 & 11 & 18 & 7 & 11 & 5054.0817 & 0.0096 \\
\hline 19 & 7 & 12 & 18 & 6 & 12 & 5055.865 & -0.0061 \\
\hline 19 & 6 & 13 & 18 & 5 & 13 & 5057.2458 & -0.0149 \\
\hline 19 & 5 & 14 & 18 & 4 & 14 & 5058.3632 & 0.0043 \\
\hline 19 & 4 & 16 & 18 & 3 & 16 & 5059.9731 & 0.0077 \\
\hline 19 & 17 & 2 & 18 & 16 & 2 & 5124.1502 & -0.0156 \\
\hline 19 & 17 & 3 & 18 & 16 & 3 & 5125.3941 & -0.0072 \\
\hline 19 & 19 & 0 & 18 & 18 & 0 & 5218.9132 & 0.0043 \\
\hline 20 & 10 & 10 & 19 & 9 & 10 & 5315.9609 & -0.0066 \\
\hline 20 & 9 & 11 & 19 & 8 & 11 & 5318.7793 & -0.0054 \\
\hline 20 & 8 & 12 & 19 & 7 & 12 & 5320.8926 & -0.0007 \\
\hline 20 & 7 & 13 & 19 & 6 & 13 & 5322.515 & -0.0043 \\
\hline 20 & 6 & 14 & 19 & 5 & 14 & 5323.8154 & 0.0129 \\
\hline 20 & 17 & 4 & 19 & 16 & 4 & 5355.121 & -0.0054 \\
\hline 20 & 18 & 2 & 19 & 17 & 2 & 5400.5308 & -0.0109 \\
\hline 20 & 18 & 3 & 19 & 17 & 3 & 5401.2575 & 0.026 \\
\hline 20 & 20 & 0 & 19 & 19 & 0 & 5494.7928 & -0.0102 \\
\hline 21 & 10 & 11 & 20 & 9 & 11 & 5583.2926 & 0.0034 \\
\hline 21 & 9 & 12 & 20 & 8 & 12 & 5585.754 & 0.0092 \\
\hline
\end{tabular}


A. Appendix

\begin{tabular}{cccccccc}
$\mathrm{J}^{\prime}$ & $\mathrm{K}_{a}^{\prime}$ & $\mathrm{K}_{c}^{\prime}$ & $\mathrm{J}^{\prime \prime}$ & $\mathrm{K}_{a}^{\prime \prime}$ & $\mathrm{K}_{c}^{\prime \prime}$ & $\nu_{\exp .} / \mathrm{MHz}$ & $\Delta \nu / \mathrm{MHz}$ \\
\hline 21 & 8 & 13 & 20 & 7 & 13 & 5587.633 & -0.0011 \\
\hline
\end{tabular}
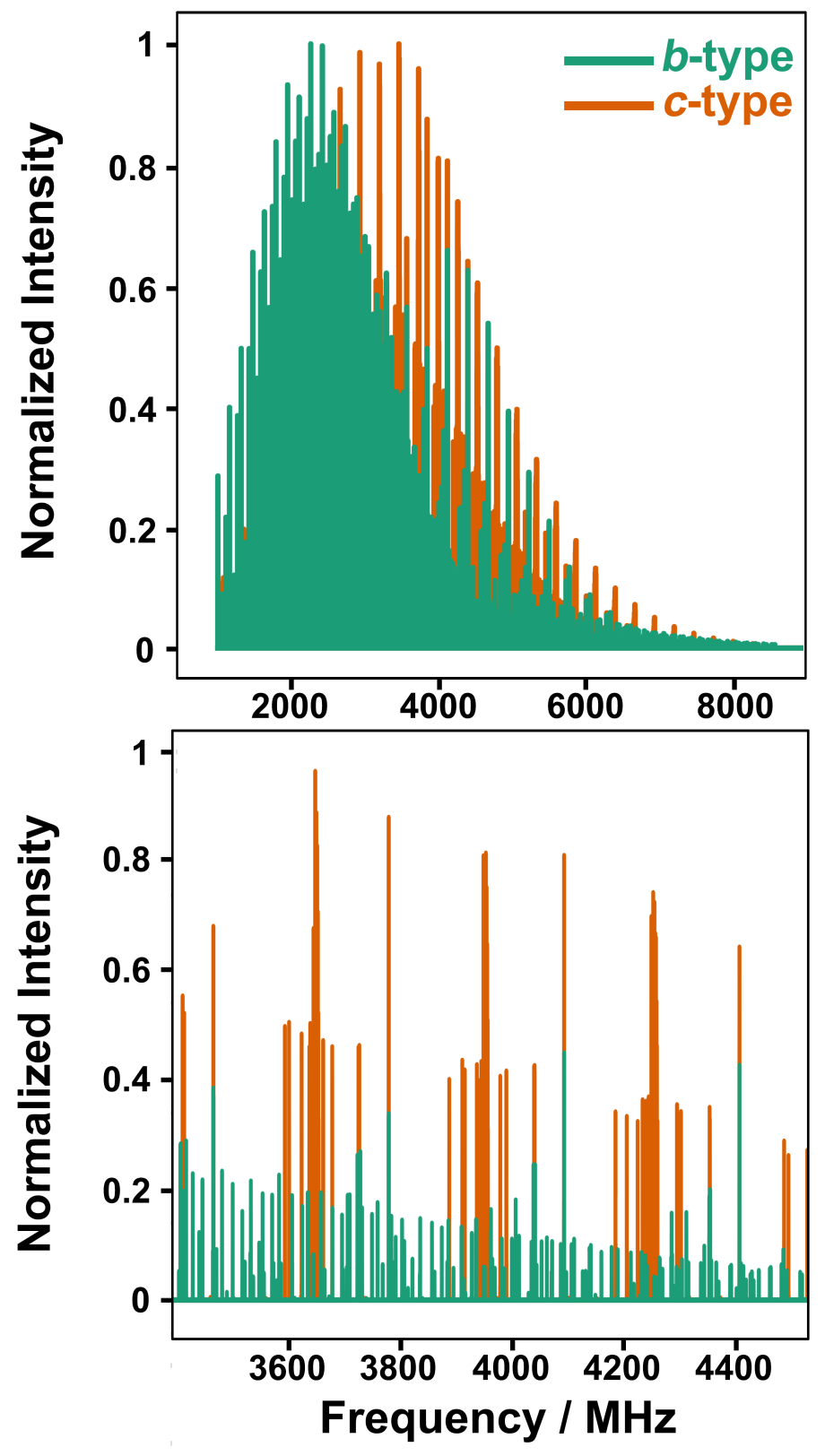

Figure A.11.: Boltzmann-weighted relative intensity distributions for $\mu_{b^{-}}$(green) and $\mu_{c^{-}}$ type (orange) transitions of $\left(\mathrm{H}_{g} \mathrm{H}_{g} \mathrm{H}_{g^{\prime}}\right)$ assuming a rotational temperature of $T_{\text {rot }}=100 \mathrm{~K}$ (reprinted with adaptations from reference 235 with permission from John Wiley and Sons). 


\section{A. Appendix}

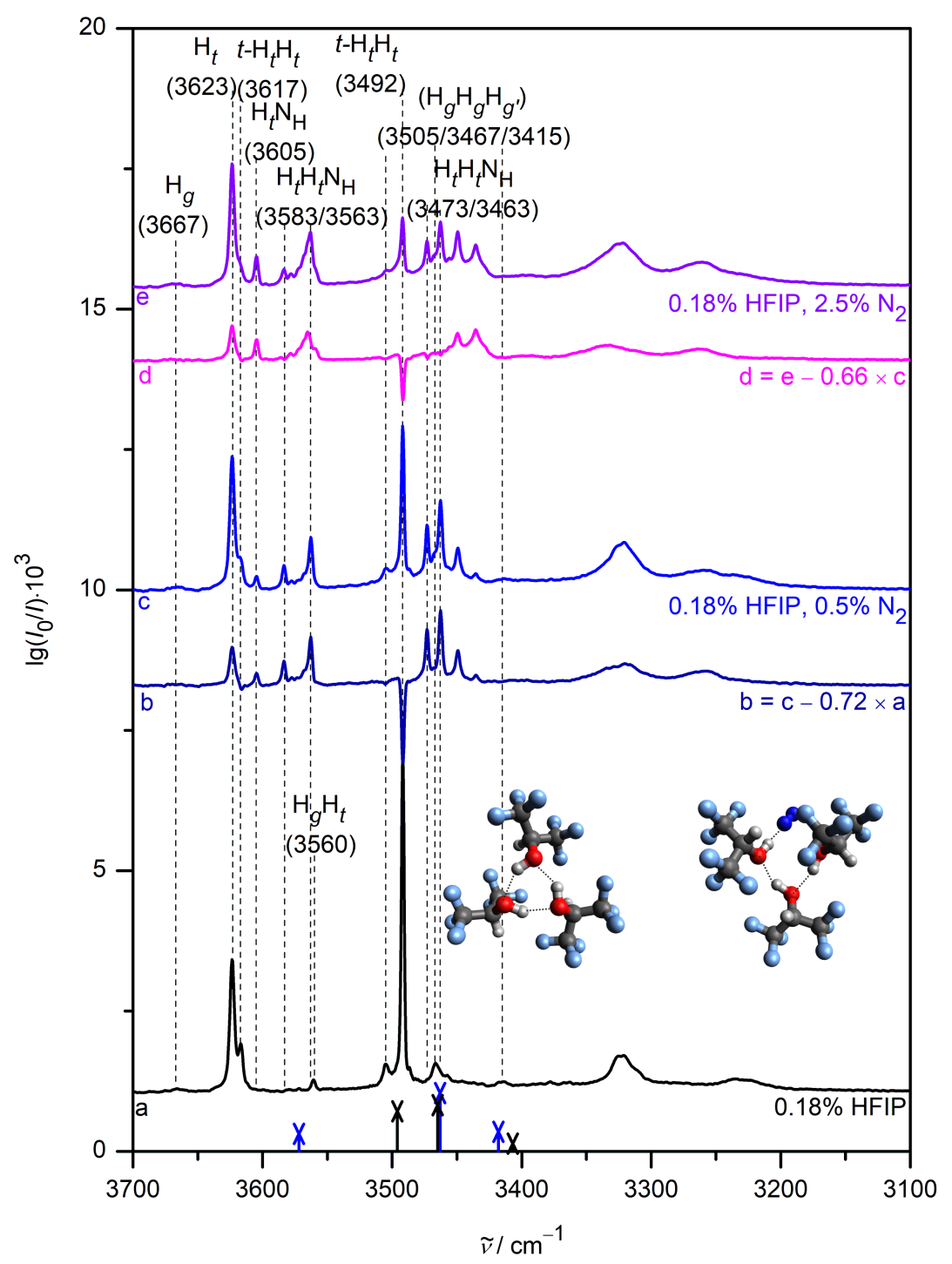

Figure A.12.: OH-stretching FTIR spectra of $\mathrm{H}$ (reprinted with adaptations from reference 235 with permission from John Wiley and Sons) expanded in helium with increasing $\mathrm{N}$ admixture from bottom to top (traces a, $\mathrm{c}$ and e). Trimer-corrected difference spectra (traces $\mathrm{b}$ and $\mathrm{d}$ ) are included. Wavenumbers and labels for (tentative) assignments are provided. Theoretical B3LYP-D3(BJ)/def2-QZVP predictions for the most stable trimer without (black) and with (blue) $\mathrm{N}$ complexation are included as stick spectra and structures. Theoretically predicted harmonic wavenumbers were scaled to the $t-\mathrm{H}_{t} \mathrm{H}_{t}$ donor vibration at $3492 \mathrm{~cm}^{-1}$. Intensities were scaled to the vibrations of $\left(\mathrm{H}_{g} \mathrm{H}_{g} \mathrm{H}_{g^{\prime}}\right)$ with the highest predicted intensity. In contrast to monomers and dimers, trimers do not appear to attach $\mathrm{N}$, providing further evidence for their closed cycle hydrogen bond topology. 
A. Appendix

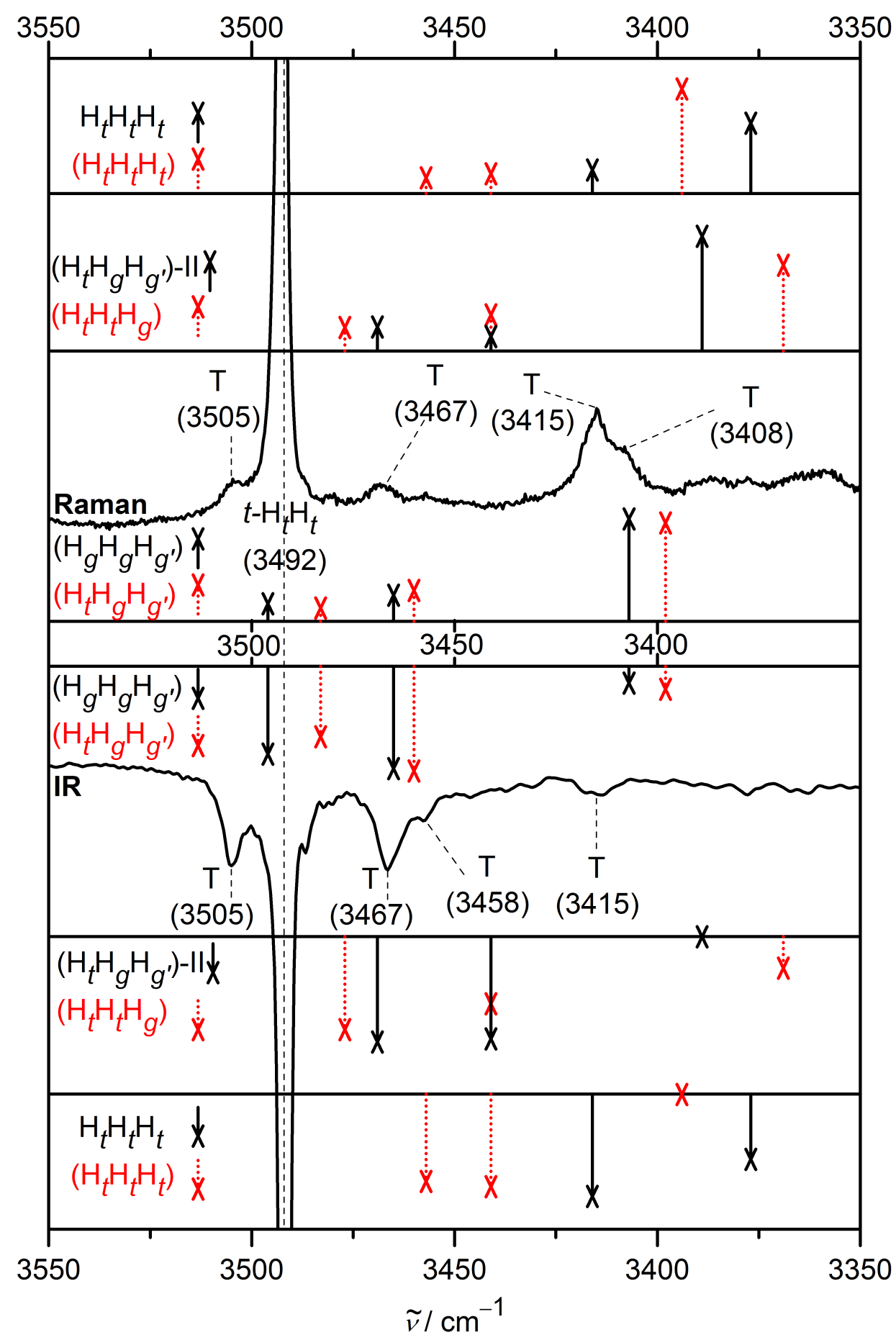

Figure A.13.: OH-stretching Raman (top) and inverted FTIR (bottom) spectra of jetcooled $\mathrm{H}$ (reprinted with adaptations from reference 235 with permission from John Wiley and Sons). Wavenumbers and labels for proposed assignments (dimer $t-\mathrm{H}_{t} \mathrm{H}_{t}$, trimers $\mathrm{T}$ ) are provided. The harmonic IR and Raman stick spectra of the six most stable trimers at the B3LYP-D3(BJ)/def2-QZVP level are scaled to the experimental $t-\mathrm{H}_{t} \mathrm{H}_{t}$ donor vibration. Only the predictions in the central panels are consistent with experiment. 


\section{A. Appendix}

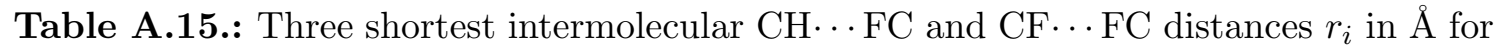
the six most stable trimers of $\mathrm{H}$ calculated at the B3LYP-D3(BJ)/def2-QZVP level. The sum of $\mathrm{CH} \cdots \mathrm{F}$ distances correlates with the calculated relative zero-point corrected energy $\Delta E_{0}$ in $\mathrm{kJ} \mathrm{mol}^{-1}$, whereas minimisation of $\mathrm{CF} \cdots \mathrm{F}$ distances is significantly less influential.

\begin{tabular}{ccccccc}
\hline Structure & Type of contact & $r_{1}$ & $r_{2}$ & $r_{3}$ & $\sum_{i} r_{i}$ & $\Delta E_{0}$ \\
\hline \multirow{2}{*}{$\left(\mathrm{H}_{g} \mathrm{H}_{g} \mathrm{H}_{g^{\prime}}\right)$} & $\mathrm{CH} \cdots \mathrm{FC}$ & 2.49 & 2.58 & 2.64 & 7.71 & \multirow{2}{*}{0.0} \\
& $\mathrm{CF} \cdots \mathrm{FC}$ & 3.06 & 3.18 & 3.27 & 9.51 & \\
$\left(\mathrm{H}_{t} \mathrm{H}_{g} \mathrm{H}_{g^{\prime}}\right)$ & $\mathrm{CH} \cdots \mathrm{FC}$ & 2.62 & 2.65 & 3.60 & 8.88 & \multirow{2}{*}{1.5} \\
& $\mathrm{CF} \cdots \mathrm{FC}$ & 2.94 & 3.07 & 3.10 & 9.10 & \\
$\left(\mathrm{H}_{t} \mathrm{H}_{g} \mathrm{H}_{g^{\prime}}\right)-\mathrm{II}$ & $\mathrm{CH} \cdots \mathrm{FC}$ & 2.57 & 2.67 & 3.95 & 9.19 & \multirow{2}{*}{2.2} \\
& $\mathrm{CF} \cdots \mathrm{FC}$ & 3.09 & 3.30 & 4.29 & 10.69 & \\
$\left(\mathrm{H}_{t} \mathrm{H}_{t} \mathrm{H}_{g}\right)$ & $\mathrm{CH} \cdots \mathrm{FC}$ & 2.53 & 3.68 & 4.20 & 10.41 & \multirow{2}{*}{2.4} \\
& $\mathrm{CF} \cdots \mathrm{FC}$ & 3.13 & 3.52 & 3.75 & 10.41 & \\
$\mathrm{H}_{t} \mathrm{H}_{t} \mathrm{H}_{t}$ & $\mathrm{CH} \cdots \mathrm{FC}$ & 2.83 & 2.83 & 5.36 & 11.02 & \multirow{2}{*}{2.5} \\
& $\mathrm{CF} \cdots \mathrm{FC}$ & 3.00 & 3.15 & 3.23 & 9.39 & \\
$\left(\mathrm{H}_{t} \mathrm{H}_{t} \mathrm{H}_{t}\right)$ & $\mathrm{CH} \cdots \mathrm{FC}$ & 3.83 & 3.98 & 4.06 & 11.87 & \multirow{2}{*}{2.9} \\
& $\mathrm{CF} \cdots \mathrm{FC}$ & 3.05 & 3.26 & 3.74 & 10.05 & \\
\hline
\end{tabular}

Table A.16.: Theoretically predicted harmonic $\left(\omega_{\mathrm{OH}}\right) \mathrm{OH}$ stretching wavenumber, hydrogen bond induced downshift $\left(\Delta \omega_{\mathrm{OH}}\right)$ and lowest predicted wavenumber $\left(\omega_{\mathrm{l}}\right.$, typically $\mathrm{CF}_{3}$ torsion in $\mathrm{H}_{t}$ and intermolecular torsion in $\left.\mathrm{H}_{t} \mathrm{~N}_{\mathrm{H}}\right)$, in $\mathrm{cm}^{-1}$, IR intensity $\left(S_{\mathrm{OH}}\right)$ in $\mathrm{km} \mathrm{mol}^{-1}$, as well as equilibrium rotational constants $\left(A_{\mathrm{e}}, B_{\mathrm{e}}, C_{\mathrm{e}}\right)$ in $\mathrm{MHz}$ and magnitudes of the electric dipole moment components $\left(\left|\mu_{a}\right|,\left|\mu_{b}\right|,\left|\mu_{c}\right|\right)$ in D calculated at different levels of approximation.

\begin{tabular}{cccccccccccc}
\hline Level of theory & Structure & $\omega_{\mathrm{OH}}$ & $S_{\mathrm{OH}}$ & $\Delta \omega_{\mathrm{OH}}$ & $\omega_{\mathrm{l}}$ & $A_{\mathrm{e}}$ & $B_{\mathrm{e}}$ & $C_{\mathrm{e}}$ & $\left|\mu_{a}\right|$ & $\left|\mu_{b}\right|$ & $\left|\mu_{c}\right|$ \\
\hline B3LYP-D3/ & $\mathrm{H}_{t}$ & 3789 & 63 & - & 25 & 2089.8 & 1042.2 & 922.9 & 0.0 & 0.6 & 0.1 \\
def2-QZVP & $\mathrm{H}_{t} \mathrm{~N}_{\mathrm{H}}$ & 3753 & 281 & 36 & 18 & 958.2 & 743.0 & 535.7 & 0.8 & 0.0 & 0.4 \\
\hline \multirow{2}{*}{ PBEh-3c } & $\mathrm{H}_{t}$ & 3895 & 69 & - & 34 & 2112.5 & 1050.1 & 930.0 & - & - & - \\
& $\mathrm{H}_{t} \mathrm{~N}_{\mathrm{H}}$ & 3883 & 240 & 12 & 20 & 956.3 & 763.3 & 549.7 & - & - & - \\
\hline B2PLYP-D3/ & $\mathrm{H}_{t}$ & 3807 & 65 & - & 26 & 2104.0 & 1055.3 & 933.8 & - & - & - \\
VTZ & $\mathrm{H}_{t} \mathrm{~N}_{\mathrm{H}}$ & 3778 & 282 & 29 & 21 & 967.2 & 758.9 & 547.2 & - & - & - \\
\hline B2PLYP-D3/ & $\mathrm{H}_{t}$ & 3796 & 66 & - & 5 & 2099.8 & 1051.9 & 931.0 & - & - & - \\
aVTZ & $\mathrm{H}_{t} \mathrm{~N}_{\mathrm{H}}$ & 3762 & 273 & 34 & 10 & 965.7 & 752.6 & 542.9 & - & - & - \\
\hline B2PLYP-D3/ & $\mathrm{H}_{t}$ & 3807 & 70 & - & 24 & 2104.1 & 1052.2 & 931.5 & - & - & - \\
VQZ & $\mathrm{H}_{t} \mathrm{~N}_{\mathrm{H}}$ & 3775 & 280 & 32 & 18 & 966.5 & 748.4 & 540.5 & - & - & - \\
\hline B2PLYP-D3/ & $\mathrm{H}_{t}$ & 3809 & 65 & - & 18 & 2103.1 & 1052.0 & 931.2 & - & - & - \\
aVQZ & $\mathrm{H}_{t} \mathrm{~N}_{\mathrm{H}}$ & 3771 & 278 & 38 & 19 & 966.2 & 748.2 & 540.4 & - & - & - \\
\hline \multirow{2}{*}{ B97-3c } & $\mathrm{H}_{t}$ & 3716 & 43 & - & 20 & 2072.9 & 1031.0 & 914.2 & - & - & - \\
& $\mathrm{H}_{t} \mathrm{~N}_{\mathrm{H}}$ & 3688 & 189 & 28 & 14 & 952.6 & 695.4 & 507.7 & - & - & - \\
\hline M06-2X/ & $\mathrm{H}_{t}$ & 3828 & 82 & - & 16 & 2119.3 & 1062.9 & 940.0 & 0.0 & 0.6 & 0.1
\end{tabular}




\section{A. Appendix}

\begin{tabular}{|c|c|c|c|c|c|c|c|c|c|c|c|}
\hline Level of theory & Structure & $\omega_{\mathrm{OH}}$ & $S_{\mathrm{OH}}$ & $\Delta \omega_{\mathrm{OH}}$ & $\omega_{1}$ & $A_{\mathrm{e}}$ & $B_{\mathrm{e}}$ & $C_{\mathrm{e}}$ & $\left|\mu_{a}\right|$ & $\left|\mu_{b}\right|$ & $\left|\mu_{c}\right|$ \\
\hline aVTZ & $\mathrm{H}_{t} \mathrm{~N}_{\mathrm{H}}$ & 3798 & 283 & 30 & 15 & 970.0 & 769.9 & 555.4 & 0.6 & 0.0 & 0.4 \\
\hline MP2/ & $\mathrm{H}_{t}$ & 3818 & 67 & - & 28 & 2109.1 & 1059.2 & 937.0 & - & - & - \\
\hline VTZ & $\mathrm{H}_{t} \mathrm{~N}_{\mathrm{H}}$ & 3787 & 270 & 31 & 13 & 971.0 & 754.3 & 545.4 & - & - & - \\
\hline MP2/ & $\mathrm{H}_{t}$ & 3794 & 70 & - & 15 & 2103.7 & 1057.2 & 935.2 & - & - & - \\
\hline aVTZ & $\mathrm{H}_{t} \mathrm{~N}_{\mathrm{H}}$ & 3758 & 278 & 36 & 11 & 967.0 & 765.2 & 551.9 & - & - & - \\
\hline MP2/ & $\mathrm{H}_{t}$ & 3819 & 73 & - & 25 & 2114.0 & 1059.6 & 937.7 & - & - & - \\
\hline VQZ & $\mathrm{H}_{t} \mathrm{~N}_{\mathrm{H}}$ & 3784 & 275 & 35 & 17 & 972.1 & 753.1 & 544.6 & - & - & - \\
\hline MP2/ & $\mathrm{H}_{t}$ & 3810 & 73 & - & 24 & 2112.0 & 1059.7 & 937.6 & - & - & - \\
\hline aVQZ & $\mathrm{H}_{t} \mathrm{~N}_{\mathrm{H}}$ & 3772 & 276 & 38 & 15 & 970.0 & 762.2 & 550.2 & - & - & - \\
\hline \multirow{2}{*}{$\begin{array}{c}\text { PBE0-D3/ } \\
\text { aVTZ }\end{array}$} & $\mathrm{H}_{t}$ & 3830 & 65 & - & 24 & 2112.9 & 1053.2 & 932.6 & 0.0 & 0.5 & 0.1 \\
\hline & $\mathrm{H}_{t} \mathrm{~N}_{\mathrm{H}}$ & 3786 & 274 & 44 & 18 & 968.9 & 741.7 & 536.3 & 0.7 & 0.0 & 0.3 \\
\hline \multirow{2}{*}{$\begin{array}{c}\text { SCS-MP2/ } \\
\text { VTZ }\end{array}$} & $\mathrm{H}_{t}$ & 3822 & 62 & - & 28 & 2103.3 & 1053.1 & 931.9 & - & - & - \\
\hline & $\mathrm{H}_{t} \mathrm{~N}_{\mathrm{H}}$ & 3802 & 232 & 20 & 13 & 967.5 & 733.7 & 532.8 & - & - & - \\
\hline \multirow{2}{*}{$\begin{array}{c}\text { SCS-MP2/ } \\
\text { aVTZ }\end{array}$} & $\mathrm{H}_{t}$ & 3801 & 65 & - & 16 & 2098.3 & 1051.1 & 930.1 & - & - & - \\
\hline & $\mathrm{H}_{t} \mathrm{~N}_{\mathrm{H}}$ & 3778 & 242 & 23 & 13 & 965.3 & 737.9 & 535.0 & - & - & - \\
\hline \multirow{2}{*}{$\begin{array}{c}\text { SCS-MP2/ } \\
\text { VQZ }\end{array}$} & $\mathrm{H}_{t}$ & 3827 & 67 & - & 26 & 2108.4 & 1053.5 & 932.6 & - & - & - \\
\hline & $\mathrm{H}_{t} \mathrm{~N}_{\mathrm{H}}$ & 3803 & 237 & 24 & 19 & 969.7 & 728.4 & 529.4 & - & - & - \\
\hline \multirow{2}{*}{$\begin{array}{c}\text { SCS-MP2/ } \\
\text { aVQZ }\end{array}$} & $\mathrm{H}_{t}$ & 3818 & 68 & - & 24 & 2106.5 & 1053.5 & 932.4 & - & - & - \\
\hline & $\mathrm{H}_{t} \mathrm{~N}_{\mathrm{H}}$ & 3792 & 241 & 26 & 14 & 968.0 & 735.9 & 534.0 & - & - & - \\
\hline \multirow{2}{*}{$\begin{array}{c}\omega \mathrm{B} 97-\mathrm{XD} / \\
\mathrm{aVTZ}\end{array}$} & $\mathrm{H}_{t}$ & 3860 & 63 & - & 21 & 2108.1 & 1046.5 & 927.2 & 0.0 & 0.5 & 0.1 \\
\hline & $\mathrm{H}_{t} \mathrm{~N}_{\mathrm{H}}$ & 3816 & 256 & 44 & 17 & 965.8 & 726.3 & 526.1 & 0.8 & 0.0 & 0.3 \\
\hline
\end{tabular}

Table A.17.: Theoretically predicted harmonic $\left(\omega_{\mathrm{OH}}\right) \mathrm{OH}$ stretching wavenumber and lowest predicted wavenumber $\left(\omega_{1}\right)$, typically hydrogen bond torsion in $\mathrm{cm}^{-1}$, IR intensity $\left(S_{\mathrm{OH}}\right)$ in $\mathrm{km} \mathrm{mol}^{-1}$, spectroscopic downshift $\left(\Delta \omega_{\mathrm{OH}}\right)$ relative to the corresponding vibration in the pure $\mathrm{H}_{t}$ or $\mathrm{H}_{t} \mathrm{H}_{t}$ fragment in $\mathrm{cm}^{-1}$, relative electronic and harmonic vibrational zero-point corrected energies $\Delta E_{\mathrm{e}}$ and $\Delta E_{0}$ compared to the most stable cluster conformation, equilibrium rotational constants $\left(A_{\mathrm{e}}, B_{\mathrm{e}}, C_{\mathrm{e}}\right)$ in $\mathrm{MHz}$ and magnitudes of the electric dipole moment components $\left(\left|\mu_{a}\right|,\left|\mu_{b}\right|,\left|\mu_{c}\right|\right)$ in D calculated at different levels of approximation. Roman structure symbols denote the $\mathrm{H}$ monomer unit involved in the vibration, italic symbols the passive monomer. Clusters with $C_{s}$-symmetry at the respective level of theory are marked by asterisks.

\begin{tabular}{|c|c|c|c|c|c|c|c|c|c|c|c|c|c|}
\hline Level of theory & Structure & $\omega_{\mathrm{OH}}$ & $S_{\mathrm{OH}}$ & $\Delta \omega_{\mathrm{OH}}$ & $\omega_{1}$ & $\Delta E_{\mathrm{e}}$ & $\Delta E_{0}$ & $A_{\mathrm{e}}$ & $B_{\mathrm{e}}$ & $C_{\mathrm{e}}$ & $\left|\mu_{a}\right|$ & $\left|\mu_{b}\right|$ & $\left|\mu_{c}\right|$ \\
\hline \multirow{9}{*}{ B3LYP/VTZ } & $\mathrm{H}_{t} *$ & 3780 & 57 & - & 29 & 0.0 & 0.0 & 2088.7 & 1041.8 & 922.5 & 0.0 & 0.6 & 0.1 \\
\hline & $\mathrm{H}_{g}$ & 3819 & 69 & - & 38 & 5.2 & 4.5 & 2092.1 & 1043.0 & 923.2 & 1.1 & 1.3 & 1.8 \\
\hline & $t-H_{t} \mathrm{H}_{t} *$ & 3780 & 75 & 0 & 3 & 0.0 & 0.0 & 480.4 & 198.5 & 164.4 & 0.4 & 0.0 & 0.9 \\
\hline & $c-H_{t} \mathrm{H}_{t}$ & 3769 & 75 & 11 & 6 & 0.3 & 0.6 & 521.1 & 179.5 & 151.2 & 0.7 & 0.2 & 0.6 \\
\hline & $H_{g} \mathrm{H}_{t}$ & 3776 & 73 & 4 & 6 & 2.2 & 2.4 & 545.1 & 147.3 & 143.4 & 2.8 & 0.5 & 1.4 \\
\hline & $t-H_{t} \mathrm{H}_{t} \mathrm{~N}_{\mathrm{H}} *$ & 3730 & 318 & 50 & 2 & 0.0 & 0.0 & 336.8 & 155.3 & 151.6 & 0.9 & 0.0 & 1.3 \\
\hline & $c-H_{t} \mathrm{H}_{t} \mathrm{~N}_{\mathrm{H}}$ & 3717 & 310 & 52 & 9 & 0.3 & 0.6 & 383.6 & 155.4 & 144.2 & 0.6 & 0.3 & 0.0 \\
\hline & $t-\mathrm{H}_{t} H_{t} *$ & 3635 & 557 & 145 & 3 & 0.0 & 0.0 & 480.4 & 198.5 & 164.4 & 0.4 & 0.0 & 0.9 \\
\hline & $c-\mathrm{H}_{t} H_{t}$ & 3623 & 599 & 157 & 6 & 0.3 & 0.6 & 521.1 & 179.5 & 151.2 & 0.7 & 0.2 & 0.6 \\
\hline
\end{tabular}




\section{A. Appendix}

\begin{tabular}{|c|c|c|c|c|c|c|c|c|c|c|c|c|c|}
\hline \multirow[t]{4}{*}{ Level of theory } & Structure & $\omega_{\mathrm{OH}}$ & $S_{\mathrm{OH}}$ & $\Delta \omega_{\mathrm{OH}}$ & $\omega_{1}$ & $\Delta E_{\mathrm{e}}$ & $\Delta E_{0}$ & $A_{\mathrm{e}}$ & $B_{\mathrm{e}}$ & $C_{\mathrm{e}}$ & $\left|\mu_{a}\right|$ & $\left|\mu_{b}\right|$ & $\mid \mu_{c}$ \\
\hline & $\mathrm{H}_{g} H_{t}$ & 3688 & 634 & 131 & 6 & 2.2 & 2.4 & 545.1 & 147.3 & 143.4 & 2.8 & 0.5 & 1.4 \\
\hline & $t-\mathrm{H}_{t} H_{t} \mathrm{~N}_{\mathrm{H}} *$ & 3615 & 636 & 20 & 2 & 0.0 & 0.0 & 336.8 & 155.3 & 151.6 & 0.9 & 0.0 & 1.3 \\
\hline & $c-\mathrm{H}_{t} H_{t} \mathrm{~N}_{\mathrm{H}}$ & 3601 & 645 & 22 & 9 & 0.3 & 0.6 & 383.6 & 155.4 & 144.2 & 0.6 & 0.3 & 0.0 \\
\hline \multirow{7}{*}{ B3LYP/aVTZ } & $\mathrm{H}_{t}{ }^{*}$ & 3776 & 63 & - & 24 & 0.0 & 0.0 & 2084.9 & 1037.8 & 919.3 & 0.0 & 0.6 & 0.1 \\
\hline & $\mathrm{H}_{g}$ & 3814 & 75 & - & 36 & 4.3 & 3.7 & 2088.5 & 1039.6 & 920.3 & 1.0 & 1.3 & 1.9 \\
\hline & $t-H_{t} \mathrm{H}_{t}$ & 3771 & 76 & 5 & 4 & 0.0 & 0.2 & 490.5 & 179.2 & 156.0 & 0.6 & 0.0 & 0.7 \\
\hline & $c-H_{t} \mathrm{H}_{t}$ & 3770 & 74 & 6 & 3 & 0.0 & 0.0 & 502.6 & 162.9 & 138.6 & 0.7 & 0.1 & 0.4 \\
\hline & $H_{g} \mathrm{H}_{t}$ & 3768 & 75 & 8 & 7 & 1.2 & 1.5 & 547.9 & 142.3 & 138.9 & 2.8 & 0.9 & 1.2 \\
\hline & $t-H_{t} \mathrm{H}_{t} \mathrm{~N}_{\mathrm{H}}$ & 3722 & 298 & 49 & 4 & 0.0 & 0.1 & 328.5 & 154.5 & 140.3 & 0.8 & 0.9 & 0.7 \\
\hline & $c-H_{t} \mathrm{H}_{t} \mathrm{~N}_{\mathrm{H}}{ }^{*}$ & 3716 & 296 & 54 & 3 & 0.1 & 0.0 & 364.9 & 143.4 & 135.7 & 0.6 & 0.0 & 0.1 \\
\hline \multirow{5}{*}{ B3LYP/aVTZ } & $t-\mathrm{H}_{t} H_{t}$ & 3630 & 558 & 146 & 4 & 0.0 & 0.2 & 490.5 & 179.2 & 156.0 & 0.6 & 0.0 & 0.7 \\
\hline & $c-\mathrm{H}_{t} H_{t}$ & 3624 & 606 & 152 & 3 & 0.0 & 0.0 & 502.6 & 162.9 & 138.6 & 0.7 & 0.1 & 0.4 \\
\hline & $\mathrm{H}_{g} H_{t}$ & 3667 & 678 & 147 & 7 & 1.2 & 1.5 & 547.9 & 142.3 & 138.9 & 2.8 & 0.9 & 1.2 \\
\hline & $t-\mathrm{H}_{t} H_{t} \mathrm{~N}_{\mathrm{H}}$ & 3609 & 622 & 21 & 4 & 0.0 & 0.1 & 328.5 & 154.5 & 140.3 & 0.8 & 0.9 & 0.7 \\
\hline & $c-\mathrm{H}_{t} H_{t} \mathrm{~N}_{\mathrm{H}} *$ & 3604 & 640 & 20 & 3 & 0.1 & 0.0 & 364.9 & 143.4 & 135.7 & 0.6 & 0.0 & 0.1 \\
\hline \multirow{7}{*}{ B3LYP/VQZ } & $\mathrm{H}_{t} *$ & 3784 & 62 & - & 27 & 0.0 & 0.0 & 2088.7 & 1039.4 & 920.7 & 0.0 & 0.6 & 0.1 \\
\hline & $\mathrm{H}_{g}$ & 3822 & 74 & - & 37 & 4.4 & 3.8 & 2092.3 & 1040.9 & 921.6 & 1.0 & 1.3 & 1.9 \\
\hline & $t-H_{t} \mathrm{H}_{t}$ & 3779 & 76 & 5 & 4 & 0.1 & 0.3 & 493.8 & 176.1 & 156.2 & 0.6 & 0.0 & 0.7 \\
\hline & $c-H_{t} \mathrm{H}_{t}$ & 3778 & 75 & 6 & 3 & 0.0 & 0.0 & 505.7 & 166.5 & 141.2 & 0.7 & 0.2 & 0.5 \\
\hline & $H_{g} \mathrm{H}_{t}$ & 3777 & 75 & 7 & 8 & 1.4 & 1.6 & 547.8 & 142.9 & 139.2 & 2.8 & 0.7 & 1.3 \\
\hline & $t-H_{t} \mathrm{H}_{t} \mathrm{~N}_{\mathrm{H}}$ & 3730 & 304 & 49 & 4 & 0.0 & 0.2 & 330.8 & 155.0 & 139.3 & 0.9 & 0.9 & 0.7 \\
\hline & $c-H_{t} \mathrm{H}_{t} \mathrm{~N}_{\mathrm{H}}$ & 3725 & 302 & 53 & 3 & 0.0 & 0.0 & 367.1 & 145.6 & 136.5 & 0.6 & 0.2 & 0.1 \\
\hline \multirow{5}{*}{ B3LYP/VQZ } & $t-\mathrm{H}_{t} H_{t}$ & 3641 & 562 & 143 & 4 & 0.1 & 0.3 & 493.8 & 176.1 & 156.2 & 0.6 & 0.0 & 0.7 \\
\hline & $c-\mathrm{H}_{t} H_{t}$ & 3636 & 607 & 148 & 3 & 0.0 & 0.0 & 505.7 & 166.5 & 141.2 & 0.7 & 0.2 & 0.5 \\
\hline & $\mathrm{H}_{g} H_{t}$ & 3681 & 670 & 141 & 8 & 1.4 & 1.6 & 547.8 & 142.9 & 139.2 & 2.8 & 0.7 & 1.3 \\
\hline & $t-\mathrm{H}_{t} H_{t} \mathrm{~N}_{\mathrm{H}}$ & 3621 & 626 & 20 & 4 & 0.0 & 0.2 & 330.8 & 155.0 & 139.3 & 0.9 & 0.9 & 0.7 \\
\hline & $c-\mathrm{H}_{t} H_{t} \mathrm{~N}_{\mathrm{H}}$ & 3615 & 641 & 21 & 3 & 0.0 & 0.0 & 367.1 & 145.6 & 136.5 & 0.6 & 0.2 & 0.1 \\
\hline \multirow{7}{*}{ B3LYP-D3/VTZ } & $\mathrm{H}_{t} *$ & 3781 & 57 & - & 28 & 0.0 & 0.0 & 2090.7 & 1045.9 & 925.7 & 0.0 & 0.6 & 0.1 \\
\hline & $\mathrm{H}_{g}$ & 3820 & 68 & - & 38 & 5.6 & 4.9 & 2093.9 & 1046.9 & 926.3 & 1.1 & 1.3 & 1.8 \\
\hline & $t-H_{t} \mathrm{H}_{t}$ & 3784 & 76 & -3 & 1 & 0.0 & 0.0 & 480.1 & 210.1 & 172.7 & 0.4 & 0.0 & 0.9 \\
\hline & $c-H_{t} \mathrm{H}_{t}$ & 3766 & 83 & 15 & 11 & 0.3 & 0.9 & 531.2 & 191.5 & 162.0 & 0.6 & 0.2 & 0.6 \\
\hline & $H_{g} \mathrm{H}_{t}$ & 3784 & 66 & 36 & 16 & 3.2 & 3.4 & 539.5 & 169.4 & 163.8 & 2.7 & 0.7 & 1.8 \\
\hline & $t-H_{t} \mathrm{H}_{t} \mathrm{~N}_{\mathrm{H}}$ & 3725 & 366 & 59 & 5 & 1.7 & 1.0 & 354.9 & 163.0 & 155.5 & 1.0 & 0.3 & 1.3 \\
\hline & $c-H_{t} \mathrm{H}_{t} \mathrm{~N}_{\mathrm{H}}$ & 3702 & 359 & 64 & 19 & 0.0 & 0.0 & 404.4 & 168.8 & 155.1 & 0.6 & 0.3 & 0.1 \\
\hline \multirow{5}{*}{ B3LYP-D3/VTZ } & $t-\mathrm{H}_{t} H_{t}$ & 3616 & 622 & 165 & 1 & 0.0 & 0.0 & 480.1 & 210.1 & 172.7 & 0.4 & 0.0 & 0.9 \\
\hline & $c-\mathrm{H}_{t} H_{t}$ & 3604 & 637 & 177 & 11 & 0.3 & 0.9 & 531.2 & 191.5 & 162.0 & 0.6 & 0.2 & 0.6 \\
\hline & $\mathrm{H}_{g} H_{t}$ & 3722 & 412 & 98 & 16 & 3.2 & 3.4 & 539.5 & 169.4 & 163.8 & 2.7 & 0.7 & 1.8 \\
\hline & $t-\mathrm{H}_{t} H_{t} \mathrm{~N}_{\mathrm{H}}$ & 3590 & 740 & 26 & 5 & 1.7 & 1.0 & 354.9 & 163.0 & 155.5 & 1.0 & 0.3 & 1.3 \\
\hline & $c-\mathrm{H}_{t} H_{t} \mathrm{~N}_{\mathrm{H}}$ & 3575 & 695 & 29 & 19 & 0.0 & 0.0 & 404.4 & 168.8 & 155.1 & 0.6 & 0.3 & 0.1 \\
\hline \multirow{7}{*}{ B3LYP-D3/aVTZ } & $\mathrm{H}_{t} *$ & 3777 & 62 & - & 24 & 0.0 & 0.0 & 2086.9 & 1042.0 & 922.6 & 0.0 & 0.6 & 0.1 \\
\hline & $\mathrm{H}_{g}$ & 3815 & 74 & - & 36 & 4.7 & 4.1 & 2090.3 & 1043.7 & 923.6 & 1.1 & 1.3 & 1.9 \\
\hline & $t-H_{t} \mathrm{H}_{t} *$ & 3776 & 78 & 1 & 6 & 0.0 & 0.0 & 479.6 & 206.8 & 170.3 & 0.4 & 0.0 & 0.9 \\
\hline & $c-H_{t} \mathrm{H}_{t}$ & 3762 & 80 & 15 & 10 & 1.3 & 1.7 & 525.5 & 187.1 & 157.2 & 0.6 & 0.3 & 0.6 \\
\hline & $H_{g} \mathrm{H}_{t}$ & 3771 & 76 & 6 & 13 & 2.6 & 2.8 & 572.0 & 155.1 & 153.5 & 3.2 & 0.9 & 0.7 \\
\hline & $t-H_{t} \mathrm{H}_{t} \mathrm{~N}_{\mathrm{H}} *$ & 3715 & 366 & 61 & 5 & 0.5 & 0.1 & 347.0 & 160.9 & 155.0 & 1.0 & 0.0 & 1.3 \\
\hline & $c-H_{t} \mathrm{H}_{t} \mathrm{~N}_{\mathrm{H}}$ & 3693 & 355 & 69 & 16 & 0.0 & 0.0 & 398.3 & 164.6 & 151.3 & 0.5 & 0.3 & 0.1 \\
\hline \multirow{5}{*}{ B3LYP-D3/aVTZ } & $t-\mathrm{H}_{t} H_{t} *$ & 3607 & 622 & 170 & 6 & 0.0 & 0.0 & 479.6 & 206.8 & 170.3 & 0.4 & 0.0 & 0.9 \\
\hline & $c-\mathrm{H}_{t} H_{t}$ & 3594 & 668 & 183 & 10 & 1.3 & 1.7 & 525.5 & 187.1 & 157.2 & 0.6 & 0.3 & 0.6 \\
\hline & $\mathrm{H}_{g} H_{t}$ & 3671 & 643 & 144 & 13 & 2.6 & 2.8 & 572.0 & 155.1 & 153.5 & 3.2 & 0.9 & 0.7 \\
\hline & $t-\mathrm{H}_{t} H_{t} \mathrm{~N}_{\mathrm{H}} *$ & 3581 & 732 & 26 & 5 & 0.5 & 0.1 & 347.0 & 160.9 & 155.0 & 1.0 & 0.0 & 1.3 \\
\hline & $c-\mathrm{H}_{t} H_{t} \mathrm{~N}_{\mathrm{H}}$ & 3564 & 717 & 30 & 16 & 0.0 & 0.0 & 398.3 & 164.6 & 151.3 & 0.5 & 0.3 & 0.1 \\
\hline \multirow{7}{*}{ B3LYP-D3/VQZ } & $\mathrm{H}_{t}^{*}$ & 3785 & 62 & - & 26 & 0.0 & 0.0 & 2090.7 & 1043.4 & 924.0 & 0.0 & 0.6 & 0.1 \\
\hline & $\mathrm{H}_{g}$ & 3823 & 74 & - & 37 & 4.9 & 4.2 & 2094.1 & 1044.9 & 924.8 & 1.1 & 1.3 & 1.8 \\
\hline & $t-H_{t} \mathrm{H}_{t}{ }^{*}$ & 3785 & 78 & 0 & 6 & 0.0 & 0.0 & 480.0 & 207.4 & 170.7 & 0.4 & 0.0 & 0.9 \\
\hline & $c-H_{t} \mathrm{H}_{t}$ & 3769 & 81 & 16 & 10 & 0.9 & 1.3 & 526.9 & 187.9 & 158.1 & 0.6 & 0.2 & 0.6 \\
\hline & $H_{g} \mathrm{H}_{t}$ & 3780 & 76 & 5 & 12 & 2.8 & 2.9 & 569.9 & 155.5 & 154.2 & 3.1 & 0.8 & 0.9 \\
\hline & $t-H_{t} \mathrm{H}_{t} \mathrm{~N}_{\mathrm{H}} *$ & 3726 & 365 & 59 & 5 & 1.0 & 0.4 & 349.8 & 161.1 & 154.7 & 1.0 & 0.0 & 1.3 \\
\hline & $c-H_{t} \mathrm{H}_{t} \mathrm{~N}_{\mathrm{H}}$ & 3704 & 356 & 65 & 16 & 0.0 & 0.0 & 399.9 & 165.1 & 151.9 & 0.5 & 0.3 & 0.1 \\
\hline
\end{tabular}


A. Appendix

\begin{tabular}{|c|c|c|c|c|c|c|c|c|c|c|c|c|c|}
\hline Level of theory & Structure & $\omega_{\mathrm{OH}}$ & $S_{\mathrm{OH}}$ & $\Delta \omega_{\mathrm{OH}}$ & $\omega_{1}$ & $\Delta E_{\mathrm{e}}$ & $\Delta E_{0}$ & $A_{\mathrm{e}}$ & $B_{\mathrm{e}}$ & $C_{\mathrm{e}}$ & $\left|\mu_{a}\right|$ & $\left|\mu_{b}\right|$ & $\left|\mu_{c}\right|$ \\
\hline \multirow{5}{*}{ B3LYP-D3/VQZ } & $t-\mathrm{H}_{t} H_{t}{ }^{*}$ & 3620 & 619 & 165 & 6 & 0.0 & 0.0 & 480.0 & 207.4 & 170.7 & 0.4 & 0.0 & 0.9 \\
\hline & $c-\mathrm{H}_{t} H_{t}$ & 3607 & 653 & 178 & 10 & 0.9 & 1.3 & 526.9 & 187.9 & 158.1 & 0.6 & 0.2 & 0.6 \\
\hline & $\mathrm{H}_{g} H_{t}$ & 3687 & 628 & 5 & 12 & 2.8 & 2.9 & 569.9 & 155.5 & 154.2 & 3.1 & 0.8 & 0.9 \\
\hline & $t-\mathrm{H}_{t} H_{t} \mathrm{~N}_{\mathrm{H}} *$ & 3595 & 729 & 25 & 5 & 1.0 & 0.4 & 349.8 & 161.1 & 154.7 & 1.0 & 0.0 & 1.3 \\
\hline & $c-\mathrm{H}_{t} H_{t} \mathrm{~N}_{\mathrm{H}}$ & 3578 & 704 & 29 & 16 & 0.0 & 0.0 & 399.9 & 165.1 & 151.9 & 0.5 & 0.3 & 0.1 \\
\hline \multirow{7}{*}{$\begin{array}{l}\text { B3LYP-D3/ } \\
\text { def2-QZVP }\end{array}$} & $\mathrm{H}_{t}^{*}$ & 3789 & 63 & - & 25 & 0.0 & 0.0 & 2089.8 & 1042.2 & 922.9 & 0.0 & 0.6 & 0.1 \\
\hline & $\mathrm{H}_{g}$ & 3827 & 75 & - & 37 & 4.7 & 4.1 & 2093.2 & 1043.8 & 923.8 & 1.1 & 1.3 & 1.9 \\
\hline & $t-H_{t} \mathrm{H}_{t} *$ & 3788 & 79 & 1 & 10 & 0.0 & 0.0 & 479.7 & 206.1 & 169.8 & 0.4 & 0.0 & 0.9 \\
\hline & $c-H_{t} \mathrm{H}_{t}$ & 3774 & 80 & 15 & 7 & 1.2 & 1.5 & 525.5 & 186.3 & 156.6 & 0.6 & 0.3 & 0.6 \\
\hline & $H_{g} \mathrm{H}_{t}$ & 3783 & 77 & 6 & 12 & 2.6 & 2.7 & 570.6 & 154.7 & 153.3 & 3.1 & 0.9 & 0.8 \\
\hline & $t-H_{t} \mathrm{H}_{t} \mathrm{~N}_{\mathrm{H}} *$ & 3729 & 362 & 59 & 6 & 0.5 & 0.2 & 348.1 & 160.3 & 154.2 & 1.0 & 0.0 & 1.3 \\
\hline & $c-H_{t} \mathrm{H}_{t} \mathrm{~N}_{\mathrm{H}}$ & 3708 & 352 & 66 & 15 & 0.0 & 0.0 & 397.3 & 163.7 & 150.7 & 0.6 & 0.3 & 0.1 \\
\hline \multirow{5}{*}{$\begin{array}{l}\text { B3LYP-D3/ } \\
\text { def2-QZVP }\end{array}$} & $t-\mathrm{H}_{t} H_{t} *$ & 3624 & 612 & 165 & 10 & 0.0 & 0.0 & 479.7 & 206.1 & 169.8 & 0.4 & 0.0 & 0.9 \\
\hline & $c-\mathrm{H}_{t} H_{t}$ & 3611 & 654 & 178 & 7 & 1.2 & 1.5 & 525.5 & 186.3 & 156.6 & 0.6 & 0.3 & 0.6 \\
\hline & $\mathrm{H}_{g} H_{t}$ & 3688 & 632 & 139 & 12 & 2.6 & 2.7 & 570.6 & 154.7 & 153.3 & 3.1 & 0.9 & 0.8 \\
\hline & $t-\mathrm{H}_{t} \stackrel{H}{H}_{t} \mathrm{~N}_{\mathrm{H}} *$ & 3599 & 719 & 25 & 6 & 0.5 & 0.2 & 348.1 & 160.3 & 154.2 & 1.0 & 0.0 & 1.3 \\
\hline & $c-\mathrm{H}_{t} H_{t} \mathrm{~N}_{\mathrm{H}}$ & 3582 & 704 & 29 & 15 & 0.0 & 0.0 & 397.3 & 163.7 & 150.7 & 0.6 & 0.3 & 0.1 \\
\hline \multirow{7}{*}{ PBE0-D3/aVTZ } & $\mathrm{H}_{t}^{*}$ & 3830 & 65 & - & 24 & 0.0 & 0.0 & 2112.9 & 1053.2 & 932.6 & 0.0 & 0.5 & 0.1 \\
\hline & $\mathrm{H}_{g}$ & 3868 & 78 & - & 36 & 4.7 & 4.1 & 2115.1 & 1055.2 & 933.8 & 1.1 & 1.3 & 1.8 \\
\hline & $t-H_{t} \mathrm{H}_{t} *$ & 3826 & 82 & 4 & 7 & 0.0 & 0.0 & 485.4 & 205.9 & 169.9 & 0.5 & 0.0 & 0.8 \\
\hline & $c-H_{t} \mathrm{H}_{t}$ & 3812 & 80 & 18 & 10 & 0.9 & 1.3 & 532.6 & 187.1 & 157.4 & 0.7 & 0.2 & 0.5 \\
\hline & $H_{g} \mathrm{H}_{t}$ & 3822 & 80 & 8 & 12 & 3.0 & 3.1 & 577.3 & 154.3 & 152.8 & 3.2 & 0.9 & 0.7 \\
\hline & $t-H_{t} \mathrm{H}_{t} \mathrm{~N}_{\mathrm{H}} *$ & 3757 & 367 & 69 & 6 & 0.9 & 0.6 & 348.3 & 160.8 & 155.3 & 1.0 & 0.0 & 1.2 \\
\hline & $c-H_{t} \mathrm{H}_{t} \mathrm{~N}_{\mathrm{H}}$ & 3737 & 349 & 75 & 16 & 0.0 & 0.0 & 399.5 & 165.0 & 151.9 & 0.6 & 0.3 & 0.0 \\
\hline \multirow{5}{*}{ PBE0-D3/aVTZ } & $t-\mathrm{H}_{t} H_{t}{ }^{*}$ & 3650 & 622 & 180 & 7 & 0.0 & 0.0 & 485.4 & 205.9 & 169.9 & 0.5 & 0.0 & 0.8 \\
\hline & $c-\mathrm{H}_{t} H_{t}$ & 3633 & 673 & 197 & 10 & 0.9 & 1.3 & 532.6 & 187.1 & 157.4 & 0.7 & 0.2 & 0.5 \\
\hline & $\mathrm{H}_{g} H_{t}$ & 3710 & 662 & 158 & 12 & 3.0 & 3.1 & 577.3 & 154.3 & 152.8 & 3.2 & 0.9 & 0.7 \\
\hline & $t-\mathrm{H}_{t} H_{t} \mathrm{~N}_{\mathrm{H}} *$ & 3623 & 727 & 27 & 6 & 0.9 & 0.6 & 348.3 & 160.8 & 155.3 & 1.0 & 0.0 & 1.2 \\
\hline & $c-\mathrm{H}_{t} H_{t} \mathrm{~N}_{\mathrm{H}}$ & 3604 & 721 & 29 & 16 & 0.0 & 0.0 & 399.5 & 165.0 & 151.9 & 0.6 & 0.3 & 0.0 \\
\hline \multirow{7}{*}{ M06-2X/aVTZ } & $\mathrm{H}_{t}^{*}$ & 3828 & 82 & - & 16 & 0.0 & 0.0 & 2119.3 & 1062.9 & 940.0 & 0.0 & 0.6 & 0.1 \\
\hline & $\mathrm{H}_{g}$ & 3871 & 98 & - & 40 & 4.6 & 4.1 & 2119.5 & 1066.5 & 942.1 & 1.1 & 1.3 & 1.9 \\
\hline & $t-H_{t} \mathrm{H}_{t}^{*}$ & 3817 & 97 & 11 & 8 & 0.2 & 0.0 & 487.9 & 214.0 & 176.0 & 0.1 & 0.0 & 0.8 \\
\hline & $c-H_{t} \mathrm{H}_{t}$ & 3796 & 111 & 32 & 16 & 0.0 & 0.4 & 540.9 & 198.2 & 167.9 & 0.4 & 0.3 & 0.7 \\
\hline & $H_{g} \mathrm{H}_{t}$ & 3813 & 88 & 15 & 20 & 3.7 & 3.9 & 602.2 & 162.7 & 158.7 & 3.2 & 0.3 & 1.0 \\
\hline & $t-H_{t} \mathrm{H}_{t} \mathrm{~N}_{\mathrm{H}} *$ & 3773 & 346 & 44 & 8 & 2.2 & 1.8 & 361.0 & 165.2 & 157.7 & 0.7 & 0.0 & 1.2 \\
\hline & $c-H_{t} \mathrm{H}_{t} \mathrm{~N}_{\mathrm{H}}$ & 3744 & 344 & 52 & 19 & 0.0 & 0.0 & 411.7 & 175.8 & 161.4 & 0.4 & 0.2 & 0.3 \\
\hline \multirow{5}{*}{ M06-2X/aVTZ } & $t-\mathrm{H}_{t} H_{t} *$ & 3685 & 588 & 143 & 8 & 0.2 & 0.0 & 487.9 & 214.0 & 176.0 & 0.1 & 0.0 & 0.8 \\
\hline & $c-\mathrm{H}_{t} H_{t}$ & 3681 & 565 & 147 & 16 & 0.0 & 0.4 & 540.9 & 198.2 & 167.9 & 0.4 & 0.3 & 0.7 \\
\hline & $\mathrm{H}_{g} H_{t}$ & 3792 & 431 & 79 & 20 & 3.7 & 3.9 & 602.2 & 162.7 & 158.7 & 3.2 & 0.3 & 1.0 \\
\hline & $t-\mathrm{H}_{t} H_{t} \mathrm{~N}_{\mathrm{H}} *$ & 3665 & 686 & 20 & 8 & 2.2 & 1.8 & 361.0 & 165.2 & 157.7 & 0.7 & 0.0 & 1.2 \\
\hline & $c-\mathrm{H}_{t} H_{t} \mathrm{~N}_{\mathrm{H}}$ & 3653 & 618 & 28 & 19 & 0.0 & 0.0 & 411.7 & 175.8 & 161.4 & 0.4 & 0.2 & 0.3 \\
\hline \multirow{7}{*}{ M06-2X-D3/aVTZ } & $\mathrm{H}_{t}^{*}$ & 3828 & 82 & - & 16 & 0.0 & 0.0 & 2119.2 & 1062.7 & 939.9 & 0.0 & 0.6 & 0.1 \\
\hline & $\mathrm{H}_{g}$ & 3871 & 98 & - & 40 & 4.5 & 4.1 & 2119.4 & 1066.3 & 942.0 & 1.1 & 1.3 & 1.9 \\
\hline & $t-H_{t} \mathrm{H}_{t} *$ & 3817 & 97 & 11 & 8 & 0.0 & 0.0 & 487.8 & 214.5 & 176.3 & 0.1 & 0.0 & 0.8 \\
\hline & $c-H_{t} \mathrm{H}_{t}$ & 3795 & 112 & 33 & 16 & 0.0 & 0.6 & 540.4 & 198.8 & 168.3 & 0.4 & 0.3 & 0.7 \\
\hline & $H_{g} \mathrm{H}_{t}$ & 3813 & 88 & 15 & 20 & 3.7 & 4.0 & 601.4 & 163.0 & 159.1 & 3.2 & 0.3 & 1.0 \\
\hline & $t-H_{t} \mathrm{H}_{t} \mathrm{~N}_{\mathrm{H}} *$ & 3773 & 348 & 44 & 7 & 2.3 & 1.9 & 360.2 & 165.5 & 158.1 & 0.7 & 0.0 & 1.2 \\
\hline & $c-H_{t} \mathrm{H}_{t} \mathrm{~N}_{\mathrm{H}}$ & 3744 & 343 & 51 & 19 & 0.0 & 0.0 & 411.6 & 176.2 & 161.6 & 0.4 & 0.2 & 0.3 \\
\hline \multirow{5}{*}{ M06-2X-D3/aVTZ } & $t-\mathrm{H}_{t} H_{t} *$ & 3684 & 589 & 144 & 8 & 0.0 & 0.0 & 487.8 & 214.5 & 176.3 & 0.1 & 0.0 & 0.8 \\
\hline & $c-\mathrm{H}_{t} H_{t}$ & 3680 & 566 & 148 & 16 & 0.0 & 0.6 & 540.4 & 198.8 & 168.3 & 0.4 & 0.3 & 0.7 \\
\hline & $\mathrm{H}_{g} H_{t}$ & 3793 & 426 & 78 & 20 & 3.7 & 4.0 & 601.4 & 163.0 & 159.1 & 3.2 & 0.3 & 1.0 \\
\hline & $t-\mathrm{H}_{t} H_{t} \mathrm{~N}_{\mathrm{H}} *$ & 3664 & 688 & 20 & 7 & 2.3 & 1.9 & 360.2 & 165.5 & 158.1 & 0.7 & 0.0 & 1.2 \\
\hline & $c-\mathrm{H}_{t} H_{t} \mathrm{~N}_{\mathrm{H}}$ & 3652 & 618 & 28 & 19 & 0.0 & 0.0 & 411.6 & 176.2 & 161.6 & 0.4 & 0.2 & 0.3 \\
\hline \multirow[b]{4}{*}{$\omega \mathrm{B} 97-\mathrm{XD} / \mathrm{aVTZ}$} & $\mathrm{H}_{t}^{*}$ & 3860 & 63 & - & 22 & 0.0 & 0.0 & 2108.1 & 1046.5 & 927.2 & 0.0 & 0.5 & 0.1 \\
\hline & $\mathrm{H}_{g}$ & 3900 & 77 & - & 37 & 4.9 & 4.2 & 2111.4 & 1048.2 & 928.1 & 1.0 & 1.3 & 1.9 \\
\hline & $t-H_{t} \mathrm{H}_{t} *$ & 3857 & 80 & 3 & 8 & 0.0 & 0.0 & 482.7 & 204.9 & 168.9 & 0.5 & 0.0 & 0.8 \\
\hline & $c-H_{t} \mathrm{H}_{t}$ & 3841 & 78 & 19 & 14 & 0.4 & 1.5 & 531.2 & 186.0 & 156.9 & 0.7 & 0.2 & 0.5 \\
\hline
\end{tabular}




\section{A. Appendix}

\begin{tabular}{|c|c|c|c|c|c|c|c|c|c|c|c|c|c|}
\hline Level of theory & Structure & $\omega_{\mathrm{OH}}$ & $S_{\mathrm{OH}}$ & $\Delta \omega_{\mathrm{OH}}$ & $\omega_{1}$ & $\Delta E_{\mathrm{e}}$ & $\Delta E_{0}$ & $A_{\mathrm{e}}$ & $B_{\mathrm{e}}$ & $C_{\mathrm{e}}$ & $\left|\mu_{a}\right|$ & $\left|\mu_{b}\right|$ & $\left|\mu_{c}\right|$ \\
\hline & $H_{g} \mathrm{H}_{t}$ & 3854 & 78 & 6 & 12 & 2.9 & 2.8 & 565.8 & 158.5 & 156.7 & 3.2 & 0.1 & 1.3 \\
\hline & $t-H_{t} \mathrm{H}_{t} \mathrm{~N}_{\mathrm{H}} *$ & 3791 & 342 & 66 & 7 & 1.0 & 0.1 & 343.6 & 159.9 & 154.9 & 1.0 & 0.0 & 1.2 \\
\hline & $c-H_{t} \mathrm{H}_{t} \mathrm{~N}_{\mathrm{H}}$ & 3773 & 321 & 68 & 17 & 0.0 & 0.0 & 393.9 & 163.8 & 151.0 & 0.6 & 0.3 & 0.0 \\
\hline \multirow{5}{*}{$\omega \mathrm{B} 97-\mathrm{XD} / \mathrm{aVTZ}$} & $t-\mathrm{H}_{t} H_{t} *$ & 3690 & 605 & 170 & 8 & 0.0 & 0.0 & 28.3 & 482.7 & 204.9 & 168.9 & 0.5 & 0.0 \\
\hline & $c-\mathrm{H}_{t} H_{t}$ & 3683 & 621 & 177 & 14 & 0.4 & 1.5 & 531.2 & 186.0 & 156.9 & 0.7 & 0.2 & 0.5 \\
\hline & $\mathrm{H}_{g} H_{t}$ & 3768 & 565 & 132 & 12 & 2.9 & 2.8 & 565.8 & 158.5 & 156.7 & 3.2 & 0.1 & 1.3 \\
\hline & $t-\mathrm{H}_{t} H_{t} \mathrm{~N}_{\mathrm{H}} *$ & 3671 & 691 & 19 & 7 & 1.0 & 0.1 & 343.6 & 159.9 & 154.9 & 1.0 & 0.0 & 1.2 \\
\hline & $c-\mathrm{H}_{t} H_{t} \mathrm{~N}_{\mathrm{H}}$ & 3656 & 673 & 27 & 17 & 0.0 & 0.0 & 393.9 & 163.8 & 151.0 & 0.6 & 0.3 & 0.0 \\
\hline \multirow{7}{*}{ B97-D3/aVTZ } & $\mathrm{H}_{t}^{*}$ & 3708 & 48 & - & 24 & 0.0 & 0.0 & 2058.0 & 1022.7 & 906.6 & 0.0 & 0.6 & 0.1 \\
\hline & $\mathrm{H}_{g}$ & 3744 & 57 & - & 35 & 5.4 & 4.7 & 2063.2 & 1024.0 & 907.4 & 1.0 & 1.2 & 1.8 \\
\hline & $t-H_{t} \mathrm{H}_{t}$ & 3711 & 61 & 3 & 3 & 0.0 & 0.0 & 474.8 & 191.9 & 160.3 & 0.6 & 0.1 & 0.8 \\
\hline & $c-H_{t} \mathrm{H}_{t}$ & 3703 & 59 & 5 & 9 & 0.1 & 0.4 & 517.2 & 174.9 & 147.7 & 0.8 & 0.2 & 0.5 \\
\hline & $H_{g} \mathrm{H}_{t}$ & 3709 & 61 & -1 & 11 & 3.1 & 3.1 & 549.0 & 149.9 & 147.9 & 3.1 & 1.0 & 0.6 \\
\hline & $t-H_{t} \mathrm{H}_{t} \mathrm{~N}_{\mathrm{H}}$ & 3655 & 303 & 56 & 6 & 1.5 & 1.3 & 330.4 & 153.9 & 147.3 & 1.1 & 0.8 & 1.0 \\
\hline & $c-H_{t} \mathrm{H}_{t} \mathrm{~N}_{\mathrm{H}}$ & 3641 & 292 & 62 & 11 & 0.0 & 0.0 & 379.1 & 154.3 & 143.6 & 0.7 & 0.3 & 0.0 \\
\hline \multirow{5}{*}{ B97-D3/aVTZ } & $t-\mathrm{H}_{t} H_{t}$ & 3544 & 562 & 164 & 3 & 0.0 & 0.0 & 474.8 & 191.9 & 160.3 & 0.6 & 0.1 & 0.8 \\
\hline & $c-\mathrm{H}_{t} H_{t}$ & 3518 & 641 & 190 & 9 & 0.1 & 0.4 & 517.2 & 174.9 & 147.7 & 0.8 & 0.2 & 0.5 \\
\hline & $H_{g} \mathrm{H}_{t}$ & 3600 & 604 & 144 & 11 & 3.1 & 3.1 & 549.0 & 149.9 & 147.9 & 3.1 & 1.0 & 0.6 \\
\hline & $t-\mathrm{H}_{t} H_{t} \mathrm{~N}_{\mathrm{H}}$ & 3514 & 661 & 30 & 6 & 1.5 & 1.3 & 330.4 & 153.9 & 147.3 & 1.1 & 0.8 & 1.0 \\
\hline & $c-\mathrm{H}_{t} H_{t} \mathrm{~N}_{\mathrm{H}}$ & 3495 & 683 & 23 & 11 & 0.0 & 0.0 & 379.1 & 154.3 & 143.6 & 0.7 & 0.3 & 0.0 \\
\hline \multirow{7}{*}{$\mathrm{HF} / \mathrm{aVTZ}$} & $\mathrm{H}_{t} *$ & 4120 & 97 & - & 29 & 0.0 & 0.0 & 2169.3 & 1065.6 & 944.1 & 0.0 & 0.6 & 0.1 \\
\hline & $\mathrm{H}_{g}$ & 4157 & 115 & - & 40 & 3.0 & 2.4 & 2170.0 & 1067.4 & 944.8 & 1.0 & 1.4 & 2.1 \\
\hline & $t-H_{t} \mathrm{H}_{t} *$ & 4110 & 107 & 10 & 4 & 0.0 & 0.0 & 493.9 & 186.9 & 156.4 & 0.3 & 0.0 & 0.8 \\
\hline & $c-H_{t} \mathrm{H}_{t} *$ & 4107 & 104 & 13 & 3 & 0.3 & 0.2 & 516.1 & 160.1 & 135.6 & 0.5 & 0.0 & 0.4 \\
\hline & $H_{g} \mathrm{H}_{t}$ & 4108 & 99 & 2 & 6 & 0.7 & 0.7 & 572.2 & 142.1 & 139.8 & 2.8 & 0.8 & 1.0 \\
\hline & $t-H_{t} \mathrm{H}_{t} \mathrm{~N}_{\mathrm{H}}{ }^{*}$ & 4096 & 231 & 14 & 4 & 0.0 & 0.0 & 325.7 & 146.6 & 146.5 & 0.6 & 1.1 & 0.0 \\
\hline & $c-H_{t} \mathrm{H}_{t} \mathrm{~N}_{\mathrm{H}} *$ & 4092 & 227 & 15 & 5 & 0.1 & 0.1 & 363.4 & 140.7 & 134.7 & 0.4 & 0.0 & 0.1 \\
\hline \multirow{5}{*}{$\mathrm{HF} / \mathrm{aVTZ}$} & $t-\mathrm{H}_{t} H_{t} *$ & 4045 & 410 & 75 & 4 & 0.0 & 0.0 & 493.9 & 186.9 & 156.4 & 0.3 & 0.0 & 0.8 \\
\hline & $c-\mathrm{H}_{t} H_{t} *$ & 4043 & 433 & 77 & 3 & 0.3 & 0.2 & 516.1 & 160.1 & 135.6 & 0.5 & 0.0 & 0.4 \\
\hline & $\mathrm{H}_{g} H_{t}$ & 4089 & 471 & 68 & 6 & 0.7 & 0.7 & 572.2 & 142.1 & 139.8 & 2.8 & 0.8 & 1.0 \\
\hline & $t-\mathrm{H}_{t} H_{t} \mathrm{~N}_{\mathrm{H}} *$ & 4037 & 440 & 8 & 4 & 0.0 & 0.0 & 325.7 & 146.6 & 146.5 & 0.6 & 1.1 & 0.0 \\
\hline & $c-\mathrm{H}_{t} H_{t} \mathrm{~N}_{\mathrm{H}} *$ & 4034 & 450 & 9 & 5 & 0.1 & 0.1 & 363.4 & 140.7 & 134.7 & 0.4 & 0.0 & 0.1 \\
\hline \multirow{7}{*}{ AM1 } & $\mathrm{H}_{t}^{*}$ & 3413 & 91 & - & 12 & 0.0 & 0.0 & 2051.3 & 988.7 & 876.3 & 0.0 & 0.8 & 0.5 \\
\hline & $\mathrm{H}_{g}$ & 3433 & 109 & - & 22 & 11.0 & 10.1 & 2055.1 & 983.9 & 873.8 & 0.9 & 1.5 & 2.2 \\
\hline & $t-H_{t} \mathrm{H}_{t}{ }^{*}$ & 3410 & 130 & 3 & 6 & 4.1 & 3.4 & 454.8 & 193.8 & 159.6 & 1.0 & 0.0 & 1.3 \\
\hline & $c-H_{t} \mathrm{H}_{t} *$ & 3411 & 181 & 2 & 7 & 0.0 & 0.0 & 468.9 & 185.7 & 152.4 & 0.9 & 0.0 & 1.6 \\
\hline & $H_{g} \mathrm{H}_{t}$ & 3425 & 183 & -12 & 11 & 12.7 & 11.7 & 564.5 & 146.2 & 140.8 & 2.3 & 0.7 & 0.5 \\
\hline & $t-H_{t} \stackrel{\mathrm{H}}{t}_{t} \mathrm{~N}_{\mathrm{H}} *$ & 3412 & 140 & 1 & 5 & 3.9 & 3.0 & 368.8 & 144.2 & 132.9 & 0.6 & 0.0 & 1.6 \\
\hline & $c-H_{t} \mathrm{H}_{t} \mathrm{~N}_{\mathrm{H}} *$ & 3412 & 193 & 1 & 7 & 0.0 & 0.0 & 360.8 & 166.4 & 152.9 & 0.9 & 0.0 & 1.6 \\
\hline \multirow{5}{*}{ AM1 } & $t-\mathrm{H}_{t} H_{t}{ }^{*}$ & 3408 & 147 & 5 & 6 & 4.1 & 3.4 & 454.8 & 193.8 & 159.6 & 1.0 & 0.0 & 1.3 \\
\hline & $c-\mathrm{H}_{t} H_{t} *$ & 3385 & 104 & 28 & 7 & 0.0 & 0.0 & 468.9 & 185.7 & 152.4 & 0.9 & 0.0 & 1.6 \\
\hline & $\mathrm{H}_{g} H_{t}$ & 3407 & 107 & 26 & 11 & 12.7 & 11.7 & 564.5 & 146.2 & 140.8 & 2.3 & 0.7 & 0.5 \\
\hline & $t-\mathrm{H}_{t} H_{t} \mathrm{~N}_{\mathrm{H}} *$ & 3408 & 182 & 0 & 5 & 3.9 & 3.0 & 368.8 & 144.2 & 132.9 & 0.6 & 0.0 & 1.6 \\
\hline & $c-\mathrm{H}_{t} H_{t} \mathrm{~N}_{\mathrm{H}} *$ & 3387 & 140 & -2 & 7 & 0.0 & 0.0 & 360.8 & 166.4 & 152.9 & 0.9 & 0.0 & 1.6 \\
\hline \multirow{7}{*}{ PM6 } & $\mathrm{H}_{t}$ & 2531 & 237 & - & 9 & 0.0 & 0.0 & 2111.9 & 1000.3 & 885.2 & 0.0 & 0.8 & 0.2 \\
\hline & $\mathrm{H}_{g}$ & 2536 & 298 & - & 22 & 11.1 & 9.5 & 2146.7 & 1001.5 & 889.0 & 0.5 & 1.5 & 2.8 \\
\hline & $t-H_{t} \mathrm{H}_{t}$ & 2515 & 249 & 16 & 5 & 0.3 & 0.4 & 469.4 & 183.6 & 153.8 & 0.1 & 0.1 & 1.0 \\
\hline & $H_{g} \mathrm{H}_{t}$ & 2629 & 177 & -98 & 8 & 5.7 & 6.3 & 502.8 & 177.6 & 158.1 & 1.6 & 1.1 & 2.1 \\
\hline & $c-H_{t} \mathrm{H}_{t}$ & 2510 & 282 & 21 & 6 & 0.0 & 0.0 & 504.6 & 165.3 & 139.0 & 0.1 & 0.2 & 0.8 \\
\hline & $t-H_{t} \mathrm{H}_{t} \mathrm{~N}_{\mathrm{H}}$ & 2489 & 380 & 26 & 4 & 1.0 & 1.0 & 344.7 & 146.2 & 140.3 & 0.3 & 0.1 & 1.3 \\
\hline & $c-H_{t} \mathrm{H}_{t} \mathrm{~N}_{\mathrm{H}}$ & 2483 & 527 & 27 & 5 & 0.0 & 0.0 & 366.7 & 149.1 & 138.4 & 0.0 & 0.3 & 0.5 \\
\hline \multirow{6}{*}{ PM6 } & $t-\mathrm{H}_{t} H_{t}$ & 2468 & 544 & 63 & 5 & 0.3 & 0.4 & 469.4 & 183.6 & 153.8 & 0.1 & 0.1 & 1.0 \\
\hline & $c-\mathrm{H}_{t} H_{t}$ & 2470 & 537 & 61 & 6 & 0.0 & 0.0 & 504.6 & 165.3 & 139.0 & 0.1 & 0.2 & 0.8 \\
\hline & $\mathrm{H}_{g} H_{t}$ & 2589 & 270 & -53 & 8 & 5.7 & 6.3 & 502.8 & 177.6 & 158.1 & 1.6 & 1.1 & 2.1 \\
\hline & $t-\mathrm{H}_{t} H_{t} \mathrm{~N}_{\mathrm{H}}$ & 2456 & 649 & -12 & 4 & 1.0 & 1.0 & 344.7 & 146.2 & 140.3 & 0.3 & 0.1 & 1.3 \\
\hline & $c-\mathrm{H}_{t} H_{t} \mathrm{~N}_{\mathrm{H}}$ & 2455 & 520 & 15 & 5 & 0.0 & 0.0 & 366.7 & 149.1 & 138.4 & 0.0 & 0.3 & 0.5 \\
\hline & $\mathrm{H}_{t} *$ & 3649 & 47 & - & 22 & 0.0 & 0.0 & 2051.5 & 1025.8 & 908.6 & 0.0 & 0.5 & 0.1 \\
\hline
\end{tabular}


A. Appendix

\begin{tabular}{|c|c|c|c|c|c|c|c|c|c|c|c|c|c|}
\hline Level of theory & Structure & $\omega_{\mathrm{OH}}$ & $S_{\mathrm{OH}}$ & $\Delta \omega_{\mathrm{OH}}$ & $\omega_{1}$ & $\Delta E_{\mathrm{e}}$ & $\Delta E_{0}$ & $A_{\mathrm{e}}$ & $B_{\mathrm{e}}$ & $C_{\mathrm{e}}$ & $\left|\mu_{a}\right|$ & $\left|\mu_{b}\right|$ & $\left|\mu_{c}\right|$ \\
\hline & $\mathrm{H}_{g}$ & 3686 & 57 & - & 35 & 5.4 & 4.8 & 2056.0 & 1027.3 & 909.6 & 1.1 & 1.2 & 1.7 \\
\hline & $t-H_{t} \mathrm{H}_{t}$ & 3647 & 62 & 2 & 4 & 0.0 & 0.0 & 478.0 & 196.1 & 164.8 & 0.7 & 0.1 & 0.8 \\
\hline & $c-H_{t} \mathrm{H}_{t}$ & 3638 & 62 & 11 & 7 & 0.1 & 0.4 & 515.2 & 180.2 & 151.6 & 0.8 & 0.1 & 0.5 \\
\hline & $H_{g} \mathrm{H}_{t}$ & 3647 & 63 & 2 & 11 & 2.5 & 2.5 & 552.0 & 154.4 & 151.9 & 3.2 & 1.1 & 0.5 \\
\hline & $t-H_{t} \mathrm{H}_{t} \mathrm{~N}_{\mathrm{H}}$ & 3553 & 393 & 94 & 3 & 0.8 & 0.4 & 336.5 & 159.1 & 148.9 & 1.2 & 0.9 & 0.9 \\
\hline & $c-H_{t} \mathrm{H}_{t} \mathrm{~N}_{\mathrm{H}}$ & 3536 & 388 & 111 & 11 & 0.0 & 0.0 & 386.4 & 158.5 & 146.6 & 0.7 & 0.3 & 0.1 \\
\hline \multirow{5}{*}{ BP86-D3/aVTZ } & $t-\mathrm{H}_{t} H_{t}$ & 3432 & 696 & 217 & 4 & 0.0 & 0.0 & 478.0 & 196.1 & 164.8 & 0.7 & 0.1 & 0.8 \\
\hline & $c-\mathrm{H}_{t} H_{t}$ & 3410 & 764 & 239 & 7 & 0.1 & 0.4 & 515.2 & 180.2 & 151.6 & 0.8 & 0.1 & 0.5 \\
\hline & $\mathrm{H}_{g} H_{t}$ & 3502 & 714 & 166 & 11 & 2.5 & 2.5 & 552.0 & 154.4 & 151.9 & 3.2 & 1.1 & 0.5 \\
\hline & $t-\mathrm{H}_{t} H_{t} \mathrm{~N}_{\mathrm{H}}$ & 3393 & 825 & 39 & 3 & 0.8 & 0.4 & 336.5 & 159.1 & 148.9 & 1.2 & 0.9 & 0.9 \\
\hline & $c-\mathrm{H}_{t} H_{t} \mathrm{~N}_{\mathrm{H}}$ & 3377 & 826 & 33 & 11 & 0.0 & 0.0 & 386.4 & 158.5 & 146.6 & 0.7 & 0.3 & 0.1 \\
\hline \multirow{7}{*}{ BLYP-D3/aVTZ } & $\mathrm{H}_{t}^{*}$ & 3632 & 48 & - & 22 & 0.0 & 0.0 & 2031.9 & 1017.0 & 900.7 & 0.0 & 0.6 & 0.1 \\
\hline & $\mathrm{H}_{g}$ & 3669 & 57 & - & 35 & 5.2 & 4.6 & 2037.3 & 1018.4 & 901.6 & 1.0 & 1.3 & 1.8 \\
\hline & $t-H_{t} \mathrm{H}_{t}$ & 3635 & 61 & -3 & 1 & 0.0 & 0.0 & 468.5 & 201.2 & 165.9 & 0.4 & 0.0 & 0.9 \\
\hline & $c-H_{t} \mathrm{H}_{t}$ & 3622 & 64 & 10 & 8 & 0.9 & 1.3 & 512.0 & 181.5 & 152.5 & 0.7 & 0.2 & 0.7 \\
\hline & $H_{g} \mathrm{H}_{t}$ & 3630 & 60 & 2 & 12 & 2.6 & 2.8 & 554.4 & 152.7 & 150.3 & 3.1 & 1.0 & 0.5 \\
\hline & $t-H_{t} \mathrm{H}_{t} \mathrm{~N}_{\mathrm{H}}$ & 3569 & 359 & 66 & 1 & 1.5 & 1.4 & 337.1 & 157.2 & 151.8 & 1.0 & 0.0 & 1.4 \\
\hline & $c-H_{t} \mathrm{H}_{t} \mathrm{~N}_{\mathrm{H}}$ & 3546 & 351 & 76 & 13 & 0.0 & 0.0 & 387.5 & 159.2 & 146.6 & 0.6 & 0.3 & 0.1 \\
\hline \multirow{5}{*}{ BLYP-D3/aVTZ } & $t-\mathrm{H}_{t} H_{t}$ & 3459 & 609 & 177 & 1 & 0.0 & 0.0 & 468.5 & 201.2 & 165.9 & 0.4 & 0.0 & 0.9 \\
\hline & $c-\mathrm{H}_{t} H_{t}$ & 3436 & 684 & 196 & 8 & 0.9 & 1.3 & 512.0 & 181.5 & 152.5 & 0.7 & 0.2 & 0.7 \\
\hline & $\mathrm{H}_{g} H_{t}$ & 3517 & 647 & 152 & 12 & 2.6 & 2.8 & 554.4 & 152.7 & 150.3 & 3.1 & 1.0 & 0.5 \\
\hline & $t-\mathrm{H}_{t} H_{t} \mathrm{~N}_{\mathrm{H}}$ & 3430 & 724 & 29 & 1 & 1.5 & 1.4 & 337.1 & 157.2 & 151.8 & 1.0 & 0.0 & 1.4 \\
\hline & $c-\mathrm{H}_{t} H_{t} \mathrm{~N}_{\mathrm{H}}$ & 3406 & 738 & 30 & 13 & 0.0 & 0.0 & 387.5 & 159.2 & 146.6 & 0.6 & 0.3 & 0.1 \\
\hline \multirow{7}{*}{ PBEh-3c } & $\mathrm{H}_{t}^{*}$ & 3895 & 69 & - & 34 & 0.0 & 0.0 & 2112.5 & 1050.1 & 930.0 & - & - & - \\
\hline & $\mathrm{H}_{g}$ & 3942 & 90 & - & 39 & 4.8 & 3.6 & 2121.8 & 1051.5 & 931.3 & - & - & - \\
\hline & $t-H_{t} \mathrm{H}_{t} *$ & 3890 & 92 & -5 & 5 & 0.3 & 0.5 & 484.3 & 196.9 & 163.4 & - & - & - \\
\hline & $c-H_{t} \mathrm{H}_{t}$ & 3884 & 90 & 11 & 9 & 1.6 & 2.1 & 529.3 & 176.7 & 149.4 & - & - & - \\
\hline & $H_{g} \mathrm{H}_{t}$ & 3889 & 71 & 6 & 14 & 0.0 & 0.0 & 531.3 & 162.5 & 158.1 & - & - & - \\
\hline & $t-H_{t} \mathrm{H}_{t} \mathrm{~N}_{\mathrm{H}} *$ & 3857 & 341 & 33 & 4 & 0.4 & 0.0 & 356.7 & 154.6 & 147.7 & - & - & - \\
\hline & $c-H_{t} \mathrm{H}_{t} \mathrm{~N}_{\mathrm{H}}$ & 3844 & 352 & 40 & 14 & 0.0 & 0.0 & 395.0 & 160.4 & 148.5 & - & - & - \\
\hline \multirow{5}{*}{ PBEh-3c } & $t-\mathrm{H}_{t} H_{t} *$ & 3769 & 500 & 126 & 5 & 0.3 & 0.3 & 484.3 & 196.9 & 163.4 & - & - & - \\
\hline & $c-\mathrm{H}_{t} H_{t}$ & 3760 & 533 & 135 & 9 & 1.6 & 2.1 & 529.3 & 176.7 & 149.4 & - & - & - \\
\hline & $\mathrm{H}_{g} H_{t}$ & 3853 & 393 & 89 & 14 & 0.0 & 0.0 & 531.3 & 162.5 & 158.1 & - & - & - \\
\hline & $t-\mathrm{H}_{t} H_{t} \mathrm{~N}_{\mathrm{H}} *$ & 3742 & 608 & 27 & 4 & 0.4 & 0.0 & 356.7 & 154.6 & 147.7 & - & - & - \\
\hline & $c-\mathrm{H}_{t} H_{t} \mathrm{~N}_{\mathrm{H}}$ & 3734 & 584 & 26 & 14 & 0.0 & 0.0 & 395.0 & 160.4 & 148.5 & - & - & - \\
\hline \multirow{7}{*}{ MP2/VTZ } & $\mathrm{H}_{t}$ & 3818 & 67 & - & 28 & 0.0 & 0.0 & 2109.1 & 1059.2 & 937.0 & - & - & - \\
\hline & $\mathrm{H}_{g}$ & 3853 & 78 & - & 37 & 4.9 & 4.3 & 2113.0 & 1060.9 & 938.2 & - & - & - \\
\hline & $t-H_{t} \mathrm{H}_{t} *$ & 3810 & 84 & 8 & 4 & 0.0 & 0.0 & 486.4 & 211.1 & 173.9 & - & - & - \\
\hline & $c-H_{t} \mathrm{H}_{t}$ & 3794 & 87 & 24 & 10 & 1.2 & 1.5 & 539.4 & 191.6 & 162.1 & - & - & - \\
\hline & $H_{g} \mathrm{H}_{t}$ & 3805 & 80 & 13 & 12 & 3.4 & 3.4 & 578.7 & 160.4 & 158.0 & - & - & - \\
\hline & $t-H_{t} \mathrm{H}_{t} \mathrm{~N}_{\mathrm{H}} *$ & 3757 & 346 & 53 & 4 & 1.5 & 1.0 & 354.2 & 163.9 & 157.5 & - & - & - \\
\hline & $c-H_{t} \mathrm{H}_{t} \mathrm{~N}_{\mathrm{H}}$ & 3733 & 344 & 61 & 18 & 0.0 & 0.0 & 406.7 & 170.5 & 156.7 & - & - & - \\
\hline \multirow{5}{*}{ MP2/VTZ } & $t-\mathrm{H}_{t} H_{t} *$ & 3670 & 581 & 148 & 4 & 0.0 & 0.0 & 486.4 & 211.1 & 173.9 & - & - & - \\
\hline & $c-\mathrm{H}_{t} H_{t}$ & 3652 & 609 & 166 & 10 & 1.2 & 1.5 & 539.4 & 191.6 & 162.1 & - & - & - \\
\hline & $\mathrm{H}_{g} H_{t}$ & 3781 & 446 & 72 & 12 & 3.4 & 3.4 & 578.7 & 160.4 & 158.0 & - & - & - \\
\hline & $t-\mathrm{H}_{t} H_{t} \mathrm{~N}_{\mathrm{H}} *$ & 3645 & 689 & 25 & 4 & 1.5 & 1.0 & 354.2 & 163.9 & 157.5 & - & - & - \\
\hline & $c-\mathrm{H}_{t} H_{t} \mathrm{~N}_{\mathrm{H}}$ & 3623 & 655 & 29 & 18 & 0.0 & 0.0 & 406.7 & 170.5 & 156.7 & - & - & - \\
\hline \multirow{7}{*}{ SCS-MP2/VTZ } & $\mathrm{H}_{t}$ & 3822 & 62 & - & 28 & 0.0 & 0.0 & 2103.3 & 1053.1 & 931.9 & - & - & - \\
\hline & $\mathrm{H}_{g}$ & 3857 & 72 & - & 37 & 4.7 & 4.1 & 2107.0 & 1054.8 & 933.0 & - & - & - \\
\hline & $t-H_{t} \mathrm{H}_{t} *$ & 3818 & 77 & 4 & 6 & 0.0 & 0.0 & 484.0 & 206.3 & 170.3 & - & - & - \\
\hline & $c-H_{t} \mathrm{H}_{t}$ & 3804 & 80 & 18 & 9 & 1.1 & 1.4 & 536.3 & 186.9 & 158.3 & - & - & - \\
\hline & $H_{g} \mathrm{H}_{t}$ & 3813 & 72 & 9 & 11 & 2.9 & 2.8 & 575.1 & 156.8 & 154.6 & - & - & - \\
\hline & $t-H_{t} \mathrm{H}_{t} \mathrm{~N}_{\mathrm{H}} *$ & 3781 & 296 & 36 & 5 & 0.9 & 0.4 & 347.8 & 160.2 & 154.8 & - & - & - \\
\hline & $c-H_{t} \mathrm{H}_{t} \mathrm{~N}_{\mathrm{H}}$ & 3761 & 295 & 43 & 17 & 0.0 & 0.0 & 400.2 & 166.1 & 153.1 & - & - & - \\
\hline \multirow{4}{*}{ SCS-MP2/VTZ } & $t-\mathrm{H}_{t} H_{t} *$ & 3708 & 499 & 114 & 6 & 0.0 & 0.0 & 484.0 & 206.3 & 170.3 & - & - & - \\
\hline & $c-\mathrm{H}_{t} H_{t}$ & 3694 & 520 & 128 & 9 & 1.1 & 1.4 & 536.3 & 186.9 & 158.3 & - & - & - \\
\hline & $\mathrm{H}_{g} H_{t}$ & 3781 & 446 & 76 & 11 & 2.9 & 2.8 & 575.1 & 156.8 & 154.6 & - & - & - \\
\hline & $t-\mathrm{H}_{t} H_{t} \mathrm{~N}_{\mathrm{H}} *$ & 3689 & 582 & 19 & 5 & 0.9 & 0.4 & 347.8 & 160.2 & 154.8 & - & - & - \\
\hline
\end{tabular}




\section{A. Appendix}

\begin{tabular}{|c|c|c|c|c|c|c|c|c|c|c|c|c|c|}
\hline Level of theory & Structure & $\omega_{\mathrm{OH}}$ & $S_{\mathrm{OH}}$ & $\Delta \omega_{\mathrm{OH}}$ & $\omega_{1}$ & $\Delta E_{\mathrm{e}}$ & $\Delta E_{0}$ & $A_{\mathrm{e}}$ & $B_{\mathrm{e}}$ & $C_{\mathrm{e}}$ & $\left|\mu_{a}\right|$ & $\left|\mu_{b}\right|$ & $\left|\mu_{c}\right|$ \\
\hline & $c-\mathrm{H}_{t} H_{t} \mathrm{~N}_{\mathrm{H}}$ & 3672 & 558 & 22 & 17 & 0.0 & 0.0 & 400.2 & 166.1 & 153.1 & - & - & - \\
\hline \multirow{7}{*}{ B97-3c } & $\mathrm{H}_{t}$ & 3716 & 43 & - & 20 & 0.0 & 0.0 & 2072.9 & 1031.0 & 914.2 & - & - & - \\
\hline & $\mathrm{H}_{g}$ & 3759 & 50 & - & 35 & 5.5 & 4.8 & 2077.0 & 1033.4 & 915.8 & - & - & - \\
\hline & $t-H_{t} \mathrm{H}_{t} *$ & 3722 & 56 & -6 & 3 & 0.0 & 0.0 & 478.0 & 191.3 & 159.0 & - & - & - \\
\hline & $c-H_{t} \mathrm{H}_{t}$ & 3712 & 55 & 4 & 13 & 0.5 & 0.9 & 521.4 & 174.0 & 147.0 & - & - & - \\
\hline & $H_{g} \mathrm{H}_{t}$ & 3720 & 56 & -4 & 10 & 2.8 & 2.9 & 546.9 & 150.5 & 148.9 & - & - & - \\
\hline & $t-H_{t} \mathrm{H}_{t} \mathrm{~N}_{\mathrm{H}} *$ & 3674 & 265 & 48 & 3 & 1.0 & 0.6 & 331.4 & 151.3 & 147.6 & - & - & - \\
\hline & $c-H_{t} \mathrm{H}_{t} \mathrm{~N}_{\mathrm{H}}$ & 3660 & 258 & 52 & 7 & 0.0 & 0.0 & 380.3 & 153.8 & 143.3 & - & - & - \\
\hline \multirow{5}{*}{ B97-3c } & $t-\mathrm{H}_{t} H_{t} *$ & 3580 & 490 & 136 & 3 & 0.0 & 0.0 & 478.0 & 191.3 & 159.0 & - & - & - \\
\hline & $c-\mathrm{H}_{t} H_{t}$ & 3545 & 577 & 171 & 13 & 0.5 & 0.9 & 521.4 & 174.0 & 147.0 & - & - & - \\
\hline & $\mathrm{H}_{g} H_{t}$ & 3644 & 511 & 115 & 10 & 2.8 & 2.9 & 546.9 & 150.5 & 148.9 & - & - & - \\
\hline & $t-\mathrm{H}_{t} H_{t} \mathrm{~N}_{\mathrm{H}}{ }^{*}$ & 3557 & 570 & 23 & 3 & 1.0 & 0.6 & 331.4 & 151.3 & 147.6 & - & - & - \\
\hline & $c-\mathrm{H}_{t} H_{t} \mathrm{~N}_{\mathrm{H}}$ & 3526 & 602 & 19 & 7 & 0.0 & 0.0 & 380.3 & 153.8 & 143.3 & - & - & - \\
\hline \multirow{7}{*}{ B2PLYP-D3/VTZ } & $\mathrm{H}_{t}$ & 3807 & 65 & - & 26 & 0.0 & 0.0 & 2104.0 & 1055.3 & 933.8 & - & - & - \\
\hline & $\mathrm{H}_{g}$ & 3845 & 76 & - & 25 & 5.2 & 4.6 & 2107.1 & 1056.6 & 934.5 & - & - & - \\
\hline & $t-H_{t} \mathrm{H}_{t}$ & 3807 & 80 & 0 & 10 & 0.0 & 0.0 & 484.3 & 211.8 & 174.2 & - & - & - \\
\hline & $c-H_{t} \mathrm{H}_{t}$ & 3789 & 85 & 18 & 8 & 0.9 & 1.5 & 536.7 & 191.9 & 162.3 & - & - & - \\
\hline & $H_{g} \mathrm{H}_{t}$ & 3802 & 80 & 5 & 4 & 3.6 & 3.7 & 572.0 & 159.7 & 157.7 & - & - & - \\
\hline & $t-H_{t} \mathrm{H}_{t} \mathrm{~N}_{\mathrm{H}}$ & 3753 & 367 & 54 & 13 & 1.3 & 0.7 & 355.1 & 164.1 & 157.1 & - & - & - \\
\hline & $c-H_{t} \mathrm{H}_{t} \mathrm{~N}_{\mathrm{H}}$ & 3729 & 360 & 60 & 9 & 0.0 & 0.0 & 407.2 & 170.4 & 156.4 & - & - & - \\
\hline \multirow{5}{*}{ B2PLYP-D3/VTZ } & $t-\mathrm{H}_{t} H_{t}$ & 3656 & 599 & 151 & 10 & 0.0 & 0.0 & 484.3 & 211.8 & 174.2 & - & - & - \\
\hline & $c-\mathrm{H}_{t} H_{t}$ & 3641 & 624 & 166 & 8 & 0.9 & 1.5 & 536.7 & 191.9 & 162.3 & - & - & - \\
\hline & $\mathrm{H}_{g} H_{t}$ & 3734 & 566 & 111 & 4 & 3.6 & 3.7 & 572.0 & 159.7 & 157.7 & - & - & - \\
\hline & $t-\mathrm{H}_{t} H_{t} \mathrm{~N}_{\mathrm{H}}$ & 3632 & 703 & 175 & 13 & 1.3 & 0.7 & 355.1 & 164.1 & 157.1 & - & - & - \\
\hline & $c-\mathrm{H}_{t} H_{t} \mathrm{~N}_{\mathrm{H}}$ & 3613 & 670 & 28 & 9 & 0.0 & 0.0 & 407.2 & 170.4 & 156.4 & - & - & - \\
\hline
\end{tabular}


A. Appendix

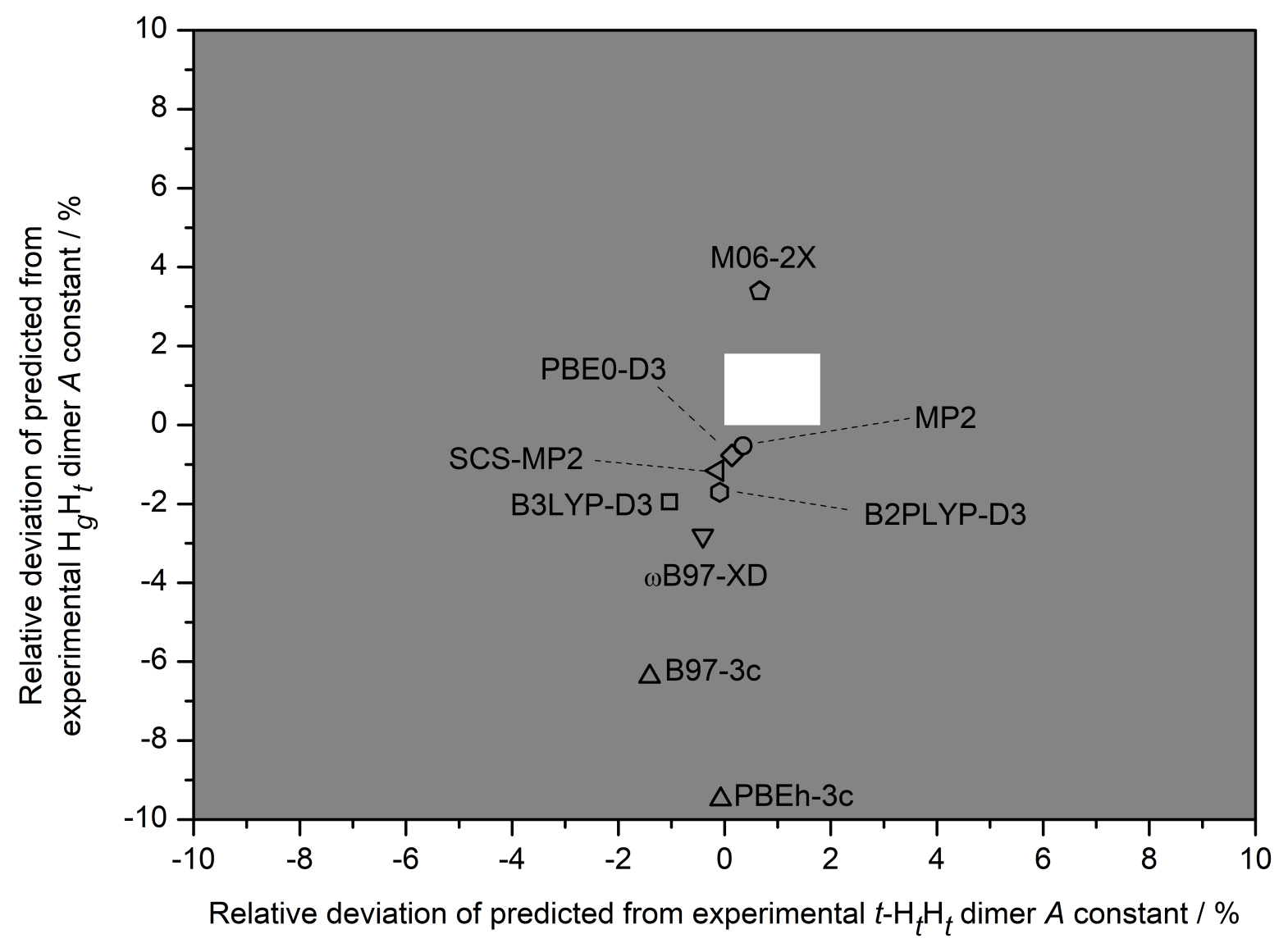

Figure A.14.: Relative deviation of the theoretically predicted $\left(A_{\mathrm{e}}\right)$ from the experimental rotational constant $(A)$ for the $t-\mathrm{H}_{t} \mathrm{H}_{t}$ and $\mathrm{H}_{g} \mathrm{H}_{t}$ dimers at different levels of electronic structure calculation. The zone of estimated incompatibility with experiment is grey. Only predictions within the white area are compatible with experiment for both species. 


\section{A. Appendix}

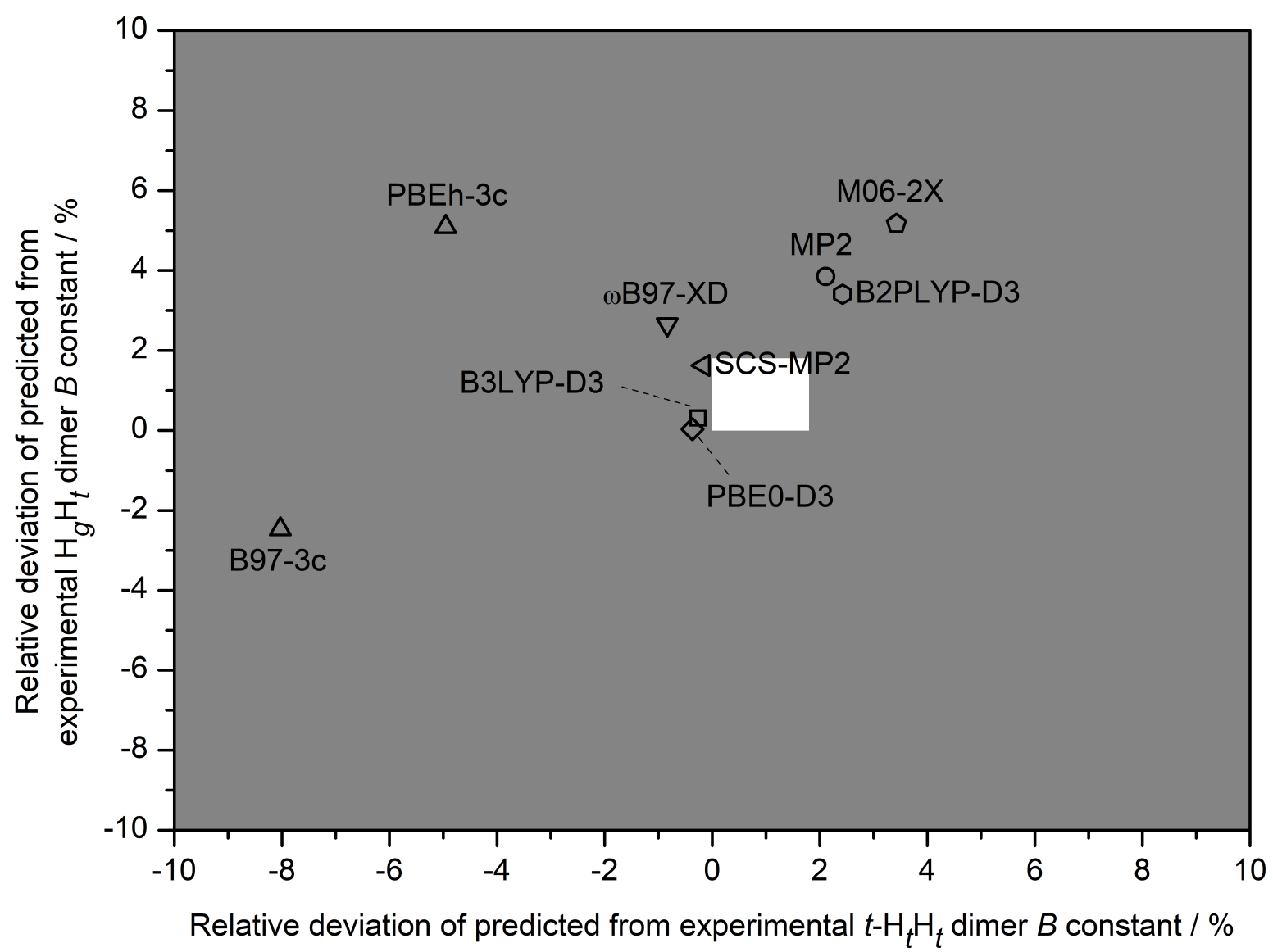

Figure A.15.: Relative deviation of the theoretically predicted $\left(B_{\mathrm{e}}\right)$ from the experimental rotational constant $(B)$ for the $t-\mathrm{H}_{t} \mathrm{H}_{t}$ and $\mathrm{H}_{g} \mathrm{H}_{t}$ dimers at different levels of electronic structure calculation. The zone of estimated incompatibility with experiment is grey. Only predictions within the white area are compatible with experiment for both species. 
A. Appendix

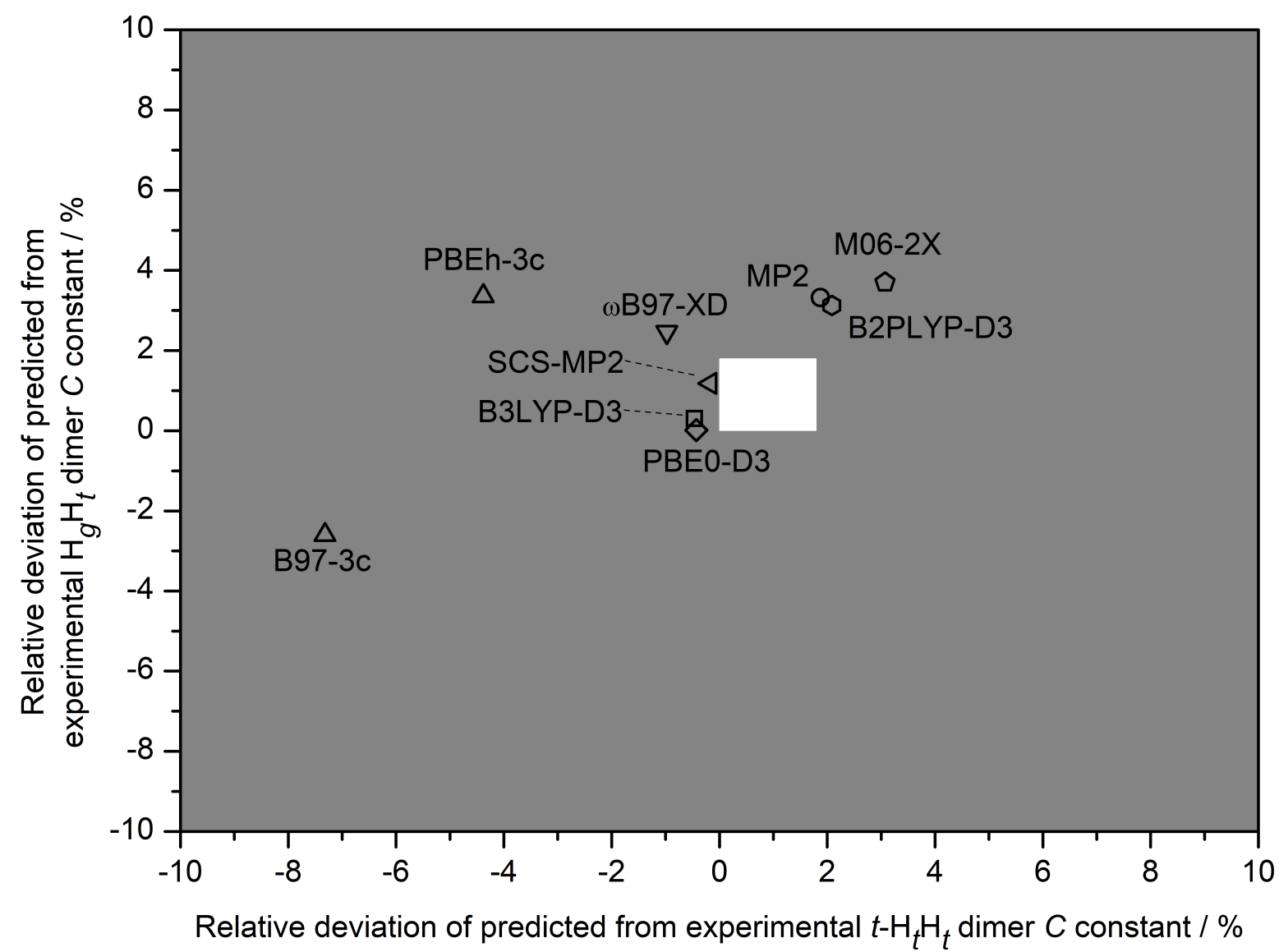

Figure A.16.: Relative deviation of the theoretically predicted $\left(C_{\mathrm{e}}\right)$ from the experimental rotational constant $(C)$ for the $t-\mathrm{H}_{t} \mathrm{H}_{t}$ and $\mathrm{H}_{g} \mathrm{H}_{t}$ dimers at different levels of electronic structure calculation. The zone of estimated incompatibility with experiment is grey. Only predictions within the white area are compatible with experiment for both species.

Table A.18.: Integration bounds in $\mathrm{cm}^{-1}$ for evaluation of relative $t-\mathrm{H}_{t} \mathrm{H}_{t}$ and $\mathrm{H}_{g} \mathrm{H}_{t}$ abundances from a trimer corrected difference spectrum (Figure A.3, trace $c-0.46 \times$ trace e). Integration bounds for the intensity ratio of both $\mathrm{H}_{t} \mathrm{H}_{t} \mathrm{~N}_{\mathrm{H}}$ isomers from a $\mathrm{HHH}$ trimer-corrected difference spectrum (Figure 3.8, trace $\mathrm{b}-1.99 \times$ trace a) are included. The Bruker OPUS (Version 7.0) program package was used for linear baseline corrections interpolated from the integral bounds and subsequent integrations (OPUS integration method B).

\begin{tabular}{ccc}
\hline & Upper bound & Lower bound \\
\hline$t-\mathrm{H}_{t} \mathrm{H}_{t}$ & 3497.3 & 3487.8 \\
$\mathrm{H}_{g} \mathrm{H}_{t}$ & 3567.4 & 3554.5 \\
$\mathrm{H}_{t} \mathrm{H}_{t} \mathrm{~N}_{\mathrm{H}-\mathrm{I}}$ & 3588.5 & 3579.7 \\
& 3477.4 & 3469.4 \\
$\mathrm{H}_{t} \mathrm{H}_{t} \mathrm{~N}_{\mathrm{H}}-\mathrm{II}$ & 3568.4 & 3560.0 \\
& 3467.9 & 3458.9 \\
\hline
\end{tabular}




\section{A. Appendix}

\section{A.4. Aliphatic Alcohols}

Table A.19.: OH stretching band centres of alcohol clusters in supersonic expansions and in bulk nitrogen matrices together with the resulting matrix induced downshift $\left(\Delta \tilde{\nu}_{\mathrm{mi}}\right)$. Molecules are abbreviated with N (nitrogen), M (methanol), B (tert-butyl alcohol) and $\mathrm{E}$ (ethanol). To describe cluster composition, single letters are repeated according to the number of units present in donor-acceptor sequence. Roman structure symbols denote the monomer unit involved in the vibration, italic symbols the passive monomer. If several signals arise in the matrix due to site splitting, dominant ones are written in bold font.

\begin{tabular}{cccc}
\hline & & $\tilde{\nu} / \mathrm{cm}^{-1}$ & \\
Structure & Jet-expansion & N-matrix & $\Delta \tilde{\nu}_{\mathrm{mi}} / \mathrm{cm}^{-1}$ \\
\hline $\mathrm{M}$ & $3685^{30}$ & $3660^{56} / \mathbf{3 6 6 4}^{58,117,118} / 3665^{119}$ & $20-25$ \\
$M \mathrm{M}$ & $3684^{92} / 3675^{103}$ & $3659,3655,3654,{ }^{118} \mathbf{3 6 5 1} \mathbf{1}^{58,118}$ & $16-33$ \\
$\mathrm{M} M$ & $3575^{30}$ & $3520, \mathbf{3 5 0 4}, 3496,3489^{58,59,118} / 3490^{56}$ & $55-86$ \\
$\mathrm{~B}$ & $3642^{30}$ & $3628^{122} / \mathbf{3 6 2 9}^{123}$ & $13-14$ \\
$B \mathrm{~B}$ & $3630^{30}$ & - & - \\
$\mathrm{B} B$ & $3497^{30,296}$ & $3501, \mathbf{3 4 7 4}, \mathbf{3 4 6 6}, 3425^{122}$ & $-4-72$ \\
$\mathrm{E}_{t}$ & $3678^{295}$ & $3653^{60}$ & 25 \\
$\mathrm{E}_{g}$ & $3660^{295}$ & $3650^{60}$ & 10 \\
$E_{g} \mathrm{E}_{g}$ & $3654^{295}$ & & \\
$E \mathrm{E}$ & - & $\left\{3654, \mathbf{3 6 4 6}, \mathbf{3 6 4 2}, \mathbf{3 6 3 7}, 3640,3635^{62}\right\}$ & $0-37$ \\
$E_{g} \mathrm{E}_{t}$ & $3672^{295}$ & & \\
$\mathrm{E}_{g} E_{g}$ & $3531^{31,297}$ & $\left\{\mathbf{3 5 1 7}, 3501,3494,3490,3486,3477,3470^{62}\right\}$ & $14-78$ \\
$\mathrm{E} E$ & $3540^{31,297}$ & & \\
$\mathrm{E}_{g} E_{t}$ & $3548^{31,297}$ & & \\
\hline
\end{tabular}




\section{A. Appendix}

Table A.20.: $\mathrm{OH}$ stretching band centres of alcohol clusters in supersonic expansions and in bulk argon matrices together with the resulting matrix induced downshift $\left(\Delta \tilde{\nu}_{\mathrm{mi}}\right)$. Molecules are abbreviated with N (nitrogen), M (methanol), B (tert-butyl alcohol) and E (ethanol). To describe cluster composition, single letters are repeated according to the number of units present in donor-acceptor sequence. Roman structure symbols denote the monomer unit involved in the vibration, italic symbols the passive monomer. If several signals arise in the matrix due to site splitting, dominant ones are written in bold font.

\begin{tabular}{|c|c|c|c|}
\hline \multicolumn{4}{|c|}{$\tilde{\nu} / \mathrm{cm}^{-1}$} \\
\hline Structure & Jet-expansion & Ar-matrix & $\Delta \tilde{\nu}_{\mathrm{mi}} / \mathrm{cm}^{-1}$ \\
\hline $\mathrm{M}$ & $3685^{30}$ & $3667,{ }^{386,387} 3666^{58,386}$ & 18-19 \\
\hline$M \mathrm{M}$ & $3684^{92} / 3675^{103}$ & $3679^{58,386}$ & $-4-5$ \\
\hline $\mathrm{M} M$ & $3575^{30}$ & $\begin{array}{c}3541,{ }^{386,387} 3534,{ }^{386} 3533,,^{387} 3530,{ }^{58} \\
\mathbf{3 5 2 7}^{386} \mathbf{3 5 2 6}^{387} 3519,{ }^{387} 3518^{386}\end{array}$ & $34-57$ \\
\hline B & $3642^{30}$ & $3627,3622^{122,123}$ & $15-20$ \\
\hline$B \mathrm{~B}$ & $3630^{30}$ & - & - \\
\hline $\mathrm{B} B$ & $3497^{30,296}$ & $3506,3479^{122}$ & $-9-18$ \\
\hline $\mathrm{E}_{t}$ & $3678^{295}$ & $3661,3656^{60}$ & $17-22$ \\
\hline $\mathrm{E}_{g}$ & $3660^{295}$ & - & \\
\hline$E_{g} \mathrm{E}_{g}$ & $3654^{295}$ & & \\
\hline$E \mathrm{E}$ & - & $\mathbf{3 6 6 0}, 3646,3665^{62}$ & $-6-11$ \\
\hline$E_{g} \mathrm{E}_{t}$ & $3672^{295}$ & & \\
\hline $\mathrm{E}_{g} E_{g}$ & $3531^{31,297}$ & & \\
\hline $\mathrm{E} E$ & $3540^{31,297}$ & $\left.3536,3531, \mathbf{3 5 2 7}, 3520,3507^{62}\right\}$ & $-5-41$ \\
\hline $\mathrm{E}_{g} E_{t}$ & $3548^{31,297}$ & & \\
\hline
\end{tabular}


A. Appendix

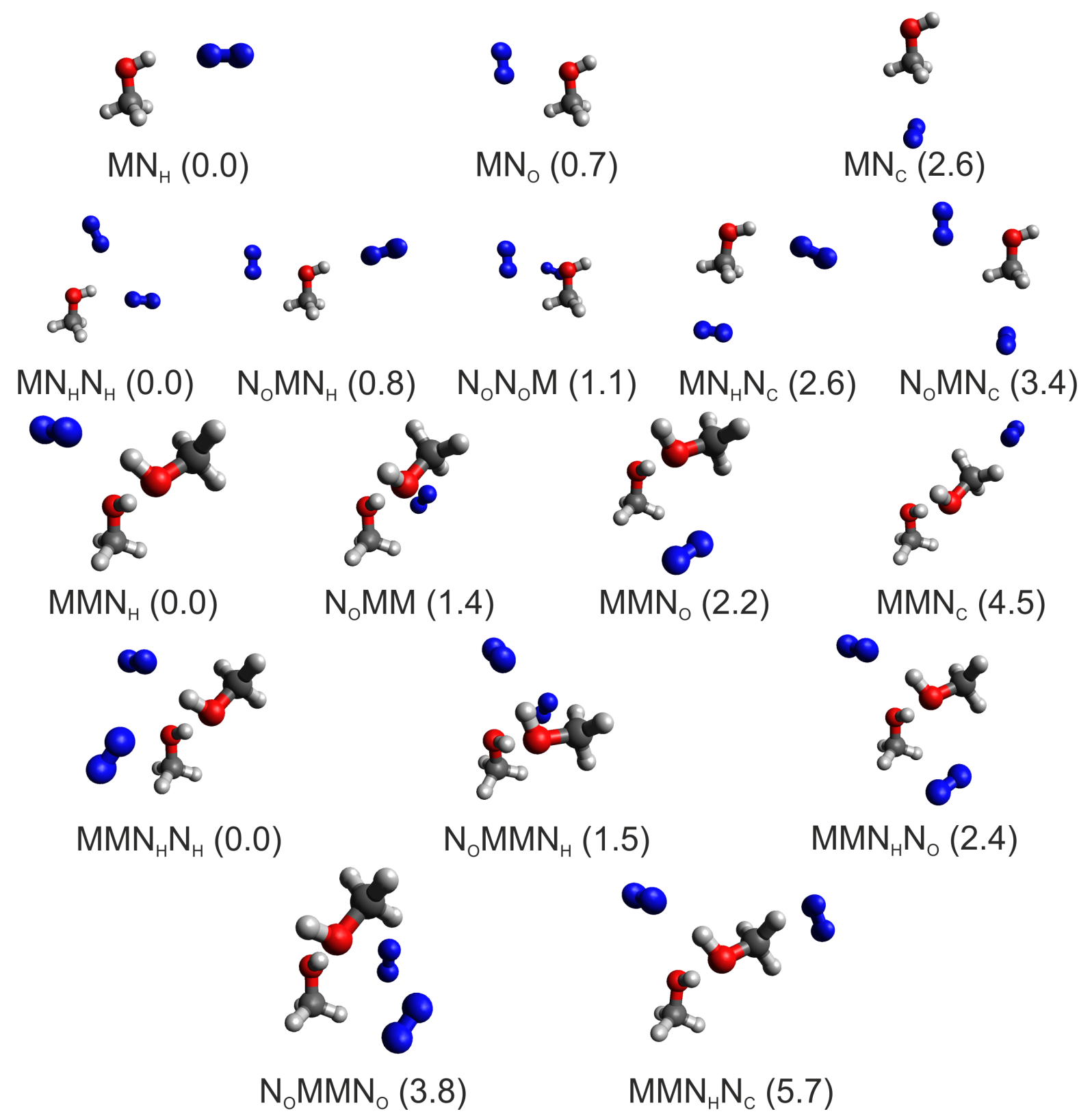

Figure A.17.: Stable structures of mixed MN aggregates optimised at the B3LYPD3(BJ)/aVTZ level (reprinted from reference 287 licensed under CC BY 4.0). Relative energies in $\mathrm{kJ} \mathrm{mol}^{-1}$ for each composition obtained from $\operatorname{CCSD}(\mathrm{T}) / \mathrm{aVTZ}$ single-point energies with added harmonic zero-point vibrational energy from B3LYP-D3(BJ)/aVTZ calculations are given in parentheses. 
A. Appendix
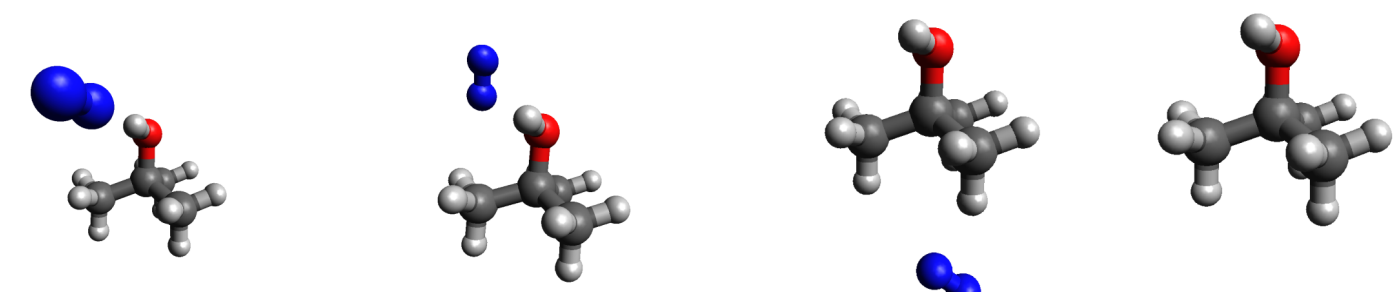

$\mathrm{BN}_{\mathrm{H}}(0.0)$

$\mathrm{BN}_{\mathrm{O}}(0.5)$

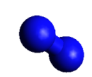

$\mathrm{BN}_{\mathrm{C} 3}(1.7)$

$\mathrm{BN}_{\mathrm{C}}(2.2)$
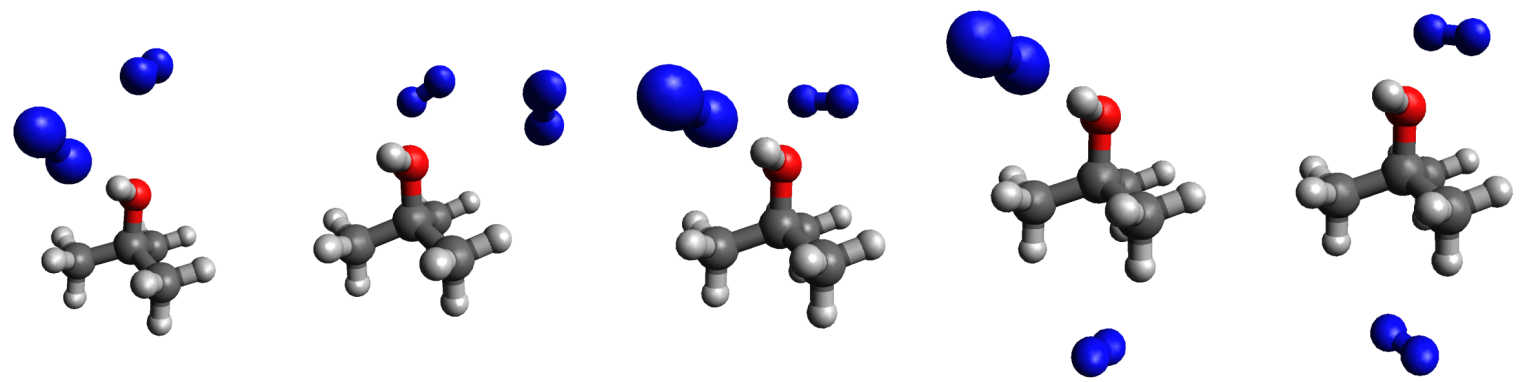

$\mathrm{BN}_{\mathrm{H}} \mathrm{N}_{\mathrm{H}}(0.0)$

$\mathrm{N}_{\mathrm{O}} \mathrm{N}_{\mathrm{O}} \mathrm{B}(0.4)$

$\mathrm{N}_{\circ} \mathrm{BN}_{\mathrm{H}}(0.5)$

$\mathrm{BN}_{\mathrm{H}} \mathrm{N}_{\mathrm{C} 3}(1.6)$

$\mathrm{N}_{\circ} \mathrm{BN}_{\mathrm{C} 3}(2.4)$

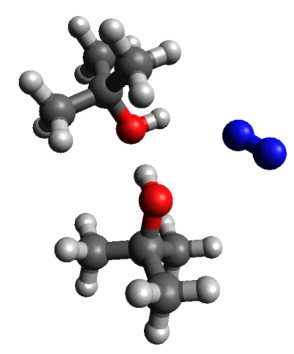

$\mathrm{BBN}_{H}(0.0)$

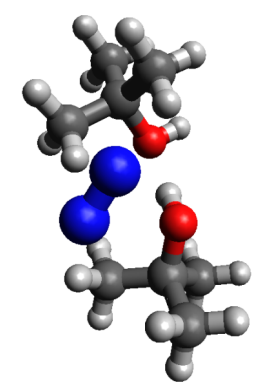

$\mathrm{N}_{0} \mathrm{BB}(1.9)$

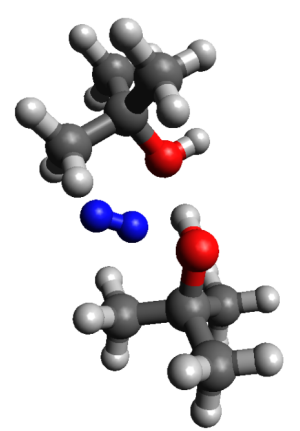

$\mathrm{BBN}_{\circ}(2.6)$
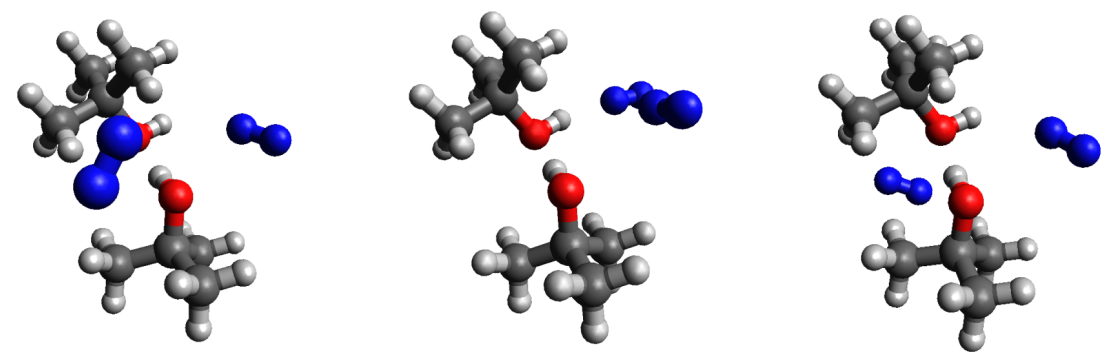

$\mathrm{N}_{\mathrm{O}} \mathrm{BBN}_{\mathrm{H}}(0.0)$

$\operatorname{BBN}_{H} N_{H}(0.5)$

$\mathrm{BBN}_{\mathrm{H}} \mathrm{N}_{\mathrm{O}}(0.5)$

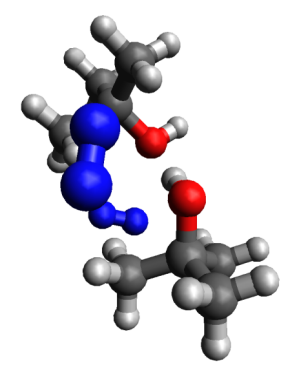

$\mathrm{N}_{\circ} \mathrm{BBN}_{\circ}(2.6)$

Figure A.18.: Stable structures of mixed BN aggregates optimised at the B3LYPD3(BJ)/aVTZ level (reprinted from reference 287 licensed under CC BY 4.0). Relative energies in $\mathrm{kJ} \mathrm{mol}^{-1}$ for each composition including harmonic zero-point vibrational energy are given in parentheses. 


\section{A. Appendix}

Table A.21.: Theoretically predicted harmonic $\left(\omega_{\mathrm{OH}}\right) \mathrm{OH}$ stretching band centre positions and integrated infrared band strengths $S_{\omega}$ of $\mathrm{M}$ and MN aggregates as well as spectral harmonic downshifts relative to the corresponding vibration in the pure methanol fragments $\left(\Delta \omega_{\mathrm{OH}}\right)$ obtained at the B3LYP-D3(BJ)/aVTZ level. Roman M symbols denote the monomer unit involved in the vibration, italic symbols the passive monomer.

\begin{tabular}{|c|c|c|c|}
\hline Structure & $\omega_{\mathrm{OH}} / \mathrm{cm}^{-1}$ & $S_{\omega} / \mathrm{km} \mathrm{mol}^{-1}$ & $\Delta \omega_{\mathrm{OH}} / \mathrm{cm}^{-1}$ \\
\hline M & 3829 & 31 & - \\
\hline $\mathrm{MN}_{\mathrm{H}}$ & 3831 & 103 & -2 \\
\hline $\mathrm{MN}_{\mathrm{O}}$ & 3830 & 31 & -1 \\
\hline $\mathrm{MN}_{\mathrm{C}}$ & 3829 & 33 & 0 \\
\hline $\mathrm{MN}_{\mathrm{H}} \mathrm{N}_{\mathrm{H}}$ & 3829 & 98 & 0 \\
\hline $\mathrm{N}_{\mathrm{O}} \mathrm{MN}_{\mathrm{H}}$ & 3831 & 137 & -2 \\
\hline $\mathrm{N}_{\mathrm{O}} \mathrm{N}_{\mathrm{O}} \mathrm{M}$ & 3830 & 32 & -1 \\
\hline $\mathrm{MN}_{\mathrm{H}} \mathrm{N}_{\mathrm{C}}$ & 3830 & 84 & -1 \\
\hline $\mathrm{N}_{\mathrm{O}} \mathrm{MN}_{\mathrm{C}}$ & 3830 & 33 & -1 \\
\hline$M \mathrm{M}$ & 3825 & 42 & - \\
\hline$M \mathrm{MN}_{\mathrm{H}}$ & 3807 & 129 & 18 \\
\hline $\mathrm{N}_{\mathrm{O}} M \mathrm{M}$ & 3827 & 42 & -2 \\
\hline$M \mathrm{MN}_{\mathrm{O}}$ & 3823 & 44 & 2 \\
\hline$M \mathrm{MN}_{\mathrm{C}}$ & 3826 & 44 & -1 \\
\hline$M \mathrm{MN}_{\mathrm{H}} \mathrm{N}_{\mathrm{H}}$ & 3810 & 78 & 15 \\
\hline $\mathrm{N}_{\mathrm{O}} M \mathrm{MN}_{\mathrm{H}}$ & 3809 & 84 & 16 \\
\hline$M \mathrm{MN}_{\mathrm{H}} \mathrm{N}_{\mathrm{O}}$ & 3806 & 130 & 19 \\
\hline $\mathrm{N}_{\mathrm{O}} M \mathrm{MN}_{\mathrm{O}}$ & 3824 & 43 & 1 \\
\hline$M \mathrm{MN}_{\mathrm{H}} \mathrm{N}_{\mathrm{C}}$ & 3807 & 115 & 18 \\
\hline $\mathrm{M} M$ & 3653 & 551 & - \\
\hline $\mathrm{M} M \mathrm{~N}_{\mathrm{H}}$ & 3629 & 552 & 24 \\
\hline $\mathrm{N}_{\mathrm{O}} \mathrm{M} M$ & 3645 & 551 & 8 \\
\hline $\mathrm{M} M \mathrm{~N}_{\mathrm{O}}$ & 3650 & 521 & 3 \\
\hline $\mathrm{M} M \mathrm{~N}_{\mathrm{C}}$ & 3649 & 560 & 4 \\
\hline $\mathrm{M} M \mathrm{~N}_{\mathrm{H}} \mathrm{N}_{\mathrm{H}}$ & 3618 & 534 & 35 \\
\hline $\mathrm{N}_{\mathrm{O}} \mathrm{M} M \mathrm{~N}_{\mathrm{H}}$ & 3622 & 519 & 31 \\
\hline $\mathrm{M} M \mathrm{~N}_{\mathrm{H}} \mathrm{N}_{\mathrm{O}}$ & 3629 & 509 & 24 \\
\hline $\mathrm{N}_{\mathrm{O}} \mathrm{M} M \mathrm{~N}_{\mathrm{O}}$ & 3642 & 523 & 11 \\
\hline $\mathrm{M} M \mathrm{~N}_{\mathrm{H}} \mathrm{N}_{\mathrm{C}}$ & 3630 & 538 & 23 \\
\hline
\end{tabular}




\section{A. Appendix}

Table A.22.: Theoretically predicted harmonic $\left(\omega_{\mathrm{OH}}\right) \mathrm{OH}$ stretching band centre positions and integrated infrared band strengths $S_{\omega}$ of B and BN aggregates as well as spectral harmonic downshifts relative to the corresponding vibration in the pure $\mathrm{B}$ fragments $\left(\Delta \omega_{\mathrm{OH}}\right)$ obtained at the B3LYP-D3(BJ)/aVTZ level. Roman B symbols denote the monomer unit involved in the vibration, italic symbols the passive monomer.

\begin{tabular}{|c|c|c|c|}
\hline Structure & $\omega_{\mathrm{OH}} / \mathrm{cm}^{-1}$ & $S_{\omega} / \mathrm{km} \mathrm{mol}^{-1}$ & $\Delta \omega_{\mathrm{OH}} / \mathrm{cm}^{-1}$ \\
\hline $\mathrm{B}$ & 3801 & 14 & - \\
\hline $\mathrm{BN}_{\mathrm{H}}$ & 3810 & 87 & -9 \\
\hline $\mathrm{BN}_{\mathrm{O}}$ & 3801 & 14 & 0 \\
\hline $\mathrm{BN}_{\mathrm{C} 3}$ & 3801 & 15 & 0 \\
\hline $\mathrm{BN}_{\mathrm{C}}$ & 3801 & 14 & 0 \\
\hline $\mathrm{BN}_{\mathrm{H}} \mathrm{N}_{\mathrm{H}}$ & 3805 & 94 & -4 \\
\hline $\mathrm{N}_{\mathrm{O}} \mathrm{N}_{\mathrm{O}} \mathrm{B}$ & 3801 & 15 & 0 \\
\hline $\mathrm{N}_{\mathrm{O}} \mathrm{BN}_{\mathrm{H}}$ & 3810 & 92 & -9 \\
\hline $\mathrm{BN}_{\mathrm{H}} \mathrm{N}_{\mathrm{C} 3}$ & 3809 & 89 & -8 \\
\hline $\mathrm{N}_{\mathrm{O}} \mathrm{BN}_{\mathrm{C} 3}$ & 3801 & 15 & 0 \\
\hline$B \mathrm{~B}$ & 3795 & 19 & - \\
\hline$B \mathrm{BN}_{\mathrm{H}}$ & 3792 & 57 & 3 \\
\hline $\mathrm{N}_{\mathrm{O}} B \mathrm{~B}$ & 3795 & 20 & 0 \\
\hline$B \mathrm{BN}_{\mathrm{O}}$ & 3795 & 20 & 0 \\
\hline $\mathrm{N}_{\mathrm{O}} B \mathrm{BN}_{\mathrm{H}}$ & 3792 & 55 & 3 \\
\hline$B \mathrm{BN}_{\mathrm{H}} \mathrm{N}_{\mathrm{H}}$ & 3792 & 128 & 3 \\
\hline$B \mathrm{BN}_{\mathrm{H}} \mathrm{N}_{\mathrm{O}}$ & 3791 & 63 & 4 \\
\hline $\mathrm{N}_{\mathrm{O}} B B \mathrm{~N}_{\mathrm{O}}$ & 3795 & 20 & 0 \\
\hline $\mathrm{B} B$ & 3614 & 525 & - \\
\hline $\mathrm{B} B \mathrm{~N}_{\mathrm{H}}$ & 3593 & 543 & 21 \\
\hline $\mathrm{N}_{\mathrm{O}} \mathrm{B} B$ & 3611 & 513 & 3 \\
\hline $\mathrm{B} B \mathrm{~N}_{\mathrm{O}}$ & 3617 & 521 & -3 \\
\hline $\mathrm{N}_{\mathrm{O}} \mathrm{B} B \mathrm{~N}_{\mathrm{H}}$ & 3590 & 530 & 24 \\
\hline $\mathrm{B} B \mathrm{~N}_{\mathrm{H}} \mathrm{N}_{\mathrm{H}}$ & 3586 & 571 & 28 \\
\hline $\mathrm{B} B \mathrm{~N}_{\mathrm{H}} \mathrm{N}_{\mathrm{O}}$ & 3596 & 533 & 18 \\
\hline $\mathrm{N}_{\mathrm{O}} B B N_{\mathrm{O}}$ & 3614 & 512 & 0 \\
\hline
\end{tabular}




\section{A. Appendix}

Table A.23.: Theoretically predicted harmonic $\left(\omega_{\mathrm{OH}}\right) \mathrm{OH}$ stretching wavenumber and lowest predicted wavenumber $\left(\omega_{l}\right)$ in $\mathrm{cm}^{-1}$, IR intensity $\left(S_{\omega}\right)$ in $\mathrm{km} \mathrm{mol}^{-1}$, spectroscopic downshift $\left(\Delta \omega_{\mathrm{OH}}\right)$ relative to the corresponding vibration in the respective monomer (for homodimers) or homodimer (for heteroaggregates) in $\mathrm{cm}^{-1}$, relative electronic and harmonic vibrational zero-point corrected energies $\left(\Delta E_{\mathrm{e}}\right.$ and $\left.\Delta E_{0}\right)$ compared to the most stable cluster conformation, dissociation energies into the most stable monomers $\left(D_{\mathrm{e}}\right.$ and $\left.D_{0}\right)$ as well as energies required for isolated $\mathrm{N}$ abstraction from mixed $\mathrm{N}$ clusters excluding and including zero-point corrections $\left(N_{\mathrm{e}}\right.$ and $\left.N_{0}\right)$ in $\mathrm{kJ} \mathrm{mol}^{-1}$ calculated at the B3LYP$\mathrm{D} 3(\mathrm{BJ}) / \mathrm{aVTZ}$ level for various $\mathrm{E}$ and EN aggregates.

\begin{tabular}{|c|c|c|c|c|c|c|c|c|c|c|}
\hline Structure & $\omega_{\mathrm{OH}}$ & $S_{\omega}$ & $\Delta \omega_{\mathrm{OH}}$ & $\Delta E_{\mathrm{e}}$ & $\Delta E_{0}$ & $D_{\mathrm{e}}$ & $D_{0}$ & $N_{\mathrm{e}}$ & $N_{0}$ & $\omega_{1}$ \\
\hline $\mathrm{E}_{t}$ & 3828 & 29 & - & 0.0 & 0.0 & - & - & - & - & 231 \\
\hline $\mathrm{E}_{g}$ & 3811 & 22 & - & 0.2 & 0.3 & - & - & - & - & 257 \\
\hline $\mathrm{E}_{t} \mathrm{~N}_{\mathrm{H}}$ & 3830 & 112 & -2 & 0.1 & 0.0 & 5.9 & 3.7 & 5.9 & 3.7 & 10 \\
\hline $\mathrm{E}_{t} \mathrm{~N}_{\mathrm{O}}$ & 3826 & 29 & 2 & 1.1 & 0.5 & 4.8 & 3.2 & 4.8 & 3.2 & 24 \\
\hline $\mathrm{E}_{t} \mathrm{~N}_{\mathrm{C}}$ & 3827 & 29 & 1 & 3.6 & 2.1 & 2.3 & 1.6 & 2.3 & 1.6 & 2 \\
\hline $\mathrm{E}_{g} \mathrm{~N}_{\mathrm{H}}$ & 3818 & 62 & -7 & 0.0 & 0.2 & 6.0 & 3.5 & 6.0 & 3.9 & 29 \\
\hline $\mathrm{E}_{g} \mathrm{~N}_{\mathrm{O}}$ & 3812 & 23 & -1 & 1.4 & 0.5 & 4.6 & 3.2 & 4.6 & 3.2 & 24 \\
\hline \multirow{2}{*}{$\mathrm{E}_{g} \mathrm{E}_{g}$} & 3807 & 30 & 4 & \multirow[t]{2}{*}{0.0} & 0.0 & \multirow{2}{*}{28.2} & 22.6 & \multirow[t]{2}{*}{-} & - & \multirow[t]{2}{*}{5} \\
\hline & $\begin{array}{l}3635 \\
3822\end{array}$ & $\begin{array}{c}449 \\
39\end{array}$ & $\begin{array}{c}176 \\
6\end{array}$ & & \multirow[b]{2}{*}{0.5} & & \multirow[b]{2}{*}{22.1} & & \multirow[b]{2}{*}{ - } & \\
\hline $\mathrm{E}_{g} \mathrm{E}_{t}$ & 3638 & 471 & 173 & 0.4 & & 27.8 & & - & & 12 \\
\hline $\mathrm{E}_{g} \mathrm{E}_{t}$ & $\begin{array}{l}3824 \\
3645\end{array}$ & $\begin{array}{c}39 \\
445\end{array}$ & $\begin{array}{c}4 \\
166\end{array}$ & 0.3 & 0.7 & 27.9 & 21.9 & - & - & 24 \\
\hline \multirow{2}{*}{$\mathrm{E}_{t} \mathrm{E}_{t}$} & 3823 & 39 & 5 & \multirow{2}{*}{0.7} & \multirow{2}{*}{0.8} & & & & & \\
\hline & 3645 & 554 & 183 & & & 27.5 & 21.8 & - & - & 31 \\
\hline $\mathrm{E}_{q} \mathrm{E}_{g^{\prime}}$ & 3806 & 30 & 5 & 0.9 & 0.9 & 27.3 & 21.7 & - & - & 8 \\
\hline $\mathrm{L}_{g} \mathrm{\Sigma}_{g^{\prime}}$ & 3630 & 497 & 181 & 0.9 & 0.9 & 21.0 & 21.1 & 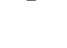 & & \\
\hline $\mathrm{E}_{t} \mathrm{E}_{g}$ & $\begin{array}{l}3806 \\
3637\end{array}$ & $\begin{array}{c}30 \\
574\end{array}$ & $\begin{array}{c}5 \\
191\end{array}$ & 1.0 & 0.9 & 27.2 & 21.7 & - & - & 11 \\
\hline & 3807 & 31 & 4 & 10 & & & & & & \\
\hline $\mathrm{E}_{g} \mathrm{E}_{g^{\prime}}$ & 3647 & 374 & 164 & 1.0 & 1.2 & 27.2 & 21.4 & - & - & 17 \\
\hline $\mathrm{E}_{g} \mathrm{E}_{g^{\prime}}$ & $\begin{array}{l}3811 \\
3648\end{array}$ & $\begin{array}{c}32 \\
495\end{array}$ & $\begin{array}{c}0 \\
163\end{array}$ & 2.0 & 1.7 & 26.2 & 20.9 & - & - & 9 \\
\hline & $\begin{array}{l}5040 \\
3822\end{array}$ & $\begin{array}{l}490 \\
36\end{array}$ & 6 & & & & & & & \\
\hline $\mathrm{E}_{g} \mathrm{E}_{t}$ & 3650 & 369 & 161 & 1.9 & 1.9 & 26.3 & 20.7 & - & - & 25 \\
\hline $\mathrm{E}_{t} \mathrm{E}_{g}$ & $\begin{array}{l}3813 \\
3662\end{array}$ & $\begin{array}{c}31 \\
485\end{array}$ & $\begin{array}{l}-2 \\
166\end{array}$ & 2.9 & 2.1 & 25.3 & 20.5 & - & - & 18 \\
\hline & $\begin{array}{l}3662 \\
3808\end{array}$ & $\begin{array}{l}485 \\
31\end{array}$ & $\begin{array}{c}166 \\
3\end{array}$ & & & & & & & \\
\hline $\mathrm{E}_{t} \mathrm{E}_{g}$ & 3645 & 665 & 183 & 3.1 & 2.1 & 25.1 & 20.5 & - & - & 5 \\
\hline $\mathrm{E}_{q} \mathrm{E}_{q^{\prime}} \mathrm{N}_{\mathrm{H}}$ & 3798 & 78 & 8 & 0.0 & 0.0 & 36.7 & 28.4 & 9.4 & 6.7 & 17 \\
\hline $\mathrm{E}_{g} \mathrm{~L}_{g^{\prime}} \mathrm{N}_{\mathrm{H}}$ & 3605 & 519 & 25 & 0.0 & & & & & & \\
\hline $\mathrm{E}_{g} \mathrm{E}_{t} \mathrm{~N}_{\mathrm{H}}$ & $\begin{array}{l}3816 \\
3613\end{array}$ & $\begin{array}{c}80 \\
499\end{array}$ & $\begin{array}{c}6 \\
25\end{array}$ & 0.4 & 0.0 & 36.3 & 28.4 & 8.6 & 6.4 & 8 \\
\hline & 3817 & 90 & 7 & 01 & & & 283 & & & 18 \\
\hline $\mathrm{E}_{g} \mathrm{E}_{t} \mathrm{~N}_{\mathrm{H}}$ & 3615 & 507 & 30 & 0.1 & 0.1 & 36.6 & 28.3 & 8.7 & 0.5 & 18 \\
\hline $\mathrm{E}_{g} \mathrm{E}_{g} \mathrm{~N}_{\mathrm{H}}$ & $\begin{array}{l}3799 \\
3604\end{array}$ & $\begin{array}{c}69 \\
512\end{array}$ & $\begin{array}{c}8 \\
31\end{array}$ & 0.5 & 0.1 & 36.2 & 28.3 & 8.0 & 5.8 & 3 \\
\hline & $\begin{array}{l}5004 \\
3814\end{array}$ & $\begin{array}{l}712 \\
72\end{array}$ & $\begin{array}{c}01 \\
9\end{array}$ & & & & & & & \\
\hline $\mathrm{E}_{t} \mathrm{E}_{t} \mathrm{~N}_{\mathrm{H}}$ & 3616 & 577 & 29 & 0.9 & 0.5 & 35.8 & 27.9 & 8.4 & 6.2 & 20 \\
\hline
\end{tabular}




\section{A. Appendix}

\begin{tabular}{|c|c|c|c|c|c|c|c|c|c|c|}
\hline Structure & $\omega_{\mathrm{OH}}$ & $S_{\omega}$ & $\Delta \omega_{\mathrm{OH}}$ & $\Delta E_{\mathrm{e}}$ & $\Delta E_{0}$ & $D_{\mathrm{e}}$ & $D_{0}$ & $N_{\mathrm{e}}$ & $N_{0}$ & $\omega_{1}$ \\
\hline \multirow{2}{*}{$\mathrm{E}_{t} \mathrm{E}_{g} \mathrm{~N}_{\mathrm{H}}$} & 3799 & 67 & 7 & \multirow{2}{*}{0.6} & \multirow{2}{*}{0.6} & \multirow{2}{*}{36.1} & \multirow{2}{*}{27.8} & \multirow{2}{*}{8.9} & \multirow{2}{*}{6.2} & \multirow{2}{*}{21} \\
\hline & 3616 & 586 & 21 & & & & & & & \\
\hline \multirow{2}{*}{$\mathrm{N}_{\mathrm{O}} \mathrm{E}_{t} \mathrm{E}_{g}$} & 3807 & 31 & -1 & \multirow{2}{*}{1.4} & \multirow{2}{*}{0.8} & \multirow{2}{*}{35.3} & \multirow{2}{*}{27.6} & \multirow{2}{*}{8.2} & \multirow{2}{*}{6.0} & \multirow{2}{*}{13} \\
\hline & 3629 & 571 & 8 & & & & & & & \\
\hline \multirow{2}{*}{$\mathrm{N}_{\mathrm{O}} \mathrm{E}_{g} \mathrm{E}_{g}$} & 3807 & 29 & 0 & \multirow{2}{*}{1.5} & \multirow{2}{*}{0.9} & \multirow{2}{*}{35.2} & \multirow{2}{*}{27.5} & 7.0 & 5.0 & 19 \\
\hline & $\begin{array}{l}3632 \\
3814\end{array}$ & 430 & $\begin{array}{l}3 \\
8\end{array}$ & & & & & & & \\
\hline $\mathrm{E}_{g} \mathrm{E}_{t} \mathrm{~N}_{\mathrm{H}}$ & $\begin{array}{l}3814 \\
3628\end{array}$ & $\begin{array}{l}111 \\
422\end{array}$ & $\begin{array}{c}8 \\
22\end{array}$ & 1.3 & 1.1 & 35.4 & 27.3 & 9.1 & 6.6 & 8 \\
\hline $\mathrm{N}_{\mathrm{O}} \mathrm{E}_{a} \mathrm{E}_{a^{\prime}}$ & 3807 & 30 & -1 & 10 & 11 & 348 & 273 & 75 & 57 & 10 \\
\hline $\mathrm{N}^{-} \mathrm{O}^{\mathrm{L}} \mathrm{g}^{\prime}$ & 3623 & 487 & 7 & 1.9 & 1.1 & 84.0 & 21.0 & 1.0 & 0.1 & 10 \\
\hline $\mathrm{E}_{g} \mathrm{E}_{g^{\prime}} \mathrm{N}_{\mathrm{H}}$ & 3795 & 62 & 16 & 1.6 & 1.2 & 35.1 & 27.2 & 8.9 & 6.4 & 4 \\
\hline & 3615 & 512 & 33 & & & & & & & \\
\hline $\mathrm{E}_{t} \mathrm{E}_{g} \mathrm{~N}_{\mathrm{H}}$ & $\begin{array}{l}3800 \\
3640\end{array}$ & 83 & 13 & 2.1 & 1.5 & 34.6 & 26.9 & 9.4 & 6.5 & 10 \\
\hline & 3795 & 93 & 13 & & & & & & & \\
\hline $\mathrm{E}_{t} \mathrm{E}_{g} \mathrm{~N}_{\mathrm{H}}$ & 3626 & 661 & 19 & 2.3 & 1.6 & 34.4 & 26.8 & 9.3 & 6.5 & 8 \\
\hline $\mathrm{N}_{O} \mathrm{E}_{o} \mathrm{E}_{+}$ & 3823 & 40 & 1 & 2.8 & 1.6 & 33.9 & 26.8 & 6.0 & 4.9 & 9 \\
\hline & 3636 & 464 & 9 & 2.8 & & 5.9 & 20.0 & 0.0 & & \\
\hline $\mathrm{E}_{g} \mathrm{E}_{g} \mathrm{~N}_{\mathrm{O}}$ & 3806 & 33 & 1 & 2.3 & 1.8 & 34.4 & 26.6 & 6.2 & 4.2 & 25 \\
\hline & 3633 & 449 & 2 & & & & & & & \\
\hline $\mathrm{E}_{g} \mathrm{E}_{g^{\prime}} \mathrm{N}_{\mathrm{O}}$ & $\begin{array}{l}3807 \\
3631\end{array}$ & $\begin{array}{c}31 \\
461\end{array}$ & $\begin{array}{c}0 \\
16\end{array}$ & 2.4 & 1.8 & 34.3 & 26.6 & 7.1 & 5.4 & 17 \\
\hline $\mathrm{N}_{0} \mathrm{E}_{a} \mathrm{E}_{t}$ & 3822 & 38 & 0 & 2.5 & 1.8 & 34.2 & 26.6 & 6.4 & 4.6 & 19 \\
\hline & 3634 & 471 & 4 & & & & & & & \\
\hline $\mathrm{E}_{g} \mathrm{E}_{t} \mathrm{~N}_{\mathrm{O}}$ & 3823 & 38 & 2 & 2.5 & 1.8 & 34.2 & 26.6 & 6.3 & 4.7 & 20 \\
\hline & 3813 & $\begin{array}{c}459 \\
30\end{array}$ & 0 & & & & & & & \\
\hline $\mathrm{N}_{\mathrm{O}} \mathrm{E}_{t} \mathrm{E}_{g}$ & 3648 & 513 & 14 & 3.2 & 2.0 & 33.5 & 26.4 & 8.2 & 6.0 & 13 \\
\hline $\mathrm{E}_{a} \mathrm{E}_{q^{\prime}} \mathrm{N}_{\mathrm{O}}$ & 3809 & 34 & 2 & 36 & 2.6 & 33.1 & 25.8 & 6.9 & 5.0 & 25 \\
\hline & 3643 & 525 & 5 & & & & & & & \\
\hline $\mathrm{N}_{\mathrm{O}} \mathrm{E}_{g} \mathrm{E}_{g^{\prime}}$ & $\begin{array}{l}3810 \\
3638\end{array}$ & $\begin{array}{c}32 \\
497\end{array}$ & $\begin{array}{c}1 \\
10\end{array}$ & 4.2 & 2.7 & 32.5 & 25.7 & 6.3 & 4.8 & 11 \\
\hline $\mathrm{F}, \mathrm{F}_{\mathrm{N}} \mathrm{N}$ & 3819 & 43 & 3 & 38 & 29 & 329 & 25.5 & 6.5 & 49 & 18 \\
\hline $\mathrm{L}_{g^{1}}$ & 3641 & 429 & 9 & & & & & & & \\
\hline $\mathrm{N}_{\mathrm{O}} \mathrm{E}_{g} \mathrm{E}_{t}$ & 3822 & 36 & 0 & 4.4 & 3.2 & 32.3 & 25.2 & 6.0 & 4.6 & 7 \\
\hline & 3642 & 392 & 8 & & & & & & & \\
\hline $\mathrm{E}_{t} \mathrm{E}_{t} \mathrm{~N}_{\mathrm{H}} \mathrm{N}_{\mathrm{H}}$ & 3812 & 62 & 9 & 01 & 00 & 443 & 34.9 & 84 & 6.9 & 8 \\
\hline & 3602 & 588 & 43 & & & & & & & \\
\hline $\mathrm{N}_{\mathrm{O}} \mathrm{E}_{g} \mathrm{E}_{g} \mathrm{~N}_{\mathrm{H}}$ & 3797 & 60 & 10 & 0.4 & 0.8 & 43.9 & 34.1 & 7.7 & 5.7 & 13 \\
\hline & $\begin{array}{l}3606 \\
3813\end{array}$ & $\begin{array}{c}458 \\
82\end{array}$ & $\begin{array}{c}29 \\
9\end{array}$ & & & & & & & \\
\hline $\mathrm{E}_{g} \mathrm{E}_{t} \mathrm{~N}_{\mathrm{H}} \mathrm{N}_{\mathrm{O}}$ & 3615 & 478 & 23 & 0.6 & 0.9 & 43.7 & 34.0 & 7.5 & 5.6 & 9 \\
\hline $\mathrm{N}_{O} \mathrm{E}_{t} \mathrm{E}_{q} \mathrm{~N}_{\mathrm{H}}$ & 3796 & 78 & 10 & 10 & 1.1 & 43.3 & 33.8 & 7.2 & 5.9 & 6 \\
\hline & 3612 & 576 & 25 & & 1.1 & & & & & \\
\hline $\mathrm{E}_{t} \mathrm{E}_{g} \mathrm{~N}_{\mathrm{H}} \mathrm{N}_{\mathrm{H}}$ & 3790 & 66 & 18 & 1.0 & 1.1 & 43.3 & 33.8 & 9.0 & 6.8 & 13 \\
\hline & $\begin{array}{l}3613 \\
3798\end{array}$ & $\begin{array}{c}635 \\
75\end{array}$ & $\begin{array}{c}32 \\
8\end{array}$ & & & & & & & \\
\hline $\mathrm{N}_{\mathrm{O}} \mathrm{E}_{g} \mathrm{E}_{g^{\prime}} \mathrm{N}_{\mathrm{H}}$ & 3601 & 526 & 29 & 0.7 & 1.2 & 43.6 & 33.7 & 6.9 & 5.3 & 8 \\
\hline $\mathrm{E}_{g} \mathrm{E}_{g} \mathrm{~N}_{\mathrm{H}} \mathrm{N}_{\mathrm{O}}$ & 3797 & 81 & 10 & 0.5 & 1.3 & 43.8 & 33.6 & 7.6 & 5.3 & 8 \\
\hline & $\begin{array}{l}3611 \\
3814\end{array}$ & $\begin{array}{l}445 \\
109\end{array}$ & $\begin{array}{c}24 \\
8\end{array}$ & & & & & & & \\
\hline $\mathrm{E}_{g} \mathrm{E}_{t} \mathrm{~N}_{\mathrm{H}} \mathrm{N}_{\mathrm{H}}$ & & & & 1.0 & 1.7 & 43.3 & 33.2 & 7.9 & 5.9 & 16 \\
\hline
\end{tabular}




\section{A. Appendix}

\begin{tabular}{|c|c|c|c|c|c|c|c|c|c|c|}
\hline Structure & $\omega_{\mathrm{OH}}$ & $S_{\omega}$ & $\Delta \omega_{\mathrm{OH}}$ & $\Delta E_{\mathrm{e}}$ & $\Delta E_{0}$ & $D_{\mathrm{e}}$ & $D_{0}$ & $N_{\mathrm{e}}$ & $N_{0}$ & $\omega_{1}$ \\
\hline \multirow{3}{*}{$\mathrm{N}_{\mathrm{O}} \mathrm{E}_{g} \mathrm{E}_{g^{\prime}} \mathrm{N}_{\mathrm{H}}$} & 3619 & 410 & 31 & \multirow{3}{*}{1.7} & \multirow{3}{*}{1.8} & \multirow{3}{*}{42.6} & \multirow{3}{*}{33.1} & \multirow{3}{*}{7.5} & \multirow{3}{*}{5.8} & \multirow{3}{*}{0.110} \\
\hline & 3794 & 56 & 17 & & & & & & & \\
\hline & 3611 & 486 & 37 & & & & & & & \\
\hline $\mathrm{E}_{g} \mathrm{E}_{g^{\prime}} \mathrm{N}_{\mathrm{H}} \mathrm{N}_{\mathrm{O}}$ & $\begin{array}{l}3806 \\
3619\end{array}$ & 31 & 1 & 1.8 & 1.9 & 42.5 & 33.0 & 5.8 & 4.5 & 20 \\
\hline \multirow{2}{*}{$\mathrm{N}_{\mathrm{O}} \mathrm{E}_{g} \mathrm{E}_{t} \mathrm{~N}_{\mathrm{H}}$} & $\begin{array}{l}3619 \\
3810\end{array}$ & $\begin{array}{c}459 \\
80\end{array}$ & $\begin{array}{l}28 \\
12\end{array}$ & \multirow[b]{2}{*}{2.1} & \multirow{2}{*}{2.3} & \multirow{2}{*}{42.2} & \multirow{2}{*}{32.6} & \multirow{2}{*}{6.8} & \multirow[b]{2}{*}{5.2} & \multirow{2}{*}{6} \\
\hline & 3625 & 461 & 25 & & & & & & & \\
\hline \multirow{2}{*}{$\mathrm{E}_{t} \mathrm{E}_{g} \mathrm{~N}_{\mathrm{H}} \mathrm{N}_{\mathrm{O}}$} & 3802 & 84 & 11 & \multirow{2}{*}{2.1} & \multirow{2}{*}{2.3} & \multirow{2}{*}{42.2} & \multirow{2}{*}{32.6} & \multirow{2}{*}{7.5} & \multirow{2}{*}{5.6} & \multirow{2}{*}{12} \\
\hline & 3626 & 497 & 36 & & & & & & & \\
\hline \multirow{2}{*}{$\mathrm{N}_{\mathrm{O}} \mathrm{E}_{t} \mathrm{E}_{g} \mathrm{~N}_{\mathrm{H}}$} & 3798 & 86 & 15 & \multirow{2}{*}{3.6} & \multirow{2}{*}{3.4} & \multirow{2}{*}{40.7} & \multirow{2}{*}{31.5} & \multirow{2}{*}{6.0} & 4.6 & 6 \\
\hline & 3635 & 515 & 27 & & & & & & 4.0 & \\
\hline
\end{tabular}

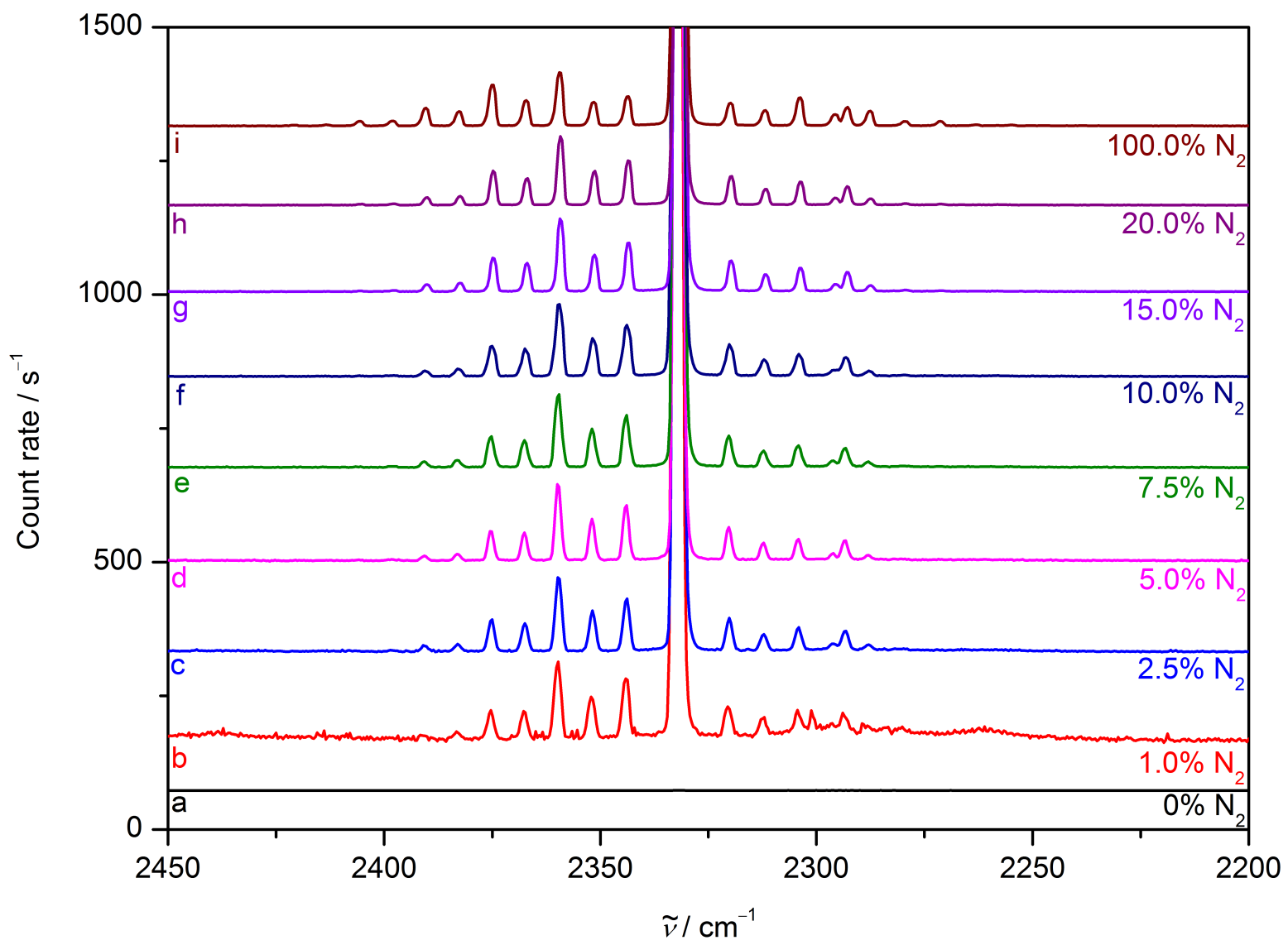

Figure A.19.: NN stretching Raman spectra of E expanded in helium (trace a), N (trace i), $1.0 \%, 2.5 \%, 5.0 \%, 7.5 \%, 10.0 \%, 15.0 \%$ and $20.0 \% \mathrm{~N}$ in helium (traces b to h). E was kept at a temperature of $273 \mathrm{~K}$, the carrier gas pressure in the gas line at 1.5 bar and the stagnation pressure in the reservoir at $0.75 \mathrm{bar}$, while the laser probed the expansion at a $2 \mathrm{~mm}$ nozzle distance. All spectra were scaled to matching height of the NN stretching Q-branch at $2330 \mathrm{~cm}^{-1}$. 


\section{A. Appendix}

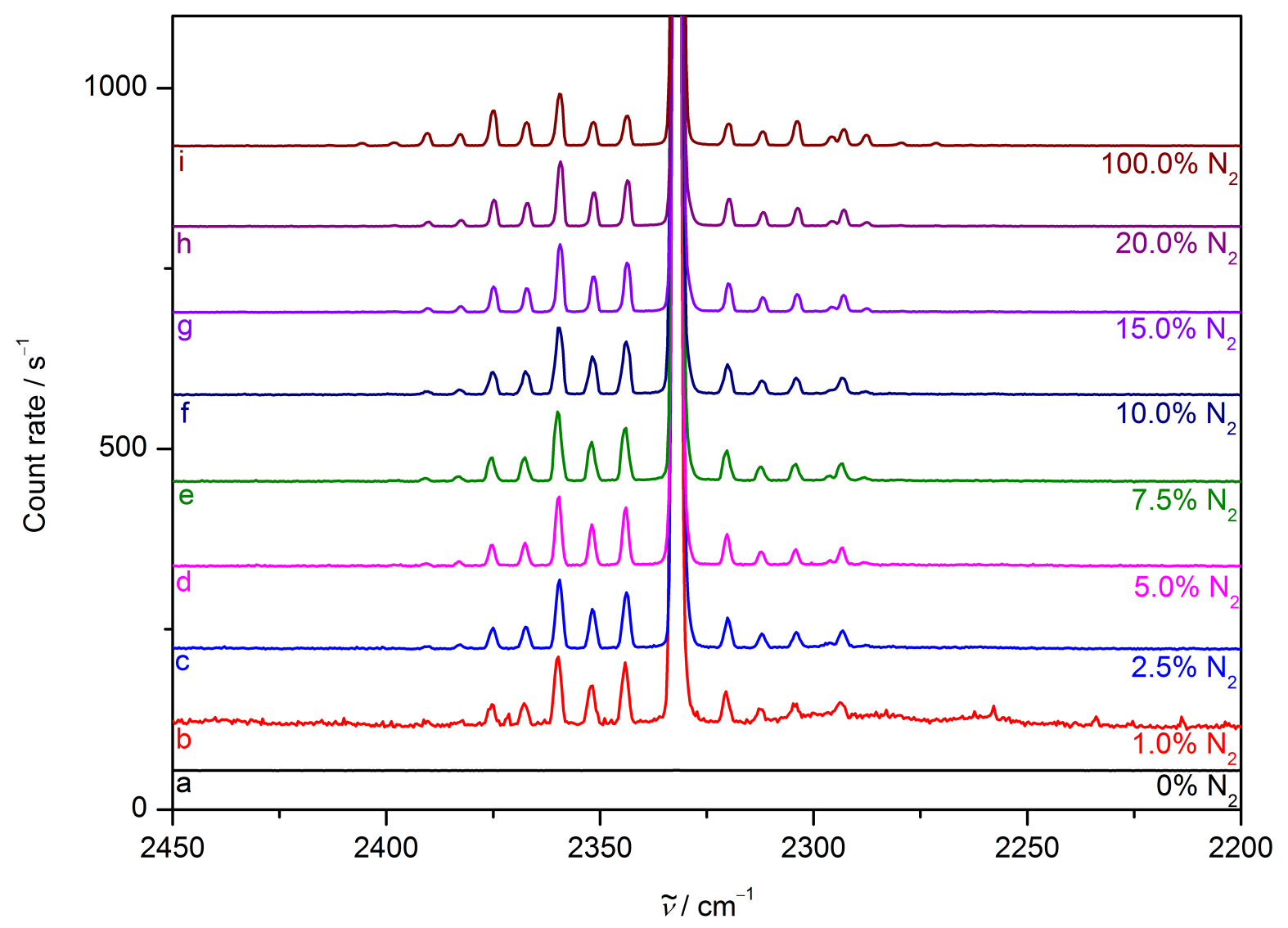

Figure A.20.: NN stretching Raman spectra of E expanded in helium (trace a), $\mathrm{N}$ (trace i), $1.0 \%, 2.5 \%, 5.0 \%, 7.5 \%, 10.0 \%, 15.0 \%$ and $20.0 \% \mathrm{~N}$ in helium (traces b to h). E was kept at a temperature of $273 \mathrm{~K}$, the carrier gas pressure in the gas line at 1.5 bar and the stagnation pressure in the reservoir at $0.75 \mathrm{bar}$, while the laser probed the expansion at a $3 \mathrm{~mm}$ nozzle distance. All spectra were scaled to matching height of the NN stretching Q-branch at $2330 \mathrm{~cm}^{-1}$. 


\section{A. Appendix}

Table A.24.: Rotational temperature $\left(T_{\text {rot }}\right)$ obtained from $S$-branch rovibrational lines of NN stretching Raman spectra of $\mathrm{E}$ and $\mathrm{N}$ mixtures. Temperatures were obtained for various $\mathrm{N}$ molar fractions $\left(x\left(\mathrm{~N}_{2}\right)\right)$ in the expansion and with the laser probing the expansion at different distances $d$ from the nozzle. Separate analysis was performed for even and odd J quantum numbers. Integrals of rovibrational lines were obtained with the OriginPro (Version 8.5) program package and linear baseline corrections were interpolated from the respective integral bounds of each spectral line. A plot of $\frac{\left(\mathrm{J}^{\prime \prime}+1\right)\left(\mathrm{J}^{\prime \prime}+2\right)}{\left(2 \mathrm{~J}^{\prime \prime}+1\right)\left(2 \mathrm{~J}^{\prime \prime}+3\right)} I$ against $\mathrm{J}^{\prime \prime}\left(\mathrm{J}^{\prime \prime}+1\right)$ yields a linear function with a slope of $-\frac{h c \tilde{B^{\prime \prime}}}{k_{\mathrm{B}} T_{\text {rot }}}$. The slope was obtained by a least squares linear regression in OriginPro (Version 8.5) and from its error the uncertainties of $T_{\text {rot,even }}$ and $T_{\text {rot,odd }}$ were calculated by Gaussian error propagation.

\begin{tabular}{ccccc}
\hline$d / \mathrm{mm}$ & $x\left(\mathrm{~N}_{2}\right) / \%$ & $T_{\text {rot,even }} / \mathrm{K}$ & $T_{\text {rot,odd }} / \mathrm{K}$ & $T_{\text {rot }} / \mathrm{K}$ \\
\hline & 1.0 & $44(15)$ & $77(23)$ & $57(17)$ \\
& 2.5 & $15(5)$ & $26(9)$ & $19(6)$ \\
1 & 5.0 & $8(3)$ & $13(5)$ & $10(3)$ \\
& 7.5 & $6(2)$ & $10(4)$ & $7(3)$ \\
& 10.0 & $5(2)$ & $8(3)$ & $6(2)$ \\
& 15.0 & $4(2)$ & $8(3)$ & $6(2)$ \\
& 20.0 & $3(2)$ & $6(3)$ & $4(2)$ \\
& 100.0 & $2(1)$ & $3(2)$ & $3(1)$ \\
\hline \multirow{4}{*}{2} & 1.0 & $34(12)$ & $114(42)$ & $51(34)$ \\
& 2.5 & $13(5)$ & $20(8)$ & $16(4)$ \\
& 5.0 & $7(3)$ & $11(5)$ & $8(3)$ \\
& 7.5 & $4(2)$ & $7(3)$ & $5(2)$ \\
& 10.0 & $4(2)$ & $6(3)$ & $5(2)$ \\
& 15.0 & $4(2)$ & $7(4)$ & $5(2)$ \\
& 20.0 & $3(2)$ & $6(3)$ & $4(2)$ \\
& 100.0 & $2(1)$ & $4(2)$ & $3(1)$ \\
\hline \multirow{6}{*}{3} & 1.0 & $50(19)$ & $165(76)$ & $73(46)$ \\
& 2.5 & $16(7)$ & $28(13)$ & $20(6)$ \\
& 5.0 & $9(4)$ & $15(7)$ & $11(4)$ \\
& 7.5 & $6(3)$ & $10(5)$ & $8(2)$ \\
& 10.0 & $5(3)$ & $8(4)$ & $6(2)$ \\
& 15.0 & $3(2)$ & $5(3)$ & $4(2)$ \\
& 20.0 & $2(1)$ & $8(4)$ & $4(3)$ \\
& 100.0 & $2(1)$ & $6(3)$ & $3(2)$ \\
\hline
\end{tabular}


A. Appendix

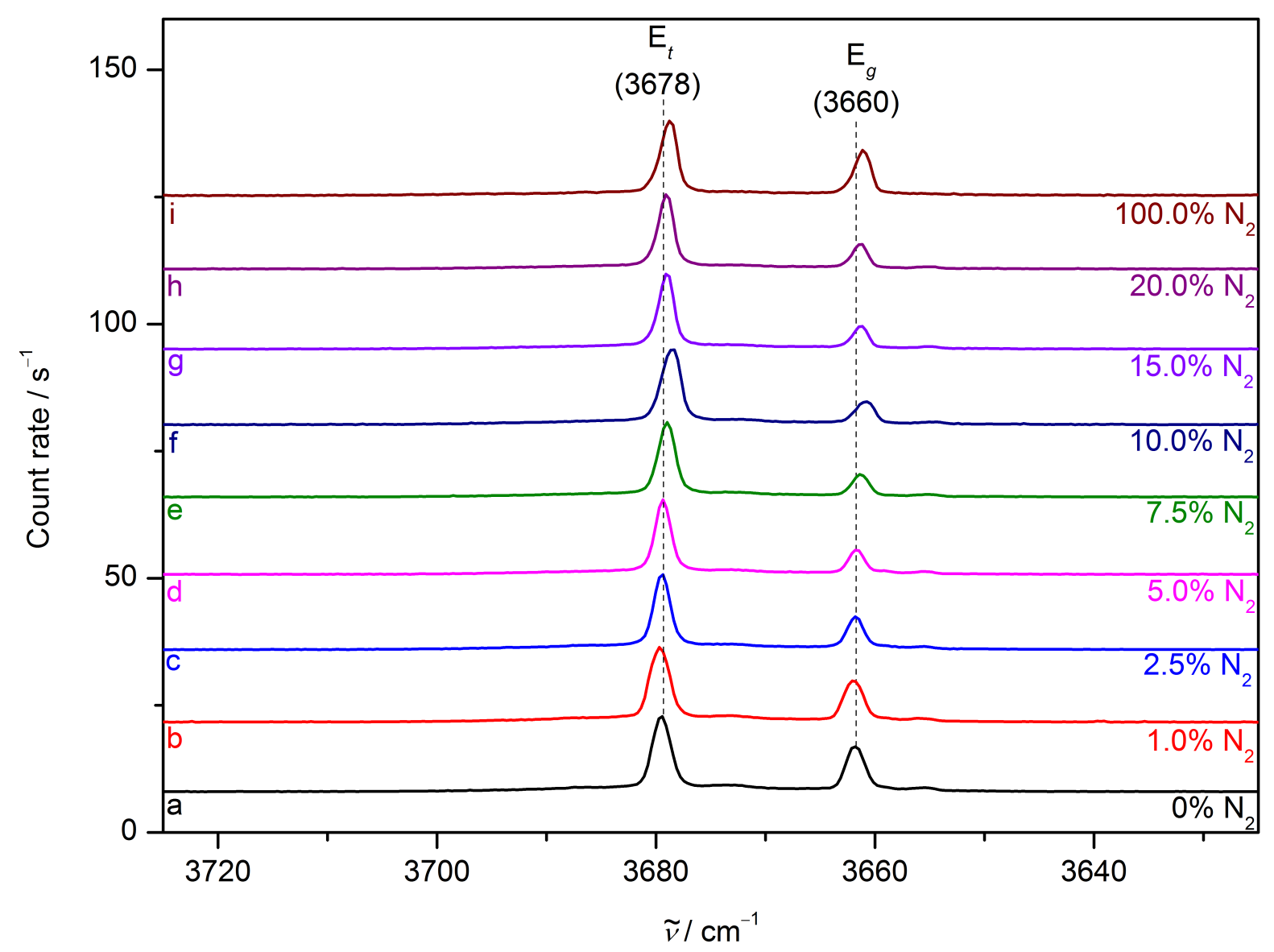

Figure A.21.: OH stretching Raman spectra of E expanded in helium (trace a), N (trace i), $1.0 \%, 2.5 \%, 5.0 \%, 7.5 \%, 10.0 \%, 15.0 \%$ and $20.0 \% \mathrm{~N}$ in helium (traces b to $\mathrm{h}$ ). $\mathrm{E}$ was kept at a temperature of $273 \mathrm{~K}$, the carrier gas pressure in the gas line at $1.5 \mathrm{bar}$ and the stagnation pressure in the reservoir at $0.75 \mathrm{bar}$, while the laser probed the expansion at a $2 \mathrm{~mm}$ nozzle distance. All spectra were scaled to matching height of the $\mathrm{E}_{t}$ band at $3678 \mathrm{~cm}^{-1}$. 
A. Appendix

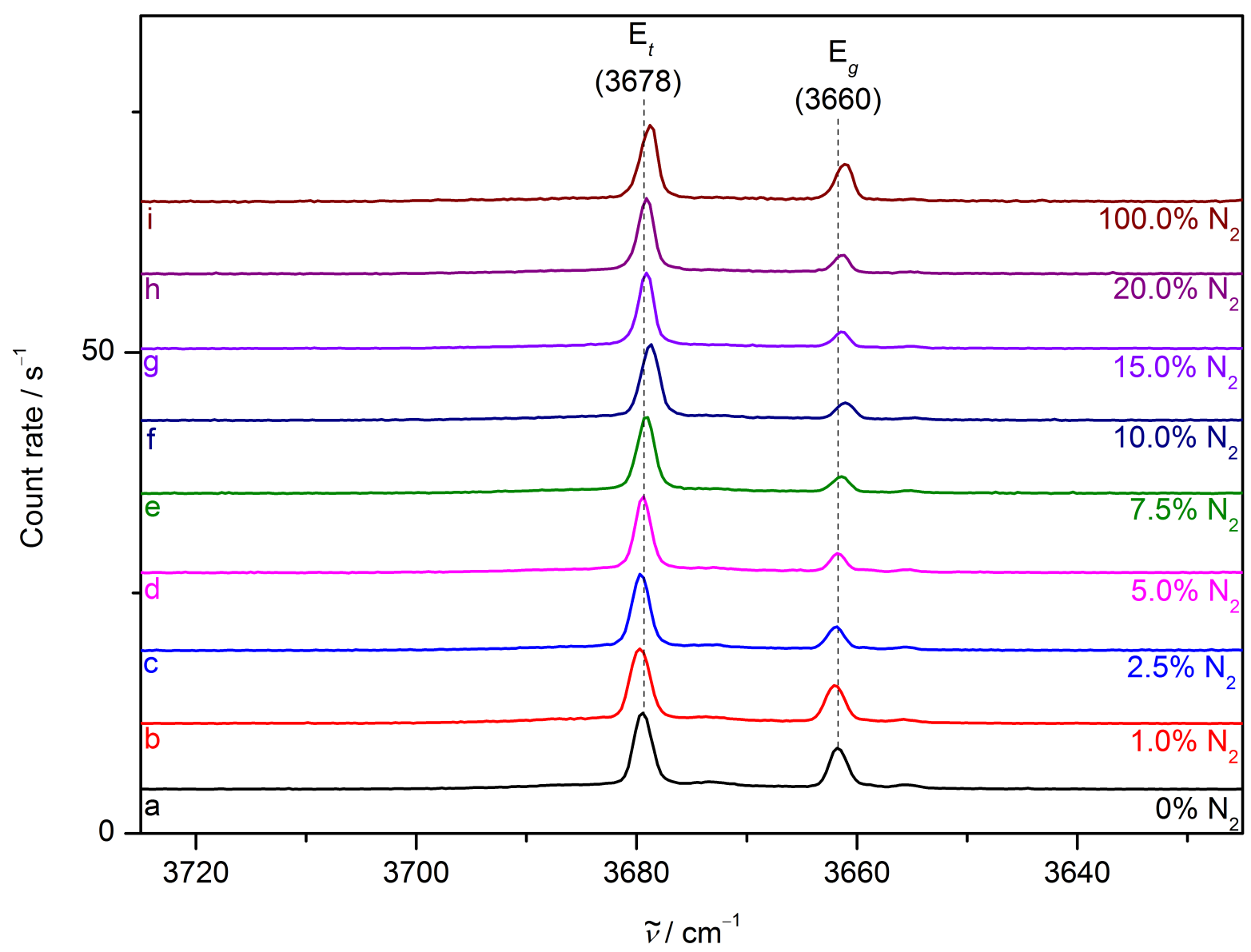

Figure A.22.: $\mathrm{OH}$ stretching Raman spectra of E expanded in helium (trace a), N (trace i), $1.0 \%, 2.5 \%, 5.0 \%, 7.5 \%, 10.0 \%, 15.0 \%$ and $20.0 \% \mathrm{~N}$ in helium (traces b to h). $\mathrm{E}$ was kept at a temperature of $273 \mathrm{~K}$, the carrier gas pressure in the gas line at $1.5 \mathrm{bar}$ and the stagnation pressure in the reservoir at $0.75 \mathrm{bar}$, while the laser probed the expansion at a $3 \mathrm{~mm}$ nozzle distance. All spectra were scaled to matching height of the $\mathrm{E}_{t}$ band at $3678 \mathrm{~cm}^{-1}$. 


\section{A. Appendix}

\section{A.5. Pyrrole}

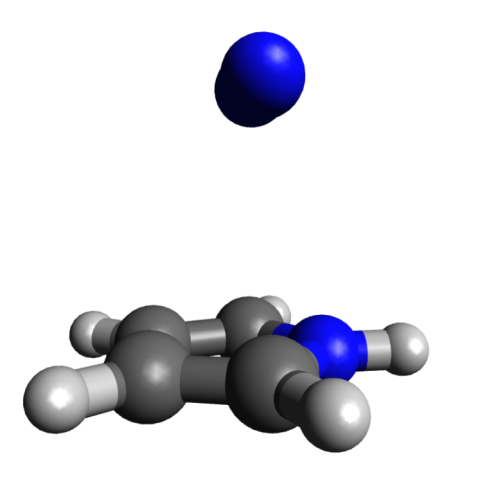

$\mathrm{PN}_{\pi}(0.0)$

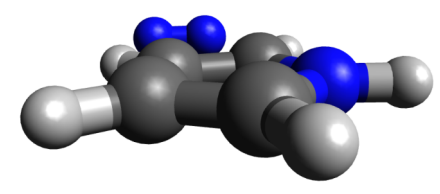

$\mathrm{PN}_{\mathrm{c}}(3.2)$
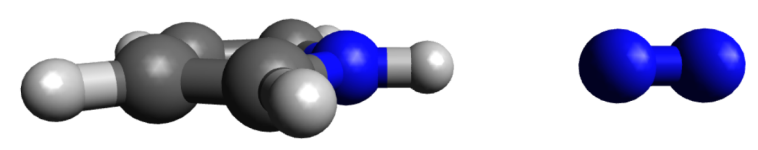

$$
\mathrm{PN}_{\mathrm{H}}(0.7)
$$
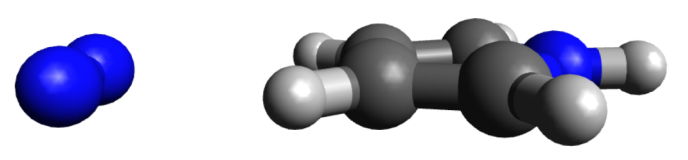

$\mathrm{PN}_{\mathrm{C}}(3.4)$

Figure A.23.: Stable structures found for the mixed dimer of $\mathrm{P}$ and $\mathrm{N}$ optimised at the B3LYP-D3(BJ)/aVTZ level (reprinted from reference 313 licensed under CC BY 3.0). Relative energies in $\mathrm{kJ} \mathrm{mol}^{-1}$ calculated from $\operatorname{CCSD}(\mathrm{T}) / \mathrm{aVTZ}$ single-point calculations and harmonic zero-point vibrational energy from B3LYP-D3(BJ)/aVTZ are given in parentheses. 
A. Appendix

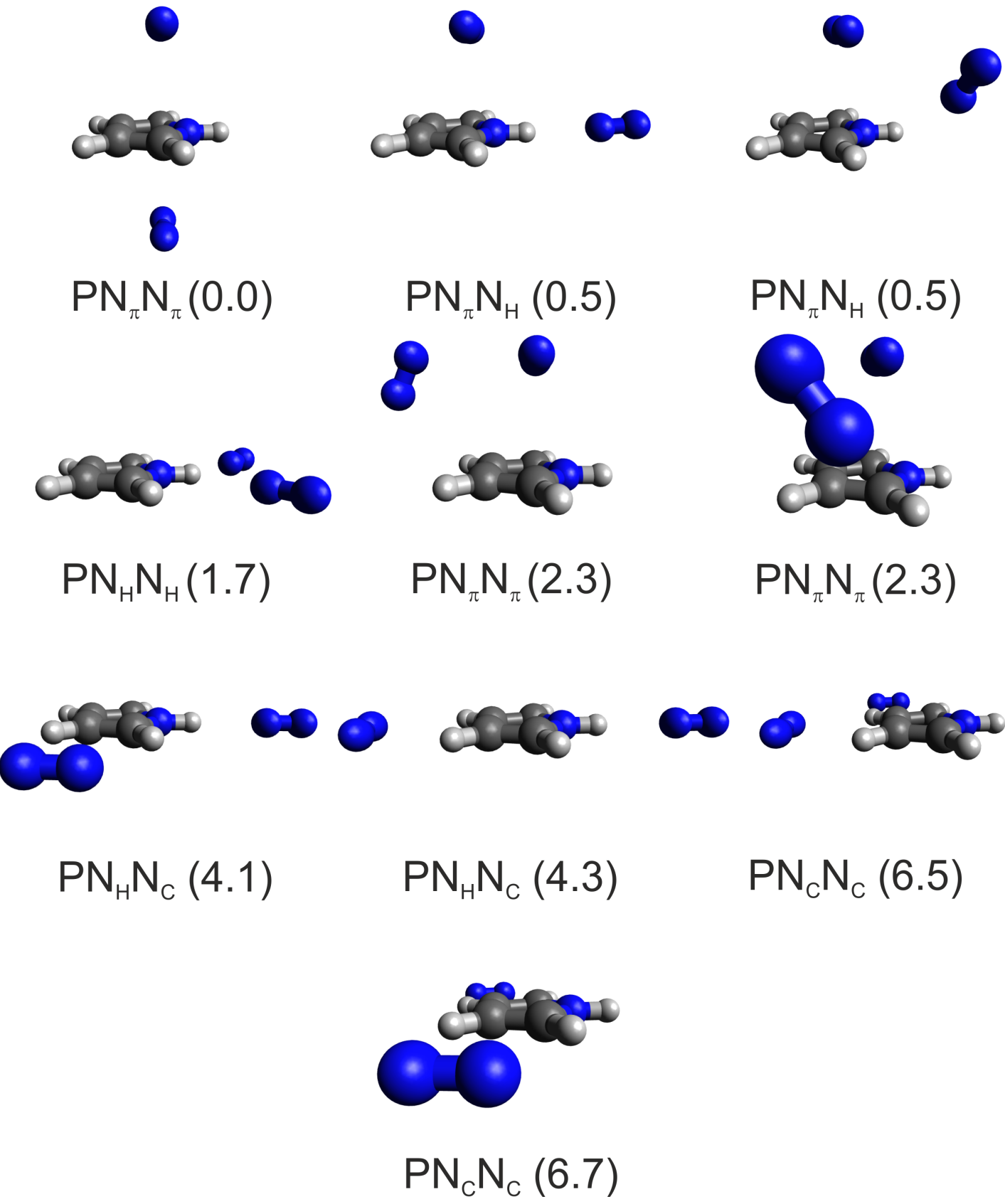

Figure A.24.: Stable structures found for the mixed trimer of $\mathrm{P}$ and two $\mathrm{N}$ molecules optimised at the B3LYP-D3(BJ)/aVTZ level (reprinted from reference 313 licensed under CC BY 3.0). Relative energies in $\mathrm{kJ} \mathrm{mol}^{-1}$ calculated from $\mathrm{CCSD}(\mathrm{T}) / \mathrm{aVTZ}$ single-point calculations and harmonic zero-point vibrational energy from B3LYP-D3(BJ)/aVTZ are given in parentheses. 
A. Appendix

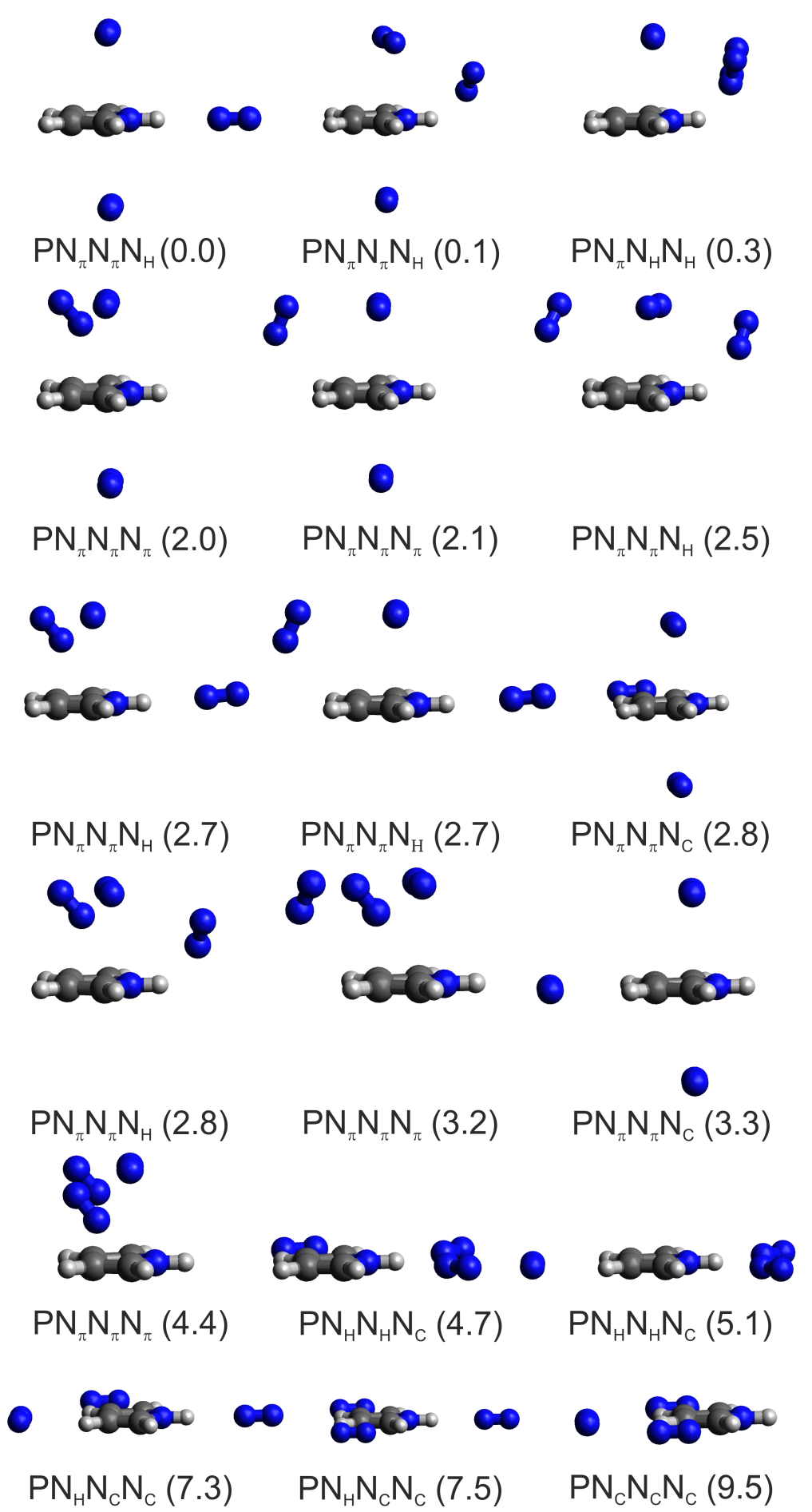

Figure A.25.: Stable structures found for the mixed tetramer of $\mathrm{P}$ and three $\mathrm{N}$ molecules optimised at the B3LYP-D3(BJ)/aVTZ level (reprinted from reference 313 licensed under CC BY 3.0). Relative energies in $\mathrm{kJ} \mathrm{mol}^{-1}$ calculated from CCSD(T)/aVTZ single-point calculations and harmonic zero-point vibrational energy from B3LYP-D3(BJ)/aVTZ are given in parentheses. 
A. Appendix

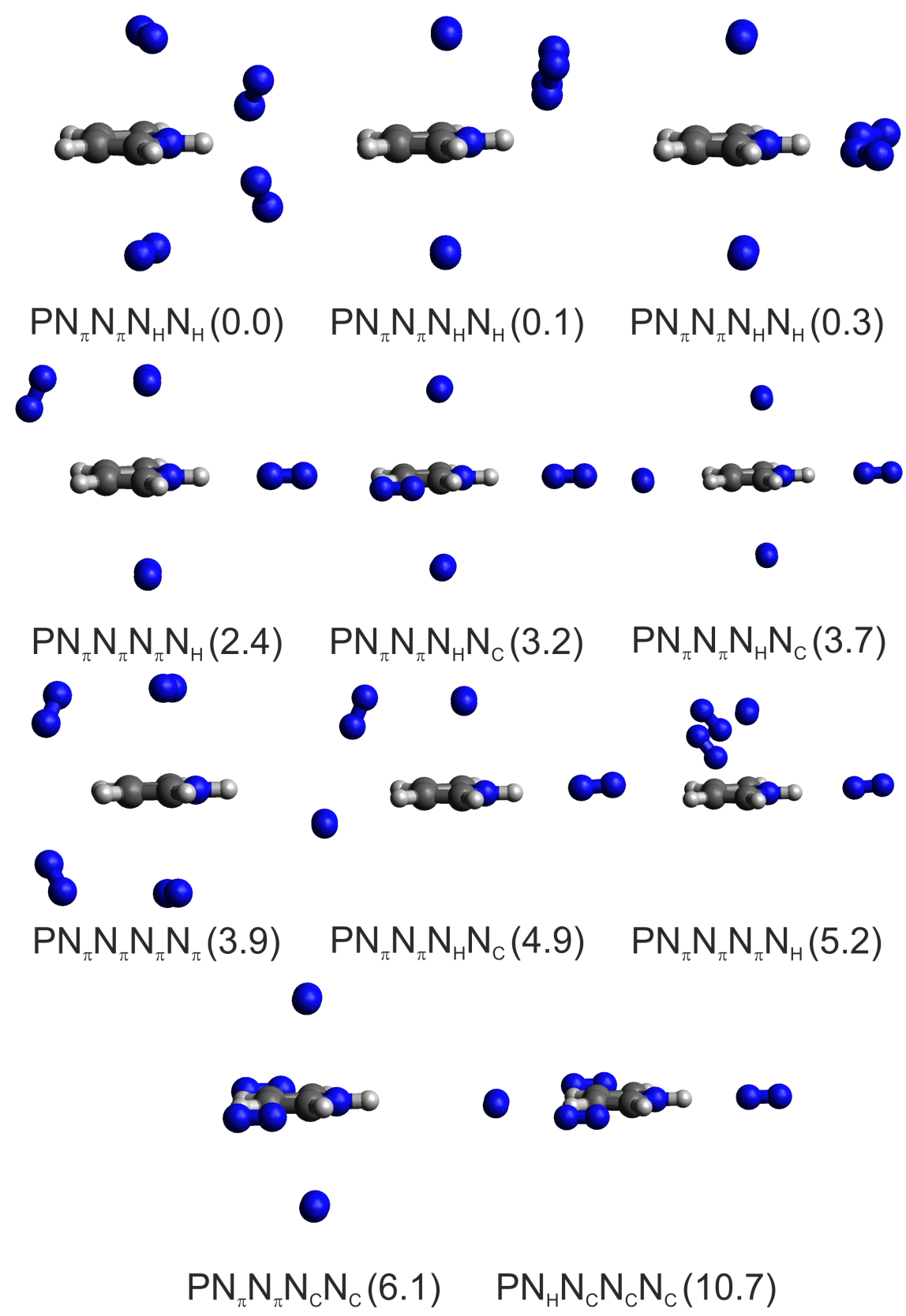

Figure A.26.: Stable structures found for the mixed pentamer of $\mathrm{P}$ and four N molecules optimised at the B3LYP-D3(BJ)/aVTZ level (reprinted from reference 313 licensed under CC BY 3.0). Relative energies in $\mathrm{kJ} \mathrm{mol}^{-1}$ calculated from $\operatorname{CCSD}(\mathrm{T}) / \mathrm{aVTZ}$ single-point calculations and harmonic zero-point vibrational energy from B3LYP-D3(BJ)/aVTZ are given in parentheses. 
A. Appendix

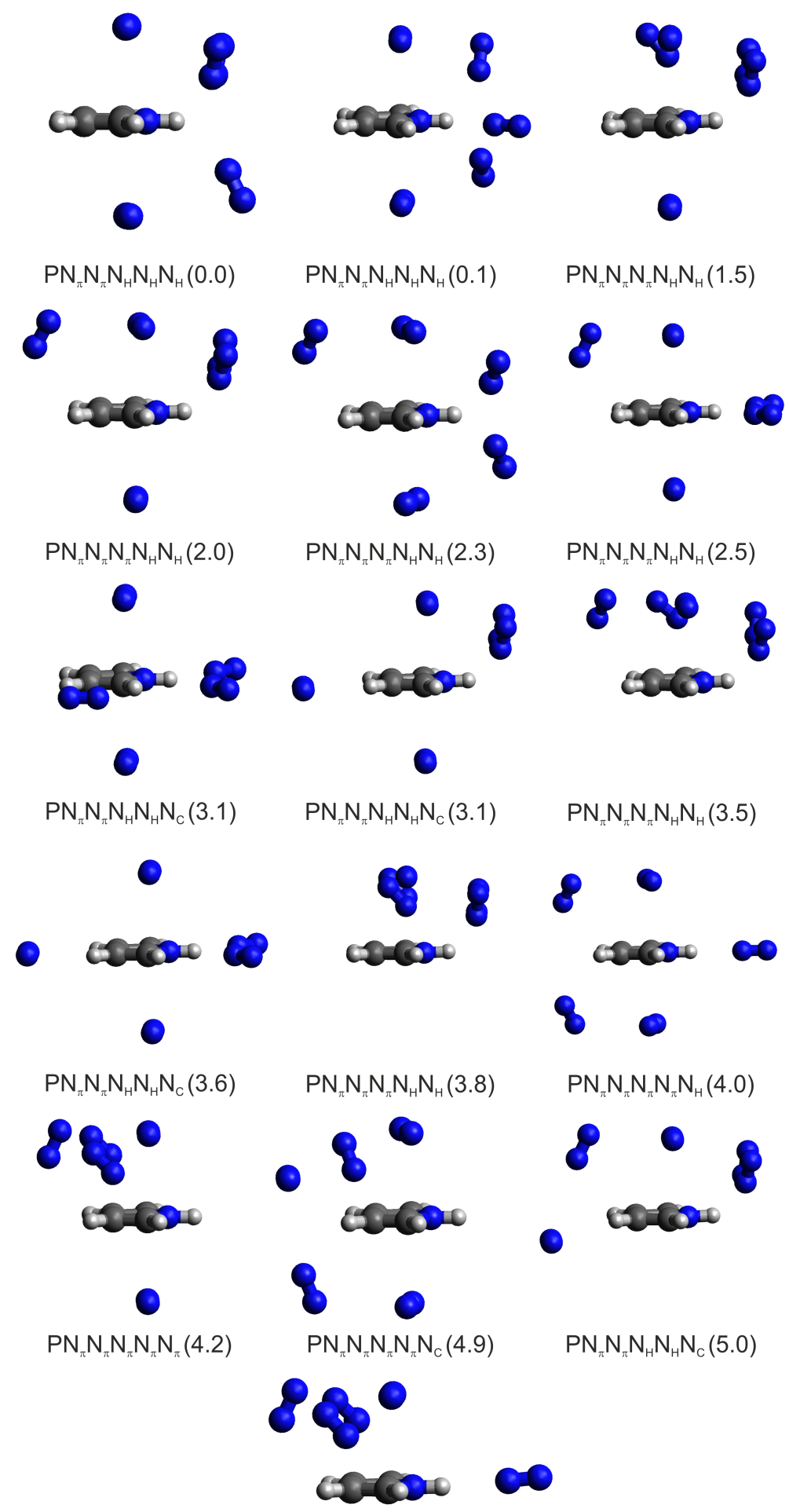

$\mathrm{PN}_{\pi} \mathrm{N}_{\pi} \mathrm{N}_{\pi} \mathrm{N}_{\pi} \mathrm{N}_{\mathrm{H}}(5.6)$

Figure A.27.: Stable structures found for the mixed hexamer of $\mathrm{P}$ and five $\mathrm{N}$ molecules optimised at the B3LYP-D3(BJ)/aVTZ level (reprinted from reference 313 licensed under $\mathrm{CC}$ BY 3.0). Relative energies in $\mathrm{kJ} \mathrm{mol}^{-1}$ including harmonic zero-point vibrational energy are given in parentheses. 


\section{A. Appendix}

Table A.25.: Theoretically predicted properties of stable $\mathrm{P}$ and $\mathrm{N}$ aggregates such as harmonic $\left(\omega_{\mathrm{NH}}\right)$ and anharmonic $\left(\tilde{\nu}_{\mathrm{NH}}\right) \mathrm{NH}$ stretching wavenumbers, lowest predicted harmonic $\left(\omega_{1}\right)$ and anharmonic $\left(\tilde{\nu}_{1}\right)$ wavenumbers in $\mathrm{cm}^{-1}$, harmonic $\left(S_{\omega}\right)$ and anharmonic $\left(S_{\nu}\right)$ infrared band strengths in $\mathrm{km} \mathrm{mol}^{-1}$, spectroscopic downshifts $\left(\Delta \omega_{\mathrm{NH}}, \Delta \tilde{\nu}_{\mathrm{NH}}\right)$ relative to the pyrrole monomer in $\mathrm{cm}^{-1}$, as well as relative electronic and harmonically zero-point corrected energies $\Delta E_{\mathrm{e}}$ and $\Delta E_{0}$ compared to the most stable cluster conformation in $\mathrm{kJ} \mathrm{mol}^{-1}$. All properties were calculated at the B3LYP-D3(BJ)/aVTZ level. Only the electronic energy was taken from $\operatorname{CCSD}(\mathrm{T}) / \mathrm{aVTZ}$ single-point calculations for all clusters except hexamers.

\begin{tabular}{|c|c|c|c|c|c|c|c|c|c|c|}
\hline Structure & $\omega_{\mathrm{NH}}$ & $S_{\omega}$ & $\Delta \omega_{\mathrm{NH}}$ & $\omega_{1}$ & $\tilde{\nu}_{\mathrm{NH}}$ & $S_{\nu}$ & $\Delta \tilde{\nu}_{\mathrm{NH}}$ & $\tilde{\nu}_{1}$ & $\Delta E_{\mathrm{e}}$ & $\Delta E_{0}$ \\
\hline $\mathrm{P}$ & 3674 & 65 & - & 496 & 3508 & 51 & - & 492 & - & - \\
\hline PP & $\begin{array}{l}3669 \\
3598\end{array}$ & $\begin{array}{c}72 \\
324\end{array}$ & $\begin{array}{c}5 \\
76\end{array}$ & 17 & - & - & - & - & - & - \\
\hline PPP & $\begin{array}{l}3553 \\
3553 \\
3536\end{array}$ & $\begin{array}{c}631 \\
631 \\
0\end{array}$ & $\begin{array}{l}121 \\
121 \\
138\end{array}$ & 22 & - & - & - & - & - & - \\
\hline $\begin{array}{l}\mathrm{PN}_{\pi} \\
\mathrm{PN}_{\mathrm{H}} \\
\mathrm{PN}_{\mathrm{C}} \\
\mathrm{PN}_{\mathrm{C}}\end{array}$ & $\begin{array}{l}3674 \\
3669 \\
3674 \\
3674\end{array}$ & $\begin{array}{c}65 \\
206 \\
65 \\
67\end{array}$ & $\begin{array}{l}0 \\
5 \\
0 \\
0\end{array}$ & $\begin{array}{l}21 \\
11 \\
19 \\
16\end{array}$ & $\begin{array}{l}3507 \\
3506 \\
3510 \\
3517\end{array}$ & $\begin{array}{c}51 \\
133 \\
52 \\
52\end{array}$ & $\begin{array}{c}1 \\
2 \\
-2 \\
-9\end{array}$ & $\begin{array}{c}18810 \\
20 \\
-58 \\
-24\end{array}$ & $\begin{array}{l}0.0 \\
0.8 \\
4.1 \\
4.3\end{array}$ & $\begin{array}{l}0.0 \\
0.7 \\
3.2 \\
3.4\end{array}$ \\
\hline $\begin{array}{l}\mathrm{PN}_{\pi} \mathrm{N}_{\pi} \\
\mathrm{PN}_{\pi} \mathrm{N}_{\mathrm{H}} \\
\mathrm{PN}_{\pi} \mathrm{N}_{\mathrm{H}} \\
\mathrm{PN}_{\mathrm{H}} \mathrm{N}_{\mathrm{H}} \\
\mathrm{PN}_{\pi} \mathrm{N}_{\pi} \\
\mathrm{PN}_{\pi} \mathrm{N}_{\pi} \\
\mathrm{PN}_{\mathrm{H}} \mathrm{N}_{\mathrm{C}} \\
\mathrm{PN}_{\mathrm{H}} \mathrm{N}_{\mathrm{C}} \\
\mathrm{PN}_{\mathrm{C}} \mathrm{N}_{\mathrm{C}} \\
\mathrm{PN}_{\mathrm{C}} \mathrm{N}_{\mathrm{C}}\end{array}$ & $\begin{array}{l}3673 \\
3668 \\
3675 \\
3679 \\
3674 \\
3673 \\
3669 \\
3669 \\
3674 \\
3674\end{array}$ & $\begin{array}{c}65 \\
210 \\
94 \\
166 \\
67 \\
65 \\
207 \\
213 \\
67 \\
65\end{array}$ & $\begin{array}{c}1 \\
6 \\
-1 \\
-5 \\
0 \\
1 \\
5 \\
5 \\
0 \\
0\end{array}$ & $\begin{array}{c}18 \\
10 \\
8 \\
16 \\
7 \\
17 \\
6 \\
7 \\
10 \\
11\end{array}$ & $\begin{array}{l}3517 \\
3505 \\
3508 \\
3530 \\
3517 \\
3513 \\
3510 \\
3514 \\
3521 \\
3526\end{array}$ & $\begin{array}{c}52 \\
131 \\
69 \\
121 \\
54 \\
51 \\
123 \\
139 \\
53 \\
49\end{array}$ & $\begin{array}{c}-9 \\
3 \\
0 \\
-22 \\
-9 \\
-5 \\
-2 \\
-6 \\
-13 \\
-18\end{array}$ & $\begin{array}{c}132 \\
-22 \\
23 \\
137 \\
-667 \\
-13 \\
-146 \\
-92 \\
-11 \\
25\end{array}$ & $\begin{array}{l}0.0 \\
0.4 \\
0.7 \\
2.2 \\
3.0 \\
2.9 \\
4.9 \\
5.1 \\
8.2 \\
8.4\end{array}$ & $\begin{array}{l}0.0 \\
0.5 \\
0.5 \\
1.7 \\
2.3 \\
2.3 \\
4.1 \\
4.3 \\
6.5 \\
6.7\end{array}$ \\
\hline $\mathrm{PN}_{\pi} \mathrm{N}_{\pi} \mathrm{N}_{\mathrm{H}}$ & 3667 & 214 & 7 & 9 & - & - & - & - & 0.0 & 0.0 \\
\hline $\mathrm{PN}_{\pi} \mathrm{N}_{\pi} \mathrm{N}_{\mathrm{H}}$ & 3674 & 98 & 0 & 12 & - & - & - & - & 0.5 & 0.1 \\
\hline $\mathrm{PN}_{\pi} \mathrm{N}_{\mathrm{H}} \mathrm{N}_{\mathrm{H}}$ & 3677 & 100 & -3 & 11 & - & - & - & - & 0.8 & 0.3 \\
\hline $\mathrm{PN}_{\pi} \mathrm{N}_{\pi} \mathrm{N}_{\pi}$ & 3672 & 65 & 2 & 15 & - & - & - & - & 2.9 & 2.0 \\
\hline $\mathrm{PN}_{\pi} \mathrm{N}_{\pi} \mathrm{N}_{\pi}$ & 3673 & 67 & 1 & 7 & - & - & - & - & 3.0 & 2.1 \\
\hline $\mathrm{PN}_{\pi} \mathrm{N}_{\pi} \mathrm{N}_{\mathrm{H}}$ & 3675 & 97 & -1 & 10 & - & - & - & - & 3.6 & 2.5 \\
\hline $\mathrm{PN}_{\pi} \mathrm{N}_{\pi} \mathrm{N}_{\mathrm{H}}$ & 3668 & 209 & 6 & 6 & - & - & - & - & 3.4 & 2.7 \\
\hline $\mathrm{PN}_{\pi} \mathrm{N}_{\pi} \mathrm{N}_{\mathrm{H}}$ & 3668 & 214 & 6 & 7 & - & - & - & - & 3.5 & 2.7 \\
\hline $\mathrm{PN}_{\pi} \mathrm{N}_{\pi} \mathrm{N}_{\mathrm{C}}$ & 3672 & 65 & 2 & 1 & - & - & - & - & 3.7 & 2.8 \\
\hline $\mathrm{PN}_{\pi} \mathrm{N}_{\pi} \mathrm{N}_{\mathrm{H}}$ & 3675 & 92 & -1 & 10 & - & - & - & - & 3.8 & 2.8 \\
\hline $\mathrm{PN}_{\pi} \mathrm{N}_{\pi} \mathrm{N}_{\pi}$ & 3673 & 67 & 1 & 17 & - & - & - & - & 4.4 & 3.2 \\
\hline $\mathrm{PN}_{\pi} \mathrm{N}_{\pi} \mathrm{N}_{\mathrm{C}}$ & 3672 & 67 & 2 & 4 & - & - & - & - & 4.3 & 3.3 \\
\hline $\mathrm{PN}_{\pi} \mathrm{N}_{\pi} \mathrm{N}_{\pi}$ & 3673 & 65 & 1 & 13 & - & - & - & - & 5.9 & 4.4 \\
\hline $\mathrm{PN}_{\mathrm{H}} \mathrm{N}_{\mathrm{H}} \mathrm{N}_{\mathrm{C}}$ & 3678 & 165 & -4 & 8 & - & - & - & - & 6.1 & 4.7 \\
\hline $\mathrm{PN}_{\mathrm{H}} \mathrm{N}_{\mathrm{H}} \mathrm{N}_{\mathrm{C}}$ & 3679 & 172 & -5 & 7 & - & - & - & - & 6.7 & 5.1 \\
\hline $\mathrm{PN}_{\mathrm{H}} \mathrm{N}_{\mathrm{C}} \mathrm{N}_{\mathrm{C}}$ & 3668 & 214 & 6 & 6 & - & - & - & - & 9.1 & 7.3 \\
\hline $\mathrm{PN}_{\mathrm{H}} \mathrm{N}_{\mathrm{C}} \mathrm{N}_{\mathrm{C}}$ & 3668 & 208 & 6 & 5 & - & - & - & - & 9.1 & 7.5 \\
\hline $\mathrm{PN}_{\mathrm{C}} \mathrm{N}_{\mathrm{C}} \mathrm{N}_{\mathrm{C}}$ & 3673 & 67 & 1 & 5 & - & - & - & - & 12.2 & 9.5 \\
\hline
\end{tabular}


A. Appendix

\begin{tabular}{|c|c|c|c|c|c|c|c|c|c|c|}
\hline Structure & $\omega_{\mathrm{NH}}$ & $S_{\omega}$ & $\Delta \omega_{\mathrm{NH}}$ & $\omega_{l}$ & $\tilde{\nu}_{\mathrm{NH}}$ & $S_{\nu}$ & $\Delta \tilde{\nu}_{\mathrm{NH}}$ & $\tilde{\nu}_{1}$ & $\Delta E_{\mathrm{e}}$ & $\Delta E_{0}$ \\
\hline $\mathrm{PN}_{\pi} \mathrm{N}_{\pi} \mathrm{N}_{\mathrm{H}} \mathrm{N}_{\mathrm{H}}$ & 3676 & 122 & -2 & 10 & - & - & - & - & 0.1 & 0.0 \\
\hline $\mathrm{PN}_{\pi} \mathrm{N}_{\pi} \mathrm{N}_{\mathrm{H}} \mathrm{N}_{\mathrm{H}}$ & 3676 & 104 & -2 & 9 & - & - & - & - & 0.0 & 0.1 \\
\hline $\mathrm{PN}_{\pi} \mathrm{N}_{\pi} \mathrm{N}_{\mathrm{H}} \mathrm{N}_{\mathrm{H}}$ & 3678 & 168 & -4 & 4 & - & - & - & - & 0.1 & 0.3 \\
\hline $\mathrm{PN}_{\pi} \mathrm{N}_{\pi} \mathrm{N}_{\pi} \mathrm{N}_{\mathrm{H}}$ & 3667 & 219 & -3 & 7 & - & - & - & - & 2.5 & 2.4 \\
\hline $\mathrm{PN}_{\pi} \mathrm{N}_{\pi} \mathrm{N}_{\mathrm{H}} \mathrm{N}_{\mathrm{C}}$ & 3666 & 214 & 8 & 2 & - & - & - & - & 3.3 & 3.2 \\
\hline $\mathrm{PN}_{\pi} \mathrm{N}_{\pi} \mathrm{N}_{\mathrm{H}} \mathrm{N}_{\mathrm{C}}$ & 3666 & 221 & 8 & 5 & - & - & - & - & 3.9 & 3.7 \\
\hline $\mathrm{PN}_{\pi} \mathrm{N}_{\pi} \mathrm{N}_{\pi} \mathrm{N}_{\pi}$ & 3673 & 69 & 1 & 6 & - & - & - & - & 4.9 & 3.9 \\
\hline $\mathrm{PN}_{\pi} \mathrm{N}_{\pi} \mathrm{N}_{\mathrm{H}} \mathrm{N}_{\mathrm{C}}$ & 3668 & 221 & 6 & 6 & - & - & - & - & 5.7 & 4.9 \\
\hline $\mathrm{PN}_{\pi} \mathrm{N}_{\pi} \mathrm{N}_{\pi} \mathrm{N}_{\mathrm{H}}$ & 3667 & 211 & 7 & 5 & - & - & - & - & 5.9 & 5.2 \\
\hline $\mathrm{PN}_{\pi} \mathrm{N}_{\pi} \mathrm{N}_{\mathrm{C}} \mathrm{N}_{\mathrm{C}}$ & 3672 & 65 & 2 & 1 & - & - & - & - & 7.2 & 6.1 \\
\hline $\mathrm{PN}_{\mathrm{H}} \mathrm{N}_{\mathrm{C}} \mathrm{N}_{\mathrm{C}} \mathrm{N}_{\mathrm{C}}$ & 3668 & 215 & 6 & 5 & - & - & - & - & 12.6 & 10.7 \\
\hline $\mathrm{PN}_{\pi} \mathrm{N}_{\pi} \mathrm{N}_{\mathrm{H}} \mathrm{N}_{\mathrm{H}} \mathrm{N}_{\mathrm{H}}$ & 3674 & 142 & 0 & 3 & - & - & - & - & 0.0 & 0.0 \\
\hline $\mathrm{PN}_{\pi} \mathrm{N}_{\pi} \mathrm{N}_{\mathrm{H}} \mathrm{N}_{\mathrm{H}} \mathrm{N}_{\mathrm{H}}$ & 3674 & 174 & 0 & 8 & - & - & - & - & 0.1 & 0.1 \\
\hline $\mathrm{PN}_{\pi} \mathrm{N}_{\pi} \mathrm{N}_{\pi} \mathrm{N}_{\mathrm{H}} \mathrm{N}_{\mathrm{H}}$ & 3675 & 98 & -1 & 9 & - & - & - & - & 1.5 & 1.5 \\
\hline $\mathrm{PN}_{\pi} \mathrm{N}_{\pi} \mathrm{N}_{\pi} \mathrm{N}_{\mathrm{H}} \mathrm{N}_{\mathrm{H}}$ & 3676 & 106 & -2 & 4 & - & - & - & - & 2.2 & 2.0 \\
\hline $\mathrm{PN}_{\pi} \mathrm{N}_{\pi} \mathrm{N}_{\pi} \mathrm{N}_{\mathrm{H}} \mathrm{N}_{\mathrm{H}}$ & 3676 & 126 & -2 & 10 & - & - & - & - & 2.6 & 2.3 \\
\hline $\mathrm{PN}_{\pi} \mathrm{N}_{\pi} \mathrm{N}_{\pi} \mathrm{N}_{\mathrm{H}} \mathrm{N}_{\mathrm{H}}$ & 3678 & 171 & -4 & 1 & - & - & - & - & 2.7 & 2.5 \\
\hline $\mathrm{PN}_{\pi} \mathrm{N}_{\pi} \mathrm{N}_{\mathrm{H}} \mathrm{N}_{\mathrm{H}} \mathrm{N}_{\mathrm{C}}$ & 3677 & 166 & -3 & 4 & - & - & - & - & 3.2 & 3.1 \\
\hline $\mathrm{PN}_{\pi} \mathrm{N}_{\pi} \mathrm{N}_{\mathrm{H}} \mathrm{N}_{\mathrm{H}} \mathrm{N}_{\mathrm{C}}$ & 3676 & 107 & -2 & 4 & - & - & - & - & 3.4 & 3.1 \\
\hline $\mathrm{PN}_{\pi} \mathrm{N}_{\pi} \mathrm{N}_{\pi} \mathrm{N}_{\mathrm{H}} \mathrm{N}_{\mathrm{H}}$ & 3675 & 100 & -1 & 11 & - & - & - & - & 3.9 & 3.5 \\
\hline $\mathrm{PN}_{\pi} \mathrm{N}_{\pi} \mathrm{N}_{\mathrm{H}} \mathrm{N}_{\mathrm{H}} \mathrm{N}_{\mathrm{C}}$ & 3678 & 173 & -4 & 5 & - & - & - & - & 3.8 & 3.6 \\
\hline $\mathrm{PN}_{\pi} \mathrm{N}_{\pi} \mathrm{N}_{\pi} \mathrm{N}_{\mathrm{H}} \mathrm{N}_{\mathrm{H}}$ & 3675 & 91 & -1 & 8 & - & - & - & - & 4.3 & 3.8 \\
\hline $\mathrm{PN}_{\pi} \mathrm{N}_{\pi} \mathrm{N}_{\pi} \mathrm{N}_{\pi} \mathrm{N}_{\mathrm{H}}$ & 3666 & 224 & 8 & 6 & - & - & - & - & 4.4 & 4.0 \\
\hline $\mathrm{PN}_{\pi} \mathrm{N}_{\pi} \mathrm{N}_{\pi} \mathrm{N}_{\pi} \mathrm{N}_{\pi}$ & 3672 & 67 & 2 & 14 & - & - & - & - & 4.9 & 4.2 \\
\hline $\mathrm{PN}_{\pi} \mathrm{N}_{\pi} \mathrm{N}_{\pi} \mathrm{N}_{\pi} \mathrm{N}_{\mathrm{C}}$ & 3672 & 69 & 2 & 7 & - & - & - & - & 5.9 & 4.9 \\
\hline $\mathrm{PN}_{\pi} \mathrm{N}_{\pi} \mathrm{N}_{\mathrm{H}} \mathrm{N}_{\mathrm{H}} \mathrm{N}_{\mathrm{C}}$ & 3677 & 107 & -3 & 1 & - & - & - & - & 5.7 & 5.0 \\
\hline $\mathrm{PN}_{\pi} \mathrm{N}_{\pi} \mathrm{N}_{\pi} \mathrm{N}_{\pi} \mathrm{N}_{\mathrm{H}}$ & 3667 & 215 & 7 & 5 & - & - & - & - & 6.2 & 5.6 \\
\hline
\end{tabular}


A. Appendix

A.6. Chloroform
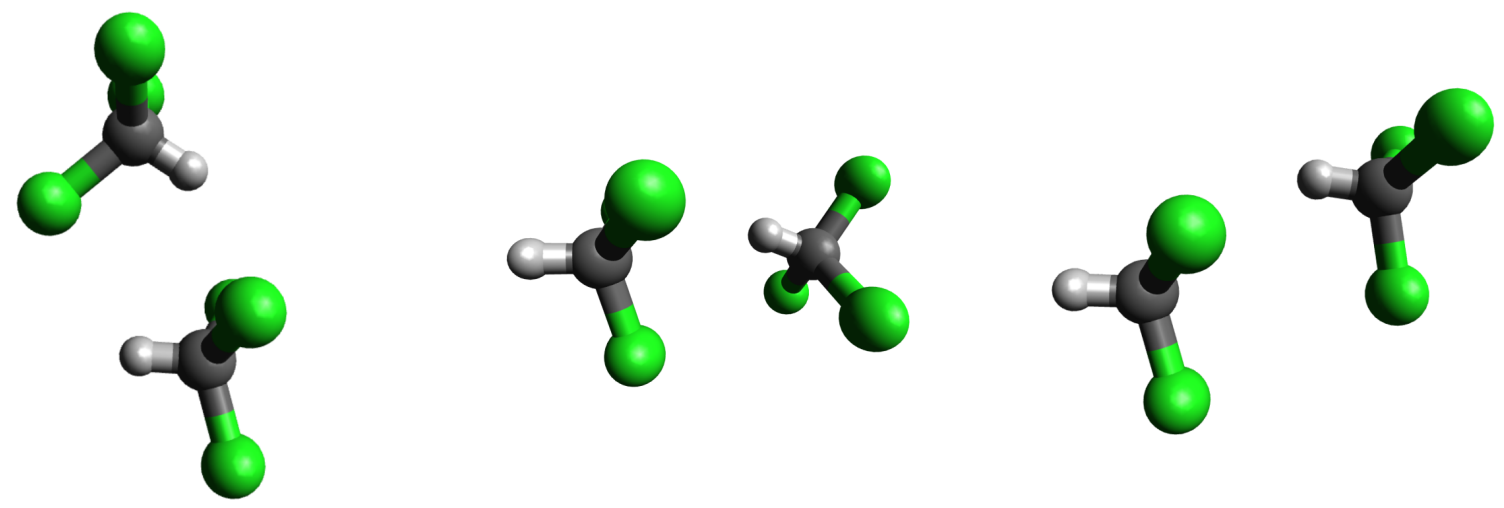

$\mathrm{C}_{\mathrm{Cl}} \mathrm{C}_{\mathrm{CI}}\left[\mathrm{C}_{s}\right](0.0)$

$\mathrm{CC}_{\mathrm{C}}\left[\mathrm{C}_{1}\right]$

$(2.4)$

$\mathrm{CC}_{\mathrm{Cl}}\left[\mathrm{C}_{\mathrm{s}}\right]$

$(2.5)$

Figure A.28.: Stable structures of the CC dimer optimised at the B3LYP-D3(BJ)/def2QZVP level (reprinted from reference 337). Molecular symmetry point groups are given in brackets, relative energies including harmonic zero-point vibrational energy in $\mathrm{kJ} \mathrm{mol}^{-1}$ in parentheses

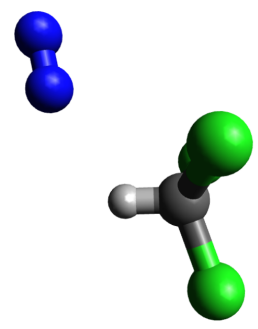

$\mathrm{CN}_{\mathrm{H}}\left[C_{s}\right](0.0)$

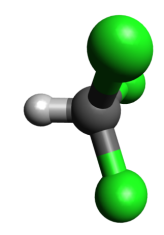

$\mathrm{CN}_{\mathrm{C}}\left[C_{s}\right](1.7)$
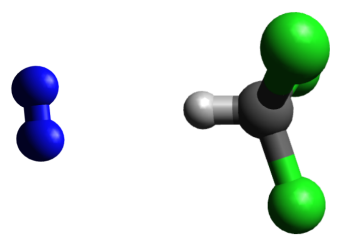

0

$$
\mathrm{CN}_{\mathrm{Cl}}\left[C_{s}\right](3.4)
$$

Figure A.29.: Stable structures of the mixed CN dimer optimised at the B3LYPD3(BJ)/def2-QZVP level of theory. Molecular symmetry point groups are given in trackts, relative energies including harmonic zero-point vibrational energy in $\mathrm{kJ} \mathrm{mol}^{-1}$ in paretheses.

240 
A. Appendix

8
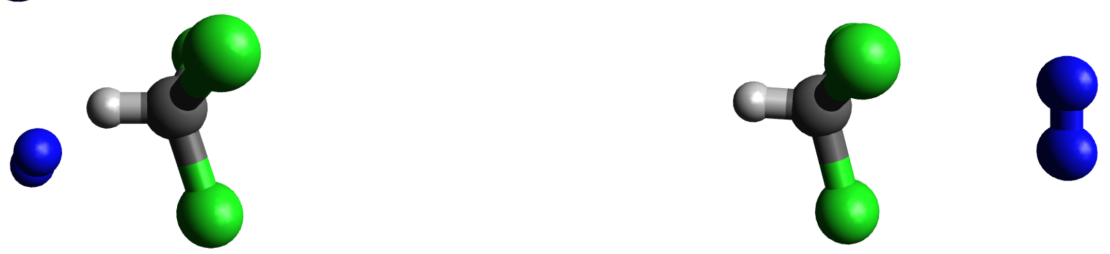

$\mathrm{CN}_{\mathrm{H}} \mathrm{N}_{\mathrm{H}}\left[C_{s}\right](0.0)$

$\mathrm{CN}_{\mathrm{H}} \mathrm{N}_{\mathrm{C}}\left[C_{s}\right](2.1)$

9
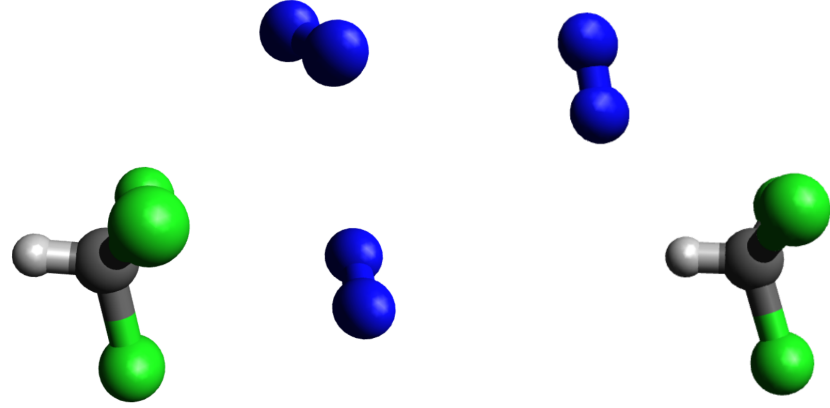

$$
\mathrm{CN}_{\mathrm{C}} \mathrm{N}_{\mathrm{Cl}}\left[C_{1}\right](3.6)
$$

$$
\mathrm{CN}_{\mathrm{H}} \mathrm{N}_{\mathrm{Cl}}\left[\mathrm{C}_{s}\right](3.8)
$$

Figure A.30.: Stable structures of the mixed CNN trimer optimised at the B3LYPD3(BJ)/def2-QZVP level of theory. Molecular symmetry point groups are given in brackets, relative energies including harmonic zero-point vibrational energy in $\mathrm{kJ} \mathrm{mol}^{-1}$ in parentheses. 


\section{A. Appendix}
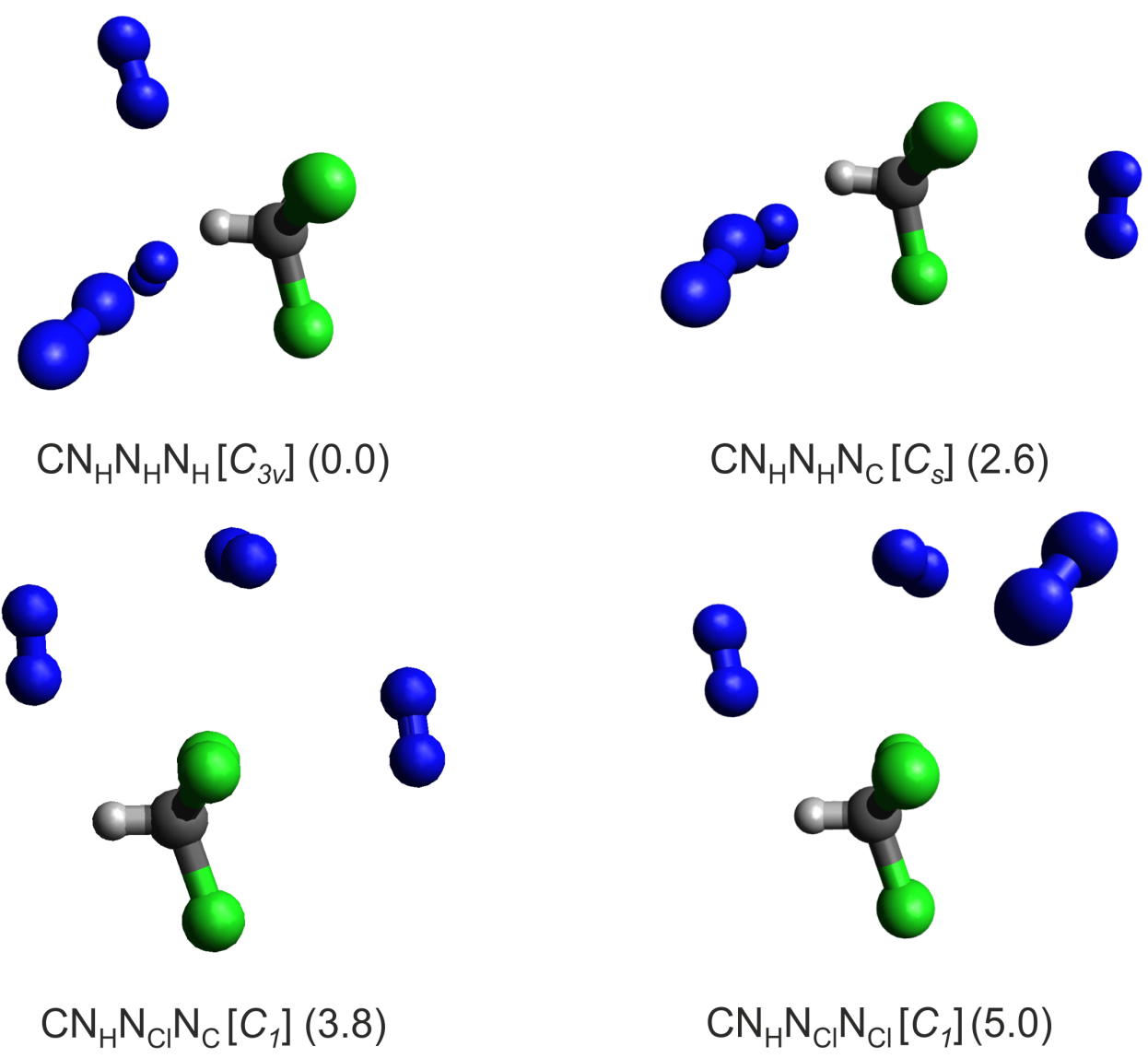

$$
\mathrm{CN}_{\mathrm{H}} \mathrm{N}_{\mathrm{Cl}} \mathrm{N}_{\mathrm{Cl}}\left[C_{1}\right](5.0)
$$
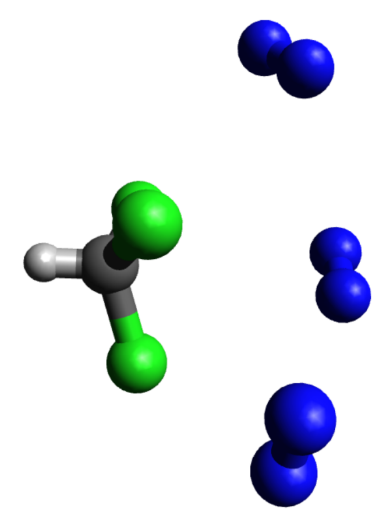

$$
\mathrm{CN}_{\mathrm{C}} \mathrm{N}_{\mathrm{Cl}} \mathrm{N}_{\mathrm{Cl}}\left[C_{1}\right] \text { (6.0) }
$$

Figure A.31.: Stable structures of the mixed CNNN tetramer optimised at the B3LYPD3(BJ)/def2-QZVP level of theory. Molecular symmetry point groups are given in brackets, relative energies including harmonic zero-point vibrational energy in $\mathrm{kJ} \mathrm{mol}^{-1}$ in parentheses. 
A. Appendix
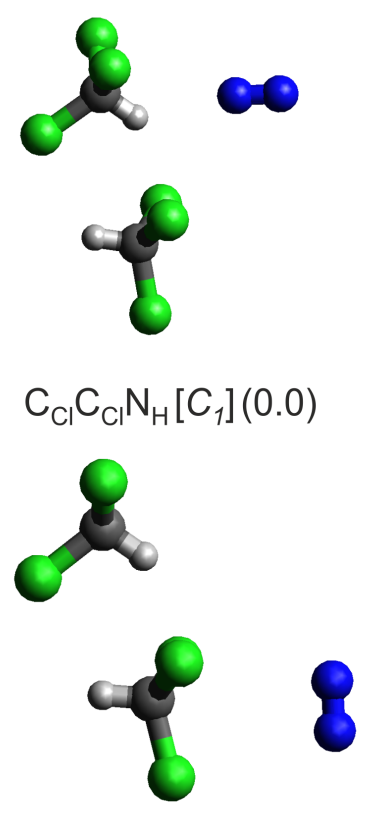

$\mathrm{C}_{\mathrm{Cl}} \mathrm{N}_{\mathrm{C}} \mathrm{C}_{\mathrm{Cl}}\left[\mathrm{C}_{1}\right](3.2)$
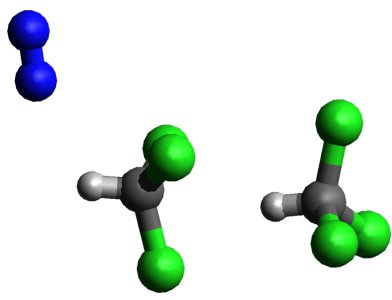

$\mathrm{CC}_{\mathrm{C}} \mathrm{N}_{\mathrm{H}}\left[C_{1}\right](3.8)$
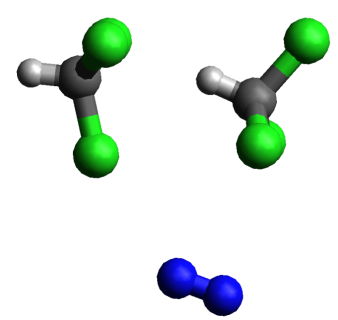

$\mathrm{CN}_{\mathrm{Cl}} \mathrm{C}_{\mathrm{C}}\left[C_{1}\right](4.2)$
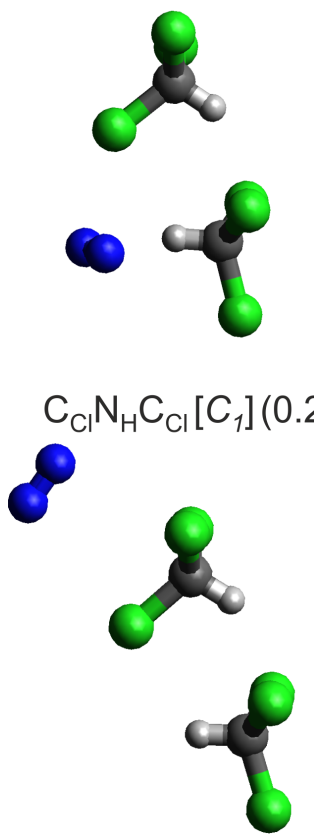

$\mathrm{C}_{\mathrm{Cl}} \mathrm{C}_{\mathrm{Cl}} \mathrm{N}_{\mathrm{C}}\left[C_{1}\right](3.3)$

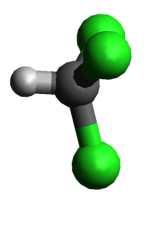

$\mathrm{CN}_{\mathrm{H}} \mathrm{C}_{\mathrm{C}}\left[\mathrm{C}_{1}\right](3.9)$

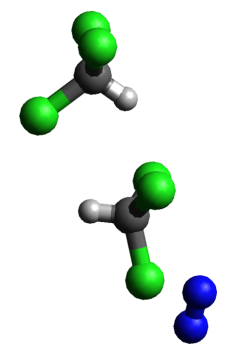

$\mathrm{C}_{\mathrm{Cl}} \mathrm{N}_{\mathrm{Cl}} \mathrm{C}_{\mathrm{Cl}}\left[\mathrm{C}_{1}\right](4.3)$

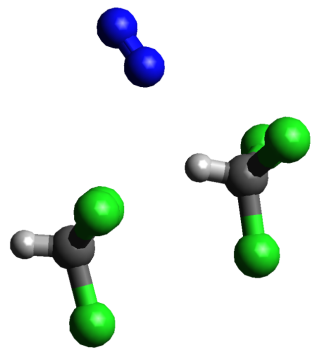

$\mathrm{CN}_{\mathrm{H}} \mathrm{C}_{\mathrm{Cl}}\left[\mathrm{C}_{1}\right](2.5)$

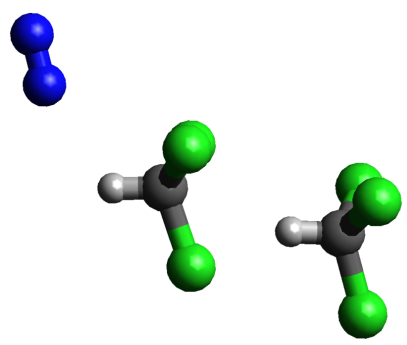

$\mathrm{CC}_{\mathrm{C}} \mathrm{N}_{\mathrm{H}}\left[\mathrm{C}_{1}\right](3.7)$
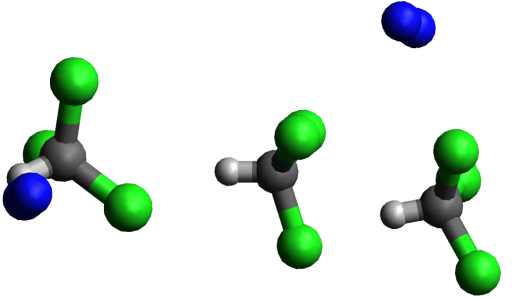

$\mathrm{CN}_{\mathrm{Cl}} \mathrm{C}_{\mathrm{C}}\left[C_{1}\right](3.9)$

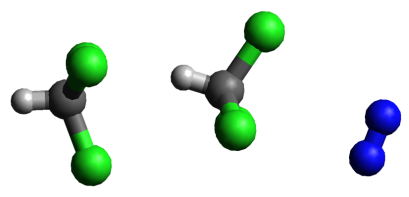

$\mathrm{CN}_{\mathrm{C}} \mathrm{C}_{\mathrm{C}}\left[C_{1}\right](5.6)$

Figure A.32.: Stable structures of the mixed CCN trimer optimised at the B3LYPD3(BJ)/def2-QZVP level of theory. Molecular symmetry point groups are given in backcts, relative energies including harmonic zero-point vibrational energy in $\mathrm{kJ} \mathrm{mol}^{-1}$ in paretheses.

243 
A. Appendix
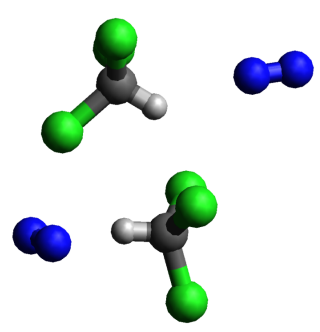

$\mathrm{C}_{\mathrm{Cl}} \mathrm{N}_{\mathrm{H}} \mathrm{C}_{\mathrm{Cl}} \mathrm{N}_{\mathrm{H}}\left[\mathrm{C}_{1}\right](0.0)$

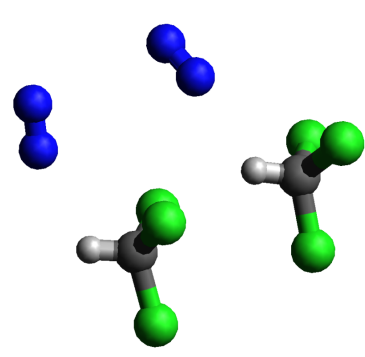

$\mathrm{CN}_{\mathrm{H}} \mathrm{C}_{\mathrm{Cl}} \mathrm{N}_{\mathrm{H}}\left[\mathrm{C}_{1}\right](2.8)$
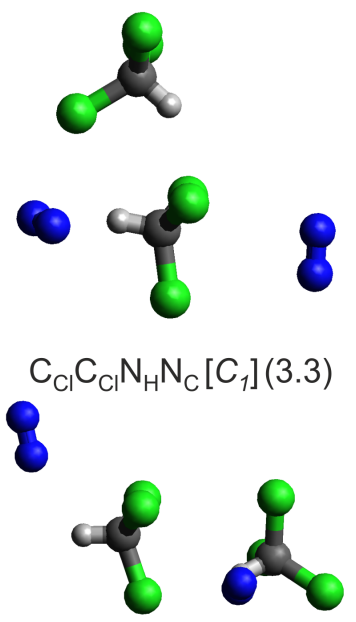

$$
\mathrm{CN}_{\mathrm{H}} \mathrm{C}_{\mathrm{C}} \mathrm{N}_{\mathrm{H}}\left[\mathrm{C}_{1}\right](5.3)
$$

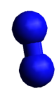

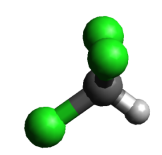

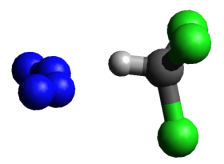

$\mathrm{C}_{\mathrm{Cl}} \mathrm{C}_{\mathrm{Cl}} \mathrm{N}_{\mathrm{H}} \mathrm{N}_{\mathrm{H}}\left[\mathrm{C}_{1}\right](0.0)$

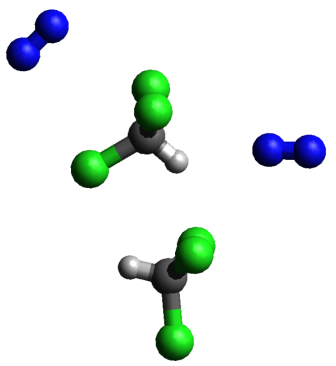

$\mathrm{N}_{\mathrm{C}} \mathrm{C}_{\mathrm{Cl}} \mathrm{N}_{\mathrm{H}} \mathrm{C}_{\mathrm{Cl}}\left[\mathrm{C}_{1}\right](3.1)$

8
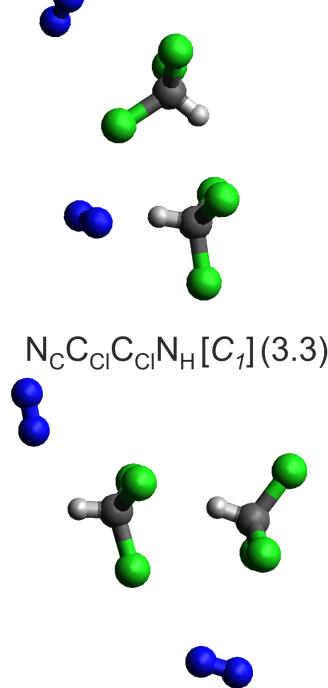

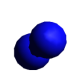

$\mathrm{C}_{\mathrm{Cl}} \mathrm{N}_{\mathrm{H}} \mathrm{C}_{\mathrm{Cl}} \mathrm{N}_{\mathrm{C}}\left[\mathrm{C}_{1}\right](2.0)$
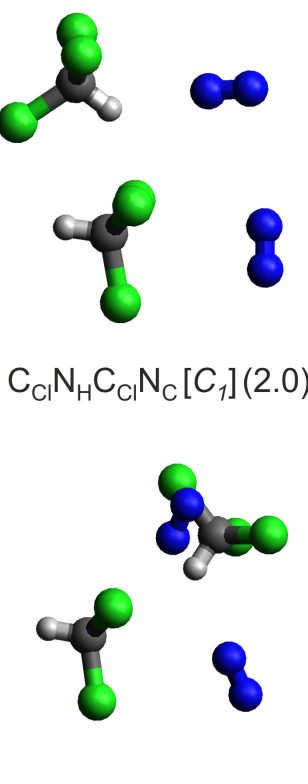

$\mathrm{CN}_{\mathrm{H}} \mathrm{C}_{\mathrm{Cl}} \mathrm{N}_{\mathrm{Cl}}\left[\mathrm{C}_{1}\right](3.2)$

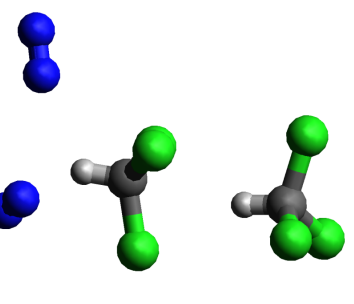

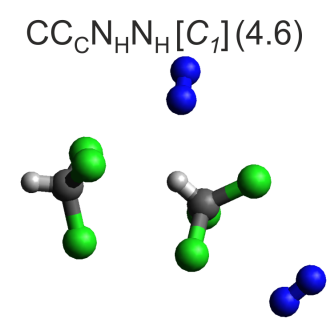

$\mathrm{N}_{\mathrm{C}} \mathrm{CN}_{\mathrm{H}} \mathrm{C}_{\mathrm{Cl}}\left[C_{1}\right](7.0)$

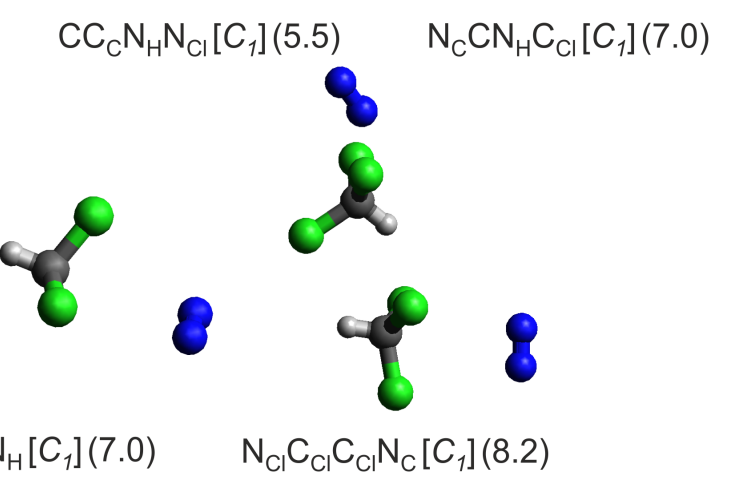

Figure A.33.: Stable structures of the mixed CCNN tetramer optimised at the B3LYPD3(BJ)/def2-QZVP level of theory. Molecular symmetry point groups are given in backts, relative energies including harmonic zero-point vibrational energy in $\mathrm{kJ} \mathrm{mol}^{-1}$ in paretheses.

244 
A. Appendix

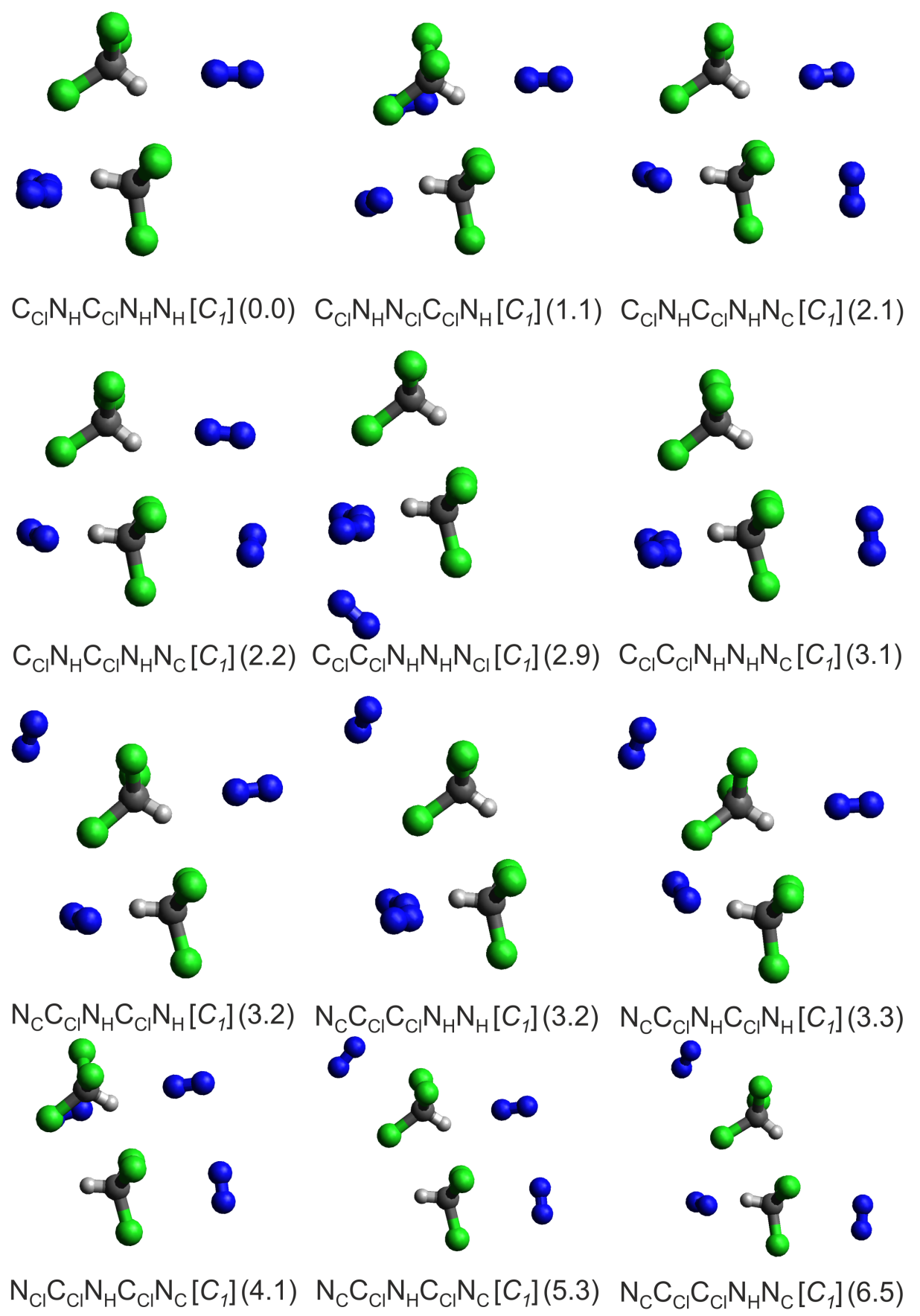

Figure A.34.: Stable structures of the mixed CCNNN pentamer optimised at the B3LYPD3(BJ)/def2-QZVP level of theory. Molecular symmetry point groups are given in brackets, relative energies including harmonic zero-point vibrational energy in $\mathrm{kJ} \mathrm{mol}^{-1}$ in parentheses. 


\section{A. Appendix}

Table A.26.: Theoretically predicted harmonic $\left(\omega_{\mathrm{CH}}\right)$ and anharmonic $\left(\tilde{\nu}_{\mathrm{CH}}\right) \mathrm{CH}$ stretching wavenumber in $\mathrm{cm}^{-1}$, IR intensity $(S)$ in $\mathrm{km} \mathrm{mol}^{-1}$, spectroscopic downshifts $\left(\Delta \omega_{\mathrm{CH}}\right.$, $\left.\Delta \tilde{\nu}_{\mathrm{CH}}\right)$ relative to the corresponding vibration in the pure $\mathrm{C}$ monomers or $\mathrm{CC}$ dimers in $\mathrm{cm}^{-1}$, lowest predicted harmonic $\left(\omega_{1}\right)$ and anharmonic $\left(\tilde{\nu}_{1}\right)$ wavenumbers, dissociation energy into the most stable monomers without $\left(D_{\mathrm{e}}\right)$ and with $\left(D_{0}\right)$ harmonic vibrational zeropoint energy in $\mathrm{kJ} \mathrm{mol}^{-1}$. All properties were calculated at the B3LYP-D3(BJ)/def2QZVP level of theory. Anharmonic vibrational frequencies calculated with second order vibrational perturbation theory (Gaussian09 Rev. E01, ${ }^{141}$ VPT2 ${ }^{148}$ ) are unreliable due to unphysical predictions for low frequency large amplitude motions (indicated by parentheses) which affect localised high frequency modes like the $\mathrm{CH}$ stretching vibration through their respective coupling constants.

\begin{tabular}{|c|c|c|c|c|c|c|c|c|c|c|}
\hline Structure & $\omega_{\mathrm{CH}}$ & $S_{\omega}$ & $\tilde{\nu}_{\mathrm{CH}}$ & $S_{\nu}$ & $\Delta \omega_{\mathrm{CH}}$ & $\Delta \tilde{\nu}_{\mathrm{CH}}$ & $\omega_{1}$ & $\tilde{\nu}_{1}$ & $D_{\mathrm{e}}$ & $D_{0}$ \\
\hline $\mathrm{C}$ & 3169 & 1 & 3030 & 0 & - & - & 258 & 249 & - & - \\
\hline $\mathrm{C}_{\mathrm{Cl}} \mathrm{C}_{\mathrm{Cl}}$ & $\begin{array}{l}3183 \\
3174\end{array}$ & $\begin{array}{c}13 \\
5\end{array}$ & $\begin{array}{l}3039 \\
3034\end{array}$ & $\begin{array}{c}19 \\
4\end{array}$ & $\begin{array}{c}-14 \\
-5\end{array}$ & $\begin{array}{l}-9 \\
-4\end{array}$ & 2 & $(-2876)$ & 17.6 & 16.0 \\
\hline $\mathrm{CC}_{\mathrm{C}}$ & $\begin{array}{l}3185 \\
3170 \\
\end{array}$ & $\begin{array}{c}13 \\
2\end{array}$ & $\begin{array}{l}3044 \\
3036\end{array}$ & $\begin{array}{l}9 \\
3\end{array}$ & $\begin{array}{c}-16 \\
-1\end{array}$ & $\begin{array}{c}-14 \\
-6\end{array}$ & 12 & $(-54)$ & 15.0 & 13.6 \\
\hline $\mathrm{CC}_{\mathrm{Cl}}$ & $\begin{array}{l}3187 \\
3171 \\
\end{array}$ & $\begin{array}{c}12 \\
2 \\
\end{array}$ & $\begin{array}{l}3047 \\
3041 \\
\end{array}$ & $\begin{array}{c}21 \\
5 \\
\end{array}$ & $\begin{array}{c}-18 \\
-2 \\
\end{array}$ & $\begin{array}{l}-17 \\
-11 \\
\end{array}$ & 12 & $(-35)$ & 14.7 & 13.5 \\
\hline $\mathrm{CN}_{\mathrm{H}}$ & 3185 & 4 & 3038 & 2 & -16 & -8 & 21 & $(38)$ & 7.2 & 5.6 \\
\hline $\mathrm{CN}_{\mathrm{C}}$ & 3170 & 1 & 3022 & 2 & -1 & 8 & 9 & $(861)$ & 4.9 & 3.9 \\
\hline $\mathrm{CN}_{\mathrm{Cl}}$ & 3169 & 1 & 3068 & $(38)$ & 0 & -38 & 4 & $(517)$ & 3.0 & 2.2 \\
\hline $\mathrm{CN}_{\mathrm{H}} \mathrm{N}_{\mathrm{H}}$ & 3199 & 9 & 3048 & 2 & -30 & -18 & 11 & $(633)$ & 14.8 & 11.7 \\
\hline $\mathrm{CN}_{\mathrm{H}} \mathrm{N}_{\mathrm{C}}$ & 3185 & 5 & 3052 & 6 & -16 & -22 & 9 & $(921)$ & 12.2 & 9.6 \\
\hline $\mathrm{CN}_{\mathrm{C}} \mathrm{N}_{\mathrm{Cl}}$ & 3170 & 1 & 3041 & 3 & -1 & -11 & 18 & 5 & 10.4 & 8.1 \\
\hline $\mathrm{CN}_{\mathrm{H}} \mathrm{N}_{\mathrm{Cl}}$ & 3184 & 4 & 3022 & 3 & -15 & 8 & 3 & 3556 & 10.3 & 7.9 \\
\hline $\mathrm{CN}_{\mathrm{H}} \mathrm{N}_{\mathrm{H}} \mathrm{N}_{\mathrm{H}}$ & 3216 & 17 & 3078 & $(726540)$ & -47 & -48 & 10 & $(-3326)$ & 22.8 & 18.4 \\
\hline $\mathrm{CN}_{\mathrm{H}} \mathrm{N}_{\mathrm{H}} \mathrm{N}_{\mathrm{C}}$ & 3199 & 10 & 3053 & 12 & -30 & -23 & 8 & $(-536)$ & 19.8 & 15.8 \\
\hline $\mathrm{CN}_{\mathrm{H}} \mathrm{N}_{\mathrm{Cl}} \mathrm{N}_{\mathrm{C}}$ & 3180 & 3 & - & - & -11 & - & 7 & - & 18.4 & 14.6 \\
\hline $\mathrm{CN}_{\mathrm{H}} \mathrm{N}_{\mathrm{Cl}} \mathrm{N}_{\mathrm{Cl}}$ & 3183 & 4 & - & - & -14 & - & 7 & - & 17.3 & 13.4 \\
\hline $\mathrm{CN}_{\mathrm{C}} \mathrm{N}_{\mathrm{Cl}} \mathrm{N}_{\mathrm{Cl}}$ & 3170 & 1 & - & - & -1 & - & 13 & - & 15.8 & 12.4 \\
\hline $\mathrm{C}_{\mathrm{Cl}} \mathrm{C}_{\mathrm{Cl}} \mathrm{N}_{\mathrm{H}}$ & $\begin{array}{l}3201 \\
3174 \\
\end{array}$ & $\begin{array}{c}19 \\
5 \\
\end{array}$ & $\begin{array}{l}3056 \\
3036 \\
\end{array}$ & $\begin{array}{c}19 \\
5 \\
\end{array}$ & $\begin{array}{c}-18 \\
0 \\
\end{array}$ & $\begin{array}{c}-17 \\
-2 \\
\end{array}$ & 10 & (138) & 26.6 & 23.3 \\
\hline $\mathrm{C}_{\mathrm{Cl}} \mathrm{N}_{\mathrm{H}} \mathrm{C}_{\mathrm{Cl}}$ & $\begin{array}{l}3189 \\
3180\end{array}$ & $\begin{array}{c}9 \\
13\end{array}$ & $\begin{array}{l}3045 \\
3043\end{array}$ & $\begin{array}{c}9 \\
11\end{array}$ & $\begin{array}{l}-6 \\
-6\end{array}$ & $\begin{array}{c}-11 \\
-9\end{array}$ & 9 & $(-199)$ & 26.3 & 23.1 \\
\hline
\end{tabular}


A. Appendix

\begin{tabular}{|c|c|c|c|c|c|c|c|c|c|c|}
\hline Structure & $\omega_{\mathrm{CH}}$ & $S_{\omega}$ & $\tilde{\nu}_{\mathrm{CH}}$ & $S_{\nu}$ & $\Delta \omega_{\mathrm{CH}}$ & $\Delta \tilde{\nu}_{\mathrm{CH}}$ & $\omega_{l}$ & $\tilde{\nu}_{1}$ & $D_{\mathrm{e}}$ & $D_{0}$ \\
\hline \multirow{2}{*}{$\mathrm{CN}_{\mathrm{H}} \mathrm{C}_{\mathrm{Cl}}$} & 3200 & 16 & 3045 & 11 & -13 & 2 & \multirow{2}{*}{13} & \multirow{2}{*}{$(324)$} & \multirow{2}{*}{23.8} & \multirow{2}{*}{20.8} \\
\hline & 3170 & 2 & 3027 & 3 & 1 & 14 & & & & \\
\hline \multirow{2}{*}{$\mathrm{C}_{\mathrm{Cl}} \mathrm{N}_{\mathrm{C}} \mathrm{C}_{\mathrm{Cl}}$} & 3184 & 13 & 3027 & 2 & -1 & 12 & \multirow{2}{*}{2} & \multirow{2}{*}{$(-2490)$} & \multirow{2}{*}{22.7} & \multirow{2}{*}{20.1} \\
\hline & 3175 & 5 & 3043 & 12 & -1 & -9 & & & & \\
\hline \multirow{2}{*}{$\mathrm{C}_{\mathrm{Cl}} \mathrm{C}_{\mathrm{Cl}} \mathrm{N}_{\mathrm{C}}$} & 3184 & 14 & 3043 & 17 & -1 & -4 & \multirow{2}{*}{3} & \multirow{2}{*}{$(-312)$} & \multirow{2}{*}{22.6} & \multirow{2}{*}{20.0} \\
\hline & 3174 & 5 & 3032 & 5 & 0 & 2 & & & & \\
\hline \multirow{2}{*}{$\mathrm{CC}_{\mathrm{C}} \mathrm{N}_{\mathrm{H}}$} & 3186 & 16 & 3044 & 5 & -1 & 0 & \multirow{2}{*}{9} & \multirow{2}{*}{$(-227)$} & \multirow{2}{*}{22.5} & \multirow{2}{*}{19.6} \\
\hline & 3185 & 5 & 3045 & 106 & -15 & -9 & & & & \\
\hline \multirow{2}{*}{$\mathrm{CC}_{\mathrm{C}} \mathrm{N}_{\mathrm{H}}$} & 3187 & 16 & 3048 & 14 & -2 & -4 & \multirow{2}{*}{10} & \multirow{2}{*}{$(-121)$} & \multirow{2}{*}{22.2} & \multirow{2}{*}{19.5} \\
\hline & 3186 & 2 & 3052 & 4 & -1 & -16 & & & & \\
\hline \multirow{2}{*}{$\mathrm{CN}_{\mathrm{H}} \mathrm{C}_{\mathrm{C}}$} & 3185 & 16 & 3047 & 5 & 0 & -3 & & & 223 & 194 \\
\hline & 3170 & 2 & 3037 & 4 & 0 & -1 & 13 & & 22.3 & 19.4 \\
\hline & 3182 & 12 & 3039 & 5 & 3 & 5 & & & & \\
\hline $\mathrm{CN}$ & 3170 & 2 & 3038 & 3 & 0 & -2 & 19 & $(61)$ & 22.1 & 19.4 \\
\hline $\mathrm{CN}_{\mathrm{CH}} \mathrm{C}$ & 3190 & 12 & 3034 & 20 & -5 & 10 & 9 & (116) & 215 & 191 \\
\hline $\cos c$ & 3170 & 1 & 3030 & 0 & 0 & 6 & $y$ & $(110)$ & 21.0 & 19.1 \\
\hline $\mathrm{C}_{C l} \mathrm{~N}$ & 3183 & 13 & 3044 & 17 & 0 & -5 & 3 & (297) & 214 & 190 \\
\hline 40 & 3174 & 5 & 3038 & 6 & 0 & -4 & $J$ & $(291)$ & 21.4 & 19.0 \\
\hline & 3187 & 13 & 3035 & 7 & -2 & 4 & 9 & & & \\
\hline $\mathrm{Cl}_{\mathrm{C}}$ & 3171 & 2 & 3031 & 0 & -1 & 5 & y & ) & 19.8 & 16.8 \\
\hline 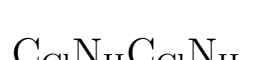 & 3199 & 18 & - & - & -16 & - & 0 & & & \\
\hline $\mathrm{Cl}^{+} \mathrm{H} \cup \mathrm{Cl}$ & 3189 & 10 & - & - & -15 & - & 9 & - & 35.4 & 30.6 \\
\hline & 3206 & 16 & - & - & -23 & - & & & & \\
\hline $\mathrm{C}_{\mathrm{Cl}} \mathrm{C}_{\mathrm{Cl}} \mathrm{N}_{\mathrm{H}} \mathrm{N}_{\mathrm{H}}$ & 3182 & 13 & - & - & -8 & - & 4 & - & 35.3 & 30.6 \\
\hline $\mathrm{C}_{C 1} \mathrm{~N}_{H} \mathrm{C}_{C 1} \mathrm{~N}_{C}$ & 3200 & 20 & - & - & -17 & - & & & & 286 \\
\hline $\mathrm{Cl}^{N_{\mathrm{H}}} \cup_{\mathrm{Cl}}{ }^{\mathrm{N}} \mathrm{C}$ & 3175 & 5 & - & - & -1 & - & 10 & - & 33.1 & 28.0 \\
\hline $\mathrm{CN}$ & 3198 & 16 & - & - & -11 & - & 12 & & 325 & 278 \\
\hline $\mathrm{H}_{\mathrm{H}}$ & 3183 & 5 & - & - & -9 & - & 12 & 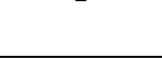 & 02.0 & 21.0 \\
\hline & 3201 & 20 & - & - & -18 & - & 7 & - & 17 & 27.5 \\
\hline $\mathrm{N}_{\mathrm{C}} \cup \mathrm{Cl} \mathrm{N}_{\mathrm{H}} \cup_{\mathrm{Cl}}$ & 3175 & 5 & - & - & -1 & - & $r$ & - & 31.8 & 26.5 \\
\hline & 3197 & 21 & - & - & -10 & - & & & 318 & 274 \\
\hline $\mathrm{CN}_{\mathrm{H}} \mathrm{U}$ & 3170 & 1 & - & - & 1 & - & 11 & - & 31.8 & 26.4 \\
\hline $\mathrm{C}_{8}$ & 3190 & 10 & - & - & -7 & - & 0 & & 316 & 273 \\
\hline $\mathrm{Cl}_{\mathrm{Cl}} \mathrm{Cl}_{\mathrm{H}} \mathrm{N}_{\mathrm{C}}$ & 3181 & 13 & - & - & -7 & - & 9 & - & 31.0 & 26.3 \\
\hline $\mathrm{N}_{\mathrm{C}} \mathrm{C}_{\mathrm{Cl}} \mathrm{C}_{\mathrm{Cl}} \mathrm{N}_{\mathrm{H}}$ & 3188 & 9 & - & - & -5 & - & 7 & - & 31.5 & 27.3 \\
\hline
\end{tabular}




\begin{tabular}{|c|c|c|c|c|c|c|c|c|c|c|}
\hline Structure & $\omega_{\mathrm{CH}}$ & $S_{\omega}$ & $\tilde{\nu}_{\mathrm{CH}}$ & $S_{\nu}$ & $\Delta \omega_{\mathrm{CH}}$ & $\Delta \tilde{\nu}_{\mathrm{CH}}$ & $\omega_{1}$ & $\tilde{\nu}_{1}$ & $D_{\mathrm{e}}$ & $D_{0}$ \\
\hline & 3180 & 13 & - & - & -6 & - & & & & \\
\hline \multirow{2}{*}{$\mathrm{CC}_{\mathrm{C}} \mathrm{N}_{\mathrm{H}} \mathrm{N}_{\mathrm{H}}$} & 3201 & 13 & - & - & -16 & - & \multirow{2}{*}{9} & & \multirow{2}{*}{30.0} & \multirow{2}{*}{26.0} \\
\hline & 3189 & 13 & - & - & -19 & - & & & & \\
\hline \multirow{2}{*}{$\mathrm{CN}_{\mathrm{H}} \mathrm{C}_{\mathrm{C}} \mathrm{N}_{\mathrm{H}}$} & 3185 & 2 & - & - & 0 & - & \multirow{2}{*}{10} & \multirow{2}{*}{ - } & \multirow{2}{*}{29.8} & \multirow{2}{*}{25.3} \\
\hline & 3184 & 21 & - & - & -14 & - & & & & \\
\hline \multirow{2}{*}{$\mathrm{CC}_{\mathrm{C}} \mathrm{N}_{\mathrm{H}} \mathrm{N}_{\mathrm{Cl}}$} & 3192 & 14 & - & - & -7 & - & \multirow{2}{*}{7} & \multirow{2}{*}{ - } & \multirow{2}{*}{29.0} & \multirow{2}{*}{25.1} \\
\hline & 3186 & 4 & - & - & -16 & - & & & & \\
\hline \multirow{2}{*}{$\mathrm{N}_{\mathrm{C}} \mathrm{CN}_{\mathrm{H}} \mathrm{C}_{\mathrm{Cl}}$} & 3186 & 17 & - & - & 1 & - & \multirow{2}{*}{10} & \multirow{2}{*}{ - } & \multirow{2}{*}{27.5} & \multirow{2}{*}{23.6} \\
\hline & 3170 & 2 & - & - & 1 & - & & & & \\
\hline \multirow{2}{*}{$\mathrm{N}_{\mathrm{C}} \mathrm{CC}_{\mathrm{Cl}} \mathrm{N}_{\mathrm{H}}$} & 3189 & 17 & - & - & -2 & - & \multirow{2}{*}{8} & \multirow{2}{*}{ - } & \multirow{2}{*}{27.3} & \multirow{2}{*}{23.6} \\
\hline & 3186 & 4 & - & - & -15 & - & & & & \\
\hline \multirow{2}{*}{$\mathrm{N}_{\mathrm{Cl}} \mathrm{C}_{\mathrm{Cl}} \mathrm{C}_{\mathrm{Cl}} \mathrm{N}_{\mathrm{C}}$} & 3182 & 13 & - & - & 1 & - & 1 & & 258 & 22.4 \\
\hline & 3175 & 5 & - & - & -1 & - & 1 & - & 20.0 & 22.4 \\
\hline $\mathrm{C}_{\mathrm{Cl}} \mathrm{N}_{\mathrm{H}} \mathrm{C}_{\mathrm{Cl}} \mathrm{N}_{\mathrm{H}} \mathrm{N}_{\mathrm{H}}$ & 3206 & 17 & - & - & -23 & - & 7 & & 444 & \\
\hline 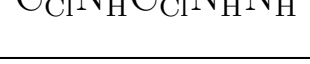 & 3200 & 18 & - & - & -26 & - & $r$ & - & 44.4 & 38.0 \\
\hline 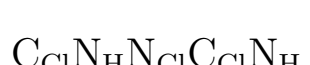 & 3198 & 18 & - & - & -15 & - & 11 & - & 431 & 36.9 \\
\hline 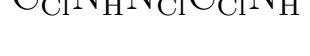 & 3189 & 10 & - & - & -15 & - & 11 & - & 40.1 & 0.9 \\
\hline $\mathrm{C}_{\mathrm{Cl}} \mathrm{N}_{\mathrm{H}} \mathrm{C}_{\mathrm{Cl}} \mathrm{N}_{\mathrm{H}} \mathrm{N}_{\mathrm{C}}$ & 3198 & 19 & - & - & -15 & - & 10 & & 420 & 359 \\
\hline 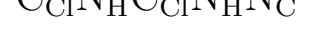 & 3190 & 10 & - & - & -16 & - & 10 & - & 42.0 & 30.9 \\
\hline $\mathrm{C}_{\mathrm{C}} \mathrm{N}_{\mathrm{H}} \mathrm{C}_{\mathrm{C}} \mathrm{N}_{\mathrm{H}} \mathrm{N}_{\mathrm{C}}$ & 3201 & 19 & - & - & -18 & - & 10 & & 410 & 358 \\
\hline $\mathrm{U}_{\mathrm{Cl}} \mathrm{N}_{\mathrm{H}}$ & 3190 & 9 & - & - & -16 & - & 10 & - & 41.9 & 0.0 .0 \\
\hline $\mathrm{C}_{\mathrm{Cl}_{1}} \mathrm{C}_{\mathrm{ll}} \mathrm{N}_{\mathrm{N}} \mathrm{N}_{\mathrm{H}} \mathrm{N}_{\mathrm{H}}$ & 3206 & 15 & - & - & -23 & - & 4 & - & 410 & 35.1 \\
\hline $\mathrm{C}_{\mathrm{Cl}} \mathrm{Cl}_{\mathrm{Cl}} \mathrm{N}_{\mathrm{H}} \mathrm{N}_{\mathrm{H}} \mathrm{N}_{\mathrm{Cl}}$ & 3182 & 13 & - & - & -8 & - & 4 & - & 41.0 & 0.11 \\
\hline 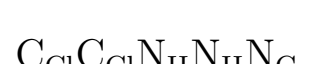 & 3207 & 17 & - & - & -24 & - & 4 & & 406 & 34.9 \\
\hline$\cup_{\mathrm{Cl}} \cup_{\mathrm{Cl}} \mathrm{N}_{\mathrm{H}} \mathrm{N}_{\mathrm{H}} \mathrm{N}_{\mathrm{C}}$ & 3183 & 14 & - & - & -9 & - & 4 & - & 40.0 & 34.9 \\
\hline $\mathrm{N}_{G} \mathrm{C}_{4} \mathrm{~N}_{H} \mathrm{C}_{\mathrm{C}} \mathrm{N}_{H}$ & 3198 & 19 & - & - & -15 & - & 8 & & 407 & 348 \\
\hline 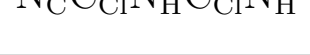 & 3189 & 10 & - & - & -15 & - & 0 & & 0.1 & (0.0 \\
\hline $\mathrm{N}_{C} \mathrm{C}_{\mathrm{Cl}} \mathrm{C}_{\mathrm{Cl}} \mathrm{N}_{\mathrm{H}} \mathrm{N}_{\mathrm{H}}$ & 3206 & 16 & - & - & -23 & - & 6 & - & 404 & 348 \\
\hline $\mathrm{N}_{\mathrm{C}} \mathrm{Cll}_{\mathrm{Cl}} \mathrm{Cl}_{\mathrm{H}} \mathrm{N}_{\mathrm{H}}$ & 3182 & 14 & - & - & -8 & - & 0 & - & 40.4 & \\
\hline $\mathrm{N}_{C} \mathrm{C}_{C} \mathrm{~N}_{\mathrm{H}} \mathrm{C}_{\mathrm{C}} \mathrm{N}_{\mathrm{H}}$ & 3194 & 18 & - & - & -11 & - & 8 & & 407 & 347.7 \\
\hline $\mathbb{N}_{\mathrm{C}} \cup \mathrm{Cl}^{1} \mathrm{~N}_{\mathrm{H}} \cup \mathrm{Cl}^{\mathbb{N}}$ & 3182 & 10 & - & - & -8 & - & 0 & - & 40.1 & 34.1 \\
\hline 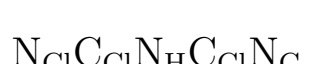 & 3197 & 19 & - & - & -14 & - & 8 & - & 396 & 339 \\
\hline 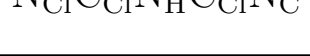 & 3174 & 4 & - & - & 0 & - & 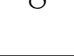 & & 0.0 & \\
\hline $\mathrm{N}_{\mathrm{C}} \mathrm{C}_{\mathrm{Cl}} \mathrm{N}_{\mathrm{H}} \mathrm{C}_{\mathrm{Cl}} \mathrm{N}_{\mathrm{C}}$ & 3200 & 21 & - & - & -17 & - & 7 & - & 38.2 & 32.8 \\
\hline & 3175 & 5 & - & - & -1 & - & 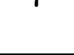 & & & \\
\hline
\end{tabular}




\begin{tabular}{ccccccccccc} 
Structure & $\omega_{\mathrm{CH}}$ & $S_{\omega}$ & $\tilde{\nu}_{\mathrm{CH}}$ & $S_{\nu}$ & $\Delta \omega_{\mathrm{CH}}$ & $\Delta \tilde{\nu}_{\mathrm{CH}}$ & $\omega_{\mathrm{l}}$ & $\tilde{\nu}_{\mathrm{l}}$ & $D_{\mathrm{e}}$ & $D_{0}$ \\
\hline $\mathrm{N}_{\mathrm{C}} \mathrm{C}_{\mathrm{Cl}} \mathrm{C}_{\mathrm{Cl}} \mathrm{N}_{\mathrm{H}} \mathrm{N}_{\mathrm{C}}$ & 3189 & 10 & - & - & -6 & - & & & & \\
& 3181 & 13 & - & - & -7 & - & 7 & - & 36.7 & 31.5 \\
\hline
\end{tabular}

Table A.27.: Theoretically predicted harmonic $\left(\omega_{\mathrm{CCl}}\right)$ and anharmonic $\left(\tilde{\nu}_{\mathrm{CCl}}\right) \mathrm{CCl}$ stretching wavenumber in $\mathrm{cm}^{-1}$, IR intensity $(S)$ in $\mathrm{km} \mathrm{mol}^{-1}$, spectroscopic downshift $\left(\Delta \omega_{\mathrm{CCl}}\right)$ relative to the corresponding vibration in the pure $\mathrm{C}$ monomers or $\mathrm{CC}$ dimers in $\mathrm{cm}^{-1}$, lowest predicted harmonic $\left(\omega_{1}\right)$ and anharmonic $\left(\tilde{\nu}_{1}\right)$ wavenumbers, dissociation energy into the most stable monomers without $\left(D_{\mathrm{e}}\right)$ and with $\left(D_{0}\right)$ harmonic vibrational zero-point energy in $\mathrm{kJ} \mathrm{mol}^{-1}$. All properties were calculated at the B3LYP-D3(BJ)/def2QZVP level of theory. Anharmonic vibrational frequencies calculated with second order vibrational perturbation theory (Gaussian09 Rev. E01, ${ }^{141} \mathrm{VPT} 2{ }^{148}$ ) are unreliable due to unphysical predictions for low frequency large amplitude motions which affect localised high frequency modes like the $\mathrm{CH}$ stretching vibration through their respective coupling constants.

\begin{tabular}{|c|c|c|c|c|c|c|c|c|c|}
\hline Structure & $\omega_{\mathrm{CCl}}$ & $S_{\omega}$ & $\tilde{\nu}_{\mathrm{CCl}}$ & $S_{\nu}$ & $\Delta \omega_{\mathrm{CCl}}$ & $\omega_{1}$ & $\tilde{\nu}_{1}$ & $D_{\mathrm{e}}$ & $D_{0}$ \\
\hline \multirow{2}{*}{$\mathrm{C}$} & 733 & 161 & 699 & 161 & \multirow{2}{*}{ - } & \multirow{2}{*}{258} & \multirow{2}{*}{249} & \multirow{2}{*}{-} & \multirow{2}{*}{ - } \\
\hline & 733 & 161 & 727 & 168 & & & & & \\
\hline \multirow{4}{*}{$\mathrm{C}_{\mathrm{Cl}} \mathrm{C}_{\mathrm{Cl}}$} & 744 & 228 & 725 & 203 & -11 & \multirow{4}{*}{2} & \multirow{4}{*}{$(-2876)$} & \multirow{4}{*}{17.6} & \multirow{4}{*}{16.0} \\
\hline & 742 & 123 & 735 & 25 & -9 & & & & \\
\hline & 725 & 220 & 706 & 195 & 8 & & & & \\
\hline & 723 & 38 & 701 & 53 & 10 & & & & \\
\hline \multirow{4}{*}{$\mathrm{CC}_{\mathrm{C}}$} & 740 & 260 & 722 & 254 & -7 & \multirow{4}{*}{12} & \multirow{4}{*}{$(-54)$} & \multirow{4}{*}{15.0} & \multirow{4}{*}{13.6} \\
\hline & 737 & 261 & 718 & 279 & -4 & & & & \\
\hline & 734 & 28 & 716 & 28 & -1 & & & & \\
\hline & 722 & 16 & 702 & 28 & 11 & & & & \\
\hline \multirow{4}{*}{$\mathrm{CC}_{\mathrm{Cl}}$} & 738 & 243 & 719 & 214 & -5 & \multirow{4}{*}{12} & \multirow{4}{*}{$(-35)$} & \multirow{4}{*}{14.7} & \multirow{4}{*}{13.5} \\
\hline & 738 & 263 & 721 & 218 & -5 & & & & \\
\hline & 729 & 15 & 712 & 44 & 4 & & & & \\
\hline & 723 & 23 & 704 & 28 & 10 & & & & \\
\hline \multirow{2}{*}{$\mathrm{CN}_{\mathrm{H}}$} & 736 & 169 & 716 & 165 & -3 & \multirow{2}{*}{21} & \multirow{2}{*}{ (38) } & \multirow{2}{*}{7.2} & \multirow{2}{*}{5.6} \\
\hline & 732 & 153 & 714 & 151 & 1 & & & & \\
\hline \multirow{2}{*}{$\mathrm{CN}_{\mathrm{C}}$} & 733 & 150 & 720 & 165 & 0 & \multirow{2}{*}{9} & \multirow{2}{*}{$(861)$} & \multirow{2}{*}{4.9} & \multirow{2}{*}{3.9} \\
\hline & 732 & 155 & 716 & 170 & 1 & & & & \\
\hline \multirow{2}{*}{$\mathrm{CN}_{\mathrm{Cl}}$} & 735 & 166 & 706 & 110 & -2 & \multirow{2}{*}{4} & & & \\
\hline & 732 & 161 & 709 & 104 & 1 & & $(5 \perp 7)$ & 3.0 & 2.2 \\
\hline & 737 & 167 & 714 & 147 & -4 & 11 & (633) & 148 & 117 \\
\hline $\mathrm{CN}_{\mathrm{H}^{1}} \mathrm{~N}_{\mathrm{H}}$ & 732 & 155 & 713 & 159 & 1 & 11 & (1003) & 14.0 & 11.1 \\
\hline
\end{tabular}




\section{A. Appendix}

\begin{tabular}{|c|c|c|c|c|c|c|c|c|c|}
\hline Structure & $\omega_{\mathrm{CCl}}$ & $S_{\omega}$ & $\tilde{\nu}_{\mathrm{CCl}}$ & $S_{\nu}$ & $\Delta \omega_{\mathrm{CCl}}$ & $\omega_{1}$ & $\tilde{\nu}_{1}$ & $D_{\mathrm{e}}$ & $D_{0}$ \\
\hline \multirow{2}{*}{$\mathrm{CN}_{\mathrm{H}} \mathrm{N}_{\mathrm{C}}$} & 736 & 158 & 716 & 133 & -3 & \multirow{2}{*}{9} & \multirow{2}{*}{$(921)$} & \multirow{2}{*}{12.2} & \multirow{2}{*}{9.6} \\
\hline & 731 & 147 & 710 & 128 & 2 & & & & \\
\hline \multirow{2}{*}{$\mathrm{CN}_{\mathrm{C}} \mathrm{N}_{\mathrm{Cl}}$} & 733 & 151 & 712 & 137 & 0 & \multirow{2}{*}{18} & \multirow{2}{*}{5} & \multirow{2}{*}{10.4} & \multirow{2}{*}{8.1} \\
\hline & 732 & 146 & 711 & 133 & 1 & & & & \\
\hline \multirow{2}{*}{$\mathrm{CN}_{\mathrm{H}} \mathrm{N}_{\mathrm{Cl}}$} & 738 & 174 & 725 & 202 & -5 & \multirow{2}{*}{3} & \multirow{2}{*}{$(3556)$} & \multirow{2}{*}{10.3} & \multirow{2}{*}{7.9} \\
\hline & 731 & 153 & 719 & 179 & 2 & & & & \\
\hline \multirow{2}{*}{$\mathrm{CN}_{\mathrm{H}} \mathrm{N}_{\mathrm{H}} \mathrm{N}_{\mathrm{H}}$} & 735 & 160 & $(2487)$ & $(59829)$ & -2 & \multirow{2}{*}{10} & \multirow{2}{*}{$(-3326)$} & \multirow{2}{*}{22.8} & \multirow{2}{*}{18.4} \\
\hline & 735 & 160 & $(-1058)$ & $(25455)$ & -2 & & & & \\
\hline \multirow{2}{*}{$\mathrm{CN}_{\mathrm{H}} \mathrm{N}_{\mathrm{H}} \mathrm{N}_{\mathrm{C}}$} & 736 & 160 & 716 & 131 & -3 & \multirow{2}{*}{8} & \multirow{2}{*}{$(-536)$} & \multirow{2}{*}{19.8} & \multirow{2}{*}{15.8} \\
\hline & 732 & 144 & 716 & 128 & 1 & & & & \\
\hline \multirow{2}{*}{$\mathrm{CN}_{\mathrm{H}} \mathrm{N}_{\mathrm{Cl}} \mathrm{N}_{\mathrm{C}}$} & 737 & 170 & - & - & -4 & 7 & & & \\
\hline & 730 & 133 & - & - & 3 & $r$ & - & 18.4 & 14.6 \\
\hline & 737 & 178 & - & - & -4 & & & & \\
\hline $\mathrm{CN}_{\mathrm{H}} \mathrm{N}_{\mathrm{Cl}} \mathrm{N}_{\mathrm{Cl}}$ & 732 & 142 & - & - & 1 & 7 & - & 17.3 & 13.4 \\
\hline $\mathrm{CN}_{\mathrm{C}} \mathrm{N}_{\mathrm{C}} \mathrm{N}_{\mathrm{C}}$ & 733 & 149 & - & - & 0 & & & 158 & 124 \\
\hline $\mathrm{CN}_{\mathrm{CN}} \mathrm{N}_{\mathrm{Cl}} \mathrm{N}_{\mathrm{Cl}}$ & 732 & 139 & - & - & 1 & 13 & - & 15.8 & 12.4 \\
\hline & 744 & 193 & 727 & 212 & 0 & & & & \\
\hline & 741 & 151 & 724 & 120 & 1 & & $(138)$ & & 233 \\
\hline $\mathrm{Cl}_{\mathrm{Cl}}$ & 727 & 208 & 710 & 184 & -2 & 10 & $(138)$ & 26.6 & 23.3 \\
\hline & 723 & 53 & 705 & 59 & 0 & & & & \\
\hline & 744 & 207 & 728 & 281 & 0 & & & & \\
\hline & 742 & 142 & 726 & 112 & 0 & 0 & $(-100)$ & 263 & 221 \\
\hline $\mathrm{C}_{\mathrm{Cl}} \mathrm{N}$ & 726 & 205 & 710 & 183 & -1 & $y$ & $(-199)$ & 20.3 & 23.1 \\
\hline & 723 & 54 & 708 & 50 & 0 & & & & \\
\hline & 741 & 292 & 723 & 401 & -3 & & & & \\
\hline$C N-C$ & 737 & 178 & 723 & 648 & 1 & 12 & $(2,4)$ & 228 & 208 \\
\hline $\mathrm{N}_{\mathrm{H}} \cup \mathrm{Cl}$ & 734 & 81 & 716 & 88 & -5 & 13 & $(324)$ & 23.8 & 20.8 \\
\hline & 722 & 14 & 707 & 56 & 1 & & & & \\
\hline & 744 & 221 & 729 & 198 & 0 & & & & \\
\hline & 742 & 113 & 734 & 45 & 0 & 2 & h) & 20 & 1 \\
\hline $\mathrm{C}_{\mathrm{Cl}} \mathrm{N}_{\mathrm{C}} \mathrm{C}_{\mathrm{Cl}}$ & 725 & 217 & 707 & 288 & 0 & 2 & $(-2490)$ & 22.1 & 20.1 \\
\hline & 722 & 38 & 704 & 63 & 1 & & & & \\
\hline & 743 & 211 & 725 & 567 & 1 & & & & \\
\hline & 742 & 139 & 731 & 508 & 0 & 3 & & 226 & 200 \\
\hline $\mathrm{C}_{\mathrm{Cl}} \mathrm{Cl}_{\mathrm{Cl}} \mathrm{N}_{\mathrm{C}}$ & 725 & 204 & 707 & 216 & 0 & 3 & $(-312)$ & 22.0 & 20.0 \\
\hline & 722 & 39 & 705 & 123 & 1 & & & & \\
\hline
\end{tabular}


A. Appendix

\begin{tabular}{|c|c|c|c|c|c|c|c|c|c|}
\hline Structure & $\omega_{\mathrm{CCl}}$ & $S_{\omega}$ & $\tilde{\nu}_{\mathrm{CCl}}$ & $S_{\nu}$ & $\Delta \omega_{\mathrm{CCl}}$ & $\omega_{1}$ & $\tilde{\nu}_{1}$ & $D_{\mathrm{e}}$ & $D_{0}$ \\
\hline \multirow{4}{*}{$\mathrm{CC}_{\mathrm{C}} \mathrm{N}_{\mathrm{H}}$} & 741 & 263 & 721 & 452 & -1 & \multirow{4}{*}{9} & \multirow{4}{*}{$(-227)$} & \multirow{4}{*}{22.5} & \multirow{4}{*}{19.6} \\
\hline & 737 & 261 & 717 & 200 & 0 & & & & \\
\hline & 734 & 27 & 716 & 265 & 0 & & & & \\
\hline & 723 & 15 & 688 & 610 & -1 & & & & \\
\hline \multirow{4}{*}{$\mathrm{CC}_{\mathrm{C}} \mathrm{N}_{\mathrm{H}}$} & 739 & 261 & 721 & 255 & 1 & \multirow{4}{*}{10} & \multirow{4}{*}{$(-121)$} & \multirow{4}{*}{22.2} & \multirow{4}{*}{19.5} \\
\hline & 738 & 251 & 723 & 235 & -1 & & & & \\
\hline & 729 & 12 & 712 & 16 & 5 & & & & \\
\hline & 724 & 19 & 707 & 27 & -2 & & & & \\
\hline \multirow{4}{*}{$\mathrm{CN}_{\mathrm{H}} \mathrm{C}_{\mathrm{C}}$} & 742 & 247 & 724 & 258 & -2 & \multirow{4}{*}{13} & \multirow{4}{*}{$(-152)$} & \multirow{4}{*}{22.3} & \multirow{4}{*}{19.4} \\
\hline & 735 & 171 & 718 & 143 & 2 & & & & \\
\hline & 731 & 110 & 714 & 103 & 3 & & & & \\
\hline & 723 & 33 & 707 & 48 & -1 & & & & \\
\hline \multirow{4}{*}{$\mathrm{CN}_{\mathrm{Cl}} \mathrm{C}_{\mathrm{C}}$} & 740 & 241 & 725 & 231 & 0 & \multirow{4}{*}{19} & \multirow{4}{*}{ (61) } & \multirow{4}{*}{22.1} & \multirow{4}{*}{19.4} \\
\hline & 737 & 272 & 718 & 257 & 0 & & & & \\
\hline & 734 & 24 & 717 & 30 & 0 & & & & \\
\hline & 722 & 14 & 704 & 35 & 0 & & & & \\
\hline \multirow{4}{*}{$\mathrm{CN}_{\mathrm{Cl}} \mathrm{C}_{\mathrm{C}}$} & 740 & 264 & 728 & 601 & 0 & \multirow{4}{*}{9} & \multirow{4}{*}{ (116) } & \multirow{4}{*}{21.5} & \multirow{4}{*}{19.1} \\
\hline & 737 & 233 & 727 & 234 & 0 & & & & \\
\hline & 730 & 19 & 719 & 577 & 4 & & & & \\
\hline & 722 & 27 & 704 & 194 & 0 & & & & \\
\hline & 744 & 222 & 700 & 377 & 0 & & & & \\
\hline & 742 & 122 & 731 & 93 & 0 & 2 & & & \\
\hline $\mathrm{C}_{\mathrm{Cl}} \mathrm{N}_{\mathrm{Cl}} \mathrm{Cl}_{\mathrm{Cl}}$ & 725 & 207 & 713 & 207 & 0 & 3 & $(291)$ & 21.4 & 19.0 \\
\hline & 723 & 49 & 698 & 48 & 0 & & & & \\
\hline & 738 & 252 & 722 & 379 & 2 & & & & \\
\hline $\mathrm{CN}_{\mathrm{C}} \mathrm{C}_{\mathrm{H}}$ & 738 & 238 & 724 & 278 & 1 & 9 & $(-263)$ & 198 & 177 \\
\hline $\mathrm{N}_{\mathrm{C}} \cup \mathrm{C}$ & 729 & 15 & 713 & 142 & 5 & 9 & $(-203)$ & 19.8 & 16.8 \\
\hline & 722 & 21 & 705 & 25 & 1 & & & & \\
\hline & 743 & 180 & - & - & 1 & & & & \\
\hline $\mathrm{C}_{\mathrm{Cl}} \mathrm{N}_{\mathrm{H}} \mathrm{C}_{\mathrm{Cl}} \mathrm{N}_{\mathrm{H}}$ & 741 & 160 & - & - & 1 & 0 & & 354 & 306 \\
\hline$\cup_{\mathrm{Cl}} \mathrm{N}_{\mathrm{H}} \cup_{\mathrm{Cl}} \mathrm{N}_{\mathrm{H}}$ & 727 & 215 & - & - & -2 & 9 & - & 30.4 & 30.0 \\
\hline & 723 & 51 & - & - & 0 & & & & \\
\hline & 744 & 222 & - & - & 0 & & & & \\
\hline $\mathrm{C}_{\mathrm{Cl}} \mathrm{C}_{\mathrm{Cl}} \mathrm{N}_{\mathrm{U}} \mathrm{N}_{\mathrm{H}}$ & 742 & 127 & - & - & 0 & 4 & & 353 & 306 \\
\hline$\cup_{\mathrm{Cl}} \cup \mathrm{Cl}^{N} \mathrm{H}^{N}{ }_{\mathrm{H}}$ & 726 & 211 & - & - & -1 & 4 & - & 35.3 & 30.0 \\
\hline & 724 & 51 & - & - & -1 & & & & \\
\hline & 743 & 191 & - & - & 1 & & & & \\
\hline $\mathrm{C}_{\mathrm{Cl}} \mathrm{N}_{\mathrm{H}} \mathrm{C}_{\mathrm{Cl}} \mathrm{N}_{\mathrm{C}}$ & 741 & 133 & - & - & 1 & 10 & - & 33.1 & 28.6 \\
\hline
\end{tabular}




\section{A. Appendix}

\begin{tabular}{|c|c|c|c|c|c|c|c|c|c|}
\hline Structure & $\omega_{\mathrm{CCl}}$ & $S_{\omega}$ & $\tilde{\nu}_{\mathrm{CCl}}$ & $S_{\nu}$ & $\Delta \omega_{\mathrm{CCl}}$ & $\omega_{1}$ & $\tilde{\nu}_{1}$ & $D_{\mathrm{e}}$ & $D_{0}$ \\
\hline & 727 & 211 & - & - & -2 & & & & \\
\hline & 722 & 50 & - & - & 1 & & & & \\
\hline \multirow{4}{*}{$\mathrm{CN}_{\mathrm{H}} \mathrm{C}_{\mathrm{Cl}} \mathrm{N}_{\mathrm{H}}$} & 742 & 272 & - & - & -4 & \multirow{4}{*}{12} & \multirow{4}{*}{-} & \multirow{4}{*}{32.5} & \multirow{4}{*}{27.8} \\
\hline & 737 & 131 & - & - & 1 & & & & \\
\hline & 735 & 142 & - & - & -6 & & & & \\
\hline & 721 & 17 & - & - & 2 & & & & \\
\hline \multirow{4}{*}{$\mathrm{N}_{\mathrm{C}} \mathrm{C}_{\mathrm{Cl}} \mathrm{N}_{\mathrm{H}} \mathrm{C}_{\mathrm{Cl}}$} & 743 & 172 & - & - & 1 & \multirow{4}{*}{7} & \multirow{4}{*}{-} & \multirow{4}{*}{31.7} & \multirow{4}{*}{27.5} \\
\hline & 741 & 170 & - & - & 1 & & & & \\
\hline & 726 & 198 & - & - & -1 & & & & \\
\hline & 722 & 48 & - & - & 1 & & & & \\
\hline \multirow{4}{*}{$\mathrm{CN}_{\mathrm{H}} \mathrm{C}_{\mathrm{Cl}} \mathrm{N}_{\mathrm{Cl}}$} & 740 & 260 & - & - & -2 & \multirow{4}{*}{11} & \multirow{4}{*}{-} & \multirow{4}{*}{31.8} & \multirow{4}{*}{27.4} \\
\hline & 737 & 194 & - & - & 1 & & & & \\
\hline & 732 & 107 & - & - & -3 & & & & \\
\hline & 725 & 10 & - & - & -2 & & & & \\
\hline \multirow{4}{*}{$\mathrm{C}_{\mathrm{Cl}} \mathrm{C}_{\mathrm{Cl}} \mathrm{N}_{\mathrm{H}} \mathrm{N}_{\mathrm{C}}$} & 744 & 208 & - & - & 0 & \multirow{4}{*}{9} & \multirow{4}{*}{-} & \multirow{4}{*}{31.6} & \multirow{4}{*}{27.3} \\
\hline & 742 & 124 & - & - & 0 & & & & \\
\hline & 726 & 208 & - & - & -1 & & & & \\
\hline & 722 & 51 & - & - & 1 & & & & \\
\hline \multirow{4}{*}{$\mathrm{N}_{\mathrm{C}} \mathrm{C}_{\mathrm{Cl}} \mathrm{C}_{\mathrm{Cl}} \mathrm{N}_{\mathrm{H}}$} & 743 & 159 & - & - & 1 & \multirow{4}{*}{7} & \multirow{4}{*}{-} & \multirow{4}{*}{31.5} & \multirow{4}{*}{27.3} \\
\hline & 742 & 186 & - & - & 0 & & & & \\
\hline & 726 & 185 & - & - & -1 & & & & \\
\hline & 722 & 63 & - & - & 1 & & & & \\
\hline & 740 & 257 & - & - & 0 & & & & \\
\hline $\mathrm{CC}_{C} \mathrm{~N}_{U} \mathrm{~N}_{U}$ & 738 & 235 & - & - & -1 & 0 & - & 300 & 260 \\
\hline $\mathrm{C}_{\mathrm{C}} \mathrm{N}_{\mathrm{H}} \mathrm{N}_{\mathrm{H}}$ & 730 & 23 & - & - & 4 & $y$ & - & 30.0 & 20.0 \\
\hline & 722 & 24 & - & - & 0 & & & & \\
\hline & 741 & 247 & - & - & -1 & & & & \\
\hline $\mathrm{CN}_{\mathrm{N}} \mathrm{C}_{\odot} \mathrm{N}_{2}$ & 735 & 201 & - & - & 2 & 10 & & 208 & 253 \\
\hline$N_{H}$ & 730 & 94 & - & - & 4 & 10 & - & 29.0 & 20.0 \\
\hline & 725 & 20 & - & - & -3 & & & & \\
\hline & 742 & 260 & - & - & -2 & & & & \\
\hline$C C \mathrm{~N}_{\mathrm{N}} \mathrm{N}$ & 737 & 221 & - & - & 0 & 7 & & 200 & 251 \\
\hline $\mathrm{C}_{\mathrm{C}} \mathrm{N}_{\mathrm{H}} \mathrm{N}_{\mathrm{Cl}}$ & 731 & 29 & - & - & 3 & 1 & - & 29.0 & 20.1 \\
\hline & 721 & 31 & - & - & 1 & & & & \\
\hline & 742 & 237 & - & - & -4 & & & & \\
\hline & 734 & 165 & - & - & 4 & 10 & $-{ }_{-1}+2$ & 275 & 236 \\
\hline $\mathrm{N}_{\mathrm{C}} \mathrm{CN} \mathrm{N}_{\mathrm{H}} \mathrm{Cl}_{\mathrm{Cl}}$ & 731 & 111 & - & - & -2 & 10 & - & 21.5 & 23.0 \\
\hline & 723 & 31 & - & - & 0 & & & & \\
\hline
\end{tabular}




\section{A. Appendix}

\begin{tabular}{|c|c|c|c|c|c|c|c|c|c|}
\hline Structure & $\omega_{\mathrm{CCl}}$ & $S_{\omega}$ & $\tilde{\nu}_{\mathrm{CCl}}$ & $S_{\nu}$ & $\Delta \omega_{\mathrm{CCl}}$ & $\omega_{1}$ & $\tilde{\nu}_{1}$ & $D_{\mathrm{e}}$ & $D_{0}$ \\
\hline \multirow{4}{*}{$\mathrm{N}_{\mathrm{C}} \mathrm{CC}_{\mathrm{Cl}} \mathrm{N}_{\mathrm{H}}$} & 740 & 248 & - & - & -2 & \multirow{4}{*}{8} & \multirow{4}{*}{-} & \multirow{4}{*}{27.3} & \multirow{4}{*}{23.6} \\
\hline & 738 & 221 & - & - & 0 & & & & \\
\hline & 729 & 28 & - & - & 0 & & & & \\
\hline & 721 & 28 & - & - & 2 & & & & \\
\hline \multirow{4}{*}{$\mathrm{N}_{\mathrm{Cl}} \mathrm{C}_{\mathrm{Cl}} \mathrm{C}_{\mathrm{Cl}} \mathrm{N}_{\mathrm{C}}$} & 745 & 221 & - & - & -1 & \multirow{4}{*}{1} & \multirow{4}{*}{-} & \multirow{4}{*}{25.8} & \multirow{4}{*}{22.4} \\
\hline & 742 & 114 & - & - & 0 & & & & \\
\hline & 725 & 211 & - & - & 0 & & & & \\
\hline & 722 & 51 & - & - & 1 & & & & \\
\hline \multirow{4}{*}{$\mathrm{C}_{\mathrm{Cl}} \mathrm{N}_{\mathrm{H}} \mathrm{C}_{\mathrm{Cl}} \mathrm{N}_{\mathrm{H}} \mathrm{N}_{\mathrm{H}}$} & 744 & 191 & - & - & 0 & \multirow{4}{*}{7} & \multirow{4}{*}{-} & \multirow{4}{*}{44.4} & \multirow{4}{*}{38.0} \\
\hline & 741 & 149 & - & - & 1 & & & & \\
\hline & 728 & 210 & - & - & -3 & & & & \\
\hline & 724 & 57 & - & - & -1 & & & & \\
\hline \multirow{4}{*}{$\mathrm{C}_{\mathrm{Cl}} \mathrm{N}_{\mathrm{H}} \mathrm{N}_{\mathrm{Cl}} \mathrm{C}_{\mathrm{Cl}} \mathrm{N}_{\mathrm{H}}$} & 744 & 186 & - & - & 0 & \multirow{4}{*}{11} & \multirow{4}{*}{-} & \multirow{4}{*}{43.1} & \multirow{4}{*}{36.9} \\
\hline & 740 & 176 & - & - & 2 & & & & \\
\hline & 728 & 161 & - & - & -3 & & & & \\
\hline & 723 & 84 & - & - & 0 & & & & \\
\hline \multirow{4}{*}{$\mathrm{C}_{\mathrm{Cl}} \mathrm{N}_{\mathrm{H}} \mathrm{C}_{\mathrm{Cl}} \mathrm{N}_{\mathrm{H}} \mathrm{N}_{\mathrm{C}}$} & 743 & 187 & - & - & 1 & \multirow{4}{*}{10} & \multirow{4}{*}{-} & \multirow{4}{*}{42.0} & \multirow{4}{*}{35.9} \\
\hline & 741 & 132 & - & - & 1 & & & & \\
\hline & 727 & 220 & - & - & -2 & & & & \\
\hline & 723 & 47 & - & - & 0 & & & & \\
\hline & 743 & 201 & - & - & 1 & & & & \\
\hline & 740 & 118 & - & - & 2 & 10 & & 419 & 358 \\
\hline $\mathrm{C}_{\mathrm{Cl}} \mathrm{N}_{\mathrm{H}} \mathrm{Cl}_{\mathrm{Cl}} \mathrm{N}_{\mathrm{H}} \mathrm{N}_{\mathrm{C}}$ & 727 & 219 & - & - & -2 & 10 & - & 41.9 & 35.8 \\
\hline & 723 & 46 & - & - & 0 & & & & \\
\hline & 744 & 222 & - & - & 0 & & & & \\
\hline 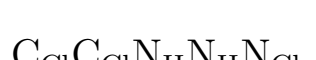 & 742 & 128 & - & - & 0 & 4 & & & \\
\hline$\cup_{\mathrm{Cl}} \cup \mathrm{Cl}^{1} \mathrm{~N}^{1} \mathrm{H}^{1} \mathrm{Cl}$ & 726 & 202 & - & - & -1 & 4 & - & 41.0 & 35.1 \\
\hline & 724 & 62 & - & - & -1 & & & & \\
\hline & 744 & 221 & - & - & 0 & & & & \\
\hline & 742 & 110 & - & - & 0 & 4 & & 406 & 340 \\
\hline $\mathrm{C}_{\mathrm{Cl}} \mathrm{Cl}_{\mathrm{Cl}} \mathrm{N}_{\mathrm{H}} \mathrm{N}_{\mathrm{H}} \mathrm{N}_{\mathrm{C}}$ & 726 & 217 & - & - & -1 & 4 & - & 40.0 & 34.9 \\
\hline & 723 & 44 & - & - & 0 & & & & \\
\hline & 743 & 139 & - & - & 1 & & & & \\
\hline $\mathrm{N}_{a} \mathrm{C}_{\mathrm{C}} \mathrm{N}_{U} \mathrm{C}_{\mathrm{Cu}} \mathrm{N}_{\mathrm{H}}$ & 741 & 197 & - & - & 1 & 8 & & 407 & 248 \\
\hline $\mathrm{N}_{\mathrm{C}} \cup \mathrm{Cl}^{N_{\mathrm{H}}} \cup_{\mathrm{Cl}} \mathrm{N}_{\mathrm{H}}$ & 728 & 199 & - & - & -3 & 8 & - & 40.1 & 34.8 \\
\hline & 723 & 54 & - & - & 0 & & & & \\
\hline & 744 & 177 & - & - & 0 & & & & \\
\hline $\mathrm{N}_{\mathrm{C}} \mathrm{C}_{\mathrm{Cl}} \mathrm{C}_{\mathrm{Cl}} \mathrm{N}_{\mathrm{H}} \mathrm{N}_{\mathrm{H}}$ & 742 & 169 & - & - & 0 & 6 & - & 40.4 & 34.8 \\
\hline
\end{tabular}


A. Appendix

\begin{tabular}{|c|c|c|c|c|c|c|c|c|c|}
\hline Structure & $\omega_{\mathrm{CCl}}$ & $S_{\omega}$ & $\tilde{\nu}_{\mathrm{CCl}}$ & $S_{\nu}$ & $\Delta \omega_{\mathrm{CCl}}$ & $\omega_{l}$ & $\tilde{\nu}_{1}$ & $D_{\mathrm{e}}$ & $D_{0}$ \\
\hline & 727 & 176 & - & - & -2 & & & & \\
\hline & 723 & 73 & - & - & 0 & & & & \\
\hline \multirow{4}{*}{$\mathrm{N}_{\mathrm{C}} \mathrm{C}_{\mathrm{Cl}} \mathrm{N}_{\mathrm{H}} \mathrm{C}_{\mathrm{Cl}} \mathrm{N}_{\mathrm{H}}$} & 742 & 94 & - & - & 2 & \multirow{4}{*}{8} & \multirow{4}{*}{-} & \multirow{4}{*}{40.7} & \multirow{4}{*}{34.7} \\
\hline & 740 & 241 & - & - & 2 & & & & \\
\hline & 730 & 205 & - & - & -5 & & & & \\
\hline & 720 & 39 & - & - & -7 & & & & \\
\hline \multirow{4}{*}{$\mathrm{N}_{\mathrm{Cl}} \mathrm{C}_{\mathrm{Cl}} \mathrm{N}_{\mathrm{H}} \mathrm{C}_{\mathrm{Cl}} \mathrm{N}_{\mathrm{C}}$} & 743 & 177 & - & - & 1 & \multirow{4}{*}{8} & \multirow{4}{*}{-} & \multirow{4}{*}{39.6} & \multirow{4}{*}{33.9} \\
\hline & 740 & 163 & - & - & 2 & & & & \\
\hline & 727 & 182 & - & - & -2 & & & & \\
\hline & 722 & 64 & - & - & 1 & & & & \\
\hline \multirow{4}{*}{$\mathrm{N}_{\mathrm{C}} \mathrm{C}_{\mathrm{Cl}} \mathrm{N}_{\mathrm{H}} \mathrm{C}_{\mathrm{Cl}} \mathrm{N}_{\mathrm{C}}$} & 743 & 174 & - & - & 1 & \multirow{4}{*}{7} & \multirow{4}{*}{-} & \multirow{4}{*}{38.2} & \multirow{4}{*}{32.8} \\
\hline & 741 & 148 & - & - & 1 & & & & \\
\hline & 726 & 202 & - & - & -1 & & & & \\
\hline & 722 & 45 & - & - & 1 & & & & \\
\hline \multirow{4}{*}{$\mathrm{N}_{\mathrm{C}} \mathrm{C}_{\mathrm{Cl}} \mathrm{C}_{\mathrm{Cl}} \mathrm{N}_{\mathrm{H}} \mathrm{N}_{\mathrm{C}}$} & 743 & 168 & - & - & 1 & \multirow{4}{*}{7} & \multirow{4}{*}{-} & \multirow{4}{*}{36.7} & \multirow{4}{*}{31.5} \\
\hline & 742 & 158 & - & - & 0 & & & & \\
\hline & 726 & 190 & - & - & -1 & & & & \\
\hline & 722 & 57 & - & - & -1 & & & & \\
\hline
\end{tabular}

\section{A.7. Cavity-enhanced Raman Spectroscopy}

Table A.28.: Measured transmission $(T)$ of several optical components used for cavityenhancement.

Wavelength

$635 \mathrm{~nm} \quad 532 \mathrm{~nm}$

\begin{tabular}{|c|c|c|}
\hline Optical component & \multicolumn{2}{|c|}{$T / \%$} \\
\hline Wedged window & \multicolumn{2}{|c|}{87} \\
\hline Front cavity mirror (SM) & $6 \times 10^{-3}$ & $7 \times 10^{-4}$ \\
\hline Rear cavity mirror (PSM) & $7 \times 10^{-3}$ & $8 \times 10^{-4}$ \\
\hline
\end{tabular}




\section{A. Appendix}

Table A.29.: Conversion factors from optical power to photovoltages supplied by a Si photodiode (Thorlabs, PDA36A) for different wavelengths and internal amplification settings monitored with a $1 \mathrm{M} \Omega$ input impedance oscilloscope.

\begin{tabular}{cccc}
\hline & \multicolumn{3}{c}{ Conversion factor $/ \mathrm{V} \mathrm{mW}^{-1}$} \\
Wavelength & $635 \mathrm{~nm}$ & $532 \mathrm{~nm}$ & $405 \mathrm{~nm}$ \\
\hline $0 \mathrm{~dB}$ & $2.45(1) \times 10^{-1}$ & $3.02(4) \times 10^{-1}$ & $9.1(5) \times 10^{-2}$ \\
$10 \mathrm{~dB}$ & $7.52(2) \times 10^{-1}$ & $9.5(2) \times 10^{-1}$ & $2.70(5) \times 10^{-1}$ \\
$20 \mathrm{~dB}$ & $1.436(8)$ & $3.02(4)$ & $8.9(1) \times 10^{-1}$ \\
$30 \mathrm{~dB}$ & $4.78(3)$ & $9.6(1)$ & $2.82(2)$ \\
$40 \mathrm{~dB}$ & $1.50(2) \times 10^{1}$ & $3.04(4) \times 10^{1}$ & $8.98(8)$ \\
$50 \mathrm{~dB}$ & $1.32(1) \times 10^{2}$ & $9.6(2) \times 10^{1}$ & $2.83(3) \times 10^{1}$ \\
$60 \mathrm{~dB}$ & $4.20(2) \times 10^{2}$ & $2.93(3) \times 10^{2}$ & $8.64(7) \times 10^{1}$ \\
$70 \mathrm{~dB}$ & $1.25(1) \times 10^{3}$ & $8.7(2) \times 10^{2}$ & $2.64(2) \times 10^{2}$ \\
\hline
\end{tabular}

\section{A.8. Conclusions and Outlook}




\section{A. Appendix}

Table A.30.: 23 experimental observables obtained as quantities employed for quantum chemistry benchmarking over the course of this work and their respective error bounds.

\begin{tabular}{|c|c|c|c|c|}
\hline Observable & Molecular structure & Unit & Value & Error \\
\hline Harmonic $\mathrm{OH}$ stretching band centre & Formic acid monomer & $\mathrm{cm}^{-1}$ & 3764 & 14 \\
\hline $\begin{array}{l}\text { Harmonic downshift of the } \mathrm{OH} \\
\text { stretching band centre }\end{array}$ & Formic acid nitrogen dimer & $\mathrm{cm}^{-1}$ & 17 & 13 \\
\hline Harmonic $\mathrm{OH}$ stretching band centre & Acetic acid monomer & $\mathrm{cm}^{-1}$ & 3765 & 32 \\
\hline $\begin{array}{l}\text { Harmonic downshift of the } \mathrm{OH} \\
\text { stretching band centre }\end{array}$ & Acetic acid nitrogen dimer & $\mathrm{cm}^{-1}$ & 13 & 13 \\
\hline $\begin{array}{l}\text { Equilibrium rotational } A \text { con- } \\
\text { stant } 272\end{array}$ & $\begin{array}{l}\text { trans-monomer of } 1,1,1,3,3,3 \text {-hexafluoro-2- } \\
\text { propanol }\end{array}$ & $\mathrm{MHz}$ & 2124 & 15 \\
\hline $\begin{array}{l}\begin{array}{l}\text { Equilibrium rotational } \\
\text { stant }\end{array}{ }^{272} \\
\end{array}$ & $\begin{array}{l}\text { trans-monomer of } 1,1,1,3,3,3 \text {-hexafluoro-2- } \\
\text { propanol }\end{array}$ & $\mathrm{MHz}$ & 1063 & 8 \\
\hline $\begin{array}{l}\text { Equilibrium rotational } C \quad \text { con- } \\
\text { stant }^{272}\end{array}$ & $\begin{array}{l}\text { trans-monomer of } 1,1,1,3,3,3 \text {-hexafluoro- } 2 \text { - } \\
\text { propanol }\end{array}$ & $\mathrm{MHz}$ & 941 & 7 \\
\hline Equilibrium rotational $A$ constant & $\begin{array}{l}\text { Binary nitrogen complex of the trans-monomer } \\
\text { of 1,1,1,3,3,3-hexafluoro-2-propanol }\end{array}$ & $\mathrm{MHz}$ & 977 & 9 \\
\hline Equilibrium rotational $B$ constant & $\begin{array}{l}\text { Binary nitrogen complex of the trans-monomer } \\
\text { of 1,1,1,3,3,3-hexafluoro-2-propanol }\end{array}$ & $\mathrm{MHz}$ & 741 & 7 \\
\hline Equilibrium rotational $C$ constant & $\begin{array}{l}\text { Binary nitrogen complex of the trans-monomer } \\
\text { of } 1,1,1,3,3,3 \text {-hexafluoro-2-propanol }\end{array}$ & $\mathrm{MHz}$ & 538 & 5 \\
\hline Equilibrium rotational $A$ constant & $\begin{array}{l}\text { Most stable dimer of two trans-monomers of } \\
1,1,1,3,3,3 \text {-hexafluoro-2-propanol }\end{array}$ & $\mathrm{MHz}$ & 489 & 5 \\
\hline Equilibrium rotational $B$ constant & $\begin{array}{l}\text { Most stable dimer of two trans-monomers of } \\
1,1,1,3,3,3 \text {-hexafluoro-2-propanol }\end{array}$ & $\mathrm{MHz}$ & 209 & 2 \\
\hline Equilibrium rotational $C$ constant & $\begin{array}{l}\text { Most stable dimer of two trans-monomers of } \\
1,1,1,3,3,3 \text {-hexafluoro-2-propanol }\end{array}$ & $\mathrm{MHz}$ & 172 & 2 \\
\hline Equilibrium rotational $A$ constant & $\begin{array}{l}\text { Metastable dimer of one trans- and one gauche- } \\
\text { monomer of 1,1,1,3,3,3-hexafluoro-2-propanol }\end{array}$ & $\mathrm{MHz}$ & 587 & 6 \\
\hline Equilibrium rotational $B$ constant & $\begin{array}{l}\text { Metastable dimer of one trans- and one gauche- } \\
\text { monomer of 1,1,1,3,3,3-hexafluoro-2-propanol }\end{array}$ & $\mathrm{MHz}$ & 156 & 2 \\
\hline Equilibrium rotational $C$ constant & $\begin{array}{l}\text { Metastable dimer of one trans- and one gauche- } \\
\text { monomer of 1,1,1,3,3,3-hexafluoro-2-propanol }\end{array}$ & $\mathrm{MHz}$ & 154 & 2 \\
\hline Harmonic $\mathrm{OH}$ stretching band centre & $\begin{array}{l}\text { trans-monomer of } 1,1,1,3,3,3 \text {-hexafluoro-2- } \\
\text { propanol }\end{array}$ & $\mathrm{cm}^{-1}$ & 3791 & 17 \\
\hline Harmonic $\mathrm{OH}$ stretching band centre & $\begin{array}{l}\text { gauche-monomer of } 1,1,1,3,3,3 \text {-hexafluoro-2- } \\
\text { propanol }\end{array}$ & $\mathrm{cm}^{-1}$ & 3836 & 17 \\
\hline $\begin{array}{l}\text { Harmonic OH stretching wavenum- } \\
\text { ber splitting }\end{array}$ & $\begin{array}{l}\text { trans- and gauche monomer of } 1,1,1,3,3,3- \\
\text { hexafluoro-2-propanol }\end{array}$ & $\mathrm{cm}^{-1}$ & 45 & 6 \\
\hline $\begin{array}{l}\text { Harmonic downshift of the } \mathrm{OH} \\
\text { stretching band centre }\end{array}$ & $\begin{array}{l}\text { trans-monomer of } 1,1,1,3,3,3 \text {-hexafluoro-2- } \\
\text { propanol with nitrogen }\end{array}$ & $\mathrm{cm}^{-1}$ & 18 & 9 \\
\hline $\begin{array}{l}\text { Harmonic spectral wavenumber } \\
\text { splitting of the hydrogen bond } \\
\text { acceptor OH stretching vibration }\end{array}$ & $\begin{array}{l}\text { Two most stable isomers of nitrogen complexated } \\
\text { dimers of 1,1,1,3,3,3-hexafluoro-2-propanol }\end{array}$ & $\mathrm{cm}^{-1}$ & 20 & 10 \\
\hline $\begin{array}{l}\text { Harmonic spectral wavenumber } \\
\text { splitting of the hydrogen bond } \\
\text { donor OH stretching vibration }\end{array}$ & $\begin{array}{l}\text { Two most stable isomers of nitrogen complexated } \\
\text { dimers of 1,1,1,3,3,3-hexafluoro-2-propanol }\end{array}$ & $\mathrm{cm}^{-1}$ & 10 & 5 \\
\hline $\begin{array}{l}\text { Harmonically zero-point-corrected } \\
\text { conformational energy difference }\end{array}$ & $\begin{array}{l}\text { Two most stable isomers of nitrogen complexated } \\
\text { dimers of 1,1,1,3,3,3-hexafluoro-2-propanol }\end{array}$ & $\mathrm{kJ} \mathrm{mol}^{-1}$ & 0.3 & 0.8 \\
\hline
\end{tabular}




\section{A. Appendix}

\section{Curriculum Vitae}

\author{
Sönke Oswald \\ Nationality
}

2009

2010-2013

2013-2015

$09 / 2014-12 / 2014$

2015-2019

$07 / 2016-07 / 2018$

02/2018-04/2018

\section{$\mathrm{D}$}

* 28th March 1990, Henstedt-Ulzburg, Germany
German

Diploma from secondary school qualifying for university admission or matriculation (Abitur), Coppernicus-Gymnasium Norderstedt

Bachelor of Science in chemistry at the Georg-August-University Göttingen, bachelor thesis: 'Aromatische und aliphatische Sechsringe in Wasserstoffbrückenkonkurrenz'

Master of Science in chemistry at the Georg-August-University Göttingen, master thesis: 'Acetone trace gas detection and aggregation studies by linear IR spectroscopy and cavity-enhanced Raman scattering'

05/2018-06/2018 Guest stay at Aix Marseille Universite, Marseille, France with Dr. Stéphane Coussan

\section{Publications}

[1] Altnöder, J., Oswald, S., Suhm, M. A., Phenyl- vs CyclohexylSubstitution in Methanol: Implications for the $\mathrm{OH}$ Conformation and for Dispersion-Affected Aggregation from Vibrational Spectra in Supersonic Jets, J. Phys. Chem. A, 2014, 118, 3266-3279. 
[2] Oswald, S., Wallrabe, M., Suhm, M. A., Cooperativity in AlcoholNitrogen Complexes: Understanding Cryomatrices through Slit Jet Expansions, J. Phys. Chem. A, 2017, 121, 3411-3422.

[3] Oswald, S. Suhm, M. A., Experimental Reference Data for Hexafluorinated Propanol by Exploring an Unusual Intermolecular Torsional Balance, Angew. Chem. Int. Ed., 2017, 56, 12672-12676.

[4] Oswald, S., Meyer, E., Suhm, M. A., Dinitrogen as a Sensor for Metastable Carboxylic Acid Dimers and a Weak Hydrogen Bond Benchmarking Tool, J. Phys. Chem. A, 2018, 122, 2933-2946.

[5] Oswald, S., Suhm, M. A., Coussan, S., Incremental NH Stretching Downshift through Stepwise Nitrogen Complexation of Pyrrole: A Combined Jet Expansion and Matrix Isolation Study, Phys. Chem. Chem. Phys., 2019, 21, 1277-1284.

[6] Oswald, S., Seifert, N. A., Bohle, F., Gawrilow, M., Grimme, S., Jäger, W., Xu, Y., Suhm, M. A., The Chiral Trimer and a Metastable Chiral Dimer of Achiral Hexafluoroisopropanol: A Multi Messenger Study, Angew. Chem. Int. Ed., 2019, accepted, DOI:10.1002/anie.201813881.

[7] Oswald, S., Coussan, S., Chloroform-Nitrogen Aggregates: Upshifted $\mathrm{CH}$ and Downshifted $\mathrm{CCl}$ Stretching Vibrations Observed by Matrix Isolation and Jet Expansion Infrared Spectroscopy, Low. Temp. Phys., 2019, accepted. 